Supporting Information

\title{
Fluorous-Tag Assisted Syntheses of Sulfated Keratan Sulfate Oligosaccharide Fragments
}

Sayantan Bhaduri and Nicola L. B. Pohl* 


\section{Table of Contents}

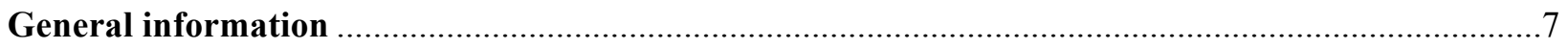

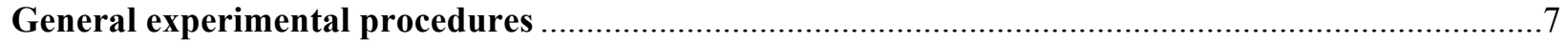

General procedure for FSPE (Fluorous solid phase extraction) ….....................................................

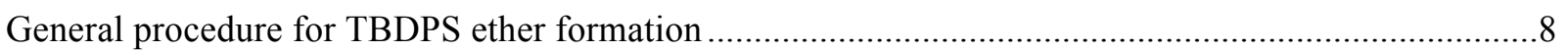

General procedure for 6-OH protection with Levulinate ester (Lev) …........................................... 8

General procedure for OMP ether deprotection using ceric ammonium nitrate (CAN) .........................

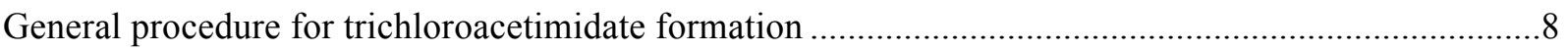

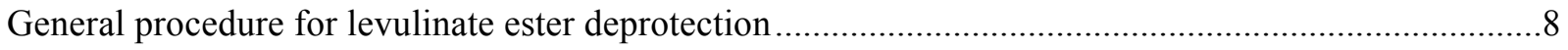

General procedure for conversion of $\mathrm{N}$-trichloroacetamide (NHTCA) to $\mathrm{N}$-acetyl (NHAc) ...................9

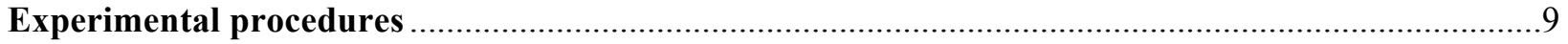

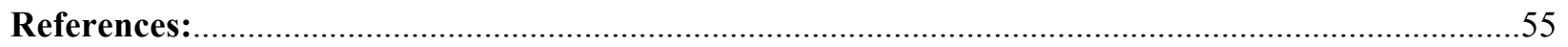

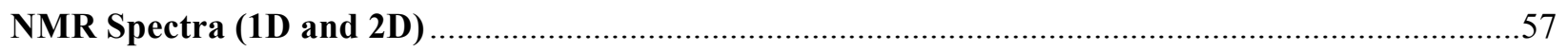

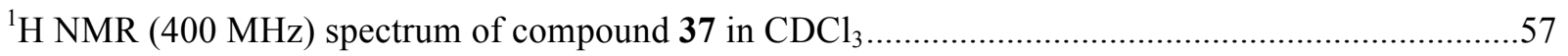

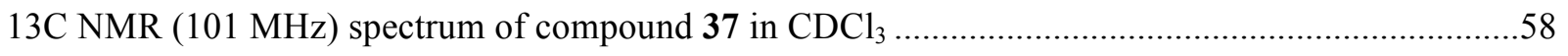

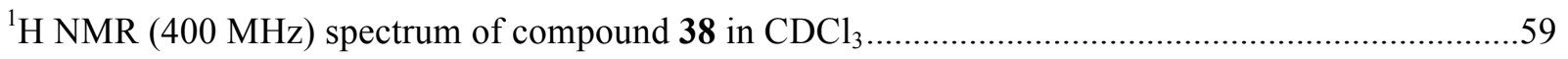

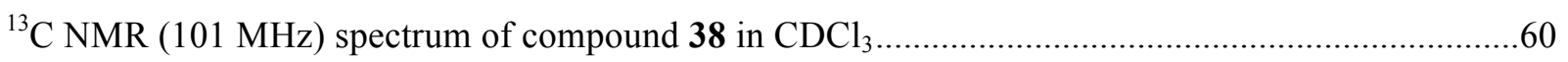

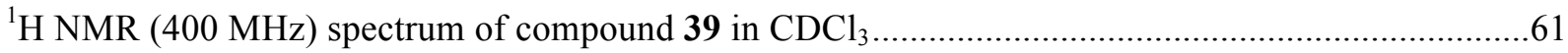

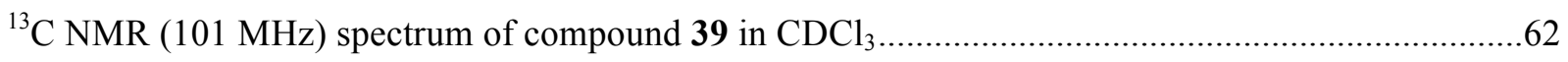

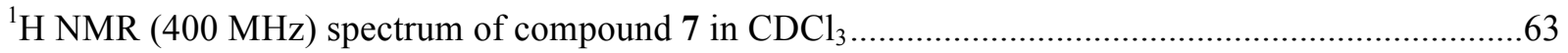

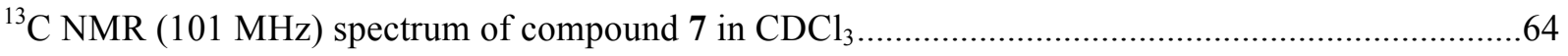

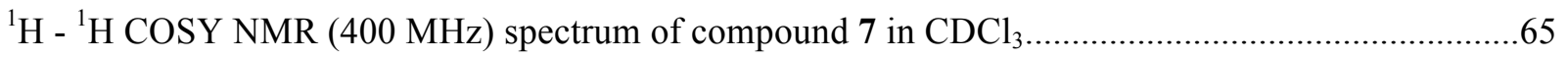

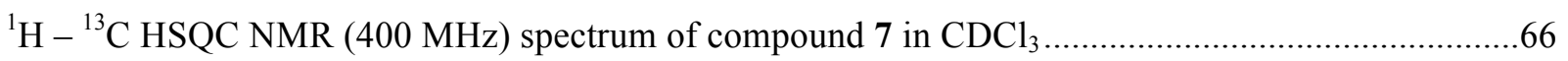

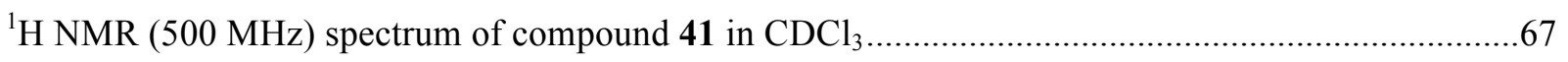

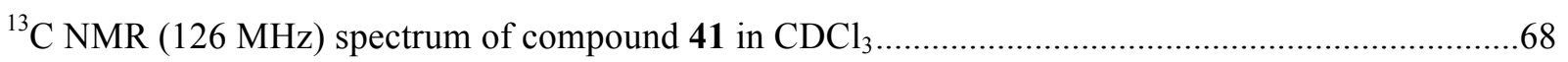

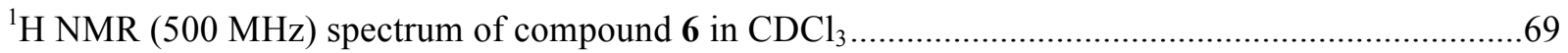

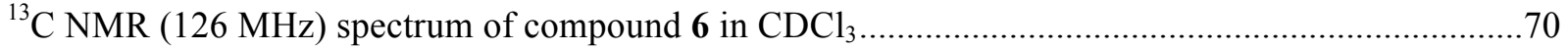

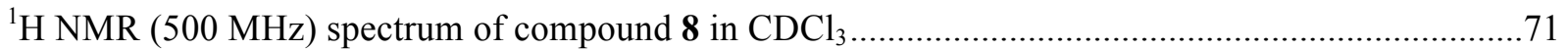

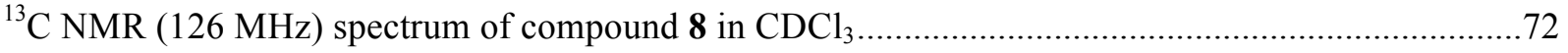

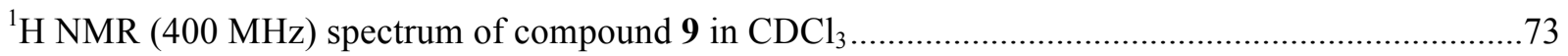

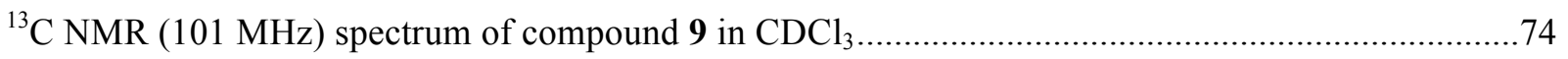




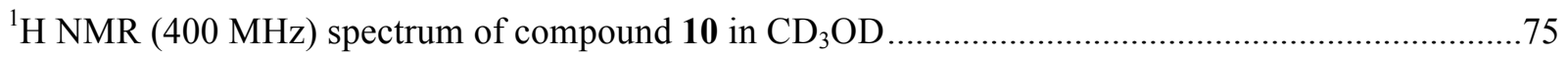

${ }^{1} \mathrm{H}$ NMR (500 MHz) spectrum of compound 35 in $\mathrm{CD}_{3} \mathrm{OD}$................................................................76

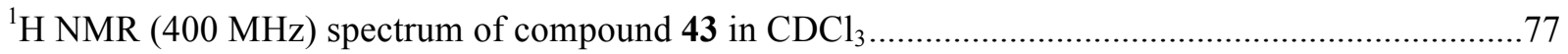

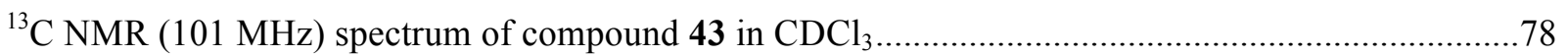

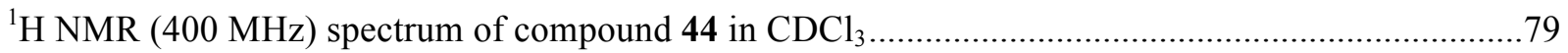

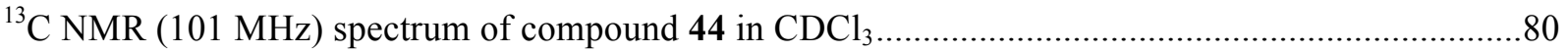

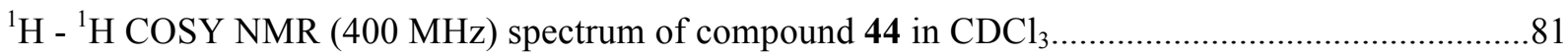

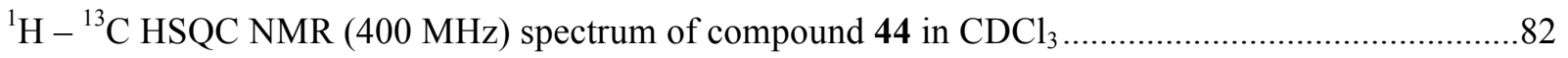

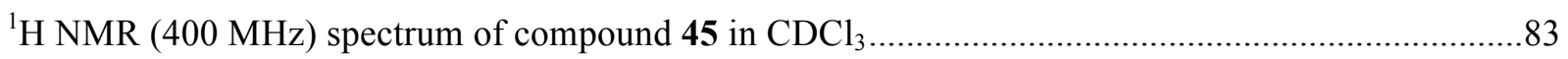

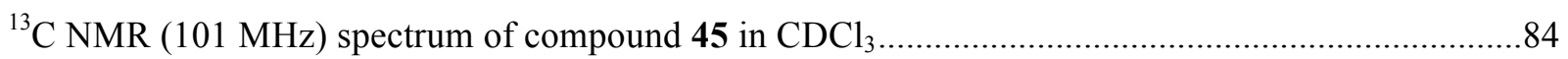

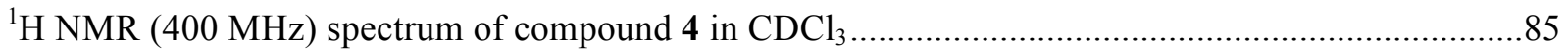

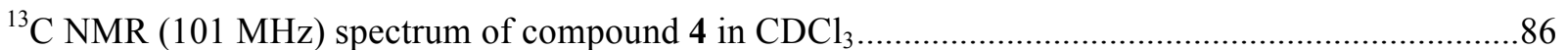

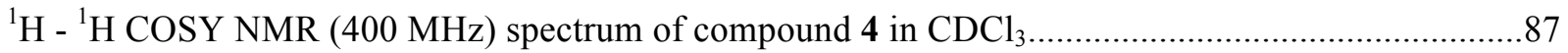

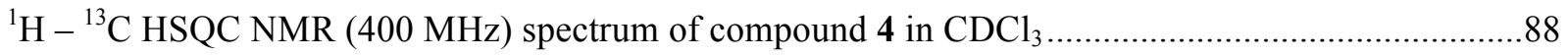

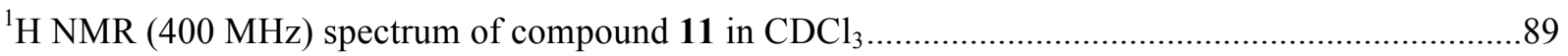

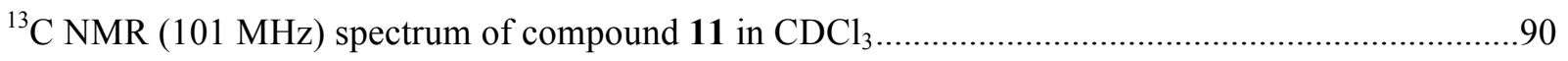

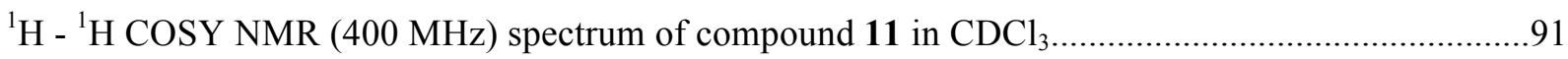

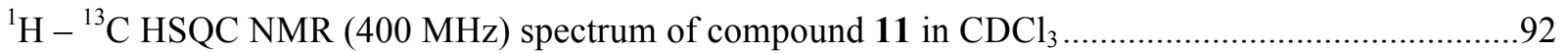

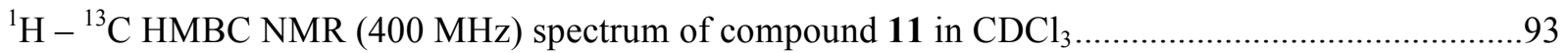

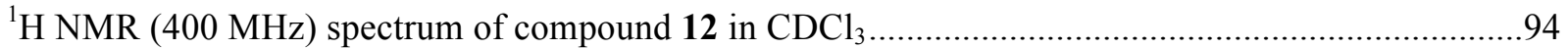

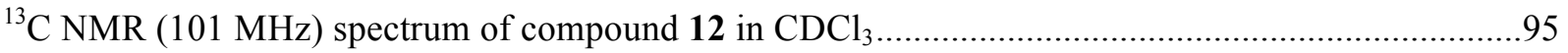

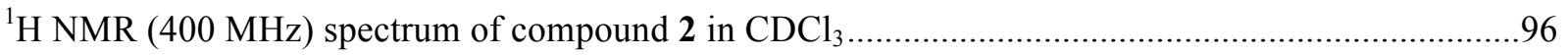

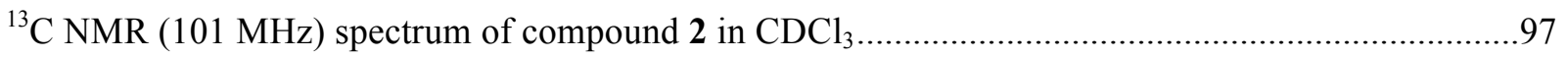

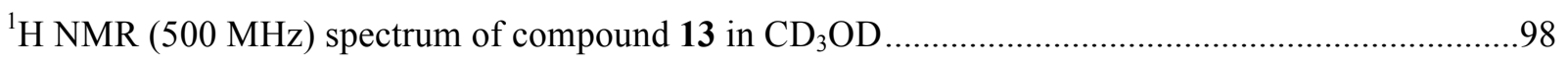

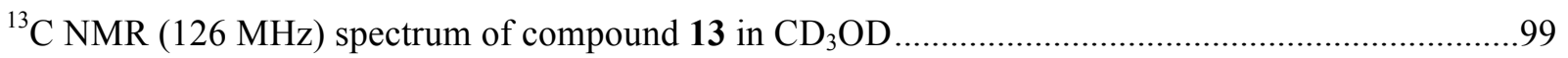

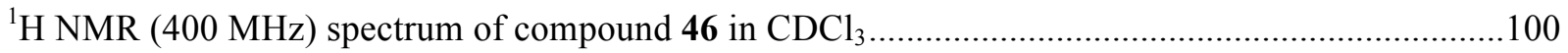

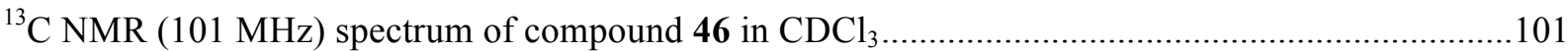

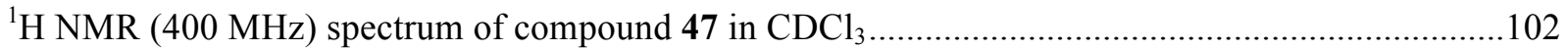

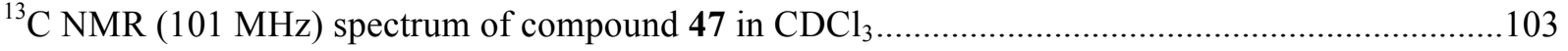

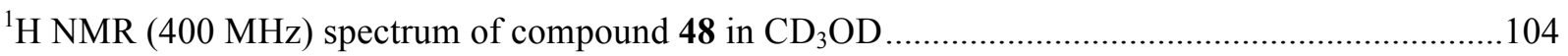

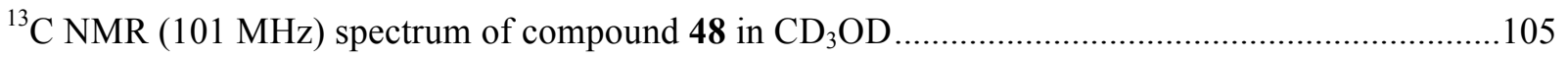

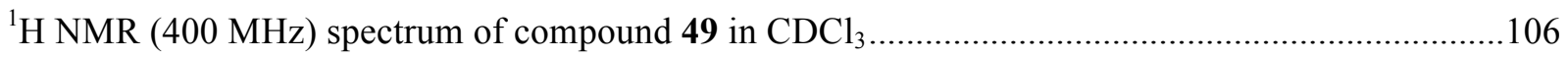

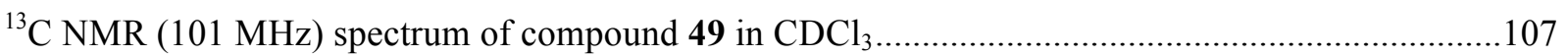




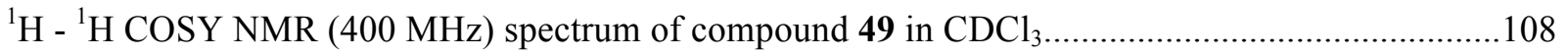

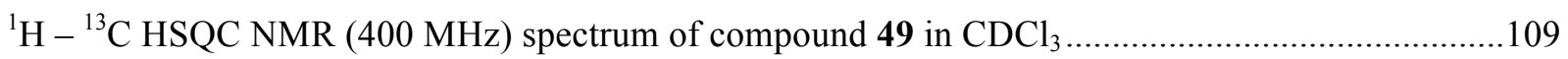

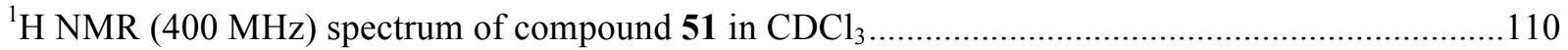

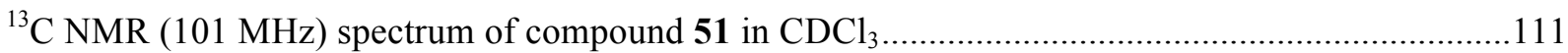

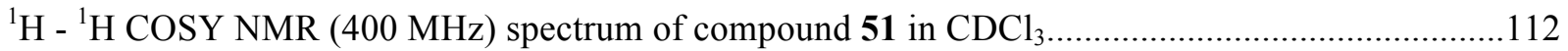

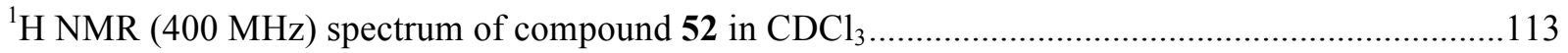

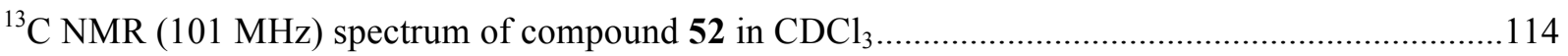

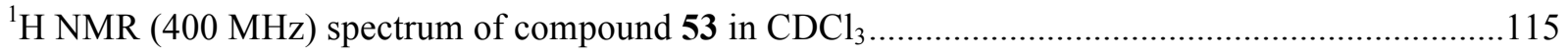

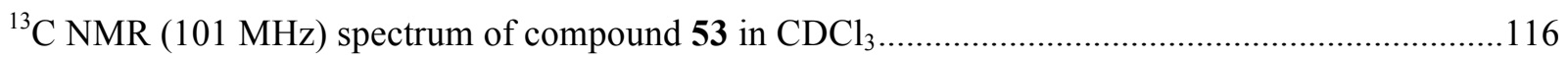

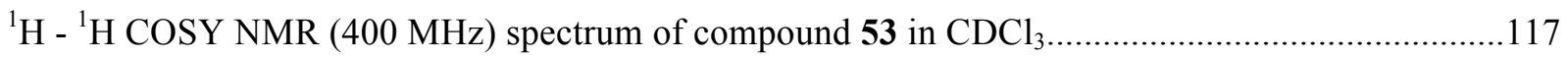

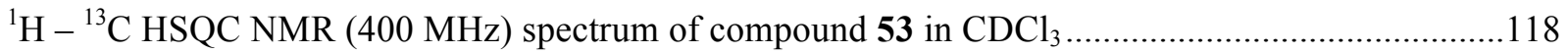

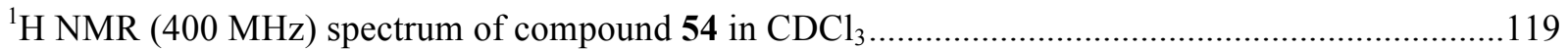

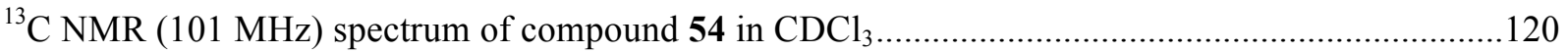

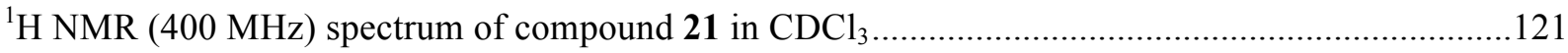

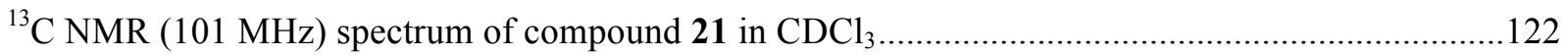

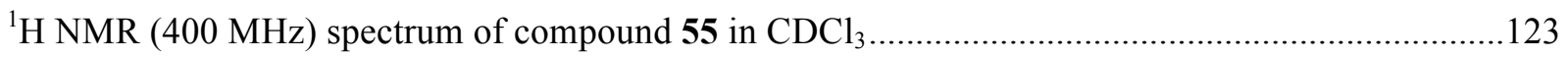

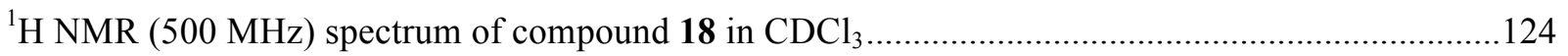

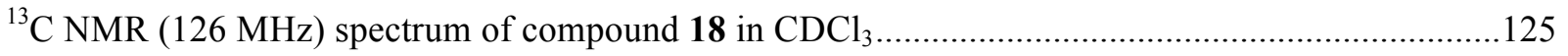

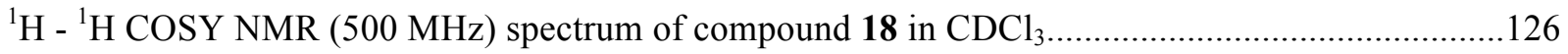

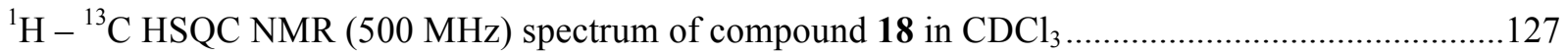

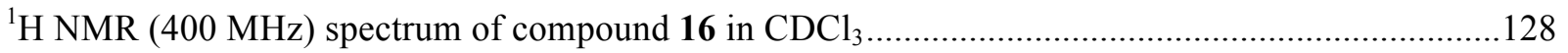

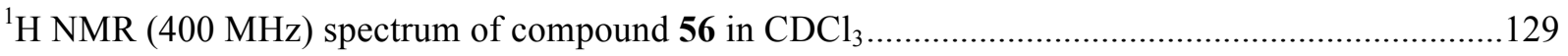

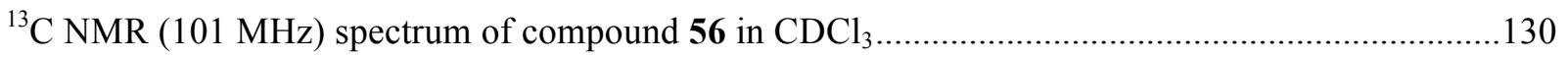

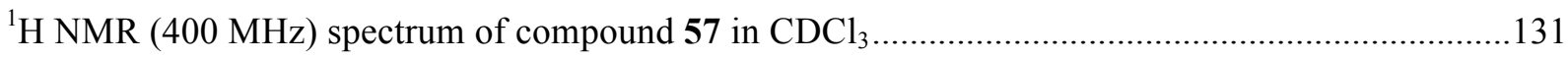

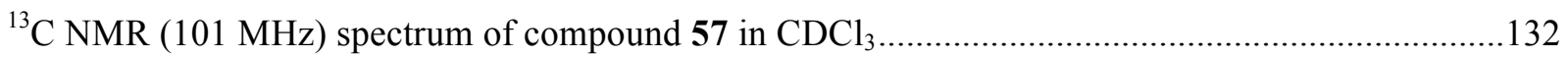

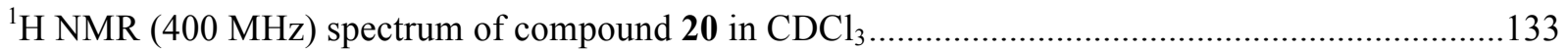

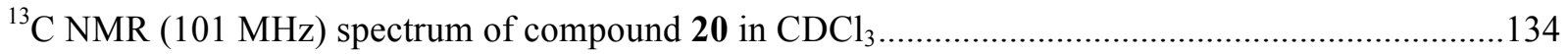

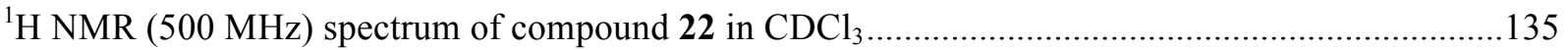

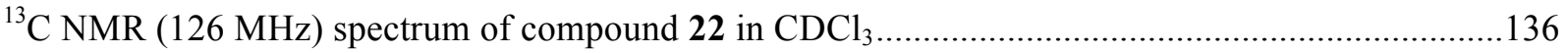

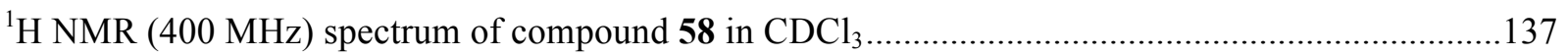

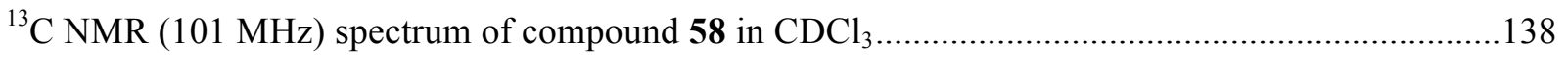

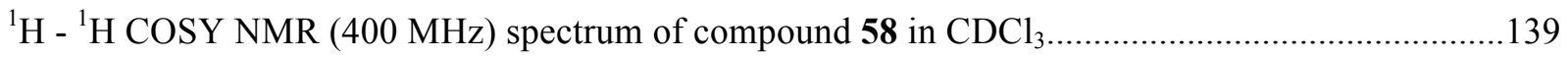

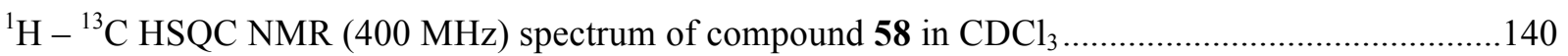




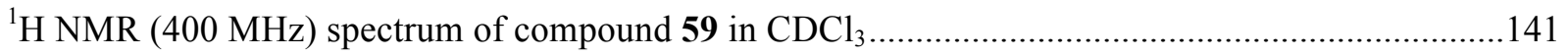

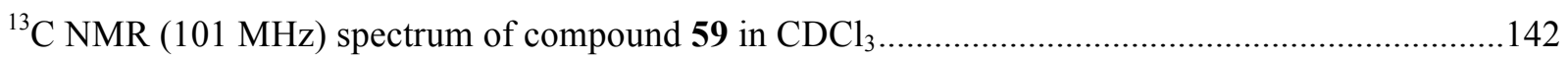

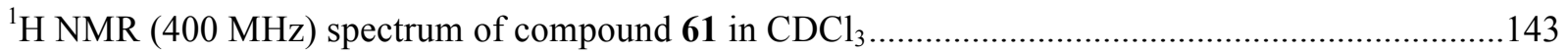

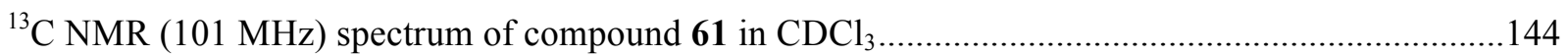

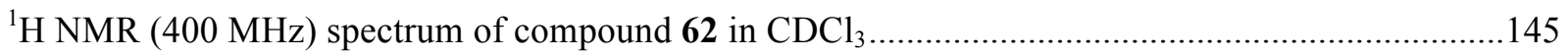

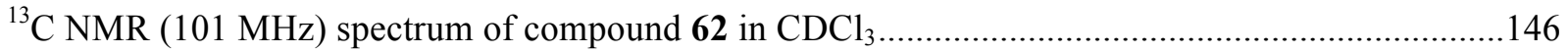

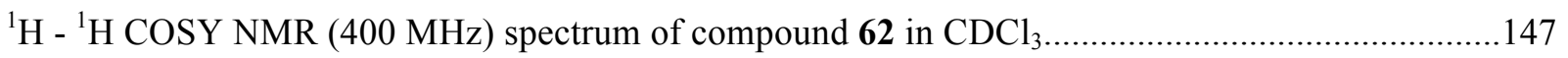

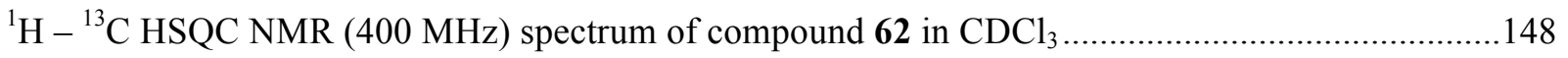

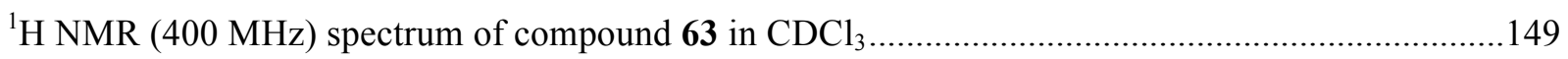

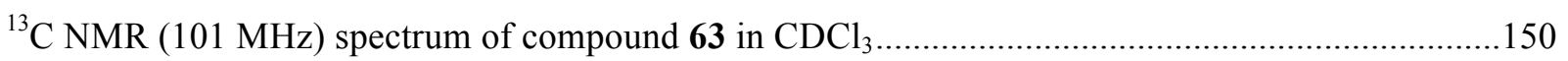

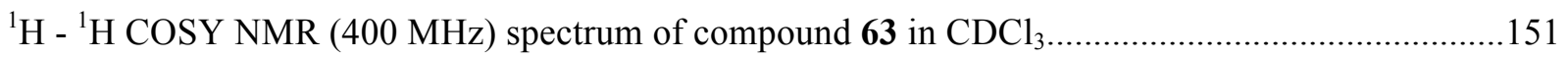

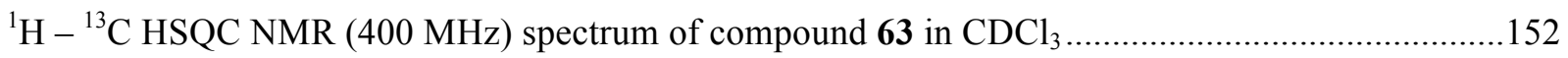

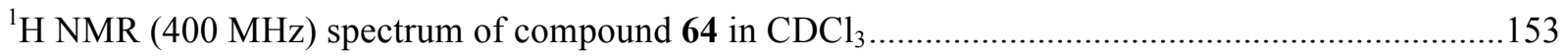

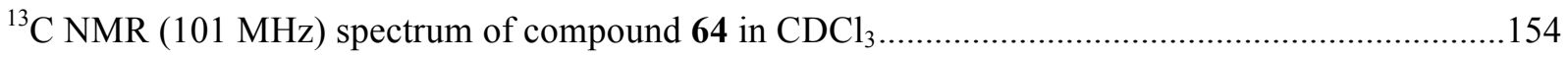

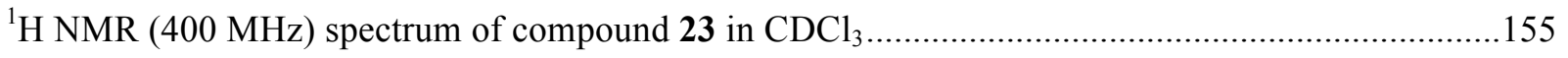

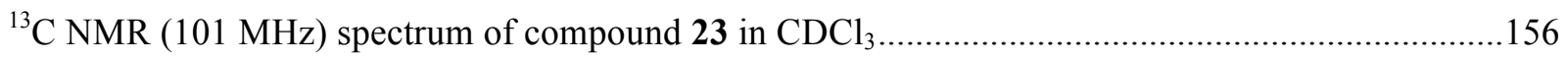

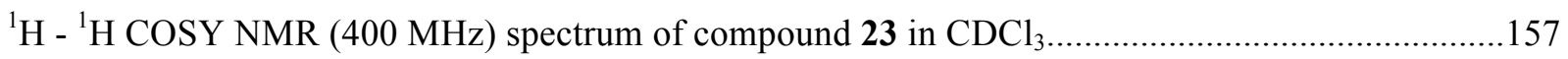

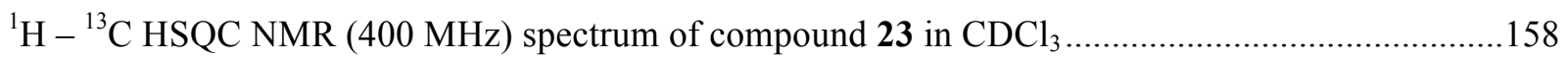

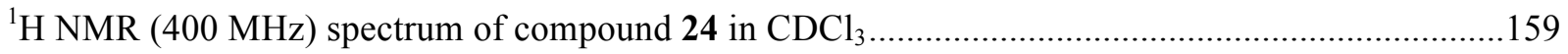

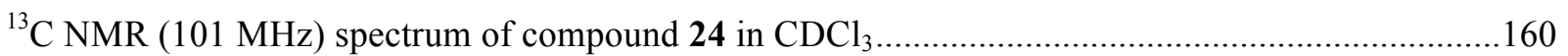

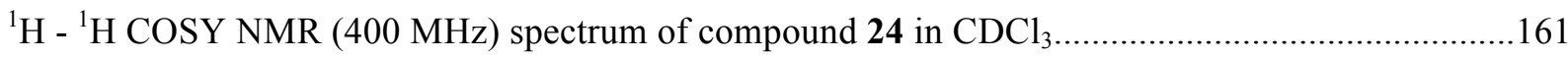

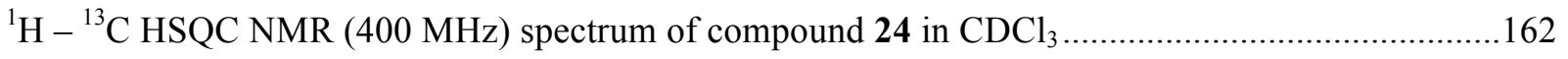

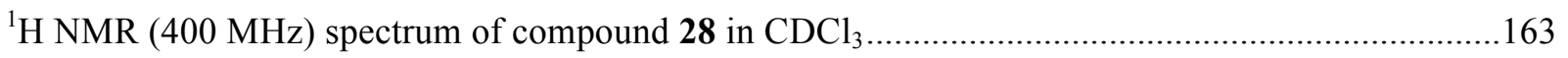

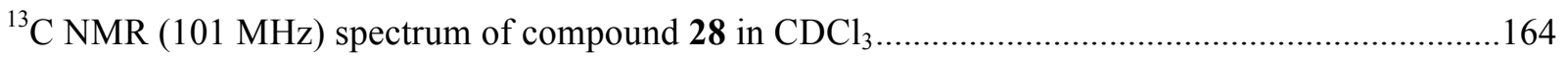

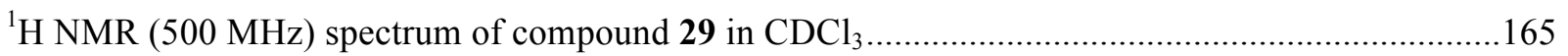

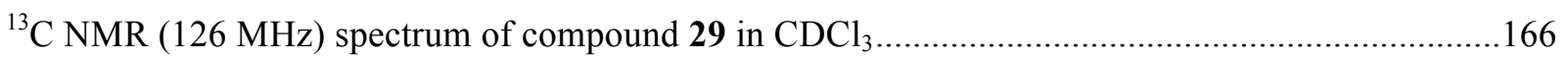

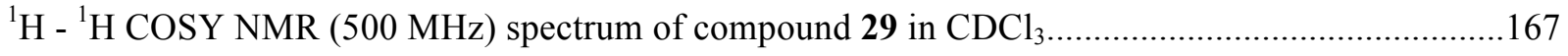

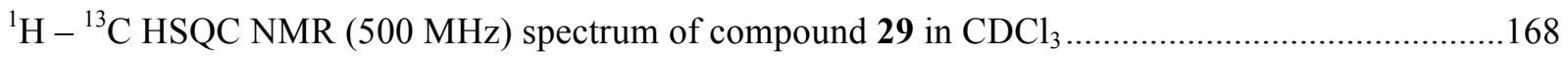

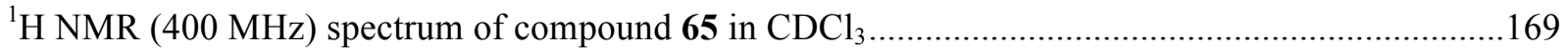

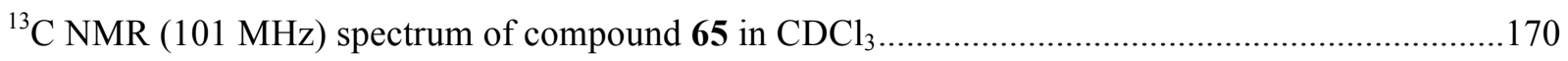

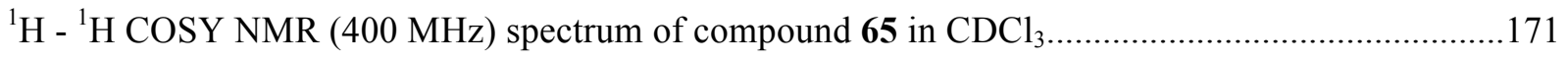

${ }^{1} \mathrm{H}-{ }^{13} \mathrm{C}$ HSQC NMR (400 MHz) spectrum (expanded) of compound $\mathbf{6 5}$ in $\mathrm{CDCl}_{3} \ldots \ldots \ldots \ldots \ldots \ldots \ldots \ldots . . . . . . . . .172$

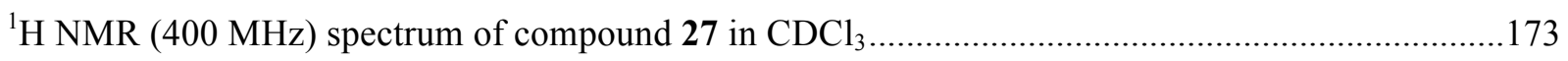




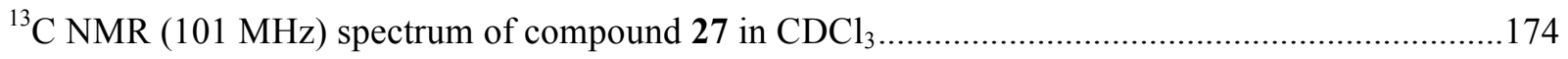

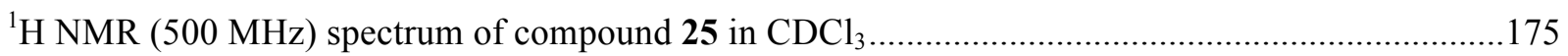

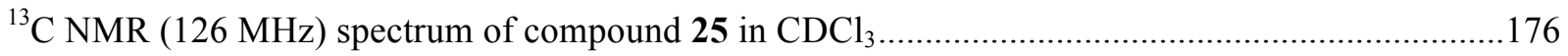

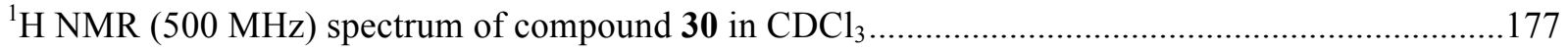

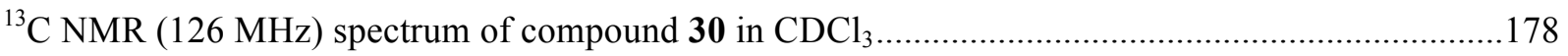

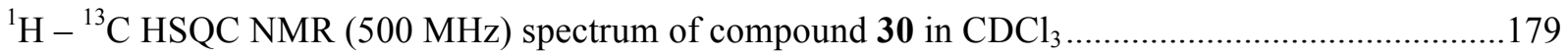

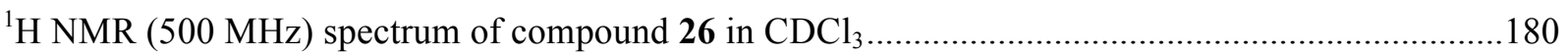

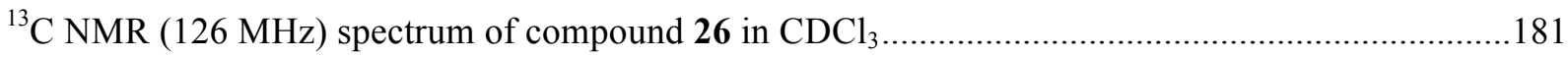

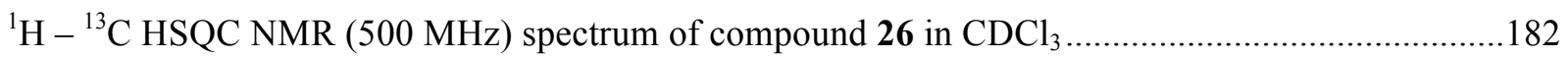

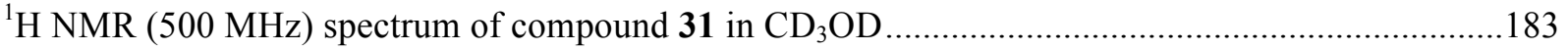

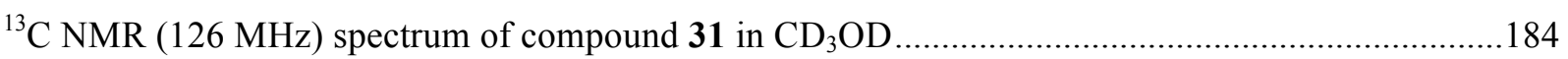

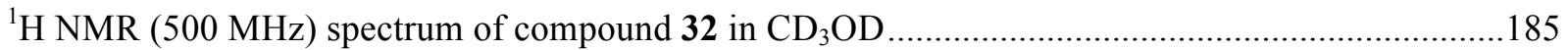

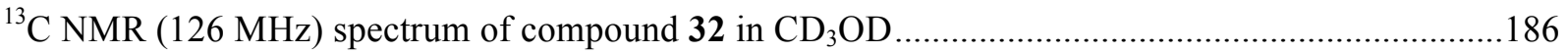

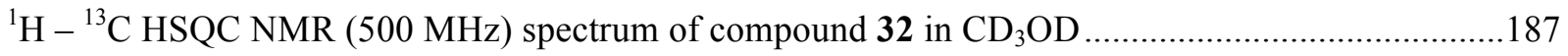

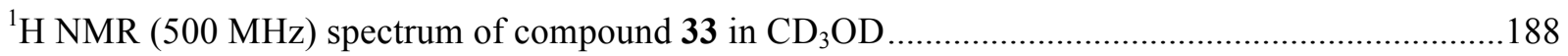

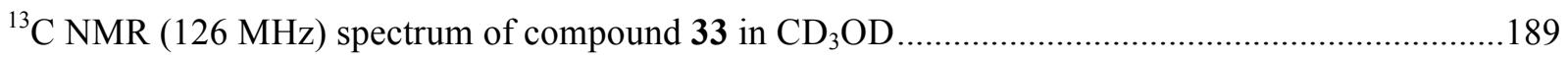

High-resolution Mass Spectrometry Data (HRMS).....................................................................190

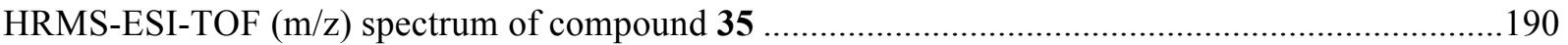

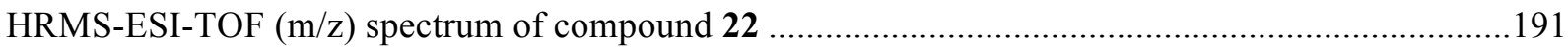

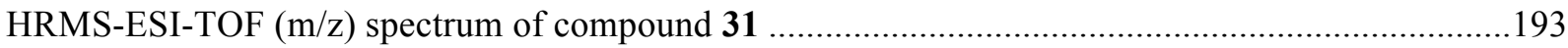

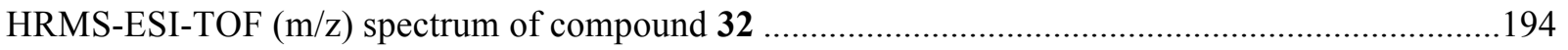

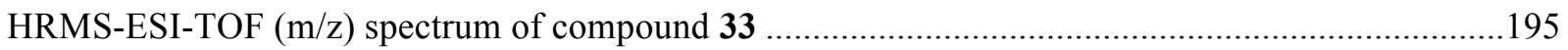

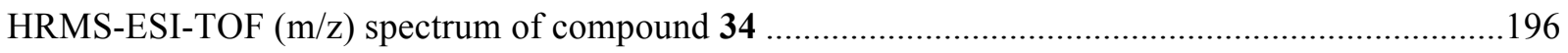




\section{General information}

All the reactions were performed using oven-dried glassware under argon atmosphere using anhydrous solvents unless otherwise noted. Anhydrous solvents [tetrahydrofuran (THF), dichloromethane (DCM) and toluene] were used from a solvent purification system purchased from Innovative Technology Inc. (Newburyport, MA). Methanol (MeOH), acetonitrile and $N, N$ Dimethylformamide (DMF) were obtained anhydrous by degassing with argon. Dowex50WX8 hydrogen form resin (Sigma Aldrich) was used for neutralizing de-esterification reactions. The hydrogenation reaction was operated in the H-Cube Pro $^{\mathrm{TM}}$ reactor (ThalesNano). Non-aqueous reagents were transferred using syringe under argon atmosphere. All other commercial solvents and reagents (obtained from Aldrich, Fisher or TCI) were reagent grade and used as received without further purification.

The reactions were monitored by thin layer chromatography (TLC) with $250 \mu \mathrm{m}$ Sorbent Technologies silica gel HL TLC plates. Developed TLC plates were visualized under UV light and by using a stain (ethanol: $p$-anisaldehyde: conc. $\mathrm{H}_{2} \mathrm{SO}_{4}=18: 1: 1$ ) followed by heating on a hot plate. Silica gel flash column chromatography was performed with Zeochem ZEOprep 60 silica gel, 40-63 $\mu \mathrm{m}$ particle sizes. Fluorous silica was obtained from SiliCycle Inc.

${ }^{1} \mathrm{H}$ and ${ }^{13} \mathrm{C}$ NMR spectra were recorded on a Varian VXR $400(400 \mathrm{MHz} / 101 \mathrm{MHz})$, Varian INOVA $400(400 \mathrm{MHz} / 101 \mathrm{MHz})$ or Varian $500(500 \mathrm{MHz} / 126 \mathrm{MHz})$ instruments. NMR coupling constants and signal patterns are reported as $\mathrm{J}$ values in $\mathrm{Hz}$ and $\delta$ values in parts per million (ppm). ${ }^{1} \mathrm{H}$ and ${ }^{13} \mathrm{C}$ spectra recorded in $\mathrm{CDCl}_{3}$ were referenced at $7.26 \mathrm{ppm}\left({ }^{1} \mathrm{H}\right)$ and 77.23 ppm $\left({ }^{13} \mathrm{C}\right)$ as solvent peak. ${ }^{1} \mathrm{H}$ and ${ }^{13} \mathrm{C}$ spectra recorded in $\mathrm{CD}_{3} \mathrm{OD}$ were referenced at 3.31 ppm $\left({ }^{1} \mathrm{H}\right)$ and $49 \mathrm{ppm}\left({ }^{13} \mathrm{C}\right)$ as solvent peak. The following abbreviations are used for the multiplicities: $\mathrm{s}=$ singlet, $\mathrm{d}=$ doublet, $\mathrm{dd}=$ double of doublet, $\mathrm{t}=$ triplet, $\mathrm{q}=$ quartet, $\mathrm{m}=$ multiplet, bs $=$ broad singlet. ${ }^{1} \mathrm{H}$ NMR peaks were assigned primarily from $2 \mathrm{D}{ }^{1} \mathrm{H}-{ }^{1} \mathrm{H}$ COSY and ${ }^{1} \mathrm{H}-{ }^{13} \mathrm{C}$ HSQC spectra. In some cases, ${ }^{1} \mathrm{H}_{-}{ }^{13} \mathrm{C}$ HMBC spectra were found to be necessary. For the fluorous-tagged compounds, some overlaps in the ${ }^{13} \mathrm{C}$ spectra are observed due to extensive ${ }^{19} \mathrm{~F}$ and ${ }^{13} \mathrm{C}$ coupling and these coupling constants are not reported. High-resolution mass measurements (HRMS) were obtained in a Waters/Micromass LCT classic instrument.

\section{General experimental procedures}

\section{General procedure for FSPE (Fluorous solid phase extraction)}

The crude reaction mixture (less than $0.3 \mathrm{~g}$ ) was dissolved in $0.4 \mathrm{~mL}$ DMF and loaded onto the cartridge filled with fluorous silica gel. Non-fluorous compounds were eluted with $10 \mathrm{~mL} 80 \%$ $\mathrm{MeOH}(\mathrm{aq})$ and fluorous compounds were eluted with $10 \mathrm{~mL} \mathrm{MeOH}$. The fluorous fraction was concentrated to obtain the desired product. 


\section{General procedure for TBDPS ether formation}

To a stirred solution of the compound (1.0 equiv.) in dry DMF $(6.0 \mathrm{~mL}), t$ butylchloro(diphenyl)silane (1.5 equiv.) and imidazole (2.0 equiv.) were added at room temperature. The reaction mixture was stirred for $10 \mathrm{~h}$ at the same temperature. The mixture was then diluted with ethyl acetate $(15 \mathrm{~mL})$ and washed with water $(3 X 10 \mathrm{~mL})$. The organic layer was dried over $\mathrm{Na}_{2} \mathrm{SO}_{4}$ (sodium sulfate) and concentrated in vacuo. The resulting crude oil was purified by flash column chromatography.

\section{General procedure for 6-OH protection with Levulinate ester (Lev)}

To a stirred solution of the compound (1.0 equiv.), $N, N^{\prime}$-dicyclohexylcarbodiimide (DCC) (1.5 equiv.) and 4-dimethylaminopyridine (DMAP) (0.5 equiv.) in dichloromethane (8 $\mathrm{mL})$ was added levulinic acid (1.3 equiv.) at room temperature under argon. The reaction was stirred for 4 $\mathrm{h}$ at the same temperature. The reaction was diluted with dichloromethane $(10 \mathrm{~mL})$ and filtered over a pad of Celite ${ }^{\circledR}$. The organic layer was washed with water $(2 \mathrm{X} 15 \mathrm{~mL})$, saturated solution of sodium bicarbonate $(2 \mathrm{X} 15 \mathrm{~mL})$ and dried over $\mathrm{Na}_{2} \mathrm{SO}_{4}$. The solvent was removed in vacuo and the crude product was then recrystallized from absolute ethanol or purified by flash column chromatography.

\section{General procedure for OMP ether deprotection using ceric ammonium nitrate (CAN)}

The compound (1.0 equiv.) was dissolved in a mixture of acetonitrile and water (4:1 acetonitrile/water; concentration: $0.03 \mathrm{M}$ ) and the solution was stirred in a $0{ }^{\circ} \mathrm{C}$ ice bath. Solid ceric ammonium nitrate (3.0 equiv.) was added portion-wise to the reaction mixture at the same temperature. The reaction mixture was then allowed to warm to ambient temperature and stirred for $0.5 \mathrm{~h}$. The reaction was diluted with water $(20 \mathrm{~mL})$ and extracted with ethyl acetate several times. The organic layer was dried over $\mathrm{Na}_{2} \mathrm{SO}_{4}$. The solvent was removed in vacuo and the resulting crude oil was purified by flash column chromatography on silica gel.

\section{General procedure for trichloroacetimidate formation}

To the compound (1.0 equiv.) in dichloromethane $(10 \mathrm{~mL})$ was added trichloroacetonitrile $(5.0$ equiv.) and DBU (1,8-Diazabicyclo[5.4.0]undec-7-ene) (0.1 equiv.) at $0{ }^{\circ} \mathrm{C}$ under argon. The reaction was stirred for $3 \mathrm{~h}$ at room temperature. The solvent was removed in vacuo and the crude oil was purified by flash column chromatography.

\section{General procedure for levulinate ester deprotection}

$\mathrm{N}_{2} \mathrm{H}_{4} \cdot \mathrm{H}_{2} \mathrm{O}$ (hydrazine monohydrate) $(0.5 \mathrm{~mL})$ was added to a mixture of pyridine $(4.8 \mathrm{~mL})$ and acetic acid $(3.2 \mathrm{~mL})$. The Compound $(0.09 \mathrm{mmol})$ was dissolved in $3.0 \mathrm{~mL}$ of this prepared solution under argon at room temperature and stirred for 2 to $3 \mathrm{~h}$ until the starting material was completely consumed as monitored by TLC. The solvent was then removed under reduced 
pressure. The resulting crude material was purified via FSPE. The fluorous fraction was concentrated to afford desired compound.

General procedure for conversion of $N$-trichloroacetamide (NHTCA) to $N$-acetyl (NHAc)

To a solution of the compound $(55.0 \mu \mathrm{mol})$ in benzene $(4 \mathrm{~mL})$ and dimethylacetamide $(1 \mathrm{~mL}$, $4: 1, \mathrm{v} / \mathrm{v})$ were added AIBN (Azobisisobutyronitrile) $(28.0 \mu \mathrm{mol})$ and tributyltin hydride $(0.55$ $\mathrm{mmol}$ ), degassed with the flow of argon and stirred at room temperature for $30 \mathrm{~min}$. The reaction mixture was then heated at $80{ }^{\circ} \mathrm{C}$ for $4 \mathrm{~h}$. TLC showed complete conversion of the starting material. The reaction mixture was then concentrated. The resulting crude solid material was purified via FSPE. The fluorous fraction was concentrated to afford desired compound.

\section{Experimental procedures}

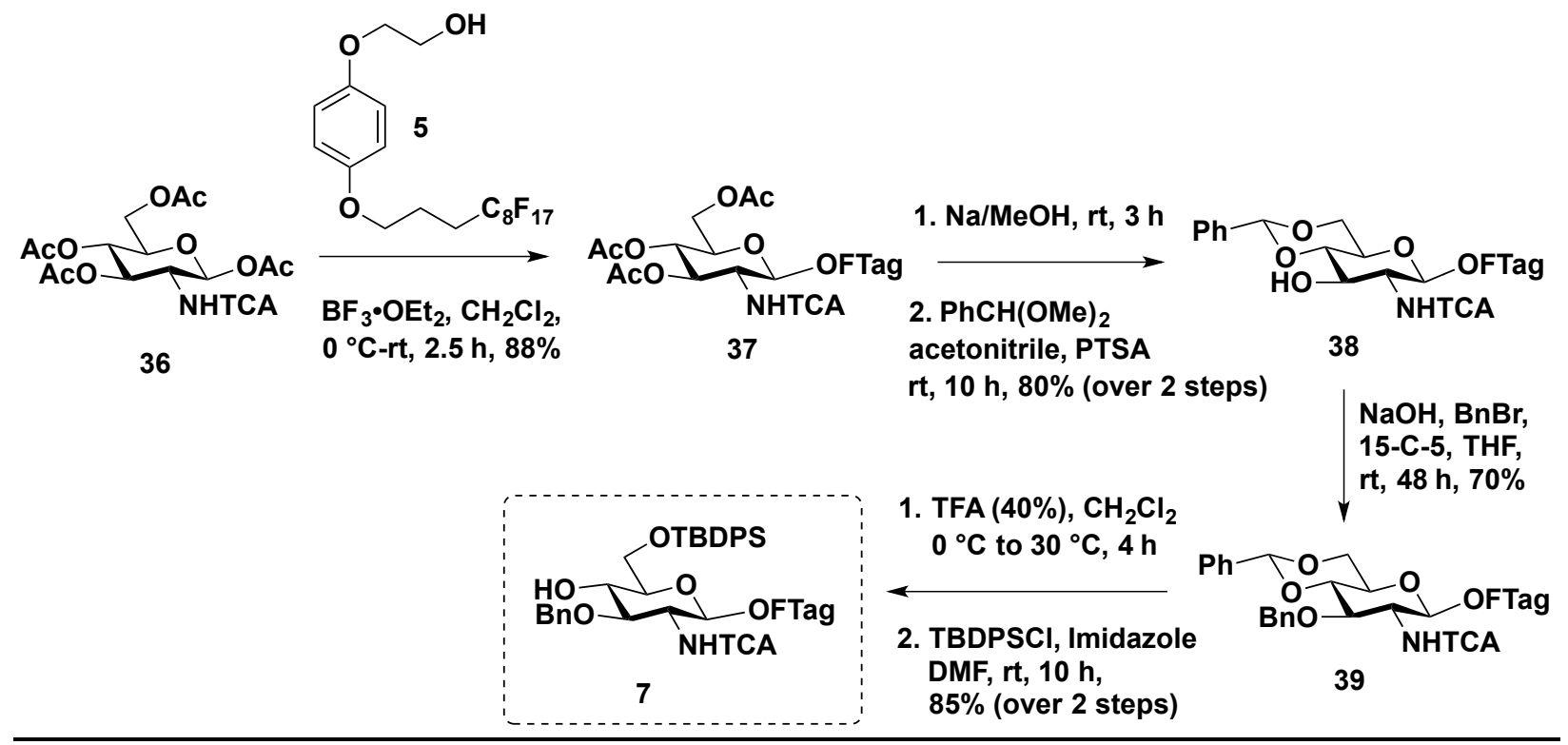

Scheme S1: Stability of fluorous tag towards commonly used protection/deprotection conditions for carbohydrate synthesis:Successful synthesis of Glucosamine building block 7

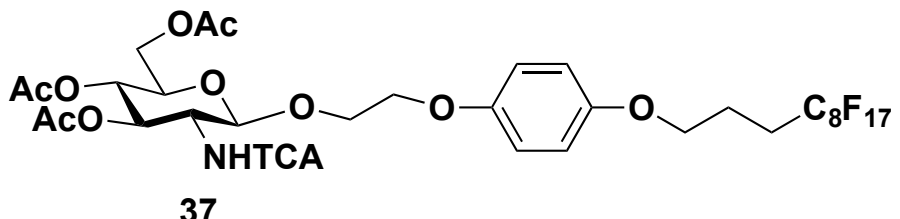

2-(4-((4,4,5,5,6,6,7,7,8,8,9,9,10,10,11,11,11-heptadecafluoroundecyl)oxy)phenoxy)ethyl

3,4,6-tri- $\boldsymbol{O}$-acetyl-2-deoxy-2-trichloroacetamido- $\boldsymbol{\beta}$-D-glucopyranoside (37): A solution of the compound $\mathbf{3 6}^{[1]}(0.36 \mathrm{~g}, 0.73 \mathrm{mmol})$ and the fluorous tagged acceptor $\mathbf{5}(0.30 \mathrm{~g}, 0.49 \mathrm{mmol})$ in 
anhydrous dichloromethane $(10 \mathrm{~mL})$ under argon was cooled to $0{ }^{\circ} \mathrm{C}$ in an ice bath while stirring vigorously. $\mathrm{BF}_{3} \cdot \mathrm{OEt}_{2}$ (boron trifluoride diethyl etherate) $(0.18 \mathrm{~mL}, 1.5 \mathrm{mmol}$ ) was added dropwise at $0{ }^{\circ} \mathrm{C}$ under argon. The reaction mixture was then allowed to warm to room temperature $\left(25^{\circ} \mathrm{C}\right)$ and stirred for $2.5 \mathrm{~h}$. The reaction mixture was quenched by the addition of triethylamine drop wise until the reaction mixture was neutral to a litmus paper and diluted with dichloromethane $(20 \mathrm{~mL})$. The organic layer was washed with water $(3 X 20 \mathrm{~mL})$, saturated solution of sodium bicarbonate $(3 \mathrm{X} 20 \mathrm{~mL})$ and brine solution $(2 \mathrm{X} 20 \mathrm{~mL})$. The organic phase was dried over $\mathrm{Na}_{2} \mathrm{SO}_{4}$ and the solvent was removed in vacuo. The resulting crude material was purified via FSPE to afford compound $37(0.45 \mathrm{~g}, 0.43 \mathrm{mmol}, 88 \%)$ as a white solid. ${ }^{1} \mathbf{H}$ NMR $\left(400 \mathrm{MHz}, \mathrm{CDCl}_{3}\right) \delta 6.80(\mathrm{~s}, 5 \mathrm{H}), 5.35(\mathrm{dd}, J=10.6,9.4 \mathrm{~Hz}, 1 \mathrm{H}), 5.14(\mathrm{t}, J=9.6 \mathrm{~Hz}, 1 \mathrm{H}), 4.89$ (d, $J=8.3 \mathrm{~Hz}, 1 \mathrm{H}), 4.30(\mathrm{dd}, J=12.3,4.7 \mathrm{~Hz}, 1 \mathrm{H}), 4.19-3.92(\mathrm{~m}, 8 \mathrm{H}), 3.76(\mathrm{~m}, 1 \mathrm{H}), 2.30(\mathrm{~m}$, 2H), $2.14-1.99(\mathrm{~m}, 11 \mathrm{H}) .{ }^{13} \mathbf{C}$ NMR $\left(101 \mathrm{MHz}, \mathrm{CDCl}_{3}\right) \delta 170.8,170.7,169.3,162.0,153.1$, 152.9, 115.5, 115.5, 100.8, 92.2, 72.2, 71.6, 68.3, 68.2, 68.1, 66.9, 61.9, 56.0, 27.9, 20.7, 20.6, 20.6. HRMS-ESI-TOF (m/z): $[\mathrm{M}+\mathrm{Na}]^{+}$Calculated for $\mathrm{C}_{33} \mathrm{H}_{31} \mathrm{Cl}_{3} \mathrm{~F}_{17} \mathrm{NO}_{11} \mathbf{1 0 6 8 . 0 5 8 8}$, Found 1068.0594.

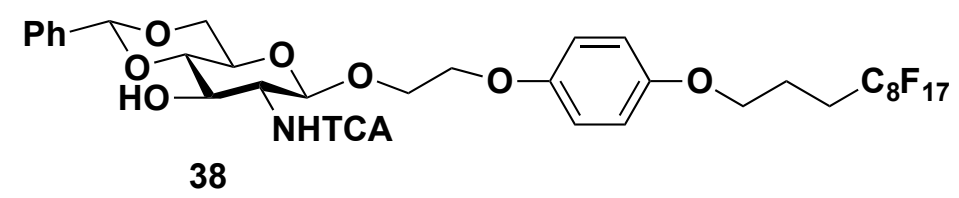

2-(4-((4,4,5,5,6,6,7,7,8,8,9,9,10,10,11,11,11-heptadecafluoroundecyl)oxy)phenoxy)ethyl $4,6-$ $\boldsymbol{O}$-benzylidene-2-deoxy-2-trichloroacetamido- $\beta$-D-glucopyranoside (38): To a solution of compound $37(0.40 \mathrm{~g}, 0.38 \mathrm{mmol})$ in methanol $(15 \mathrm{~mL})$ was added a pinch of solid sodium metal at room temperature under argon. The reaction mixture was stirred for $3 \mathrm{~h}$ at room temperature and quenched by addition Dowex ${ }^{\circledR}(50 \mathrm{~W} X 8,200-400 \mathrm{mesh})\left(\mathrm{H}^{+}\right)$cation exchange resin. The resin was filtered over a pad of Celite ${ }^{\circledR}$, rinsed with methanol. The methanol was removed in vacuo and trace methanol or water was removed by co-evaporation with toluene $(3 \times 5 \mathrm{~mL})$. The crude white solid product $(0.34 \mathrm{~g}, 0.37 \mathrm{mmol})$ was used directly in the next step. Benzaldehyde dimethyl acetal $(0.170 \mathrm{~mL}, 1.11 \mathrm{mmol})$ and p-toluene sulfonic acid monohydrate $(0.014 \mathrm{~g}, 0.074$ mmol) were added to a solution of the crude solid material $(0.34 \mathrm{~g}, 0.37 \mathrm{mmol})$ in anhydrous acetonitrile $(15 \mathrm{~mL})$ under argon. The reaction mixture was stirred at room temperature until TLC confirmed the formation of the product $(10 \mathrm{~h})$. The reaction was quenched by addition of triethylamine drop wise until the reaction mixture was neutral to a litmus paper. After concentrating the reaction mixture under reduce pressure, the resulting crude material was purified via FSPE to afford compound $38(0.31 \mathrm{~g}, 0.30 \mathrm{mmol}, 82 \%)$ as a white solid. ${ }^{1} \mathbf{H}$ NMR $\left(400 \mathrm{MHz}, \mathrm{CDCl}_{3}\right) \delta 7.49(\mathrm{dd}, J=6.6,3.1 \mathrm{~Hz}, 2 \mathrm{H}), 7.38(\mathrm{dd}, J=5.0,1.9$ $\mathrm{Hz}, 3 \mathrm{H}), 7.02(\mathrm{~d}, J=6.8 \mathrm{~Hz}, 1 \mathrm{H}), 6.81(\mathrm{~s}, 4 \mathrm{H}), 5.56(\mathrm{~s}, 1 \mathrm{H}), 5.05(\mathrm{~d}, J=8.3 \mathrm{~Hz}, 1 \mathrm{H}), 4.40-4.30$ $(\mathrm{m}, 2 \mathrm{H}), 4.18-3.91(\mathrm{~m}, 7 \mathrm{H}), 3.80(\mathrm{t}, J=10.0 \mathrm{~Hz}, 1 \mathrm{H}), 3.60-3.53(\mathrm{~m}, 2 \mathrm{H}), 3.06(\mathrm{~s}, 1 \mathrm{H}), 2.30$ (m, 2H), 2.07 (m, 2H). ${ }^{13} \mathbf{C}$ NMR (101 MHz, $\left.\mathrm{CDCl}_{3}\right) \delta 162.6,153.1,152.8,136.8,129.3,128.3$, 126.2, 115.6, 115.5, 115.48, 110.0, 101.9, 100.3, 92.3, 81.5, 69.9, 68.5, 67.8, 66.9, 66.3, 61.5, 
59.6, 29.7, 27.9, 20.6. HRMS-ESI-TOF (m/z): $[\mathrm{M}+\mathrm{Na}]^{+}$Calculated for $\mathrm{C}_{34} \mathrm{H}_{29} \mathrm{Cl}_{3} \mathrm{~F}_{17} \mathrm{NO}_{8}$ 1030.0585, Found 1030.0582.

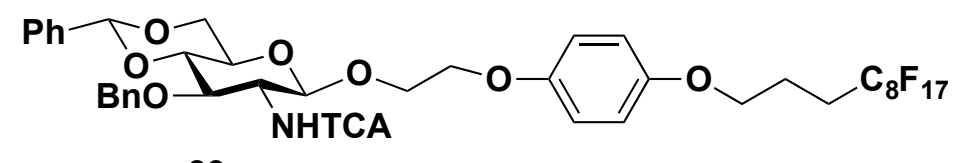

39

2-(4-((4,4,5,5,6,6,7,7,8,8,9,9,10,10,11,11,11-heptadecafluoroundecyl)oxy)phenoxy)ethyl 3-Obenzyl-4,6- $O$-benzylidene-2-deoxy-2-trichloroacetamido- $\beta$-D-glucopyranoside (39): To a solution of compound $\mathbf{3 8}(0.30 \mathrm{~g}, 0.29 \mathrm{mmol})$ in dry THF $(15 \mathrm{~mL})$ were added crushed $\mathrm{NaOH}$ pellets $^{[2]}(0.12 \mathrm{~g}, 2.9 \mathrm{mmol})$ and $15-\mathrm{C}-5(30 \mu \mathrm{L}, 0.15 \mathrm{mmol})$ at $25{ }^{\circ} \mathrm{C}$ under argon. The reaction mixture was vigorously stirred for $1 \mathrm{~h}$ at the same temperature. $\mathrm{BnBr}$ (benzyl bromide) $(0.350 \mathrm{~mL}, 2.97 \mathrm{mmol})$ was then added drop wise to the reaction mixture and allowed to stir for $48 \mathrm{~h}$ at the same temperature. TLC showed complete consumption of the starting material. Then, the reaction mixture was filtered through celite and concentrated. The resulting crude solid was taken in dichloromethane $(20 \mathrm{~mL})$ and washed with brine solution (3X20 mL). The organic phase was dried over $\mathrm{Na}_{2} \mathrm{SO}_{4}$ and the solvent was removed in vacuo. The resulting crude material was purified via FSPE. The fluorous fraction was concentrated and further purified by flash column chromatography on silica gel $5 \%$ to $10 \%$ ethyl acetate gradient in hexane) to afford compound $\mathbf{3 9}(0.23 \mathrm{~g}, 0.21 \mathrm{mmol}, 70 \%)$ as a white solid. ${ }^{1} \mathbf{H}$ NMR $\left(400 \mathrm{MHz}, \mathrm{CDCl}_{3}\right) \delta 7.50(\mathrm{dd}, J=7.3,2.0 \mathrm{~Hz}, 2 \mathrm{H}), 7.44-7.34(\mathrm{~m}, 3 \mathrm{H}), 7.27(\mathrm{~d}, J=11.8 \mathrm{~Hz}$, $5 \mathrm{H}), 6.90(\mathrm{~d}, J=7.5 \mathrm{~Hz}, 1 \mathrm{H}), 6.80$ (s, 4H), $5.60(\mathrm{~s}, 1 \mathrm{H}), 5.13$ (d, $J=8.4 \mathrm{~Hz}, 1 \mathrm{H}), 4.90$ (d, $J=$ $11.2 \mathrm{~Hz}, 1 \mathrm{H}), 4.70(\mathrm{~d}, J=11.2 \mathrm{~Hz}, 1 \mathrm{H}), 4.40-4.27(\mathrm{~m}, 2 \mathrm{H}), 4.15-3.89(\mathrm{~m}, 6 \mathrm{H}), 3.78(\mathrm{~m}, 2 \mathrm{H})$, $3.61-3.48(\mathrm{~m}, 2 \mathrm{H}), 2.29(\mathrm{~m}, 2 \mathrm{H}), 2.07(\mathrm{~m}, 2 \mathrm{H}) .{ }^{13} \mathbf{C}$ NMR $\left(101 \mathrm{MHz}, \mathrm{CDCl}_{3}\right) \delta 161.9,152.9$, 137.7, 137.1, 129.1, 128.4, 128.3, 128.3, 127.9, 126.0, 115.6, 115.4, 101.3, 100.1, 92.4, 82.7, 75.8, 74.8, 68.6, 68.5, 67.8, 66.9, 66.2, 58.9, 29.7, 27.9, 20.6. HRMS-ESI-TOF (m/z): $[\mathrm{M}+\mathrm{Na}]^{+}$ Calculated for $\mathrm{C}_{41} \mathrm{H}_{35} \mathrm{Cl}_{3} \mathrm{~F}_{17} \mathrm{NO}_{8}$ 1122.1035, Found 1122.1064.

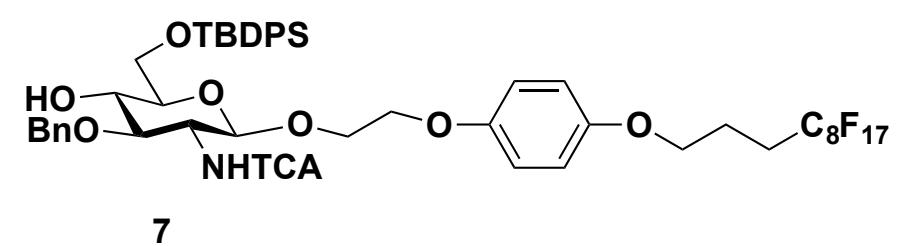

2-(4-((4,4,5,5,6,6,7,7,8,8,9,9,10,10,11,11,11-heptadecafluoroundecyl)oxy)phenoxy)ethyl $3-O$ benzyl-6- $O$ - $t$-butyldiphenylsilyl-2-deoxy-2-trichloroacetamido- $\beta$-D-glucopyranoside (7): A solution of compound $39(0.20 \mathrm{~g}, 0.18 \mathrm{mmol})$ in dichloromethane $(10 \mathrm{~mL})$ was cooled to $0{ }^{\circ} \mathrm{C}$ in an ice bath. Aqueous trifluoroacetic acid solution $(50 \% \mathrm{v} / \mathrm{v}, 5 \mathrm{~mL}$ was added to the reaction mixture at $0{ }^{\circ} \mathrm{C}$. The solution was allowed to warm to room temperature and stirred for $4 \mathrm{~h}$ at 30 ${ }^{\circ} \mathrm{C}$ until the starting material was completely consumed by TLC. The reaction mixture was diluted with dichloromethane $(20 \mathrm{~mL})$ and water $(20 \mathrm{~mL})$. The layers were separated and the water layer was extracted twice with dichloromethane $(2 \times 20 \mathrm{~mL})$. The combined organic layer 
was washed with water $(3 \times 20 \mathrm{~mL})$, saturated solution of sodium bicarbonate $(3 \times 20 \mathrm{~mL})$ and dried over $\mathrm{Na}_{2} \mathrm{SO}_{4}$. The organic solvent was removed in vacuo to afford white solid $(0.17 \mathrm{~g}, 0.17$ $\mathrm{mmol})$ and trace water was removed by co evaporation with toluene $(3 \times 5 \mathrm{~mL})$. The crude product was successfully converted to compound $7(0.19 \mathrm{~g}, 0.15 \mathrm{mmol}, 85 \%$ over 2 steps $)$ as a white solid following the general procedure for TBDPS ether formation. ${ }^{1} \mathbf{H}$ NMR (400 MHz, $\left.\mathrm{CDCl}_{3}\right) \delta 7.70(\mathrm{~d}, J=6.6 \mathrm{~Hz}, 4 \mathrm{H}), 7.49-7.25(\mathrm{~m}, 11 \mathrm{H}), 6.97(\mathrm{~d}, J=7.6 \mathrm{~Hz}, 1 \mathrm{H}), 6.78(\mathrm{~s}, 4 \mathrm{H})$, $5.00(\mathrm{~d}, J=8.3 \mathrm{~Hz}, 1 \mathrm{H}), 4.80(\mathrm{q}, J=11.3 \mathrm{~Hz}, 2 \mathrm{H}), 4.11-3.89(\mathrm{~m}, 8 \mathrm{H}), 3.89-3.75(\mathrm{~m}, 2 \mathrm{H})$, $3.57-3.45(\mathrm{~m}, 2 \mathrm{H}), 2.85(\mathrm{~d}, J=2.4 \mathrm{~Hz}, 1 \mathrm{H}), 2.29(\mathrm{~m}, 2 \mathrm{H}), 2.06(\mathrm{~m}, 2 \mathrm{H}), 1.08(\mathrm{~s}, 9 \mathrm{H}) .{ }^{13} \mathrm{C}$ NMR $\left(101 \mathrm{MHz}, \mathrm{CDCl}_{3}\right) \delta 161.9,152.9,152.9,138.0,135.6,135.5,132.9,132.7,129.9,128.6$, $128.1,127.9,127.8,127.7,115.5,115.3,99.4,92.4,79.8,74.7,74.6,73.4,67.8,67.8,66.9,64.7$, 58.2, 27.9, 26.8, 20.6, 19.2. HRMS-ESI-TOF (m/z): $[\mathrm{M}+\mathrm{Na}]^{+}$Calculated for $\mathrm{C}_{50} \mathrm{H}_{49} \mathrm{Cl}_{3} \mathrm{~F}_{17} \mathrm{NO}_{8} \mathrm{Si}$ 1272.1906, Found 1272.1895.

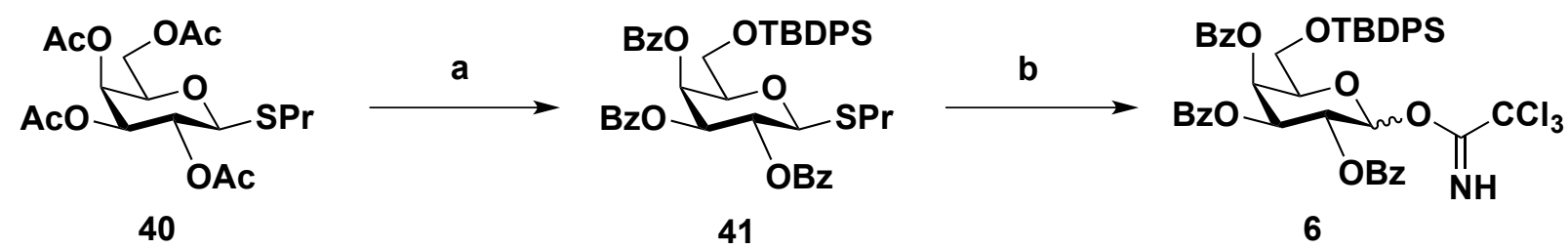

Scheme S2: Synthesis of Galactose donor 6

Reagents and conditions: (a) (i) Na, MeOH, $25^{\circ} \mathrm{C}, 2 \mathrm{~h}$; (ii) TBDPSCl, imidazole DMF, $25^{\circ} \mathrm{C}$, $8 \mathrm{~h}$; (iii) $\mathrm{BzCl}$, pyridine, DMAP, $0{ }^{\circ} \mathrm{C}$ to $25{ }^{\circ} \mathrm{C}, 8 \mathrm{~h}, 72 \%$ (over 3 steps); (b) (i) NBS, Acetone: $\mathrm{H}_{2} \mathrm{O}(9: 1), 0{ }^{\circ} \mathrm{C}$ to $25^{\circ} \mathrm{C}, 2 \mathrm{~h}, 96 \%$; (ii) $\mathrm{CCl}_{3} \mathrm{CN}, \mathrm{DBU}, \mathrm{CH}_{2} \mathrm{Cl}_{2}, 0{ }^{\circ} \mathrm{C}$ to $25{ }^{\circ} \mathrm{C}, 3 \mathrm{~h}$, 93\%.

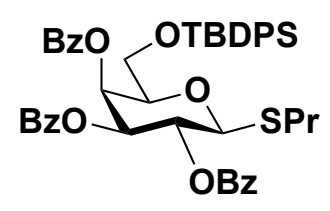

41

Propyl 2,3,4-tri-O-benzoyl-6- $\boldsymbol{O}$-t-butyldiphenylsilyl-1-thio- $\boldsymbol{\beta}$-D-galactopyranoside (41): To a solution of compound $\mathbf{4 0}^{[3]}(0.40 \mathrm{~g}, 0.98 \mathrm{mmol})$ in methanol $(15 \mathrm{~mL})$ was added a pinch of solid sodium metal at room temperature under argon. The reaction mixture was stirred for $3 \mathrm{~h}$ at room temperature and quenched by addition Dowex ${ }^{\circledR}\left(50 \mathrm{~W}\right.$ X8, 200-400 mesh) $\left(\mathrm{H}^{+}\right)$cation exchange resin. The resin was filtered over a pad of Celite ${ }^{\circledR}$, rinsed with methanol. The methanol was removed in vacuo and trace methanol or water was removed by co-evaporation with toluene $(3 \times 5$ $\mathrm{mL})$. The crude white solid product $(0.22 \mathrm{~g}, 0.94 \mathrm{mmol})$ was used directly in the next step. To a stirred solution of the resulting crude solid $(0.22 \mathrm{~g}, 0.94 \mathrm{mmol})$ in dry DMF $(5 \mathrm{~mL})$, tbutylchloro(diphenyl)silane $(0.360 \mathrm{~mL}, 1.42 \mathrm{mmol})$ and imidazole $(0.130 \mathrm{~g}, 1.89 \mathrm{mmol})$ were added at $0{ }^{\circ} \mathrm{C}$. The reaction mixture was stirred for $10 \mathrm{~h}$ at the room temperature $\left(25^{\circ} \mathrm{C}\right)$. The 
mixture was then diluted with ethyl acetate $(20 \mathrm{~mL})$ and washed with water $(3 \mathrm{X} 30 \mathrm{~mL})$. The organic layer was dried over $\mathrm{Na}_{2} \mathrm{SO}_{4}$ and concentrated in vacuo. The resulting crude oil was dissolved in anhydrous pyridine $(5 \mathrm{~mL})$ and cooled to $0{ }^{\circ} \mathrm{C}$. Benzoyl chloride $(0.660 \mathrm{~mL}, 5.66$ $\mathrm{mmol}$ ) and 4-(dimethylamino)pyridine $(60 \mathrm{mg}, 0.47 \mathrm{mmol})$ were added to the reaction mixture. The solution was allowed to warm to room temperature and stirred for $8 \mathrm{~h}$ until the starting material was completely consumed by TLC. The organic solvent was removed in vacuo and trace of pyridine was removed by co-evaporation with toluene $(3 \times 10 \mathrm{~mL})$. The resulting oil was dissolved in dichloromethane $(20 \mathrm{~mL})$. The organic layer was washed with water $(3 \mathrm{X} 20 \mathrm{~mL}), 1$ $\mathrm{M} \mathrm{HCl}$ solution $(3 \mathrm{X} 20 \mathrm{~mL})$ and brine solution $(2 \mathrm{X} 20 \mathrm{~mL})$. The organic phase was dried over $\mathrm{Na}_{2} \mathrm{SO}_{4}$ and the solvent was removed in vacuo. The crude material was purified by flash column chromatography on silica gel $(10 \%$ to $20 \%$ ethyl acetate gradient in hexane) to afford compound 41 (0.56 g, $0.72 \mathrm{mmol}, 72 \%$ over 3 steps) as a white solid. ${ }^{1} \mathbf{H}$ NMR (500 MHz, $\left.\mathrm{CDCl}_{3}\right) \delta 8.05(\mathrm{dd}, J=8.2,1.1 \mathrm{~Hz}, 2 \mathrm{H}), 7.98(\mathrm{dd}, J=8.2,1.1 \mathrm{~Hz}, 2 \mathrm{H}), 7.83(\mathrm{dd}, J=8.3,1.1 \mathrm{~Hz}$, $2 \mathrm{H}), 7.72-7.62(\mathrm{~m}, 3 \mathrm{H}), 7.54-7.36(\mathrm{~m}, 11 \mathrm{H}), 7.33-7.24(\mathrm{~m}, 3 \mathrm{H}), 7.15(\mathrm{t}, J=7.6 \mathrm{~Hz}, 2 \mathrm{H})$, $6.13(\mathrm{~d}, J=3.2 \mathrm{~Hz}, 1 \mathrm{H}), 5.81(\mathrm{t}, J=9.9 \mathrm{~Hz}, 1 \mathrm{H}), 5.68(\mathrm{dd}, J=9.9,3.3 \mathrm{~Hz}, 1 \mathrm{H}), 4.80(\mathrm{~d}, J=9.9$ $\mathrm{Hz}, 1 \mathrm{H}), 4.12(\mathrm{t}, J=6.9 \mathrm{~Hz}, 1 \mathrm{H}), 3.93-3.78(\mathrm{~m}, 2 \mathrm{H}), 2.78(\mathrm{~m}, 2 \mathrm{H}), 1.75-1.58(\mathrm{~m}, 2 \mathrm{H}), 1.04$ $(\mathrm{s}, 9 \mathrm{H}), 0.99(\mathrm{t}, J=7.3 \mathrm{~Hz}, 3 \mathrm{H}) .{ }^{13} \mathrm{C}$ NMR $\left(126 \mathrm{MHz}, \mathrm{CDCl}_{3}\right) \delta 165.6,165.4,165.3,135.6$, $135.4,133.3,133.2,133.1,132.9,132.6,129.9,129.8,129.8,129.6,129.5,129.4,129.1,128.6$, $128.3,128.2,127.8,127.6,84.0,77.6,73.1,68.4,68.1,61.3,31.8,26.7,23.2,19.1,13.5$. HRMS-ESI-TOF (m/z): $[\mathrm{M}+\mathrm{Na}]^{+}$Calculated for $\mathrm{C}_{46} \mathrm{H}_{48} \mathrm{O}_{8} \mathrm{SSi}$ 811.2737, Found 811.2751.

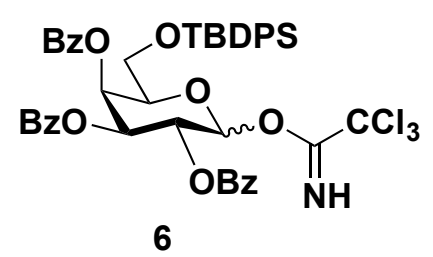

2,3,4-tri-O-benzoyl-6- $O$ - $t$-butyldiphenylsilyl- $\alpha / \beta$-D-galactopyranosyl trichloroacetimidate (6): To a solution of compound $41(0.40 \mathrm{~g}, 0.51 \mathrm{mmol})$ in acetone and water $(10 \mathrm{~mL}, 9: 1$ acetone/water $)$ was added NBS $(0.360 \mathrm{~g}, 2.03 \mathrm{mmol})$ at $0{ }^{\circ} \mathrm{C}$. The reaction mixture was stirred for $2 \mathrm{~h}$ at room temperature until the starting material was completely consumed. The reaction mixture was diluted with dichloromethane $(30 \mathrm{~mL})$. The organic layer was washed with water $(3 X 20 \mathrm{~mL})$, saturated solution of sodium bicarbonate $(3 \mathrm{X} 20 \mathrm{~mL})$ and brine solution $(2 \mathrm{X} 20 \mathrm{~mL})$. The organic phase was dried over $\mathrm{Na}_{2} \mathrm{SO}_{4}$ and the solvent was removed in vacuo. The crude material was purified by flash column chromatography on silica gel $(10 \%$ to $30 \%$ ethyl acetate gradient in hexane) to afford the hemiacetal $(0.35 \mathrm{~g}, 0.48 \mathrm{mmol}, 96 \%)$ as white foam. The hemiacetal was successfully converted compound $6(0.39 \mathrm{~g}, 0.45 \mathrm{mmol}, 93 \%)$ as pale yellow oil, which turned to white foam under high vacuum following the general procedure for trichloroacetimidate formation. ${ }^{1} \mathbf{H}$ NMR $\left(500 \mathrm{MHz}, \mathrm{CDCl}_{3}\right) \delta 8.61(\mathrm{~s}, 1 \mathrm{H}), 8.06(\mathrm{~d}, J=7.2 \mathrm{~Hz}$, 2H), $7.97(\mathrm{~d}, J=7.3 \mathrm{~Hz}, 2 \mathrm{H}), 7.84(\mathrm{~d}, J=7.2 \mathrm{~Hz}, 2 \mathrm{H}), 7.69-7.62(\mathrm{~m}, 3 \mathrm{H}), 7.56-7.46(\mathrm{~m}, 6 \mathrm{H})$, $7.44-7.35(\mathrm{~m}, 5 \mathrm{H}), 7.30(\mathrm{dd}, J=15.4,7.4 \mathrm{~Hz}, 4 \mathrm{H}), 7.18(\mathrm{t}, J=7.6 \mathrm{~Hz}, 2 \mathrm{H}), 6.86(\mathrm{~d}, J=3.6 \mathrm{~Hz}$, 
$1 \mathrm{H}), 6.23(\mathrm{~d}, J=2.7 \mathrm{~Hz}, 1 \mathrm{H}), 6.12(\mathrm{dd}, J=10.7,3.3 \mathrm{~Hz}, 1 \mathrm{H}), 5.88(\mathrm{dd}, J=10.7,3.7 \mathrm{~Hz}, 1 \mathrm{H})$, $4.62(\mathrm{t}, J=7.0 \mathrm{~Hz}, 1 \mathrm{H}), 3.84(\mathrm{~d}, J=7.0 \mathrm{~Hz}, 2 \mathrm{H}), 1.01(\mathrm{~s}, 9 \mathrm{H}) .{ }^{13} \mathbf{C}$ NMR $\left(126 \mathrm{MHz}, \mathrm{CDCl}_{3}\right) \delta$ $165.6,165.5,165.3,160.7,135.5,135.4,133.5,133.4,133.2,132.8,132.6,129.9,129.8,129.7$, 129.6, 129.4, 129.1, 128.8, 128.6, 128.4, 128.2, 127.7, 127.6, 93.8, 90.9, 72.0, 68.5, 68.3, 68.2, 61.3, 26.6, 19.0. HRMS-ESI-TOF (m/z): $[\mathrm{M}+\mathrm{Na}]^{+}$Calculated for $\mathrm{C}_{45} \mathrm{H}_{42} \mathrm{Cl}_{3} \mathrm{NO}_{9} \mathrm{Si}$ 898.1576, Found 898.1545.

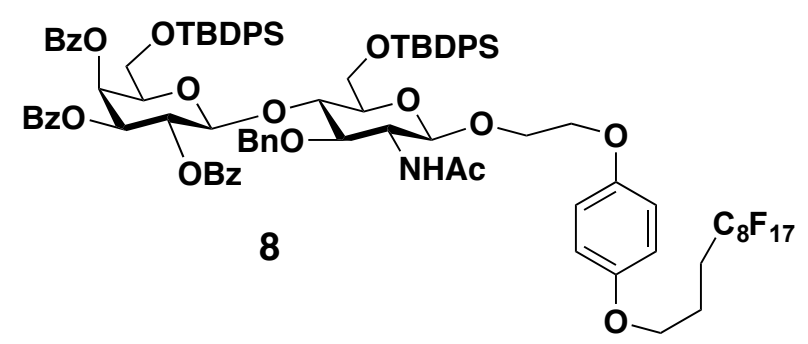

\section{2-(4-((4,4,5,5,6,6,7,7,8,8,9,9,10,10,11,11,11-heptadecafluoroundecyl)oxy)phenoxy)ethyl} (2,3,4-tri- $O$-benzoyl-6-O-t-butyldiphenylsilyl- $\beta$-D-galactopyranosyl)-( $1 \rightarrow 4)$-2-acetamido-3$\boldsymbol{O}$-benzyl-2-deoxy-6-O-t-butyldiphenylsilyl- $\boldsymbol{\beta}$-D-glucopyranoside (8): To a vigorously stirred solution of glycosyl donor $6(42.0 \mathrm{mg}, 48.0 \mu \mathrm{mol})$ and glycosyl acceptor $7(0.030 \mathrm{~g}, 24 \mu \mathrm{mol})$ in dry dichloromethane $(1.0 \mathrm{~mL})$ under argon, catalytic TMSOTf (Trimethylsilyl trifluoromethanesulfonate) $(0.87 \mu \mathrm{L}, 4.8 \mu \mathrm{mol})$ was added at $0{ }^{\circ} \mathrm{C}$ and the reaction mixture was stirred for $30 \mathrm{~min}$ at the same temperature until the acceptor was completely consumed shown by TLC. The reaction mixture quenched by the addition of triethylamine drop wise until the reaction mixture was neutral to a litmus paper. The reaction mixture was then diluted with dichloromethane $(3 \mathrm{~mL})$ and the solvent was removed under reduced pressure. The resulting crude material was purified via FSPE. The fluorous fraction was concentrated to afford the disaccharide (37 mg, $0.020 \mathrm{mmol}$ ) as white foam. NHTCA group of the resulting disaccharide (37 $\mathrm{mg}, 0.020 \mathrm{mmol}$ ) successfully converted to NHAc by following the general procedure for conversion of $\mathrm{N}$-trichloroacetamide (NHTCA) to $\mathrm{N}$-acetyl (NHAc) and was purified via FSPE to afford compound 8 as white foam (30.0 mg, $16.0 \mu \mathrm{mol}, 72 \%$ over 2 steps). ${ }^{1} \mathbf{H}$ NMR $(500 \mathrm{MHz}$, $\left.\mathrm{CDCl}_{3}\right) \delta 7.99-7.91(\mathrm{~m}, 2 \mathrm{H}), 7.88-7.72(\mathrm{~m}, 8 \mathrm{H}), 7.65-7.50(\mathrm{~m}, 6 \mathrm{H}), 7.47-7.19(\mathrm{~m}, 20 \mathrm{H})$, $7.12-6.98(\mathrm{~m}, 5 \mathrm{H}), 6.76(\mathrm{~s}, 4 \mathrm{H}), 6.14(\mathrm{~d}, J=3.1 \mathrm{~Hz}, 1 \mathrm{H}), 5.77-5.61(\mathrm{~m}, 3 \mathrm{H}), 5.14(\mathrm{~d}, J=7.9$ $\mathrm{Hz}, 1 \mathrm{H}), 4.96(\mathrm{~d}, J=11.1 \mathrm{~Hz}, 1 \mathrm{H}), 4.72(\mathrm{~d}, J=7.4 \mathrm{~Hz}, 1 \mathrm{H}), 4.65(\mathrm{~d}, J=11.2 \mathrm{~Hz}, 1 \mathrm{H}), 4.28(\mathrm{t}, J$ $=7.7 \mathrm{~Hz}, 1 \mathrm{H}), 4.04-3.91(\mathrm{~m}, 7 \mathrm{H}), 3.89-3.76(\mathrm{~m}, 4 \mathrm{H}), 3.66-3.57(\mathrm{~m}, 1 \mathrm{H}), 3.50(\mathrm{q}, J=7.9$ $\mathrm{Hz}, 1 \mathrm{H}), 3.28-3.17(\mathrm{~m}, 1 \mathrm{H}), 2.30(\mathrm{~m}, 2 \mathrm{H}), 2.06(\mathrm{~m}, 2 \mathrm{H}), 1.86(\mathrm{~s}, 3 \mathrm{H}), 1.11(\mathrm{~s}, 9 \mathrm{H}), 0.98(\mathrm{~s}$, 9H). ${ }^{13} \mathbf{C}$ NMR $\left(126 \mathrm{MHz}, \mathrm{CDCl}_{3}\right) \delta 170.2,165.5,165.3,153.2,152.9,138.5,135.9,135.5$, $135.4,133.6,133.2,133.2,133.1,132.6,132.5,132.3,130.3,129.9,129.8,129.6,129.6,129.2$, $129.0,128.5,128.4,128.3,128.2,128.1,128.1,127.8,127.7,127.6,127.5,115.7,115.4,100.0$, $99.8,77.7,75.5,74.8,74.1,73.5,71.8,70.7,67.8,67.6,67.3,66.9,61.8,60.5,55.3,27.8,26.9$, 26.7, 23.5, 20.7, 19.4, 18.9. HRMS-ESI-TOF (m/z): $[\mathrm{M}+\mathrm{Na}]^{+}$Calculated for $\mathrm{C}_{93} \mathrm{H}_{92} \mathrm{~F}_{17} \mathrm{NO}_{16} \mathrm{Si}_{2}$ 1881.5612, Found 1881.5643. 


\section{$\underline{\text { Specific Goal }}$}

To develop a suitable deprotection condition for the TBDPS group (extremely stable in both acidic and basic conditions) in presence of ester functionality, different standard deprotection protocols were tested on monosaccharide. The applicability of these optimized protocols were also tested on disaccharides containing multiple TBDPS groups.

Deprotection of the bulky TBDPS ( $t$-butyldiphenylsilyl) group in presence of ester functionality is one of the trickiest reactions in organic chemistry. TBDPS ether is a widely used protecting group due to its high tolerance towards both acidic and basic reaction conditions. On the other hand, it is also very much challenging to deprotect this extremely robust protecting group, especially in presence of esters. So, our main objective was to figure out the optimized condition for its fast and clean removal without affecting the esters. Initially, different reaction conditions were tested on monosaccharide residue $\mathbf{S 1}$ available in the literature for deprotection of the silyl ethers. ${ }^{[4]}$ Our observations are shown in Table S1.

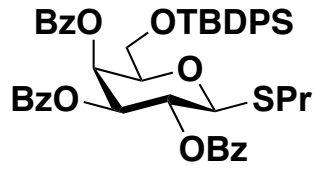

S1

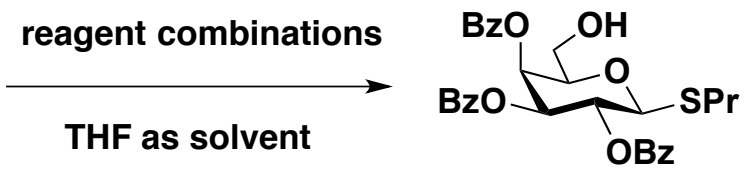

S2

Table S1. Standardization of deprotection protocol of TBDPS group in presence of benzoyl esters (Bz) on monosaccharide

\begin{tabular}{|c|c|c|c|c|c|}
\hline Entry & Reagent $[a]$ & $\begin{array}{l}\text { Temp } \\
\left({ }^{\circ} \mathrm{C}\right)\end{array}$ & $\begin{array}{l}\text { Time } \\
\text { (h) }\end{array}$ & $\begin{array}{l}\text { Concentration } \\
\text { (M) }\end{array}$ & Result [b] \\
\hline 1. & $\begin{array}{l}\text { TBAF } 1 \mathrm{M} \text { in THF } \\
\text { (1.1 equiv.) }\end{array}$ & 25 & 1 & 0.03 & Decomposed \\
\hline 2. & $\begin{array}{l}\text { TBAF } 1 \mathrm{M} \text { in THF } \\
\text { (1.1 equiv.) }\end{array}$ & 0 & 3 & 0.03 & Decomposed \\
\hline 3. & $\begin{array}{l}\text { TBAF } 1 \mathrm{M} \text { in THF } \\
\text { ( } 6 \text { equiv.)/ } \mathrm{AcOH} \text { ( } 30 \\
\text { equiv.) }\end{array}$ & 25 & 30 & 0.04 & $\begin{array}{l}\text { Complete conversion, } \\
\text { clean reaction }\end{array}$ \\
\hline 4. & $\mathrm{Et}_{3} \mathrm{~N} \cdot 3 \mathrm{HF}$ (1 equiv.) & 25 & 24 & 0.04 & $\begin{array}{l}10 \% \text { conversion, no } \\
\text { decomposition }\end{array}$ \\
\hline 5. & $\mathrm{Et}_{3} \mathrm{~N} \cdot 3 \mathrm{HF}$ (10 equiv.) & 25 & 20 & 0.04 & $\begin{array}{l}\text { Complete } \\
\text { conversion, clean } \\
\text { reaction }\end{array}$ \\
\hline
\end{tabular}




\begin{tabular}{|l|l|l|l|l|l|}
\hline 6. & $\begin{array}{l}\text { TBAF 1M in THF } \\
(7 \text { equiv.)/ AcOH (14 } \\
\text { equiv.) }\end{array}$ & 45 & 3 & 0.04 & $\begin{array}{l}\text { Gradual } \\
\text { decomposition }\end{array}$ \\
\hline 7. & $\begin{array}{l}\text { TBAF 1M in THF } \\
\text { (7 equiv.)/ AcOH (14 } \\
\text { equiv.) }\end{array}$ & 25 & 20 & 0.04 & $\begin{array}{l}\text { Complete conversion, } \\
\text { clean reaction }\end{array}$ \\
\hline $\mathbf{8 .}$ & $\begin{array}{l}\text { TBAF 1M in THF } \\
\text { (7 equiv.)/ AcOH (14 } \\
\text { equiv.) }\end{array}$ & $\mathbf{3 5}$ & $\mathbf{7}$ & $\mathbf{0 . 0 4}$ & $\begin{array}{l}\text { Complete clean } \\
\text { conversion, cean } \\
\text { reaction }\end{array}$ \\
\hline $\mathbf{9 .}$ & $\begin{array}{l}\text { TBAF 1M in THF } \\
\text { (2 equiv.)/ AcOH (4 } \\
\text { equiv.) }\end{array}$ & $\mathbf{2 5}$ & $\mathbf{1 0}$ & $\mathbf{0 . 0 3}$ & $\begin{array}{l}\text { Complete conversion, clean } \\
\text { ceaction }\end{array}$ \\
\hline
\end{tabular}

Note:

[a] : HF•pyridine is the most commonly used reagent to cleave silyl ethers in presence of esters. ${ }^{[5]}$ This condition was not attempted, as it is not amenable to the automation platform. [b] : All the observations were based on TLC conversions.

TBAF: tetrabutylammonium fluoride; $\mathrm{AcOH}$ : glacial acetic acid; $\mathrm{Et}_{3} \mathrm{~N} \cdot 3 \mathrm{HF}$ : triethylamine trihydrofluoride

From our initial observations, it was concluded that entries 5, 8 and 9 are the most suitable choices for deprotection of TBDPS groups in presence of benzoyl esters. Et $3 \mathrm{~N} \cdot 3 \mathrm{HF}$ reagent ${ }^{[6]}$ produced clean conversion without affecting the esters, although the reactions were extremely sluggish (entry 4 and 5). TBAF/AcOH combination is one of the most commonly used reagents for the removal of silyl ethers and it perfectly worked in our case also (entry 3, 7, 8 and 9). Addition of $\mathrm{AcOH}$ prevented the decomposition of the benzoate esters in presence of basic fluoride ions by buffering the reaction medium, although excess equivalents of $\mathrm{AcOH}$ hindered progress of the reaction (entries 1, 2, 3 and 7). Heating the reaction also significantly accelerated progress of the reaction (entry 8 ).

Therefore, these optimized conditions were tested on a reference disaccharide molecule S3 having two TBDPS groups and the results are shown in Table S2. These results also supported the initial findings: (i) excess equivalents of $\mathrm{AcOH}$ made progress of the reaction extremely slow; only $40 \%$ mono-deprotected product was isolated even after $40 \mathrm{~h}$ (entry 1), and (ii) $\mathrm{Et}_{3} \mathrm{~N} \bullet 3 \mathrm{HF}$ was also probably not the best choice as the reaction did not give us any improved result even after 2 days (entry 2). In the case of disaccharide having two TBDPS groups on it, heating at $35{ }^{\circ} \mathrm{C}$ gave us completely decomposed product. So, it was confirmed that although heating on the monosaccharide stage accelerated progress of the reaction; the same would not be valid on the disaccharide having multiple TBDPS groups (entry 3). Entry 4 and 5 rendered the best possible results where we observed that gradual addition of the excess equivalents of TBAF 
accelerated the reaction from 40 to $20 \mathrm{~h}$ and gave us our desired product without affecting the benzoyl esters.
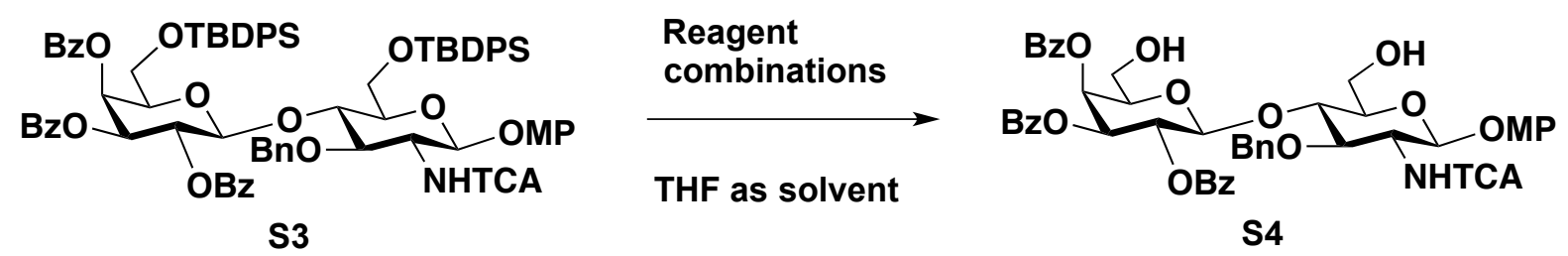

Table S2. Standardization of the deprotection protocol of TBDPS group in presence of benzoyl esters $(\mathrm{Bz})$ on the disaccharide having 2 TBDPS groups

\begin{tabular}{|c|c|c|c|c|c|}
\hline Entry & Reagent & $\begin{array}{l}\text { Temp } \\
\left({ }^{\circ} \mathrm{C}\right)\end{array}$ & $\begin{array}{l}\text { Time } \\
\text { (h) }\end{array}$ & $\begin{array}{l}\text { Concent } \\
\text { ration } \\
\text { (M) }\end{array}$ & Result [a] \\
\hline 1. & $\begin{array}{l}\text { TBAF } 1 \mathrm{M} \text { in THF } \\
(6 \text { equiv) / } \mathrm{AcOH} \\
(30 \text { equiv) }\end{array}$ & 25 & 40 & 0.04 & $\begin{array}{l}\text { Clean reaction, } 40 \% \\
\text { monodeprotected product }\end{array}$ \\
\hline 2. & $\begin{array}{l}\mathrm{Et}_{3} \mathrm{~N} \cdot 3 \mathrm{HF} \\
(40 \text { equiv) }\end{array}$ & 25 & 48 & 0.04 & $\begin{array}{l}\text { Clean reaction, } 50 \% \\
\text { monodeprotected product, } \\
20 \% \text { desired product }\end{array}$ \\
\hline 3. & $\begin{array}{l}\text { TBAF } 1 \mathrm{M} \text { in THF } \\
\text { ( } 7 \text { equiv) / } \mathrm{AcOH} \\
\text { (14 equiv) }\end{array}$ & 35 & 3 & 0.04 & Complete decomposition \\
\hline 4. & $\begin{array}{l}\text { TBAF 1M in THF } \\
\text { (4 equiv) / AcOH (8 } \\
\text { equiv) }\end{array}$ & 25 & 40 & 0.03 & $\begin{array}{l}\text { Complete conversion, } \\
\text { clean reaction }\end{array}$ \\
\hline 5. & $\begin{array}{l}\text { TBAF 1M in THF } \\
\text { ( } 6 \text { equiv) / AcOH }(8 \\
\text { equiv) }\end{array}$ & 25 & 20 & 0.03 & $\begin{array}{l}\text { Complete conversion, } \\
\text { clean reaction }\end{array}$ \\
\hline \multicolumn{6}{|c|}{$\begin{array}{l}\text { Note: } \\
{[a]: \text { All the observations were based on TLC conversions. }}\end{array}$} \\
\hline
\end{tabular}

Now, our attention was focused to test these optimized deprotection protocols on our actual fluorous tagged disaccharide having two TBDPS groups. 


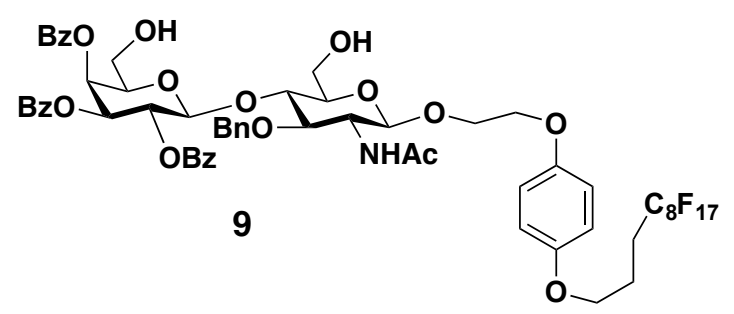

2-(4-((4,4,5,5,6,6,7,7,8,8,9,9,10,10,11,11,11-heptadecafluoroundecyl)oxy)phenoxy)ethyl $(2,3,4-$ tri- $O$-benzoyl- $\beta$-D-galactopyranosyl)-( $1 \rightarrow 4)$-2-acetamido-3- $O$-benzyl-2-deoxy- $\beta$-Dglucopyranoside (9): To a solution of compound $8(25.0 \mathrm{mg}, 13.0 \mu \mathrm{mol})$ in dry THF $(0.5 \mathrm{~mL})$ was added acetic acid $(6.00 \mu \mathrm{L}, 104 \mu \mathrm{mol})$ followed by tetrabutylammonium fluoride $(1.0 \mathrm{M}$ in THF, $0.08 \mathrm{~mL}, 0.08 \mathrm{mmol}$ ). The reaction mixture was stirred for $24 \mathrm{~h}$ at $25{ }^{\circ} \mathrm{C}$ and then concentrated. The crude product was purified via FSPE to afford the desilylated compound 9 (14.0 mg, $10.0 \mu \mathrm{mol}, 81 \%)$ as white foam. ${ }^{1} \mathbf{H}$ NMR (400 MHz, $\left.\mathrm{CDCl}_{3}\right) \delta 7.97$ (d, $J=8.2 \mathrm{~Hz}$, 4H), $7.77-7.69(\mathrm{~m}, 2 \mathrm{H}), 7.45(\mathrm{~m}, 2 \mathrm{H}), 7.31(\mathrm{~m}, 7 \mathrm{H}), 7.20-7.02(\mathrm{~m}, 5 \mathrm{H}), 6.75-6.60(\mathrm{~m}, 4 \mathrm{H})$, $6.48(\mathrm{~d}, J=6.8 \mathrm{~Hz}, 1 \mathrm{H}), 5.83-5.73(\mathrm{~m}, 2 \mathrm{H}), 5.66(\mathrm{dd}, J=10.3,3.4 \mathrm{~Hz}, 1 \mathrm{H}), 5.21-5.06(\mathrm{~m}$, $3 \mathrm{H}), 4.58(\mathrm{~d}, J=10.8 \mathrm{~Hz}, 2 \mathrm{H}), 4.42-4.34(\mathrm{~m}, 1 \mathrm{H}), 4.13-4.01(\mathrm{~m}, 2 \mathrm{H}), 3.92(\mathrm{~m}, 3 \mathrm{H}), 3.82(\mathrm{t}, J$ $=5.9 \mathrm{~Hz}, 2 \mathrm{H}), 3.72-3.53(\mathrm{~m}, 4 \mathrm{H}), 3.40(\mathrm{~d}, J=12.5 \mathrm{~Hz}, 1 \mathrm{H}), 3.10(\mathrm{dd}, J=17.0,8.8 \mathrm{~Hz}, 2 \mathrm{H})$, $2.46(\mathrm{~s}, 1 \mathrm{H}), 2.20(\mathrm{~m}, 2 \mathrm{H}), 1.97(\mathrm{~m}, 2 \mathrm{H}), 1.56(\mathrm{~s}, 3 \mathrm{H}) .{ }^{13} \mathbf{C} \mathbf{N M R}\left(101 \mathrm{MHz}, \mathrm{CDCl}_{3}\right) \delta 170.8$, $165.7,165.3,165.2,153.3,152.2$, 138.2, 133.4, 133.2, 133.0, 129.84, 129.80, 129.7, 129.1, $128.9,128.5,128.4,128.2,128.1,127.7,116.2,115.5,99.9,99.6,77.5,75.7,75.4,75.2,72.0$, 70.5, 69.1, 68.5, 67.9, 66.8, 61.7, 59.4, 58.1, 29.7, 23.2, 20.6. HRMS-ESI-TOF (m/z): [M+H] ${ }^{+}$ Calculated for $\mathrm{C}_{61} \mathrm{H}_{56} \mathrm{~F}_{17} \mathrm{NO}_{16}$ 1382.3406, Found 1382.3445.

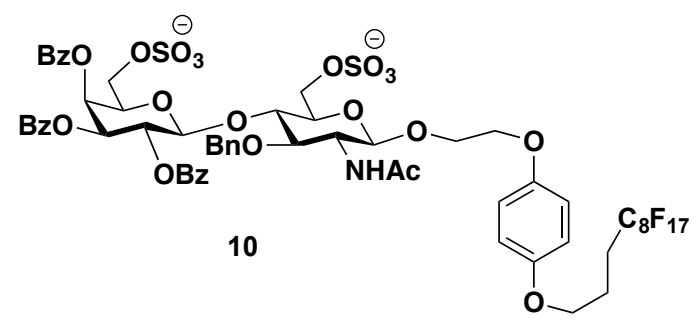

\section{2-(4-((4,4,5,5,6,6,7,7,8,8,9,9,10,10,11,11,11-heptadecafluoroundecyl)oxy)phenoxy)ethyl} $(2,3,4$-tri- $O$-benzoyl-6- $O$-sulfonate- $\beta$-D-galactopyranosyl)-( $\rightarrow 4)$-2-acetamido-3- $O$-benzyl2-deoxy-6- $\boldsymbol{O}$-sulfonate- $\boldsymbol{\beta}$-D-glucopyranoside (10): To a solution of compound $9(0.010 \mathrm{~g}, 7.0$ $\mu \mathrm{mol})$ in anhydrous pyridine $(0.4 \mathrm{~mL})$ was added sulfur trioxide•pyridine complex ( $\mathrm{SO}_{3}$.pyridine) $(22.0 \mathrm{mg}, 0.140 \mathrm{mmol})$. The reaction mixture was stirred at $60^{\circ} \mathrm{C}$ for $12 \mathrm{~h}$. Then, the reaction mixture was cooled to room temperature, quenched with methanol $(5 \mathrm{~mL})$, triethylamine $(0.05 \mathrm{~mL})$ and concentrated under reduced pressure. The crude product was purified by FSPE to afford the sulfated compound $\mathbf{1 0}(8.0 \mathrm{mg}, 5.0 \mu \mathrm{mol}, 79 \%)$ as white foam. ${ }^{1} \mathbf{H}$ NMR (400 MHz, Methanol-d4) $\delta 8.04-7.98(\mathrm{~m}, 2 \mathrm{H}), 7.90-7.79(\mathrm{~m}, 3 \mathrm{H}), 7.67-7.59(\mathrm{~m}, 2 \mathrm{H})$, $7.50-7.43(\mathrm{~m}, 2 \mathrm{H}), 7.36(\mathrm{t}, J=7.5 \mathrm{~Hz}, 5 \mathrm{H}), 7.27-7.07(\mathrm{~m}, 7 \mathrm{H}), 6.72(\mathrm{~s}, 4 \mathrm{H}), 5.86(\mathrm{~d}, J=3.0$ $\mathrm{Hz}, 1 \mathrm{H}), 5.67$ (dd, $J=10.3,7.9 \mathrm{~Hz}, 1 \mathrm{H}), 5.58$ (dd, $J=10.3,3.2 \mathrm{~Hz}, 1 \mathrm{H}), 5.37$ (d, $J=7.8 \mathrm{~Hz}$, 
1H), $5.10(\mathrm{~d}, J=11.0 \mathrm{~Hz}, 1 \mathrm{H}), 4.63(\mathrm{~d}, J=11.0 \mathrm{~Hz}, 1 \mathrm{H}), 4.42(\mathrm{~d}, J=8.1 \mathrm{~Hz}, 2 \mathrm{H}), 4.34(\mathrm{~d}, J=$ $8.4 \mathrm{~Hz}, 1 \mathrm{H}), 4.14(\mathrm{dd}, J=10.6,5.9 \mathrm{~Hz}, 1 \mathrm{H}), 4.08-3.98(\mathrm{~m}, 3 \mathrm{H}), 3.90(\mathrm{q}, J=7.2,6.0 \mathrm{~Hz}, 5 \mathrm{H})$, $3.68(\mathrm{t}, J=7.3 \mathrm{~Hz}, 2 \mathrm{H}), 3.59(\mathrm{~s}, 2 \mathrm{H}), 3.31(\mathrm{~d}, J=9.6 \mathrm{~Hz}, 1 \mathrm{H}), 2.27(\mathrm{~m}, 2 \mathrm{H}), 1.98-1.89(\mathrm{~m}$, $2 \mathrm{H}), 1.72(\mathrm{~s}, 3 \mathrm{H})$. HRMS-ESI-TOF (m/z): $[\mathrm{M}-2 \mathrm{H}]^{2-}$ Calculated for $\mathrm{C}_{61} \mathrm{H}_{56} \mathrm{~F}_{17} \mathrm{NO}_{22} \mathrm{~S}_{2}$ 769.6154, Found 769.6157.

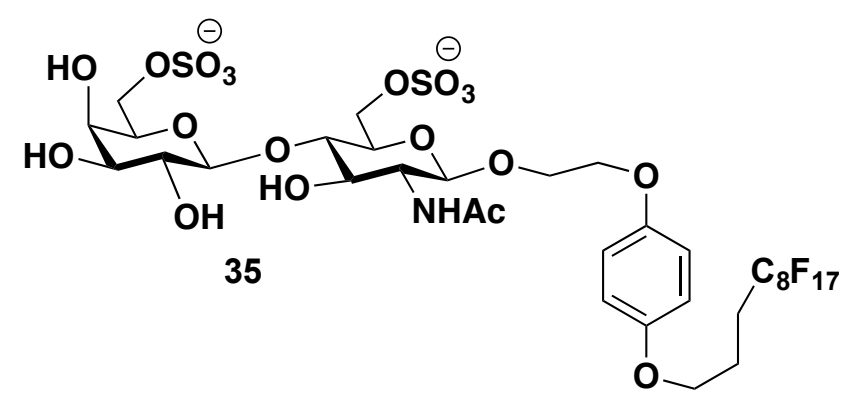

2-(4-((4,4,5,5,6,6,7,7,8,8,9,9,10,10,11,11,11-heptadecafluoroundecyl)oxy)phenoxy)ethyl (6-Osulfonate- $\beta$-D-galactopyranosyl)-( $\rightarrow 4)$-2-acetamido-2-deoxy-6- $O$-sulfonate- $\beta$-D-

glucopyranoside (35): Compound $10(6.0 \mathrm{mg}, 3.9 \mu \mathrm{mol})$, dissolved in methanol, was first subjected to hydrogenolysis in $\mathrm{H}$-Cube Pro apparatus. The reaction was run at $45{ }^{\circ} \mathrm{C}$ with the flow rate $1 \mathrm{~mL} / \mathrm{min}$. The pressure was set at 1 bar. The rate of $\mathrm{H}_{2}$-production was set at 58 $\mathrm{cm}^{3} / \mathrm{min} .30 \mathrm{~mm} \mathrm{10 \%} \mathrm{Pd/C} \mathrm{cartridge} \mathrm{was} \mathrm{used} \mathrm{for} \mathrm{the} \mathrm{reaction.} \mathrm{The} \mathrm{starting} \mathrm{concentration} \mathrm{was}$ $0.05 \mathrm{M}$. Two cycles were run followed by rinsing. The reaction completion was monitored via TLC. After the completion of the reaction, the solvent was evaporated and the crude reaction mixture was dried under vacuum for overnight. The resulting material was dissolved in $1 \mathrm{~mL}$ methanol. $0.5 \mathrm{M} \mathrm{NaOMe}$ (sodium methoxide) was added drop wise to the reaction mixture at 25 ${ }^{\circ} \mathrm{C}$ until $\mathrm{pH}$ was 9.5. The reaction was stirred for $24 \mathrm{~h}(\mathrm{pH}$ was maintained at 9.5) and was quenched with $1 \mathrm{M}$ acetic acid. The reaction mixture was concentrated and purified via FSPE. The compound was further purified via sephadex LH-20 column with methanol. Appropriate fractions containing the product were concentrated to afford compound $\mathbf{3 5}(2.7 \mathrm{mg}, 2.4 \mu \mathrm{mol}$, $61 \%$ over 2 steps) as white foam. ${ }^{1} \mathbf{H}$ NMR $(400 \mathrm{MHz}$, Methanol-d4) $\delta 6.87(\mathrm{~d}, J=2.1 \mathrm{~Hz}, 4 \mathrm{H})$, 5.35 (s, 1H), 4.57 (d, $J=8.3 \mathrm{~Hz}, 2 \mathrm{H}), 4.51-4.47(\mathrm{~m}, 1 \mathrm{H}), 4.37$ (d, $J=10.9 \mathrm{~Hz}, 1 \mathrm{H}), 4.31$ (dd, $J$ $=11.0,4.7 \mathrm{~Hz}, 1 \mathrm{H}), 4.20-4.17(\mathrm{~m}, 2 \mathrm{H}), 4.07(\mathrm{~d}, J=6.6 \mathrm{~Hz}, 2 \mathrm{H}), 4.02(\mathrm{t}, J=6.0 \mathrm{~Hz}, 2 \mathrm{H}), 3.93$ $-3.89(\mathrm{~m}, 2 \mathrm{H}), 3.87(\mathrm{~s}, 2 \mathrm{H}), 3.78-3.74(\mathrm{~m}, 2 \mathrm{H}), 3.69-3.62(\mathrm{~m}, 5 \mathrm{H}), 3.53(\mathrm{~d}, J=3.9 \mathrm{~Hz}, 2 \mathrm{H})$, $2.40-2.35(\mathrm{~m}, 1 \mathrm{H}), 2.21-2.16(\mathrm{~m}, 2 \mathrm{H}), 2.07-2.03(\mathrm{~m}, 3 \mathrm{H}), 1.90(\mathrm{~s}, 3 \mathrm{H})$. HRMS-ESI-TOF (m/z): $[\mathrm{M}-2 \mathrm{H}]^{2-}$ Calculated for $\mathrm{C}_{33} \mathrm{H}_{36} \mathrm{~F}_{17} \mathrm{NO}_{19} \mathrm{~S}_{2}$ 568.5526, Found 568.5519; $[\mathrm{M}-2 \mathrm{H}+\mathrm{Na}]^{-}$ Calculated for $\mathrm{C}_{33} \mathrm{H}_{36} \mathrm{~F}_{17} \mathrm{NO}_{19} \mathrm{~S}_{2} \mathbf{1 1 6 0 . 0 9 5 0}$, Found 1160.0990 . 


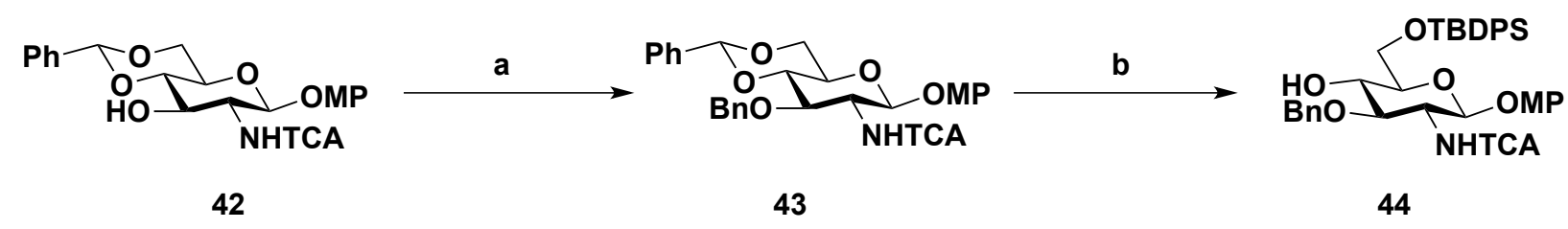

Scheme S3: Synthesis of Glucosamine acceptor 44

Reagents and conditions: (a) $\mathrm{NaH}, \mathrm{BnBr}, \mathrm{DMF},-20^{\circ} \mathrm{C}--15{ }^{\circ} \mathrm{C}, 5 \mathrm{~h}, 74 \%$; (b) (i) TFA (40\%), $\mathrm{CH}_{2} \mathrm{Cl}_{2}, 0{ }^{\circ} \mathrm{C}$ to $30^{\circ} \mathrm{C}, 4 \mathrm{~h}$; (ii) TBDPSCl, imidazole, DMF, $25^{\circ} \mathrm{C}, 10 \mathrm{~h}, 81 \%$ over 2 steps.

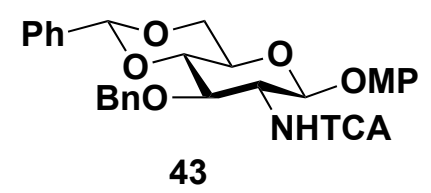

4-Methoxyphenyl 3- $\boldsymbol{O}$-benzyl-4,6- $\boldsymbol{O}$-benzylidene-2-deoxy-2-trichloroacetamido- $\boldsymbol{\beta}$-Dglucopyranoside (43): $\mathrm{NaH}$ (60\% suspension in mineral oil) $(0.390 \mathrm{~g}, 16.2 \mathrm{mmol})$ was added portion wise to absolute DMF $(15 \mathrm{~mL})$ at $-20{ }^{\circ} \mathrm{C}$ under argon. The reaction mixture was stirred until the homogeneous suspension was formed and the compound $\mathbf{4 2}^{[1]}(3.00 \mathrm{~g}, 5.78 \mathrm{mmol})$ was added portion wise during $15 \mathrm{~min}$. The stirring was continued until the gas evolution ceased $\left(1 \mathrm{~h}\right.$ at $\left.-20^{\circ} \mathrm{C}\right)$, benzyl bromide $(0.820 \mathrm{~mL}, 6.93 \mathrm{mmol})$ was added drop wise and the reaction mixture was stirred for $3 \mathrm{~h}$ at $-15^{\circ} \mathrm{C} .{ }^{[7]}$ The progress of the reaction was monitored by TLC. After $4 \mathrm{~h}$, the reaction mixture was cooled to $-40^{\circ} \mathrm{C}$ and glacial $\mathrm{AcOH}(1.63 \mathrm{~mL})$ was carefully added drop wise. The reaction mixture was allowed to attain room temperature slowly and diluted with ethyl acetate $(30 \mathrm{~mL})$, washed with saturated solution of sodium bicarbonate (3X30 $\mathrm{mL}$ ). The organic phase was dried over $\mathrm{Na}_{2} \mathrm{SO}_{4}$ and the solvent was removed in vacuo. The solid was then recrystallized from absolute ethanol to afford the compound $\mathbf{4 3}(2.60 \mathrm{~g}, 4.27 \mathrm{mmol}$, $74 \%)$ as a white solid. ${ }^{1} \mathbf{H}$ NMR $\left(400 \mathrm{MHz}, \mathrm{CDCl}_{3}\right) \delta 7.43(\mathrm{dd}, J=7.5,2.0 \mathrm{~Hz}, 2 \mathrm{H}), 7.32(\mathrm{dd}, J$ $=4.9,2.4 \mathrm{~Hz}, 3 \mathrm{H}), 7.23(\mathrm{~d}, J=2.7 \mathrm{~Hz}, 5 \mathrm{H}), 6.96-6.85(\mathrm{~m}, 3 \mathrm{H}), 6.78-6.68(\mathrm{~m}, 2 \mathrm{H}), 5.54(\mathrm{~s}$, $1 \mathrm{H}), 5.35(\mathrm{~d}, J=8.4 \mathrm{~Hz}, 1 \mathrm{H}), 4.85(\mathrm{~d}, J=11.2 \mathrm{~Hz}, 1 \mathrm{H}), 4.65(\mathrm{~d}, J=11.2 \mathrm{~Hz}, 1 \mathrm{H}), 4.38-4.24$ (m, 2H), $3.85-3.62(\mathrm{~m}, 6 \mathrm{H}), 3.55(\mathrm{~m}, 1 \mathrm{H}) .{ }^{13} \mathbf{C}$ NMR $\left(101 \mathrm{MHz}, \mathrm{CDCl}_{3}\right) \delta 162.0,155.8,150.9$, 137.7, 137.1, 129.1, 128.5, 128.3, 127.9, 126.0, 119.1, 114.6, 101.3, 99.7, 92.3, 82.5, 75.6, 74.8, 68.6, 66.2, 58.8, 55.6. HRMS-ESI-TOF $(\mathbf{m} / \mathbf{z}):[\mathrm{M}+\mathrm{Na}]^{+}$Calculated for $\mathrm{C}_{29} \mathrm{H}_{28} \mathrm{Cl}_{3} \mathrm{NO}_{7}$ 632.0805, Found 632.0825.

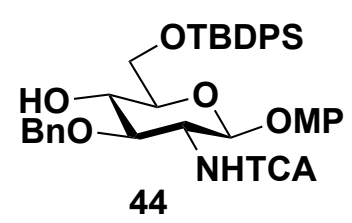

4-Methoxyphenyl 3-O-benzyl-6-O-t-butyldiphenylsilyl-2-deoxy-2-trichloroacetamido- $\beta$-Dglucopyranoside (44): A solution of the compound $43(2.00 \mathrm{~g}, 3.28 \mathrm{mmol})$ in dichloromethane $(20 \mathrm{~mL})$ was cooled to $0{ }^{\circ} \mathrm{C}$ in an ice bath. Aqueous trifluoroacetic acid solution $(40 \% \mathrm{v} / \mathrm{v}, 10$ 
$\mathrm{mL}$ ) was added to the reaction mixture at $0{ }^{\circ} \mathrm{C}$. The solution was allowed to warm to room temperature and stirred for $4 \mathrm{~h}$ at $30{ }^{\circ} \mathrm{C}$ until the starting material was completely consumed by TLC. The reaction mixture was diluted with dichloromethane $(30 \mathrm{~mL})$ and water $(30 \mathrm{~mL})$. The layers were separated and the water layer was extracted twice with dichloromethane $(2 \times 20 \mathrm{~mL})$. The combined organic layer was washed with water $(3 \times 20 \mathrm{~mL})$, saturated solution of sodium bicarbonate $(3 \times 20 \mathrm{~mL})$ and dried over $\mathrm{Na}_{2} \mathrm{SO}_{4}$. The organic solvent was removed in vacuo to afford white solid (1.52 g, $2.92 \mathrm{mmol})$ and trace water was removed by co evaporation with toluene $(3 \times 10 \mathrm{~mL})$. The crude product was successfully converted to compound $44(2.02 \mathrm{~g}, 2.66$ mmol, $81 \%$ over 2 steps) as a white solid following the general procedure for TBDPS ether formation. ${ }^{1} \mathbf{H}$ NMR $\left(400 \mathrm{MHz}, \mathrm{CDCl}_{3}\right) \delta 7.68(\mathrm{~d}, J=6.7 \mathrm{~Hz}, 4 \mathrm{H}), 7.34(\mathrm{~d}, J=3.2 \mathrm{~Hz}, 11 \mathrm{H})$, $7.09-6.92(\mathrm{~m}, 3 \mathrm{H}), 6.79-6.68(\mathrm{~m}, 2 \mathrm{H}), 5.30(\mathrm{~d}, J=8.3 \mathrm{~Hz}, 1 \mathrm{H}), 4.80(\mathrm{~d}, J=2.8 \mathrm{~Hz}, 2 \mathrm{H}), 4.10$ $(\mathrm{dd}, J=10.3,8.6 \mathrm{~Hz}, 1 \mathrm{H}), 3.93(\mathrm{~m}, 2 \mathrm{H}), 3.85-3.69(\mathrm{~m}, 5 \mathrm{H}), 3.56(\mathrm{dd}, J=9.5,4.8 \mathrm{~Hz}, 1 \mathrm{H}), 2.67$ $(\mathrm{d}, J=2.7 \mathrm{~Hz}, 1 \mathrm{H}), 1.07(\mathrm{~s}, 9 \mathrm{H}) .{ }^{13} \mathbf{C} \mathbf{N M R}\left(101 \mathrm{MHz}, \mathrm{CDCl}_{3}\right) \delta 162.0,155.5,151.2,137.9$, 135.6, 135.5, 132.9, 132.6, 129.8, 128.6, 128.1, 128.1, 127.8, 118.9, 114.5, 99.1, 92.4, 79.7, 75.1, 74.8, 72.8, 64.3, 58.2, 55.6, 26.8, 19.2. HRMS-ESI-TOF (m/z): $\left[\mathrm{M}+\mathrm{NH}_{4}\right]^{+}$Calculated for $\mathrm{C}_{38} \mathrm{H}_{42} \mathrm{Cl}_{3} \mathrm{NO}_{7} \mathrm{Si}$ 775.2140, Found 775.2112.

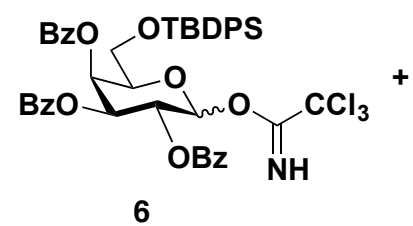

6

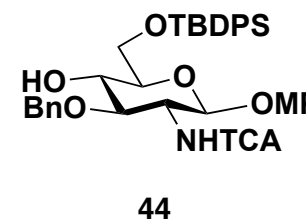

44

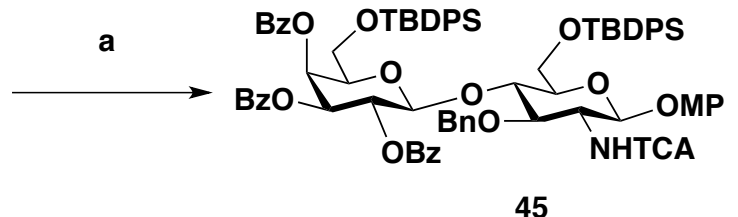

45

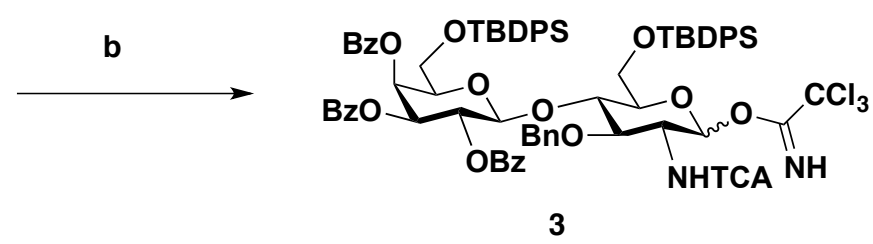

Scheme S4: Synthesis of the disaccharide donor 3

Reagents and conditions: (a) TMSOTf, $\mathrm{CH}_{2} \mathrm{Cl}_{2}, 0{ }^{\circ} \mathrm{C}, 20 \mathrm{~min}, 88 \%$; (b) (i) $\mathrm{CAN}, \mathrm{CH}_{3} \mathrm{CN}: \mathrm{H}_{2} \mathrm{O}$ (4:1), $0{ }^{\circ} \mathrm{C}$ to $25^{\circ} \mathrm{C}, 0.5 \mathrm{~h}$; (ii) $\mathrm{CCl}_{3} \mathrm{CN}, \mathrm{Cs}_{2} \mathrm{CO}_{3}, \mathrm{CH}_{2} \mathrm{Cl}_{2}, 0{ }^{\circ} \mathrm{C}$ to $25^{\circ} \mathrm{C}, 3 \mathrm{~h}, 77 \%$ (over 2 steps).

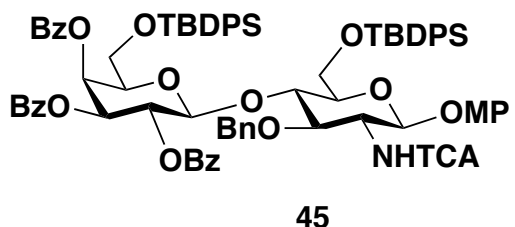

4-Methoxyphenyl

(2,3,4-tri- $O$-benzoyl-6- $O$-t-butyldiphenylsilyl- $\beta$-D-galactopyranosyl)$(1 \rightarrow 4)-3-O$-benzyl-6- $O$-t-butyldiphenylsilyl-2-deoxy-2-trichloroacetamido- $\beta$-D-

glucopyranoside (45): To a vigorously stirred solution of glycosyl donor 6 (0.64 g, $0.74 \mathrm{mmol})$ and glycosyl acceptor $44(0.40 \mathrm{~g}, 0.53 \mathrm{mmol})$ in anhydrous dichloromethane $(10 \mathrm{~mL})$ under argon, catalytic TMSOTf (Trimethylsilyl trifluoromethanesulfonate) $(9.60 \mu \mathrm{L}, 53.0 \mu \mathrm{mol}$ ) was 
added at $0{ }^{\circ} \mathrm{C}$ and the reaction mixture was stirred for $20 \mathrm{~min}$ at the same temperature until the acceptor was completely consumed shown by TLC. The reaction mixture quenched by the addition of triethylamine drop wise until the reaction mixture was neutral to a litmus paper. The reaction mixture was then diluted with dichloromethane $(10 \mathrm{~mL})$ and the solvent was removed under reduced pressure. The resulting crude material was purified by flash column chromatography on silica gel (20\% to $30 \%$ ethyl acetate gradient in hexane) to afford compound $45(0.68 \mathrm{~g}, 0.46 \mathrm{mmol}, 88 \%)$ as a white solid. ${ }^{1} \mathbf{H}$ NMR $\left(400 \mathrm{MHz}, \mathrm{CDCl}_{3}\right) \delta 7.85(\mathrm{~d}, J$ $=7.8 \mathrm{~Hz}, 2 \mathrm{H}), 7.80-7.73(\mathrm{~m}, 3 \mathrm{H}), 7.63(\mathrm{dd}, J=19.9,7.4 \mathrm{~Hz}, 4 \mathrm{H}), 7.55-7.41(\mathrm{~m}, 6 \mathrm{H}), 7.37-$ $7.23(\mathrm{~m}, 10 \mathrm{H}), 7.21-7.10(\mathrm{~m}, 9 \mathrm{H}), 7.02-6.84(\mathrm{~m}, 6 \mathrm{H}), 6.78(\mathrm{~d}, J=9.1 \mathrm{~Hz}, 2 \mathrm{H}), 6.58(\mathrm{~d}, J=$ $9.1 \mathrm{~Hz}, 3 \mathrm{H}), 6.28(\mathrm{~s}, 1 \mathrm{H}), 6.10(\mathrm{~d}, J=3.1 \mathrm{~Hz}, 1 \mathrm{H}), 5.70-5.64(\mathrm{~m}, 1 \mathrm{H}), 5.57(\mathrm{dd}, J=10.4,3.3$ $\mathrm{Hz}, 1 \mathrm{H}), 5.17$ (dd, $J=18.5,8.0 \mathrm{~Hz}, 2 \mathrm{H}), 5.06(\mathrm{~d}, J=10.2 \mathrm{~Hz}, 1 \mathrm{H}), 4.58(\mathrm{~d}, J=10.3 \mathrm{~Hz}, 1 \mathrm{H})$, $4.32(\mathrm{t}, J=8.6 \mathrm{~Hz}, 1 \mathrm{H}), 4.11-4.05(\mathrm{~m}, 1 \mathrm{H}), 3.99(\mathrm{t}, J=7.1 \mathrm{~Hz}, 2 \mathrm{H}), 3.82-3.73(\mathrm{~m}, 3 \mathrm{H}), 3.58$ (s, 3H), $3.13(\mathrm{~d}, J=8.7 \mathrm{~Hz}, 1 \mathrm{H}), 1.04(\mathrm{~s}, 9 \mathrm{H}), 0.90(\mathrm{~s}, 9 \mathrm{H}) .{ }^{13} \mathbf{C}$ NMR $\left(101 \mathrm{MHz}, \mathrm{CDCl}_{3}\right) \delta$ 165.5, 165.4, 163.7, 162.0, 155.6, 151.1, 137.7, 135.9, 135.6, 135.5, 135.4, 135.4, 133.5, 133.3, $133.2,132.5,132.3,131.9,130.4,129.9,129.8,129.6,129.6,129.4,129.1,128.8,128.6,128.5$, $128.4,128.3,128.3,127.9,127.8,127.6,119.2,114.4,100.1,99.1,92.5,77.2,75.5,75.2,75.2$, 73.6, 71.9, 70.6, 67.5, 61.2, 60.4, 60.3, 57.9, 55.6, 26.9, 26.7, 19.4, 19.0. HRMS-ESI-TOF (m/z): $\left[\mathrm{M}+\mathrm{NH}_{4}\right]^{+}$Calculated for $\mathrm{C}_{81} \mathrm{H}_{82} \mathrm{Cl}_{3} \mathrm{NO}_{15} \mathrm{Si}_{2} \mathbf{1 4 8 9 . 4 6 3 3}$, Found 1489.4540.

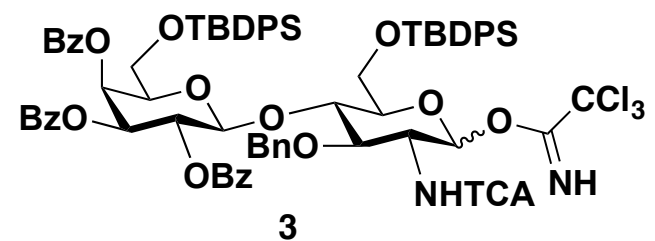

\section{2,3,4-tri- $O$-benzoyl-6- $O$ - $t$-butyldiphenylsilyl- $\beta$-D-galactopyranosyl-( $1 \rightarrow 4)-3-O$-benzyl-6- $O$ - $t$ - butyldiphenylsilyl-2-deoxy-2-trichloroacetamido- $\alpha / \beta$-D-glucopyranosyl} trichloroacetimidate (3): The compound $45(0.60 \mathrm{~g}, 0.41 \mathrm{mmol})$ was successfully converted to the hemiacetal $(0.47 \mathrm{~g}, 0.34 \mathrm{mmol}, 85 \%)$ as pale yellow foam by following the general procedure for OMP ether deprotection using ceric ammonium nitrate (CAN). Complound $\mathbf{3}$ was then synthesized by following the general procedure for trichloroacetimidate formation $(0.47 \mathrm{~g}, 0.31$ mmol, $77 \%$ over 2 steps) as pale yellow oil, which turned to white foam under high vacuum.

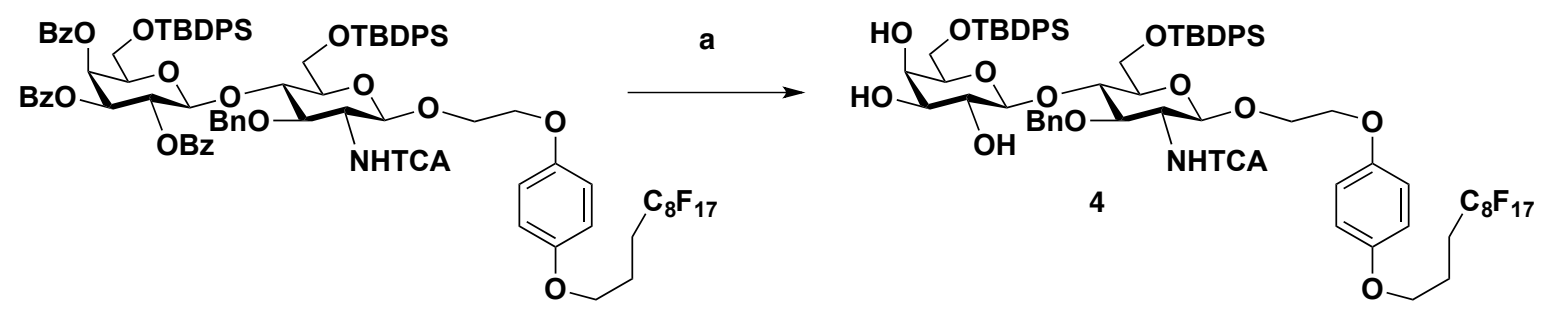

Scheme S5: Synthesis of the disaccharide acceptor 4

Reagents and conditions: (a) $\mathrm{Na}, \mathrm{MeOH}, 25^{\circ} \mathrm{C}, 3 \mathrm{~h}, 96 \%$. 
2-(4-((4,4,5,5,6,6,7,7,8,8,9,9,10,10,11,11,11-heptadecafluoroundecyl)oxy)phenoxy)ethyl (6-O$t$-butyldiphenylsilyl- $\beta$-D-galactopyranosyl)-( $\rightarrow 4)-3-O$-benzyl-6-O-t-butyldiphenylsilyl-2deoxy-2-trichloroacetamido- $\boldsymbol{\beta}$-D-glucopyranoside (4): To a solution of the disaccharide $(0.1 \mathrm{~g}$, $0.05 \mathrm{mmol})$ in methanol $(3 \mathrm{~mL})$ was added a pinch of solid sodium metal at room temperature under argon. The reaction mixture was stirred for $3 \mathrm{~h}$ at room temperature and quenched by addition Dowex ${ }^{\circledR}\left(50 \mathrm{~W}\right.$ X8, 200-400 mesh) $\left(\mathrm{H}^{+}\right)$cation exchange resin. The resin was filtered over a pad of Celite ${ }^{\circledR}$, rinsed with methanol. The methanol was removed in vacuo and trace methanol or water was removed by co-evaporation with toluene $(3 \times 5 \mathrm{~mL})$. The crude white solid product $4(0.08 \mathrm{~g}, 0.05 \mathrm{mmol})$ was used directly in the next step. ${ }^{1} \mathbf{H}$ NMR $\left(400 \mathrm{MHz}, \mathrm{CDCl}_{3}\right) \delta$ $7.74-7.57(\mathrm{~m}, 8 \mathrm{H}), 7.44-7.28(\mathrm{~m}, 12 \mathrm{H}), 7.22(\mathrm{~d}, J=6.6 \mathrm{~Hz}, 2 \mathrm{H}), 7.11-6.96(\mathrm{~m}, 4 \mathrm{H}), 6.77(\mathrm{~s}$, 4H), 4.99 (d, $J=7.7 \mathrm{~Hz}, 1 \mathrm{H}), 4.87(\mathrm{~d}, J=10.5 \mathrm{~Hz}, 1 \mathrm{H}), 4.69$ (d, $J=7.7 \mathrm{~Hz}, 1 \mathrm{H}), 4.54(\mathrm{~d}, J=$ $10.5 \mathrm{~Hz}, 1 \mathrm{H}), 4.24-4.10(\mathrm{~m}, 4 \mathrm{H}), 4.08-3.94(\mathrm{~m}, 6 \mathrm{H}), 3.87-3.73(\mathrm{~m}, 3 \mathrm{H}), 3.69-3.63(\mathrm{~m}$, $1 \mathrm{H}), 3.57-3.44(\mathrm{~m}, 4 \mathrm{H}), 2.38-2.22(\mathrm{~m}, 2 \mathrm{H}), 2.10-2.04(\mathrm{~m}, 2 \mathrm{H}), 1.03(\mathrm{~d}, J=3.7 \mathrm{~Hz}, 18 \mathrm{H})$. ${ }^{13}$ C NMR $\left(101 \mathrm{MHz}, \mathrm{CDCl}_{3}\right) \delta 161.9,153.1,152.9,137.8,135.9,135.6,135.5,135.4,133.6$, $132.9,132.9,132.8,129.9,129.8,129.8,129.7,129.6,128.3,128.2,127.8,127.8,127.8,127.7$, 127.6, 127.6, 115.6, 115.4, 102.3, 99.1, 92.4, 77.3, 75.7, 75.7, 74.4, 74.3, 73.9, 72.6, 67.9, 67.7, $67.6,66.9,62.0,61.6,57.5,29.7,26.8,26.8,20.7,19.4,19.2$. HRMS-ESI-TOF (m/z): $\left[\mathrm{M}+\mathrm{NH}_{4}\right]^{+}$Calculated for $\mathrm{C}_{72} \mathrm{H}_{77} \mathrm{Cl}_{3} \mathrm{~F}_{17} \mathrm{NO}_{13} \mathrm{Si}_{2}$ 1667.4089, Found 1667.4119.

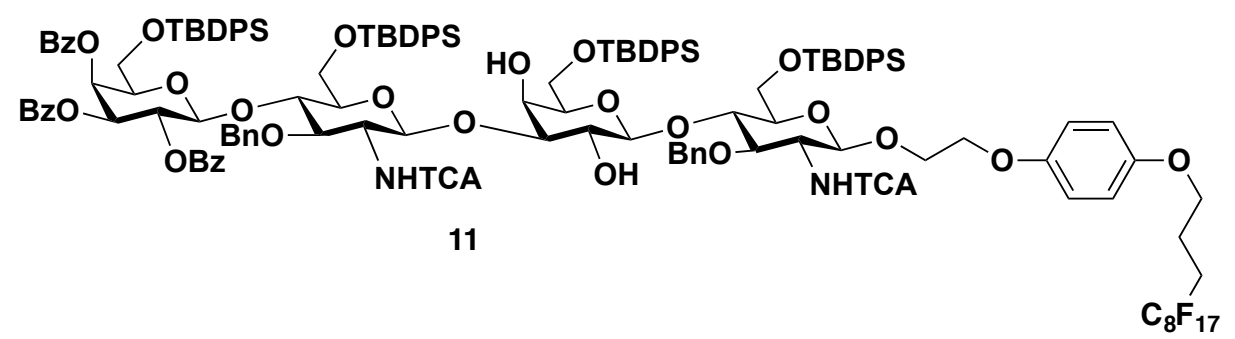

2-(4-((4,4,5,5,6,6,7,7,8,8,9,9,10,10,11,11,11-heptadecafluoroundecyl)oxy)phenoxy)ethyl $(2,3,4$-tri- $O$-benzoyl-6- $O$ - $t$-butyldiphenylsilyl- $\beta$-D-galactopyranosyl)-(1 $\rightarrow 4)-(3-O$-benzyl-6$O$-t-butyldiphenylsilyl-2-deoxy-2-trichloroacetamido- $\beta$-D-glucopyranosyl)-(1 $\rightarrow 3)-(6-O-t$ butyldiphenylsilyl- $\beta$-D-galactopyranosyl)-( $\rightarrow 4)-3-O$-benzyl-6-O-t-butyldiphenylsilyl-2deoxy-2-trichloroacetamido- $\boldsymbol{\beta}$-D-glucopyranoside (11): To a vigorously stirred solution of glycosyl donor 3 (187 mg, $0.124 \mathrm{mmol})$ and glycosyl acceptor 4 (146 $\mathrm{mg}, 88.0 \mu \mathrm{mol})$ in anhydrous dichloromethane $(3 \mathrm{~mL})$ under argon, catalytic TMSOTf (Trimethylsilyl trifluoromethanesulfonate) $(1.6 \mu \mathrm{L}, 8.8 \mu \mathrm{mol})$ was added at $0{ }^{\circ} \mathrm{C}$ and the reaction mixture was stirred for $20 \mathrm{~min}$ at the same temperature until the acceptor was completely consumed shown by TLC. The reaction mixture quenched by the addition of triethylamine drop wise until the reaction mixture was neutral to a litmus paper. The reaction mixture was then diluted with dichloromethane $(10 \mathrm{~mL})$ and the solvent was removed under reduced pressure. The resulting crude material was purified via FSPE to afford the tetrasaccharide $11(0.230 \mathrm{~g}, 77.0 \mu \mathrm{mol}, 87 \%)$ as white foam. ${ }^{1} \mathbf{H}$ NMR $\left(400 \mathrm{MHz} \mathrm{CDCl}_{3}\right) \delta 7.93(\mathrm{~d}, J=7.2 \mathrm{~Hz}, 2 \mathrm{H}), 7.85-7.77(\mathrm{~m}, 4 \mathrm{H}), 7.70$ $-7.51(\mathrm{~m}, 18 \mathrm{H}), 7.43-7.16(\mathrm{~m}, 35 \mathrm{H}), 7.10-6.96(\mathrm{~m}, 9 \mathrm{H}), 6.75(\mathrm{~s}, 4 \mathrm{H}), 6.14(\mathrm{~d}, J=3.2 \mathrm{~Hz}$, 
$1 \mathrm{H}), 5.77-5.70(\mathrm{~m}, 1 \mathrm{H}), 5.56(\mathrm{dd}, J=10.5,3.4 \mathrm{~Hz}, 1 \mathrm{H}), 5.19-5.09(\mathrm{~m}, 2 \mathrm{H}), 4.97(\mathrm{~d}, J=7.4$ $\mathrm{Hz}, 1 \mathrm{H}), 4.87-4.80(\mathrm{~m}, 1 \mathrm{H}), 4.71(\mathrm{~d}, J=10.6 \mathrm{~Hz}, 1 \mathrm{H}), 4.64(\mathrm{t}, J=7.4 \mathrm{~Hz}, 1 \mathrm{H}), 4.52(\mathrm{~d}, J=$ $10.4 \mathrm{~Hz}, 1 \mathrm{H}), 4.43(\mathrm{q}, J=9.0 \mathrm{~Hz}, 1 \mathrm{H}), 4.22-3.91(\mathrm{~m}, 13 \mathrm{H}), 3.88-3.75(\mathrm{~m}, 7 \mathrm{H}), 3.71-3.61$ $(\mathrm{m}, 2 \mathrm{H}), 3.47(\mathrm{~m}, 4 \mathrm{H}), 3.19(\mathrm{~d}, J=8.9 \mathrm{~Hz}, 1 \mathrm{H}), 2.64(\mathrm{~s}, 1 \mathrm{H}), 2.49(\mathrm{~s}, 1 \mathrm{H}), 2.37-2.21(\mathrm{~m}, 2 \mathrm{H})$, $2.06(\mathrm{~m}, 2 \mathrm{H}), 1.11(\mathrm{~s}, 9 \mathrm{H}), 0.98(\mathrm{~d}, J=11.6 \mathrm{~Hz}, 18 \mathrm{H}), 0.80(\mathrm{~s}, 9 \mathrm{H}) .{ }^{13} \mathbf{C}$ NMR $(101 \mathrm{MHz}$, $\left.\mathrm{CDCl}_{3}\right) \delta 165.4,165.2,164.8,162.1,161.7,153.1,152.8,137.7,137.6,135.8,135.7,135.6$, 135.5, 135.4, 135.4, 135.3, 133.5, 133.3, 133.2, 133.2, 133.1, 132.8, 132.7, 132.5, 132.2, 132.1, $130.5,130.1,129.8,129.7,129.6,129.1,128.7,128.6,128.5,128.4,128.4,128.2,127.9,127.8$, 127.7, 127.6, 127.5, 115.5, 115.4, 101.9, 99.9, 99.7, 99.0, 92.5, 92.4, 81.9, 77.7, 76.1, 75.7, 75.5, $75.1,74.9,74.6,74.1,73.4,71.7,71.3,70.5,67.7,67.6,67.4,67.1,66.9,62.1,61.6,60.2,57.8$, 57.4, 29.7，26.9，26.7，26.7，26.6，20.6，19.4，19.3，19.0，18.9. HRMS-ESI-TOF (m/z): $\left[\mathrm{M}+2 \mathrm{NH}_{4}\right]^{2+}$ Calculated for $\mathrm{C}_{146} \mathrm{H}_{151} \mathrm{Cl}_{6} \mathrm{~F}_{17} \mathrm{~N}_{2} \mathrm{O}_{26} \mathrm{Si}_{4}$ 1516.4095, Found 1516.4053.

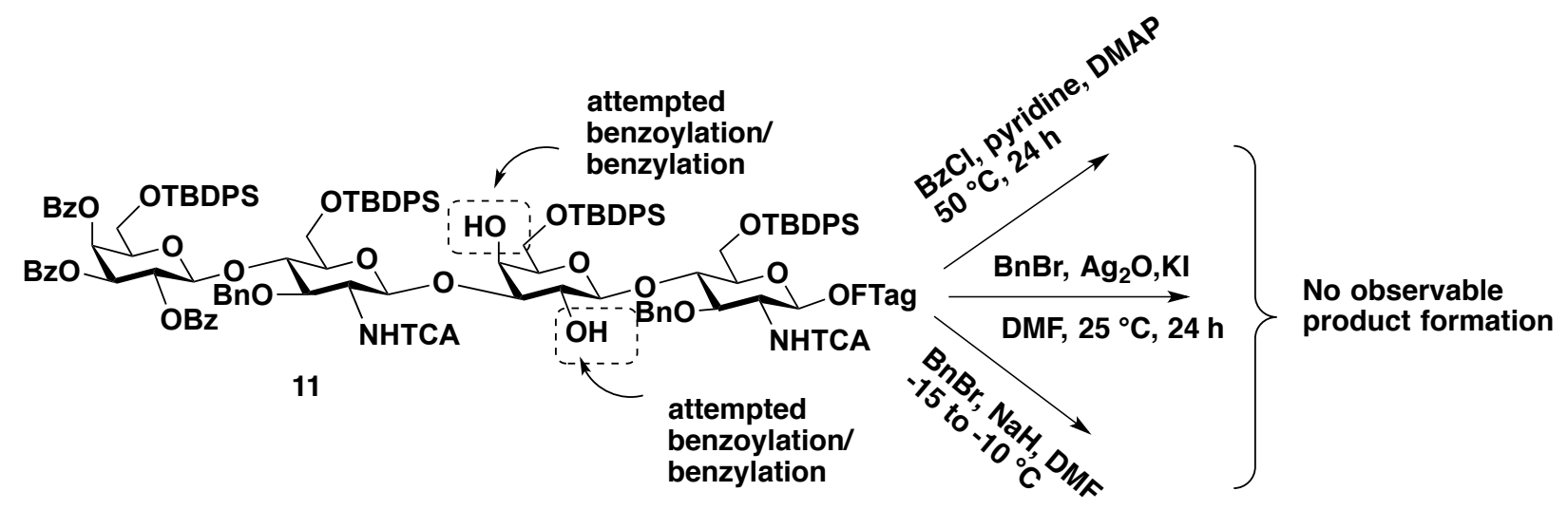

Scheme S6: Attempted benzoylation and benzylation reaction on the tetrasaccharide 11 to protect the sterically hindered secondary hydroxyl groups failed. No desired product formation was observed.

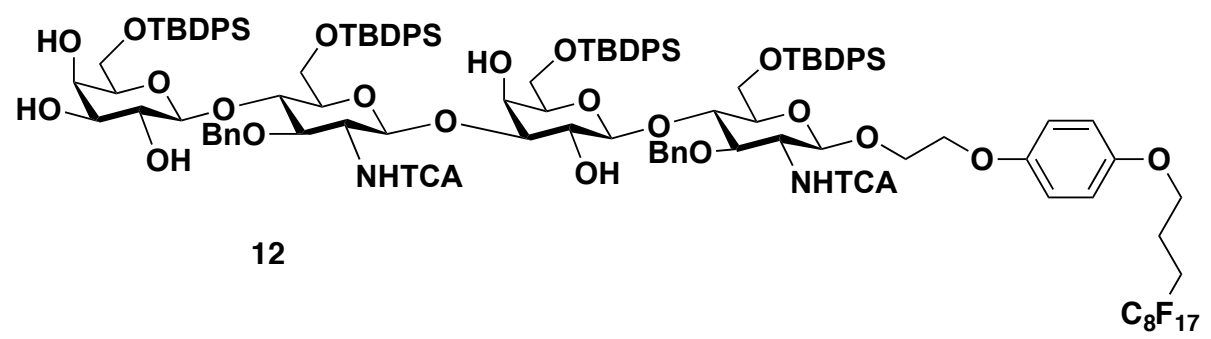

2-(4-((4,4,5,5,6,6,7,7,8,8,9,9,10,10,11,11,11-heptadecafluoroundecyl)oxy)phenoxy)ethyl (6-O$t$-butyldiphenylsilyl- $\beta$-D-galactopyranosyl)-( $1 \rightarrow 4)$-(3-O-benzyl-6-O-t-butyldiphenylsilyl-2deoxy-2-trichloroacetamido- $\beta$-D-glucopyranosyl)-(1 $\rightarrow 3)-(6-O-t$-butyldiphenylsilyl- $\beta$-Dgalactopyranosyl)-(1 $\rightarrow 4)-3-O$-benzyl-6- $O$ - $t$-butyldiphenylsilyl-2-deoxy-2-

trichloroacetamido- $\boldsymbol{\beta}$-D-glucopyranoside (12): To a solution of compound $11(0.20 \mathrm{~g}, 67$ $\mu \mathrm{mol})$ in methanol $(3 \mathrm{~mL})$ was added a pinch of solid sodium metal at room temperature under 
argon. The reaction mixture was stirred for $3 \mathrm{~h}$ at room temperature and quenched by addition Dowex ${ }^{\circledR}(50 \mathrm{~W} X 8,200-400 \mathrm{mesh})\left(\mathrm{H}^{+}\right)$cation exchange resin. The resin was filtered over a pad of Celite ${ }^{\circledR}$, rinsed with methanol. The methanol was removed in vacuo and trace methanol or water was removed by co-evaporation with toluene $(3 \times 5 \mathrm{~mL})$. The crude white solid product $12(0.176 \mathrm{~g}, 50.0 \mu \mathrm{mol}, 98 \%)$ was used directly in the next step. ${ }^{1} \mathbf{H}$ NMR $(400 \mathrm{MHz}$, $\left.\mathrm{CDCl}_{3}\right) \delta 7.63(\mathrm{~m}, 17 \mathrm{H}), 7.45-7.17(\mathrm{~m}, 30 \mathrm{H}), 7.14-6.99(\mathrm{~m}, 6 \mathrm{H}), 6.76(\mathrm{~s}, 4 \mathrm{H}), 5.28(\mathrm{~d}, J=7.8$ $\mathrm{Hz}, 1 \mathrm{H}), 4.97$ (d, $J=7.2 \mathrm{~Hz}, 1 \mathrm{H}), 4.85$ (dd, $J=16.6,10.7 \mathrm{~Hz}, 2 \mathrm{H}), 4.61$ (ddd, $J=43.7,22.6,9.0$ $\mathrm{Hz}, 3 \mathrm{H}), 4.23$ (dd, $J=19.6,11.3 \mathrm{~Hz}, 2 \mathrm{H}), 4.15-3.92(\mathrm{~m}, 13 \mathrm{H}), 3.89-3.71(\mathrm{~m}, 7 \mathrm{H}), 3.67-3.47$ (m, 7H), $3.44-3.39(\mathrm{~m}, 2 \mathrm{H}), 2.75-2.65(\mathrm{~m}, 5 \mathrm{H}), 2.29(\mathrm{~m}, 2 \mathrm{H}), 2.10-2.01(\mathrm{~m}, 2 \mathrm{H}), 1.02(\mathrm{~d}, J$ $=10.1 \mathrm{~Hz}, 27 \mathrm{H}), 0.93(\mathrm{~s}, 9 \mathrm{H}) .{ }^{13} \mathbf{C}$ NMR $\left(101 \mathrm{MHz}, \mathrm{CDCl}_{3}\right) \delta 162.2,161.7,153.1,152.8,137.8$, 135.8, 135.7, 135.6, 135.4, 135.4, 133.6, 133.4, 133.1, 133.0, 132.9, 132.8, 132.5, 129.9, 129.8, $129.7,128.6,128.3,128.2,128.2,127.8,127.8,127.8,127.7,127.7,127.7,127.6,127.5,115.6$, $115.4,102.3,101.9,99.8,99.0,92.5,92.4,81.2,78.0,76.9,76.4,75.8,75.7,75.3,74.4,74.3$, $74.2,73.9,72.6,71.6,67.9,67.7,67.5,66.9,62.2,62.1,61.9,61.7,57.6,57.0,46.0,29.7,27.1$, $26.8,26.8,26.7,26.6,20.6,19.4,19.3,19.2,19.1$.

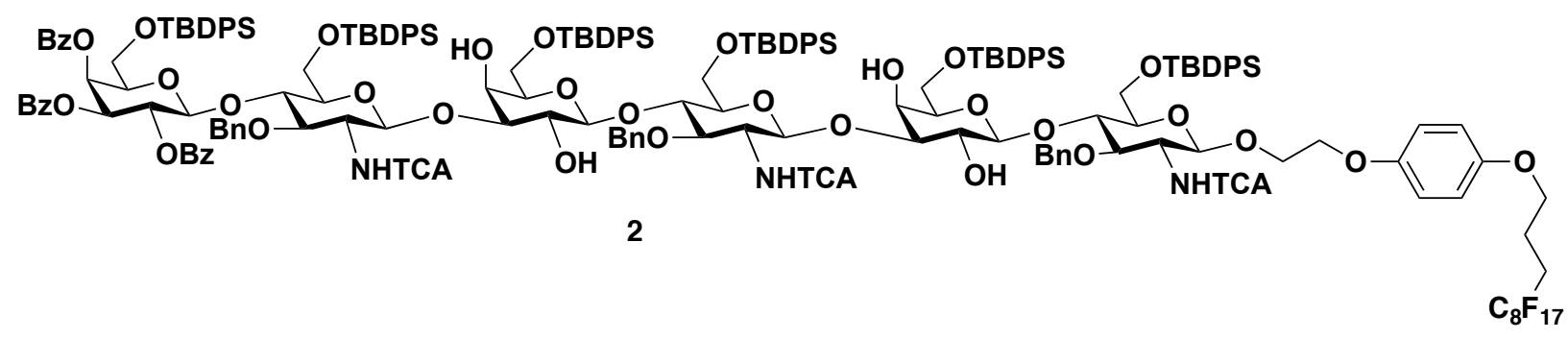

\section{2-(4-((4,4,5,5,6,6,7,7,8,8,9,9,10,10,11,11,11-heptadecafluoroundecyl)oxy)phenoxy)ethyl} (2,3,4-tri-O-benzoyl-6-O-t-butyldiphenylsilyl- $\beta$-D-galactopyranosyl)-(1 $\rightarrow 4)-(3-O$-benzyl-6$O$-t-butyldiphenylsilyl-2-deoxy-2-trichloroacetamido- $\beta$-D-glucopyranosyl)-(1 $\rightarrow 3)-(6-O-t$ butyldiphenylsilyl- $\beta$-D-galactopyranosyl)-( $\rightarrow 4)$-(3-O-benzyl-6- $O$ - $t$-butyldiphenylsilyl-2deoxy-2-trichloroacetamido- $\beta$-D-glucopyranosyl)-(1 $\rightarrow 3)-(6-O-t$-butyldiphenylsilyl- $\beta$-Dgalactopyranosyl)-(1 $\rightarrow 4)$-3- $O$-benzyl-6- $O$ - $t$-butyldiphenylsilyl-2-deoxy-2-

trichloroacetamido- $\beta$-D-glucopyranoside (2): To a vigorously stirred solution of glycosyl donor $3(0.17 \mathrm{~g}, 0.11 \mathrm{mmol})$ and glycosyl acceptor $12(0.20 \mathrm{~g}, 0.074 \mu \mathrm{mol})$ in anhydrous dichloromethane $(3 \mathrm{~mL})$ under argon, catalytic TMSOTf (Trimethylsilyl trifluoromethanesulfonate) $(1.3 \mu \mathrm{L}, 7.4 \mu \mathrm{mol})$ was added at $0{ }^{\circ} \mathrm{C}$ and the reaction mixture was stirred for $20 \mathrm{~min}$ at the same temperature until the acceptor was completely consumed shown by TLC. The reaction mixture quenched by the addition of triethylamine drop wise until the reaction mixture was neutral to a litmus paper. The reaction mixture was then diluted with dichloromethane $(10 \mathrm{~mL})$ and the solvent was removed under reduced pressure. The resulting crude material was purified via FSPE. The fluorous fraction was concentrated and further purified by flash column chromatography on silica gel (25\% to $30 \%$ ethyl acetate gradient in hexane) to afford the hexasaccharide $2(0.230 \mathrm{~g}, 58.0 \mu \mathrm{mol}, 78 \%)$ as white foam. ${ }^{1} \mathbf{H}$ NMR $\left(400 \mathrm{MHz}, \mathrm{CDCl}_{3}\right) \delta 7.93(\mathrm{~d}, J=7.5 \mathrm{~Hz}, 2 \mathrm{H}), 7.81(\mathrm{t}, J=8.4 \mathrm{~Hz}, 3 \mathrm{H}), 7.71-7.64(\mathrm{~m}, 7 \mathrm{H}), 7.57$ 
(m, 18H), $7.43-7.15(\mathrm{~m}, 48 \mathrm{H}), 7.05(\mathrm{~m}, 15 \mathrm{H}), 6.76(\mathrm{~s}, 4 \mathrm{H}), 6.14(\mathrm{~d}, J=2.7 \mathrm{~Hz}, 1 \mathrm{H}), 5.74(\mathrm{dd}, J$ $=10.3,8.1 \mathrm{~Hz}, 1 \mathrm{H}), 5.56(\mathrm{dd}, J=10.5,3.2 \mathrm{~Hz}, 1 \mathrm{H}), 5.23-5.09(\mathrm{~m}, 3 \mathrm{H}), 4.97(\mathrm{~d}, J=7.3 \mathrm{~Hz}$, $1 \mathrm{H}), 4.83(\mathrm{dd}, J=10.4,2.9 \mathrm{~Hz}, 2 \mathrm{H}), 4.68(\mathrm{dd}, J=21.9,9.1 \mathrm{~Hz}, 2 \mathrm{H}), 4.60-4.49(\mathrm{~m}, 3 \mathrm{H}), 4.42(\mathrm{t}$, $J=8.6 \mathrm{~Hz}, 1 \mathrm{H}), 4.23(\mathrm{~m}, 2 \mathrm{H}), 4.15-3.88(\mathrm{~m}, 18 \mathrm{H}), 3.86-3.77(\mathrm{~m}, 7 \mathrm{H}), 3.74-3.62(\mathrm{~m}, 4 \mathrm{H})$, $3.58-3.40(\mathrm{~m}, 6 \mathrm{H}), 3.36(\mathrm{~d}, J=6.7 \mathrm{~Hz}, 1 \mathrm{H}), 3.18(\mathrm{~d}, J=8.7 \mathrm{~Hz}, 1 \mathrm{H}), 2.67(\mathrm{~d}, J=12.9 \mathrm{~Hz}, 1 \mathrm{H})$, $2.57(\mathrm{~s}, 2 \mathrm{H}), 2.49(\mathrm{~s}, 1 \mathrm{H}), 2.29(\mathrm{~m}, 2 \mathrm{H}), 2.07(\mathrm{~m}, 2 \mathrm{H}), 1.11(\mathrm{~s}, 9 \mathrm{H}), 1.02-0.95(\mathrm{~m}, 27 \mathrm{H}), 0.91$ $(\mathrm{s}, 9 \mathrm{H}), 0.81(\mathrm{~s}, 9 \mathrm{H}) .{ }^{13} \mathrm{C}$ NMR $\left(101 \mathrm{MHz}, \mathrm{CDCl}_{3}\right) \delta 165.4,165.2,164.8,162.1,162.0,161.7$, $153.1,152.8,137.7,137.6,135.8,135.7,135.6,135.5,135.6,135.4,135.3,133.6,133.3,133.2$, $133.1,133.0,132.8,132.7,132.5,132.2,132.0,130.6,130.2,129.8,129.7,129.6,129.1,128.7$, $128.6,128.5,128.4,128.4,128.3,128.2,127.9,127.8,127.7,127.6,115.6,115.4,102.1,101.9$, $100.0,99.9,99.6,99.0,92.5,92.4,81.8,81.4,77.7,77.6,77.3,77.0,76.7,76.1,75.7,75.5,75.4$, $75.1,74.9,74.6,74.5,74.3,74.0,73.4,72.7,71.7,71.5,71.2,70.5,67.7,67.5,67.4,67.2,66.9$, $62.3,62.2,62.1,61.8,61.7,61.4,60.2,57.8,57.5,57.1,53.4,29.7,26.9,26.8,26.7,26.6,20.6$, 19.4, 19.3, 19.1, 19.0, 18.9. HRMS-ESI-TOF $(\mathbf{m} / \mathbf{z}):[\mathrm{M}+2 \mathrm{Na}]^{2+}$ Calculated for $\mathrm{C}_{199} \mathrm{H}_{211} \mathrm{Cl}_{9} \mathrm{~F}_{17} \mathrm{~N}_{3} \mathrm{O}_{36} \mathrm{Si}_{6}$ 2039.5137, Found 2039.5066.

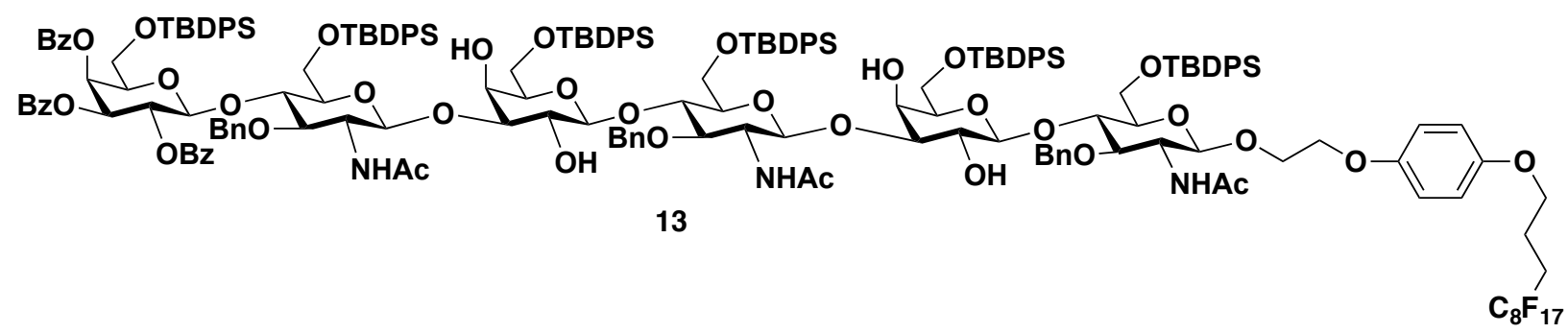

\section{2-(4-((4,4,5,5,6,6,7,7,8,8,9,9,10,10,11,11,11-heptadecafluoroundecyl)oxy)phenoxy)ethyl} $(2,3,4-$ tri- $O$-benzoyl-6-O-t-butyldiphenylsilyl- $\beta$-D-galactopyranosyl)-(1 $\rightarrow 4)$-(2-acetamido-3$O$-benzyl-6- $O$-t-butyldiphenylsilyl-2-deoxy- $\beta$-D-glucopyranosyl)-(1 $\rightarrow 3)-(6-O$ - $t$ butyldiphenylsilyl- $\beta$-D-galactopyranosyl)-( $1 \rightarrow 4)-(2$-acetamido-3-O-benzyl-6- $O$ - $t$ butyldiphenylsilyl-2-deoxy- $\beta$-D-glucopyranosyl)-(1 $\rightarrow 3)-(6-O$ - $t$-butyldiphenylsilyl- $\beta$-Dgalactopyranosyl)-(1 $\rightarrow 4)$-2-acetamido-3- $O$-benzyl-6- $O$ - $t$-butyldiphenylsilyl-2-deoxy- $\boldsymbol{\beta}$-Dglucopyranoside (13): Complound $13(18.0 \mathrm{mg}, 4.80 \mu \mathrm{mol}, 57 \%)$ as a white solid was then successfully synthesized from compound $2(35.0 \mathrm{mg}, 8.70 \mu \mathrm{mol})$ by following the general procedure for conversion of $N$-trichloroacetamide (NHTCA) to $N$-acetyl (NHAc) and was purified via FSPE. ${ }^{1} \mathbf{H}$ NMR $\left(500 \mathrm{MHz}, \mathrm{CD}_{3} \mathrm{OD}\right) \delta 7.98(\mathrm{~d}, J=8.1 \mathrm{~Hz}, 1 \mathrm{H}), 7.87(\mathrm{~d}, J=7.6 \mathrm{~Hz}, 1 \mathrm{H}), 7.81$ $(\mathrm{d}, J=8.1 \mathrm{~Hz}, 2 \mathrm{H}), 7.77-7.55(\mathrm{~m}, 24 \mathrm{H}), 7.50(\mathrm{~m}, 4 \mathrm{H}), 7.43-7.19(\mathrm{~m}, 42 \mathrm{H}), 7.18-7.12(\mathrm{~m}$, 4H), $7.09-6.95(\mathrm{~m}, 13 \mathrm{H}), 6.83(\mathrm{~s}, 4 \mathrm{H}), 6.59(\mathrm{t}, J=7.2 \mathrm{~Hz}, 1 \mathrm{H}), 6.12(\mathrm{~d}, J=2.8 \mathrm{~Hz}, 1 \mathrm{H}), 5.80-$ $5.73(\mathrm{~m}, 1 \mathrm{H}), 5.55(\mathrm{dd}, J=10.4,3.0 \mathrm{~Hz}, 1 \mathrm{H}), 5.28(\mathrm{~d}, J=8.0 \mathrm{~Hz}, 1 \mathrm{H}), 5.22-5.11(\mathrm{~m}, 2 \mathrm{H}), 5.06$ $(\mathrm{d}, J=10.9 \mathrm{~Hz}, 1 \mathrm{H}), 4.99$ (dd, $J=9.3,6.6 \mathrm{~Hz}, 2 \mathrm{H}), 4.81$ (d, $J=7.3 \mathrm{~Hz}, 2 \mathrm{H}), 4.68$ (dd, $J=20.7$, $8.9 \mathrm{~Hz}, 2 \mathrm{H}), 4.58(\mathrm{~d}, J=10.9 \mathrm{~Hz}, 1 \mathrm{H}), 4.54-4.49(\mathrm{~m}, 1 \mathrm{H}), 4.47-4.32(\mathrm{~m}, 4 \mathrm{H}), 4.31-4.21(\mathrm{~m}$, $3 \mathrm{H}), 4.15-3.84(\mathrm{~m}, 18 \mathrm{H}), 3.83-3.62(\mathrm{~m}, 15 \mathrm{H}), 3.47-3.40(\mathrm{~m}, 2 \mathrm{H}), 3.17(\mathrm{~d}, J=9.5 \mathrm{~Hz}, 1 \mathrm{H})$, $2.38(\mathrm{dd}, J=16.4,8.3 \mathrm{~Hz}, 2 \mathrm{H}), 2.33(\mathrm{~s}, 1 \mathrm{H}), 2.29(\mathrm{~s}, 1 \mathrm{H}), 2.07-2.02(\mathrm{~m}, 2 \mathrm{H}), 2.03-1.92(\mathrm{~m}$, $6 \mathrm{H}), 1.83$ (s, 3H), 1.09 (s, 9H), 1.03 (s, 9H), 0.98 (d, $J=11.3 \mathrm{~Hz}, 18 \mathrm{H}), 0.90$ (d, $J=7.3 \mathrm{~Hz}$, 
18H). ${ }^{13} \mathbf{C}$ NMR $\left(126 \mathrm{MHz}, \mathrm{CD}_{3} \mathrm{OD}\right) \delta 172.7,172.6,172.5,165.5,165.1,153.2,152.9,138.9$, 138.7, 138.5, 135.6, 135.4, 135.4, 135.3, 135.3, 135.2, 135.0, 133.6, 133.3, 133.2, 133.2, 133.1, $132.8,132.3,132.2,130.3,129.9,129.7,129.5,129.4,128.4,128.1,127.9,127.8,127.7,127.6$, 127.5, 127.4, 127.3, 126.9, 115.4, 115.2, 102.9, 102.7, 101.3, 101.3, 100.7, 99.9, 79.8, 75.9, 75.7, $75.5,75.3,75.2,74.9,74.8,74.5,73.8,73.7,73.2,72.2,72.1,71.6,70.5,67.9,67.8,67.6,67.2$, 66.7, 62.1, 60.2, 55.2, 55.1, 53.3, 31.7, 29.4, 27.5, 26.4, 26.3, 26.3, 26.1, 21.9, 20.4, 19.1, 19.0, 18.7, 18.5. HRMS-ESI-TOF (m/z): $[\mathrm{M}+2 \mathrm{H}]^{2+}$ Calculated for $\mathrm{C}_{199} \mathrm{H}_{222} \mathrm{~F}_{17} \mathrm{~N}_{3} \mathrm{O}_{36} \mathrm{Si}_{6} \mathbf{1 8 6 1 . 7 0 8 1}$, Found 1861.7097.

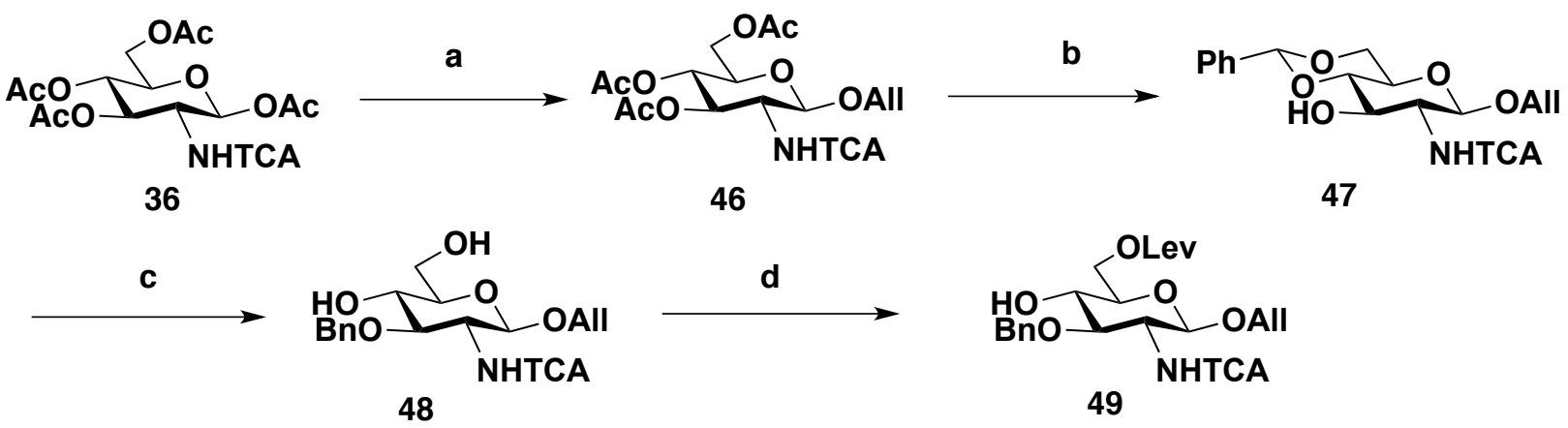

Scheme S7: Synthesis of Glucosamine acceptor 49

Reagents and conditions: (a) (i) $\mathrm{HBr} / \mathrm{AcOH}, \mathrm{CH}_{2} \mathrm{Cl}_{2}, 0{ }^{\circ} \mathrm{C}$ to $25{ }^{\circ} \mathrm{C}, 2 \mathrm{~h}$; (ii) Allyl alcohol, $\mathrm{InCl}_{3}, \mathrm{CH}_{2} \mathrm{Cl}_{2}, 25^{\circ} \mathrm{C}, 48 \mathrm{~h}, 71 \%$ (over 2 steps); (b) (i) $\mathrm{Na}, \mathrm{MeOH}, 25^{\circ} \mathrm{C}, 3 \mathrm{~h}$; (ii) $\mathrm{PhCH}(\mathrm{OMe})_{2}$, PTSA, $\mathrm{CH}_{3} \mathrm{CN}, 25{ }^{\circ} \mathrm{C}, 3 \mathrm{~h}, 78 \%$ (over 2 steps); (c) (i) $\mathrm{NaH}, \mathrm{BnBr}, \mathrm{DMF},-20{ }^{\circ} \mathrm{C}--15{ }^{\circ} \mathrm{C}, 5 \mathrm{~h}$; (ii) TFA (40\%), $\mathrm{CH}_{2} \mathrm{Cl}_{2}, 0{ }^{\circ} \mathrm{C}$ to $30{ }^{\circ} \mathrm{C}, 4 \mathrm{~h}, 64 \%$ (over 2 steps); (d) LevOH, CMPI, DABCO, $\mathrm{CH}_{2} \mathrm{Cl}_{2}, 25^{\circ} \mathrm{C}, 48 \mathrm{~h}, 83 \%$.

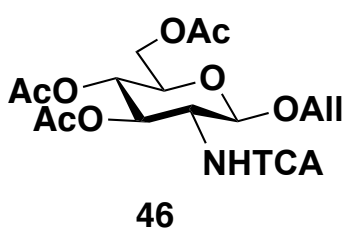

Allyl 3,4,6-tri- $O$-acetyl-2-deoxy-2-trichloroacetamido- $\beta$-D-glucopyranoside (46): Compound $36^{[1]}(5.00 \mathrm{~g}, 10.1 \mathrm{mmol})$ was dissolved in anhydrous dichloromethane $(20 \mathrm{~mL})$ and cooled to 0 ${ }^{\circ} \mathrm{C} . \mathrm{HBr} / \mathrm{AcOH}(7.10 \mathrm{~mL}, 111.6 \mathrm{mmol})$ was added drop wise and the reaction was stirred for 0.5 $\mathrm{h}$ at the same temperature. Then, the temperature was raised to room temperature and run for another $1.5 \mathrm{~h}$ until the starting material was completely consumed by TLC. The reaction was diluted with dichloromethane $(30 \mathrm{~mL})$, washed with saturated solution of sodium bicarbonate $(3 \mathrm{X} 30 \mathrm{~mL})$. The organic phase was dried over $\mathrm{Na}_{2} \mathrm{SO}_{4}$ and the solvent was removed in vacuo. The resulting crude oil $(5.10 \mathrm{~g}, 9.93 \mathrm{mmol})$ was dried under high vac and directly used for the next step. To a solution of the resulting crude oil $(5.10 \mathrm{~g}, 9.93 \mathrm{mmol})$ in anhydrous dichloromethane $(20 \mathrm{~mL})$ was added allyl alcohol $(1.35 \mathrm{~mL}, 19.9 \mathrm{mmol})$ at room temperature. Molecular sieves $\left(4 \mathrm{~A}^{\circ}\right.$ ) were added to the solution and it was stirred for $2 \mathrm{~h}$. $\mathrm{InCl}_{3}$ (Indium 
chloride) (1.09 g, $4.96 \mathrm{mmol})$ was then added in portion and the reaction was stirred for $48 \mathrm{~h}$ until the complete consumption of the starting material. The reaction mixture was diluted with dichloromethane $(30 \mathrm{~mL})$ and filtered over a pad of Celite ${ }^{\circledR}$. The filtrate was washed with water $(3 \mathrm{X} 30 \mathrm{~mL})$ and brine solution $(3 \mathrm{X} 30 \mathrm{~mL})$. The organic layer was dried over $\mathrm{Na}_{2} \mathrm{SO}_{4}$. The solvent was removed in vасио and the resulting crude oil was purified by flash column chromatography on silica gel (25\% to $35 \%$ ethyl acetate gradient in hexane) to afford compound 46 (3.54 g, $7.21 \mathrm{mmol}, 71 \%$ over 2 steps) as a white solid. ${ }^{1} \mathbf{H}$ NMR (400 MHz, $\left.\mathrm{CDCl}_{3}\right) \delta 7.02(\mathrm{~d}, J=8.9 \mathrm{~Hz}, 1 \mathrm{H}), 5.84-5.71(\mathrm{~m}, 1 \mathrm{H}), 5.34(\mathrm{dd}, J=10.6,9.4 \mathrm{~Hz}, 1 \mathrm{H}), 5.21(\mathrm{dd}$, $J=17.2,1.5 \mathrm{~Hz}, 1 \mathrm{H}), 5.15-5.01(\mathrm{~m}, 2 \mathrm{H}), 4.68(\mathrm{~d}, J=8.3 \mathrm{~Hz}, 1 \mathrm{H}), 4.26(\mathrm{~m}, 2 \mathrm{H}), 4.13-3.92$ $(\mathrm{m}, 3 \mathrm{H}), 3.69(\mathrm{~m}, 1 \mathrm{H}), 2.03(\mathrm{~s}, 3 \mathrm{H}), 1.98-1.94(\mathrm{~d}, 6 \mathrm{H}) .{ }^{13} \mathbf{C}$ NMR $\left(101 \mathrm{MHz}, \mathrm{CDCl}_{3}\right) \delta 170.8$, $170.6,169.2,161.9,133.1,117.9,99.2,92.3,71.9,71.6,70.1,68.5,62.1,55.8,20.7,20.5$.

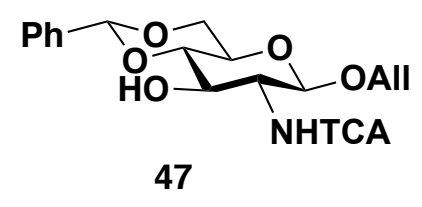

Allyl 4,6- $O$-benzylidene-2-deoxy-2-trichloroacetamido- $\beta$-D-glucopyranoside (47): To a solution of compound $46(3.0 \mathrm{~g}, 6.1 \mathrm{mmol})$ in methanol $(15 \mathrm{~mL})$ was added a pinch of solid sodium metal at room temperature under argon. The reaction mixture was stirred for $3 \mathrm{~h}$ at room temperature and quenched by addition Dowex ${ }^{\circledR}\left(50 \mathrm{~W}\right.$ X8, 200-400 mesh) $\left(\mathrm{H}^{+}\right)$cation exchange resin. The resin was filtered over a pad of Celite ${ }^{\circ}$, rinsed with methanol. The methanol was removed in vacuo and trace methanol or water was removed by co-evaporation with toluene $(3 \times 15 \mathrm{~mL})$. The crude white solid product $(2.14 \mathrm{~g}, 5.87 \mathrm{mmol})$ was used directly in the next step. Benzaldehyde dimethyl acetal $(2.64 \mathrm{~mL}, 17.6 \mathrm{mmol})$ and p-toluene sulfonic acid monohydrate $(0.560 \mathrm{~g}, 2.93 \mathrm{mmol})$ were added to a solution of the resulting crude solid (2.14 $\mathrm{g}, 5.87 \mathrm{mmol})$ in anhydrous acetonitrile $(15 \mathrm{~mL})$ under argon. The reaction mixture was stirred at room temperature until TLC confirmed the formation of the product $(3 \mathrm{~h})$. The reaction was quenched by addition of triethylamine drop wise until the reaction mixture was neutral to a litmus paper. After concentrating the reaction mixture under reduced pressure, the crude material was then purified by flash column chromatography on silica gel $(25 \%$ to $40 \%$ ethyl acetate gradient in hexane) to afford compound $47(2.16 \mathrm{~g}, 4.77 \mathrm{mmol}, 78 \%$ over 2 steps) as a white solid. ${ }^{1} \mathbf{H}$ NMR $\left(400 \mathrm{MHz}, \mathrm{CDCl}_{3}\right) \delta 7.46(\mathrm{dd}, J=6.6,3.1 \mathrm{~Hz}, 2 \mathrm{H}), 7.39-$ $7.31(\mathrm{~m}, 3 \mathrm{H}), 7.05(\mathrm{~d}, J=7.3 \mathrm{~Hz}, 1 \mathrm{H}), 5.84(\mathrm{~m}, 1 \mathrm{H}), 5.50(\mathrm{~s}, 1 \mathrm{H}), 5.27(\mathrm{dd}, J=17.2,1.5 \mathrm{~Hz}$, $1 \mathrm{H}), 5.19$ (dd, $J=10.4,1.3 \mathrm{~Hz}, 1 \mathrm{H}), 4.90(\mathrm{~d}, J=8.3 \mathrm{~Hz}, 1 \mathrm{H}), 4.35-4.27$ (m, 3H), 4.07 (dd, $J=$ $12.8,6.4 \mathrm{~Hz}, 1 \mathrm{H}), 3.75(\mathrm{t}, J=10.0 \mathrm{~Hz}, 1 \mathrm{H}), 3.57-3.42(\mathrm{~m}, 4 \mathrm{H}) .{ }^{13} \mathbf{C}$ NMR $\left(101 \mathrm{MHz}, \mathrm{CDCl}_{3}\right) \delta$ $162.2,136.8,133.1,129.3,128.3,126.2,118.4,101.8,98.9,92.4,81.5,70.6,69.5,68.5,66.1$, 59.4. HRMS-ESI-TOF (m/z): $[\mathrm{M}+\mathrm{Na}]^{+}$Calculated for $\mathrm{C}_{18} \mathrm{H}_{20} \mathrm{Cl}_{3} \mathrm{NO}_{6}$ 476.0227, Found 476.0256. 


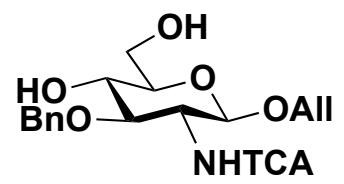

48

Allyl 3- $\boldsymbol{O}$-benzyl-2-deoxy-2-trichloroacetamido- $\boldsymbol{\beta}$-D-glucopyranoside $\quad(48): \quad \mathrm{NaH} \quad(60 \%$ suspension in mineral oil) $(74.0 \mathrm{mg}, 3.09 \mathrm{mmol})$ was added portion wise to absolute DMF (5 $\mathrm{mL}$ ) at $-20^{\circ} \mathrm{C}$ under argon. The reaction mixture was stirred until the homogeneous suspension was formed and compound $47(0.50 \mathrm{~g}, 1.1 \mathrm{mmol})$ was added portion wise during $15 \mathrm{~min}$. The stirring was continued until the gas evolution ceased $\left(1 \mathrm{~h}\right.$ at $\left.-20^{\circ} \mathrm{C}\right)$, benzyl bromide $(0.160 \mathrm{~mL}$, $1.32 \mathrm{mmol}$ ) was added drop wise and the reaction mixture was stirred for $4 \mathrm{~h}$ at $-15^{\circ} \mathrm{C}$ until most of the starting material was consumed. The reaction mixture was then neutralized by addition of methanol $(10 \mathrm{~mL})$ The reaction mixture was allowed to attain room temperature slowly and diluted with ethyl acetate $(30 \mathrm{~mL})$, washed with water $(3 \mathrm{X} 30 \mathrm{~mL})$ and brine solution $(3 \mathrm{X} 30 \mathrm{~mL})$. The organic phase was dried over $\mathrm{Na}_{2} \mathrm{SO}_{4}$ and the solvent was removed in vacuo to afford a white crude solid product. A solution of the crude solid in dichloromethane $(5 \mathrm{~mL})$ was cooled to $0{ }^{\circ} \mathrm{C}$ in an ice bath. Aqueous trifluoroacetic acid solution $(40 \% \mathrm{v} / \mathrm{v}, 3 \mathrm{~mL})$ was added to the reaction mixture at $0{ }^{\circ} \mathrm{C}$. The solution was allowed to warm to room temperature and stirred for $4 \mathrm{~h}$ at $30{ }^{\circ} \mathrm{C}$ until the starting material was completely consumed by TLC. The reaction mixture was diluted with dichloromethane $(15 \mathrm{~mL})$ and water $(15 \mathrm{~mL})$. The layers were separated and the water layer was extracted twice with dichloromethane $(2 \times 20 \mathrm{~mL})$. The combined organic layer was washed with DI water $(3 \times 20 \mathrm{~mL})$, saturated solution of sodium bicarbonate $(3 \times 20 \mathrm{~mL})$ and dried over $\mathrm{Na}_{2} \mathrm{SO}_{4}$. The organic solvent was removed in vacuo. The crude material was then purified by flash column chromatography on silica gel (50\% to $65 \%$ ethyl acetate gradient in hexane $)$ to afford compound $48(0.32 \mathrm{~g}, 0.70 \mathrm{mmol}, 64 \%$ over 2 steps) as a white solid. ${ }^{1} \mathbf{H}$ NMR (400 MHz, $\left.\mathrm{CD}_{3} \mathrm{OD}\right) \delta 7.25(\mathrm{~m}, 6 \mathrm{H}), 5.86(\mathrm{~m}, 1 \mathrm{H}), 5.30-5.22$ $(\mathrm{m}, 1 \mathrm{H}), 5.10(\mathrm{~d}, J=10.5 \mathrm{~Hz}, 1 \mathrm{H}), 4.88-4.82(\mathrm{~m}, 3 \mathrm{H}), 4.70-4.61(\mathrm{~m}, 2 \mathrm{H}), 4.33(\mathrm{dd}, J=13.2$, $4.9 \mathrm{~Hz}, 1 \mathrm{H}), 4.08(\mathrm{dd}, J=13.2,5.8 \mathrm{~Hz}, 1 \mathrm{H}), 3.88(\mathrm{dd}, J=11.9,2.0 \mathrm{~Hz}, 1 \mathrm{H}), 3.84-3.76(\mathrm{~m}$, $1 \mathrm{H}), 3.73-3.66(\mathrm{~m}, 2 \mathrm{H}), 3.52(\mathrm{t}, J=9.2 \mathrm{~Hz}, 1 \mathrm{H}), 3.32(\mathrm{~m}, 1 \mathrm{H}) .{ }^{13} \mathbf{C}$ NMR $\left(101 \mathrm{MHz}, \mathrm{CD}_{3} \mathrm{OD}\right)$ $\delta 162.7,138.4,133.9,127.7,127.3,127.0,115.8,99.7,92.7,81.6,76.6,74.4,70.7,69.5,61.2$, 57.0 .

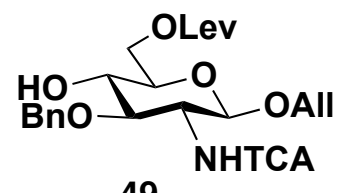

49

Allyl 3-O-benzyl-2-deoxy-6- $O$-levulinoyl-2-trichloroacetamido- $\beta$-D-glucopyranoside (49): To a solution of compound $48(0.44 \mathrm{~g}, 0.96 \mathrm{mmol})$ in anhydrous dichloromethane $(5 \mathrm{~mL})$ was added levulinic acid $(0.220 \mathrm{~g}, 1.94 \mathrm{mmol})$ and 2-chloromethyl pyridinium iodide (CMPI $)^{[8]}$ $(0.620 \mathrm{~g}, 2.42 \mathrm{mmol})$. The mixture was stirred for 15 minutes at room temperature and then 
cooled to $-20^{\circ} \mathrm{C}$ followed by the addition of 1,4-diazabicyclo[2,2,2] octane $(0.410 \mathrm{~g}, 3.68 \mathrm{mmol})$. The reaction mixture was allowed to warm up slowly to room temperature. After $24 \mathrm{~h}$, another portion of levulinic acid $(0.11 \mathrm{~g}, 0.97 \mathrm{mmol})$ and 2-chloromethyl pyridinium iodide (CMPI) $(0.310 \mathrm{~g}, 1.21 \mathrm{mmol})$ were added. Stirring was continued until TLC indicated almost complete consumption of starting material $(48 \mathrm{~h})$. The reaction mixture was filtered through Celite, diluted with EtOAc $(20 \mathrm{~mL})$ and washed with a $5 \%$ solution of $\mathrm{NaCl}(5 \times 25 \mathrm{~mL})$. The organic layers were dried over $\mathrm{Na}_{2} \mathrm{SO}_{4}$, filtered and the filtrate was concentrated in vacuo. Silica gel column chromatography of the residue (50\% to $65 \%$ ethyl acetate gradient in hexane) afforded compound 49 as a white solid (0.44 g, $0.79 \mathrm{mmol}, 83 \%) .{ }^{1} \mathbf{H}$ NMR $\left(400 \mathrm{MHz}, \mathrm{CDCl}_{3}\right) \delta 7.34-$ $7.21(\mathrm{~m}, 5 \mathrm{H}), 7.09(\mathrm{~d}, J=7.7 \mathrm{~Hz}, 1 \mathrm{H}), 5.83(\mathrm{~m}, 1 \mathrm{H}), 5.29-5.20(\mathrm{~m}, 1 \mathrm{H}), 5.19-5.10(\mathrm{~m}, 1 \mathrm{H})$, $4.86(\mathrm{~d}, J=8.3 \mathrm{~Hz}, 1 \mathrm{H}), 4.80-4.69(\mathrm{~m}, 2 \mathrm{H}), 4.48(\mathrm{dd}, J=12.1,4.3 \mathrm{~Hz}, 1 \mathrm{H}), 4.35-4.19(\mathrm{~m}$, 2H), $4.08-3.96(\mathrm{~m}, 2 \mathrm{H}), 3.60-3.43(\mathrm{~m}, 3 \mathrm{H}), 2.74(\mathrm{t}, J=6.3 \mathrm{~Hz}, 2 \mathrm{H}), 2.58(\mathrm{t}, J=6.1 \mathrm{~Hz}, 2 \mathrm{H})$, $2.16(\mathrm{~s}, 3 \mathrm{H}) .{ }^{13} \mathrm{C}$ NMR $\left(101 \mathrm{MHz}, \mathrm{CDCl}_{3}\right) \delta 207.0,173.4,161.9,137.9,133.4,128.5,128.1$, $127.9,118.0,98.4,92.4,79.2,74.7,73.8,70.9,70.3,63.2,58.2,37.9,29.8,27.9$. HRMS-ESITOF (m/z): $[\mathrm{M}+\mathrm{Na}]^{+}$Calculated for $\mathrm{C}_{23} \mathrm{H}_{28} \mathrm{Cl}_{3} \mathrm{NO}_{8} \mathbf{5 7 6 . 0 7 5 3}$, Found 576.0742.

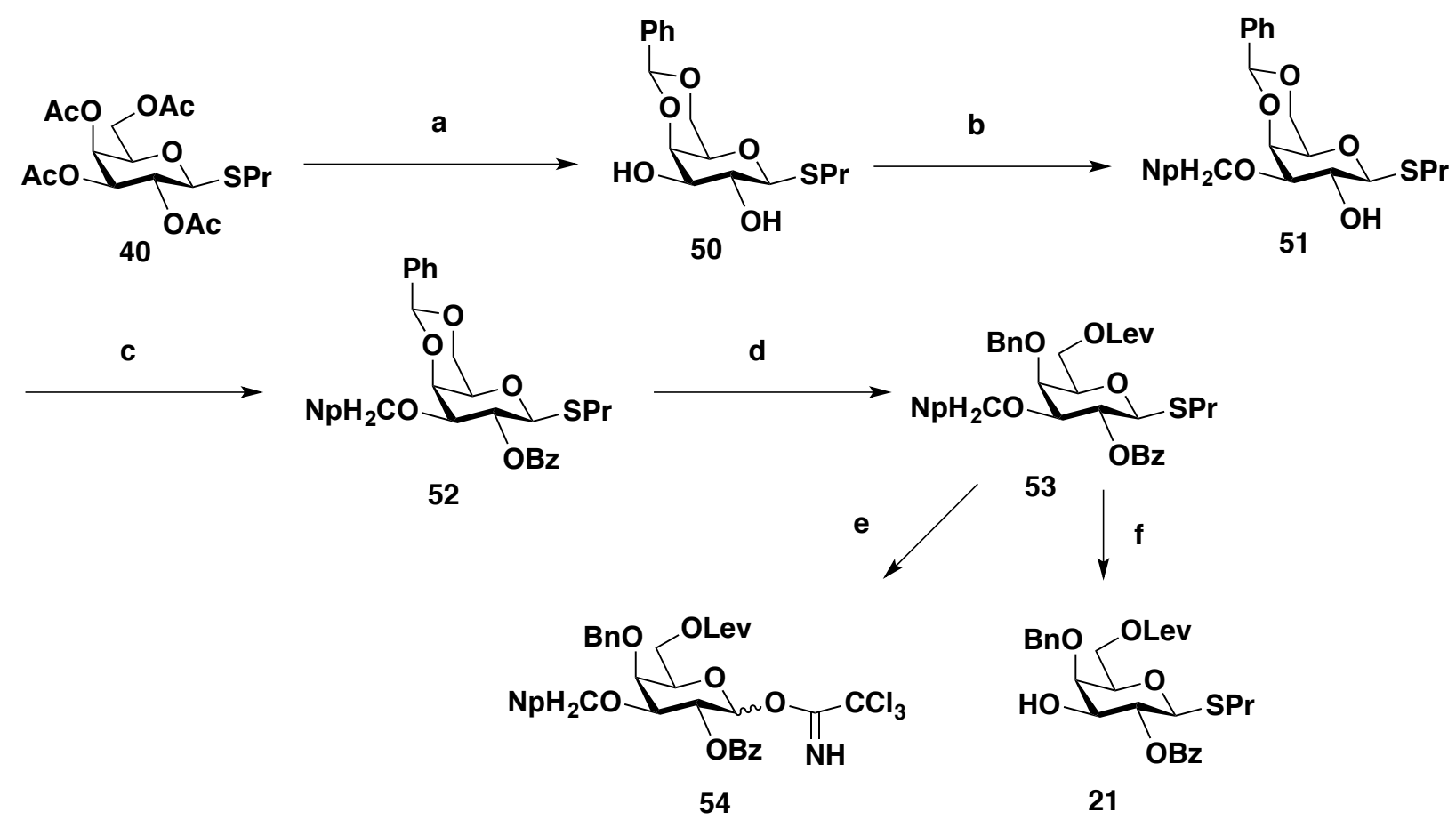

Scheme S8: Synthesis of Galactose donor 54 and acceptor 21

Reagents and conditions: (a) (i) $\mathrm{Na} / \mathrm{MeOH}, 25{ }^{\circ} \mathrm{C}, 3 \mathrm{~h}$; (ii) $\mathrm{PhCH}(\mathrm{OMe})_{2}, \mathrm{PTSA}, \mathrm{CH}_{3} \mathrm{CN}, 25$ ${ }^{\circ} \mathrm{C}, 2 \mathrm{~h}, 80 \%$ (over 2 steps); (b) (i) $\mathrm{Bu}_{2} \mathrm{SnO}$, toluene, reflux, 4 h; (ii) $\mathrm{NapBr}$, CsF, DMF, 95 ${ }^{\circ} \mathrm{C}, 10 \mathrm{~h}, 79 \%$; (c) $\mathrm{BzCl}$, pyridine, DMAP, $0{ }^{\circ} \mathrm{C}$ to $40{ }^{\circ} \mathrm{C}, 8 \mathrm{~h}, 88 \%$; (d) (i) $\mathrm{Bu}_{2} \mathrm{BOTf}(1.0 \mathrm{M}$ in methylene chloride), $\mathrm{BH}_{3} \cdot \mathrm{THF}, 0{ }^{\circ} \mathrm{C}, 1 \mathrm{~h}, 88 \%$; (ii) LevOH, DCC, DMAP, $\mathrm{CH}_{2} \mathrm{Cl}_{2}, 25^{\circ} \mathrm{C}, 2 \mathrm{~h}$, $91 \%$; (e) (i) NBS, Acetone : $\mathrm{H}_{2} \mathrm{O}(9: 1), 0{ }^{\circ} \mathrm{C}$ to $25^{\circ} \mathrm{C}, 2 \mathrm{~h}$; (ii) $\mathrm{CCl}_{3} \mathrm{CN}, \mathrm{DBU}, \mathrm{CH}_{2} \mathrm{Cl}_{2}, 0{ }^{\circ} \mathrm{C}$ to $25^{\circ} \mathrm{C}, 3 \mathrm{~h}, 84 \%$ (over 2 steps); (f) DDQ, $\mathrm{CH}_{2} \mathrm{Cl}_{2}: \mathrm{H}_{2} \mathrm{O}=18: 1,0{ }^{\circ} \mathrm{C}$ to $25{ }^{\circ} \mathrm{C}, 6 \mathrm{~h}, 86 \%$. 


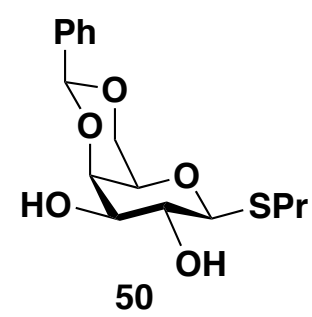

Propyl 4,6-O-benzylidene-1-thio- $\boldsymbol{\beta}$-D-galactopyranoside (50): To a solution of compound 40 $(5.00 \mathrm{~g}, 12.3 \mathrm{mmol})$ in methanol $(30 \mathrm{~mL})$ was added a pinch of solid sodium metal at room temperature under argon. The reaction mixture was stirred for $3 \mathrm{~h}$ at room temperature and quenched by addition Dowex ${ }^{\circledR}\left(50 \mathrm{~W}\right.$ X8, 200-400 mesh) $\left(\mathrm{H}^{+}\right)$cation exchange resin. The resin was filtered over a pad of Celite ${ }^{\circledR}$, rinsed with methanol. The methanol was removed in vacuo and trace methanol or water was removed by co-evaporation with toluene $(3 \times 15 \mathrm{~mL})$. The crude white solid product $(2.84 \mathrm{~g}, 11.9 \mathrm{mmol})$ was used directly in the next step. Benzaldehyde dimethyl acetal $(5.37 \mathrm{~mL}, 35.8 \mathrm{mmol})$ and p-toluene sulfonic acid monohydrate $(0.450 \mathrm{~g}, 2.38$ mmol) were added to a solution of the resulting crude solid $(2.84 \mathrm{~g}, 11.9 \mathrm{mmol})$ in anhydrous acetonitrile $(20 \mathrm{~mL})$ under argon. The reaction mixture was stirred at room temperature until TLC confirmed the formation of the product $(2.5 \mathrm{~h})$. The reaction was quenched by addition of triethylamine drop wise until the reaction mixture was neutral to a litmus paper. After concentrating the reaction mixture under reduced pressure, the crude material was purified by flash column chromatography on silica gel (40\% to $70 \%$ ethyl acetate gradient in hexane) to afford compound $\mathbf{5 0}$ (3.37 g, $10.3 \mathrm{mmol}, 84 \%$ over 2 steps) as a white solid.

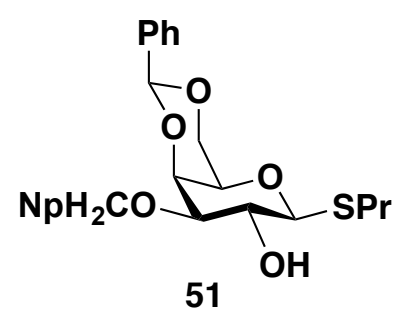

Propyl 4,6- $\boldsymbol{O}$-benzylidene-3- $\boldsymbol{O}$-(2-naphthylmethyl)-1-thio- $\boldsymbol{\beta}$-D-galactopyranoside (51): To a solution of compound $\mathbf{5 0}(5.08 \mathrm{~g}, 15.6 \mathrm{mmol})$ in dry toluene $(25 \mathrm{~mL})$ was added $\mathrm{Bu}_{2} \mathrm{SnO}(5.42$ $\mathrm{g}, 21.8 \mathrm{mmol}$ ) at room temperature under argon. The reaction mixture was refluxed for $4 \mathrm{~h}$. The solvent was then removed under reduced pressure. The resulting crude material was then dissolved in dry DMF $(20 \mathrm{~mL})$. To this solution, $\mathrm{NapBr}$ (2-Naphthylmethyl bromide) $(5.16 \mathrm{~g}$, $23.3 \mathrm{mmol}$ ) and $\mathrm{CsF}$ (cesium fluoride) $(4.73 \mathrm{~g}, 31.1 \mathrm{mmol})$ were added simultaneously and stirred for $10 \mathrm{~h}$ at $95{ }^{\circ} \mathrm{C}$ until TLC confirmed the formation of the product. The reaction mixture was cooled and the solvent was then removed under reduced pressure. The crude material was purified by flash column chromatography on silica gel $(20 \%$ to $30 \%$ ethyl acetate gradient in hexane) to afford compound $\mathbf{5 1}(5.73 \mathrm{~g}, 12.3 \mathrm{mmol}, 79 \%)$ as a white solid. ${ }^{1}$ H NMR $\left(400 \mathrm{MHz}, \mathrm{CDCl}_{3}\right) \delta 7.86-7.72(\mathrm{~m}, 4 \mathrm{H}), 7.55-7.42(\mathrm{~m}, 5 \mathrm{H}), 7.40-7.29(\mathrm{~m}, 3 \mathrm{H})$, 
$5.41(\mathrm{~s}, 1 \mathrm{H}), 4.93(\mathrm{~s}, 2 \mathrm{H}), 4.29(\mathrm{dd}, J=16.0,11.1 \mathrm{~Hz}, 2 \mathrm{H}), 4.16(\mathrm{~d}, J=2.9 \mathrm{~Hz}, 1 \mathrm{H}), 4.09$ (t, $J=$ $9.3 \mathrm{~Hz}, 1 \mathrm{H}), 3.91(\mathrm{~d}, J=12.3 \mathrm{~Hz}, 1 \mathrm{H}), 3.52(\mathrm{dd}, J=9.2,3.3 \mathrm{~Hz}, 1 \mathrm{H}), 3.35(\mathrm{~s}, 1 \mathrm{H}), 2.84-2.62$ $(\mathrm{m}, 2 \mathrm{H}), 2.58(\mathrm{~s}, 1 \mathrm{H}), 1.70(\mathrm{~m}, 2 \mathrm{H}), 0.99(\mathrm{t}, J=7.3 \mathrm{~Hz}, 3 \mathrm{H}) .{ }^{13} \mathbf{C}$ NMR $\left(101 \mathrm{MHz}, \mathrm{CDCl}_{3}\right) \delta$ 137.8, 135.6, 133.1, 133.0, 129.0, 128.2, 128.1, 127.8, 127.7, 126.6, 126.4, 126.1, 125.9, 125.7, $101.2,85.4,80.2,73.6,71.7,70.1,69.4,68.1,30.7,23.7,13.6$.

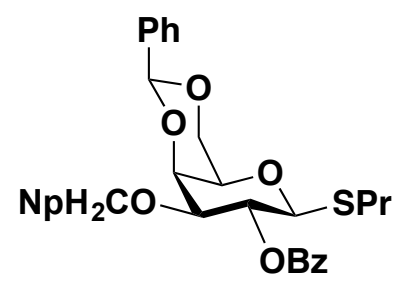

52

Propyl

2- $O$-benzoyl-4,6- $O$-benzylidene-3- $O$-(2-naphthylmethyl)-1-thio- $\beta$-Dgalactopyranoside (52): The resulting compound $\mathbf{5 1}(1.42 \mathrm{~g}, 3.04 \mathrm{mmol})$ was dissolved in anhydrous pyridine $(15 \mathrm{~mL})$ and cooled to $0{ }^{\circ} \mathrm{C}$. Benzoyl chloride $(0.710 \mathrm{~mL}, 6.08 \mathrm{mmol})$ and $4-$ (dimethylamino)pyridine $(0.180 \mathrm{~g}, 1.52 \mathrm{mmol})$ were added to the reaction mixture. The reaction was stirred for $8 \mathrm{~h}$ at $40{ }^{\circ} \mathrm{C}$ until the starting material was completely consumed by TLC. The organic solvent was removed in vacuo and trace of pyridine was removed by co-evaporation with toluene $(3 \times 15 \mathrm{~mL})$. The resulting oil was dissolved in dichloromethane $(30 \mathrm{~mL})$. The organic layer was washed with water $(5 \mathrm{X} 30 \mathrm{~mL})$ and brine solution $(2 \mathrm{X} 30 \mathrm{~mL})$. The organic phase was dried over $\mathrm{Na}_{2} \mathrm{SO}_{4}$ and the solvent was removed in vacuo. The crude material was purified by flash column chromatography on silica gel ( $20 \%$ to $30 \%$ ethyl acetate gradient in hexane) to afford compound $\mathbf{5 2}(1.53 \mathrm{~g}, 2.68 \mathrm{mmol}, 88 \%)$ as a white solid. ${ }^{1} \mathbf{H} \mathbf{~ N M R}\left(400 \mathrm{MHz}, \mathrm{CDCl}_{3}\right) \delta$ $8.02(\mathrm{~d}, J=7.3 \mathrm{~Hz}, 2 \mathrm{H}), 7.80-7.71(\mathrm{~m}, 1 \mathrm{H}), 7.69-7.51(\mathrm{~m}, 6 \mathrm{H}), 7.49-7.28(\mathrm{~m}, 8 \mathrm{H}), 5.76(\mathrm{t}, J$ $=9.7 \mathrm{~Hz}, 1 \mathrm{H}), 5.51(\mathrm{~s}, 1 \mathrm{H}), 4.89-4.70(\mathrm{~m}, 2 \mathrm{H}), 4.51(\mathrm{~d}, J=9.8 \mathrm{~Hz}, 1 \mathrm{H}), 4.37-4.24(\mathrm{~m}, 2 \mathrm{H})$, $3.98(\mathrm{~d}, J=11.4 \mathrm{~Hz}, 1 \mathrm{H}), 3.77(\mathrm{dd}, J=9.6,3.3 \mathrm{~Hz}, 1 \mathrm{H}), 3.42(\mathrm{~s}, 1 \mathrm{H}), 2.89(\mathrm{~m}, 1 \mathrm{H}), 2.69(\mathrm{~m}$, 1H), $1.66(\mathrm{~m}, 2 \mathrm{H}), 0.94(\mathrm{t}, J=7.3 \mathrm{~Hz}, 3 \mathrm{H}) .{ }^{13} \mathbf{C}$ NMR $\left(101 \mathrm{MHz}, \mathrm{CDCl}_{3}\right) \delta 165.2,137.7,135.3$, 133.0, 133.0, 132.9, 130.1, 129.8, 129.1, 128.3, 128.2, 128.1, 127.7, 127.6, 126.5, 126.4, 126.1, $125.9,125.7,101.3,82.9,78.1,73.3,71.1,70.0,69.3,68.7,30.7,23.3,13.6$.

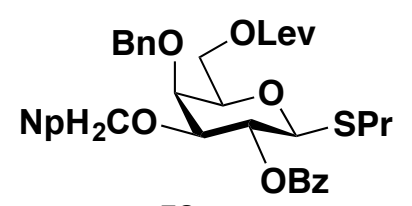

53

Propyl 2- $O$-benzoyl-4- $O$-benzyl-6- $O$-levulinoyl-3- $O$-(2-naphthylmethyl)-1-thio- $\beta$-Dgalactopyranoside (53): To a solution of compound $\mathbf{5 2}(1.10 \mathrm{~g}, 1.93 \mathrm{mmol})$ in $\mathrm{BH}_{3} \cdot \mathrm{THF}(20$ $\mathrm{mL})$ was added $\mathrm{Bu}_{2} \mathrm{BOTf}\left(1.0 \mathrm{M}\right.$ in methylene chloride) $(2.32 \mathrm{~mL}, 2.32 \mathrm{mmol})$ at $0{ }^{\circ} \mathrm{C}$. The reaction was stirred for $1 \mathrm{~h}$ until TLC showed complete conversion of the starting material. The reaction was quenched by drop wise addition of triethylamine and methanol. The resulting solution was concentrated and purified by flash column chromatography on silica gel $(30 \%$ to $45 \%$ ethyl acetate gradient in hexane) to afford $(0.97 \mathrm{~g}, 1.7 \mathrm{mmol}, 88 \%)$ a white solid. The 
resulting compound $(0.97 \mathrm{~g}, 1.7 \mathrm{mmol})$ was then successfully converted to compound $\mathbf{5 3}$ (1.04 g, $1.55 \mathrm{mmol}, 91 \%)$ as a white solid by following the general procedure for $6-\mathrm{OH}$ protection with Levulinate ester (Lev). ${ }^{1} \mathbf{H}$ NMR $\left(400 \mathrm{MHz}_{\mathrm{CDCl}}\right) \delta 8.02-7.92(\mathrm{~m}, 2 \mathrm{H}), 7.77-7.70(\mathrm{~m}, 1 \mathrm{H})$, $7.68-7.50(\mathrm{~m}, 4 \mathrm{H}), 7.46-7.25(\mathrm{~m}, 10 \mathrm{H}), 5.71(\mathrm{t}, J=9.8 \mathrm{~Hz}, 1 \mathrm{H}), 5.06(\mathrm{~d}, J=11.7 \mathrm{~Hz}, 1 \mathrm{H})$, $4.84(\mathrm{~d}, J=12.4 \mathrm{~Hz}, 1 \mathrm{H}), 4.71(\mathrm{~d}, J=11.9 \mathrm{~Hz}, 2 \mathrm{H}), 4.47(\mathrm{~d}, J=9.9 \mathrm{~Hz}, 1 \mathrm{H}), 4.30$ (dd, $J=11.1$, $6.5 \mathrm{~Hz}, 1 \mathrm{H}), 4.17(\mathrm{dd}, J=11.1,6.2 \mathrm{~Hz}, 1 \mathrm{H}), 4.03(\mathrm{~d}, J=2.1 \mathrm{~Hz}, 1 \mathrm{H}), 3.75(\mathrm{dd}, J=9.6,2.6 \mathrm{~Hz}$, $1 \mathrm{H}), 3.67(\mathrm{t}, J=6.4 \mathrm{~Hz}, 1 \mathrm{H}), 2.75-2.55(\mathrm{~m}, 4 \mathrm{H}), 2.48(\mathrm{~m}, 2 \mathrm{H}), 2.17(\mathrm{~s}, 3 \mathrm{H}), 1.57(\mathrm{~m}, 2 \mathrm{H}), 0.89$ $(\mathrm{t}, J=7.3 \mathrm{~Hz}, 3 \mathrm{H}) .{ }^{13} \mathrm{C}$ NMR $\left(101 \mathrm{MHz}, \mathrm{CDCl}_{3}\right) \delta 206.5,172.4,165.4,138.2,135.0,133.0$, $132.9,132.9,130.1,129.8,128.3,128.2,128.2,127.8,127.6,127.6,126.6,126.1,125.9,125.8$, 84.0, 80.9, 76.0, 74.3, 72.3, 71.9, 70.1, 63.2, 37.9, 31.9, 29.8, 27.8, 23.1, 13.4. HRMS-ESI-TOF (m/z): $[\mathrm{M}+\mathrm{Na}]^{+}$Calculated for $\mathrm{C}_{39} \mathrm{H}_{42} \mathrm{O}_{8} \mathrm{~S}$ 693.2498, Found 693.2522.

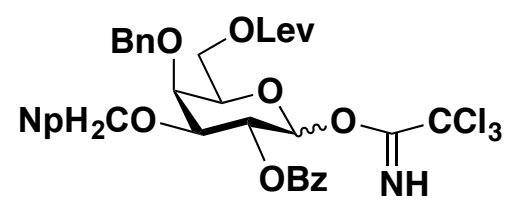

54

2-O-benzoyl-4-O-benzyl-6- $O$-levulinoyl-3- $O$-(2-naphthylmethyl)- $\quad \boldsymbol{\alpha} / \boldsymbol{\beta}$-D-galactopyranosyl trichloroacetimidate (54): To a solution of compound $\mathbf{5 3}(1.20 \mathrm{~g}, 1.79 \mathrm{mmol})$ in acetone and water $\left(20 \mathrm{~mL}, 9: 1\right.$ acetone/water) was added NBS $(1.27 \mathrm{~g}, 7.15 \mathrm{mmol})$ at $0{ }^{\circ} \mathrm{C}$. The reaction mixture was stirred for $2 \mathrm{~h}$ at room temperature until the starting material was completely consumed. The reaction mixture was diluted with dichloromethane $(30 \mathrm{~mL})$. The organic layer was washed with water $(3 \mathrm{X} 20 \mathrm{~mL})$, saturated solution of sodium bicarbonate $(3 \mathrm{X} 20 \mathrm{~mL})$ and brine solution $(2 \mathrm{X} 20 \mathrm{~mL})$. The organic phase was dried over $\mathrm{Na}_{2} \mathrm{SO}_{4}$ and the solvent was removed in vacuo. The crude material was purified by flash column chromatography on silica gel $(10 \%$ to $30 \%$ ethyl acetate gradient in hexane) to afford the hemiacetal $(1.0 \mathrm{~g}, 1.6 \mathrm{mmol})$ as white foam. Complound $\mathbf{5 4}$ was then synthesized by following the general procedure for trichloroacetimidate formation ( $1.14 \mathrm{~g}, 1.50 \mathrm{mmol}, 84 \%$ over 2 steps) as pale yellow oil, which turned to white foam under high vacuum. ${ }^{1} \mathbf{H}$ NMR $\left(400 \mathrm{MHz}, \mathrm{CDCl}_{3}\right) \delta 8.41(\mathrm{~s}, 1 \mathrm{H}), 7.92-$ $7.84(\mathrm{~m}, 2 \mathrm{H}), 7.80-7.63(\mathrm{~m}, 4 \mathrm{H}), 7.51(\mathrm{~d}, J=7.4 \mathrm{~Hz}, 1 \mathrm{H}), 7.45(\mathrm{dd}, J=6.4,2.7 \mathrm{~Hz}, 2 \mathrm{H}), 7.33$ $(\mathrm{m}, 8 \mathrm{H}), 6.63(\mathrm{~d}, J=3.5 \mathrm{~Hz}, 1 \mathrm{H}), 5.85(\mathrm{dd}, J=10.4,3.5 \mathrm{~Hz}, 1 \mathrm{H}), 5.06(\mathrm{~d}, J=11.5 \mathrm{~Hz}, 1 \mathrm{H}), 4.91$ $(\mathrm{d}, J=12.1 \mathrm{~Hz}, 1 \mathrm{H}), 4.81(\mathrm{~d}, J=12.2 \mathrm{~Hz}, 1 \mathrm{H}), 4.71(\mathrm{~d}, J=11.5 \mathrm{~Hz}, 1 \mathrm{H}), 4.29-4.18(\mathrm{~m}, 4 \mathrm{H})$, $4.12(\mathrm{~d}, J=2.3 \mathrm{~Hz}, 1 \mathrm{H}), 2.74-2.64(\mathrm{~m}, 2 \mathrm{H}), 2.48(\mathrm{td}, J=6.5,2.0 \mathrm{~Hz}, 2 \mathrm{H}), 2.14(\mathrm{~s}, 3 \mathrm{H}) .{ }^{13} \mathbf{C}$ NMR $\left(101 \mathrm{MHz}, \mathrm{CDCl}_{3}\right) \delta 206.3,172.3,165.5,160.4,137.8,134.9,133.1,133.1,132.9,129.7$, $129.4,128.4,128.4,128.3,128.3,127.9,127.8,127.6,126.9,126.2,126.0,125.9,94.3,91.0$, $77.2,75.7,74.7,73.4,72.6,71.5,69.7,63.2,37.8,29.8,27.7$. 


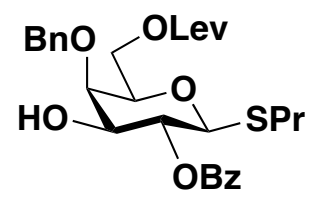

21

Propyl 2- $\boldsymbol{O}$-benzoyl-4- $\boldsymbol{O}$-benzyl-6- $\boldsymbol{O}$-levulinoyl-1-thio- $\boldsymbol{\beta}$-D-galactopyranoside (21): То a solution of compound $53(0.50 \mathrm{~g}, 0.75 \mathrm{mmol})$ in dichloromethane and water $(11.0 \mathrm{~mL}, 18: 1$ dichloromethane/water) was added DDQ $(0.260 \mathrm{~g}, 1.13 \mathrm{mmol})$ at $0{ }^{\circ} \mathrm{C}$. The reaction mixture was stirred for $6 \mathrm{~h}$ at room temperature until the starting material was completely consumed. The reaction mixture was diluted with dichloromethane $(20 \mathrm{~mL})$. The organic layer was washed with water $(3 X 20 \mathrm{~mL})$, saturated solution of sodium bicarbonate $(3 \mathrm{X} 20 \mathrm{~mL})$ and brine solution $(2 X 20$ $\mathrm{mL}$ ). The organic phase was dried over $\mathrm{Na}_{2} \mathrm{SO}_{4}$ and the solvent was removed in vacuo. The crude material was purified by flash column chromatography on silica gel (10\% to $30 \%$ ethyl acetate gradient in hexane) to afford compound $21(0.34 \mathrm{~g}, 0.65 \mathrm{mmol}, 86 \%)$ as a white solid. ${ }^{1}$ H NMR $\left(400 \mathrm{MHz}, \mathrm{CDCl}_{3}\right) \delta 8.10-7.99(\mathrm{~m}, 2 \mathrm{H}), 7.56(\mathrm{t}, J=7.4 \mathrm{~Hz}, 1 \mathrm{H}), 7.38(\mathrm{tt}, J=$ 23.8, 7.1 Hz, 7H), $5.32(\mathrm{t}, J=9.7 \mathrm{~Hz}, 1 \mathrm{H}), 4.86-4.70(\mathrm{~m}, 2 \mathrm{H}), 4.54(\mathrm{~d}, J=9.9 \mathrm{~Hz}, 1 \mathrm{H}), 4.34$ $(\mathrm{dd}, J=11.1,6.6 \mathrm{~Hz}, 1 \mathrm{H}), 4.17(\mathrm{dd}, J=11.1,6.4 \mathrm{~Hz}, 1 \mathrm{H}), 3.94-3.80(\mathrm{~m}, 2 \mathrm{H}), 3.74(\mathrm{t}, J=6.5$ $\mathrm{Hz}, 1 \mathrm{H}), 2.78-2.48(\mathrm{~m}, 7 \mathrm{H}), 2.17(\mathrm{~s}, 3 \mathrm{H}), 1.61$ (dt, $J=14.0,7.1 \mathrm{~Hz}, 2 \mathrm{H}), 0.93$ (t, $J=7.3 \mathrm{~Hz}$, 3H). ${ }^{13} \mathbf{C}$ NMR $\left(101 \mathrm{MHz}, \mathrm{CDCl}_{3}\right) \delta 206.5,172.3,166.6,137.8,133.2,129.9,129.7,128.5$, $128.3,128.02,128.0,83.6,76.5,76.1,75.5,74.4,72.3,62.8,37.9,32.0,29.7,27.8,23.2,13.4$. 


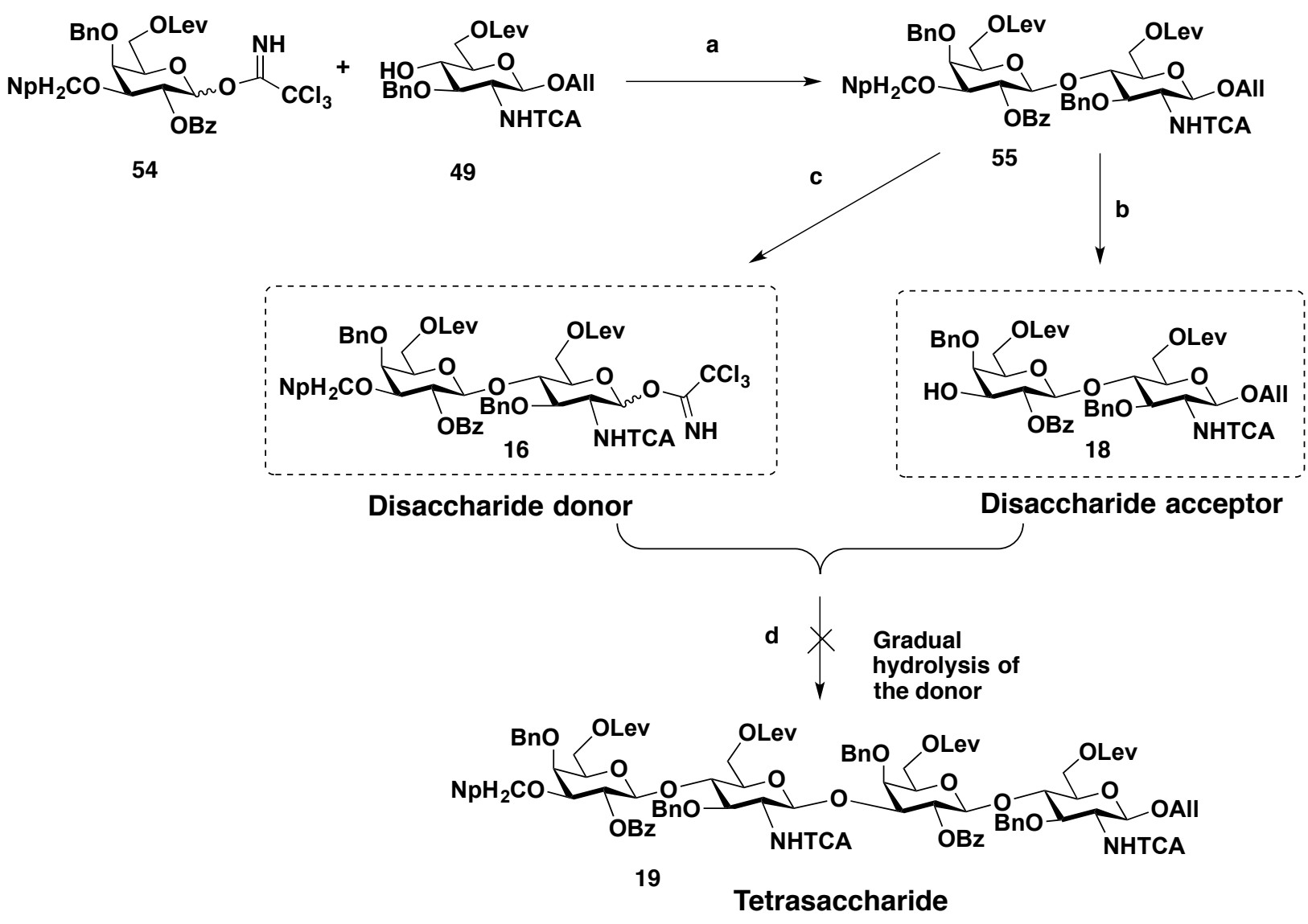

Scheme S9: Attempted synthesis of Tetrasaccharide 19

Reagents and conditions: (a) TMSOTf, $\mathrm{CH}_{2} \mathrm{Cl}_{2}, 0{ }^{\circ} \mathrm{C}, 25 \mathrm{~min}, 89 \%$; (b) DDQ, $\mathrm{CH}_{2} \mathrm{Cl}_{2}: \mathrm{H}_{2} \mathrm{O}$ (10:1), $0{ }^{\circ} \mathrm{C}$ to $25{ }^{\circ} \mathrm{C}, 6 \mathrm{~h}, 78 \%$; (c) (i) $\mathrm{PdCl}_{2}, \mathrm{AcOH}, \mathrm{NaOAc}, \mathrm{CH}_{2} \mathrm{Cl}_{2}, \mathrm{H}_{2} \mathrm{O}, 25{ }^{\circ} \mathrm{C}, 14 \mathrm{~h}$; (ii) $\mathrm{CCl}_{3} \mathrm{CN}, \mathrm{DBU}, \mathrm{CH}_{2} \mathrm{Cl}_{2}, 0{ }^{\circ} \mathrm{C}$ to $25{ }^{\circ} \mathrm{C}, 3 \mathrm{~h}, 74 \%$ (over 2 steps); (d) TMSOTf, $\mathrm{CH}_{2} \mathrm{Cl}_{2}, 0{ }^{\circ} \mathrm{C}, 30$ $\min$.

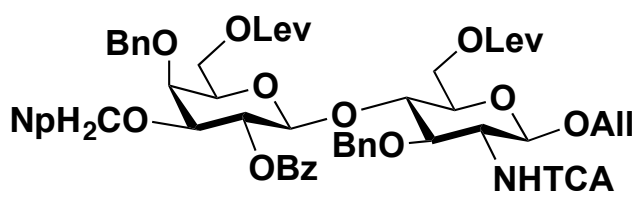

55

Allyl

[2-O-benzoyl-4-O-benzyl-6- $O$-levulinoyl-3- $O$-(2-naphthylmethyl)- $\beta$-Dgalactopyranosyl]-( $1 \rightarrow 4)$-3- $O$-benzyl-2-deoxy-6- $O$-levulinoyl-2-trichloroacetamido- $\beta$-Dglucopyranoside (55): To a vigorously stirred solution of glycosyl donor $54(0.34 \mathrm{~g}, 0.45 \mathrm{mmol})$ and glycosyl acceptor $49(0.14 \mathrm{~g}, 0.25 \mathrm{mmol})$ in anhydrous dichloromethane $(6 \mathrm{~mL})$ under argon, catalytic TMSOTf (Trimethylsilyl trifluoromethanesulfonate) $(4.60 \mu \mathrm{L}, 25.0 \mu \mathrm{mol})$ was added at $0{ }^{\circ} \mathrm{C}$ and the reaction mixture was stirred for $25 \mathrm{~min}$ at the same temperature until the acceptor was completely consumed shown by TLC. The reaction mixture quenched by the addition of 
triethylamine drop wise until the reaction mixture was neutral to a litmus paper. The reaction mixture was then diluted with dichloromethane $(10 \mathrm{~mL})$ and the solvent was removed under reduced pressure. The crude product was then purified by flash column chromatography on silica gel ( $30 \%$ to $40 \%$ ethyl acetate gradient in hexane) to afford compound $\mathbf{5 5}(0.26 \mathrm{~g}$, $0.22 \mathrm{mmol}, 89 \%)$ as a white solid. ${ }^{1} \mathbf{H}$ NMR $\left(400 \mathrm{MHz}, \mathrm{CDCl}_{3}\right) \delta 7.96(\mathrm{~d}, J=7.4 \mathrm{~Hz}, 2 \mathrm{H}), 7.67$ $(\mathrm{d}, J=7.0 \mathrm{~Hz}, 1 \mathrm{H}), 7.60-7.45(\mathrm{~m}, 4 \mathrm{H}), 7.40-7.08(\mathrm{~m}, 16 \mathrm{H}), 6.99(\mathrm{~d}, J=8.1 \mathrm{~Hz}, 1 \mathrm{H}), 5.75-$ $5.57(\mathrm{~m}, 2 \mathrm{H}), 5.15-4.95(\mathrm{~m}, 3 \mathrm{H}), 4.87(\mathrm{~d}, J=10.8 \mathrm{~Hz}, 1 \mathrm{H}), 4.78(\mathrm{~d}, J=12.4 \mathrm{~Hz}, 1 \mathrm{H}), 4.70-$ $4.53(\mathrm{~m}, 5 \mathrm{H}), 4.24(\mathrm{dd}, J=11.9,4.3 \mathrm{~Hz}, 1 \mathrm{H}), 4.13-3.99(\mathrm{~m}, 4 \mathrm{H}), 3.93-3.78(\mathrm{~m}, 5 \mathrm{H}), 3.73(\mathrm{t}, J$ $=6.4 \mathrm{~Hz}, 1 \mathrm{H}), 3.64(\mathrm{q}, J=7.8 \mathrm{~Hz}, 1 \mathrm{H}), 3.46-3.37(\mathrm{~m}, 1 \mathrm{H}), 2.76-2.53(\mathrm{~m}, 4 \mathrm{H}), 2.49(\mathrm{~d}, J=$ $13.3 \mathrm{~Hz}, 1 \mathrm{H}), 2.41-2.31(\mathrm{~m}, 3 \mathrm{H}), 2.09(\mathrm{~s}, 3 \mathrm{H}), 1.99(\mathrm{~s}, 3 \mathrm{H})$. HRMS-ESI-TOF (m/z): $[\mathrm{M}+\mathrm{Na}]^{+}$ Calculated for $\mathrm{C}_{59} \mathrm{H}_{62} \mathrm{Cl}_{3} \mathrm{NO}_{16}$ 1170.3022, Found 1170.3019.

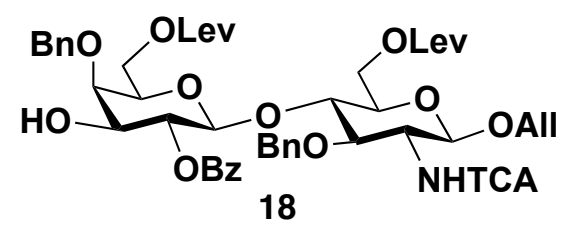

Allyl [2- $O$-benzoyl-4- $O$-benzyl-6- $O$-levulinoyl- $\beta$-D-galactopyranosyl]-(1 $\rightarrow 4)-3-O$-benzyl-2deoxy-6- $O$-levulinoyl-2-trichloroacetamido- $\beta$-D-glucopyranoside (18): To a solution of compound $55(30.0 \mathrm{mg}, 26.0 \mu \mathrm{mol})$ in dichloromethane and water $(3.0 \mathrm{~mL}, 10: 1$ dichloromethane/water) was added DDQ (2,3-Dichloro-5,6-dicyano- $p$-benzoquinone) (14.0 mg, $63.0 \mu \mathrm{mol})$ at $0{ }^{\circ} \mathrm{C}$. The reaction mixture was stirred for $6 \mathrm{~h}$ at room temperature until the starting material was completely consumed. The reaction mixture was diluted with dichloromethane $(5 \mathrm{~mL})$. The organic layer was washed with water $(5 \mathrm{X} 5 \mathrm{~mL})$, saturated solution of sodium bicarbonate $(3 \mathrm{X} 5 \mathrm{~mL})$ and brine solution $(2 \mathrm{X} 5 \mathrm{~mL})$. The organic phase was dried over $\mathrm{Na}_{2} \mathrm{SO}_{4}$ and the solvent was removed in vacuo. The crude material was purified by flash column chromatography on silica gel ( $40 \%$ to $50 \%$ ethyl acetate gradient in hexane) to afford the compound $18(0.02 \mathrm{~g}, 0.02 \mathrm{mmol}, 78 \%)$ as a white solid. ${ }^{1} \mathbf{H} \mathbf{~ N M R}\left(500 \mathrm{MHz}, \mathrm{CDCl}_{3}\right) \delta 8.02(\mathrm{~d}, J$ $=7.4 \mathrm{~Hz}, 2 \mathrm{H}), 7.51(\mathrm{t}, J=7.4 \mathrm{~Hz}, 1 \mathrm{H}), 7.40(\mathrm{t}, J=7.7 \mathrm{~Hz}, 2 \mathrm{H}), 7.33-7.15(\mathrm{~m}, 10 \mathrm{H}), 6.97(\mathrm{~d}, J$ $=8.0 \mathrm{~Hz}, 1 \mathrm{H}), 5.72(\mathrm{~m}, 1 \mathrm{H}), 5.28-5.17(\mathrm{~m}, 1 \mathrm{H}), 5.17-5.11(\mathrm{~m}, 1 \mathrm{H}), 5.05(\mathrm{~d}, J=10.3 \mathrm{~Hz}, 1 \mathrm{H})$, $4.89(\mathrm{~d}, J=10.8 \mathrm{~Hz}, 1 \mathrm{H}), 4.76-4.62(\mathrm{~m}, 4 \mathrm{H}), 4.57(\mathrm{~d}, J=10.8 \mathrm{~Hz}, 1 \mathrm{H}), 4.34(\mathrm{dd}, J=11.9,4.1$ $\mathrm{Hz}, 1 \mathrm{H}), 4.20-4.09(\mathrm{~m}, 3 \mathrm{H}), 4.03(\mathrm{dd}, J=11.1,6.5 \mathrm{~Hz}, 1 \mathrm{H}), 3.96-3.79(\mathrm{~m}, 6 \mathrm{H}), 3.62(\mathrm{q}, J=$ $7.9 \mathrm{~Hz}, 1 \mathrm{H}), 3.51-3.41(\mathrm{~m}, 1 \mathrm{H}), 2.85-2.74(\mathrm{~m}, 1 \mathrm{H}), 2.69-2.50(\mathrm{~m}, 4 \mathrm{H}), 2.43(\mathrm{t}, J=6.4 \mathrm{~Hz}$, $3 \mathrm{H}), 2.11(\mathrm{~d}, J=12.3 \mathrm{~Hz}, 6 \mathrm{H})$. MALDI-TOF (Low-resolution) $(\mathbf{m} / \mathbf{z}):[\mathrm{M}+\mathrm{Na}]^{+}$Calculated for $\mathrm{C}_{48} \mathrm{H}_{54} \mathrm{Cl}_{3} \mathrm{NO}_{16}$ 1030.29, Found 1030.09.

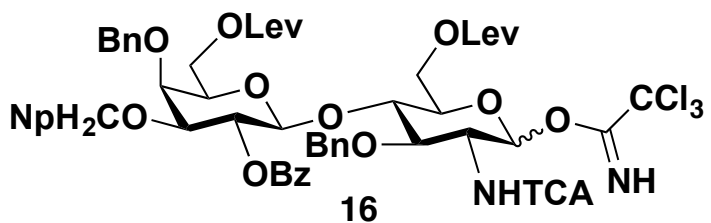




\section{[2-O-benzoyl-4- $O$-benzyl-6- $O$-levulinoyl-3- $O$-(2-naphthylmethyl)- $\beta$-D-galactopyranosyl]-}

$(1 \rightarrow 4)$-3-O-benzyl-2-deoxy-6-O-levulinoyl-2-trichloroacetamidotrichloroacetimidate (16): $\mathrm{NaOAc}$ (sodium acetate) $\left(56.0 \mathrm{mg}, 0.680 \mathrm{mmol}\right.$ ) and $\mathrm{PdCl}_{2}$ (palladium chloride) $(0.030 \mathrm{~g}, 0.17 \mathrm{mmol})$ were added to a solution of compound $\mathbf{5 5}(98.0 \mathrm{mg}$, $85.0 \mu \mathrm{mol})$ in a mixture of DCM $(1 \mathrm{~mL})$, AcOH $(1 \mathrm{~mL})$ and water $(0.05 \mathrm{~mL})$. The reaction mixture was stirred overnight $(14 \mathrm{~h})$ under an atmosphere of argon and then was concentrated under reduced pressure. The residue was diluted with dichloromethane $(3 \mathrm{~mL})$ and the organic phase was washed with water $(2 \mathrm{X} 5 \mathrm{~mL})$ and then dried over $\mathrm{Na}_{2} \mathrm{SO}_{4}$, filtered, and concentrated in vacuo. The resulting residue was purified by flash column chromatography on silica gel ( $40 \%$ to $60 \%$ ethyl acetate gradient in hexane) to afford the hemiacetal $(77 \mathrm{mg}, 0.070 \mathrm{mmol}$, $82 \%)$ as white foam. The hemiacetal $(77 \mathrm{mg}, 0.070 \mathrm{mmol})$ was then successfully converted to compound 16 ( $80.0 \mathrm{mg}, 63.0 \mu \mathrm{mol}, 74 \%$ over 2 steps) as pale yellow oil, which turned to white foam under high vacuum by following the general procedure for trichloroacetimidate formation. ${ }^{1}$ H NMR $\left(400 \mathrm{MHz}, \mathrm{CDCl}_{3}\right) \delta 8.54(\mathrm{~s}, 1 \mathrm{H}), 7.99(\mathrm{~d}, J=7.3 \mathrm{~Hz}, 2 \mathrm{H}), 7.66(\mathrm{~d}, J=8.6 \mathrm{~Hz}, 1 \mathrm{H})$, $7.59-7.46(\mathrm{~m}, 4 \mathrm{H}), 7.35(\mathrm{q}, J=11.2,9.4 \mathrm{~Hz}, 5 \mathrm{H}), 7.27-7.14(\mathrm{~m}, 11 \mathrm{H}), 6.43(\mathrm{~d}, J=7.9 \mathrm{~Hz}$, $1 \mathrm{H}), 6.27(\mathrm{~d}, J=3.4 \mathrm{~Hz}, 1 \mathrm{H}), 5.74-5.63(\mathrm{~m}, 1 \mathrm{H}), 5.00(\mathrm{dd}, J=11.4,6.9 \mathrm{~Hz}, 2 \mathrm{H}), 4.78(\mathrm{~d}, J=$ $12.4 \mathrm{~Hz}, 1 \mathrm{H}), 4.71-4.61(\mathrm{~m}, 3 \mathrm{H}), 4.61-4.53(\mathrm{~m}, 1 \mathrm{H}), 4.29$ (dd, $J=12.4,2.8 \mathrm{~Hz}, 1 \mathrm{H}), 4.16(\mathrm{~m}$, $1 \mathrm{H}), 4.09-3.97(\mathrm{~m}, 3 \mathrm{H}), 3.92(\mathrm{t}, J=8.2 \mathrm{~Hz}, 3 \mathrm{H}), 3.84-3.71(\mathrm{~m}, 2 \mathrm{H}), 3.70-3.62(\mathrm{~m}, 1 \mathrm{H}), 2.65$ $-2.47(\mathrm{~m}, 5 \mathrm{H}), 2.41-2.33(\mathrm{~m}, 3 \mathrm{H}), 2.10(\mathrm{~s}, 3 \mathrm{H}), 1.97(\mathrm{~s}, 3 \mathrm{H})$.

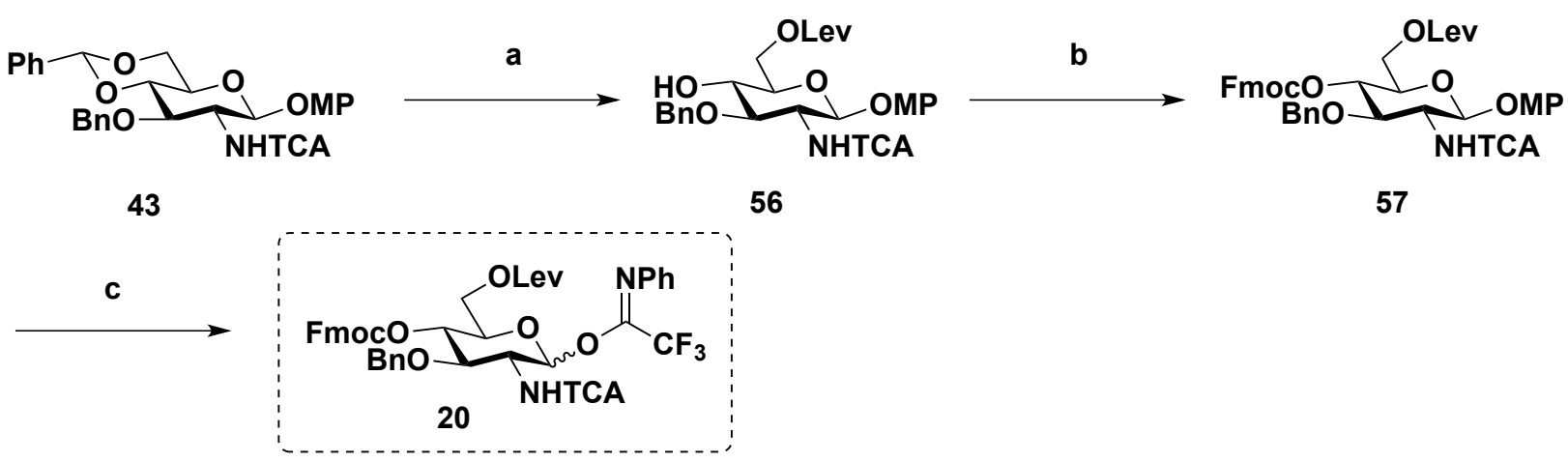

Scheme S10: Synthesis of Glucosamine donor 20

Reagents and conditions: (a) (i) TFA (40\%), $\mathrm{CH}_{2} \mathrm{Cl}_{2}, 0{ }^{\circ} \mathrm{C}$ to $30{ }^{\circ} \mathrm{C}, 4 \mathrm{~h}$; (ii) LevOH, CMPI, DABCO, $\mathrm{CH}_{2} \mathrm{Cl}_{2}, 25^{\circ} \mathrm{C}, 48 \mathrm{~h}, 76 \%$ (over 2 steps); (b) $\mathrm{FmocCl}, \mathrm{CH}_{2} \mathrm{Cl}_{2}$ : pyridine, $0{ }^{\circ} \mathrm{C}$ to $25^{\circ} \mathrm{C}$, $5 \mathrm{~h}, 87 \%$; (c) (i) $\mathrm{CAN}, \mathrm{CH}_{3} \mathrm{CN}: \mathrm{H}_{2} \mathrm{O}(4: 1), 0{ }^{\circ} \mathrm{C}$ to $25{ }^{\circ} \mathrm{C}, 0.5 \mathrm{~h}$; (ii) $\mathrm{ClC}(\mathrm{NPh}) \mathrm{CF}_{3}, \mathrm{~K}_{2} \mathrm{CO}_{3}$, acetone, $25^{\circ} \mathrm{C}, 3 \mathrm{~h}, 76 \%$ (over 2 steps).

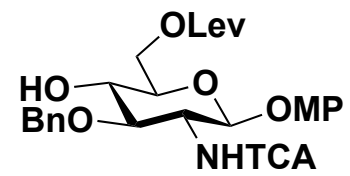



glucopyranoside (56): A solution of the compound 43 (2.0 g, $3.3 \mathrm{mmol})$ in dichloromethane (20 $\mathrm{mL})$ was cooled to $0{ }^{\circ} \mathrm{C}$ in an ice bath. Aqueous trifluoroacetic acid solution $(40 \% \mathrm{v} / \mathrm{v}, 10 \mathrm{~mL})$ was added to the reaction mixture at $0{ }^{\circ} \mathrm{C}$. The solution was allowed to warm to room temperature and stirred for $4 \mathrm{~h}$ at $30^{\circ} \mathrm{C}$ until the starting material was completely consumed by TLC. The reaction mixture was diluted with dichloromethane $(30 \mathrm{~mL})$ and water $(30 \mathrm{~mL})$. The layers were separated and the water layer was extracted twice with dichloromethane $(2 \times 20 \mathrm{~mL})$. The combined organic layer was washed with water $(3 \times 20 \mathrm{~mL})$, saturated solution of sodium bicarbonate $(3 \times 20 \mathrm{~mL})$ and dried over $\mathrm{Na}_{2} \mathrm{SO}_{4}$. The organic solvent was removed in vacuo to afford white solid $(1.52 \mathrm{~g}, 2.92 \mathrm{mmol})$ and trace water was removed by co evaporation with toluene $(3 \times 10 \mathrm{~mL})$ to afford a white solid. To a solution of the resulting compound $(1.30 \mathrm{~g}, 2.49$ $\mathrm{mmol})$ in anhydrous dichloromethane $(12 \mathrm{~mL})$ was added levulinic acid $(0.580 \mathrm{~g}, 4.98 \mathrm{mmol})$ and 2-chloromethyl pyridinium iodide (CMPI) $(1.59 \mathrm{~g}, 6.22 \mathrm{mmol})$. The mixture was stirred for 15 minutes at room temperature and then cooled to $-20^{\circ} \mathrm{C}$ followed by the addition of 1,4-diazabicyclo[2,2,2]octane $(1.06 \mathrm{~g}, 9.46 \mathrm{mmol})$. The reaction mixture was allowed to warm up slowly to room temperature. After $24 \mathrm{~h}$, another portion of levulinic acid $(0.290 \mathrm{~g}, 2.49 \mathrm{mmol})$ and 2chloromethyl pyridinium iodide (CMPI) $(0.790 \mathrm{~g}, 3.11 \mathrm{mmol})$ were added. Stirring was continued until TLC indicated almost complete consumption of starting material $(48 \mathrm{~h})$. The reaction mixture was filtered through Celite, diluted with EtOAc $(30 \mathrm{~mL})$ and washed with a $5 \%$ solution of $\mathrm{NaCl}(5 \times 25 \mathrm{~mL})$. The organic layers were dried over $\mathrm{Na}_{2} \mathrm{SO}_{4}$, filtered and the filtrate was concentrated in vacuo. Silica gel column chromatography of the residue (45\% to $50 \%$ ethyl acetate gradient in hexane) afforded compound $\mathbf{5 6}$ as a white solid (1.31 g, $2.12 \mathrm{mmol}, 85 \%)$. ${ }^{1} \mathbf{H}$ NMR $\left(400 \mathrm{MHz}, \mathrm{CDCl}_{3}\right) \delta 7.37-7.23(\mathrm{~m}, 6 \mathrm{H}), 6.98-6.90(\mathrm{~m}, 2 \mathrm{H}), 6.77(\mathrm{~d}, J=9.0 \mathrm{~Hz}$, $2 \mathrm{H}), 5.23(\mathrm{~d}, J=8.2 \mathrm{~Hz}, 1 \mathrm{H}), 4.87-4.74(\mathrm{~m}, 2 \mathrm{H}), 4.48(\mathrm{dd}, J=12.1,4.6 \mathrm{~Hz}, 1 \mathrm{H}), 4.30(\mathrm{dd}, J=$ 12.0, $1.7 \mathrm{~Hz}, 1 \mathrm{H}), 4.04(\mathrm{dd}, J=10.2,8.2 \mathrm{~Hz}, 1 \mathrm{H}), 3.90-3.79(\mathrm{~m}, 1 \mathrm{H}), 3.74(\mathrm{~s}, 3 \mathrm{H}), 3.69-3.54$ (m, 2H), 3.27 (d, $J=4.2 \mathrm{~Hz}, 1 \mathrm{H}), 2.73(\mathrm{t}, J=6.4 \mathrm{~Hz}, 2 \mathrm{H}), 2.58$ (t, $J=6.4 \mathrm{~Hz}, 2 \mathrm{H}), 2.14(\mathrm{~s}, 3 \mathrm{H})$. ${ }^{13}$ C NMR $\left(101 \mathrm{MHz}, \mathrm{CDCl}_{3}\right) \delta$ 207.2, 173.3, 162.2, 155.6, 151.2, 137.9, 128.6, 128.1, 128.0, $118.9,114.5,99.5,92.4,79.4,74.7,73.9,70.8,63.3,57.8,55.6,37.9,29.7,27.9$. HRMS-ESITOF (m/z): $[\mathrm{M}+\mathrm{Na}]^{+}$Calculated for $\mathrm{C}_{27} \mathrm{H}_{30} \mathrm{Cl}_{3} \mathrm{NO}_{9}$ 642.0860, Found 642.0864.

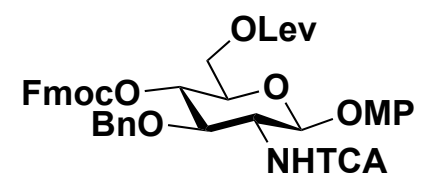

57

4-Methoxyphenyl 3-O-benzyl-2-deoxy-4-O-(9-fluorenylmethoxycarbonyl)-6- $O$-levulinoyl-2trichloroacetamido- $\beta$-D-glucopyranoside (57): The compound $56(0.780 \mathrm{~g}, 1.26 \mathrm{mmol})$ was dissolved in a mixture of dichloromethane $(7 \mathrm{~mL})$ and pyridine $(1 \mathrm{~mL})$ and the solution was stirred at $0{ }^{\circ} \mathrm{C}$ in an ice bath. Solid FmocCl (9-Fluorenylmethoxycarbonyl chloride) (0.650 g, $2.52 \mathrm{mmol}$ ) was added portion wise to the reaction mixture at the same temperature. The reaction mixture was then allowed to warm to room temperature and stirred for $5 \mathrm{~h}$. The reaction was 
diluted with water $(100 \mathrm{~mL})$ and extracted with dichloromethane several times. The organic layer was dried over $\mathrm{Na}_{2} \mathrm{SO}_{4}$. The solvent was removed in vacuo and the resulting crude oil was purified by flash column chromatography on silica gel (35\% to $45 \%$ ethyl acetate gradient in hexane) to afford compound $\mathbf{5 7}(0.920 \mathrm{~g}, 1.09 \mathrm{mmol}, 87 \%)$ as a white solid. ${ }^{1} \mathbf{H}$ NMR (400 $\left.\mathrm{MHz}, \mathrm{CDCl}_{3}\right) \delta 7.74(\mathrm{dd}, J=7.5,2.9 \mathrm{~Hz}, 2 \mathrm{H}), 7.57(\mathrm{dd}, J=15.7,7.4 \mathrm{~Hz}, 2 \mathrm{H}), 7.38(\mathrm{t}, J=7.5$ $\mathrm{Hz}, 2 \mathrm{H}), 7.30-7.16(\mathrm{~m}, 8 \mathrm{H}), 7.01-6.92(\mathrm{~m}, 2 \mathrm{H}), 6.83-6.74(\mathrm{~m}, 2 \mathrm{H}), 5.39(\mathrm{~d}, J=8.0 \mathrm{~Hz}, 1 \mathrm{H})$, $4.95(\mathrm{t}, J=9.1 \mathrm{~Hz}, 1 \mathrm{H}), 4.63(\mathrm{~s}, 2 \mathrm{H}), 4.50(\mathrm{dd}, J=10.5,6.8 \mathrm{~Hz}, 1 \mathrm{H}), 4.35(\mathrm{~m}, 3 \mathrm{H}), 4.26-4.15$ $(\mathrm{m}, 2 \mathrm{H}), 3.85-3.71(\mathrm{~m}, 5 \mathrm{H}), 2.75-2.64(\mathrm{~m}, 2 \mathrm{H}), 2.62-2.52(\mathrm{~m}, 2 \mathrm{H}), 2.13(\mathrm{~s}, 3 \mathrm{H}) .{ }^{13} \mathbf{C}$ NMR $\left(101 \mathrm{MHz}, \mathrm{CDCl}_{3}\right) \delta 206.4,172.3,172.3,162.1,155.9,155.8,154.1,150.8,143.2,142.9,141.3$, 141.2, 137.1, 128.4, 127.9, 127.8, 127.2, 125.1, 124.9, 120.1, 119.1, 114.5, 98.7, 92.2, 75.0, 74.7, 71.7, 70.3, 62.5, 58.1 , 55.6, 46.7, 37.8, 29.8, 27.8. HRMS-ESI-TOF (m/z): $[\mathrm{M}+\mathrm{Na}]^{+}$Calculated for $\mathrm{C}_{42} \mathrm{H}_{40} \mathrm{Cl}_{3} \mathrm{NO}_{11}$ 864.1547, Found 864.1581.

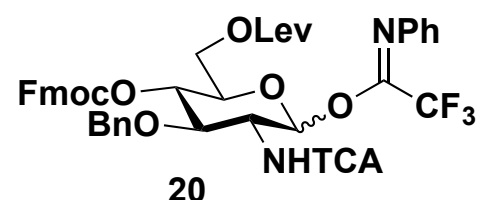

( $N$-Phenyl)-2,2,2-trifluoroacetimidate

3-O-benzyl-2-deoxy-4-O-(9fluorenylmethoxycarbonyl)-6- $O$-levulinoyl-2-trichloroacetamido- $\quad \boldsymbol{\alpha} / \boldsymbol{\beta}$-D-glucopyranoside (20): The compound $57(0.80 \mathrm{~g}, 0.95 \mathrm{mmol})$ was converted to the hemiacetal $(0.59 \mathrm{~g}, 0.81$ $\mathrm{mmol}, 85 \%)$ as white foam following the general procedure for OMP ether deprotection using ceric ammonium nitrate $(\mathrm{CAN})$. To the hemiacetal $(0.59 \mathrm{~g}, 0.81 \mathrm{mmol})$ in anhydrous acetone $(8 \mathrm{~mL})$ was added $\mathrm{CF}_{3} \mathrm{C}(\mathrm{NPh}) \mathrm{Cl}^{[1 \mathrm{~b}, 9]}$ (2,2,2-Trifluoro- $N$-phenylacetimidoyl chloride) $(0.640 \mathrm{~mL}, 4.04$ $\mathrm{mmol})$ and $\mathrm{K}_{2} \mathrm{CO}_{3}(0.560 \mathrm{~g}, 4.04 \mathrm{mmol})$ at $0{ }^{\circ} \mathrm{C}$ under argon. The reaction was stirred for $3 \mathrm{~h}$ at room temperature until the starting material was completely consumed by TLC. The reaction mixture was diluted with dichloromethane $(20 \mathrm{~mL})$ and filtered over a celite pad. The filtrate was removed in vacuo and the crude oil was purified by flash column chromatography on silica gel (30\% - 35\% EtOAc gradient in hexane) to afford compound $\mathbf{2 0}(0.65 \mathrm{~g}, 0.72 \mathrm{mmol}, 76 \%$ over 2 steps) as a pale yellow solid. ${ }^{1} \mathbf{H}$ NMR $\left(400 \mathrm{MHz}, \mathrm{CDCl}_{3}\right) \delta 7.79-7.70(\mathrm{~m}, 3 \mathrm{H}), 7.60(\mathrm{dd}, J=$ 13.6, $7.4 \mathrm{~Hz}, 3 \mathrm{H}), 7.43-7.19(\mathrm{~m}, 15 \mathrm{H}), 7.11(\mathrm{t}, J=7.5 \mathrm{~Hz}, 1 \mathrm{H}), 6.78(\mathrm{~d}, J=7.6 \mathrm{~Hz}, 2 \mathrm{H}), 6.44$ $(\mathrm{dd}, J=78.7,7.7 \mathrm{~Hz}, 2 \mathrm{H}), 5.08(\mathrm{t}, J=9.6 \mathrm{~Hz}, 1 \mathrm{H}), 4.93-4.78(\mathrm{~m}, 1 \mathrm{H}), 4.65-4.53(\mathrm{~m}, 3 \mathrm{H})$, $4.48-4.38(\mathrm{~m}, 2 \mathrm{H}), 4.33-4.17(\mathrm{~m}, 4 \mathrm{H}), 4.16-4.04(\mathrm{~m}, 1 \mathrm{H}), 3.99(\mathrm{t}, J=9.8 \mathrm{~Hz}, 1 \mathrm{H}), 2.78-$ $2.68(\mathrm{~m}, 3 \mathrm{H}), 2.65-2.56(\mathrm{~m}, 3 \mathrm{H}), 2.15(\mathrm{~d}, J=4.5 \mathrm{~Hz}, 4 \mathrm{H}) .{ }^{13} \mathbf{C}$ NMR $\left(101 \mathrm{MHz}, \mathrm{CDCl}_{3}\right) \delta$ 206.2, 172.2, 161.8, 153.9, 143.1, 142.8, 142.6, 141.4, 141.3, 137.0, 136.6, 128.8, 128.7, 128.5, $128.2,128.1,127.9,127.2,125.3,125.2,124.9,124.8,120.1,119.1,103.6,91.9,75.5,73.9,73.8$, 73.7, 71.8, 71.1, 70.4, 70.4, 70.3, 68.5, 65.6, 63.8, 61.6, 53.4, 46.7, 46.6, 37.8, 37.7, 29.7, 27.8, 27.7. HRMS-ESI-TOF (m/z): $[\mathrm{M}+\mathrm{Na}]^{+}$Calculated for $\mathrm{C}_{43} \mathrm{H}_{38} \mathrm{Cl}_{3} \mathrm{~F}_{3} \mathrm{~N}_{2} \mathrm{O}_{10}$ 929.1424, Found 929.1426. 

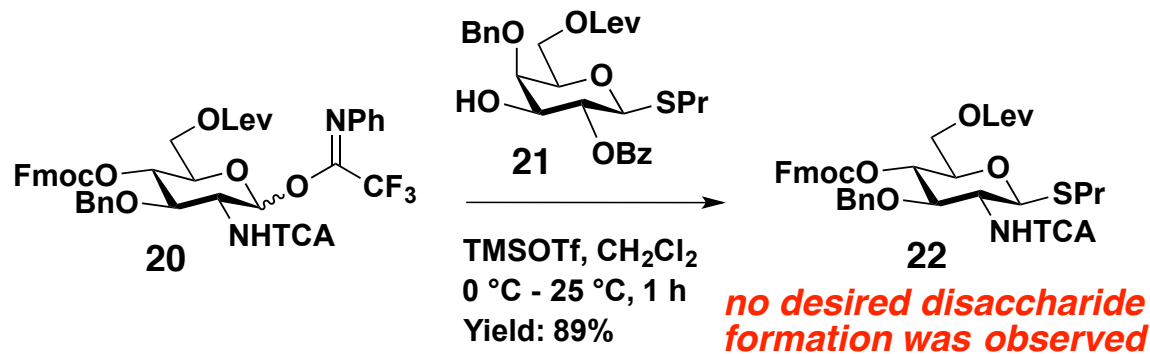

Propyl

3-O-benzyl-2-deoxy-4-O-(9-fluorenylmethoxycarbonyl)-6-O-levulinoyl-2trichloroacetamido-1-thio- $\boldsymbol{\beta}$-D-glucopyranoside (22): To a vigorously stirred solution of glycosyl donor $20(51.0 \mathrm{mg}, 56.0 \mu \mathrm{mol})$ and glycosyl acceptor 21 (15.0 mg, $28.0 \mu \mathrm{mol})$ in anhydrous dichloromethane $(1.5 \mathrm{~mL})$ under argon, catalytic TMSOTf (Trimethylsilyl trifluoromethanesulfonate) $(0.50 \mu \mathrm{L}, 2.8 \mu \mathrm{mol})$ was added at $0{ }^{\circ} \mathrm{C}$ and the reaction mixture was stirred for $1 \mathrm{~h}$ at $25^{\circ} \mathrm{C}$ until the acceptor was completely consumed shown by TLC. The reaction mixture quenched by the addition of triethylamine drop wise until the reaction mixture was neutral to a litmus paper. The reaction mixture was then diluted with dichloromethane $(3 \mathrm{~mL})$ and the solvent was removed under reduced pressure. The crude product was then purified by flash column chromatography on silica gel (25\% to $35 \%$ ethyl acetate gradient in hexane) to afford compound $22(0.020 \mathrm{~g}, 25 \mu \mathrm{mol}, 89 \%)$ as white foam. ${ }^{1} \mathbf{H}$ NMR $\left(400 \mathrm{MHz}, \mathrm{CDCl}_{3}\right) \delta$ $7.75(\mathrm{dd}, J=7.5,4.4 \mathrm{~Hz}, 2 \mathrm{H}), 7.57(\mathrm{dd}, J=20.0,7.5 \mathrm{~Hz}, 2 \mathrm{H}), 7.38(\mathrm{t}, J=7.5 \mathrm{~Hz}, 2 \mathrm{H}), 7.30-$ $7.26(\mathrm{~m}, 2 \mathrm{H}), 7.22-7.13(\mathrm{~m}, 5 \mathrm{H}), 6.91(\mathrm{~d}, J=7.7 \mathrm{~Hz}, 1 \mathrm{H}), 5.04(\mathrm{~d}, J=10.3 \mathrm{~Hz}, 1 \mathrm{H}), 4.95-$ $4.86(\mathrm{~m}, 1 \mathrm{H}), 4.62(\mathrm{~s}, 2 \mathrm{H}), 4.49(\mathrm{dd}, J=10.5,6.8 \mathrm{~Hz}, 1 \mathrm{H}), 4.34(\mathrm{dd}, J=10.5,7.4 \mathrm{~Hz}, 1 \mathrm{H}), 4.29$ - $4.15(\mathrm{~m}, 4 \mathrm{H}), 3.75(\mathrm{~m}, 1 \mathrm{H}), 3.61(\mathrm{~m}, 1 \mathrm{H}), 2.75-2.58(\mathrm{~m}, 6 \mathrm{H}), 2.17(\mathrm{~s}, 3 \mathrm{H}), 1.64(\mathrm{dt}, J=14.6$, $7.3 \mathrm{~Hz}, 2 \mathrm{H}), 0.98(\mathrm{t}, J=7.3 \mathrm{~Hz}, 3 \mathrm{H}) .{ }^{13} \mathbf{C} \mathbf{N M R}\left(126 \mathrm{MHz}, \mathrm{CDCl}_{3}\right) \delta 206.3,172.3,161.6,154.2$, 143.2, 143.0, 141.3, 137.1, 128.4, 127.9, 127.8, 127.2, 125.1, 124.9, 120.1, 82.6, 78.5, 75.7, 75.2, 74.8, 70.3, 62.7, 57.8, 46.7, 37.8, 32.7, 29.8, 29.7, 27.8, 23.2, 13.3. HRMS-ESI-TOF (m/z): $[\mathrm{M}+\mathrm{Na}]^{+}$Calculated for $\mathrm{C}_{38} \mathrm{H}_{40} \mathrm{Cl}_{3} \mathrm{NO}_{9} \mathrm{~S}$ 816.1366, Found 816.1350.

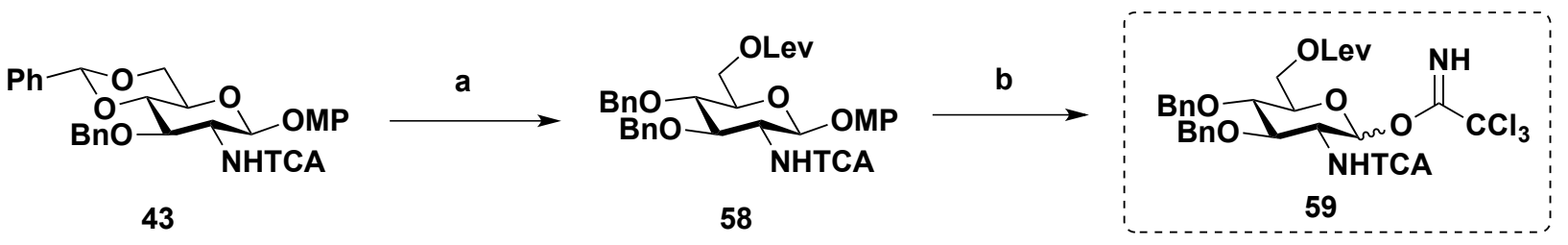

Scheme S11: Synthesis of Glucosamine donor 59

Reagents and conditions: (a) (i) $\mathrm{Bu}_{2} \mathrm{BOTf}\left(1.0 \mathrm{M}\right.$ in methylene chloride), $\mathrm{BH}_{3} \cdot \mathrm{THF}, 0{ }^{\circ} \mathrm{C}, 1.5$ h, 92\%; (ii) LevOH, DCC, DMAP, $\mathrm{CH}_{2} \mathrm{Cl}_{2}, 25^{\circ} \mathrm{C}, 2$ h, 87\%; (b) (i) $\mathrm{CAN}, \mathrm{CH}_{3} \mathrm{CN}: \mathrm{H}_{2} \mathrm{O}$ (4:1), 0 ${ }^{\circ} \mathrm{C}$ to $25^{\circ} \mathrm{C}, 0.5 \mathrm{~h}$; (ii) $\mathrm{CCl}_{3} \mathrm{CN}, \mathrm{DBU}, \mathrm{CH}_{2} \mathrm{Cl}_{2}, 0{ }^{\circ} \mathrm{C}$ to $25^{\circ} \mathrm{C}, 3 \mathrm{~h}, 72 \%$ (over 2 steps). 


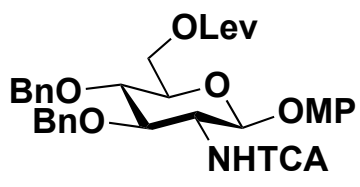

58

4-Methoxyphenyl 3,4-di- $O$-benzyl-2-deoxy-6- $O$-levulinoyl-2-trichloroacetamido- $\boldsymbol{\beta}$-Dglucopyranoside (58): To a solution of compound 43 (1.65 g, $2.71 \mathrm{mmol})$ in $\mathrm{BH}_{3} \cdot \mathrm{THF}(27$ $\mathrm{mL})$ was added $\mathrm{Bu}_{2} \mathrm{BOTf}\left(1.0 \mathrm{M}\right.$ in methylene chloride) $(3.25 \mathrm{~mL}, 3.25 \mathrm{mmol})$ at $0{ }^{\circ} \mathrm{C}$. The reaction was stirred for $1.5 \mathrm{~h}$ until TLC showed complete conversion of the starting material. The reaction was quenched by drop wise addition of triethylamine and methanol. The resulting solution was concentrated and purified by flash column chromatography on silica gel (20\% to $35 \%$ ethyl acetate gradient in hexane) to afford a white solid (1.52 g, $2.49 \mathrm{mmol}, 92 \%)$. The resulting compound (1.02 g, $1.67 \mathrm{mmol})$ was then successfully converted to compound 58 (1.03 $\mathrm{g}, 1.45 \mathrm{mmol}, 87 \%$ ) as a white solid by following the general procedure for 6-OH protection with Levulinate ester (Lev). ${ }^{1} \mathbf{H}$ NMR $\left(400 \mathrm{MHz}, \mathrm{CDCl}_{3}\right) \delta 7.42(\mathrm{~d}, J=8.1 \mathrm{~Hz}, 1 \mathrm{H}), 7.37$ $7.23(\mathrm{~m}, 10 \mathrm{H}), 6.96(\mathrm{~d}, J=9.0 \mathrm{~Hz}, 2 \mathrm{H}), 6.77(\mathrm{~d}, J=9.0 \mathrm{~Hz}, 2 \mathrm{H}), 5.27(\mathrm{~d}, J=7.2 \mathrm{~Hz}, 1 \mathrm{H}), 4.80$ $(\mathrm{d}, J=11.0 \mathrm{~Hz}, 3 \mathrm{H}), 4.61(\mathrm{~d}, J=10.9 \mathrm{~Hz}, 1 \mathrm{H}), 4.43-4.26(\mathrm{~m}, 2 \mathrm{H}), 4.17(\mathrm{t}, J=8.4 \mathrm{~Hz}, 1 \mathrm{H})$, $4.02-3.92(\mathrm{~m}, 1 \mathrm{H}), 3.84-3.77(\mathrm{~m}, 1 \mathrm{H}), 3.74(\mathrm{~s}, 3 \mathrm{H}), 3.65(\mathrm{t}, J=8.1 \mathrm{~Hz}, 1 \mathrm{H}), 2.69(\mathrm{t}, J=6.5$ $\mathrm{Hz}, 2 \mathrm{H}), 2.53(\mathrm{q}, J=6.4 \mathrm{~Hz}, 2 \mathrm{H}), 2.15(\mathrm{~s}, 3 \mathrm{H}) .{ }^{13} \mathbf{C} \mathbf{N M R}\left(101 \mathrm{MHz}, \mathrm{CDCl}_{3}\right) \delta 206.4,172.3$, $162.0,155.5,151.1,137.5,137.3,128.5,128.1,128.1,127.9,127.8,118.6,114.5,98.9,92.41$, 79.1, 77.6, 74.8, 74.6, 72.9, 63.3, 56.8, 55.6, 37.8, 29.8, 27.8. HRMS-ESI-TOF (m/z): $[\mathrm{M}+\mathrm{Na}]^{+}$Calculated for $\mathrm{C}_{34} \mathrm{H}_{36} \mathrm{Cl}_{3} \mathrm{NO}_{9}$ 732.1332, Found 732.1327.

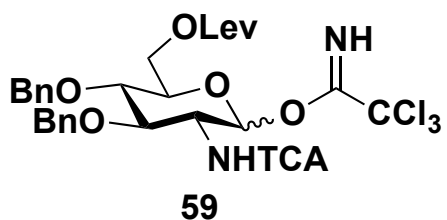

\section{3,4-di- $O$-benzyl-2-deoxy-6- $O$-levulinoyl-2-trichloroacetamido- $\alpha / \beta$-D-glucopyranosyl}

trichloroacetimidate (59): The compound $58(1.10 \mathrm{~g}, 1.55 \mathrm{mmol})$ was converted to the hemiacetal $(0.78 \mathrm{~g}, 1.3 \mathrm{mmol}, 84 \%)$ as white foam by following the general procedure for OMP ether deprotection using ceric ammonium nitrate (CAN). Compound 59 (0.830 g, 1.12 mmol, 72\% over 2 steps) as pale yellow oil was then synthesized from the hemiacetal by following the general procedure for trichloroacetimidate formation. ${ }^{1} \mathbf{H} \mathbf{~ N M R}\left(400 \mathrm{MHz}, \mathrm{CDCl}_{3}\right) \delta 8.75(\mathrm{~s}, 1 \mathrm{H}), 7.42-7.26$ $(\mathrm{m}, 10 \mathrm{H}), 6.55(\mathrm{~d}, J=8.6 \mathrm{~Hz}, 1 \mathrm{H}), 6.38(\mathrm{~d}, J=3.4 \mathrm{~Hz}, 1 \mathrm{H}), 4.90(\mathrm{dd}, J=10.9,2.9 \mathrm{~Hz}, 2 \mathrm{H}), 4.81$ $(\mathrm{d}, J=11.2 \mathrm{~Hz}, 1 \mathrm{H}), 4.66(\mathrm{~d}, J=10.7 \mathrm{~Hz}, 1 \mathrm{H}), 4.42-4.30(\mathrm{~m}, 3 \mathrm{H}), 4.08-3.92(\mathrm{~m}, 2 \mathrm{H}), 3.80(\mathrm{t}$, $J=9.4 \mathrm{~Hz}, 1 \mathrm{H}), 2.75(\mathrm{q}, J=6.1,5.5 \mathrm{~Hz}, 2 \mathrm{H}), 2.59(\mathrm{~m}, 2 \mathrm{H}), 2.19(\mathrm{~s}, 3 \mathrm{H}) .{ }^{13} \mathbf{C} \mathbf{N M R}(101 \mathrm{MHz}$, $\left.\mathrm{CDCl}_{3}\right) \delta 206.4,172.3,161.8,160.0,137.1,128.8,128.6,128.4,128.3,128.2,128.2,94.5,92.0$, 90.6, 78.2, 77.4, 77.2, 75.4, 75.1, 72.1, 62.1, 54.2, 37.8, 29.8, 27.8. HRMS-ESI-TOF (m/z): $[\mathrm{M}+\mathrm{Na}]^{+}$Calculated for $\mathrm{C}_{29} \mathrm{H}_{30} \mathrm{Cl}_{6} \mathrm{~N}_{2} \mathrm{O}_{8}$ 769.0005, Found 768.9974. 


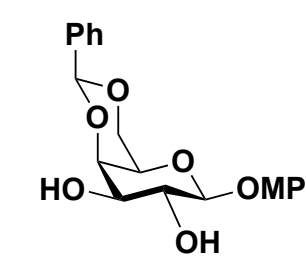

60

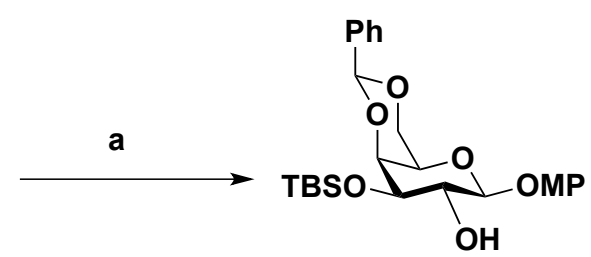

61
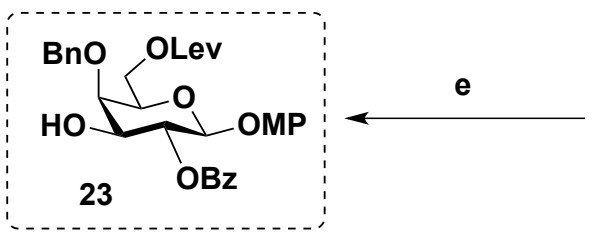

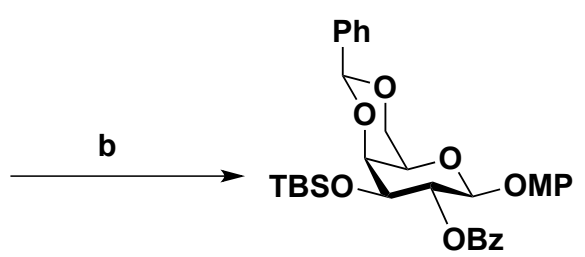

62

$\checkmark$

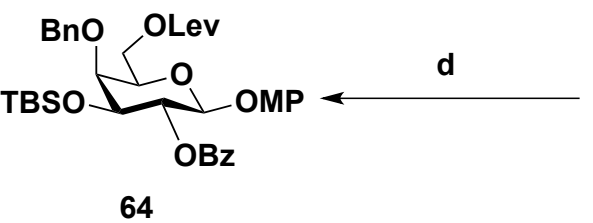

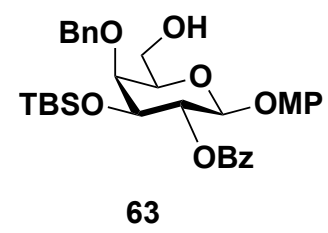

Scheme S12: Synthesis of Galactose acceptor 23

Reagents and conditions: (a) TBSCl, imidazole, DMF, $25^{\circ} \mathrm{C}, 24 \mathrm{~h}, 86 \%$; (b) $\mathrm{BzCl}$, pyridine, DMAP, $0{ }^{\circ} \mathrm{C}$ to $40{ }^{\circ} \mathrm{C}, 8 \mathrm{~h}, 87 \%$; (c) Bu 2 BOTf (1.0M in methylene chloride), $\mathrm{BH}_{3} \cdot \mathrm{THF}, 0{ }^{\circ} \mathrm{C}$, $1 \mathrm{~h}, 90 \%$; (d) LevOH, DCC, DMAP, $\mathrm{CH}_{2} \mathrm{Cl}_{2}, 25^{\circ} \mathrm{C}, 2 \mathrm{~h}, 88 \%$; (e) $\mathrm{BF}_{3} \cdot \mathrm{OEt}_{2}, \mathrm{CH}_{2} \mathrm{Cl}_{2}: \mathrm{CH}_{3} \mathrm{CN}$ $(1: 2 \mathrm{v} / \mathrm{v}), 0^{\circ} \mathrm{C}, 1 \mathrm{~h}, 91 \%$.

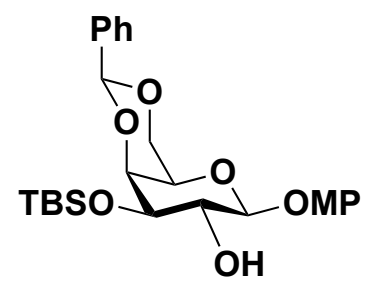

61

4-Methoxyphenyl 4,6-O-benzylidene-3- $O$-t-butyldimethylsilyl- $\boldsymbol{\beta}$-D-galactopyranoside (61): To a stirred solution of compound $\mathbf{6 0}^{[10]}(2.70 \mathrm{~g}, 7.21 \mathrm{mmol})$ in dry DMF $(20 \mathrm{~mL}), t$ butyldimethylsilyl chloride(1.19 g, $7.93 \mathrm{mmol})$ and imidazole $(0.740 \mathrm{~g}, 10.8 \mathrm{mmol})$ were added at room temperature. The reaction mixture was stirred for $24 \mathrm{~h}$ at the same temperature. The mixture was then diluted with ethyl acetate $(60 \mathrm{~mL})$ and washed with water $(5 \mathrm{X} 60 \mathrm{~mL})$. The organic layer was dried over $\mathrm{Na}_{2} \mathrm{SO}_{4}$ and concentrated in vacuo. The resulting crude oil was purified by flash column chromatography on silica gel $(10 \%$ to $20 \%$ ethyl acetate gradient in hexane) to afford compound $\mathbf{6 1}(3.03 \mathrm{~g}, 6.20 \mathrm{mmol}, 86 \%)$ as a white solid. ${ }^{1} \mathbf{H}$ NMR $\left(400 \mathrm{MHz}, \mathrm{CDCl}_{3}\right) \delta 7.40(\mathrm{dd}, J=7.6,1.8 \mathrm{~Hz}, 2 \mathrm{H}), 7.27-7.16(\mathrm{~m}, 3 \mathrm{H}), 6.97-6.88(\mathrm{~m}$, $2 \mathrm{H}), 6.71-6.61(\mathrm{~m}, 2 \mathrm{H}), 5.39(\mathrm{~s}, 1 \mathrm{H}), 4.65(\mathrm{~d}, J=7.8 \mathrm{~Hz}, 1 \mathrm{H}), 4.22(\mathrm{dd}, J=12.4,1.2 \mathrm{~Hz}, 1 \mathrm{H})$, $3.93(\mathrm{~m}, 3 \mathrm{H}), 3.68-3.58(\mathrm{~m}, 4 \mathrm{H}), 3.35$ (s, 1H), 2.27 (d, J=2.3 Hz, 1H), 0.79 (s, 9H), 0.01 (d, $J$ $=4.6 \mathrm{~Hz}, 6 \mathrm{H}) .{ }^{13} \mathrm{C}$ NMR $\left(101 \mathrm{MHz}, \mathrm{CDCl}_{3}\right) \delta 155.6,151.5,138.1,128.9,128.2,126.4,119.3$, $114.5,102.8,101.0,76.5,74.3,70.7,69.4,66.9,55.8,25.9,18.4,-4.2,-4.4$. 


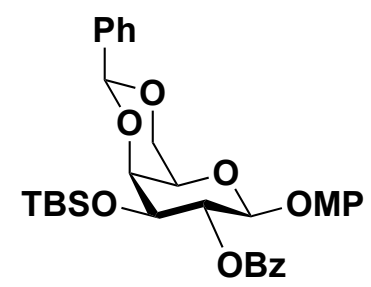

62

4-Methoxyphenyl

2-O-benzoyl-4,6- $\boldsymbol{O}$-benzylidene-3-O-t-butyldimethylsilyl- $\boldsymbol{\beta}$-Dgalactopyranoside (62): The compound 61 (1.50 g, $3.07 \mathrm{mmol})$ was dissolved in anhydrous pyridine $(15 \mathrm{~mL})$ and cooled to $0{ }^{\circ} \mathrm{C}$. Benzoyl chloride $(0.710 \mathrm{~mL}, 6.14 \mathrm{mmol})$ and 4(dimethylamino)pyridine $(0.180 \mathrm{~g}, 1.53 \mathrm{mmol})$ were added to the reaction mixture. The reaction was stirred for $8 \mathrm{~h}$ at $40{ }^{\circ} \mathrm{C}$ until the starting material was completely consumed by TLC. The organic solvent was removed in vacuo and trace of pyridine was removed by co-evaporation with toluene $(3 \times 15 \mathrm{~mL})$. The resulting oil was dissolved in dichloromethane $(30 \mathrm{~mL})$. The organic layer was washed with water $(5 \times 30 \mathrm{~mL})$ and brine solution $(2 X 30 \mathrm{~mL})$. The organic phase was dried over $\mathrm{Na}_{2} \mathrm{SO}_{4}$ and the solvent was removed in vacuo. The crude material was purified by flash column chromatography on silica gel (15\% to $25 \%$ ethyl acetate gradient in hexane) to afford compound $\mathbf{6 2}(1.58 \mathrm{~g}, 2.66 \mathrm{mmol}, 87 \%)$ as a white solid. ${ }^{\mathbf{1}} \mathbf{H}$ NMR (400 $\left.\mathrm{MHz}, \mathrm{CDCl}_{3}\right) \delta$ $8.20-8.09(\mathrm{~m}, 2 \mathrm{H}), 7.72-7.60(\mathrm{~m}, 3 \mathrm{H}), 7.56-7.40(\mathrm{~m}, 5 \mathrm{H}), 7.07-6.98(\mathrm{~m}, 2 \mathrm{H}), 6.85-6.78$ $(\mathrm{m}, 2 \mathrm{H}), 5.83(\mathrm{dd}, J=9.6,8.2 \mathrm{~Hz}, 1 \mathrm{H}), 5.66(\mathrm{~s}, 1 \mathrm{H}), 5.11(\mathrm{~d}, J=8.1 \mathrm{~Hz}, 1 \mathrm{H}), 4.50(\mathrm{dd}, J=12.3$, $1.1 \mathrm{~Hz}, 1 \mathrm{H}), 4.27-4.14(\mathrm{~m}, 3 \mathrm{H}), 3.81(\mathrm{~s}, 3 \mathrm{H}), 3.68(\mathrm{~s}, 1 \mathrm{H}), 0.87(\mathrm{~s}, 9 \mathrm{H}), 0.15(\mathrm{~s}, 3 \mathrm{H}), 0.00$ (s, 3H). ${ }^{13} \mathrm{C}$ NMR $\left(101 \mathrm{MHz}, \mathrm{CDCl}_{3}\right) \delta 165.3,155.6,151.8,137.9,133.1,130.5,129.8,128.9$, $128.5,128.2,126.4,119.5,114.5,101.7,101.1,76.6,72.5,72.2,69.2,67.1,55.7,25.6,18.1$, 4.4, -4.5. HRMS-ESI-TOF (m/z): $[\mathrm{M}+\mathrm{Na}]^{+}$Calculated for $\mathrm{C}_{33} \mathrm{H}_{40} \mathrm{O}_{8} \mathrm{Si}$ 615.2390, Found 615.2386.

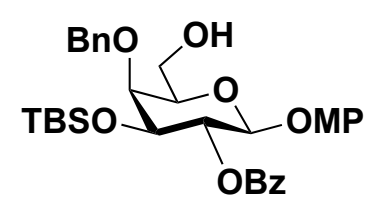

63

4-Methoxyphenyl 2-O-benzoyl-4- $O$-benzyl-3- $O$ - $t$-butyldimethylsilyl- $\boldsymbol{\beta}$-D-galactopyranoside (63): To a solution of compound $62(1.22 \mathrm{~g}, 2.06 \mathrm{mmol})$ in $\mathrm{BH}_{3} \cdot \mathrm{THF}(21 \mathrm{~mL})$ was added $\mathrm{Bu}_{2} \mathrm{BOTf}\left(1.0 \mathrm{M}\right.$ in methylene chloride) $(2.47 \mathrm{~mL}, 2.47 \mathrm{mmol})$ at $0{ }^{\circ} \mathrm{C}$. The reaction was stirred for $1 \mathrm{~h}$ until TLC showed complete conversion of the starting material. The reaction was quenched by drop wise addition of triethylamine and methanol. The resulting solution was concentrated and purified by flash column chromatography on silica gel (30\% to 35\% ethyl acetate gradient in hexane) to afford compound $\mathbf{6 3}(1.10 \mathrm{~g}, 1.85 \mathrm{mmol}, 90 \%)$ as a white solid. ${ }^{1}$ H NMR $\left(400 \mathrm{MHz}, \mathrm{CDCl}_{3}\right) \delta 8.13-8.05(\mathrm{~m}, 2 \mathrm{H}), 7.65-7.55(\mathrm{~m}, 1 \mathrm{H}), 7.52-7.31(\mathrm{~m}, 7 \mathrm{H})$, $6.95-6.85(\mathrm{~m}, 2 \mathrm{H}), 6.79-6.69(\mathrm{~m}, 2 \mathrm{H}), 5.87(\mathrm{dd}, J=9.6,7.9 \mathrm{~Hz}, 1 \mathrm{H}), 5.16(\mathrm{~d}, J=11.6 \mathrm{~Hz}$, 1H), 5.02 (d, $J=7.8 \mathrm{~Hz}, 1 \mathrm{H}), 4.68(\mathrm{~d}, J=11.6 \mathrm{~Hz}, 1 \mathrm{H}), 4.09$ (dd, $J=9.7,2.8 \mathrm{~Hz}, 1 \mathrm{H}), 3.93$ (m, 
$1 \mathrm{H}), 3.85(\mathrm{~d}, J=2.2 \mathrm{~Hz}, 1 \mathrm{H}), 3.76-3.63(\mathrm{~m}, 5 \mathrm{H}), 1.91-1.80(\mathrm{~m}, 1 \mathrm{H}), 0.87(\mathrm{~s}, 9 \mathrm{H}), 0.19(\mathrm{~s}$, $3 \mathrm{H}),-0.00(\mathrm{~s}, 3 \mathrm{H}) .{ }^{13} \mathbf{C}$ NMR $\left(101 \mathrm{MHz}, \mathrm{CDCl}_{3}\right) \delta 165.4,155.5,151.6,138.5,133.1,130.3$, 129.9, 128.6, 128.5, 128.4, 128.0, 118.7, 114.6, 101.4, 76.4, 75.4, 75.0, 74.4, 72.7, 62.1, 55.7, 25.7, 18.0, -3.9, -4.8 .

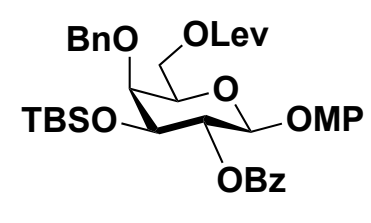

64

4-Methoxyphenyl 2- $O$-benzoyl-4- $O$-benzyl-3- $O$ - $t$-butyldimethylsilyl-6- $O$-levulinoyl- $\beta$-Dgalactopyranoside (64): Compound $\mathbf{6 3}(1.15 \mathrm{~g}, 1.93 \mathrm{mmol})$ was then successfully converted to compound $64(1.18 \mathrm{~g}, 1.70 \mathrm{mmol}, 88 \%)$ as a white solid by following the general procedure for 6-OH protection with Levulinate ester (Lev). ${ }^{1} \mathbf{H}$ NMR $\left(400 \mathrm{MHz}, \mathrm{CDCl}_{3}\right) \delta 8.14-8.04(\mathrm{~m}, 2 \mathrm{H}), 7.60$ $(\mathrm{t}, J=7.4 \mathrm{~Hz}, 1 \mathrm{H}), 7.51-7.28(\mathrm{~m}, 7 \mathrm{H}), 6.99-6.89(\mathrm{~m}, 2 \mathrm{H}), 6.80-6.71(\mathrm{~m}, 2 \mathrm{H}), 5.85(\mathrm{dd}, J=$ 9.3, $7.9 \mathrm{~Hz}, 1 \mathrm{H}), 5.17(\mathrm{~d}, J=11.4 \mathrm{~Hz}, 1 \mathrm{H}), 5.01(\mathrm{~d}, J=7.7 \mathrm{~Hz}, 1 \mathrm{H}), 4.67(\mathrm{~d}, J=11.4 \mathrm{~Hz}, 1 \mathrm{H})$, 4.41 (dd, $J=11.2,7.1 \mathrm{~Hz}, 1 \mathrm{H}), 4.28(\mathrm{dd}, J=11.2,5.5 \mathrm{~Hz}, 1 \mathrm{H}), 4.09$ (dd, $J=9.5,2.7 \mathrm{~Hz}, 1 \mathrm{H})$, $3.94-3.83(\mathrm{~m}, 2 \mathrm{H}), 3.75(\mathrm{~s}, 3 \mathrm{H}), 2.76(\mathrm{td}, J=6.2,2.0 \mathrm{~Hz}, 2 \mathrm{H}), 2.60-2.49(\mathrm{~m}, 2 \mathrm{H}), 2.20(\mathrm{~s}$, $3 \mathrm{H}), 0.86$ (s, 9H), 0.19 (s, 3H), 0.00 (s, 3H). ${ }^{13} \mathbf{C}$ NMR $\left(101 \mathrm{MHz}, \mathrm{CDCl}_{3}\right) \delta 206.6,172.6,165.3$, 155.5, 151.7, 138.4, 133.2, 130.3, 129.9, 128.5, 128.5, 128.2, 127.8, 118.9, 114.5, 101.3, 76.5, $75.1,74.2,72.6,72.6,63.5,55.7,38.1,29.9,28.0,25.7,18.0,-3.9,-4.9$. HRMS-ESI-TOF (m/z): $[\mathrm{M}+\mathrm{Na}]^{+}$Calculated for $\mathrm{C}_{38} \mathrm{H}_{48} \mathrm{O}_{10} \mathrm{Si}$ 715.2914, Found 715.2892.

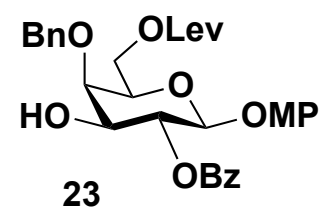

4-Methoxyphenyl 2- $O$-benzoyl-4- $O$-benzyl-6- $O$-levulinoyl- $\boldsymbol{\beta}$-D-galactopyranoside (23): The compound $64(1.0 \mathrm{~g}, 1.4 \mathrm{mmol})$ was dissolved in a mixture of dichloromethane and acetonitrile (21 mL, 1:2 dichloromethane/acetonitrile) and the solution was stirred at $0{ }^{\circ} \mathrm{C}$ in an ice bath. $\mathrm{BF}_{3} \cdot \mathrm{OEt}_{2}$ (boron trifluoride diethyl etherate) $(0.360 \mathrm{~mL}, 2.88 \mathrm{mmol})$ was added drop wise to the reaction mixture at the same temperature. The reaction mixture was stirred for $1 \mathrm{~h}$ at $0{ }^{\circ} \mathrm{C}$ until the starting material was completely consumed by TLC. The reaction was diluted with water (30 $\mathrm{mL}$ ) and extracted with dichloromethane several times. The organic layer was dried over $\mathrm{Na}_{2} \mathrm{SO}_{4}$. The solvent was removed in vacuo and the resulting crude oil was purified by flash column chromatography on silica gel ( $40 \%$ to $45 \%$ ethyl acetate gradient in hexane) to afford compound $23(0.760 \mathrm{~g}, 1.31 \mathrm{mmol}, 91 \%)$ as a white solid. ${ }^{1} \mathbf{H} \mathbf{~ N M R}\left(400 \mathrm{MHz}, \mathrm{CDCl}_{3}\right) \delta$ $8.07-7.98(\mathrm{~m}, 2 \mathrm{H}), 7.55(\mathrm{t}, J=7.4 \mathrm{~Hz}, 1 \mathrm{H}), 7.45-7.26(\mathrm{~m}, 7 \mathrm{H}), 6.97-6.88(\mathrm{~m}, 2 \mathrm{H}), 6.79-$ $6.69(\mathrm{~m}, 2 \mathrm{H}), 5.49(\mathrm{dd}, J=9.6,8.0 \mathrm{~Hz}, 1 \mathrm{H}), 4.98(\mathrm{~d}, J=7.9 \mathrm{~Hz}, 1 \mathrm{H}), 4.85(\mathrm{~d}, J=11.6 \mathrm{~Hz}, 1 \mathrm{H})$, 4.77 (d, $J=11.6 \mathrm{~Hz}, 1 \mathrm{H}), 4.37$ (dd, $J=11.2,7.0 \mathrm{~Hz}, 1 \mathrm{H}), 4.20$ (dd, $J=11.2,6.0 \mathrm{~Hz}, 1 \mathrm{H}), 3.93-$ $3.85(\mathrm{~m}, 2 \mathrm{H}), 3.82(\mathrm{t}, J=6.5 \mathrm{~Hz}, 1 \mathrm{H}), 3.72(\mathrm{~s}, 3 \mathrm{H}), 2.82(\mathrm{~d}, J=8.6 \mathrm{~Hz}, 1 \mathrm{H}), 2.72(\mathrm{~m}, 2 \mathrm{H}), 2.53$ 
(m, 2H), 2.15 (s, 3H). ${ }^{13} \mathbf{C}$ NMR $\left(101 \mathrm{MHz}, \mathrm{CDCl}_{3}\right) \delta 206.4,172.3,166.9,155.5,151.3,137.6$, 133.3, 129.8, 129.5, 128.6, 128.4, 128.1, 128.0, 118.7, 114.4, 100.5, 76.1, 75.6, 73.9, 73.5, 72.4, 62.7, 55.6, 37.8, 29.7, 27.8. HRMS-ESI-TOF (m/z): $[\mathrm{M}+\mathrm{Na}]^{+}$Calculated for $\mathrm{C}_{32} \mathrm{H}_{34} \mathrm{O}_{10}$ 601.2050, Found 601.2039.

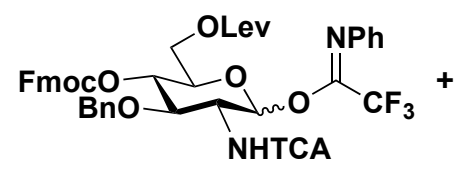

20

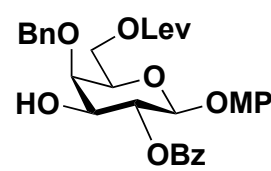

23

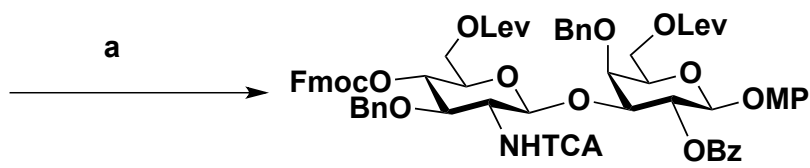

24

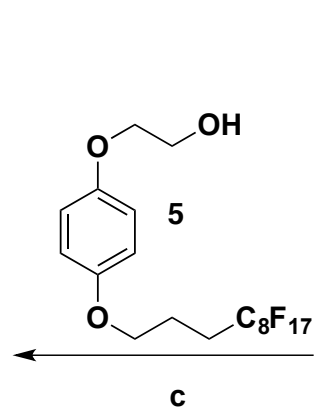

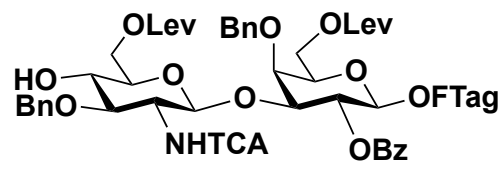

29

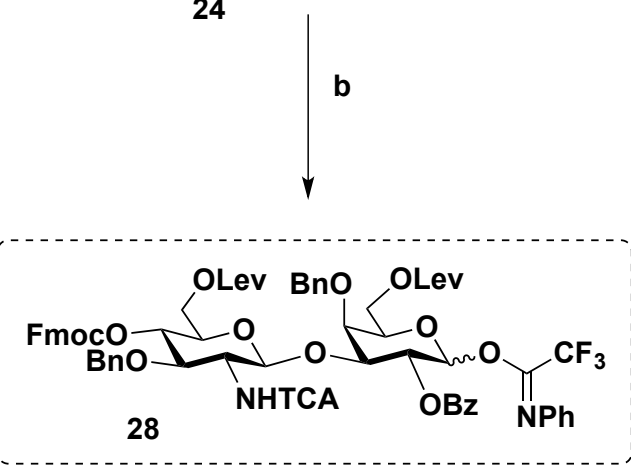

Scheme S13: Synthesis of repeating disaccharide donor 28 and disaccharide acceptor 29

Reagents and conditions: (a) TMSOTf, $\mathrm{CH}_{2} \mathrm{Cl}_{2}, 0{ }^{\circ} \mathrm{C}-25{ }^{\circ} \mathrm{C}, 1 \mathrm{~h}, 76 \%$; (b) (i) CAN, $\mathrm{CH}_{3} \mathrm{CN}: \mathrm{H}_{2} \mathrm{O}$ (4:1), $0{ }^{\circ} \mathrm{C}$ to $25{ }^{\circ} \mathrm{C}, 0.5 \mathrm{~h}$; (ii) $\mathrm{ClC}(\mathrm{NPh}) \mathrm{CF}_{3}, \mathrm{~K}_{2} \mathrm{CO}_{3}$, acetone, $25{ }^{\circ} \mathrm{C}, 3 \mathrm{~h}, 76 \%$ (over 2 steps); (c) (i) TMSOTf, $\mathrm{CH}_{2} \mathrm{Cl}_{2}, 0{ }^{\circ} \mathrm{C}-25^{\circ} \mathrm{C}, 1 \mathrm{~h}$; (ii) $\mathrm{Et}_{3} \mathrm{~N}$, THF, $25^{\circ} \mathrm{C}, 3 \mathrm{~h}, 76 \%$ (over 2 steps).

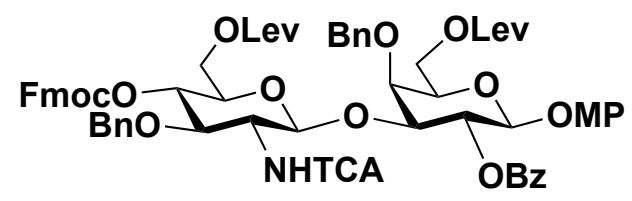

24

4-Methoxyphenyl [3-O-benzyl-2-deoxy-4-O-(9-fluorenylmethoxycarbonyl)-6- $O$-levulinoyl2-trichloroacetamido- $\beta$-D-glucopyranosyl]-( $\rightarrow 3$ )-2- $O$-benzoyl-4- $O$-benzyl-6- $O$-levulinoyl$\boldsymbol{\beta}$-D-galactopyranoside (24): To a vigorously stirred solution of glycosyl donor $\mathbf{2 0}(0.36 \mathrm{~g}, 0.39$ $\mathrm{mmol})$ and glycosyl acceptor $23(0.12 \mathrm{~g}, 0.21 \mathrm{mmol})$ in anhydrous dichloromethane $(4.0 \mathrm{~mL})$ under argon, catalytic TMSOTf (Trimethylsilyl trifluoromethanesulfonate) $(3.7 \mu \mathrm{L}, 0.020 \mathrm{mmol})$ was added at $0{ }^{\circ} \mathrm{C}$ and the reaction mixture was stirred for $1 \mathrm{~h}$ at $25{ }^{\circ} \mathrm{C}$ until the acceptor was completely consumed shown by TLC. The reaction mixture quenched by the addition of triethylamine drop wise until the reaction mixture was neutral to a litmus paper. The reaction mixture was then diluted with dichloromethane $(10 \mathrm{~mL})$ and the solvent was removed under reduced pressure. The crude product was then purified by flash column chromatography on silica gel ( $35 \%$ to $45 \%$ ethyl acetate gradient in hexane) to afford compound $\mathbf{2 4}(0.20 \mathrm{~g}$, 
$0.15 \mathrm{mmol}, 76 \%)$ as white foam. ${ }^{1} \mathbf{H}$ NMR $\left(400 \mathrm{MHz}, \mathrm{CDCl}_{3}\right) \delta 8.03(\mathrm{~d}, J=7.4 \mathrm{~Hz}, 2 \mathrm{H}), 7.73(\mathrm{~d}$, $J=7.4 \mathrm{~Hz}, 2 \mathrm{H}), 7.59-7.48(\mathrm{~m}, 3 \mathrm{H}), 7.45-7.30(\mathrm{~m}, 8 \mathrm{H}), 7.30-7.20(\mathrm{~m}, 4 \mathrm{H}), 7.13(\mathrm{dd}, J=$ 15.6, $2.9 \mathrm{~Hz}, 5 \mathrm{H}), 6.87(\mathrm{~d}, J=7.6 \mathrm{~Hz}, 1 \mathrm{H}), 6.81(\mathrm{~d}, J=9.0 \mathrm{~Hz}, 2 \mathrm{H}), 6.66(\mathrm{~d}, J=9.0 \mathrm{~Hz}, 2 \mathrm{H})$, $5.79(\mathrm{dd}, J=9.9,8.1 \mathrm{~Hz}, 1 \mathrm{H}), 5.11(\mathrm{~d}, J=8.1 \mathrm{~Hz}, 1 \mathrm{H}), 5.00(\mathrm{~d}, J=11.8 \mathrm{~Hz}, 1 \mathrm{H}), 4.94(\mathrm{~d}, J=$ $7.8 \mathrm{~Hz}, 1 \mathrm{H}), 4.87$ (t, $J=9.4 \mathrm{~Hz}, 1 \mathrm{H}), 4.70(\mathrm{~d}, J=11.8 \mathrm{~Hz}, 1 \mathrm{H}), 4.60-4.49(\mathrm{~m}, 2 \mathrm{H}), 4.45(\mathrm{dd}, J$ $=10.4,6.9 \mathrm{~Hz}, 1 \mathrm{H}), 4.35-4.23(\mathrm{~m}, 5 \mathrm{H}), 4.22-4.12(\mathrm{~m}, 4 \mathrm{H}), 3.86(\mathrm{t}, J=6.2 \mathrm{~Hz}, 1 \mathrm{H}), 3.81-$ $3.73(\mathrm{~m}, 1 \mathrm{H}), 3.68(\mathrm{~s}, 3 \mathrm{H}), 3.51(\mathrm{q}, J=8.0 \mathrm{~Hz}, 1 \mathrm{H}), 2.72(\mathrm{~m}, 4 \mathrm{H}), 2.54(\mathrm{~m}, 4 \mathrm{H}), 2.13(\mathrm{~d}, J=5.3$ $\mathrm{Hz}, 6 \mathrm{H}) .{ }^{13} \mathrm{C}$ NMR $\left(101 \mathrm{MHz}, \mathrm{CDCl}_{3}\right) \delta 206.6,206.5,172.4,172.3,165.2,161.9,155.3,154.2$, $151.4,143.1,142.9,141.3,141.2,138.5,137.2$, 133.3, 129.9, 129.7, 128.5, 128.3, 127.9, 127.7, $127.6,127.5,127.2,125.0,124.9,120.1,120.1,118.6,114.3,101.2,99.7,91.9,79.0,76.1,75.2$, 74.9, 74.5, 72.4, 71.9, 71.7, 70.3, 63.0, 62.4, 58.7, 55.5, 46.6, 37.9, 37.8, 29.8, 29.7, 27.8, 27.8. HRMS-ESI-TOF (m/z): [M+Na] $]^{+}$Calculated for $\mathrm{C}_{67} \mathrm{H}_{66} \mathrm{Cl}_{3} \mathrm{NO}_{19}$ 1318.3187, Found 1318.3148.

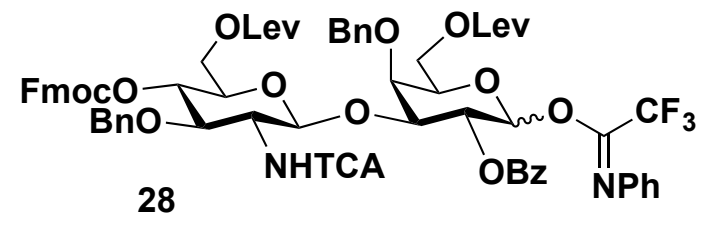

(N-Phenyl)-2,2,2-trifluoroacetimidate

[3-O-benzyl-2-deoxy-4-O-(9fluorenylmethoxycarbonyl)-6- $O$-levulinoyl-2-trichloroacetamido- $\beta$-D-glucopyranosyl]$(\mathbf{1} \rightarrow$ 3)-2-O-benzoyl-4- $\boldsymbol{O}$-benzyl-6- $\boldsymbol{O}$-levulinoyl- $\boldsymbol{\alpha} / \boldsymbol{\beta}$-D- galactopyranoside $\quad(28): \quad$ The compound $24(0.19 \mathrm{~g}, 0.15 \mathrm{mmol})$ was converted to the hemiacetal $(0.15 \mathrm{~g}, 0.12 \mathrm{mmol}, 85 \%)$ as white foam. following the general procedure for OMP ether deprotection using ceric ammonium nitrate $(\mathrm{CAN})$. To the hemiacetal $(0.15 \mathrm{~g}, 0.12 \mathrm{mmol})$ in anhydrous acetone $(5 \mathrm{~mL})$ was added $\mathrm{CF}_{3} \mathrm{C}(\mathrm{NPh}) \mathrm{Cl}\left(2,2,2-T\right.$ rifluoro- $N$-phenylacetimidoyl chloride) $(98.0 \mu \mathrm{L}, 0.620 \mathrm{mmol})$ and $\mathrm{K}_{2} \mathrm{CO}_{3}$ (potassium carbonate) $(86.0 \mathrm{mg}, 0.620 \mathrm{mmol})$ at $0{ }^{\circ} \mathrm{C}$ under argon. The reaction was stirred for 3 $\mathrm{h}$ at room temperature until the starting material was completely consumed by TLC. The reaction mixture was diluted with dichloromethane $(10 \mathrm{~mL})$ and filtered over a celite pad. The filtrate was removed in vacuo and the crude oil was purified by flash column chromatography on silica gel (35\% - 40\% EtOAc gradient in hexane and 1\% triethylamine) to afford compound $28(0.15 \mathrm{~g}$, $0.11 \mathrm{mmol}, 76 \%$ over 2 steps $)$ as white foam. ${ }^{1} \mathbf{H}$ NMR $\left(400 \mathrm{MHz}, \mathrm{CDCl}_{3}\right) \delta 8.01(\mathrm{~d}, J=7.4 \mathrm{~Hz}$, 2H), $7.74(\mathrm{dd}, J=7.5,2.9 \mathrm{~Hz}, 2 \mathrm{H}), 7.63-7.46(\mathrm{~m}, 5 \mathrm{H}), 7.37(\mathrm{~m}, 6 \mathrm{H}), 7.30-7.21(\mathrm{~m}, 4 \mathrm{H}), 7.18$ $-7.02(\mathrm{~m}, 7 \mathrm{H}), 6.97(\mathrm{~d}, J=7.3 \mathrm{~Hz}, 1 \mathrm{H}), 6.60(\mathrm{dd}, J=14.1,7.9 \mathrm{~Hz}, 1 \mathrm{H}), 6.30(\mathrm{~s}, 1 \mathrm{H}), 5.64(\mathrm{~d}, J$ $=8.6 \mathrm{~Hz}, 1 \mathrm{H}), 5.21(\mathrm{~d}, J=8.1 \mathrm{~Hz}, 1 \mathrm{H}), 4.97(\mathrm{~d}, J=11.5 \mathrm{~Hz}, 1 \mathrm{H}), 4.89(\mathrm{t}, J=9.4 \mathrm{~Hz}, 1 \mathrm{H}), 4.69$ $(\mathrm{d}, J=11.5 \mathrm{~Hz}, 1 \mathrm{H}), 4.56-4.45(\mathrm{~m}, 4 \mathrm{H}), 4.39-4.30(\mathrm{~m}, 3 \mathrm{H}), 4.29-4.14(\mathrm{~m}, 6 \mathrm{H}), 3.86(\mathrm{~m}$, 1H), $3.57-3.45(\mathrm{~m}, 1 \mathrm{H}), 2.79-2.68(\mathrm{~m}, 4 \mathrm{H}), 2.57(\mathrm{~m}, 4 \mathrm{H}), 2.15(\mathrm{~s}, 6 \mathrm{H}) .{ }^{13} \mathrm{C}$ NMR $(101 \mathrm{MHz}$, $\left.\mathrm{CDCl}_{3}\right) \delta 206.4,206.3,172.3,165.2,161.7,154.2,143.1,143.0,142.9,141.3,141.2,138.2$, $137.1,133.5,129.9,129.3,128.8,128.7,128.6,128.5,128.4,128.3,128.2,127.9,127.8,127.8$, $127.6,127.5,127.2,125.0,124.9,124.1,120.1,120.0,119.0,100.0,91.8,75.5,75.3,75.2,74.4$, $71.9,71.2,70.3,69.7,63.2,62.3,58.5,46.6,37.8,29.7,29.6,27.8,27.8$. HRMS-ESI-TOF (m/z): $[\mathrm{M}+\mathrm{Na}]^{+}$Calculated for $\mathrm{C}_{68} \mathrm{H}_{64} \mathrm{Cl}_{3} \mathrm{~F}_{3} \mathrm{~N}_{2} \mathrm{O}_{18}$ 1378.3510, Found 1378.3456. 


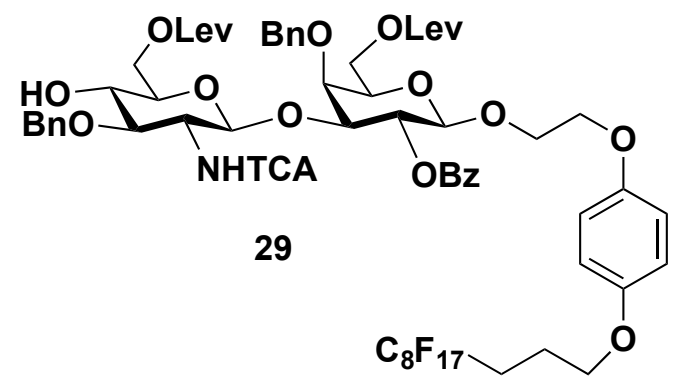

2-(4-((4,4,5,5,6,6,7,7,8,8,9,9,10,10,11,11,11-heptadecafluoroundecyl)oxy)phenoxy)ethyl (3-Obenzyl-2-deoxy-6-O-levulinoyl-2-trichloroacetamido- $\beta$-D-glucopyranosyl)-( $1 \rightarrow 3)$-2-O benzoyl-4- $O$-benzyl-6- $O$-levulinoyl- $\boldsymbol{\beta}$-D- galactopyranoside (29): To a vigorously stirred solution of glycosyl donor 28 (106 mg, $78.0 \mu \mathrm{mol})$ and glycosyl acceptor $\mathbf{5}(30.0 \mathrm{mg}, 49.0 \mu \mathrm{mol})$ in anhydrous dichloromethane $(2.0 \mathrm{~mL})$ under argon, catalytic TMSOTf (Trimethylsilyl trifluoromethanesulfonate) $(0.90 \mu \mathrm{L}, 4.9 \mu \mathrm{mol})$ was added at $0{ }^{\circ} \mathrm{C}$ and the reaction mixture was stirred for $1 \mathrm{~h}$ at $25^{\circ} \mathrm{C}$ until the acceptor was completely consumed shown by TLC. The reaction mixture quenched by the addition of triethylamine drop wise until the reaction mixture was neutral to a litmus paper. The reaction mixture was then diluted with dichloromethane $(10 \mathrm{~mL})$ and the solvent was removed under reduced pressure. The resulting crude mixture was dissolved in THF $(5 \mathrm{~mL}) . \mathrm{Et}_{3} \mathrm{~N}(2 \mathrm{~mL})$ was added to the reaction mixture at room temperature and it was stirred for $3 \mathrm{~h}$ until the starting material was completely consumed. The solvent was evaporated and the resulting crude material was purified via FSPE. The fluorous fraction was concentrated to afford compound 29 (58.0 mg, $37.0 \mu \mathrm{mol}, 76 \%$ over 2 steps) as white foam. ${ }^{1} \mathbf{H}$ NMR (500 $\left.\mathrm{MHz} \mathrm{CDCl}_{3}\right) \delta 7.90(\mathrm{~d}, J=7.8 \mathrm{~Hz}, 2 \mathrm{H}), 7.45(\mathrm{t}, J=7.4 \mathrm{~Hz}, 1 \mathrm{H}), 7.34-7.14(\mathrm{~m}, 13 \mathrm{H}), 6.71(\mathrm{~d}$, $J=7.9 \mathrm{~Hz}, 1 \mathrm{H}), 6.60(\mathrm{~d}, J=9.0 \mathrm{~Hz}, 2 \mathrm{H}), 6.47(\mathrm{~d}, J=9.0 \mathrm{~Hz}, 2 \mathrm{H}), 5.48(\mathrm{dd}, J=9.8,8.1 \mathrm{~Hz}, 1 \mathrm{H})$, $4.94-4.85(\mathrm{~m}, 2 \mathrm{H}), 4.63-4.54(\mathrm{~m}, 4 \mathrm{H}), 4.44(\mathrm{dd}, J=12.1,4.6 \mathrm{~Hz}, 1 \mathrm{H}), 4.27-4.16(\mathrm{~m}, 2 \mathrm{H})$, $4.07(\mathrm{dd}, J=11.6,5.7 \mathrm{~Hz}, 2 \mathrm{H}), 4.00(\mathrm{~d}, J=2.2 \mathrm{~Hz}, 1 \mathrm{H}), 3.97-3.92(\mathrm{~m}, 1 \mathrm{H}), 3.89-3.78(\mathrm{~m}$, $5 \mathrm{H}), 3.75-3.66(\mathrm{~m}, 2 \mathrm{H}), 3.49(\mathrm{~m}, 2 \mathrm{H}), 3.44-3.38(\mathrm{~m}, 1 \mathrm{H}), 2.79-2.74(\mathrm{~m}, 1 \mathrm{H}), 2.66(\mathrm{~m}, 4 \mathrm{H})$, $2.49(\mathrm{t}, J=5.8 \mathrm{~Hz}, 2 \mathrm{H}), 2.46-2.40(\mathrm{~m}, 2 \mathrm{H}), 2.23(\mathrm{~m}, 2 \mathrm{H}), 2.09(\mathrm{~s}, 6 \mathrm{H}), 1.98(\mathrm{~m}, 2 \mathrm{H}) .{ }^{13} \mathbf{C}$ NMR $\left(126 \mathrm{MHz}, \mathrm{CDCl}_{3}\right) \delta 206.8,206.5,173.2,172.4,165.4,161.9,153.0,152.7,138.5,137.8,133.1$, $129.9,129.8,128.5,128.4,128.2,127.9,127.9,127.6,115.4,115.3,101.6,100.0,92.1,79.5$, 78.9, 75.9, 74.8, 74.7, 73.9, 72.3, 72.1, 70.8, 67.8, 67.3, 66.9, 63.1, 63.0, 58.3, 37.9, 37.9, 29.8, 29.7, 27.9, 27.8, 20.6. HRMS-ESI-TOF (m/z): $[\mathrm{M}+\mathrm{Na}]^{+}$Calculated for $\mathrm{C}_{64} \mathrm{H}_{63} \mathrm{Cl}_{3} \mathrm{~F}_{17} \mathrm{NO}_{18}$ 1586.2729, Found 1586.2758. 


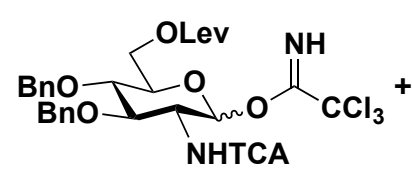

59

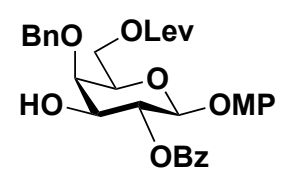

23

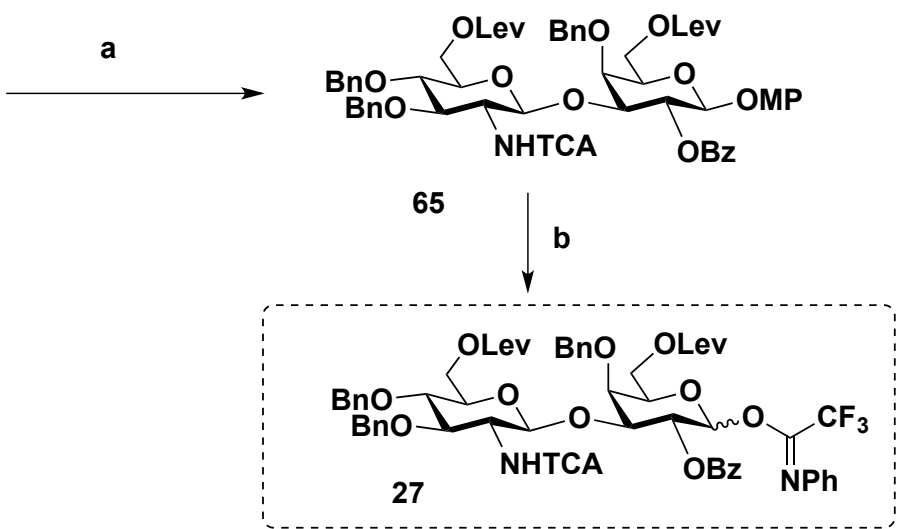

Scheme S14: Synthesis of capping disaccharide donor 27

Reagents and conditions: (a) TMSOTf, $\mathrm{CH}_{2} \mathrm{Cl}_{2}, 0{ }^{\circ} \mathrm{C}-25{ }^{\circ} \mathrm{C}, 1 \mathrm{~h}, 78 \%$; (b) (i) $\mathrm{CAN}$, $\mathrm{CH}_{3} \mathrm{CN}: \mathrm{H}_{2} \mathrm{O}(4: 1), 0{ }^{\circ} \mathrm{C}$ to $25{ }^{\circ} \mathrm{C}, 0.5 \mathrm{~h}$; (ii) $\mathrm{ClC}(\mathrm{NPh}) \mathrm{CF}_{3}, \mathrm{~K}_{2} \mathrm{CO}_{3}$, acetone, $25{ }^{\circ} \mathrm{C}, 3 \mathrm{~h}, 76 \%$ (over 2 steps).

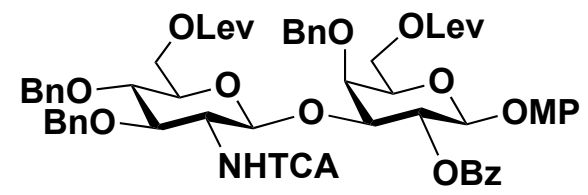

65

\section{4-Methoxyphenyl (3,4-di- $O$-benzyl-2-deoxy-6-O-levulinoyl-2-trichloroacetamido- $\beta$-D- glucopyranosyl)-( $\rightarrow 3$ )-2- $O$-benzoyl-4- $O$-benzyl-6- $O$-levulinoyl- $\beta$-D-galactopyranoside (65):} To a vigorously stirred solution of glycosyl donor $59(185 \mathrm{mg}, 0.250 \mathrm{mmol})$ and glycosyl acceptor $23(65.0 \mathrm{mg}, 0.110 \mathrm{mmol})$ in anhydrous dichloromethane $(2.5 \mathrm{~mL})$ under argon, catalytic TMSOTf (Trimethylsilyl trifluoromethanesulfonate) $(2.0 \mu \mathrm{L}, 11 \mu \mathrm{mol})$ was added at 0 ${ }^{\circ} \mathrm{C}$ and the reaction mixture was stirred for $1 \mathrm{~h}$ at $25{ }^{\circ} \mathrm{C}$ until the acceptor was completely consumed shown by TLC. The reaction mixture quenched by the addition of triethylamine drop wise until the reaction mixture was neutral to a litmus paper. The reaction mixture was then diluted with dichloromethane $(10 \mathrm{~mL})$ and the solvent was removed under reduced pressure. The crude product was then purified by flash column chromatography on silica gel (35\% to $45 \%$ ethyl acetate gradient in hexane) to afford compound $\mathbf{6 5}(0.10 \mathrm{~g}, 88 \mu \mathrm{mol}, 78 \%)$ as white foam. ${ }^{1}$ H NMR (400 MHz, $\left.\mathrm{CDCl}_{3}\right) \delta 7.95(\mathrm{~d}, J=7.5 \mathrm{~Hz}, 2 \mathrm{H}), 7.48(\mathrm{t}, J=7.3 \mathrm{~Hz}, 1 \mathrm{H}), 7.39-7.30(\mathrm{~m}$, 4H), $7.21(\mathrm{~m}, 14 \mathrm{H}), 6.74(\mathrm{t}, J=7.2 \mathrm{~Hz}, 3 \mathrm{H}), 6.60(\mathrm{~d}, J=9.0 \mathrm{~Hz}, 2 \mathrm{H}), 5.71(\mathrm{dd}, J=9.8,8.1 \mathrm{~Hz}$, $1 \mathrm{H}), 4.98-4.84(\mathrm{~m}, 3 \mathrm{H}), 4.71(\mathrm{~d}, J=11.0 \mathrm{~Hz}, 1 \mathrm{H}), 4.62(\mathrm{q}, J=12.9,11.9 \mathrm{~Hz}, 3 \mathrm{H}), 4.49(\mathrm{~d}, J=$ $11.0 \mathrm{~Hz}, 1 \mathrm{H}), 4.34(\mathrm{~d}, J=10.4 \mathrm{~Hz}, 1 \mathrm{H}), 4.27-4.10(\mathrm{~m}, 4 \mathrm{H}), 4.06-4.01(\mathrm{~m}, 1 \mathrm{H}), 3.90-3.83$ $(\mathrm{m}, 1 \mathrm{H}), 3.79(\mathrm{t}, J=6.1 \mathrm{~Hz}, 1 \mathrm{H}), 3.66-3.54(\mathrm{~m}, 5 \mathrm{H}), 3.52-3.45(\mathrm{~m}, 1 \mathrm{H}), 2.63(\mathrm{q}, J=5.3,4.7$ $\mathrm{Hz}, 4 \mathrm{H}), 2.45(\mathrm{t}, J=6.3 \mathrm{~Hz}, 4 \mathrm{H}), 2.09-2.01(\mathrm{~m}, 6 \mathrm{H}) .{ }^{13} \mathbf{C} \mathbf{N M R}\left(101 \mathrm{MHz}, \mathrm{CDCl}_{3}\right) \delta 206.5$, 206.4, 172.4, 172.3, 165.3, 161.8, 155.3, 151.4, 138.4, 137.5, 137.3, 133.3, 129.9, 129.7, 128.5, 
$128.4,128.3,128.2,128.1,127.9,127.8,127.7,127.6,118.6,114.3,101.2,99.9,92.1,79.9,78.7$, 78.1, 75.9, 74.9, 74.8, 74.7, 73.2, 72.5, 72.2, 63.1, 62.9, 58.3, 55.5, 37.8, 37.7, 29.7, 29.7, 27.8, 27.8. HRMS-ESI-TOF (m/z): $[\mathrm{M}+\mathrm{Na}]^{+}$Calculated for $\mathrm{C}_{59} \mathrm{H}_{62} \mathrm{Cl}_{3} \mathrm{NO}_{17}$ 1186.2971, Found 1186.2968 .

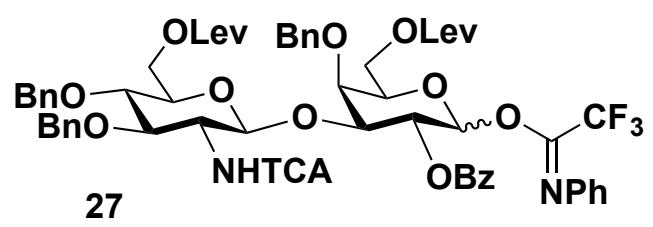

(N-Phenyl)-2,2,2-trifluoroacetimidate

(3,4-di- $O$-benzyl-2-deoxy-6-O-levulinoyl-2trichloroacetamido- $\beta$-D-glucopyranosyl)-( $1 \rightarrow 3$ )-2- $O$-benzoyl-4- $O$-benzyl-6- $O$-levulinoyl$\boldsymbol{\alpha} / \boldsymbol{\beta}$-D- galactopyranoside (27): The compound $\mathbf{6 5}(0.12 \mathrm{~g}, 0.10 \mathrm{mmol})$ was converted to the hemiacetal $(88.0 \mathrm{mg}, 83.0 \mu \mathrm{mol}, 83 \%)$ as white foam following the general procedure for OMP ether deprotection using ceric ammonium nitrate (CAN). To the hemiacetal $(88.0 \mathrm{mg}, 83.0 \mu \mathrm{mol})$ in anhydrous acetone $(3 \mathrm{~mL})$ was added $\mathrm{CF}_{3} \mathrm{C}(\mathrm{NPh}) \mathrm{Cl}$ (2,2,2-Trifluoro- $N$-phenylacetimidoyl chloride) $(66.0 \mu \mathrm{L}, 0.420 \mathrm{mmol})$ and $\mathrm{K}_{2} \mathrm{CO}_{3}$ (potassium carbonate) $(0.060 \mathrm{~g}, 0.42 \mathrm{mmol})$ at $0{ }^{\circ} \mathrm{C}$ under argon. The reaction was stirred for $3 \mathrm{~h}$ at room temperature until the starting material was completely consumed by TLC. The reaction mixture was diluted with dichloromethane $(10 \mathrm{~mL})$ and filtered over a celite pad. The filtrate was removed in vacuo and the crude oil was purified by flash column chromatography on silica gel (35\% - 40\% EtOAc gradient in hexane and 1\% triethylamine) to afford compound $27(94.0 \mathrm{mg}, 76.0 \mu \mathrm{mol}, 76 \%$ over 2 steps) as a white solid. ${ }^{1} \mathbf{H}$ NMR $\left(500 \mathrm{MHz}, \mathrm{CDCl}_{3}\right) \delta 8.04(\mathrm{~d}, J=7.4 \mathrm{~Hz}, 2 \mathrm{H}), 7.64(\mathrm{t}, J=7.4 \mathrm{~Hz}, 1 \mathrm{H}), 7.50(\mathrm{t}, J=7.7$ $\mathrm{Hz}, 2 \mathrm{H}), 7.46-7.19(\mathrm{~m}, 15 \mathrm{H}), 7.10(\mathrm{t}, J=7.7 \mathrm{~Hz}, 2 \mathrm{H}), 6.99(\mathrm{t}, J=7.3 \mathrm{~Hz}, 1 \mathrm{H}), 6.57(\mathrm{~d}, J=8.2$ $\mathrm{Hz}, 1 \mathrm{H}), 6.34(\mathrm{~s}, 2 \mathrm{H}), 5.66(\mathrm{~d}, J=7.4 \mathrm{~Hz}, 1 \mathrm{H}), 5.11(\mathrm{~d}, J=7.9 \mathrm{~Hz}, 1 \mathrm{H}), 5.01(\mathrm{~d}, J=11.4 \mathrm{~Hz}$, $1 \mathrm{H}), 4.86(\mathrm{~d}, J=11.0 \mathrm{~Hz}, 1 \mathrm{H}), 4.78(\mathrm{~d}, J=10.9 \mathrm{~Hz}, 1 \mathrm{H}), 4.71-4.62(\mathrm{~m}, 3 \mathrm{H}), 4.58-4.44(\mathrm{~m}$, 2H), $4.37-4.23(\mathrm{~m}, 5 \mathrm{H}), 4.03(\mathrm{t}, J=9.1 \mathrm{~Hz}, 1 \mathrm{H}), 3.79(\mathrm{~m}, 1 \mathrm{H}), 3.70(\mathrm{q}, J=8.2 \mathrm{~Hz}, 1 \mathrm{H}), 3.63(\mathrm{t}$, $J=8.8 \mathrm{~Hz}, 1 \mathrm{H}), 2.77(\mathrm{dd}, J=8.0,4.3 \mathrm{~Hz}, 4 \mathrm{H}), 2.59(\mathrm{~m}, 4 \mathrm{H}), 2.19(\mathrm{~d}, J=3.0 \mathrm{~Hz}, 6 \mathrm{H}) .{ }^{13} \mathbf{C} \mathbf{~ N M R}$ $\left(126 \mathrm{MHz}, \mathrm{CDCl}_{3}\right) \delta 206.3,206.2,172.4,172.4,165.2,161.7,143.1,138.3,137.4,137.3,133.6$, $129.9,129.4,128.7,128.6,128.5,128.4,128.3,128.1,128.0,127.9,127.8,127.7,119.0,100.5$, 92.0, 79.9, 78.2, 75.3, 74.9, 74.8, 73.5, 71.4, 69.9, 63.3, 62.9, 58.2, 37.9, 37.8, 29.8, 29.7, 27.8, 27.8 .

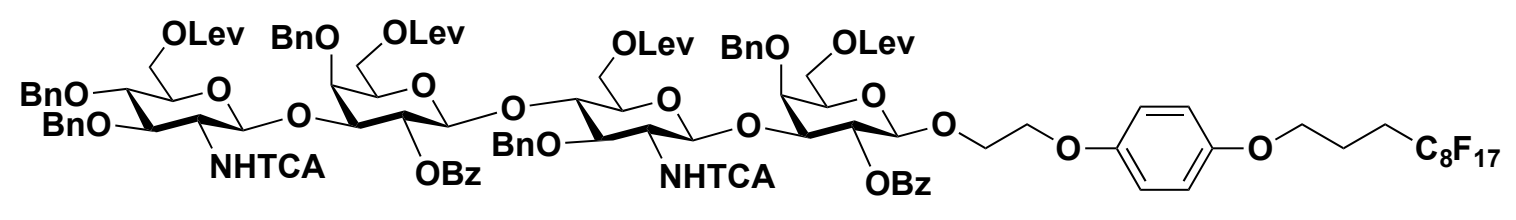

25

2-(4-((4,4,5,5,6,6,7,7,8,8,9,9,10,10,11,11,11-heptadecafluoroundecyl)oxy)phenoxy)ethyl $(3,4-$ di- $O$-benzyl-2-deoxy-6- $O$-levulinoyl-2-trichloroacetamido- $\beta$-D-glucopyranosyl)-( $1 \rightarrow 3$ )-(2$O$-benzoyl-4- $O$-benzyl-6- $O$-levulinoyl- $\beta$-D-galactopyranosyl)-(1 $\rightarrow 4)-(3-O$-benzyl-2-deoxy-6- 


\section{$O$-levulinoyl-2-trichloroacetamido- $\beta$-D-glucopyranosyl)-( $\rightarrow 3)-2-O$-benzoyl-4-O-benzyl-6-}

$\boldsymbol{O}$-levulinoyl- $\boldsymbol{\beta}$-D-galactopyranoside (25): To a vigorously stirred solution of glycosyl donor $27(64.0 \mathrm{mg}, 52.0 \mu \mathrm{mol})$ and glycosyl acceptor $29(45.0 \mathrm{mg}, 29.0 \mu \mathrm{mol})$ in anhydrous dichloromethane $(1.5 \mathrm{~mL})$ under argon, catalytic TMSOTf (Trimethylsilyl trifluoromethanesulfonate) $(0.50 \mu \mathrm{L}, 2.9 \mu \mathrm{mol})$ was added at $0{ }^{\circ} \mathrm{C}$ and the reaction mixture was stirred for $1 \mathrm{~h}$ at $25^{\circ} \mathrm{C}$ until the acceptor was completely consumed shown by TLC. The reaction mixture quenched by the addition of triethylamine drop wise until the reaction mixture was neutral to a litmus paper. The reaction mixture was then diluted with dichloromethane $(10 \mathrm{~mL})$ and the solvent was removed under reduced pressure. The resulting crude material was purified via FSPE. The fluorous fraction was concentrated to afford the tetrasaccharide 25 (63.0 $\mathrm{mg}, 24.0$ $\mu \mathrm{mol}, 83 \%)$ as white foam. ${ }^{1} \mathbf{H}$ NMR $\left(500 \mathrm{MHz} \mathrm{CDCl}_{3}\right) \delta 8.02(\mathrm{~d}, J=7.7 \mathrm{~Hz}, 2 \mathrm{H}), 7.89(\mathrm{~d}, J=$ $8.7 \mathrm{~Hz}, 2 \mathrm{H}), 7.56$ (t, $J=7.3 \mathrm{~Hz}, 1 \mathrm{H}), 7.43$ (q, $J=6.9,6.4 \mathrm{~Hz}, 3 \mathrm{H}), 7.29-7.08(\mathrm{~m}, 24 \mathrm{H}), 7.03$ (d, $J=6.7 \mathrm{~Hz}, 3 \mathrm{H}), 6.73(\mathrm{~d}, J=7.2 \mathrm{~Hz}, 1 \mathrm{H}), 6.60(\mathrm{t}, J=8.1 \mathrm{~Hz}, 3 \mathrm{H}), 6.48(\mathrm{~d}, J=8.9 \mathrm{~Hz}, 2 \mathrm{H}), 5.51$ - $5.37(\mathrm{~m}, 2 \mathrm{H}), 4.93-4.83(\mathrm{~m}, 3 \mathrm{H}), 4.78-4.56(\mathrm{~m}, 5 \mathrm{H}), 4.55-4.44(\mathrm{~m}, 6 \mathrm{H}), 4.35(\mathrm{~d}, J=10.5$ $\mathrm{Hz}, 1 \mathrm{H}), 4.23-4.08(\mathrm{~m}, 5 \mathrm{H}), 3.96(\mathrm{~m}, 5 \mathrm{H}), 3.82(\mathrm{~m}, 8 \mathrm{H}), 3.70(\mathrm{~m}, 4 \mathrm{H}), 3.59-3.47(\mathrm{~m}, 4 \mathrm{H})$, $3.34(\mathrm{~m}, 1 \mathrm{H}), 2.64(\mathrm{~m}, 5 \mathrm{H}), 2.56(\mathrm{q}, J=6.4 \mathrm{~Hz}, 3 \mathrm{H}), 2.39(\mathrm{~m}, 8 \mathrm{H}), 2.23(\mathrm{dd}, J=17.2,9.1 \mathrm{~Hz}$, 2H), 2.12 - $2.04(\mathrm{~m}, 9 \mathrm{H}), 2.03-1.95(\mathrm{~m}, 5 \mathrm{H}).) .{ }^{13} \mathbf{C}$ NMR $\left(126 \mathrm{MHz}, \mathrm{CDCl}_{3}\right) \delta$ 206.7, 206.5, 206.4, 206.4, 172.4, 172.3, 172.2, 172.2, 165.1, 162.5, 161.8, 161.7, 153.0, 152.7, 138.6, 138.4, $137.9,137.5,137.3,133.5,132.9,130.1,129.9,129.6,128.7,128.5,128.5,128.4,128.4,128.2$, $128.1,128.1,128.0,127.9,127.8,127.7,127.6,127.5,127.4,127.3,115.5,115.3,101.6,100.8$, 100.6, 100.1, 92.0, 91.9, 79.9, 79.7, 78.6, 78.1, 77.7, 76.4, 75.7, 75.6, 75.0, 74.9, 74.7, 74.5, 74.0, $73.7,73.3,72.4,72.3,71.3,67.9,67.1,66.9,63.3,62.8,58.4,56.8,37.9,37.8,37.7,37.6,31.4$, 29.8, 29.7, 29.7, 27.9, 27.8, 27.8, 27.7, 20.7. HRMS-ESI-TOF (m/z): $[\mathrm{M}+\mathrm{Na}]^{+}$Calculated for $\mathrm{C}_{116} \mathrm{H}_{117} \mathrm{Cl}_{6} \mathrm{~F}_{17} \mathrm{~N}_{2} \mathrm{O}_{33}$ 2625.5295, Found 2625.5300.

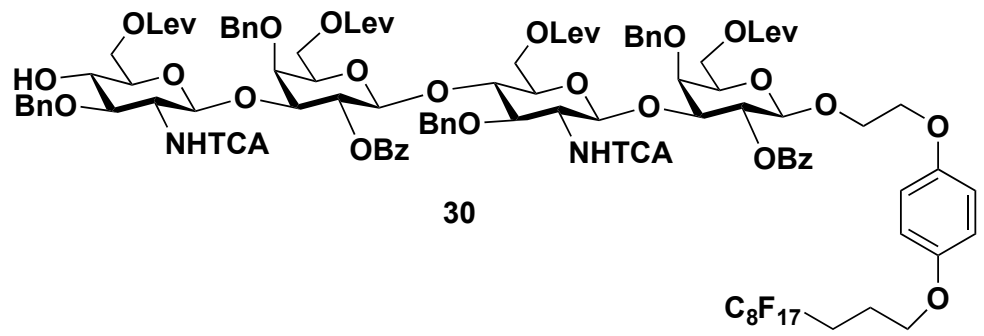

2-(4-((4,4,5,5,6,6,7,7,8,8,9,9,10,10,11,11,11-heptadecafluoroundecyl)oxy)phenoxy)ethyl (3-Obenzyl-2-deoxy-6-O-levulinoyl-2-trichloroacetamido- $\beta$-D-glucopyranosyl)-(1 $\rightarrow 3)-(2-O$ benzoyl-4- $O$-benzyl-6- $O$-levulinoyl- $\beta$-D-galactopyranosyl)-( $\rightarrow 4)$-(3-O-benzyl-2-deoxy-6- $O$ levulinoyl-2-trichloroacetamido- $\beta$-D-glucopyranosyl)-(1 $\rightarrow 3)-2-O$-benzoyl-4- $O$-benzyl-6- $O$ levulinoyl- $\boldsymbol{\beta}$-D-galactopyranoside (30): To a vigorously stirred solution of glycosyl donor 28 $(0.050 \mathrm{~g}, 36 \mu \mathrm{mol})$ and glycosyl acceptor $29(28.0 \mathrm{mg}, 18.0 \mu \mathrm{mol})$ in anhydrous dichloromethane $(1.2 \mathrm{~mL})$ under argon, catalytic TMSOTf (Trimethylsilyl trifluoromethanesulfonate) $(0.30 \mu \mathrm{L}, 1.8 \mu \mathrm{mol})$ was added at $0{ }^{\circ} \mathrm{C}$ and the reaction mixture was stirred for $1 \mathrm{~h}$ at $25^{\circ} \mathrm{C}$ until the acceptor was completely consumed shown by TLC. The reaction 
mixture quenched by the addition of triethylamine drop wise until the reaction mixture was neutral to a litmus paper. The reaction mixture was then diluted with dichloromethane $(4 \mathrm{~mL})$ and the solvent was removed under reduced pressure. The resulting crude mixture was dissolved in THF $(3 \mathrm{~mL}) . \mathrm{Et}_{3} \mathrm{~N}(1 \mathrm{~mL})$ was added to the reaction mixture at room temperature and it was stirred for $3 \mathrm{~h}$ until the starting material was completely consumed. The solvent was evaporated and the resulting crude material was purified via FSPE. The fluorous fraction was concentrated to afford tetrasaccharide acceptor $30(35.0 \mathrm{mg}, 14.0 \mu \mathrm{mol}, 77 \%$ over 2 steps $)$ as white foam. ${ }^{1} \mathbf{H}$ NMR $\left(500 \mathrm{MHz}, \mathrm{CDCl}_{3}\right) \delta 8.02(\mathrm{~d}, J=7.6 \mathrm{~Hz}, 2 \mathrm{H}), 7.89(\mathrm{~d}, J=7.5 \mathrm{~Hz}, 2 \mathrm{H}), 7.56(\mathrm{t}, J=7.3 \mathrm{~Hz}$, $1 \mathrm{H}), 7.44$ (q, $J=9.0,8.4 \mathrm{~Hz}, 3 \mathrm{H}), 7.19(\mathrm{~m}, 20 \mathrm{H}), 7.03$ (q, $J=8.4,6.6 \mathrm{~Hz}, 3 \mathrm{H}), 6.72$ (d, $J=7.3$ $\mathrm{Hz}, 1 \mathrm{H}), 6.63-6.56(\mathrm{~m}, 3 \mathrm{H}), 6.48(\mathrm{~d}, J=9.0 \mathrm{~Hz}, 2 \mathrm{H}), 5.49-5.39(\mathrm{~m}, 2 \mathrm{H}), 4.95-4.81(\mathrm{~m}, 3 \mathrm{H})$, $4.75(\mathrm{~d}, J=10.8 \mathrm{~Hz}, 1 \mathrm{H}), 4.67(\mathrm{~d}, J=6.7 \mathrm{~Hz}, 1 \mathrm{H}), 4.65-4.57(\mathrm{~m}, 2 \mathrm{H}), 4.50(\mathrm{~m}, 6 \mathrm{H}), 4.22(\mathrm{~d}, J$ $=11.2 \mathrm{~Hz}, 1 \mathrm{H}), 4.16-4.07(\mathrm{~m}, 4 \mathrm{H}), 4.01-3.90(\mathrm{~m}, 5 \mathrm{H}), 3.82(\mathrm{~m}, 8 \mathrm{H}), 3.68(\mathrm{~m}, 4 \mathrm{H}), 3.51(\mathrm{t}, J$ $=6.2 \mathrm{~Hz}, 2 \mathrm{H}), 3.46-3.38(\mathrm{~m}, 2 \mathrm{H}), 3.34(\mathrm{dd}, J=7.2,3.7 \mathrm{~Hz}, 1 \mathrm{H}), 2.85-2.79(\mathrm{~m}, 1 \mathrm{H}), 2.64(\mathrm{~m}$, $5 \mathrm{H}), 2.58-2.51(\mathrm{~m}, 3 \mathrm{H}), 2.47(\mathrm{t}, J=6.2 \mathrm{~Hz}, 2 \mathrm{H}), 2.36(\mathrm{~m}, 6 \mathrm{H}), 2.22(\mathrm{~m}, 2 \mathrm{H}), 2.10-2.04(\mathrm{~m}$, 9H), $2.02-1.96(\mathrm{~m}, 5 \mathrm{H}) .{ }^{13} \mathbf{C}$ NMR $\left(126 \mathrm{MHz}, \mathrm{CDCl}_{3}\right) \delta 206.8,206.7,206.5,206.5,173.2$, 172.4, 172.2, 172.2, 165.1, 161.8, 161.7, 153.0, 152.7, 138.6, 138.4, 137.9, 137.8, 133.6, 132.9, $130.1,129.9,129.6,128.8,128.5,128.5,128.4,128.2,128.1,128.1,128.1,127.9,127.9,127.8$, $127.5,127.5,127.4,115.5,115.3,101.6,100.8,100.6,100.2,92.1,91.9,79.9,79.3,78.6,77.7$, 76.3, 75.7, 75.6, 74.9, 74.7, 74.5, 74.0, 74.0, 73.7, 72.3, 72.3, 72.2, 71.3, 70.9, 67.9, 67.2, 66.9, $63.3,62.9,62.8,58.4,56.8,37.9,37.9,37.8,37.7,29.8,27.8,27.8,27.7,27.7,20.6$. HRMSESI-TOF (m/z): $[\mathrm{M}+\mathrm{Na}]^{+}$Calculated for $\mathrm{C}_{109} \mathrm{H}_{111} \mathrm{Cl}_{6} \mathrm{~F}_{17} \mathrm{~N}_{2} \mathrm{O}_{33}$ 2535.4822, Found 2535.4802.

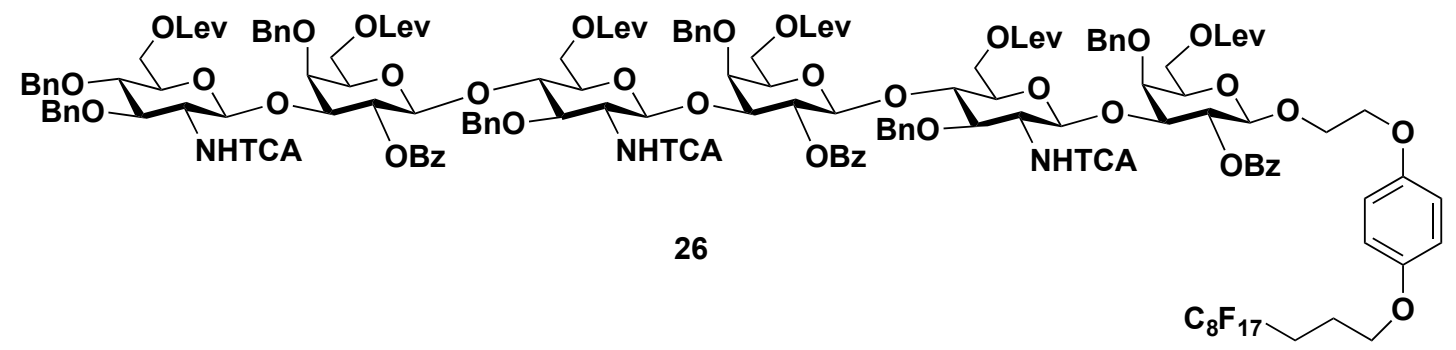

2-(4-((4,4,5,5,6,6,7,7,8,8,9,9,10,10,11,11,11-heptadecafluoroundecyl)oxy)phenoxy)ethyl $(3,4-$ di- $O$-benzyl-2-deoxy-6- $O$-levulinoyl-2-trichloroacetamido- $\beta$-D-glucopyranosyl)-(1 $\rightarrow 3)-(2$ $O$-benzoyl-4- $O$-benzyl-6- $O$-levulinoyl- $\beta$-D-galactopyranosyl)-(1 $\rightarrow 4)-(3-O$-benzyl-2-deoxy-6$O$-levulinoyl-2-trichloroacetamido- $\beta$-D-glucopyranosyl)-(1 $\rightarrow 3)-(2-O$-benzoyl-4-O-benzyl-6$O$-levulinoyl- $\beta$-D-galactopyranosyl)-(1 $\rightarrow 4)$-(3-O-benzyl-2-deoxy-6-O-levulinoyl-2trichloroacetamido- $\beta$-D-glucopyranosyl)-( $1 \rightarrow 3)-2-O$-benzoyl-4- $O$-benzyl-6- $O$-levulinoyl- $\beta$ D-galactopyranoside (26): To a vigorously stirred solution of glycosyl donor 27 (24 mg, 0.020 $\mathrm{mmol})$ and glycosyl acceptor $30(25 \mathrm{mg}, 0.010 \mathrm{mmol})$ in anhydrous dichloromethane $(1.0 \mathrm{~mL})$ under argon, catalytic TMSOTf (Trimethylsilyl trifluoromethanesulfonate) $(0.27 \mu \mathrm{L}, 1.5 \mu \mathrm{mol})$ was added at $0{ }^{\circ} \mathrm{C}$ and the reaction mixture was stirred for $1 \mathrm{~h}$ at $25{ }^{\circ} \mathrm{C}$ until the acceptor was completely consumed shown by TLC. The reaction mixture quenched by the addition of triethylamine drop wise until the reaction mixture was neutral to a litmus paper. The 
reaction mixture was then diluted with dichloromethane $(3 \mathrm{~mL})$ and the solvent was removed under reduced pressure. The resulting crude material was purified via FSPE. The fluorous fraction was concentrated to afford the hexasaccharide 26 (28 mg, $8.0 \mu \mathrm{mol}, 79 \%)$ as white foam. ${ }^{1} \mathbf{H}$ NMR $\left(500 \mathrm{MHz}, \mathrm{CDCl}_{3}\right) \delta 8.05-7.98(\mathrm{~m}, 3 \mathrm{H}), 7.92-7.86(\mathrm{~m}, 2 \mathrm{H}), 7.57-7.50(\mathrm{~m}, 2 \mathrm{H})$, $7.47-7.38(\mathrm{~m}, 5 \mathrm{H}), 7.19(\mathrm{~m}, 33 \mathrm{H}), 7.05-6.98(\mathrm{~m}, 5 \mathrm{H}), 6.66(\mathrm{~d}, J=8.1 \mathrm{~Hz}, 1 \mathrm{H}), 6.65-6.52$ (m, 4H), $6.48(\mathrm{~d}, J=8.9 \mathrm{~Hz}, 2 \mathrm{H}), 5.43(\mathrm{~m}, 3 \mathrm{H}), 4.86(\mathrm{~m}, 4 \mathrm{H}), 4.78-4.63(\mathrm{~m}, 6 \mathrm{H}), 4.61-4.33$ (m, 12H), $4.19-4.03(\mathrm{~m}, 7 \mathrm{H}), 4.01-3.93(\mathrm{~m}, 5 \mathrm{H}), 3.81(\mathrm{~m}, 12 \mathrm{H}), 3.72-3.61(\mathrm{~m}, 6 \mathrm{H}), 3.52(\mathrm{~m}$, $5 \mathrm{H}), 3.37-3.30(\mathrm{~m}, 2 \mathrm{H}), 2.66-2.58(\mathrm{~m}, 6 \mathrm{H}), 2.53(\mathrm{~m}, 6 \mathrm{H}), 2.36(\mathrm{~m}, 12 \mathrm{H}), 2.12-2.04(\mathrm{~m}$, $11 \mathrm{H}), 2.03-1.91(\mathrm{~m}, 11 \mathrm{H}) .{ }^{13} \mathrm{C}$ NMR $\left(126 \mathrm{MHz}, \mathrm{CDCl}_{3}\right) \delta$ 206.7, 206.5, 206.4, 206.3, 206.3, 206.2, 172.4, 172.3, 172.2, 172.2, 172.1, 165.1, 165.0, 164.9, 161.8, 161.7, 161.6, 153.0, 152.7, 138.6, 138.5, 138.4, 138.1, 137.9, 137.9, 137.8, 137.4, 137.3, 136.5, 133.5, 133.4, 133.0, 132.9, $131.5,130.9,130.6,130.1,130.0,129.9,129.6,129.6,129.1,128.8,128.6,128.5,128.4,128.4$, $128.2,128.1,128.1,128.1,128.0,128.0,128.0,127.9,127.8,127.7,127.7,127.6,127.5,127.4$, 127.3, 127.3, 120.2, 115.5, 115.3, 112.1, 101.6, 100.9, 100.8, 100.7, 100.6, 100.1, 92.0, 91.9, 91.9, 79.9, 79.7, 79.4, 78.7, 78.1, 77.9, 77.7, 77.3, 76.8, 76.4, 76.1, 75.8, 75.7, 75.6, 75.0, 74.9, 74.7, 74.7, 74.5, 74.1, 73.9, 73.8, 73.6, 73.3, 72.4, 72.3, 72.2, 71.9, 71.3, 67.8, 67.1, 66.9, 63.4, $63.2,62.9,62.8,62.7,62.6,58.3,58.0,56.9,56.7,56.4,37.9,37.8,37.7,37.7,37.6,29.8,29.7$, 29.7, 29.7, 29.4, 27.9, 27.8, 27.7, 27.7, 20.6. HRMS-ESI-TOF (m/z): $[\mathrm{M}+\mathrm{Na}]^{+}$Calculated for $\mathrm{C}_{161} \mathrm{H}_{165} \mathrm{Cl}_{9} \mathrm{~F}_{17} \mathrm{~N}_{3} \mathrm{O}_{48}$ 3575.7385, Found 3575.7383.

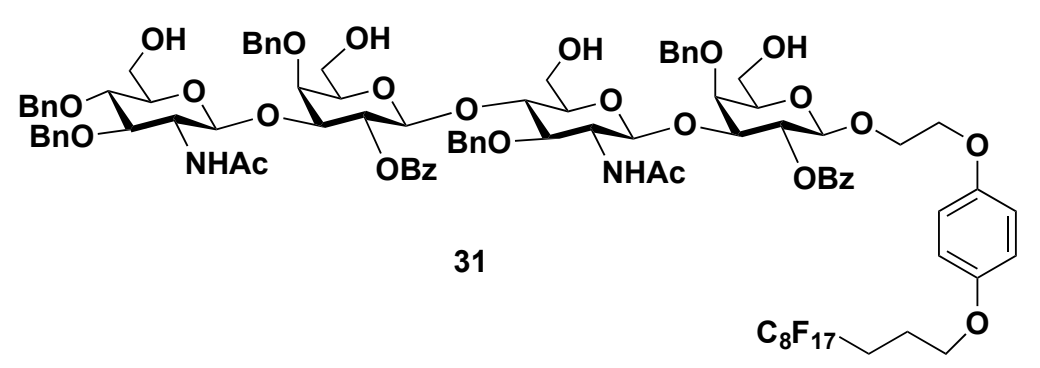

2-(4-((4,4,5,5,6,6,7,7,8,8,9,9,10,10,11,11,11-heptadecafluoroundecyl)oxy)phenoxy)ethyl (2acetamido-3,4-di- $O$-benzyl-2-deoxy- $\beta$-D-glucopyranosyl)-(1 $\rightarrow 3)$-(2-O-benzoyl-4-O-benzyl$\beta$-D-galactopyranosyl)-(1 $\rightarrow 4)$-(2-acetamido-3- $O$-benzyl-2-deoxy- $\beta$-D-glucopyranosyl)$(\mathbf{1} \rightarrow 3)-2-O$-benzoyl-4- $\boldsymbol{O}$-benzyl- $\boldsymbol{\beta}$-D-galactopyranoside $(31)$ : To a solution of compound 25 $(0.020 \mathrm{~g}, 7.7 \mu \mathrm{mol})$ in benzene $(0.48 \mathrm{~mL})$ and dimethylacetamide $(0.12 \mathrm{~mL}, 4: 1, \mathrm{v} / \mathrm{v})$ were added AIBN (Azobisisobutyronitrile) $(2 \mathrm{mg})$ and tributyltin hydride $(41.0 \mu \mathrm{L}, 0.150 \mathrm{mmol})$, degassed with the flow of argon and stirred at room temperature for $1 \mathrm{~h}$. The reaction mixture was then heated at $80{ }^{\circ} \mathrm{C}$ for $10 \mathrm{~h}$. TLC showed complete conversion of the starting material. The reaction mixture was then concentrated. The resulting crude solid material was dissolved in acetonitrile $(10 \mathrm{~mL})$ and extracted with hexane $(3 \mathrm{X} 10 \mathrm{~mL})$ to remove tin salts. The acetonitrile layer was then concentrated and the residue was purified via FSPE. The fluorous fraction was concentrated (14.0 $\mathrm{mg}, 5.80 \mu \mathrm{mol}, 78 \%$ ) as white foam and used for the next reaction. MALDI-TOF (Lowresolution) $(\mathbf{m} / \mathbf{z}):[\mathrm{M}+\mathrm{Na}]^{+}$Calculated for $\mathrm{C}_{116} \mathrm{H}_{123} \mathrm{~F}_{17} \mathrm{~N}_{2} \mathrm{O}_{33}$ 2417.76, Found 2417.68. 
$\mathrm{N}_{2} \mathrm{H}_{4} \cdot \mathrm{H}_{2} \mathrm{O}$ (hydrazine monohydrate) $(0.5 \mathrm{~mL})$ was added to a mixture of pyridine $(4.8 \mathrm{~mL})$ and acetic acid $(3.2 \mathrm{~mL})$. The resulting tetrasaccharide $(14.0 \mathrm{mg}, 5.80 \mu \mathrm{mol})$ was dissolved in $3 \mathrm{~mL}$ of this prepared solution under argon at room temperature and stirred for $3 \mathrm{~h}$ until the starting material was completely consumed by TLC. The solvent was then removed under reduced pressure. The resulting crude material was purified via FSPE. The fluorous fraction was concentrated to afford compound $\mathbf{3 1}(0.010 \mathrm{~g}, 5.0 \mu \mathrm{mol}, 66 \%$ over 2 steps $)$ as white foam. ${ }^{1} \mathbf{H}$ NMR $\left(500 \mathrm{MHz}, \mathrm{CD}_{3} \mathrm{OD}\right) \delta 8.14(\mathrm{~d}, J=7.6 \mathrm{~Hz}, 2 \mathrm{H}), 7.96(\mathrm{~d}, J=7.5 \mathrm{~Hz}, 2 \mathrm{H}), 7.68(\mathrm{~d}, J=7.3$ $\mathrm{Hz}, 1 \mathrm{H}), 7.55(\mathrm{~m}, 3 \mathrm{H}), 7.48-7.09(\mathrm{~m}, 27 \mathrm{H}), 6.66(\mathrm{~d}, J=8.7 \mathrm{~Hz}, 2 \mathrm{H}), 6.52(\mathrm{t}, J=8.4 \mathrm{~Hz}, 2 \mathrm{H})$, $5.63-5.51(\mathrm{~m}, 1 \mathrm{H}), 5.46(\mathrm{q}, J=9.6,8.4 \mathrm{~Hz}, 1 \mathrm{H}), 5.12(\mathrm{~d}, J=11.5 \mathrm{~Hz}, 1 \mathrm{H}), 4.99$ (dd, $J=23.2$, $10.9 \mathrm{~Hz}, 2 \mathrm{H}), 4.74-4.50(\mathrm{~m}, 10 \mathrm{H}), 4.39(\mathrm{~d}, J=10.3 \mathrm{~Hz}, 1 \mathrm{H}), 4.17(\mathrm{~s}, 1 \mathrm{H}), 4.13-3.80(\mathrm{~m}$, $15 \mathrm{H}), 3.77-3.53(\mathrm{~m}, 13 \mathrm{H}), 3.45(\mathrm{dd}, J=15.7,10.3 \mathrm{~Hz}, 4 \mathrm{H}), 3.09$ (d, $J=8.0 \mathrm{~Hz}, 1 \mathrm{H}), 2.33$ (dd, $J=17.5,9.4 \mathrm{~Hz}, 3 \mathrm{H}), 2.08-2.01(\mathrm{~m}, 3 \mathrm{H}), 1.38(\mathrm{~d}, J=8.5 \mathrm{~Hz}, 6 \mathrm{H}) .{ }^{13} \mathbf{C} \mathbf{N M R}(126 \mathrm{MHz}$, $\left.\mathrm{CD}_{3} \mathrm{OD}\right) \delta 171.7,171.4,165.5,165.4,152.9,152.7,139.1,138.8,138.3,137.9,137.8,133.4$, $132.9,131.0,129.7,129.6,129.6,128.6,128.4,128.3,128.2,128.1,128.0,127.9,127.8,127.7$, $127.6,127.4,127.3,127.2,127.1,115.2,115.1,102.3,102.2,101.6,100.2,81.7,80.4,80.3,79.8$, $78.5,77.1,76.6,76.3,76.2,75.9,75.5,75.4,75.4,74.7,74.7,74.6,74.3,72.5,71.9,71.7,71.1$, $67.8,67.6,66.7,61.6,61.2,60.8,59.9,56.4,55.5,29.4,21.6,21.6,20.4$. HRMS-ESI-TOF (m/z): $[\mathrm{M}+\mathrm{Na}]^{+}$Calculated for $\mathrm{C}_{96} \mathrm{H}_{99} \mathrm{~F}_{17} \mathrm{~N}_{2} \mathrm{O}_{25}$ 2026.6196, Found 2026.6177.

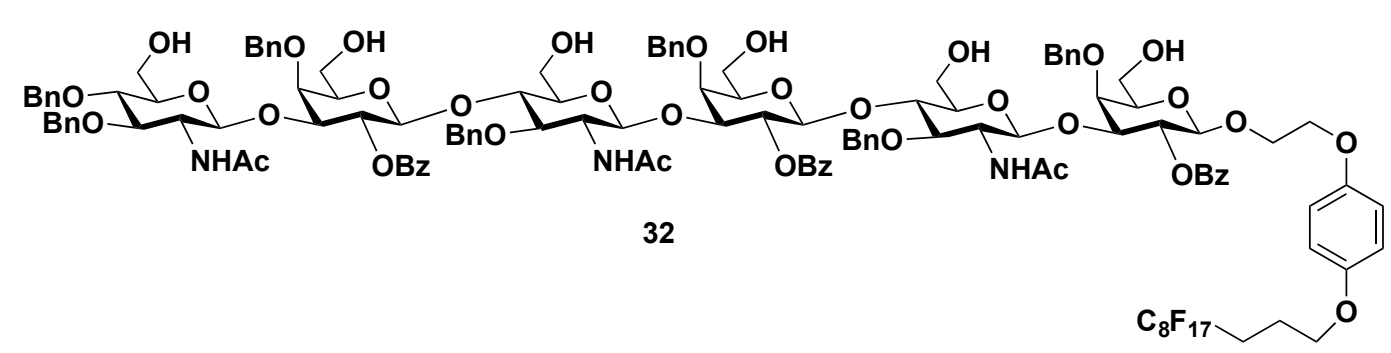

2-(4-((4,4,5,5,6,6,7,7,8,8,9,9,10,10,11,11,11-heptadecafluoroundecyl)oxy)phenoxy)ethyl (2acetamido-3,4-di- $O$-benzyl-2-deoxy- $\beta$-D-glucopyranosyl)-( $\rightarrow 3)-(2$ - $O$-benzoyl-4- $O$-benzyl$\beta$-D-galactopyranosyl)-(1 $\rightarrow 4)$-(2-acetamido-3- $O$-benzyl-2-deoxy- $\beta$-D-glucopyranosyl)$(1 \rightarrow 3)-(2-O$-benzoyl-4-O-benzyl- $\beta$-D-galactopyranosyl)-(1 $\rightarrow 4)$-(2-acetamido-3- $O$-benzyl-2deoxy- $\beta$-D-glucopyranosyl)-( $1 \rightarrow 3)-2-O$-benzoyl-4- $O$-benzyl- $\beta$-D-galactopyranoside (32): To a solution of compound $26(0.020 \mathrm{~g}, 5.6 \mu \mathrm{mol})$ in benzene $(0.48 \mathrm{~mL})$ and dimethylacetamide $(0.12 \mathrm{~mL}, 4: 1, \mathrm{v} / \mathrm{v})$ were added AIBN (Azobisisobutyronitrile) (1 mg) and tributyltin hydride $(44.0 \mu \mathrm{L}, 0.170 \mathrm{mmol})$, degassed with the flow of argon and stirred at room temperature for $1 \mathrm{~h}$. The reaction mixture was then heated at $80{ }^{\circ} \mathrm{C}$ for $10 \mathrm{~h}$. TLC showed complete conversion of the starting material. The reaction mixture was then concentrated. The resulting crude solid material was dissolved in acetonitrile $(10 \mathrm{~mL})$ and extracted with hexane $(3 \mathrm{X} 10 \mathrm{~mL})$ to remove tin salts. The acetonitrile layer was then concentrated and the residue was purified via FSPE. The fluorous fraction was concentrated (12 mg, $3.7 \mu \mathrm{mol}, 68 \%)$ as white foam and used for the next reaction. HRMS-ESI-TOF (m/z): $[\mathrm{M}+\mathrm{Na}]^{+}$Calculated for $\mathrm{C}_{161} \mathrm{H}_{174} \mathrm{~F}_{17} \mathrm{~N}_{3} \mathrm{O}_{48} \mathrm{Na}^{+}$3265.0959, Found 
3265.0896. $\mathrm{N}_{2} \mathrm{H}_{4} \cdot \mathrm{H}_{2} \mathrm{O}$ (hydrazine mohydrate) $(0.5 \mathrm{~mL})$ was added to a mixture of pyridine (4.8 $\mathrm{mL})$ and acetic acid $(3.2 \mathrm{~mL})$. The resulting hexasaccharide $(12 \mathrm{mg}, 3.7 \mu \mathrm{mol})$ was dissolved in $3.0 \mathrm{~mL}$ of this prepared solution under argon at room temperature and stirred for $3 \mathrm{~h}$ until the starting material was completely consumed by TLC. The solvent was then removed under reduced pressure. The resulting crude material was purified via FSPE. The fluorous fraction was concentrated to afford compound $\mathbf{3 2}(6.6 \mathrm{mg}, 2.5 \mu \mathrm{mol}, 45 \%$ over 2 steps $)$ as white foam. ${ }^{1} \mathbf{H}$ NMR $\left(500 \mathrm{MHz}, \mathrm{CD}_{3} \mathrm{OD}\right) \delta 8.13(\mathrm{dd}, J=26.0,7.4 \mathrm{~Hz}, 4 \mathrm{H}), 7.96(\mathrm{~d}, J=7.5 \mathrm{~Hz}, 2 \mathrm{H}), 7.74-$ $7.61(\mathrm{~m}, 2 \mathrm{H}), 7.59-7.38(\mathrm{~m}, 10 \mathrm{H}), 7.35-7.03(\mathrm{~m}, 31 \mathrm{H}), 6.67(\mathrm{~d}, J=9.0 \mathrm{~Hz}, 2 \mathrm{H}), 6.52(\mathrm{~d}, J=$ $9.0 \mathrm{~Hz}, 2 \mathrm{H}), 5.60-5.54(\mathrm{~m}, 1 \mathrm{H}), 5.54-5.47(\mathrm{~m}, 1 \mathrm{H}), 5.47-5.39(\mathrm{~m}, 1 \mathrm{H}), 5.13(\mathrm{~d}, J=11.4 \mathrm{~Hz}$, $1 \mathrm{H}), 5.00(\mathrm{~m}, 4 \mathrm{H}), 4.77(\mathrm{~d}, J=10.2 \mathrm{~Hz}, 2 \mathrm{H}), 4.73-4.47(\mathrm{~m}, 12 \mathrm{H}), 4.39(\mathrm{dd}, J=17.4,10.4 \mathrm{~Hz}$, $2 \mathrm{H}), 4.23-4.17(\mathrm{~m}, 1 \mathrm{H}), 4.14-3.81(\mathrm{~m}, 18 \mathrm{H}), 3.78-3.54(\mathrm{~m}, 19 \mathrm{H}), 3.46(\mathrm{~m}, 6 \mathrm{H}), 3.19(\mathrm{~d}, J=$ $7.3 \mathrm{~Hz}, 1 \mathrm{H}), 3.09(\mathrm{dd}, J=15.8,10.7 \mathrm{~Hz}, 2 \mathrm{H}), 2.46-2.23(\mathrm{~m}, 5 \mathrm{H}), 2.18-1.87(\mathrm{~m}, 9 \mathrm{H}) .{ }^{13} \mathbf{C}$ NMR $\left(126 \mathrm{MHz}, \mathrm{CD}_{3} \mathrm{OD}\right) \delta 171.7,171.4,171.4,165.5,165.4,165.2,152.9,152.8,139.2,138.9$, $138.4,138.1,137.8,133.3,132.8,129.6,128.5,128.3,128.2,128.2,128.1,128.0,127.9,127.8$, $127.7,127.6,127.5,127.3,127.2,127.1,115.2,115.0,102.5,102.4,101.6,101.5,100.2,100.1$, $81.9,80.4,80.0,78.4,77.1,76.9,76.3,76.2,75.9,75.4,75.2,74.7,74.6,74.5,72.5,72.3,71.7$, $67.7,66.6,61.5,61.4,61.1,60.8,59.9,56.3,55.4,55.3,29.4,22.4,21.6,21.5,20.4$. HRMSESI-TOF (m/z): $[\mathrm{M}+\mathrm{Na}]^{+}$Calculated for $\mathrm{C}_{131} \mathrm{H}_{138} \mathrm{~F}_{17} \mathrm{~N}_{3} \mathrm{O}_{36}$ 2675.8721, Found 2675.8494.

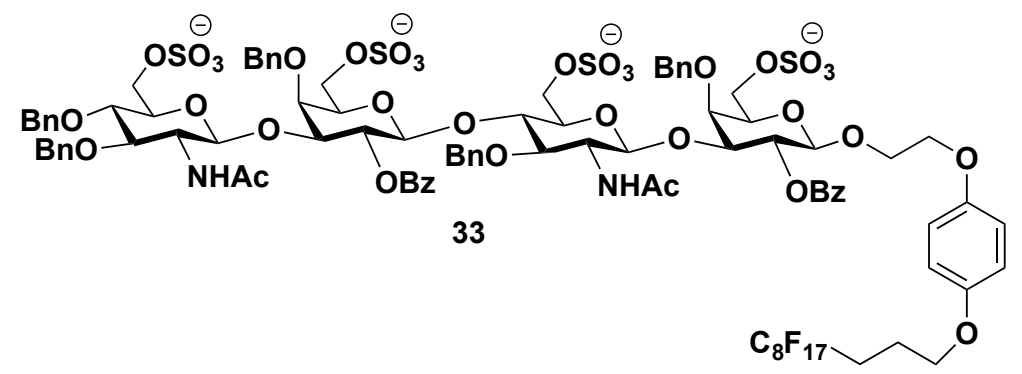

2-(4-((4,4,5,5,6,6,7,7,8,8,9,9,10,10,11,11,11-heptadecafluoroundecyl)oxy)phenoxy)ethyl (2acetamido-3,4-di- $O$-benzyl-2-deoxy-6- $O$-sulfonate- $\beta$-D-glucopyranosyl)-( $1 \rightarrow 3)-(2-O$ benzoyl-4- $O$-benzyl-6- $O$-sulfonate- $\beta$-D-galactopyranosyl)-( $1 \rightarrow 4)$-(2-acetamido-3- $O$-benzyl2-deoxy-6- $O$-sulfonate- $\beta$-D-glucopyranosyl)-( $\rightarrow 3)$-2- $O$-benzoyl-4- $O$-benzyl-6- $O$-sulfonate$\boldsymbol{\beta}$-D-galactopyranoside (33): To a solution of the compound $\mathbf{3 1}(7.0 \mathrm{mg}, 3.5 \mu \mathrm{mol})$ in dry DMF $(0.5 \mathrm{~mL})$ was added sulfur trioxide trimethylamine complex $(0.020 \mathrm{~g}, 0.14 \mathrm{mmol})$. The mixture was stirred at $55{ }^{\circ} \mathrm{C}$ for $24 \mathrm{~h}$. The reaction was quenched with methanol $(2 \mathrm{~mL})$, triethylamine $(0.05 \mathrm{~mL})$ and then concentrated. The crude product was purified via FSPE. The resulting material was further purified on Sephadex LH-20 $\left(50 \% \mathrm{CH}_{2} \mathrm{Cl}_{2}: \mathrm{MeOH}\right)$ to afford the desired compound $33(6.4 \mathrm{mg}, 2.7 \mu \mathrm{mol}, 79 \%) .{ }^{1} \mathbf{H}$ NMR $\left(500 \mathrm{MHz}, \mathrm{CD}_{3} \mathrm{OD}\right) \delta 8.21(\mathrm{~d}, J=7.3 \mathrm{~Hz}$, 2H), $8.00-7.88(\mathrm{~m}, 3 \mathrm{H}), 7.76-6.97(\mathrm{~m}, 30 \mathrm{H}), 6.65(\mathrm{~d}, J=9.0 \mathrm{~Hz}, 2 \mathrm{H}), 6.49(\mathrm{~d}, J=9.0 \mathrm{~Hz}$, 2H), $5.56-5.46(\mathrm{~m}, 1 \mathrm{H}), 5.39-5.29(\mathrm{~m}, 1 \mathrm{H}), 5.15(\mathrm{~d}, J=11.4 \mathrm{~Hz}, 1 \mathrm{H}), 5.11-4.96(\mathrm{~m}, 2 \mathrm{H})$, $4.89(\mathrm{~d}, J=10.9 \mathrm{~Hz}, 1 \mathrm{H}), 4.79-4.60(\mathrm{~m}, 6 \mathrm{H}), 4.61-4.45(\mathrm{~m}, 3 \mathrm{H}), 4.38(\mathrm{dd}, J=17.1,10.2 \mathrm{~Hz}$, 4H), 4.27 (dd, $J=10.3,4.2 \mathrm{~Hz}, 2 \mathrm{H}), 4.23-3.76(\mathrm{~m}, 20 \mathrm{H}), 3.74-3.42(\mathrm{~m}, 6 \mathrm{H}), 2.43-2.26(\mathrm{~m}$, 
2H), $2.04-1.97(\mathrm{~m}, 2 \mathrm{H}), 1.27(\mathrm{~d}, J=3.7 \mathrm{~Hz}, 6 \mathrm{H}) .{ }^{13} \mathbf{C}$ NMR $\left(126 \mathrm{MHz}, \mathrm{CD}_{3} \mathrm{OD}\right) \delta 171.7$, 171.6, 165.7, 165.6, 152.9, 152.7, 139.2, 138.8, 138.2, 137.8, 133.4, 133.0, 129.9, 129.6, 129.4, 128.6, 128.2, 128.2, 128.1, 128.0, 127.8, 127.6, 127.4, 127.3, 127.1, 126.9, 126.3, 115.3, 115.1, 102.7, 102.4, 101.3, 99.6, 82.1, 81.1, 79.2, 78.2, 76.9, 74.8, 74.6, 73.3, 72.8, 72.8, 71.9, 71.4, 67.8, 67.7, 66.8, 66.7, 66.4, 65.4, 55.7, 54.8, 29.4, 21.5, 21.4, 20.4. HRMS-ESI-TOF (m/z): [M$2 \mathrm{H}]^{2-}$ Calculated for $\mathrm{C}_{96} \mathrm{H}_{99} \mathrm{~F}_{17} \mathrm{~N}_{2} \mathrm{O}_{37} \mathrm{~S}_{4} \mathbf{1 1 6 0 . 2 1 9 1}$, Found 1160.2194.

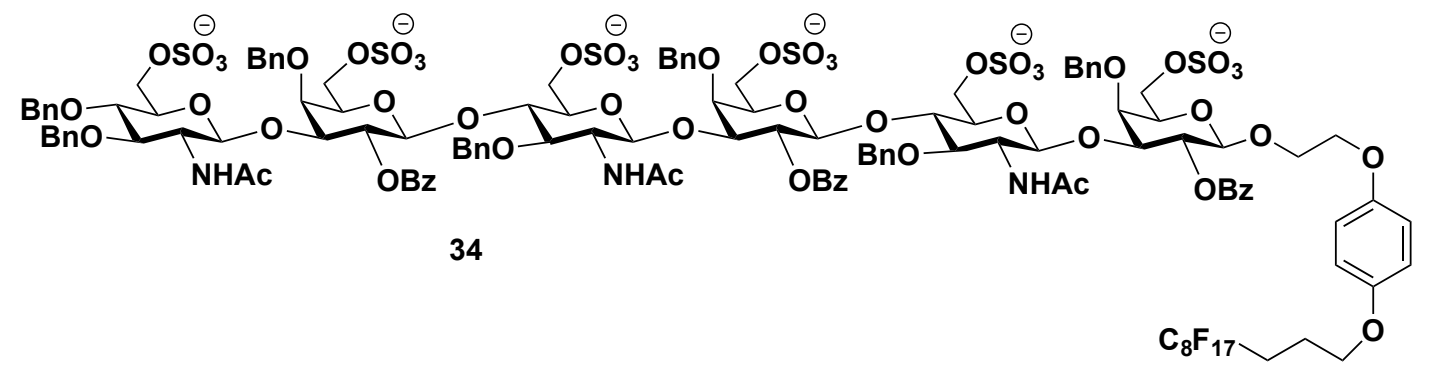

2-(4-((4,4,5,5,6,6,7,7,8,8,9,9,10,10,11,11,11-heptadecafluoroundecyl)oxy)phenoxy)ethyl (2acetamido-3,4-di- $O$-benzyl-2-deoxy-6- $O$-sulfonate- $\beta$-D-glucopyranosyl)-( $1 \rightarrow 3)-(2-O$ benzoyl-4- $O$-benzyl-6- $O$-sulfonate- $\beta$-D-galactopyranosyl)-( $\rightarrow 4)$-(2-acetamido-3- $O$-benzyl2-deoxy-6- $O$-sulfonate- $\beta$-D-glucopyranosyl)-( $1 \rightarrow 3$ )-(2- $O$-benzoyl-4- $O$-benzyl-6- $O$-sulfonate$\beta$-D-galactopyranosyl)-(1 $\rightarrow 4)$-(2-acetamido-3- $O$-benzyl-2-deoxy-6- $O$-sulfonate- $\beta$-Dglucopyranosyl)-(1 $\rightarrow 3)$-2- $O$-benzoyl-4- $O$-benzyl-6- $O$-sulfonate- $\beta$-D-galactopyranoside (34): To a solution of the compound $32(7.0 \mathrm{mg}, 2.6 \mu \mathrm{mol})$ in dry DMF $(0.5 \mathrm{~mL})$ was added sulfur trioxide•trimethylamine complex $(0.022 \mathrm{~g}, 0.16 \mathrm{mmol})$. The mixture was stirred at $55^{\circ} \mathrm{C}$ for 48 h. The reaction was quenched with methanol $(2 \mathrm{~mL})$, triethylamine $(0.05 \mathrm{~mL})$ and then concentrated. The formation of desired hexasulfated compound $\mathbf{3 4}$ was confirmed via highresolution mass spectrometry. The addition of excess reagents, higher temparature and prolonged reaction time resulted in degradation of the desired hexasulfated hexasaccharide leading to partially sulfated mixtures. This degradation was presumably attributed to the extreme lability of the sulfate esters in acidic medium. HRMS-ESI-TOF $(\mathbf{m} / \mathbf{z}):[\mathrm{M}-3 \mathrm{H}]^{3-}$ Calculated for $\mathrm{C}_{131} \mathrm{H}_{138} \mathrm{~F}_{17} \mathrm{~N}_{3} \mathrm{O}_{54} \mathrm{~S}_{6}$ 1043.5336, Found 1043.5215.

\section{References:}

[1] a) N. Barroca, J. C. Jacquinet, Carbohydr. Res. 2002, 337, 673 - 689; b) S. Hanashima, P. H. Seeberger, Chem. Asian J. 2007, 2, 1447 - 1459.

[2] C. Mukherjee, L. Liu, N. L. B. Pohl, Adv. Synth. Catal. 2014, 356, 2247 - 2256.

[3] M. Goswami, A. Ellern, N. L. B. Pohl, Angew. Chem. Int. Ed. 2013, 52, 8441- 8445.

[4] a) F. A. Jaipuri, N. L. Pohl, Org. Biomol. Chem. 2008, 6, 2686-2691; b) R. Bänteli, B. Ernst, Bioorg. Med. Chem. Lett. 2001, 11, 459-462; c) J. Tamura, J. Nishihara, J. Org. 
Chem. 2001, 66, 3074-3083; d) S. M. Chervin, J. B. Lowe, M. Koreeda, J. Org. Chem. 2002, 67, 5654-5662; e) L. Huang, Z. Wang, X. Huang, Chem. Commun. 2004, 19601961; f) S. Mandal, B. Mukhopadhyay, Tetrahedron 2007, 63, 11363-11370.

[5] a) V. Serebryany, L. Beigelman, Tetrahedron Lett. 2002, 43, 1983-1985; b) V. Derdau, T. Fey, J. Atzrodt, Tetrahedron 2010, 66, 1472-1482; c) K. C. Nicolaou, J. A. Pfefferkorn, G. Q. Cao, Angew. Chem. Int. Ed. 2000, 39, 734-739; d) T. Miura, K. Goto, H. Waragai, H. Matsumoto, Y. Hirose, M. Ohmae, H. Ishida, A. Satoh, T. Inazu, J. Org. Chem. 2004, 69, 5348-5353; e) T. Miura, Y. Hirose, M. Ohmae, T. Inazu, Org. Lett. 2001, 3, 39473950; f) B. Yang, Y. Jing, X. Huang, Eur. J. Org. Chem. 2010, 2010, 1290-1298; g) P. H. Tam, T. L. Lowary, Org. Biomol. Chem. 2010, 8, 181-192.

[6] a) D. Picq, D. Anker, Carbohydr. Res. 1987, 166, 309-313; b) B. Thollas, J. C. Jacquinet, Org. Biomol. Chem. 2004, 2, 434-442; c) J. C. Jacquinet, Carbohydr. Res. 2006, 341, 1630-1644.

[7] A. A. Sherman, O. N. Yudina, Y. V. Mironov, E. V. Sukhova, A. S. Shashkov, V. M.Menshov, N. E. Nifantieva, Carbohydr. Res. 2001, 336, 13 - 46.

[8] a) H. M. Kim, I. J. Kim, S. J. Danishefsky, J. Am. Chem. Soc. 2001, 123, 35-48; b) S. Arungundram, K. Al-Mafraji, J. Asong, F. E. Leach, I. J. Amster, A. Venot, J. E. Turnbull, G. J. Boons, J. Am. Chem. Soc. 2009, 131, 17394-17405.

[9] a) B. Yu, J. Sun, Chem. Commun. (Camb). 2010, 46, 4668-4679; b) H. Gold, R. G. Boot, J. M. F. G. Aerts, H. S. Overkleeft, J. D. C. Codée, G. A. van Der Marel, Eur. J. Org. Chem. 2011, 2011, 1652-1663.

[10] J. Ohlsson, G. Magnusson, Carbohydr. Res. 2000, 329, 49-55. 


\section{NMR Spectra (1D and 2D)}

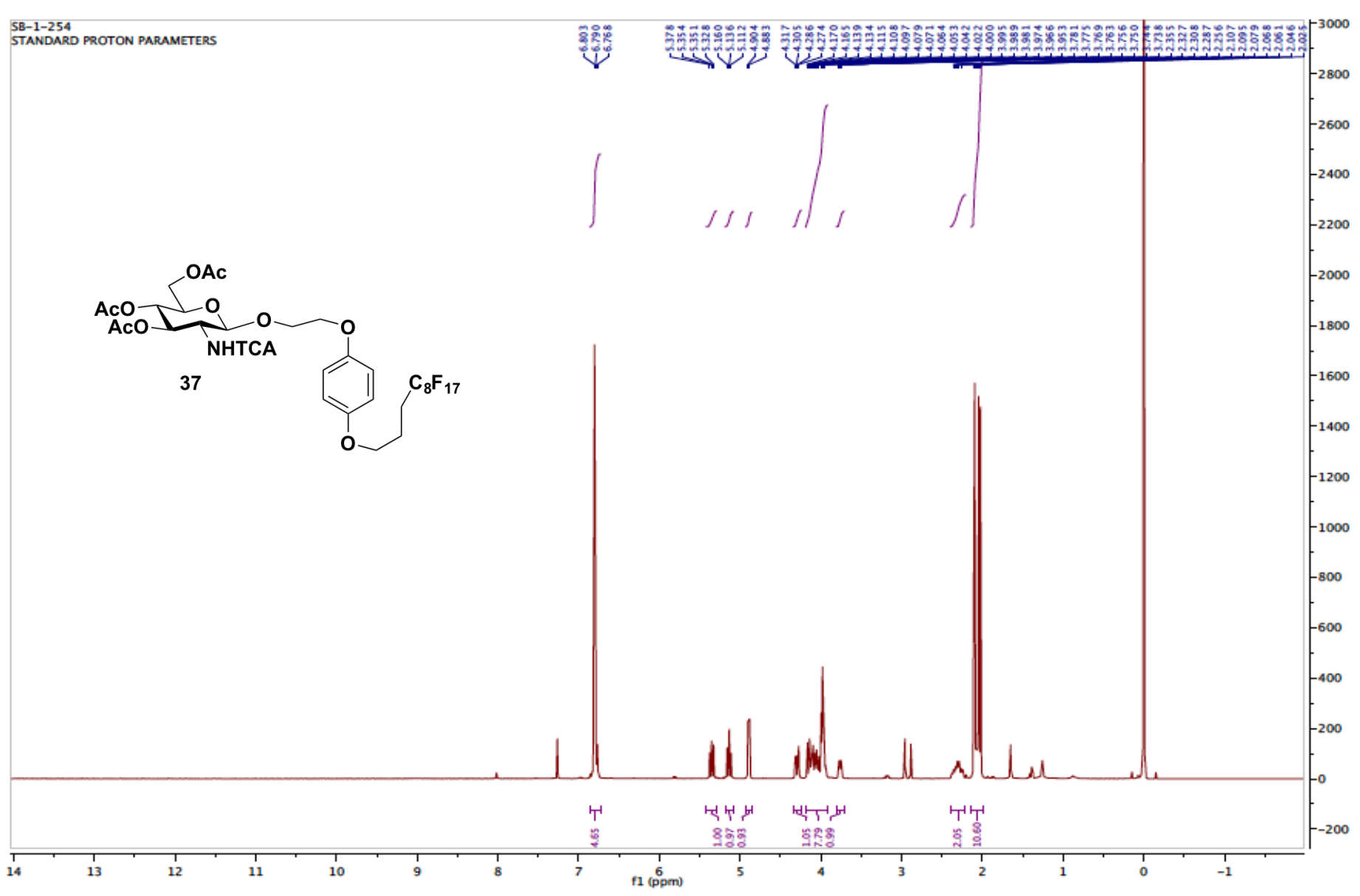

${ }^{1}$ H NMR (400 MHz) spectrum of compound 37 in $\mathrm{CDCl}_{3}$ 


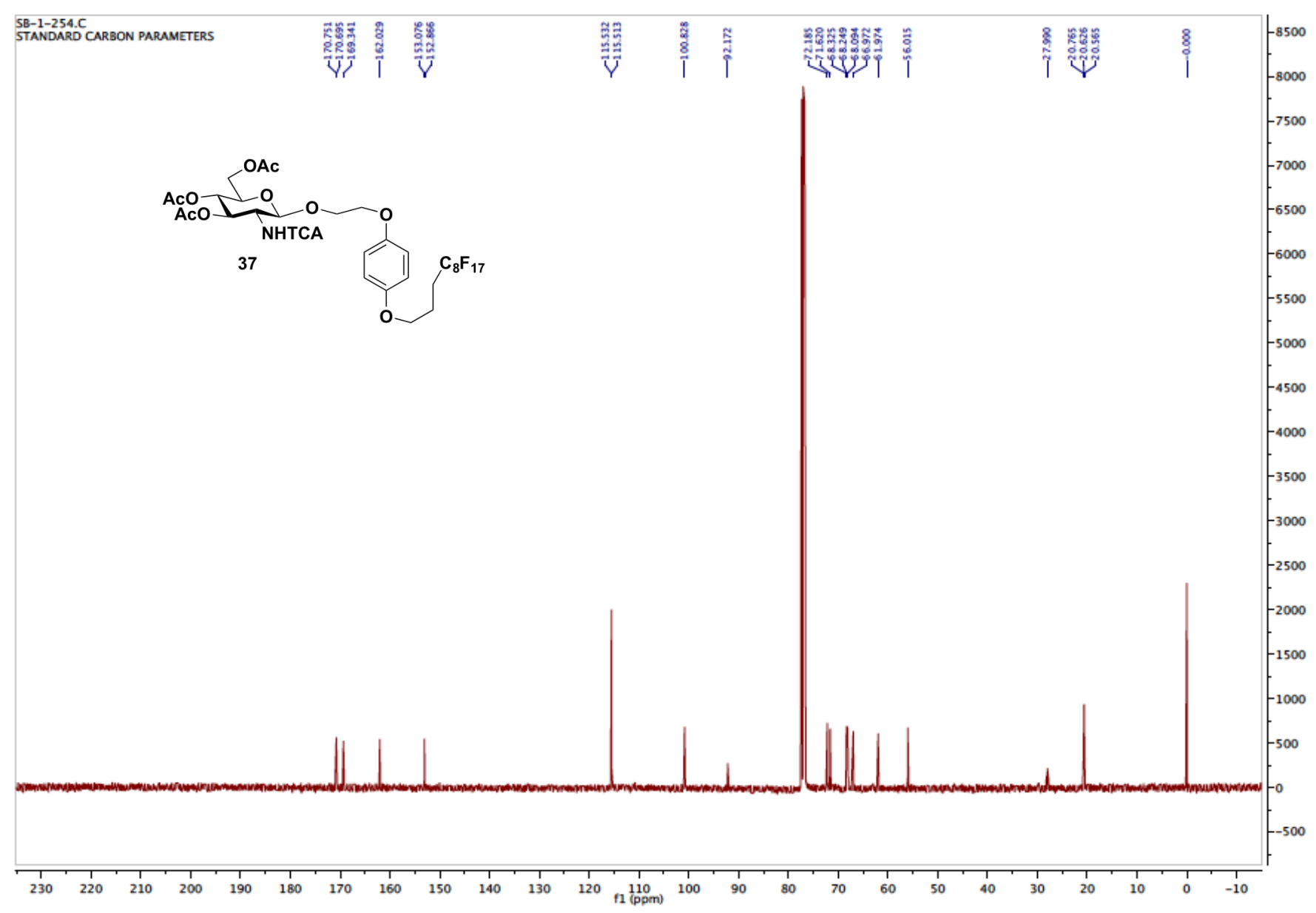

13C NMR (101 MHz) spectrum of compound 37 in $\mathrm{CDCl}_{3}$ 


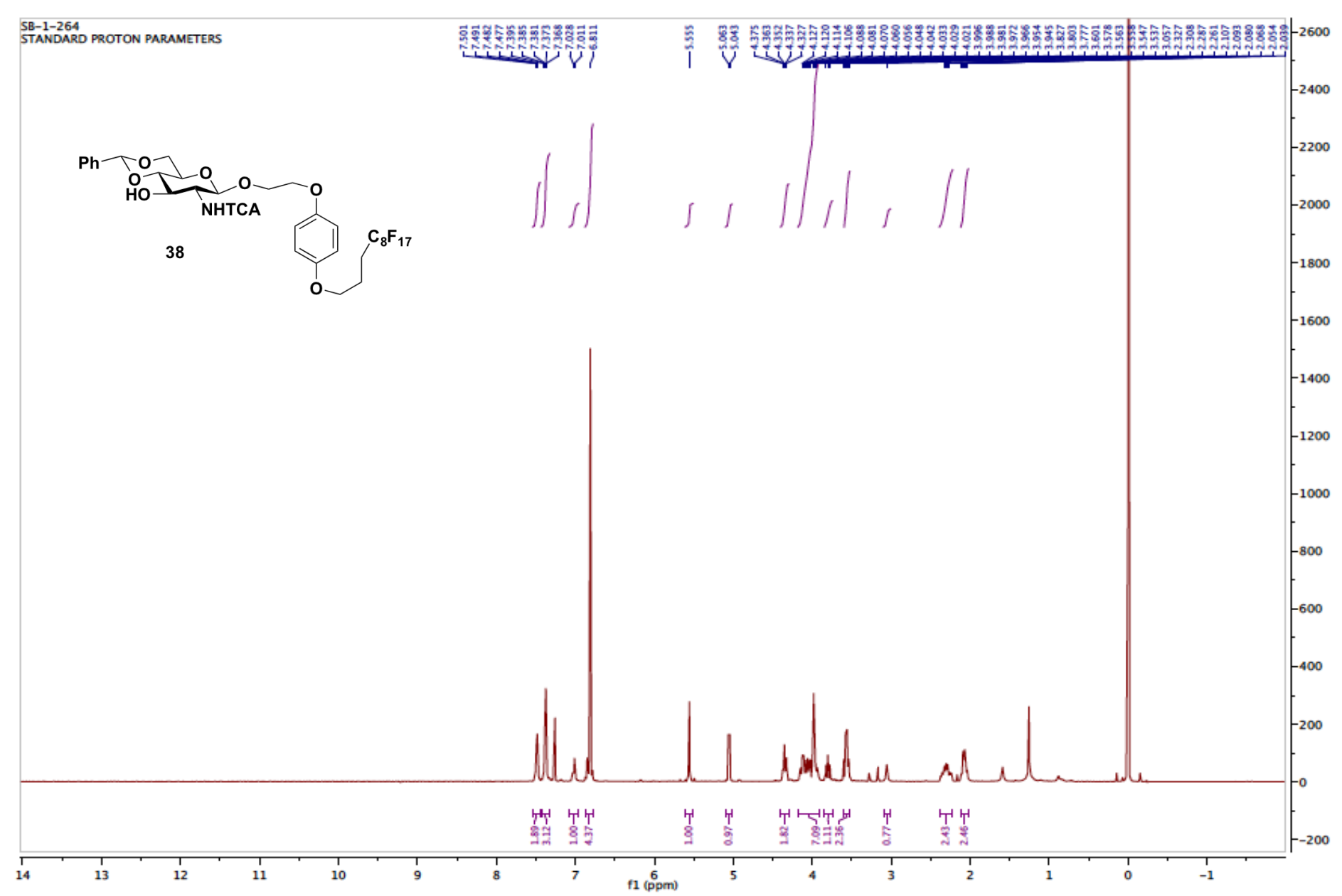

${ }^{1} \mathbf{H}$ NMR (400 MHz) spectrum of compound $\mathbf{3 8}$ in $\mathrm{CDCl}_{3}$ 


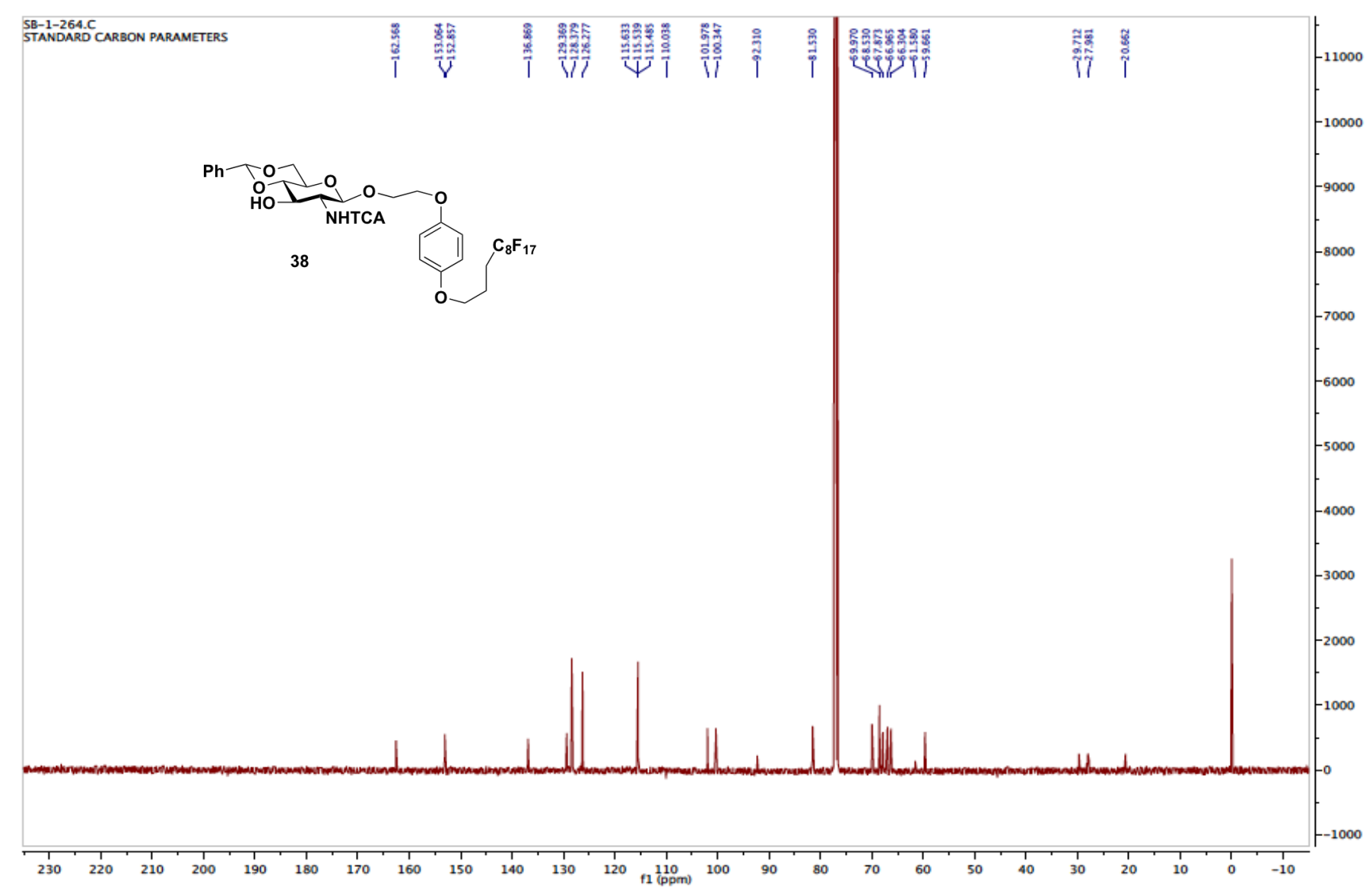

${ }^{13} \mathbf{C}$ NMR (101 MHz) spectrum of compound 38 in $\mathrm{CDCl}_{3}$ 


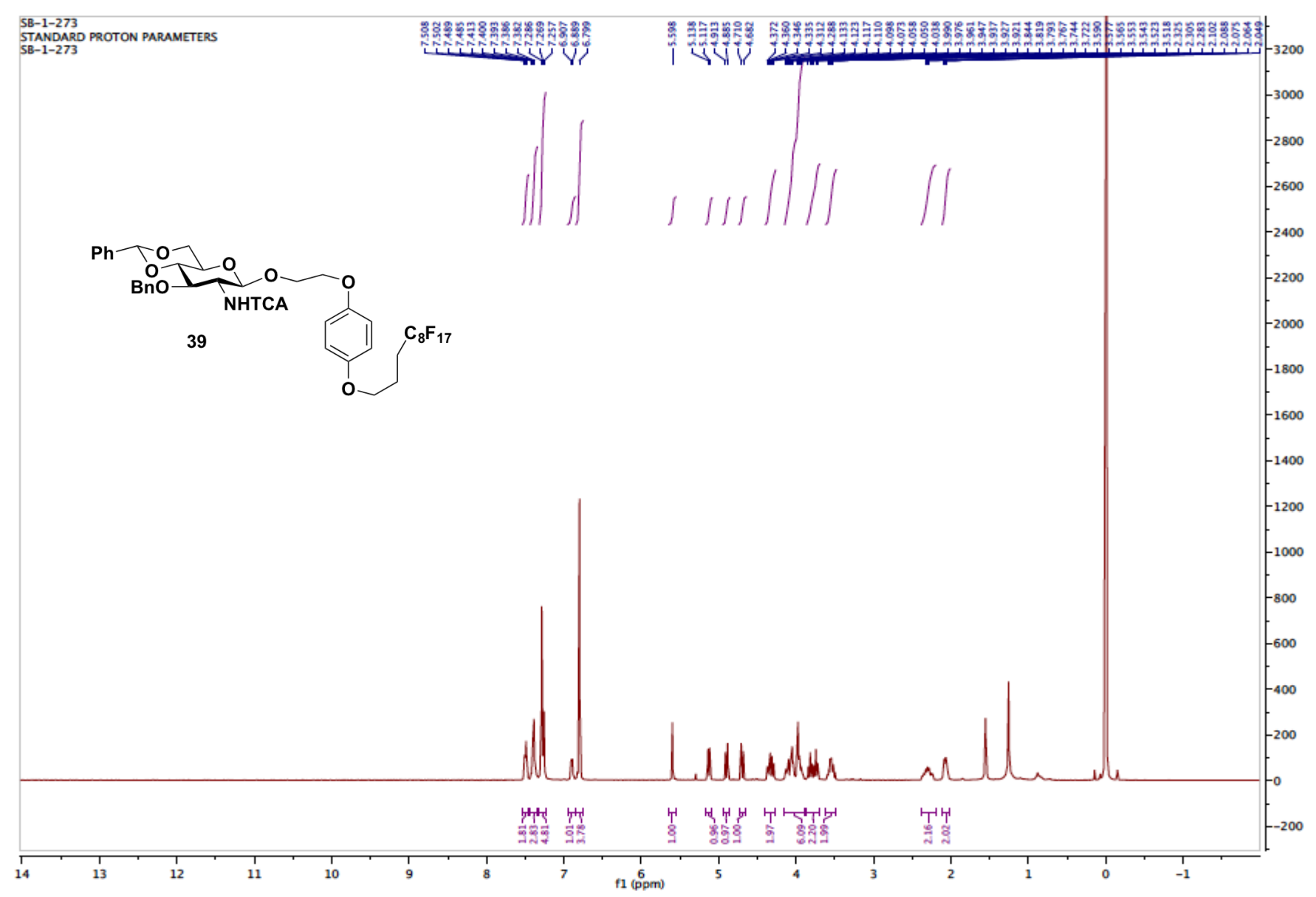

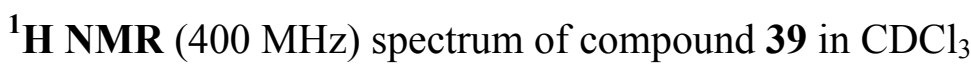




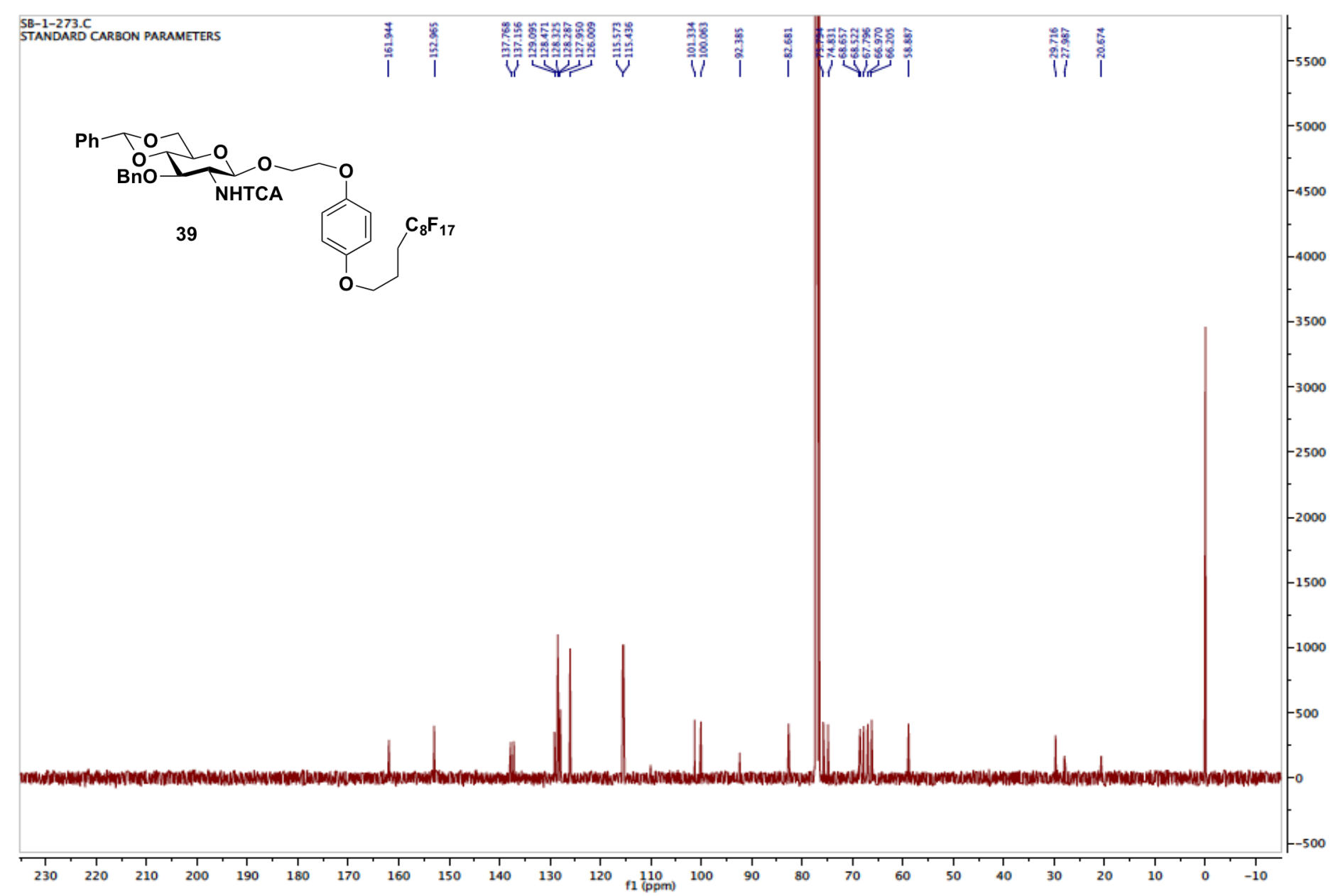

${ }^{13} \mathbf{C}$ NMR (101 MHz) spectrum of compound 39 in $\mathrm{CDCl}_{3}$ 


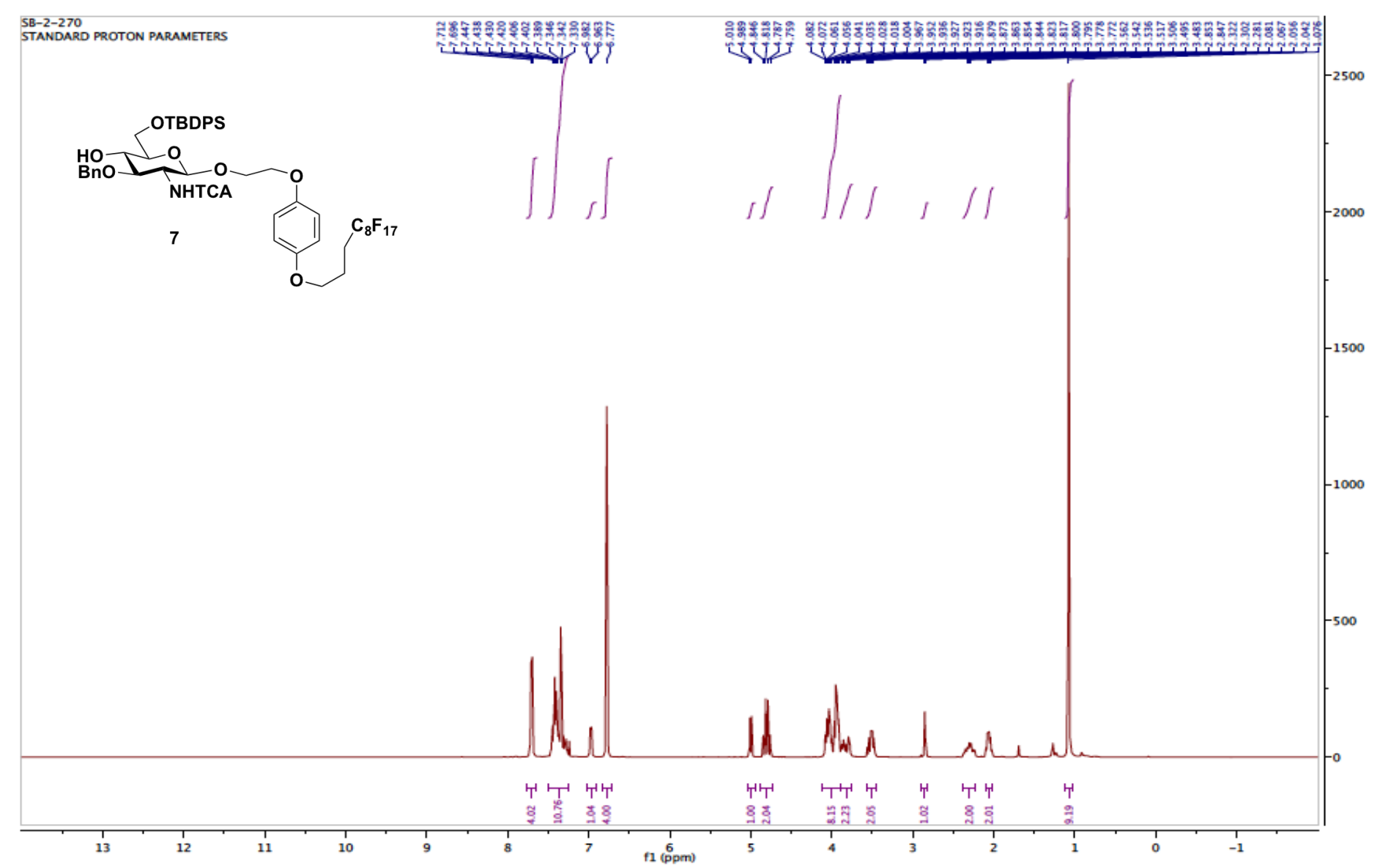

${ }^{1} \mathbf{H}$ NMR (400 MHz) spectrum of compound 7 in $\mathrm{CDCl}_{3}$ 


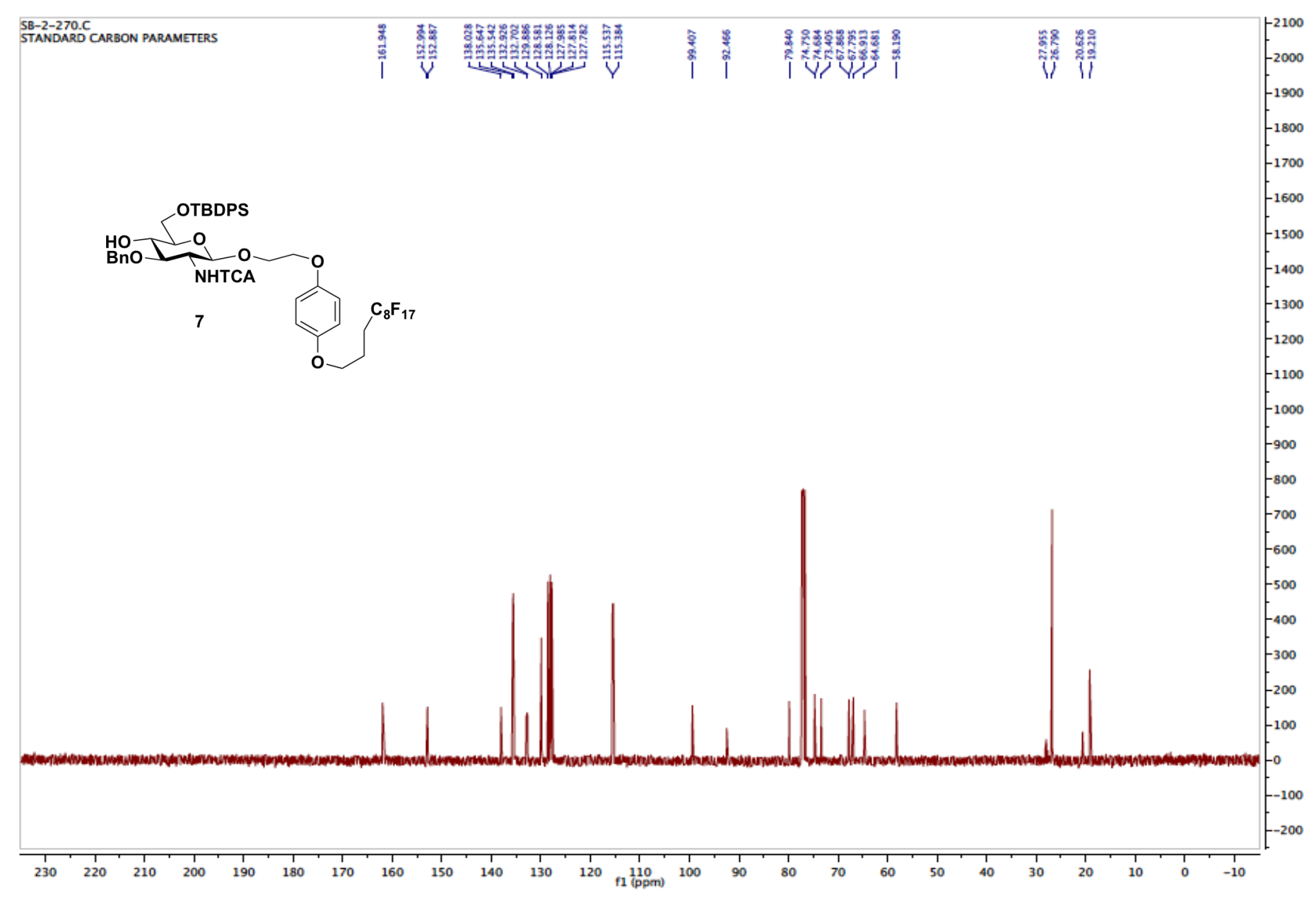

${ }^{13} \mathbf{C}$ NMR (101 MHz) spectrum of compound 7 in $\mathrm{CDCl}_{3}$ 

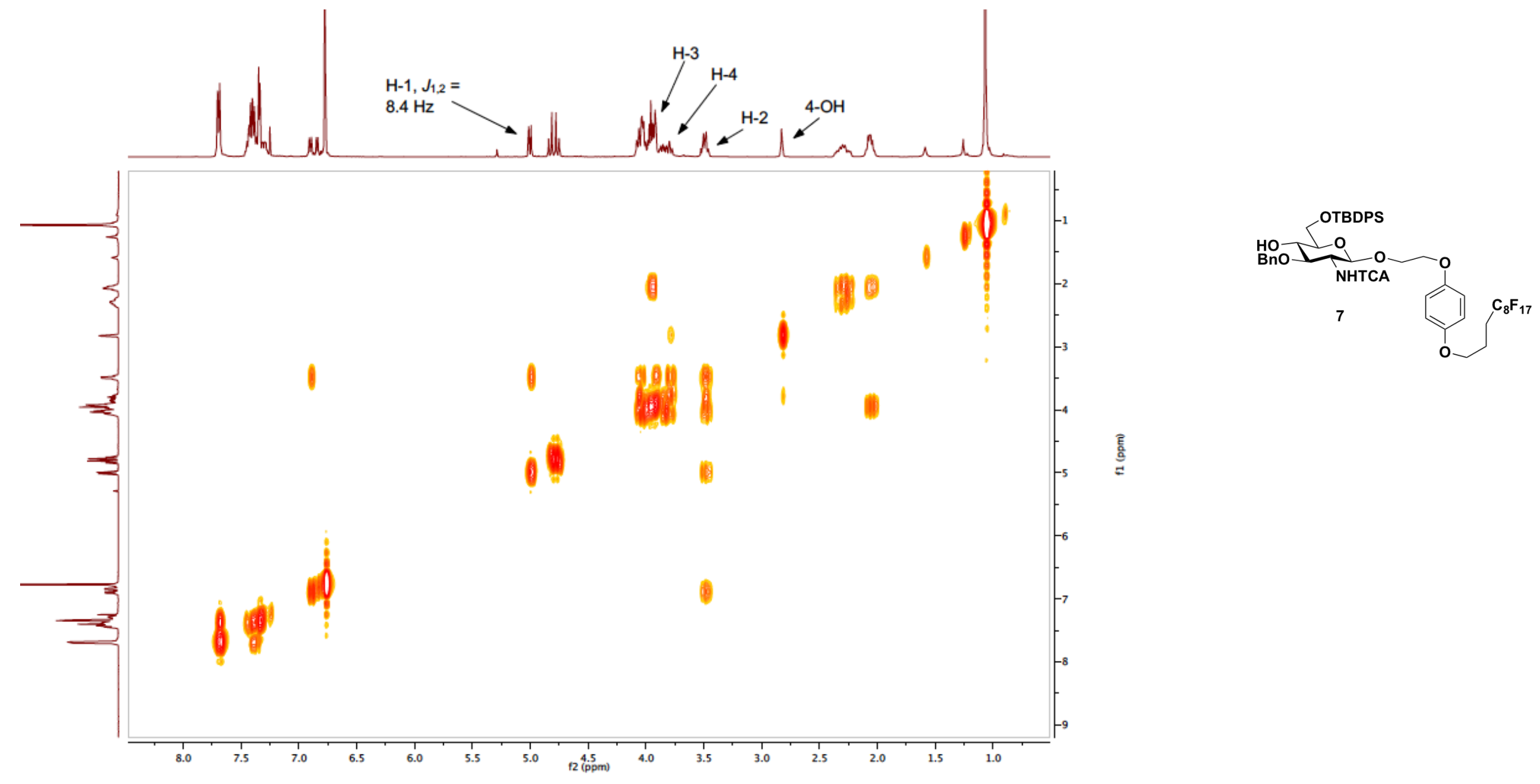

${ }^{1} \mathbf{H}$ - ${ }^{1} \mathbf{H}$ COSY NMR (400 MHz) spectrum of compound 7 in $\mathrm{CDCl}_{3}$ 


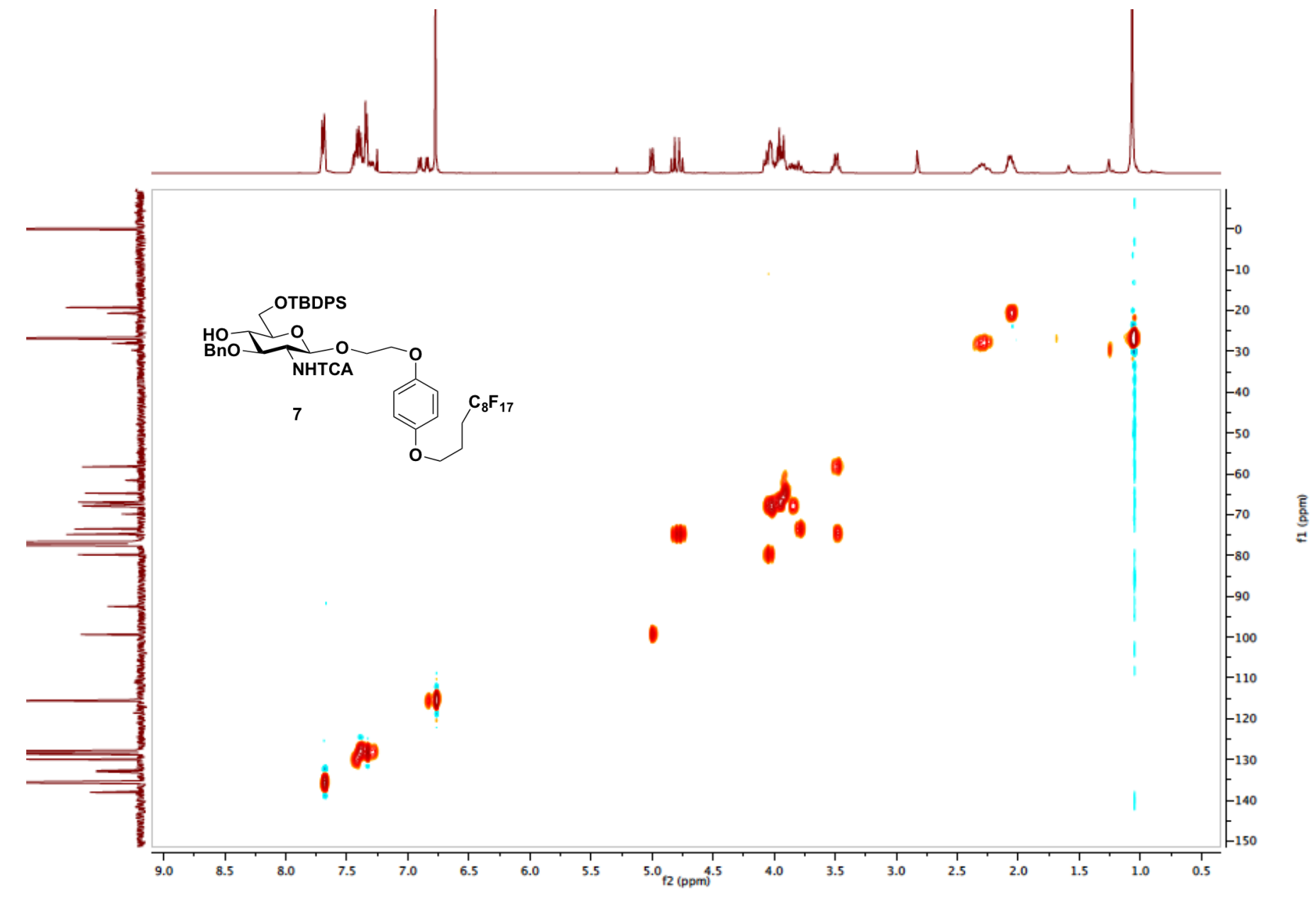

${ }^{1} \mathbf{H}-{ }^{13} \mathbf{C}$ HSQC NMR (400 MHz) spectrum of compound 7 in $\mathrm{CDCl}_{3}$ 


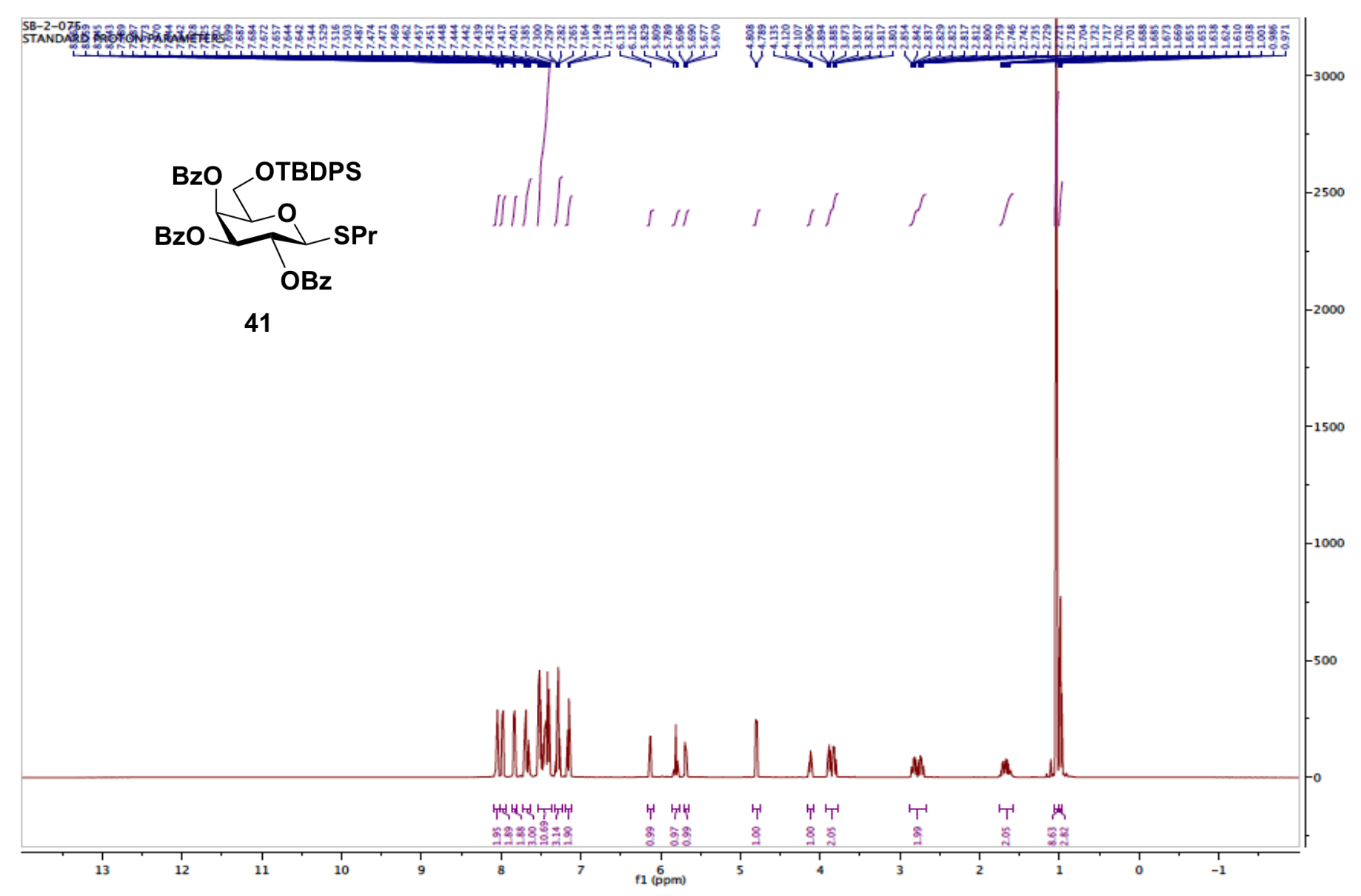

${ }^{1} \mathbf{H}$ NMR (500 MHz) spectrum of compound 41 in $\mathrm{CDCl}_{3}$ 


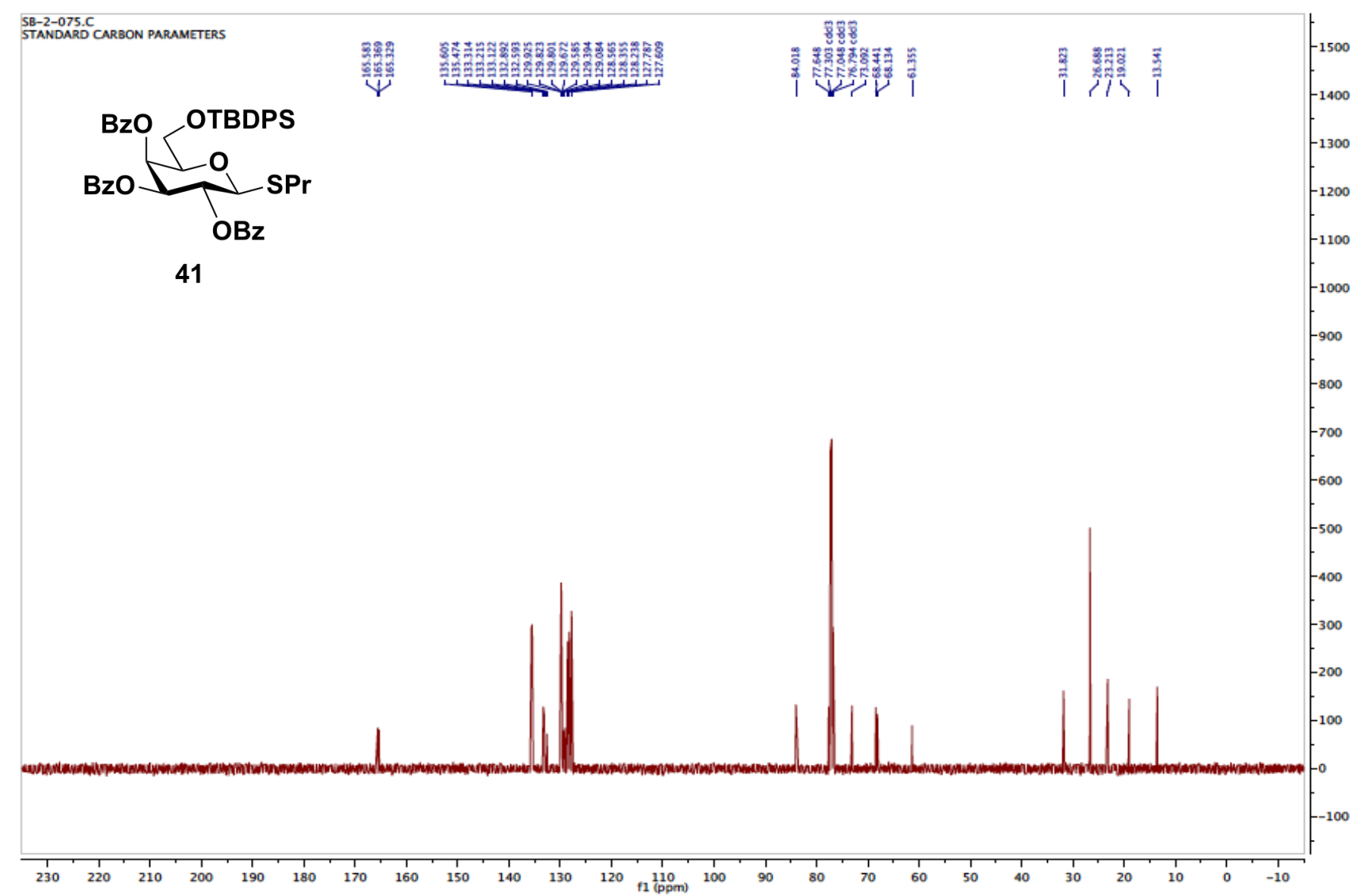

${ }^{13} \mathbf{C}$ NMR (126 MHz) spectrum of compound 41 in $\mathrm{CDCl}_{3}$ 


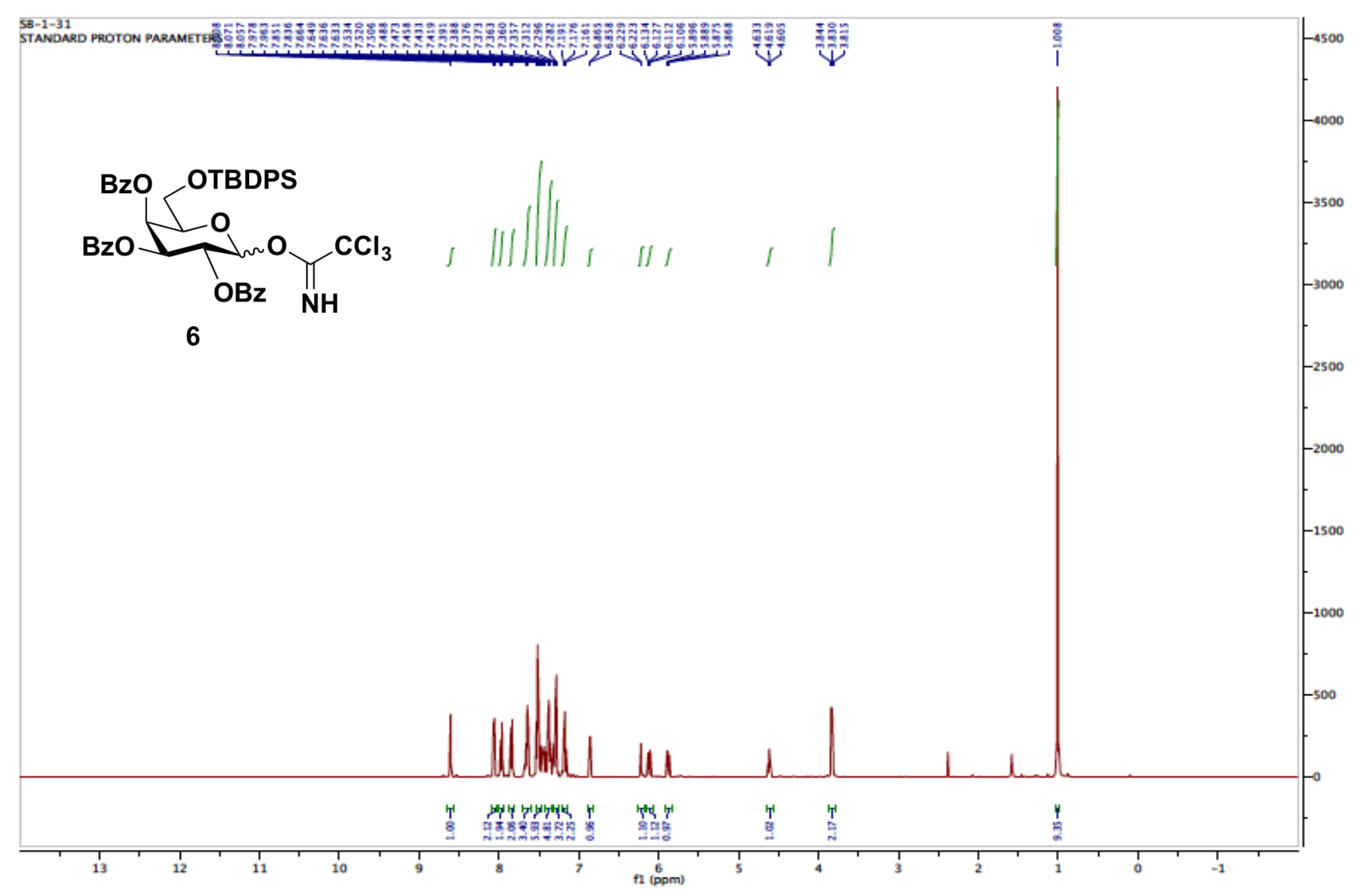

${ }^{1} \mathbf{H}$ NMR (500 MHz) spectrum of compound $\mathbf{6}$ in $\mathrm{CDCl}_{3}$ 


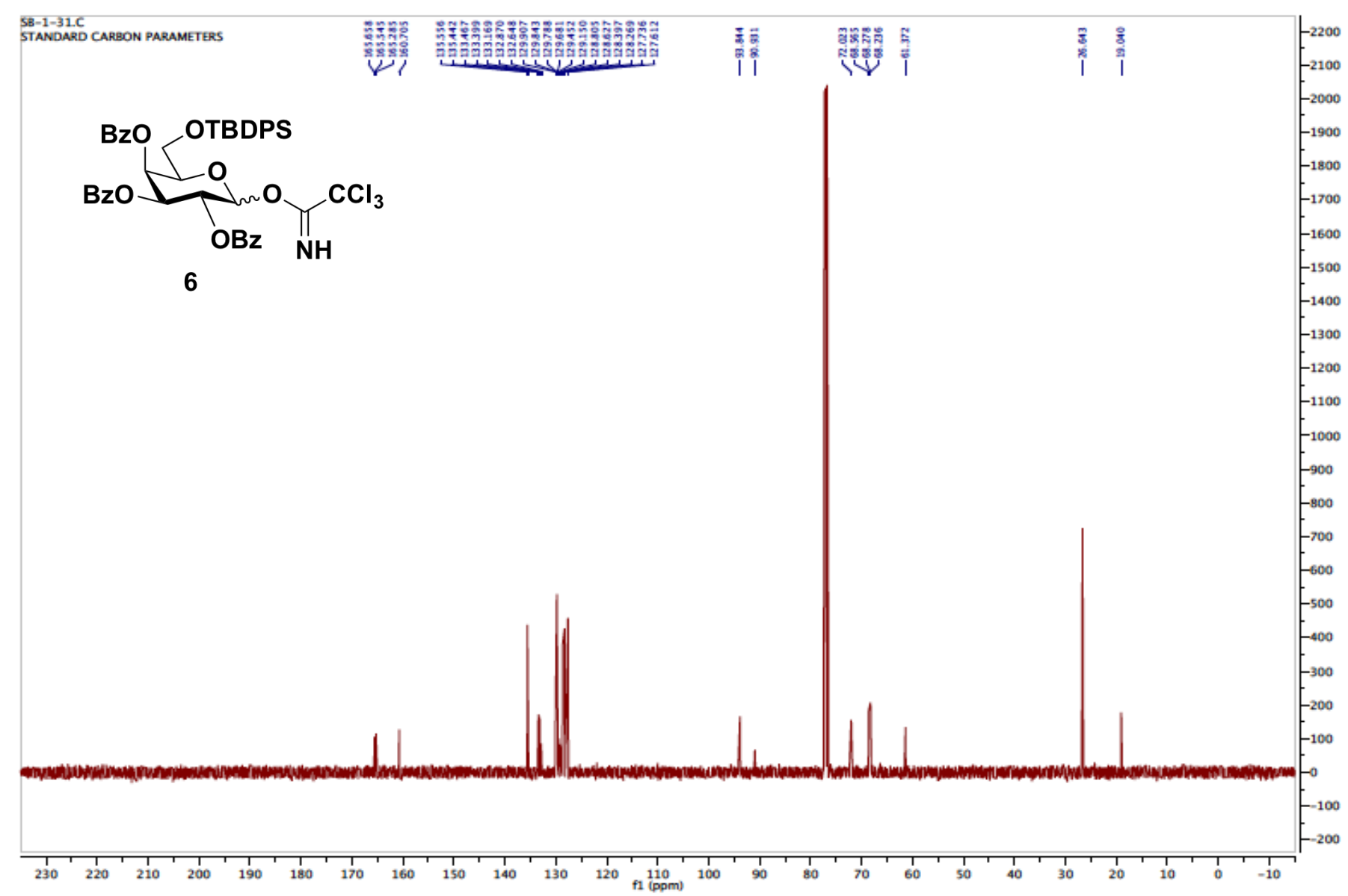

${ }^{13} \mathbf{C ~ N M R ~ ( 1 2 6 ~ M H z ) ~ s p e c t r u m ~ o f ~ c o m p o u n d ~} 6$ in $\mathrm{CDCl}_{3}$ 


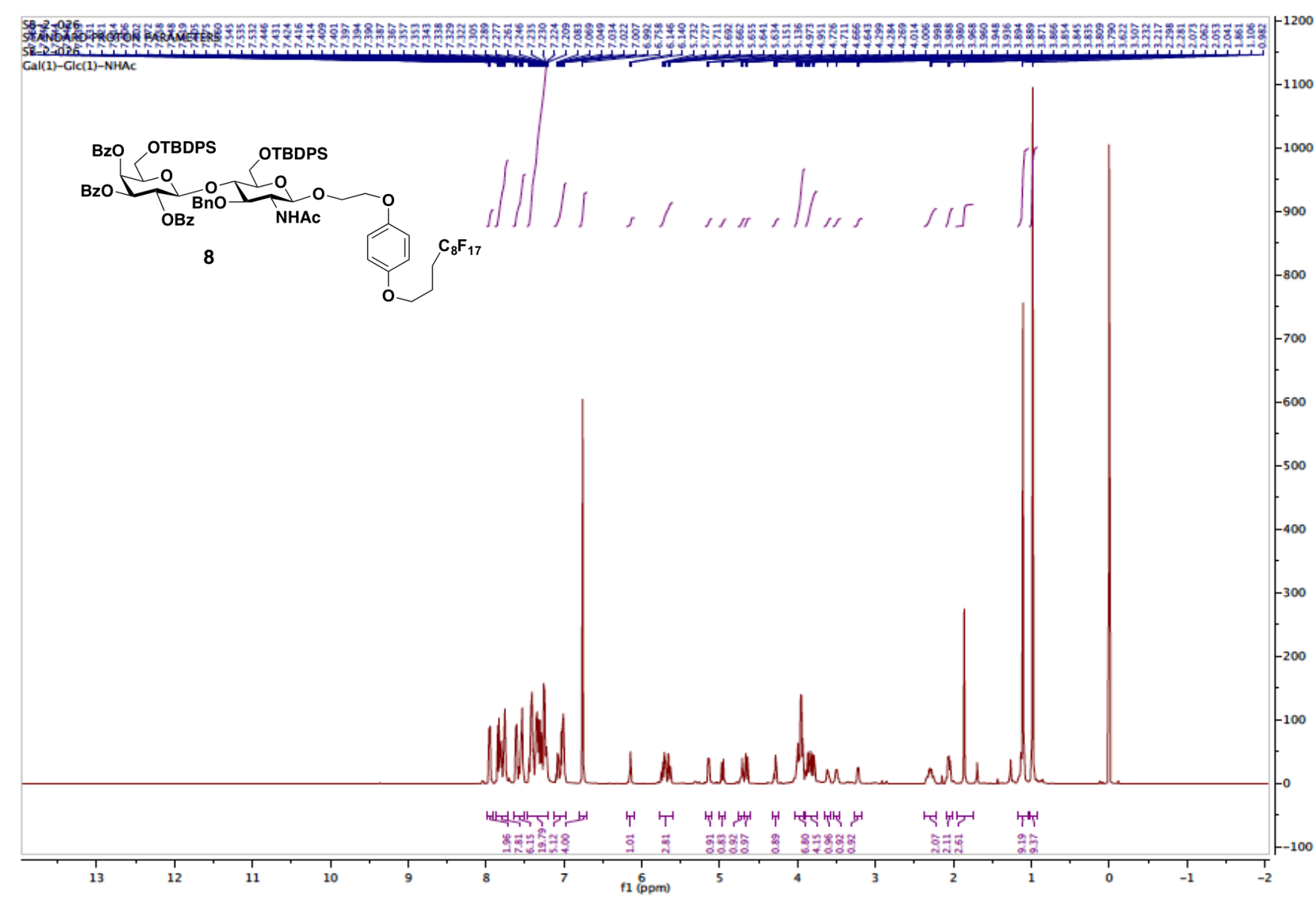

${ }^{1} \mathbf{H}$ NMR (500 MHz) spectrum of compound 8 in $\mathrm{CDCl}_{3}$ 


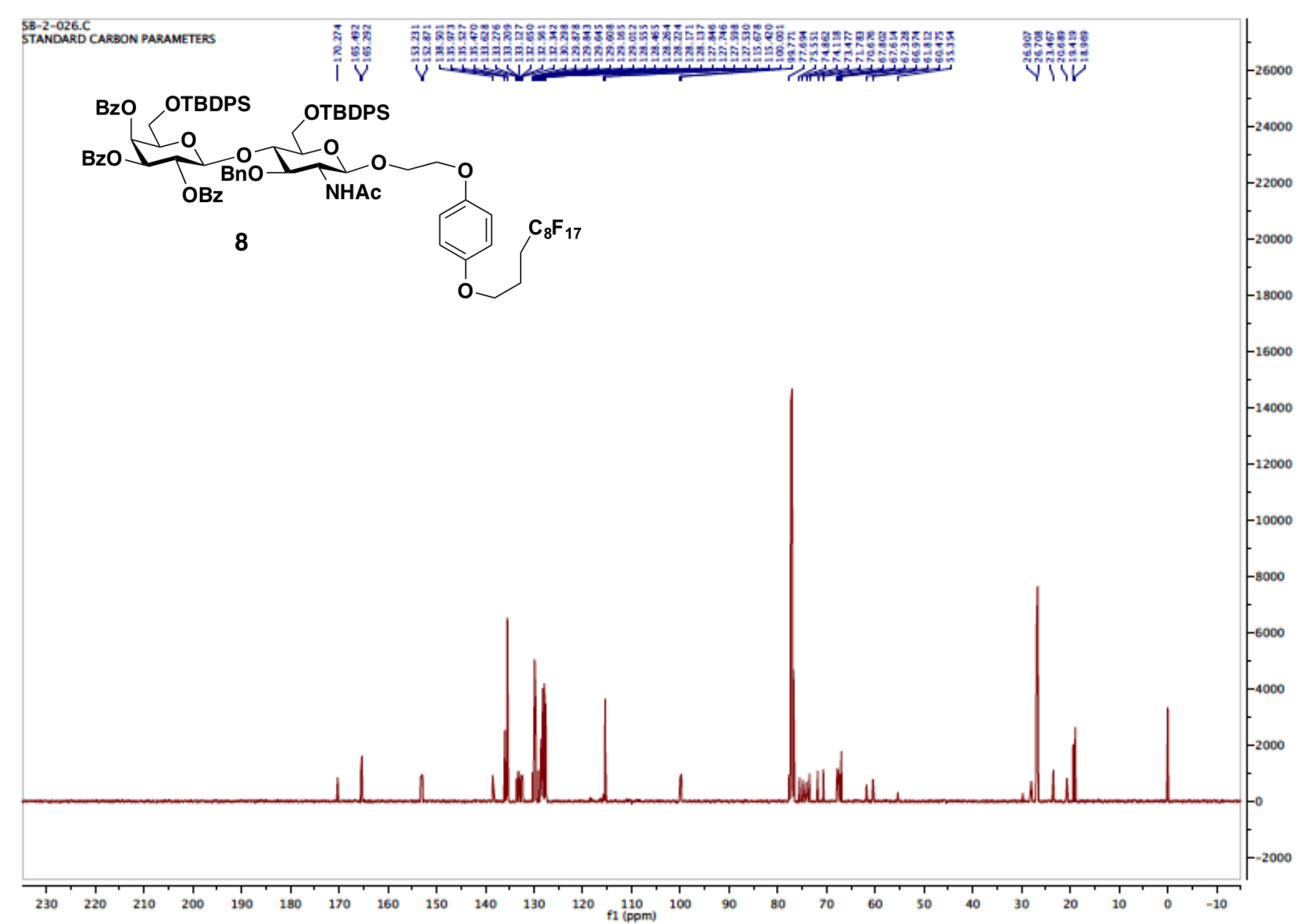

${ }^{13} \mathbf{C}$ NMR (126 MHz) spectrum of compound 8 in $\mathrm{CDCl}_{3}$ 


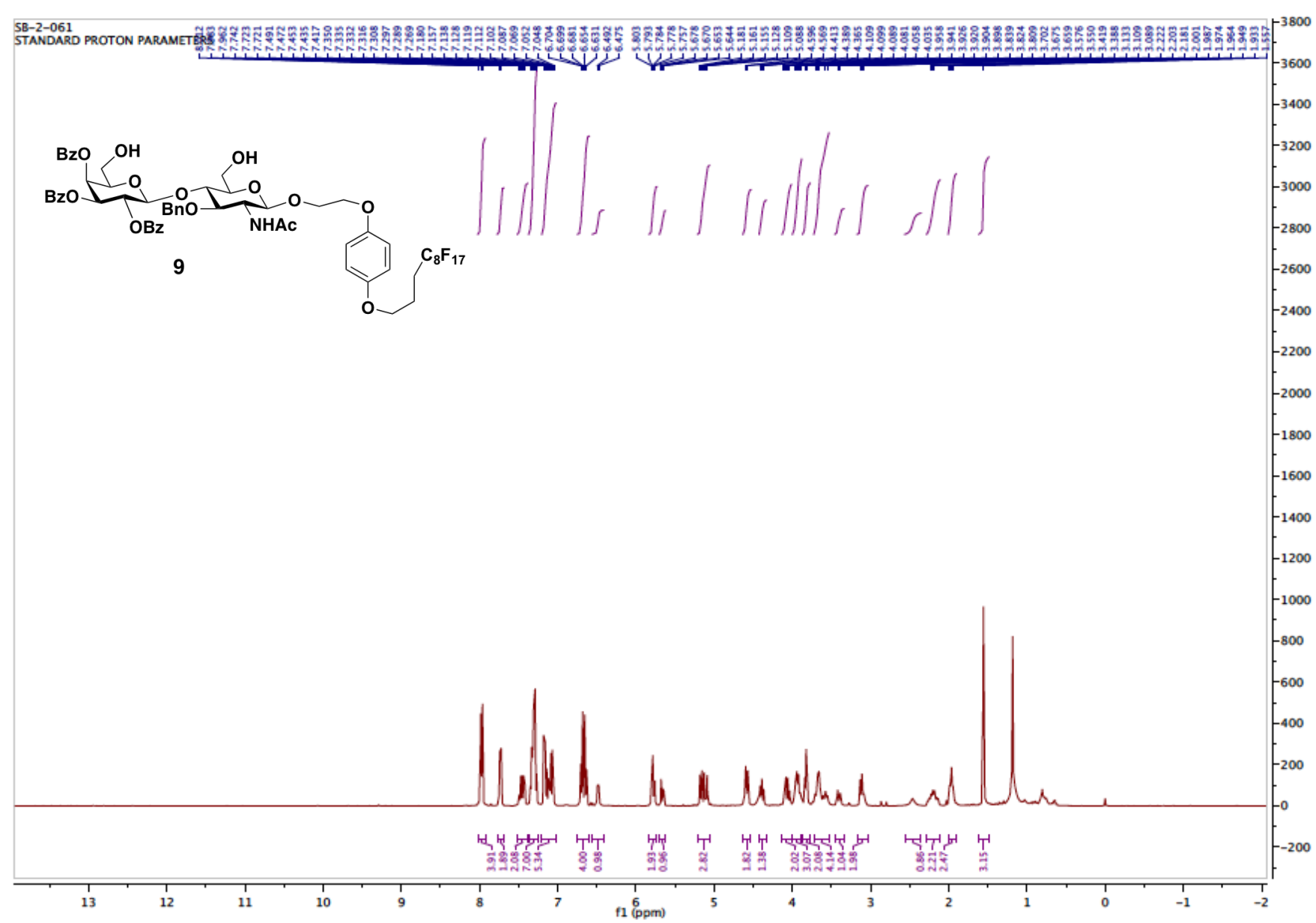

${ }^{1} \mathbf{H}$ NMR (400 MHz) spectrum of compound 9 in $\mathrm{CDCl}_{3}$ 


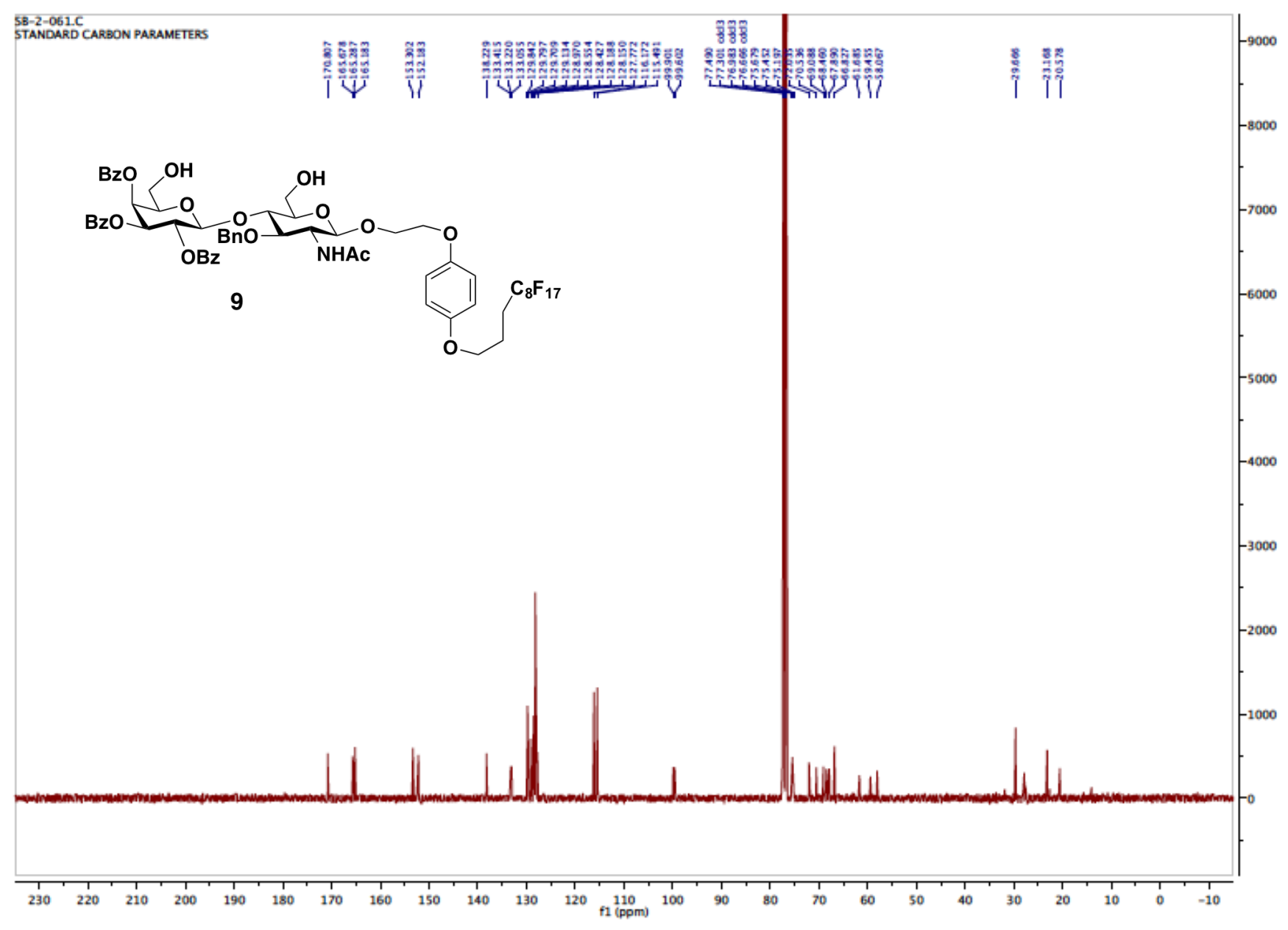

${ }^{13} \mathbf{C}$ NMR (101 MHz) spectrum of compound 9 in $\mathrm{CDCl}_{3}$ 


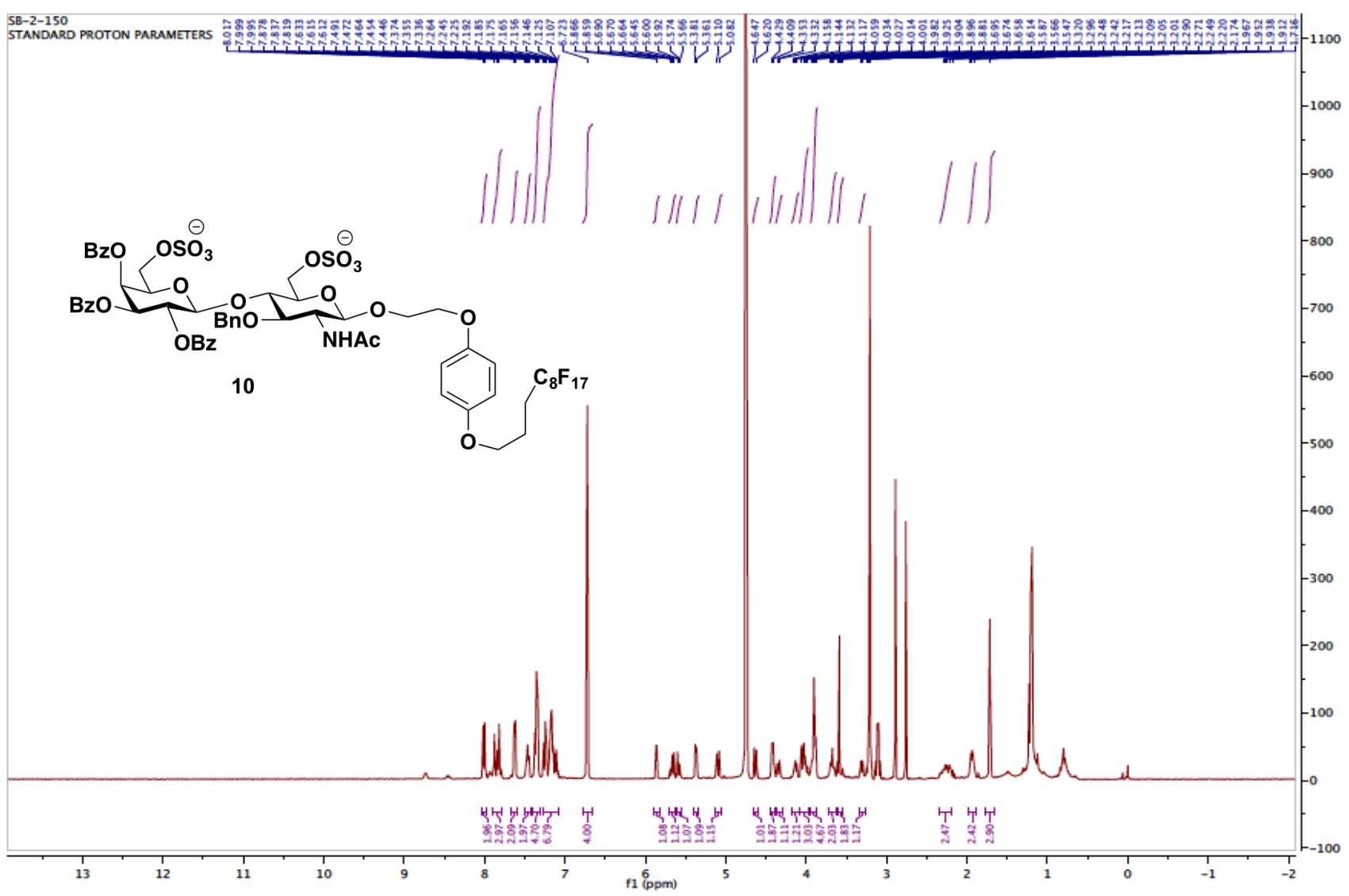

${ }^{1} \mathbf{H}$ NMR (400 MHz) spectrum of compound 10 in $\mathrm{CD}_{3} \mathrm{OD}$ 


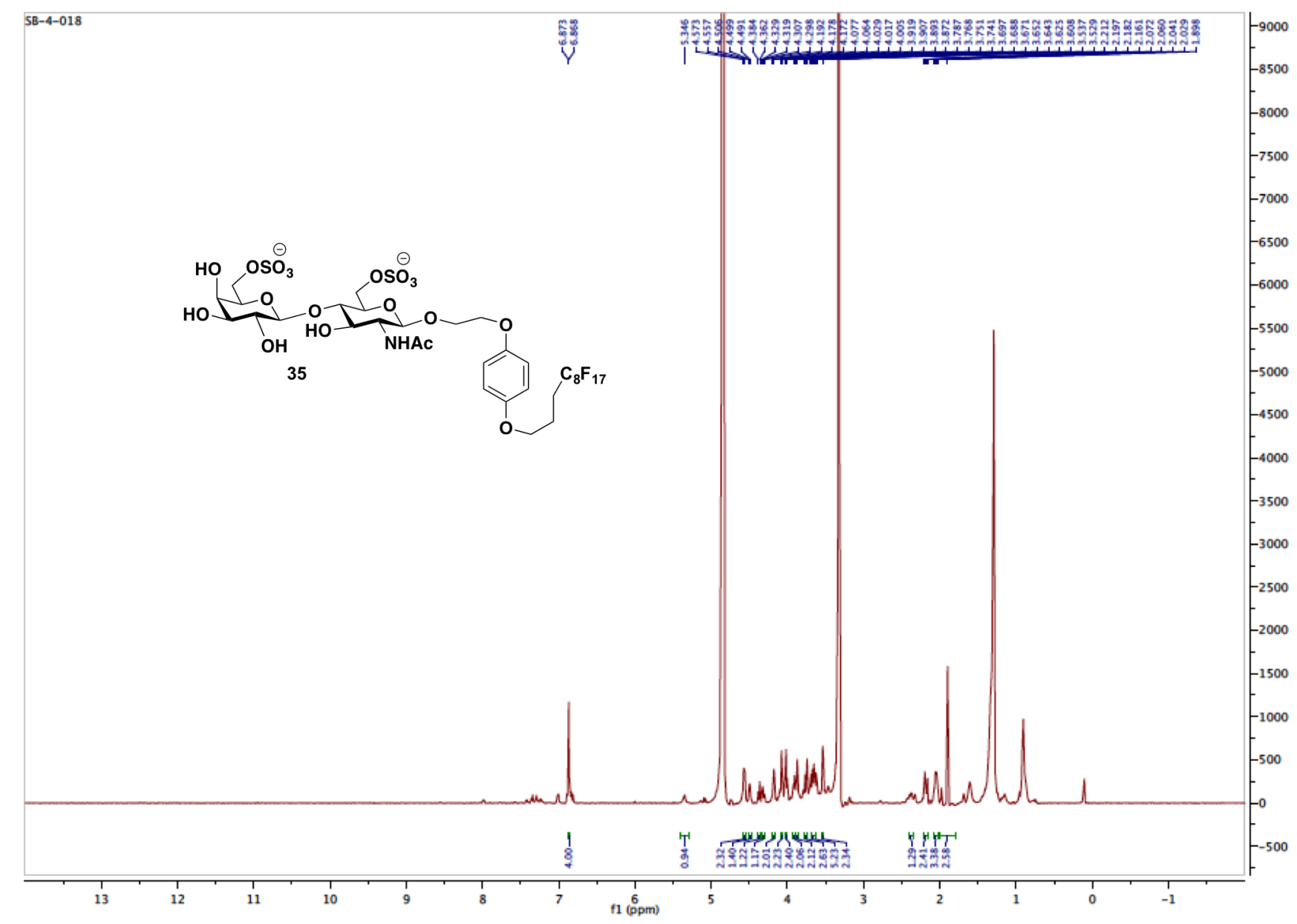

${ }^{1} \mathbf{H}$ NMR (500 MHz) spectrum of compound 35 in $\mathrm{CD}_{3} \mathrm{OD}$ 


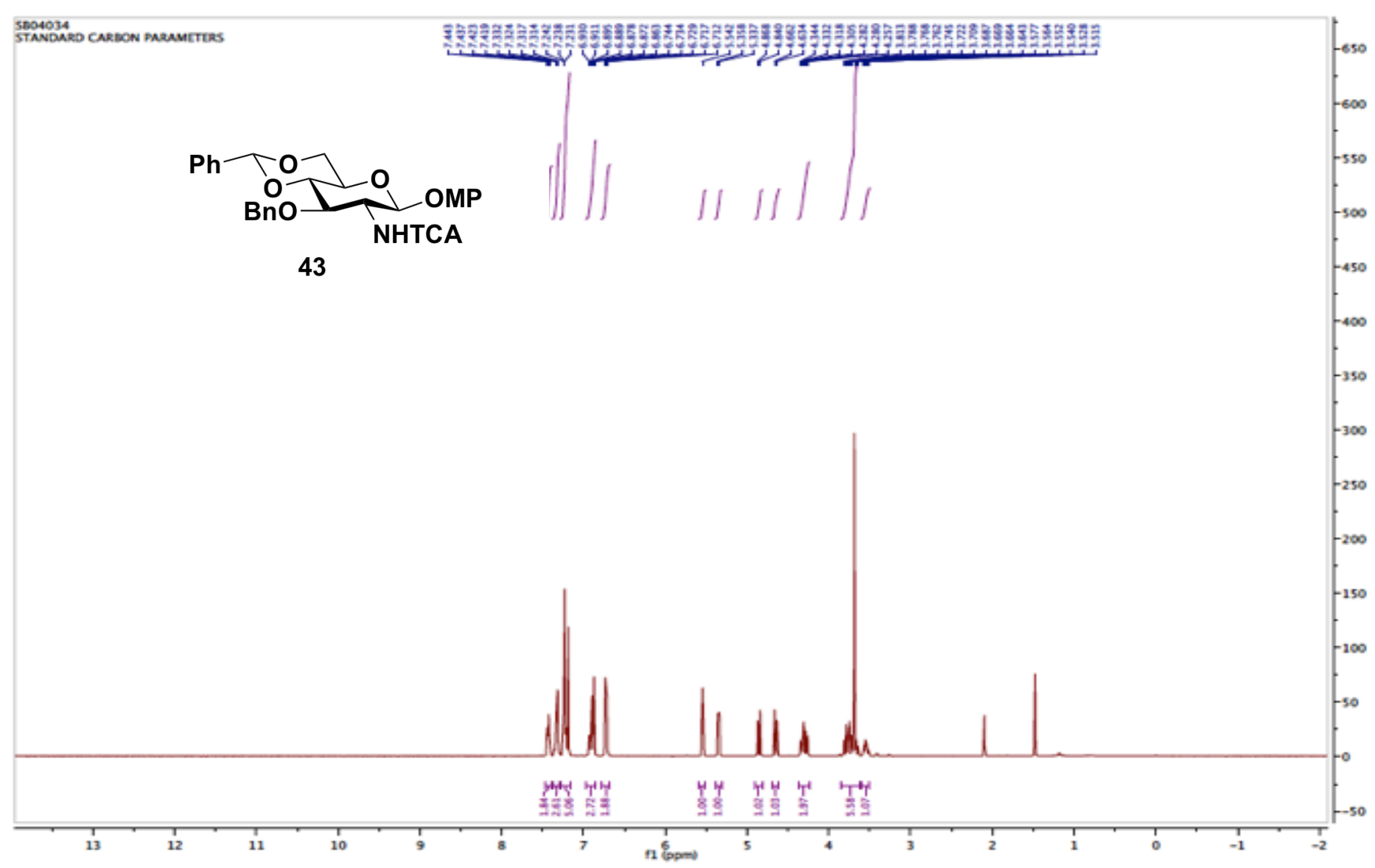

${ }^{1}$ H NMR (400 MHz) spectrum of compound 43 in $\mathrm{CDCl}_{3}$ 


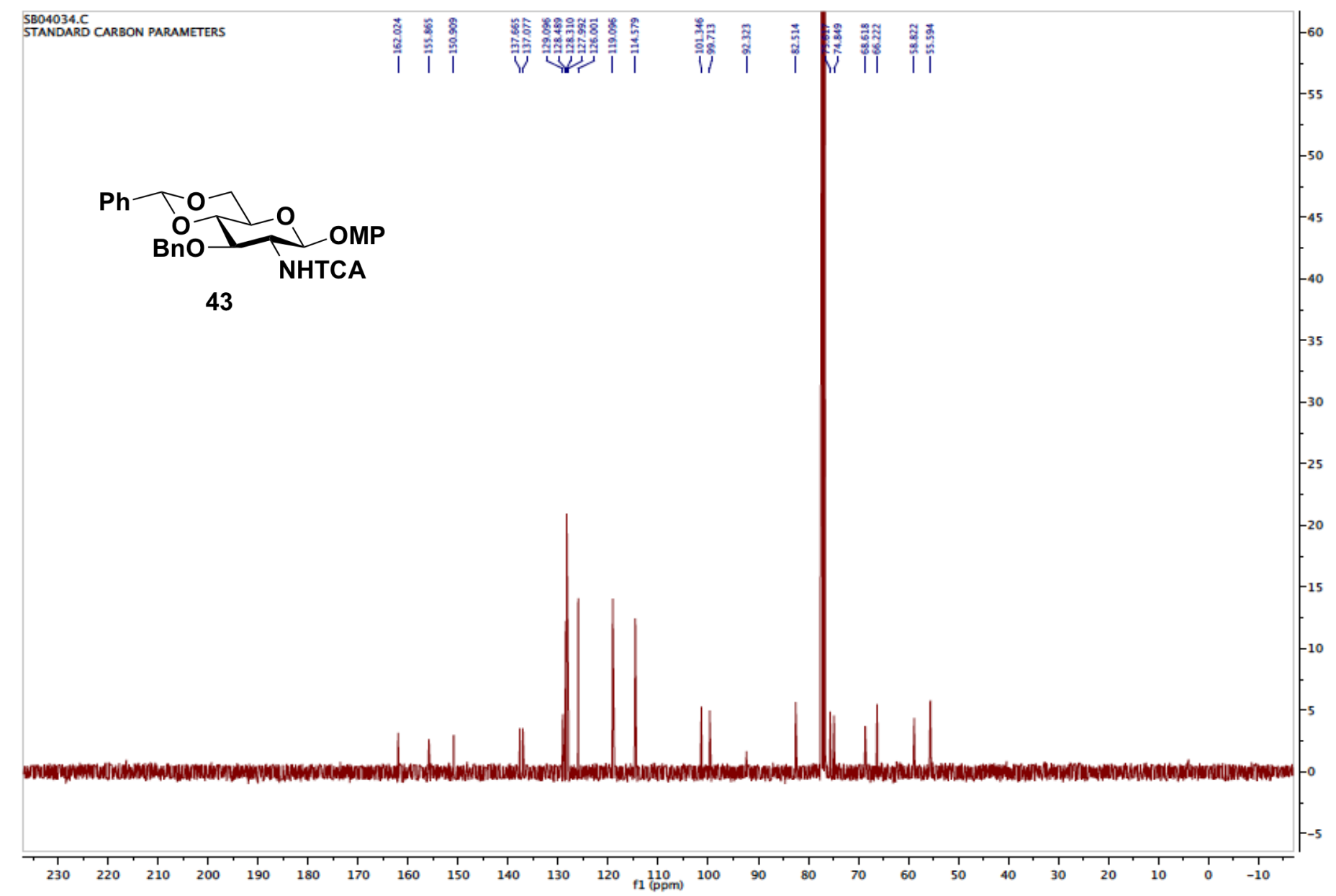

${ }^{13} \mathbf{C}$ NMR (101 MHz) spectrum of compound 43 in $\mathrm{CDCl}_{3}$ 


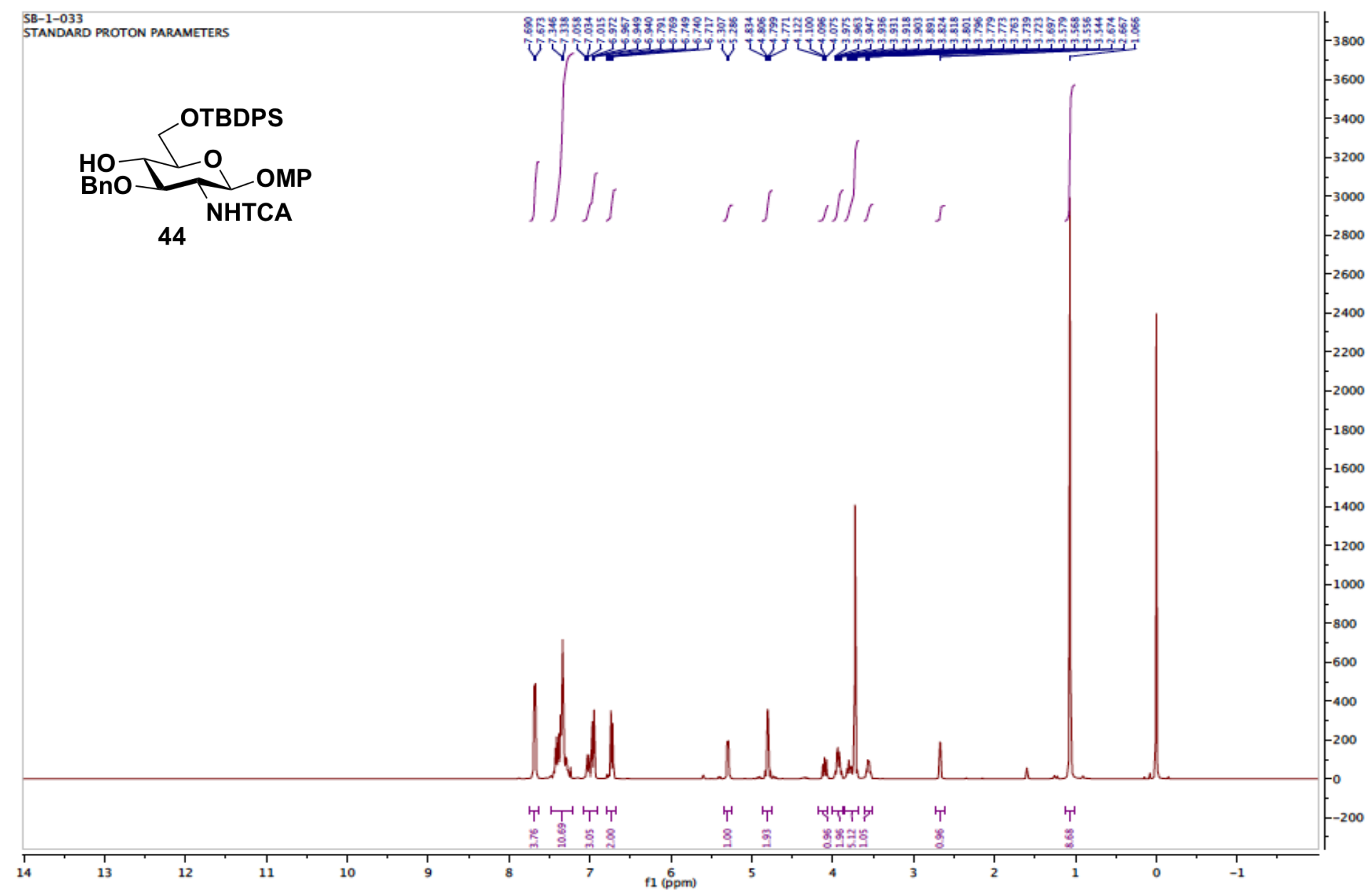

${ }^{1} \mathbf{H}$ NMR (400 MHz) spectrum of compound 44 in $\mathrm{CDCl}_{3}$ 


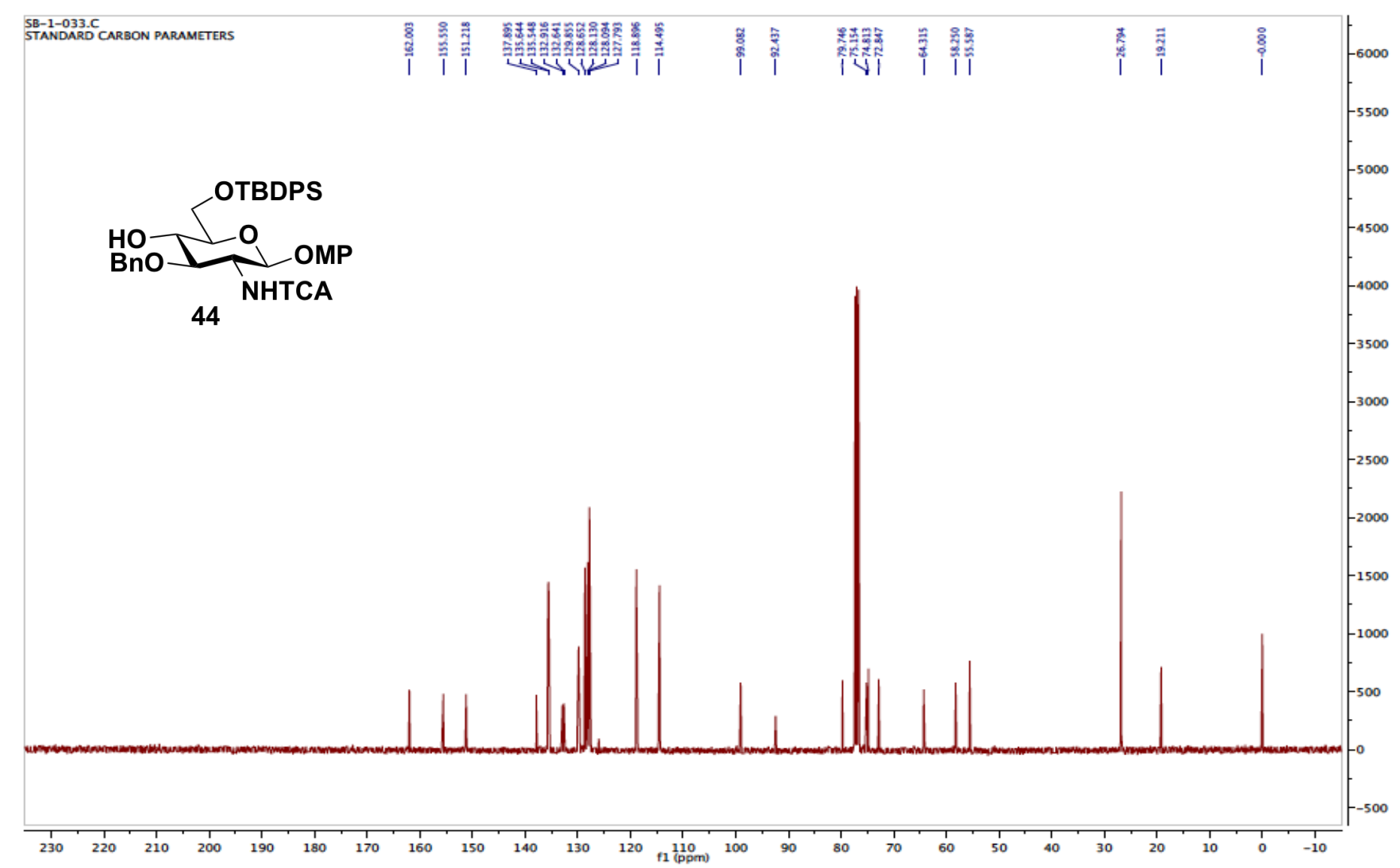

${ }^{13}$ C NMR (101 MHz) spectrum of compound 44 in $\mathrm{CDCl}_{3}$ 

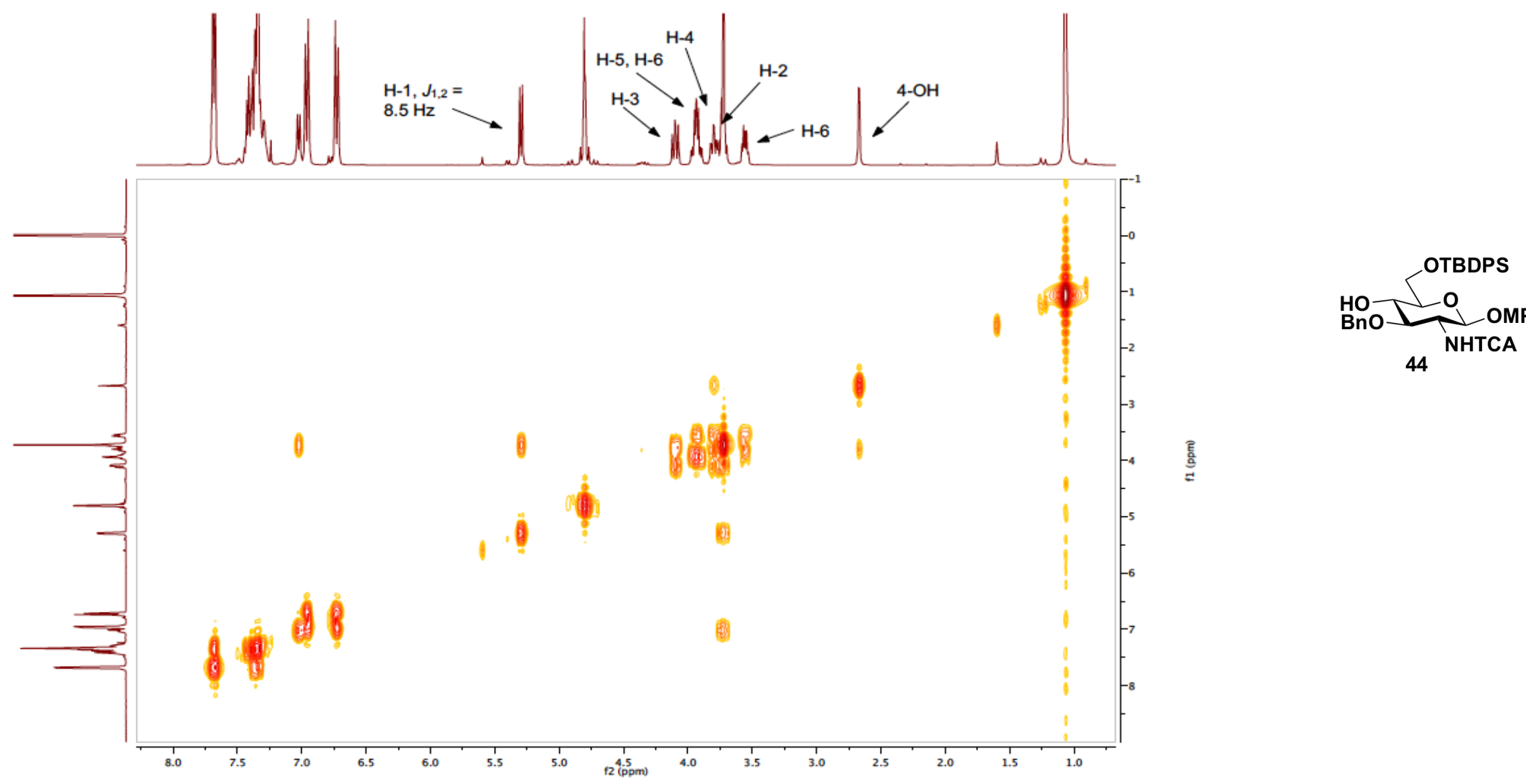

${ }^{1} \mathbf{H}$ - ${ }^{1} \mathbf{H}$ COSY NMR (400 MHz) spectrum of compound 44 in $\mathrm{CDCl}_{3}$ 

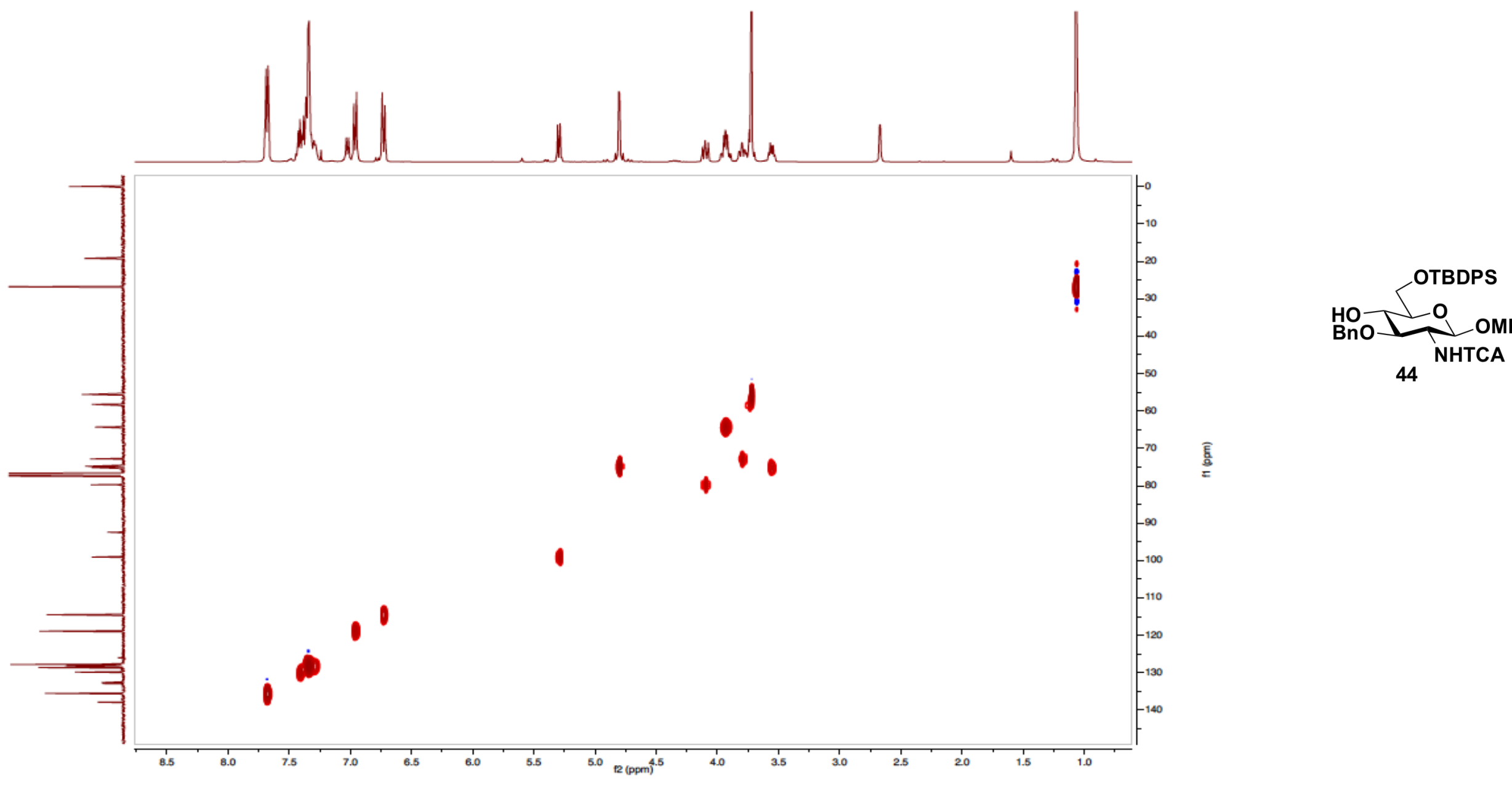

${ }^{1} \mathbf{H}-{ }^{13} \mathbf{C}$ HSQC NMR (400 MHz) spectrum of compound 44 in $\mathrm{CDCl}_{3}$ 


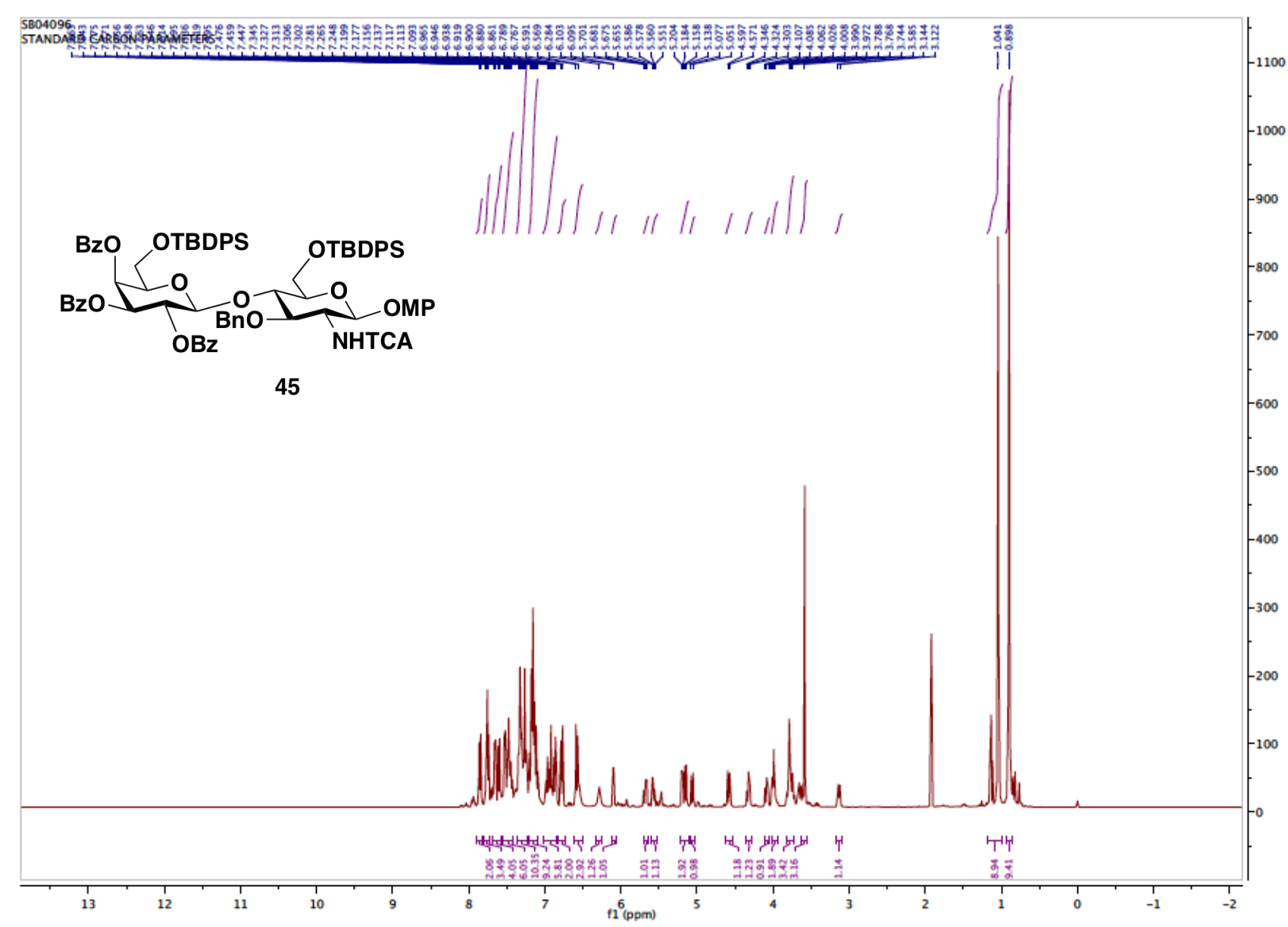

${ }^{1}$ H NMR (400 MHz) spectrum of compound 45 in $\mathrm{CDCl}_{3}$ 


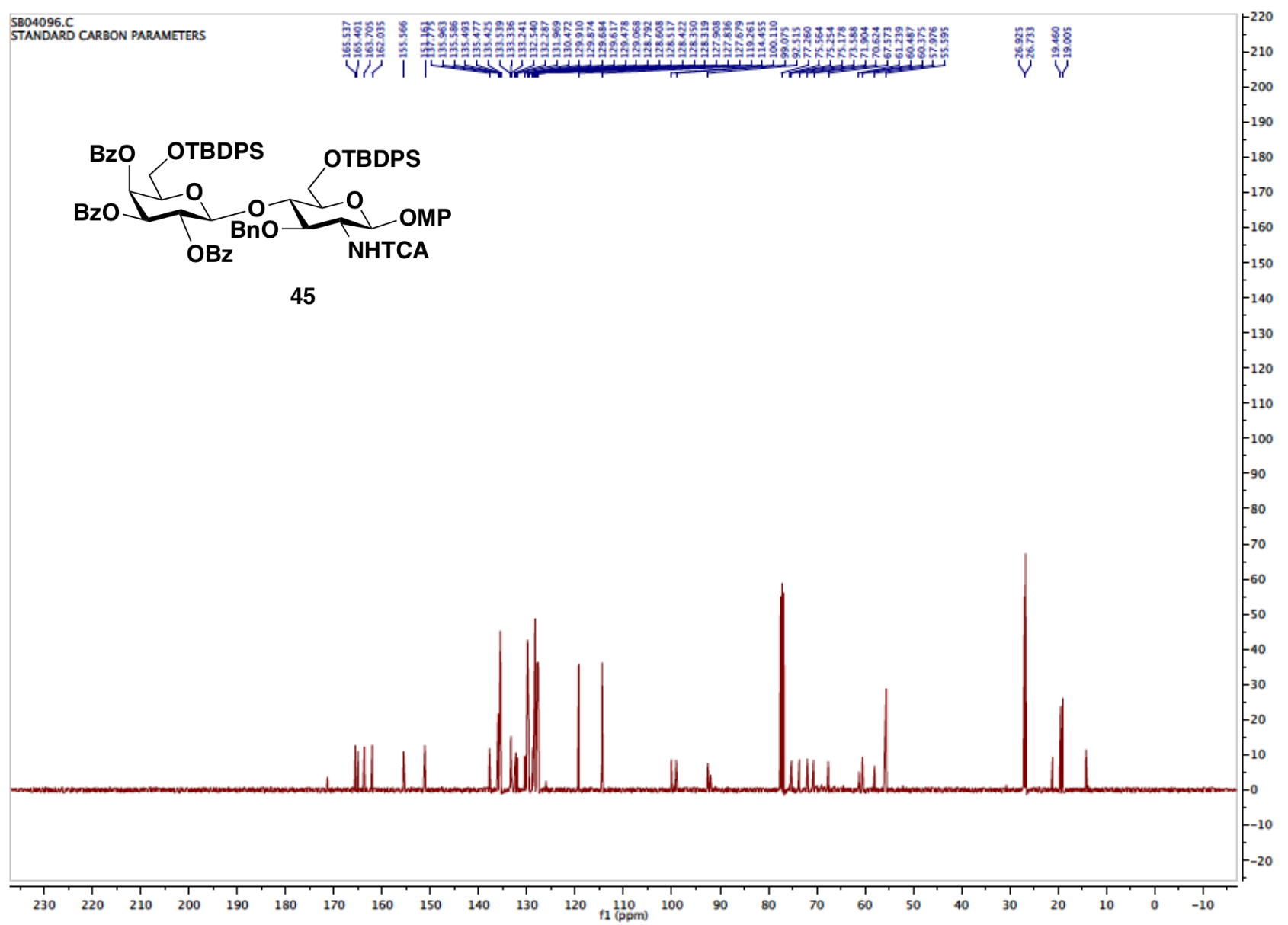

${ }^{13}$ C NMR (101 MHz) spectrum of compound 45 in $\mathrm{CDCl}_{3}$ 


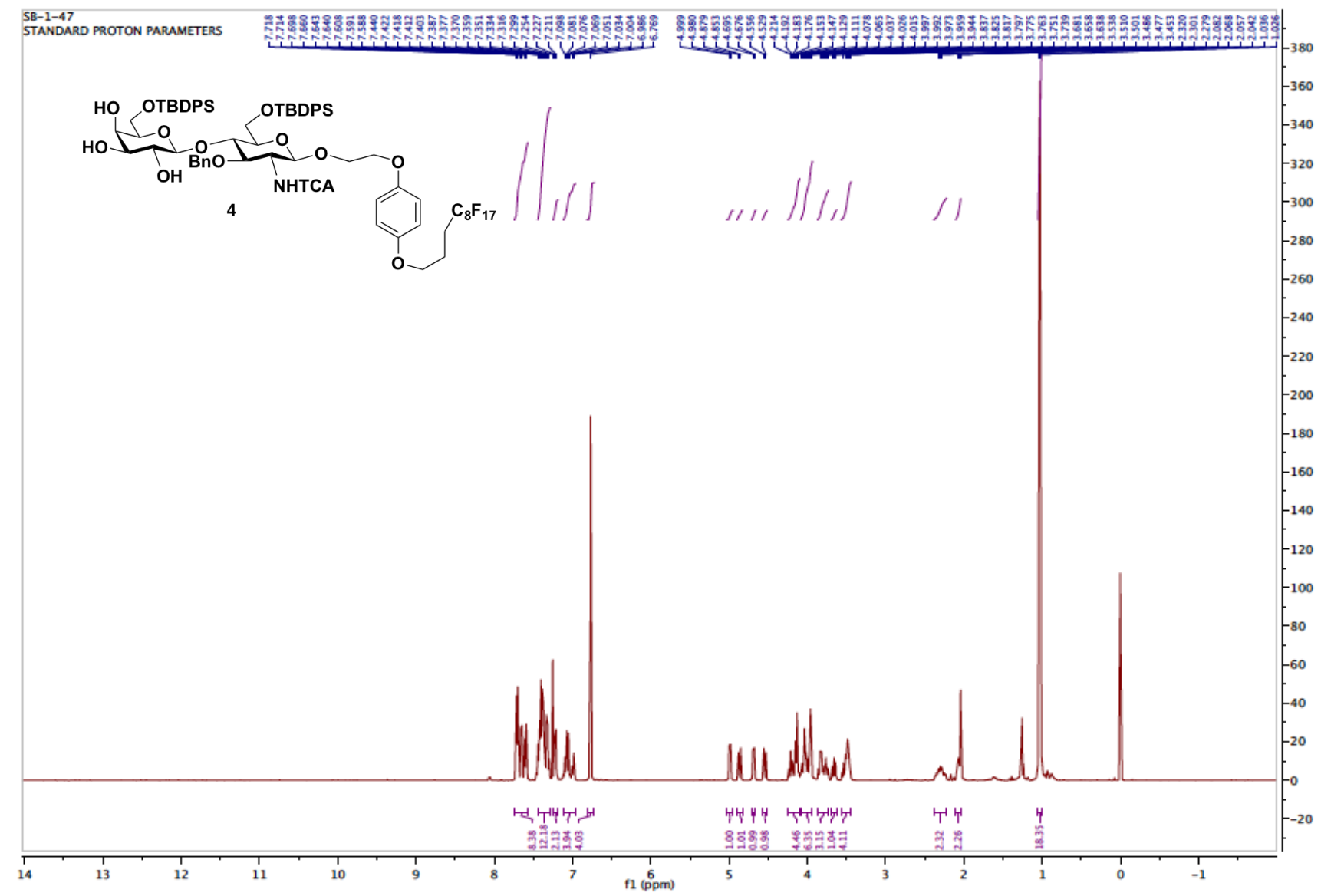

${ }^{1} \mathbf{H}$ NMR (400 MHz) spectrum of compound 4 in $\mathrm{CDCl}_{3}$ 


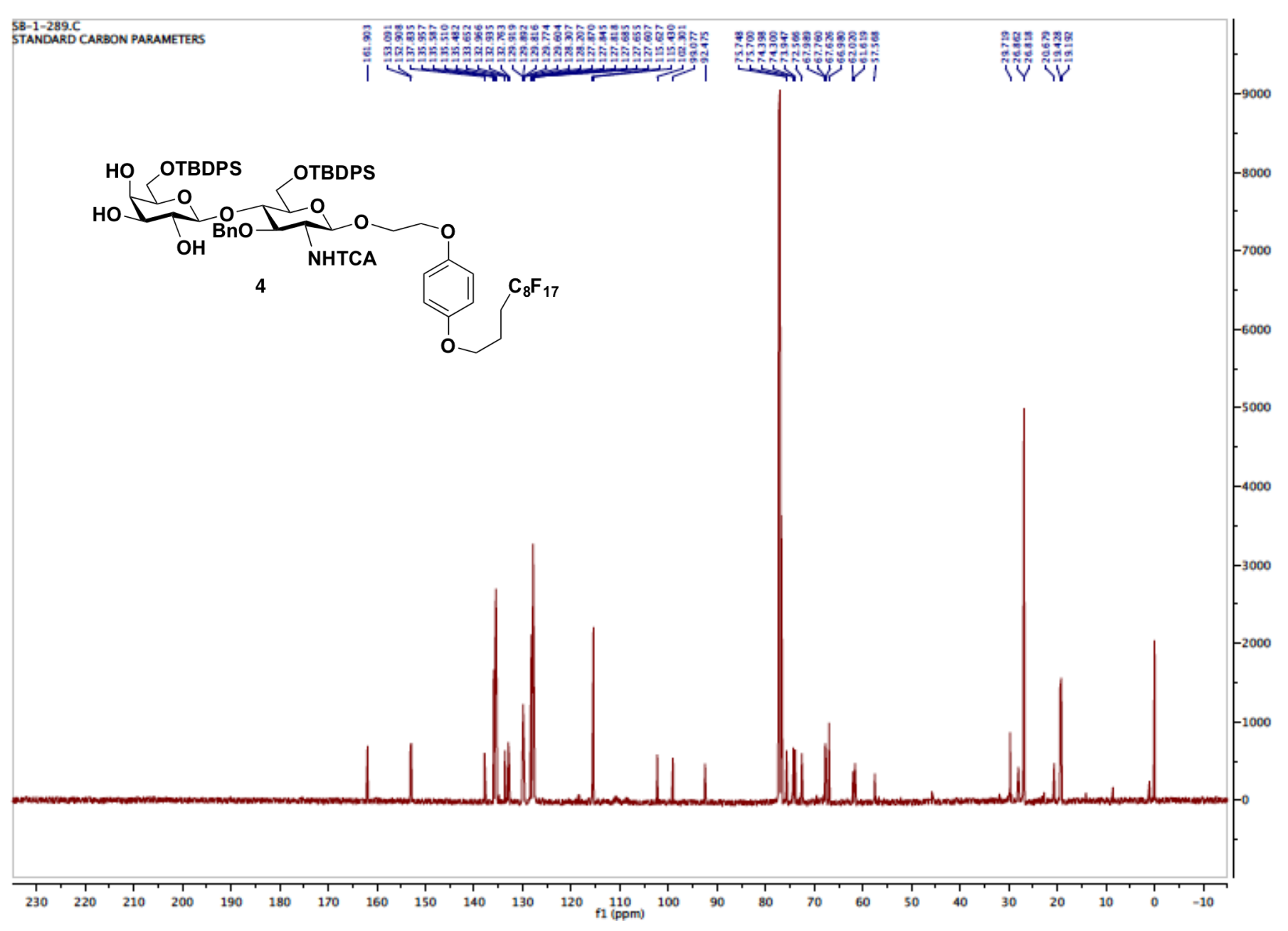

${ }^{13} \mathbf{C}$ NMR (101 MHz) spectrum of compound 4 in $\mathrm{CDCl}_{3}$ 


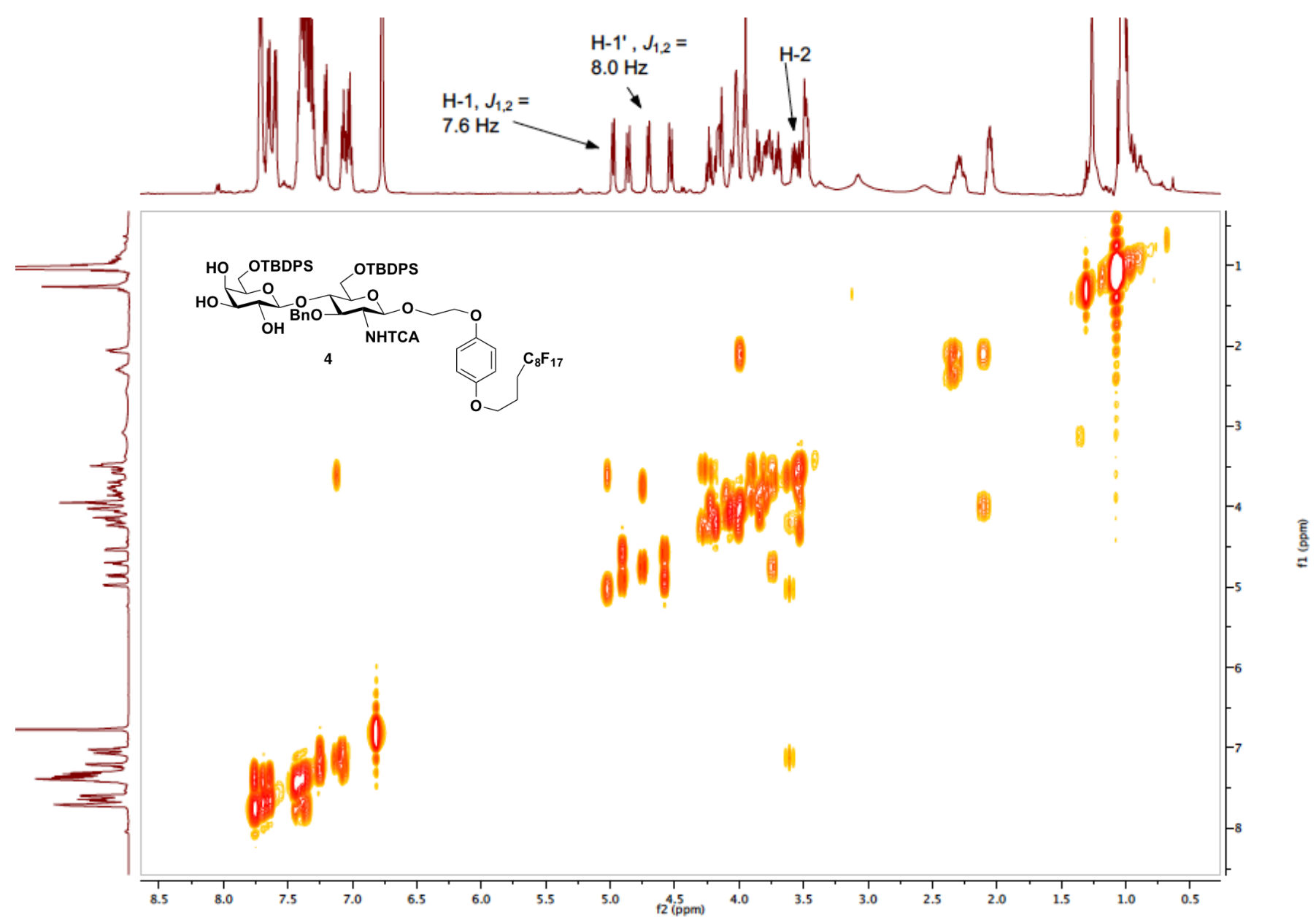

${ }^{1} \mathbf{H}$ - ${ }^{1} \mathbf{H}$ COSY NMR (400 MHz) spectrum of compound $\mathbf{4}$ in $\mathrm{CDCl}_{3}$ 


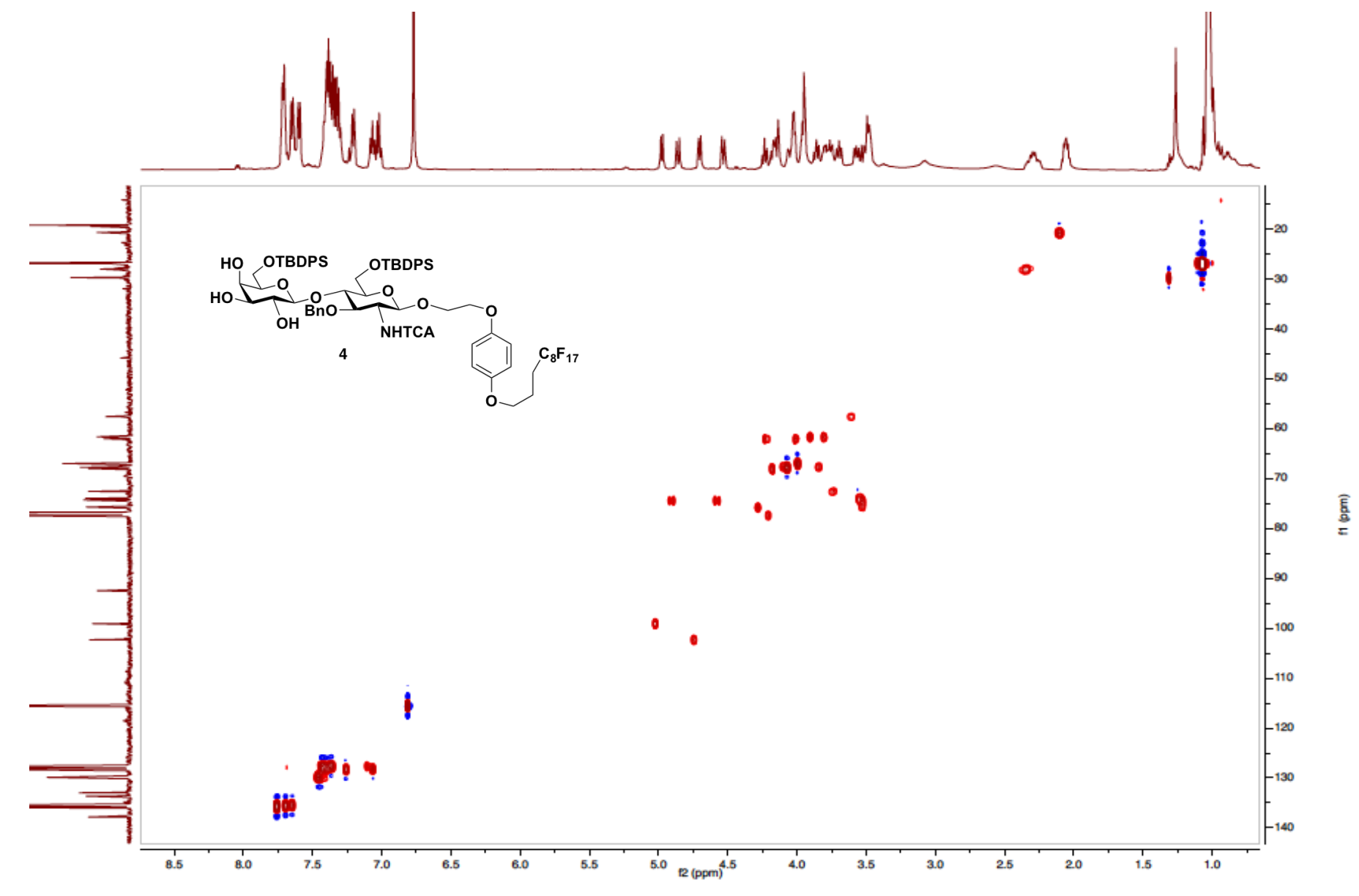

${ }^{1} \mathbf{H}-{ }^{13} \mathbf{C}$ HSQC NMR (400 MHz) spectrum of compound $\mathbf{4}$ in $\mathrm{CDCl}_{3}$ 


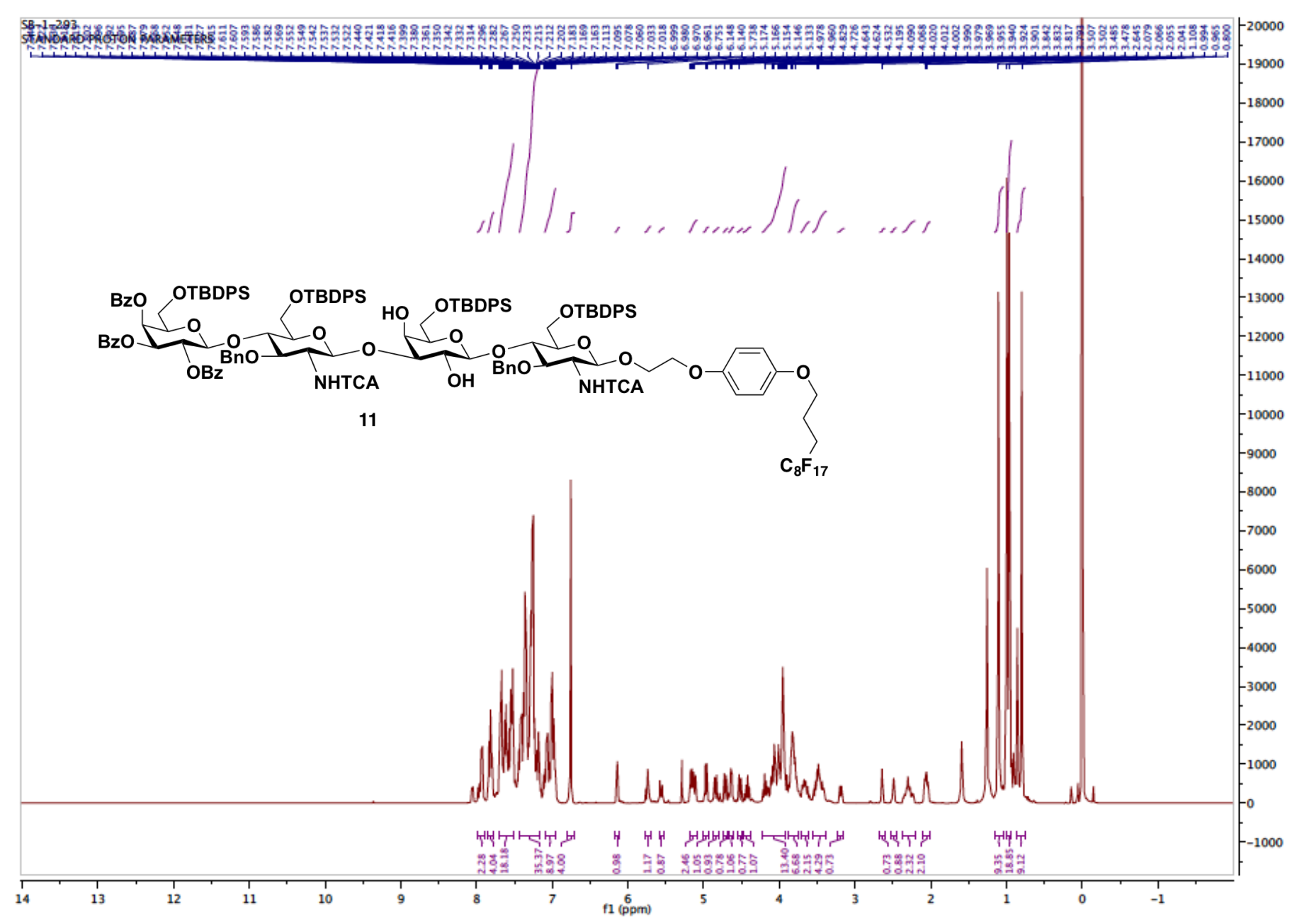

${ }^{1} \mathbf{H}$ NMR (400 MHz) spectrum of compound 11 in $\mathrm{CDCl}_{3}$ 


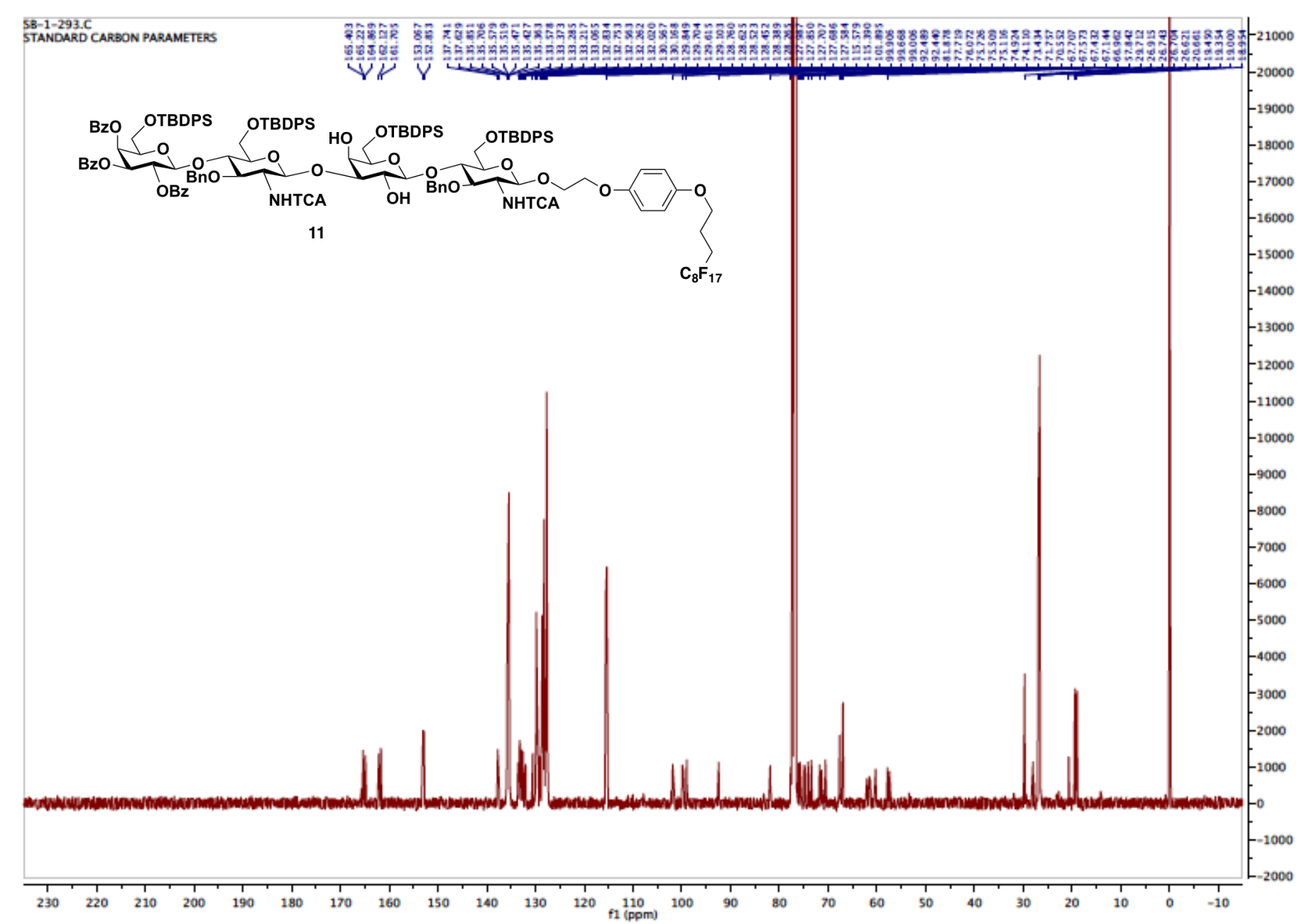

${ }^{13} \mathbf{C}$ NMR (101 MHz) spectrum of compound 11 in $\mathrm{CDCl}_{3}$ 


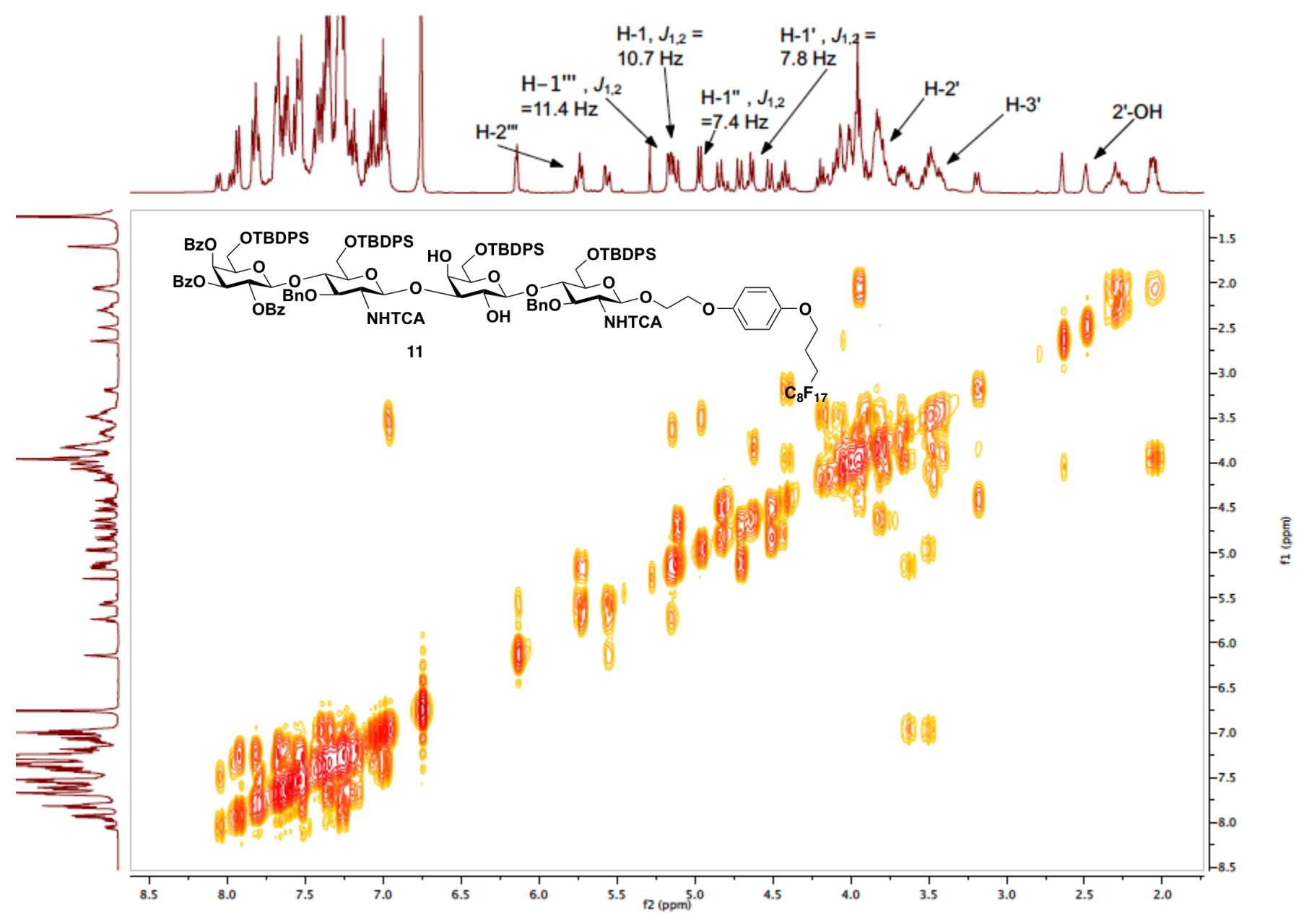

${ }^{1} \mathbf{H}$ - ${ }^{1} \mathbf{H}$ COSY NMR (400 MHz) spectrum of compound 11 in $\mathrm{CDCl}_{3}$ 


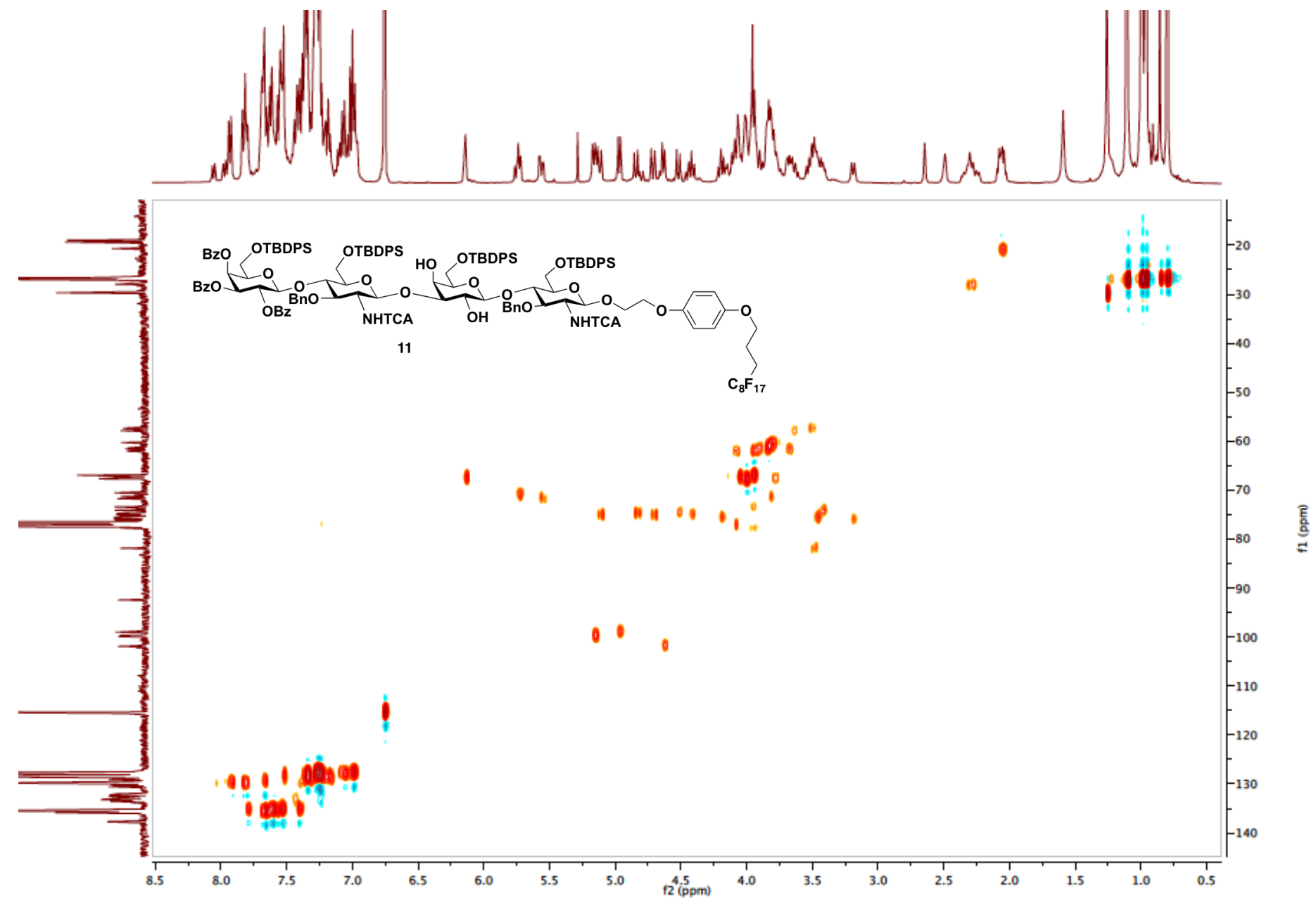

${ }^{1} \mathbf{H}-{ }^{13} \mathbf{C}$ HSQC NMR (400 MHz) spectrum of compound $\mathbf{1 1}$ in $\mathrm{CDCl}_{3}$ 


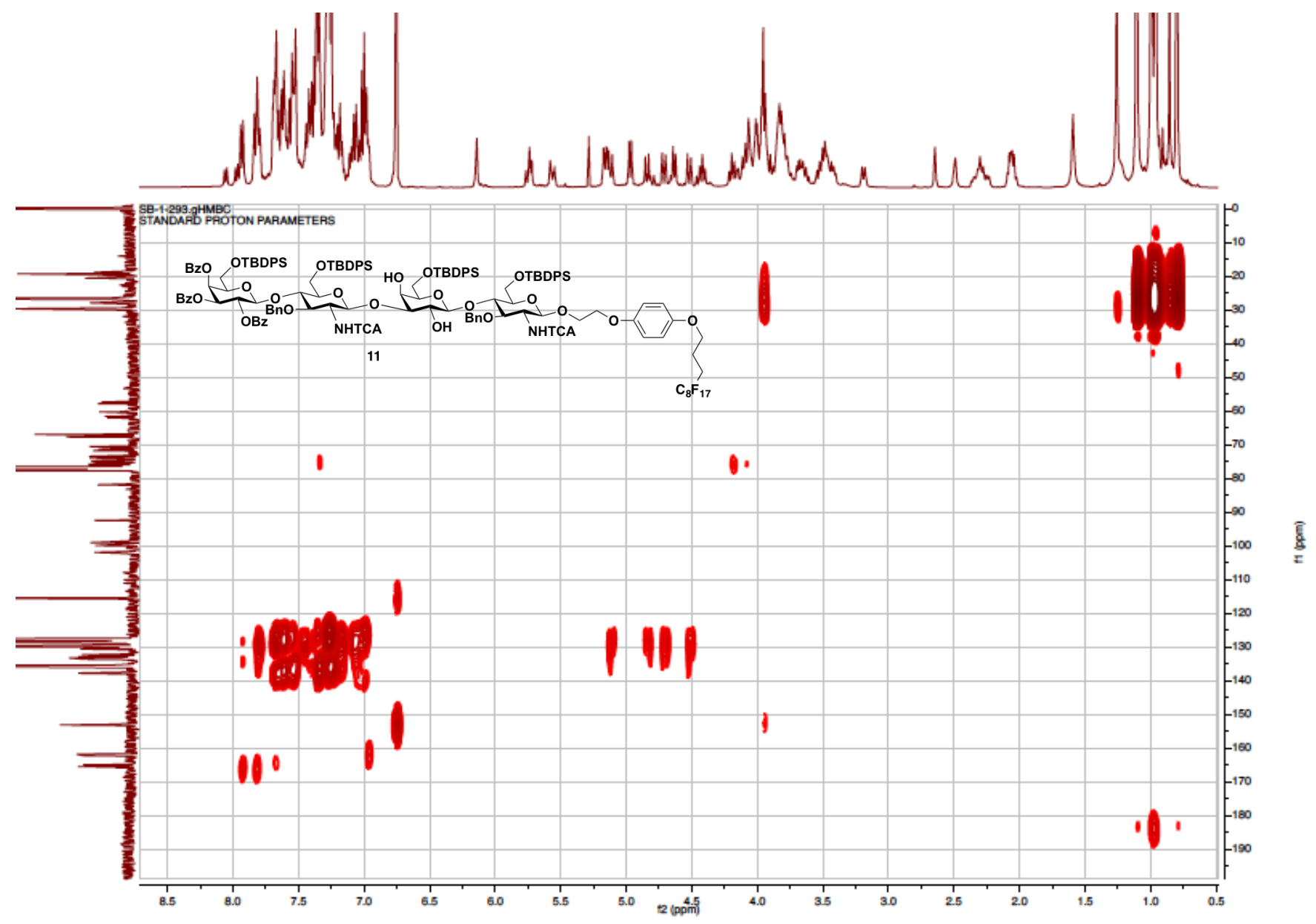

${ }^{1} \mathbf{H}-{ }^{13} \mathbf{C}$ HMBC NMR (400 MHz) spectrum of compound $\mathbf{1 1}$ in $\mathrm{CDCl}_{3}$ 


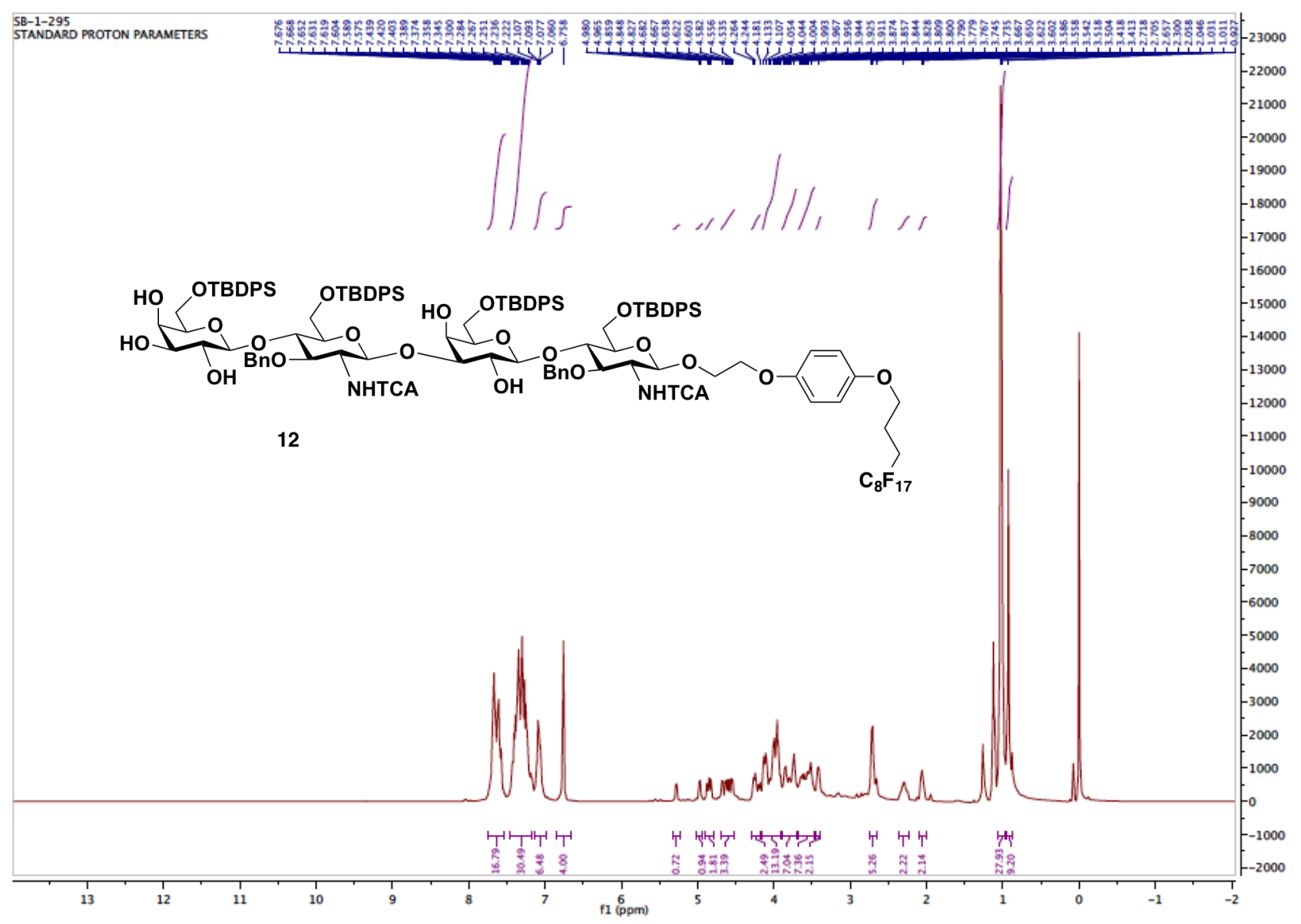

${ }^{1} \mathbf{H}$ NMR (400 MHz) spectrum of compound 12 in $\mathrm{CDCl}_{3}$ 


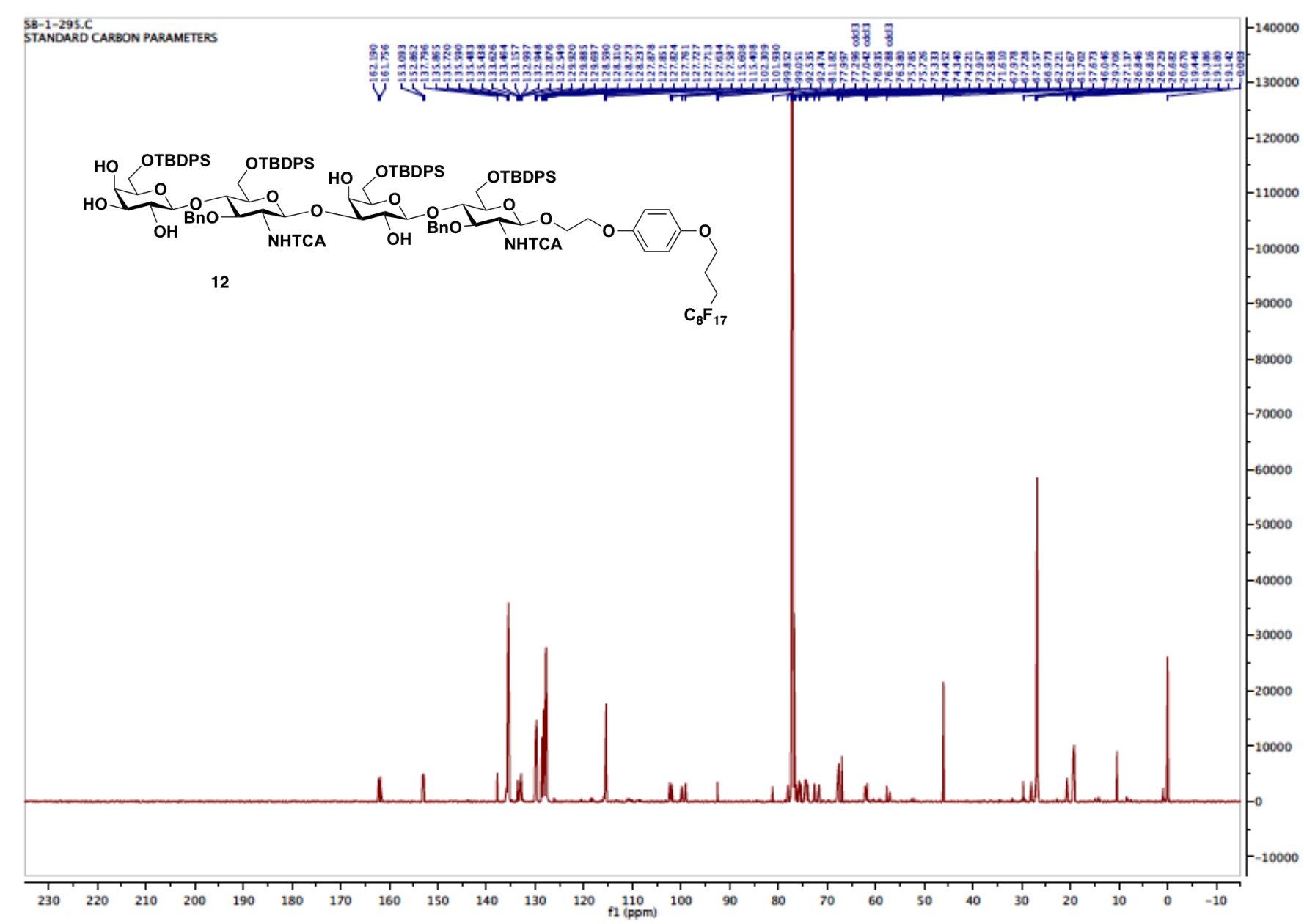

${ }^{13} \mathbf{C}$ NMR (101 MHz) spectrum of compound 12 in $\mathrm{CDCl}_{3}$ 


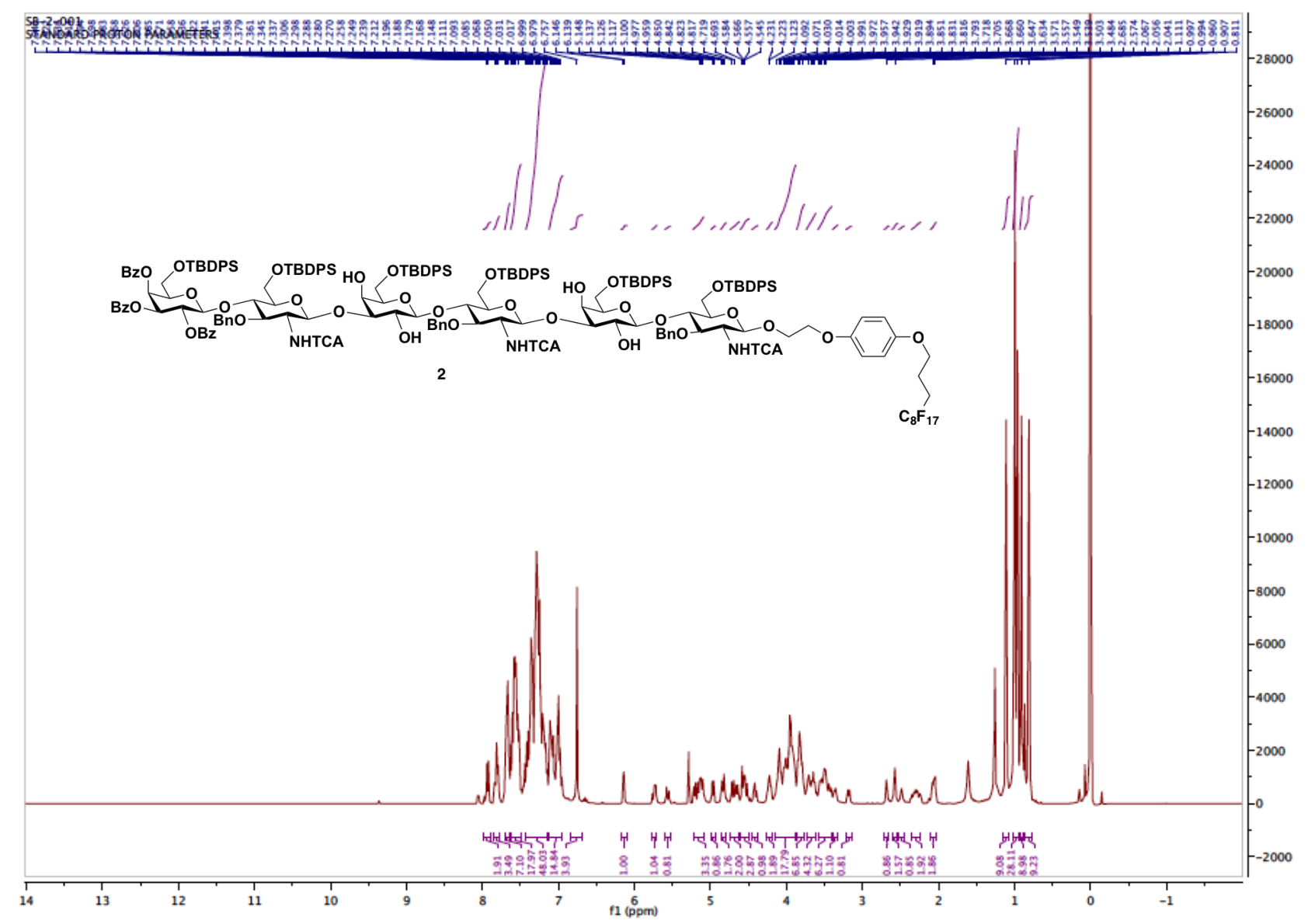

${ }^{1} \mathbf{H}$ NMR (400 MHz) spectrum of compound $\mathbf{2}$ in $\mathrm{CDCl}_{3}$ 


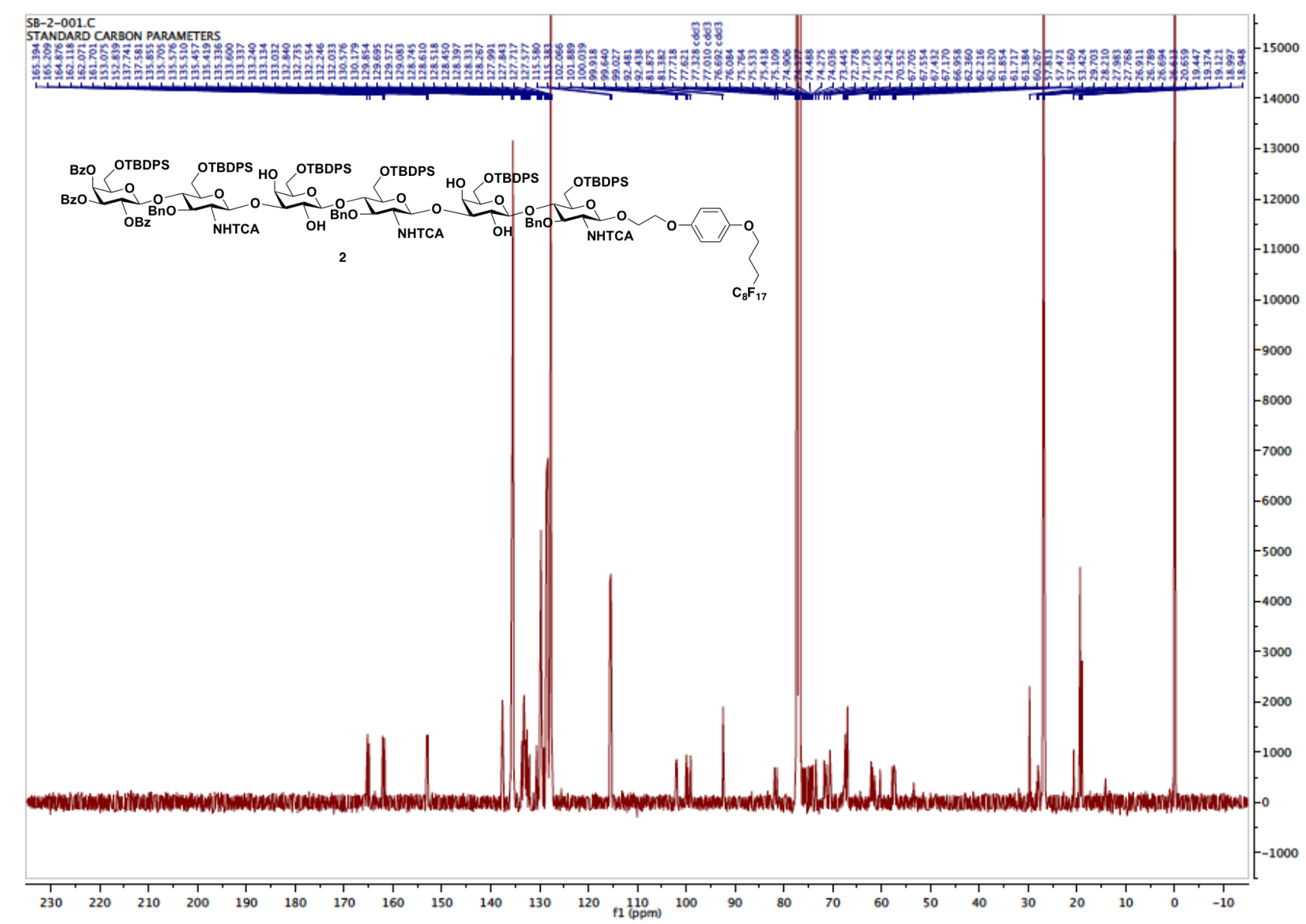

${ }^{13} \mathbf{C}$ NMR (101 MHz) spectrum of compound 2 in $\mathrm{CDCl}_{3}$ 


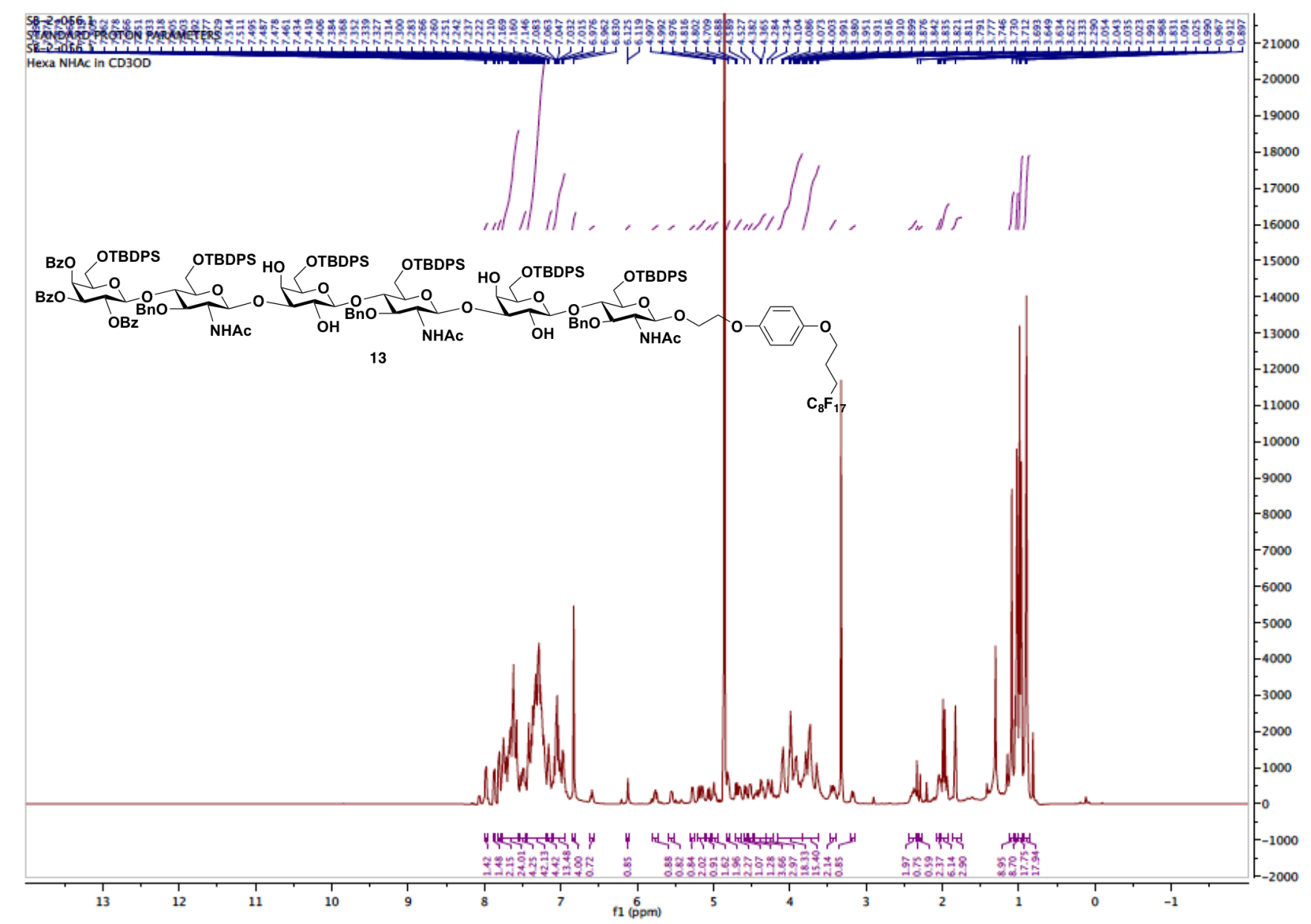

${ }^{1} \mathbf{H}$ NMR (500 MHz) spectrum of compound 13 in $\mathrm{CD}_{3} \mathrm{OD}$ 


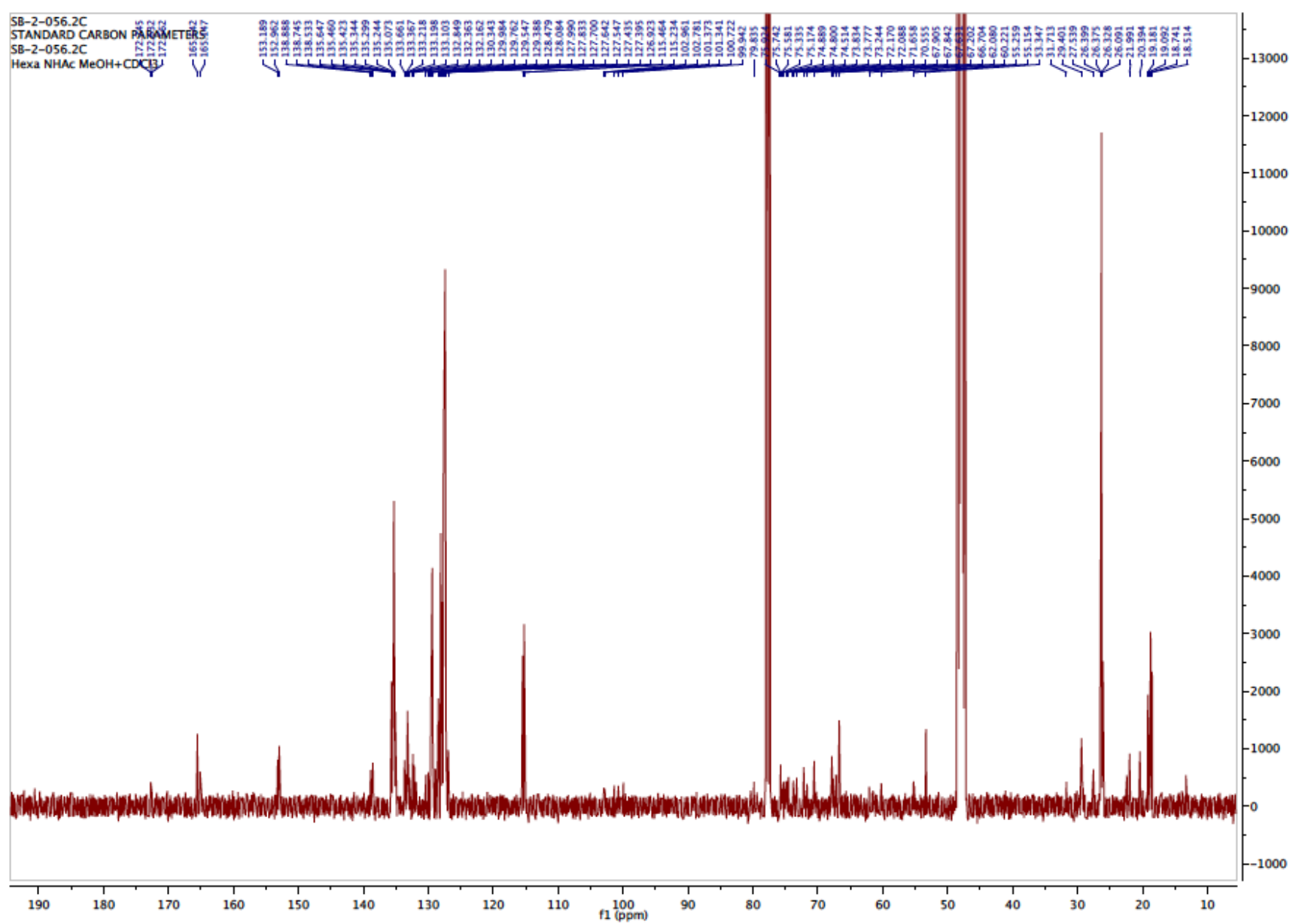

${ }^{13} \mathrm{C}$ NMR (126 MHz) spectrum of compound 13 in $\mathrm{CD}_{3} \mathrm{OD}$ 


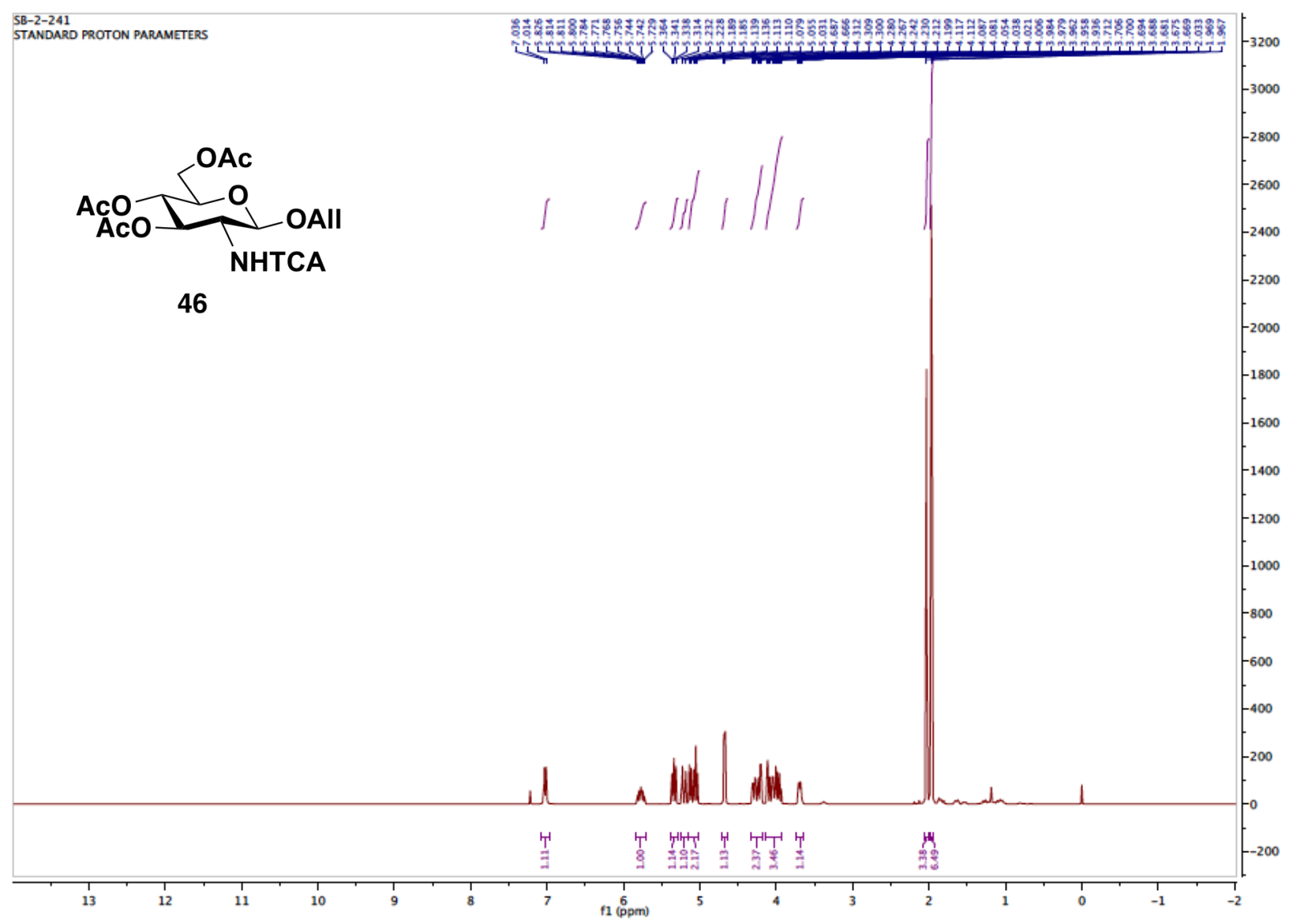

${ }^{1} \mathbf{H}$ NMR (400 MHz) spectrum of compound 46 in $\mathrm{CDCl}_{3}$ 


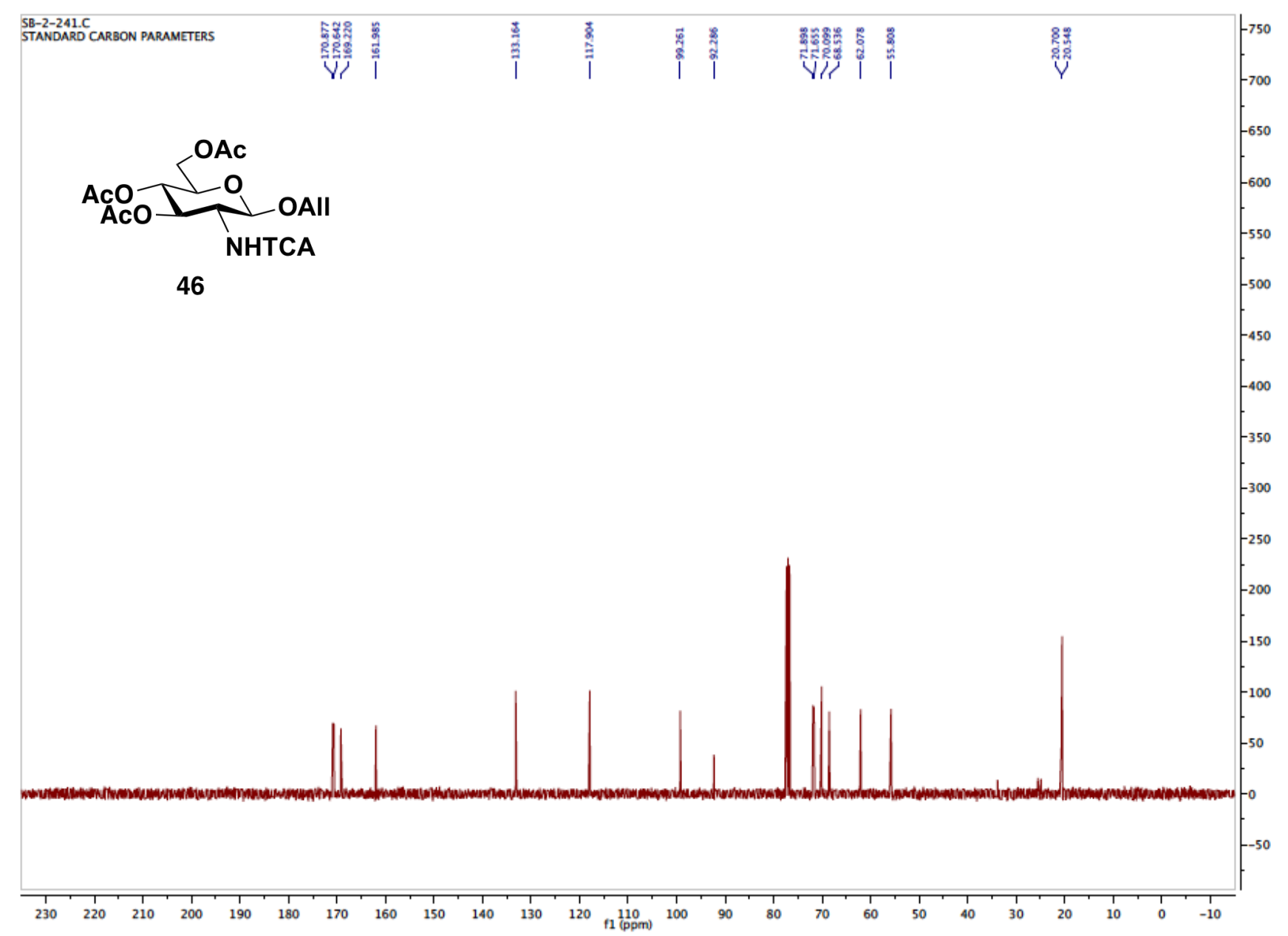

${ }^{13} \mathrm{C}$ NMR (101 MHz) spectrum of compound 46 in $\mathrm{CDCl}_{3}$ 


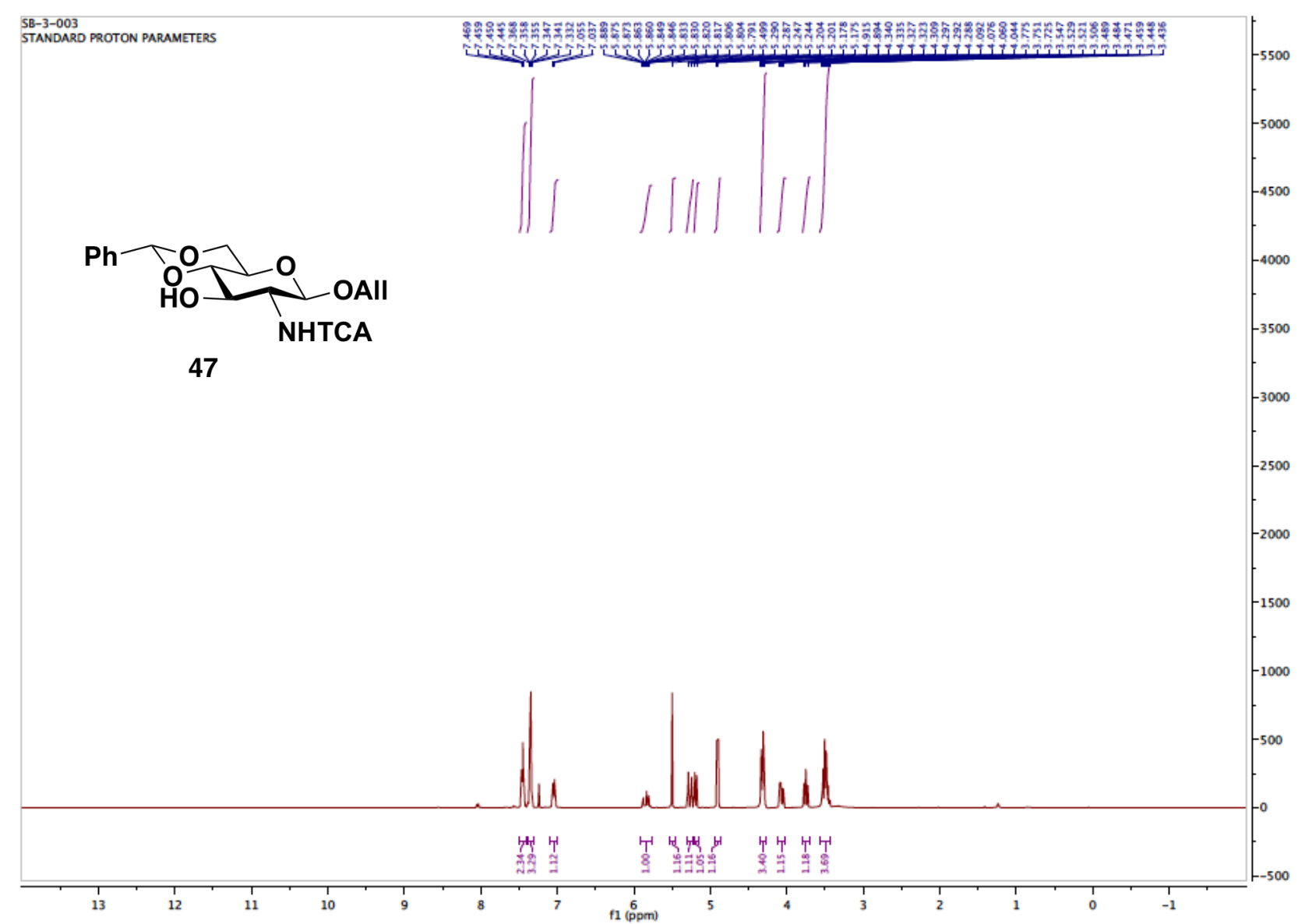

${ }^{1} \mathbf{H}$ NMR (400 MHz) spectrum of compound 47 in $\mathrm{CDCl}_{3}$ 


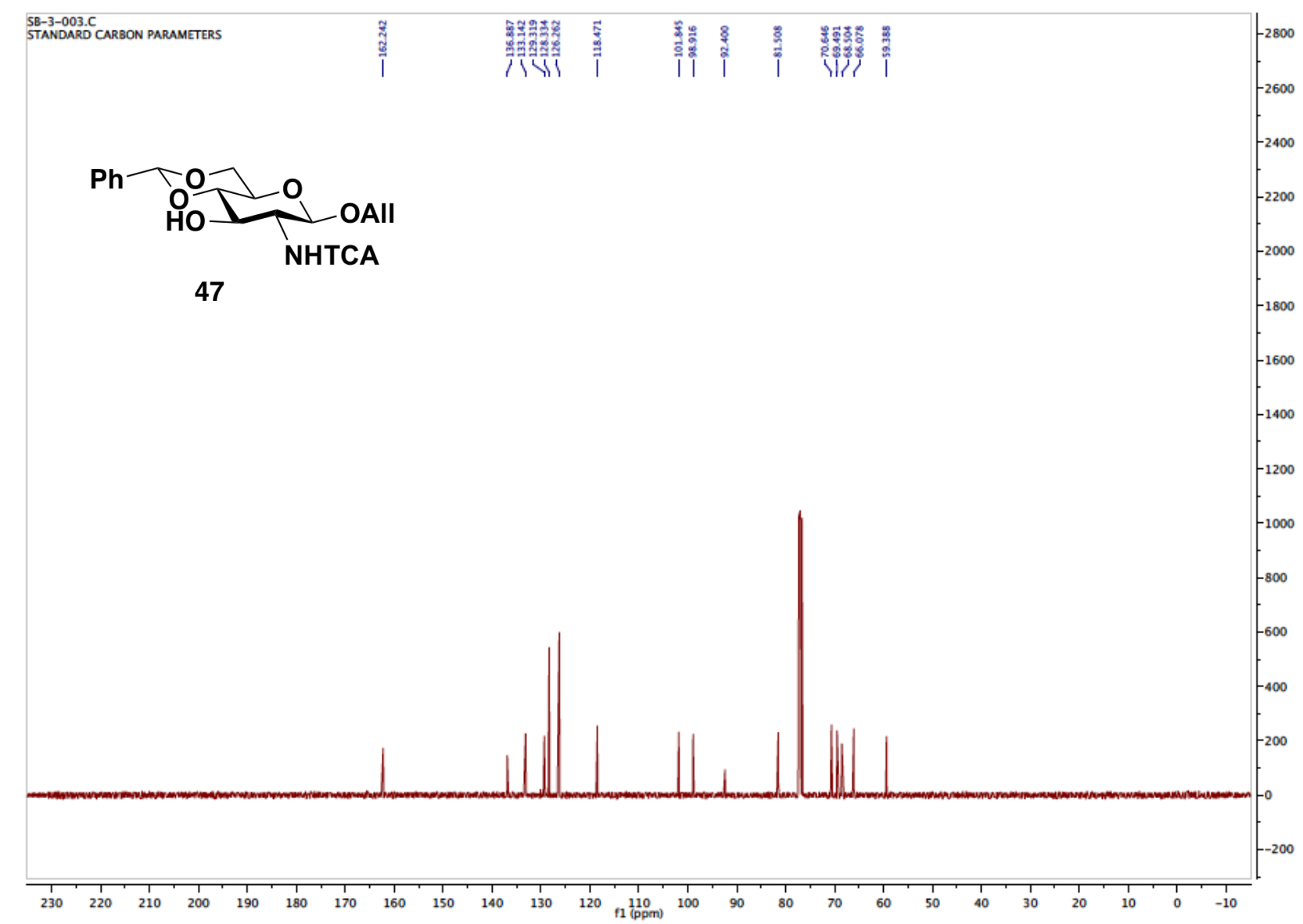

${ }^{13} \mathbf{C}$ NMR (101 MHz) spectrum of compound 47 in $\mathrm{CDCl}_{3}$ 


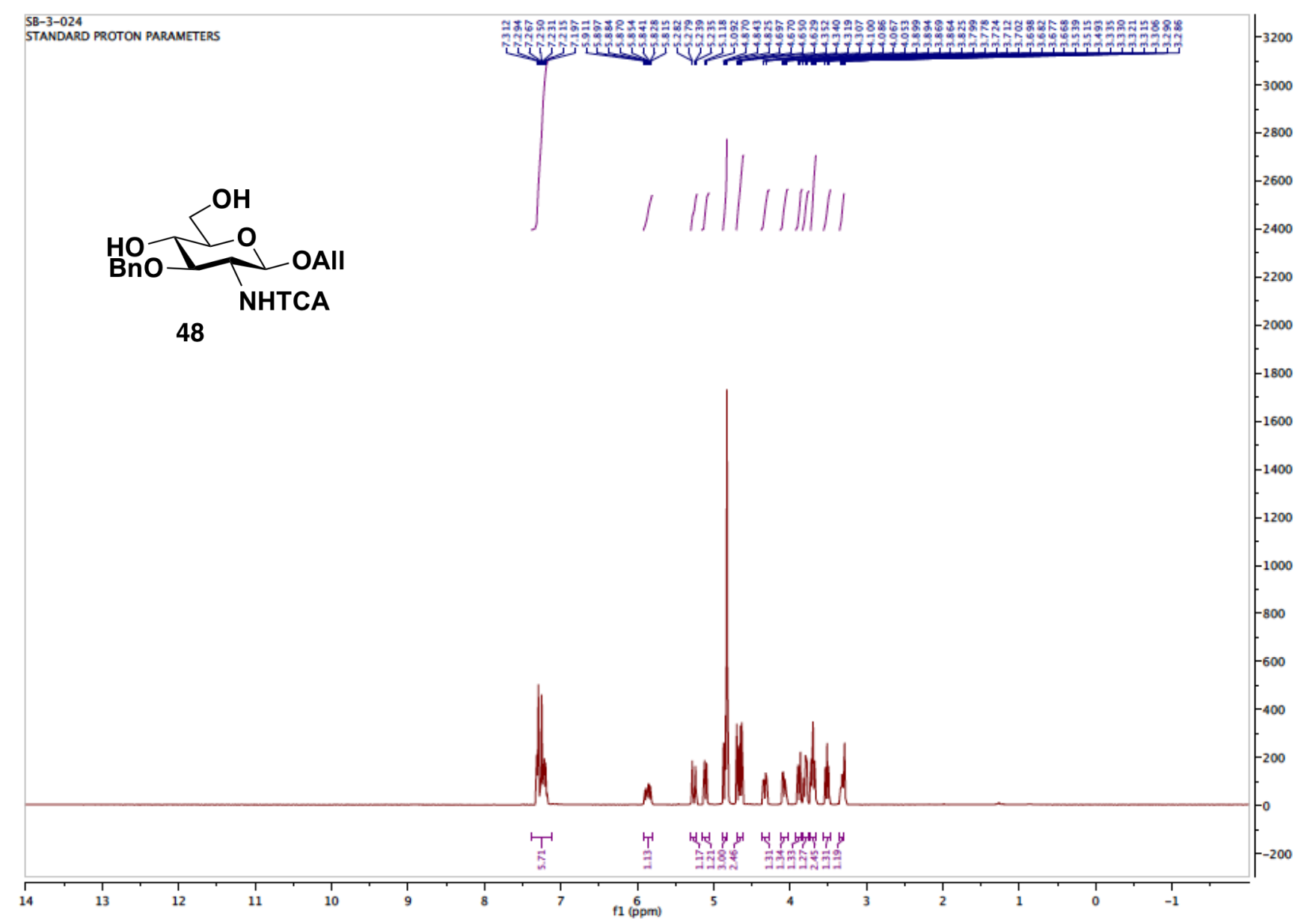

${ }^{1}$ H NMR (400 MHz) spectrum of compound 48 in $\mathrm{CD}_{3} \mathrm{OD}$ 


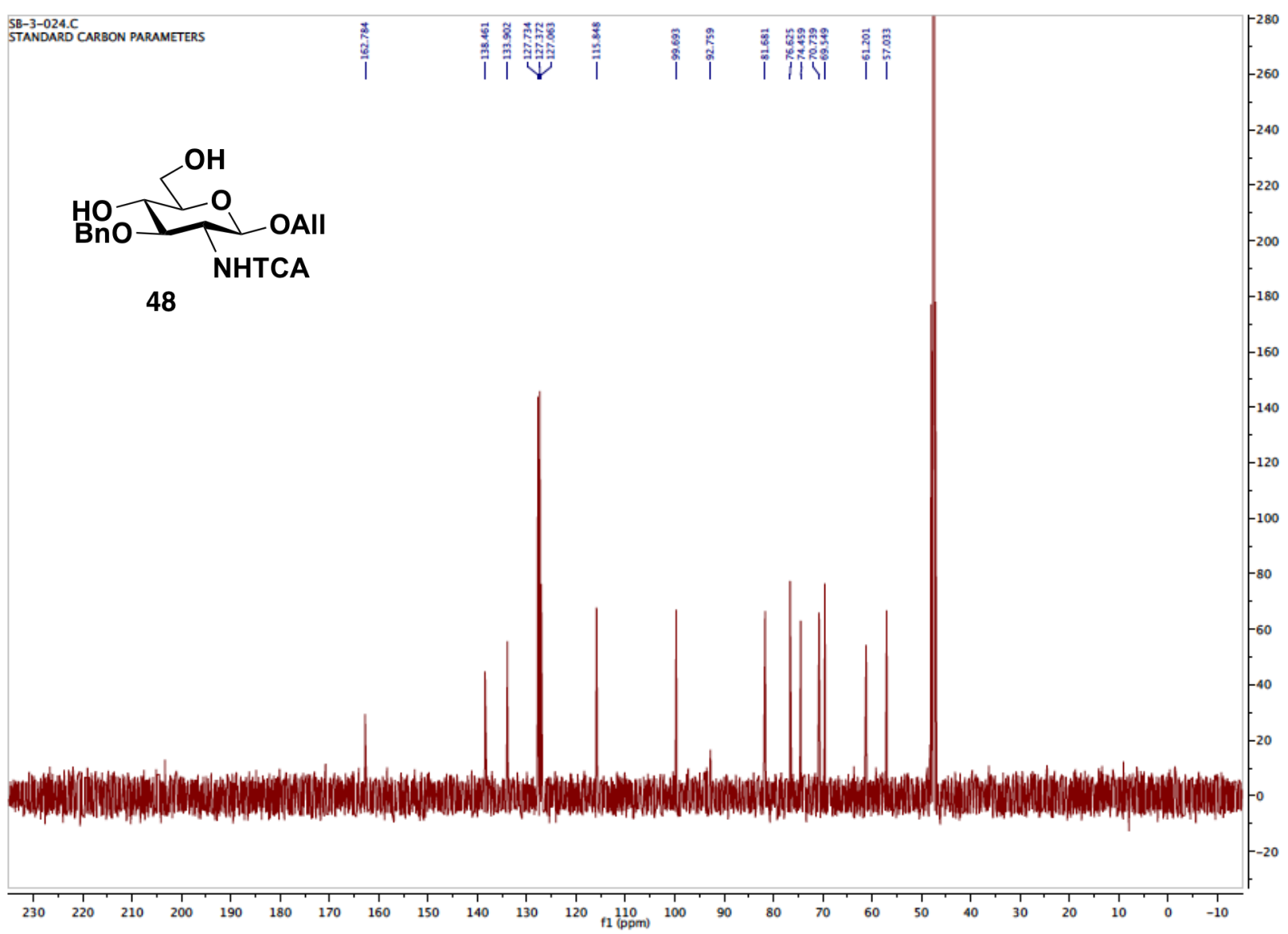

${ }^{13} \mathbf{C}$ NMR $(101 \mathrm{MHz})$ spectrum of compound 48 in $\mathrm{CD}_{3} \mathrm{OD}$ 


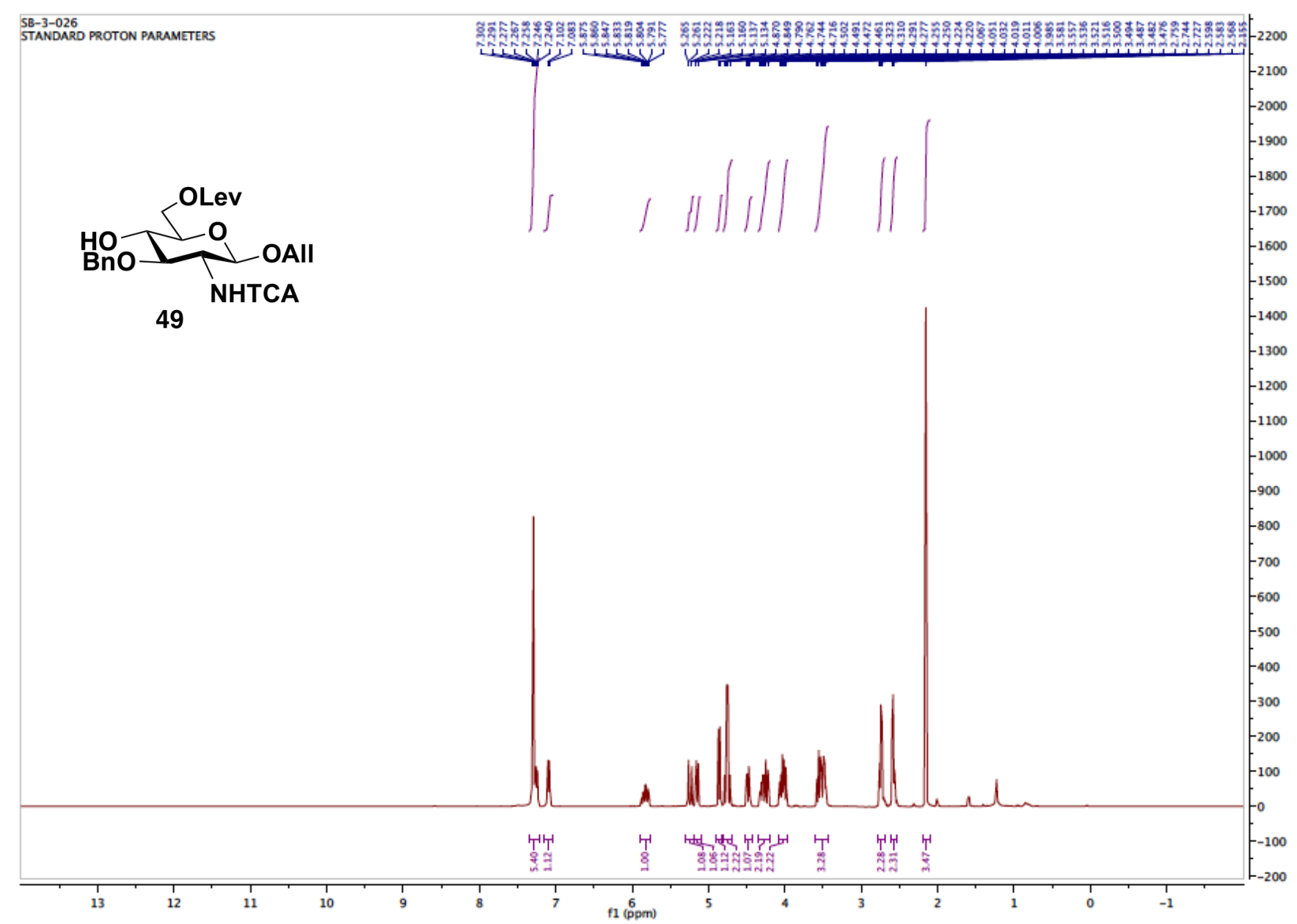

${ }^{1} \mathbf{H}$ NMR (400 MHz) spectrum of compound 49 in $\mathrm{CDCl}_{3}$ 


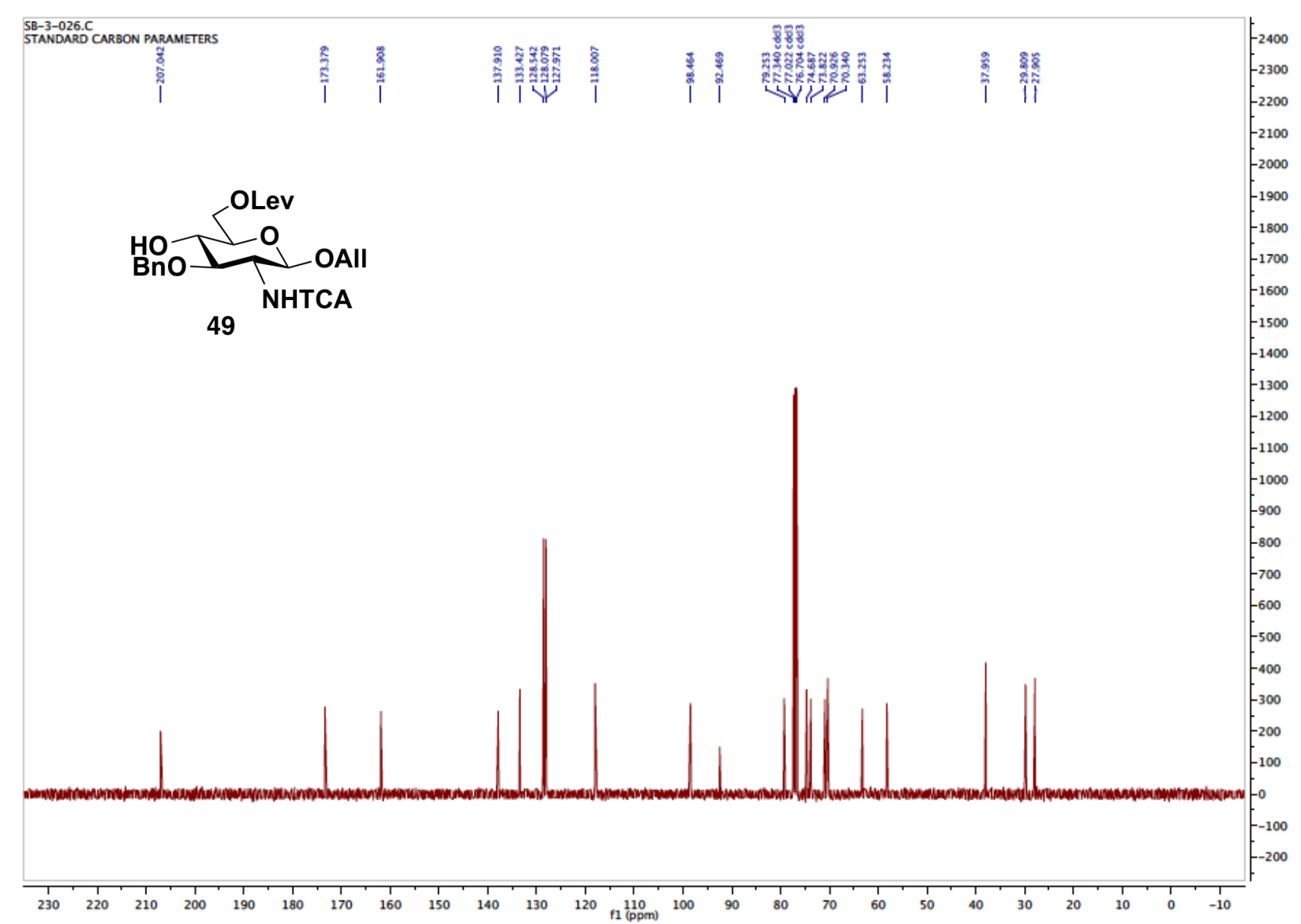

${ }^{13}$ C NMR (101 MHz) spectrum of compound 49 in $\mathrm{CDCl}_{3}$ 


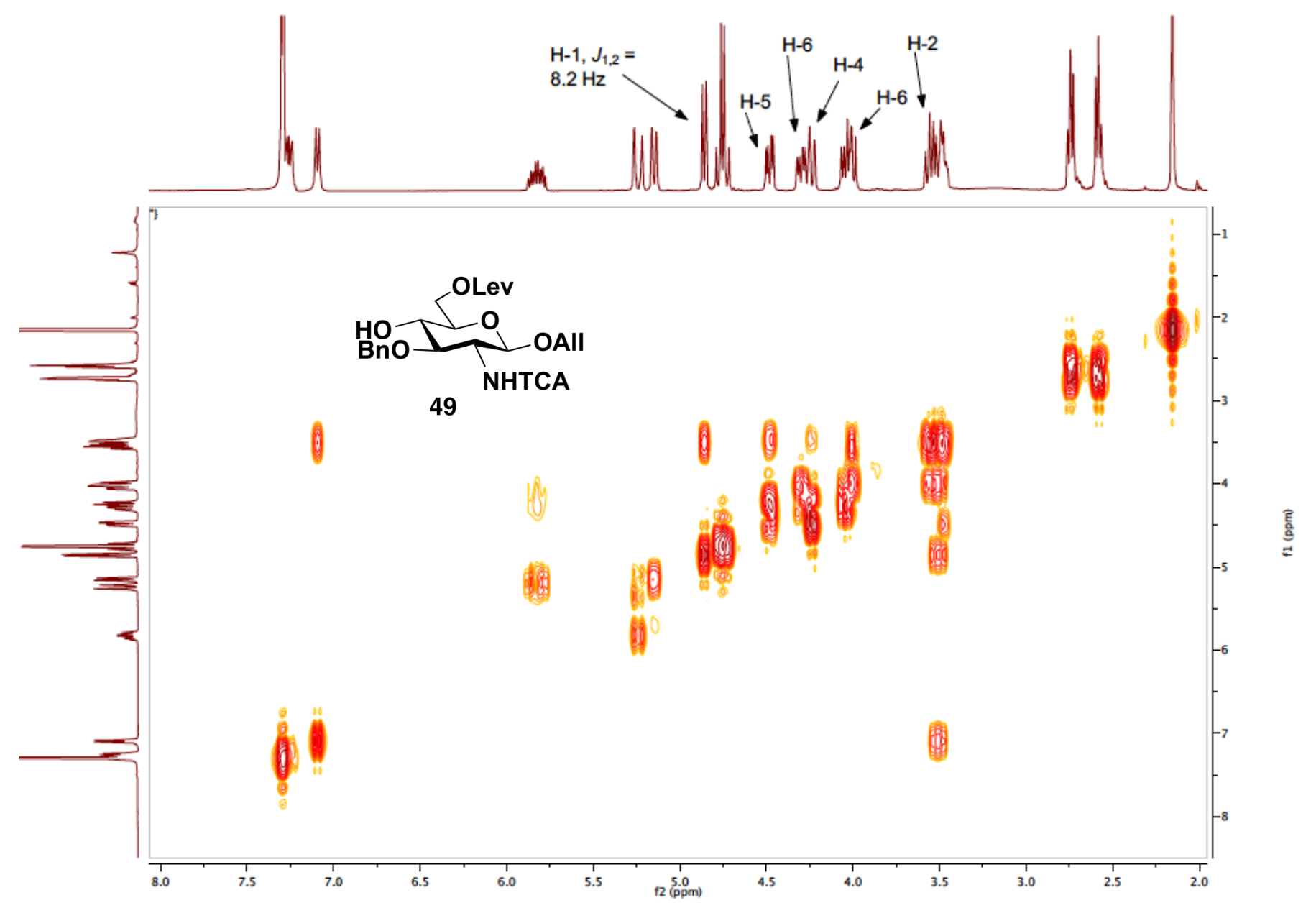

${ }^{1} \mathbf{H}$ - ${ }^{1} \mathbf{H}$ COSY NMR (400 MHz) spectrum of compound 49 in $\mathrm{CDCl}_{3}$ 


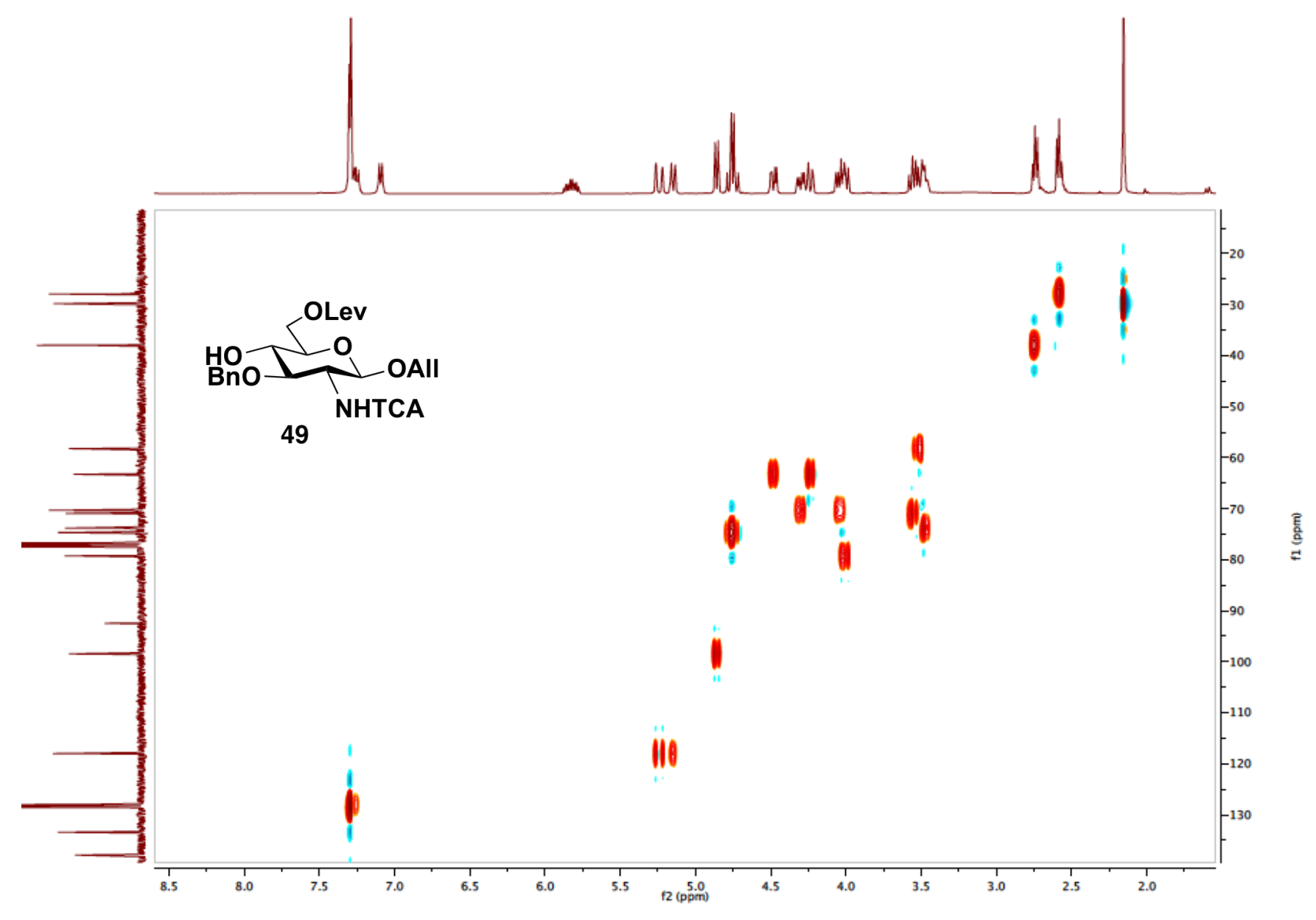

${ }^{1} \mathbf{H}-{ }^{13} \mathbf{C}$ HSQC NMR (400 MHz) spectrum of compound 49 in $\mathrm{CDCl}_{3}$ 


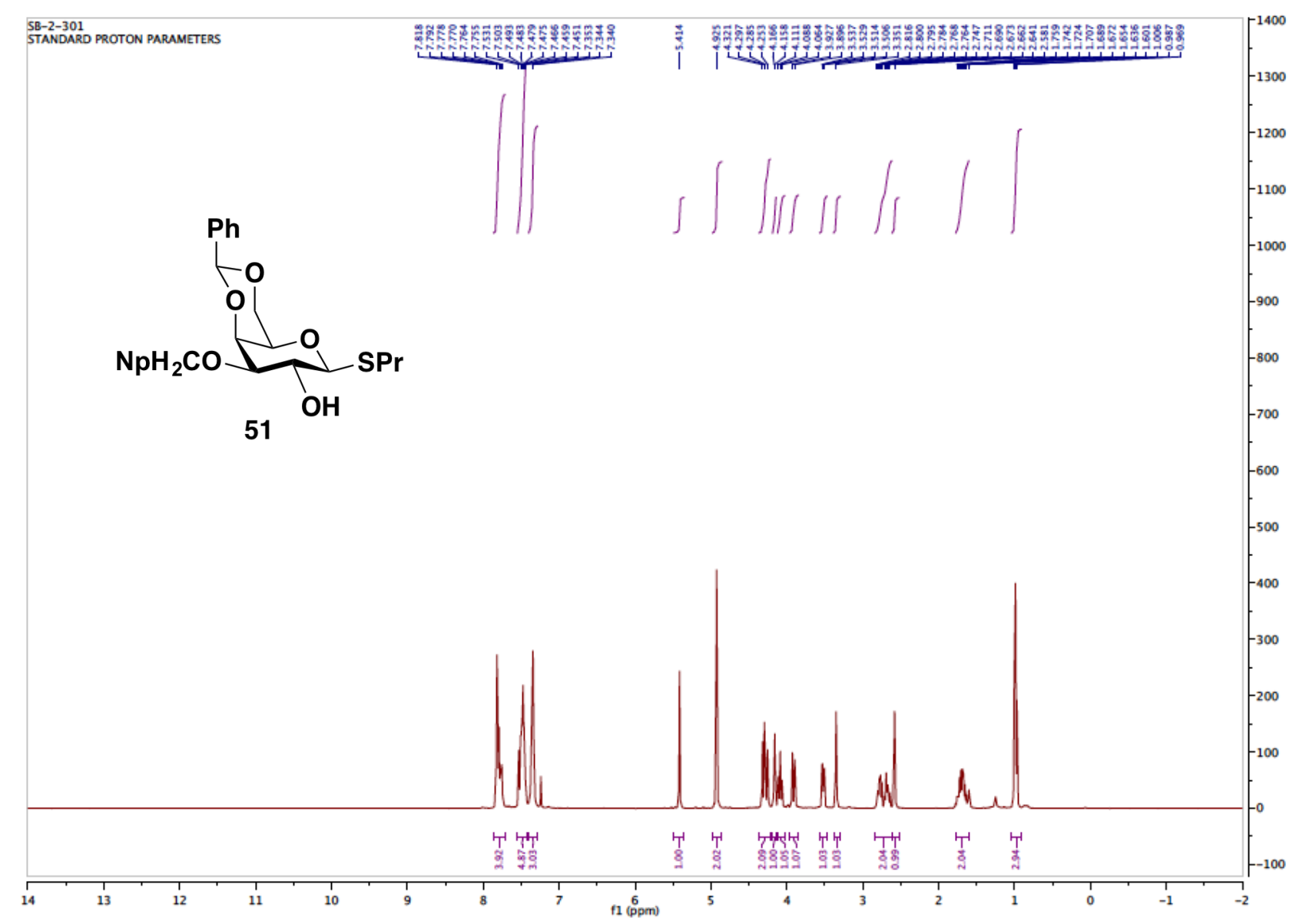

${ }^{1} \mathbf{H}$ NMR (400 MHz) spectrum of compound $\mathbf{5 1}$ in $\mathrm{CDCl}_{3}$ 


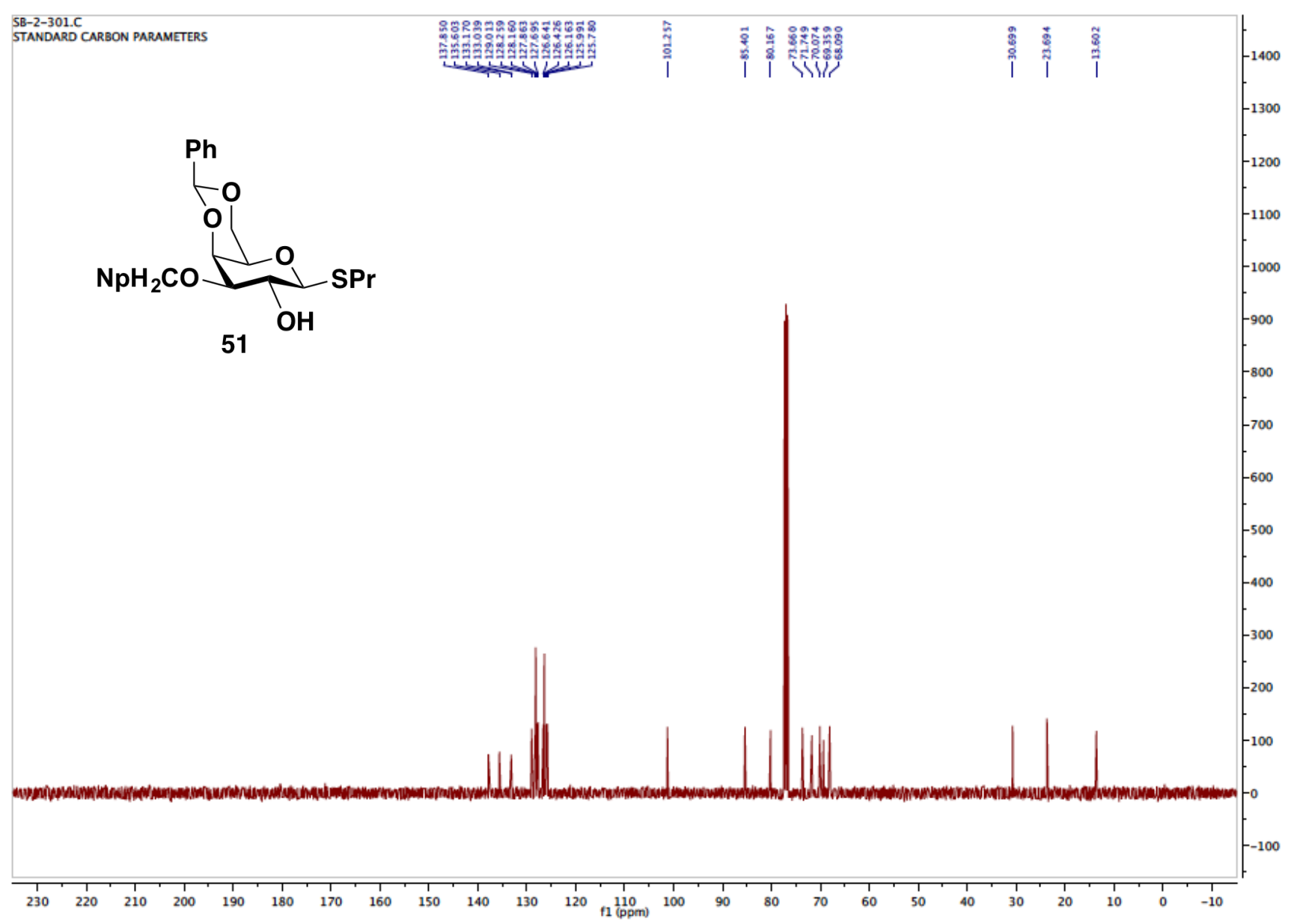

${ }^{13} \mathbf{C}$ NMR (101 MHz) spectrum of compound 51 in $\mathrm{CDCl}_{3}$ 


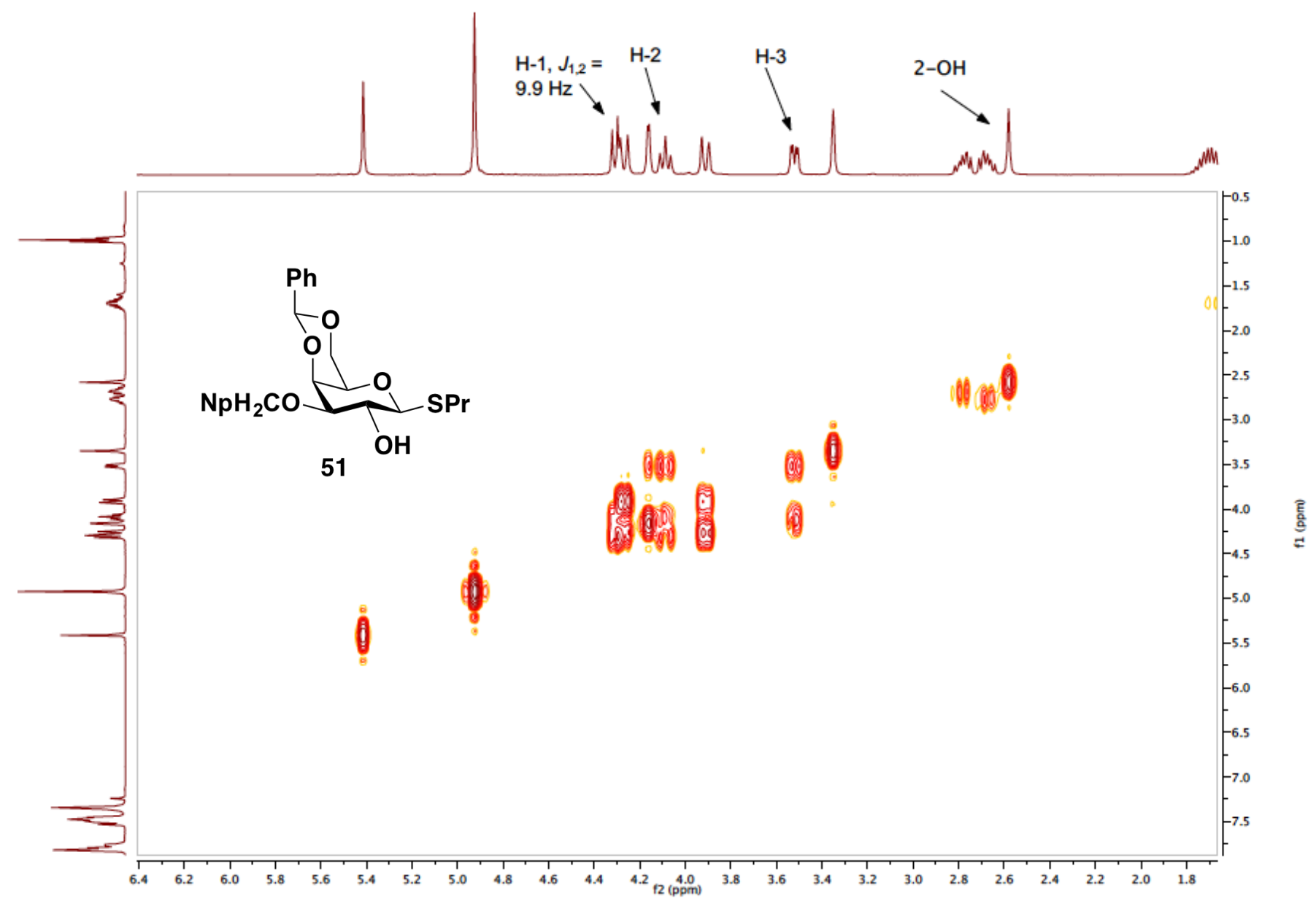

${ }^{1} \mathbf{H}$ - ${ }^{1} \mathbf{H}$ COSY NMR (400 MHz) spectrum of compound 51 in $\mathrm{CDCl}_{3}$ 


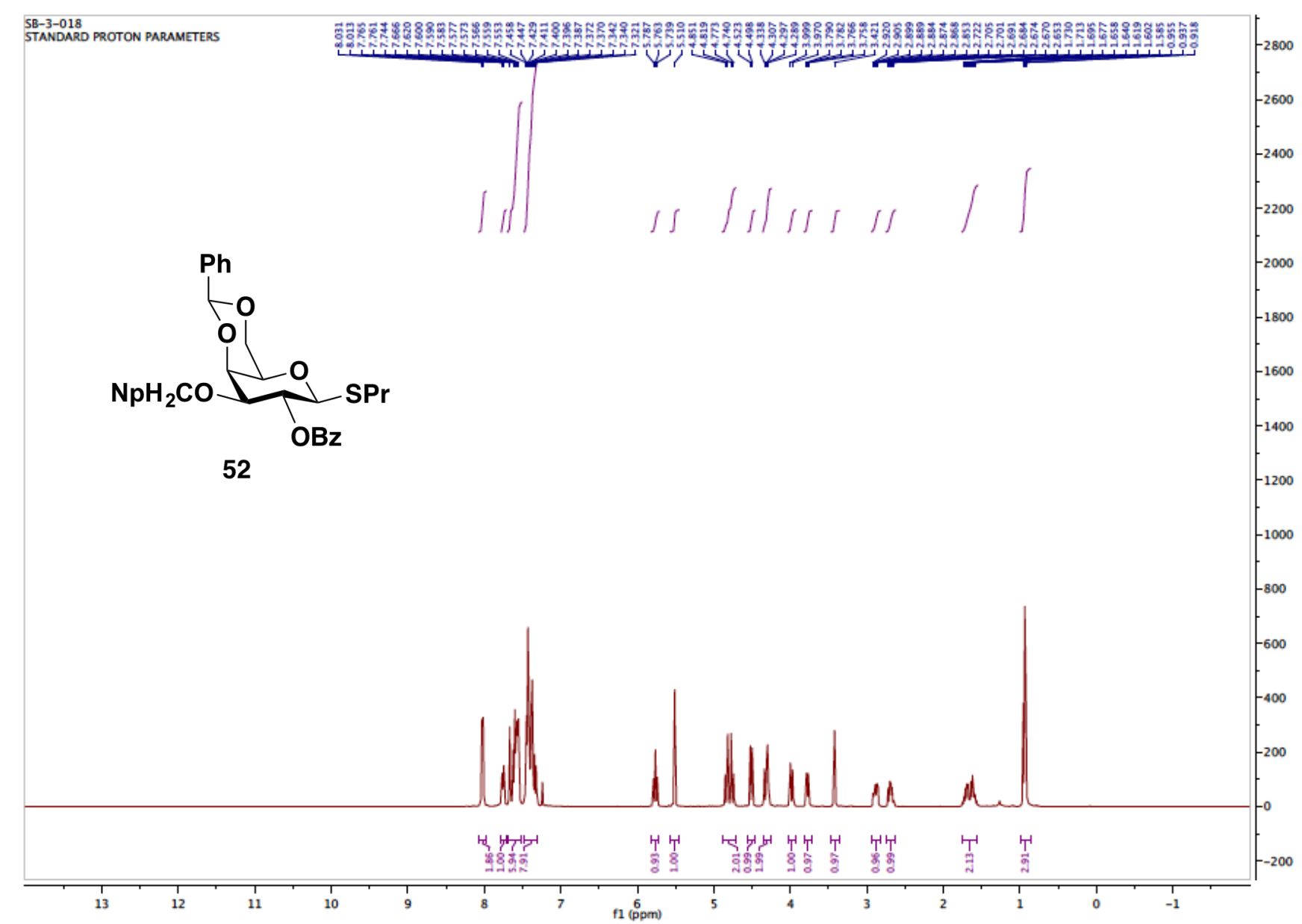

${ }^{1} \mathbf{H}$ NMR (400 MHz) spectrum of compound $\mathbf{5 2}$ in $\mathrm{CDCl}_{3}$ 


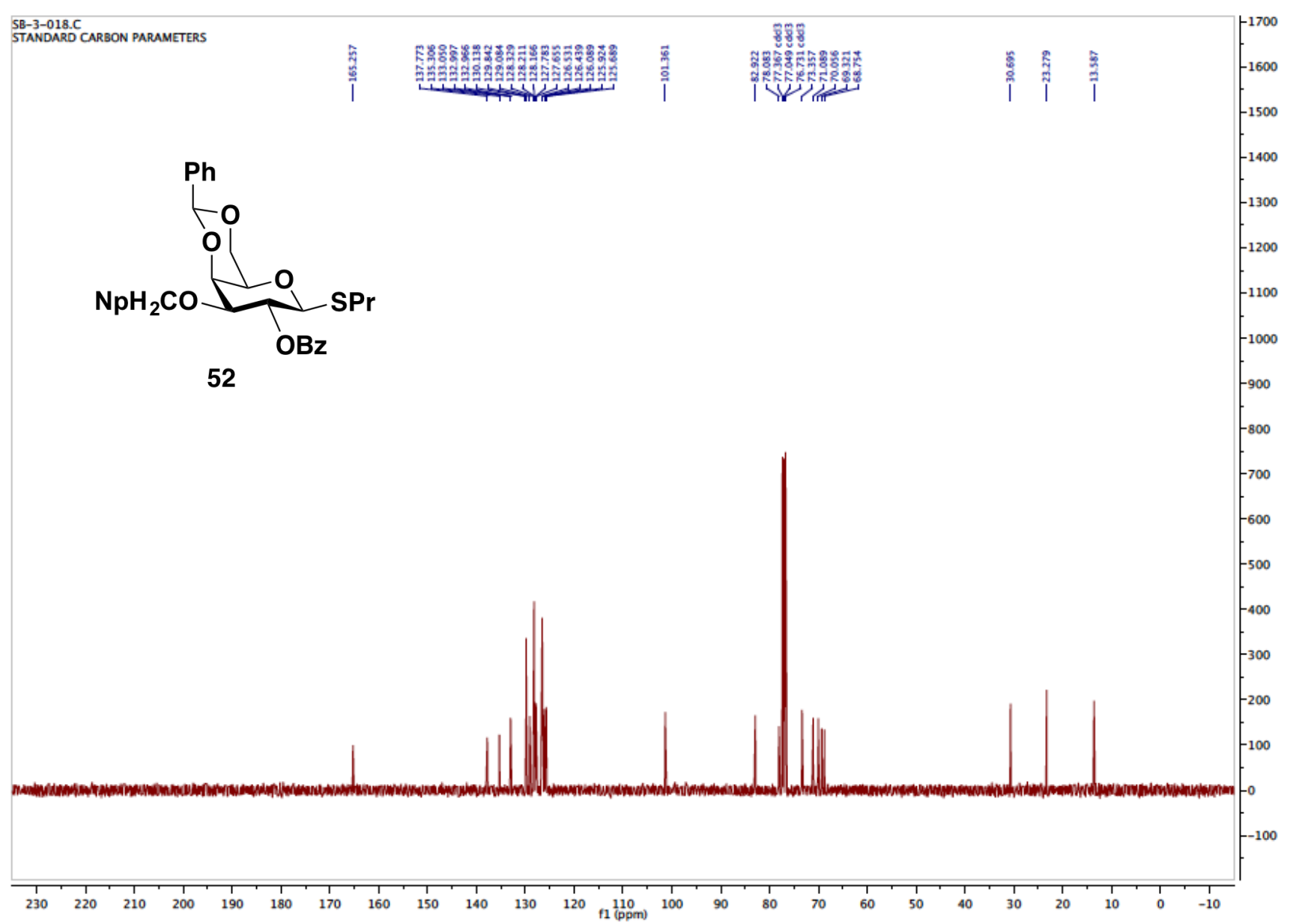

${ }^{13} \mathbf{C}$ NMR (101 MHz) spectrum of compound $\mathbf{5 2}$ in $\mathrm{CDCl}_{3}$ 


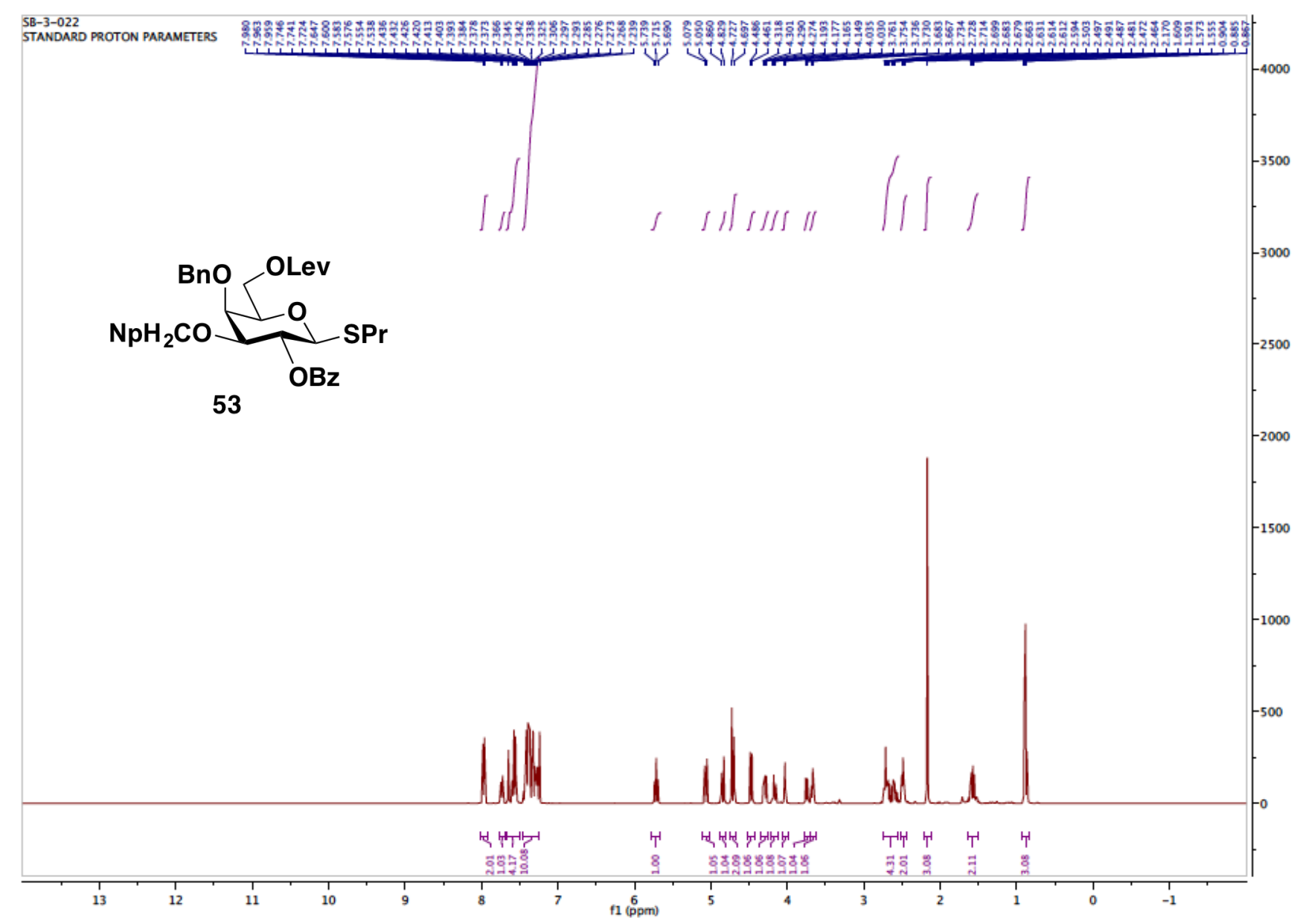

${ }^{1} \mathbf{H}$ NMR (400 MHz) spectrum of compound 53 in $\mathrm{CDCl}_{3}$ 


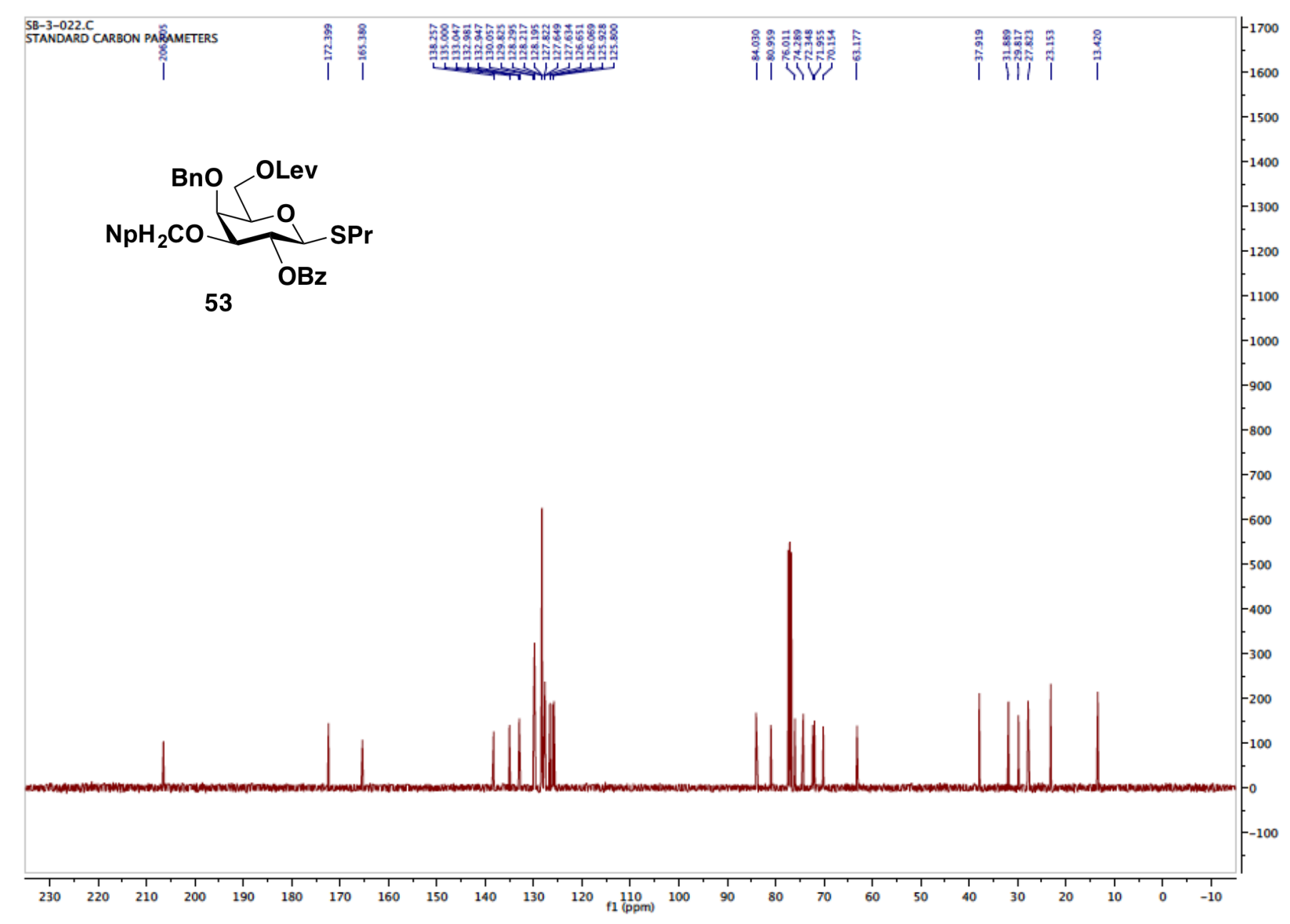

${ }^{13} \mathbf{C}$ NMR (101 MHz) spectrum of compound 53 in $\mathrm{CDCl}_{3}$ 


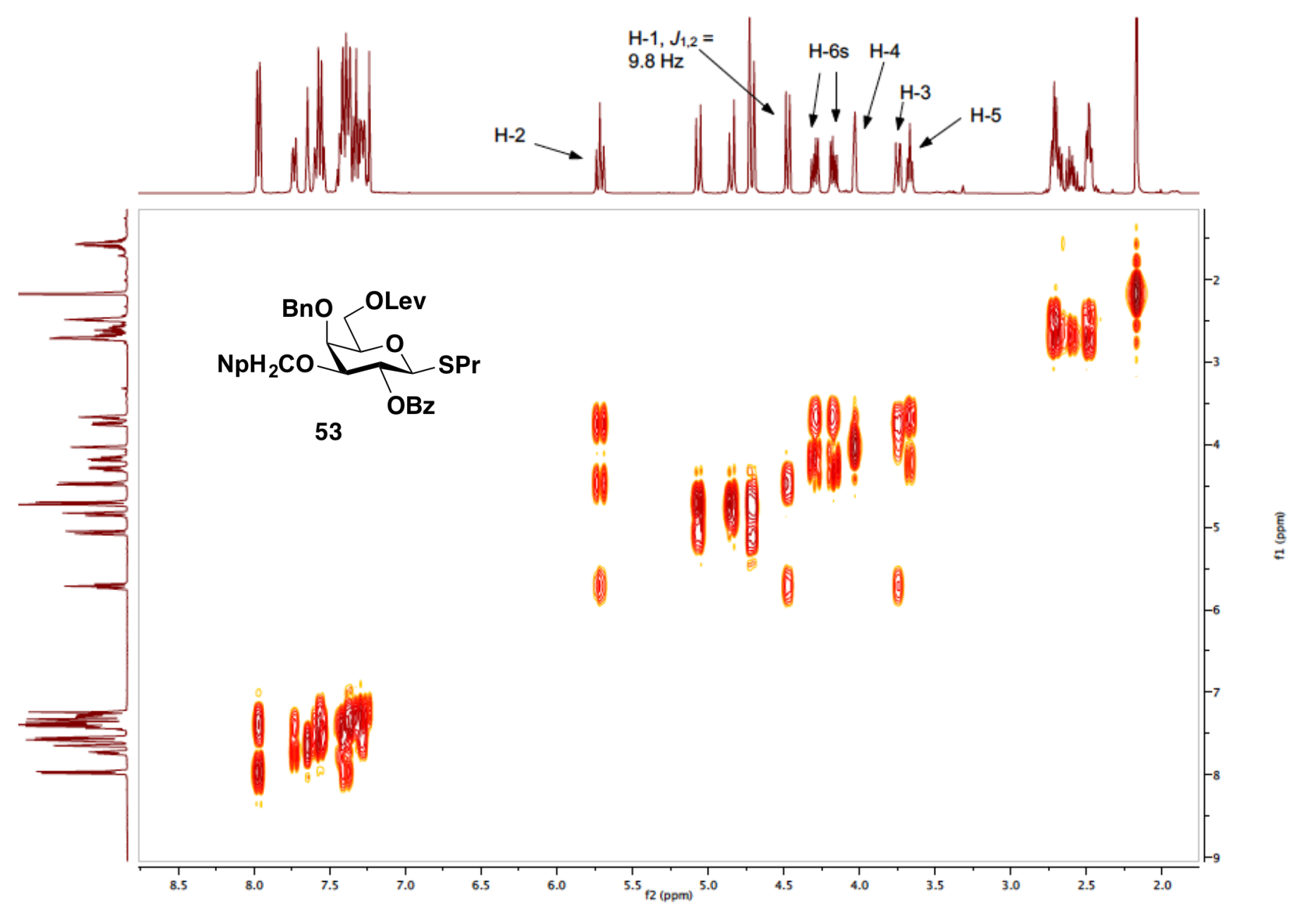

${ }^{1} \mathbf{H}$ - ${ }^{1} \mathbf{H}$ COSY NMR (400 MHz) spectrum of compound 53 in $\mathrm{CDCl}_{3}$ 


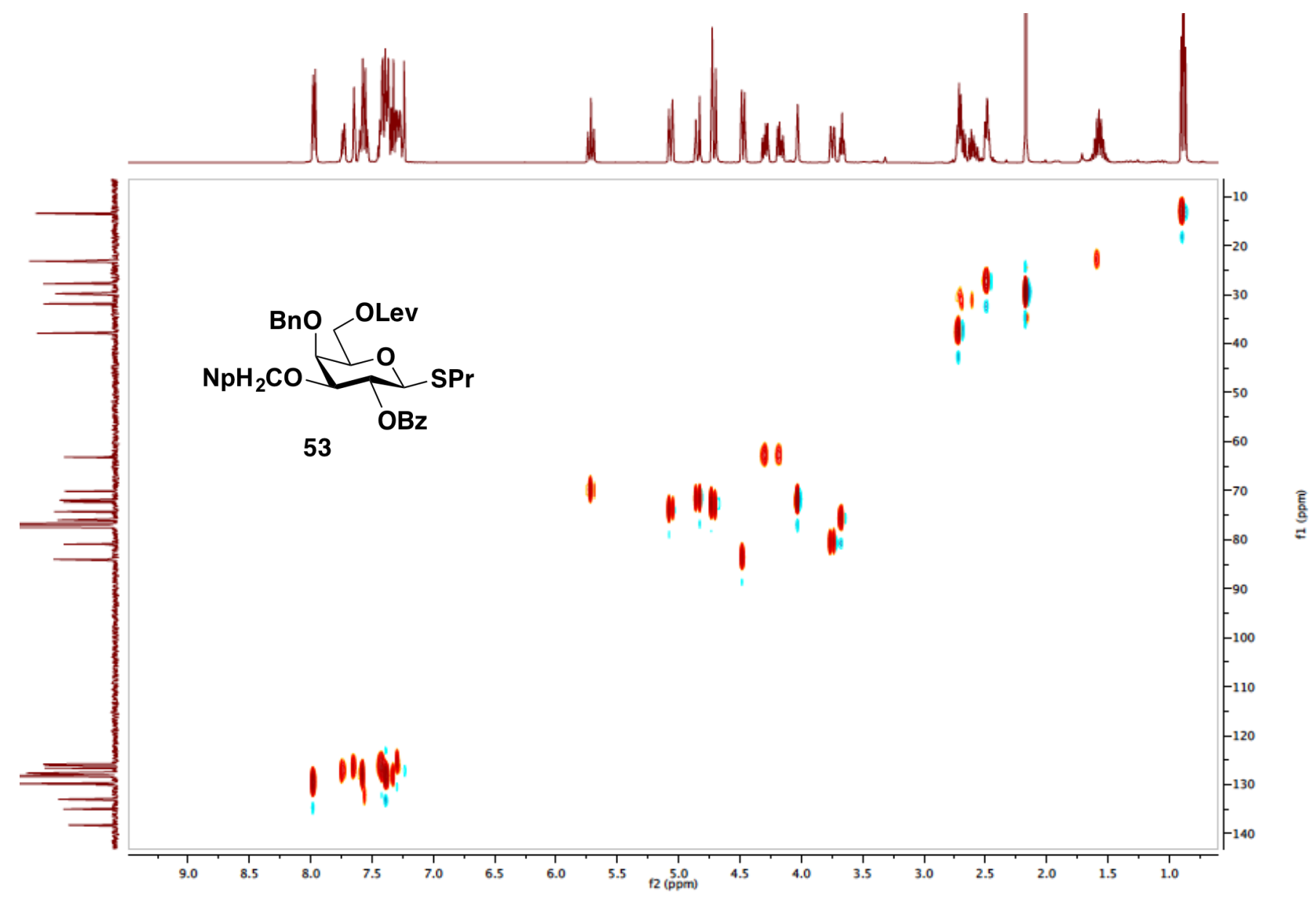

${ }^{1} \mathbf{H}-{ }^{13} \mathbf{C}$ HSQC NMR (400 MHz) spectrum of compound 53 in $\mathrm{CDCl}_{3}$ 


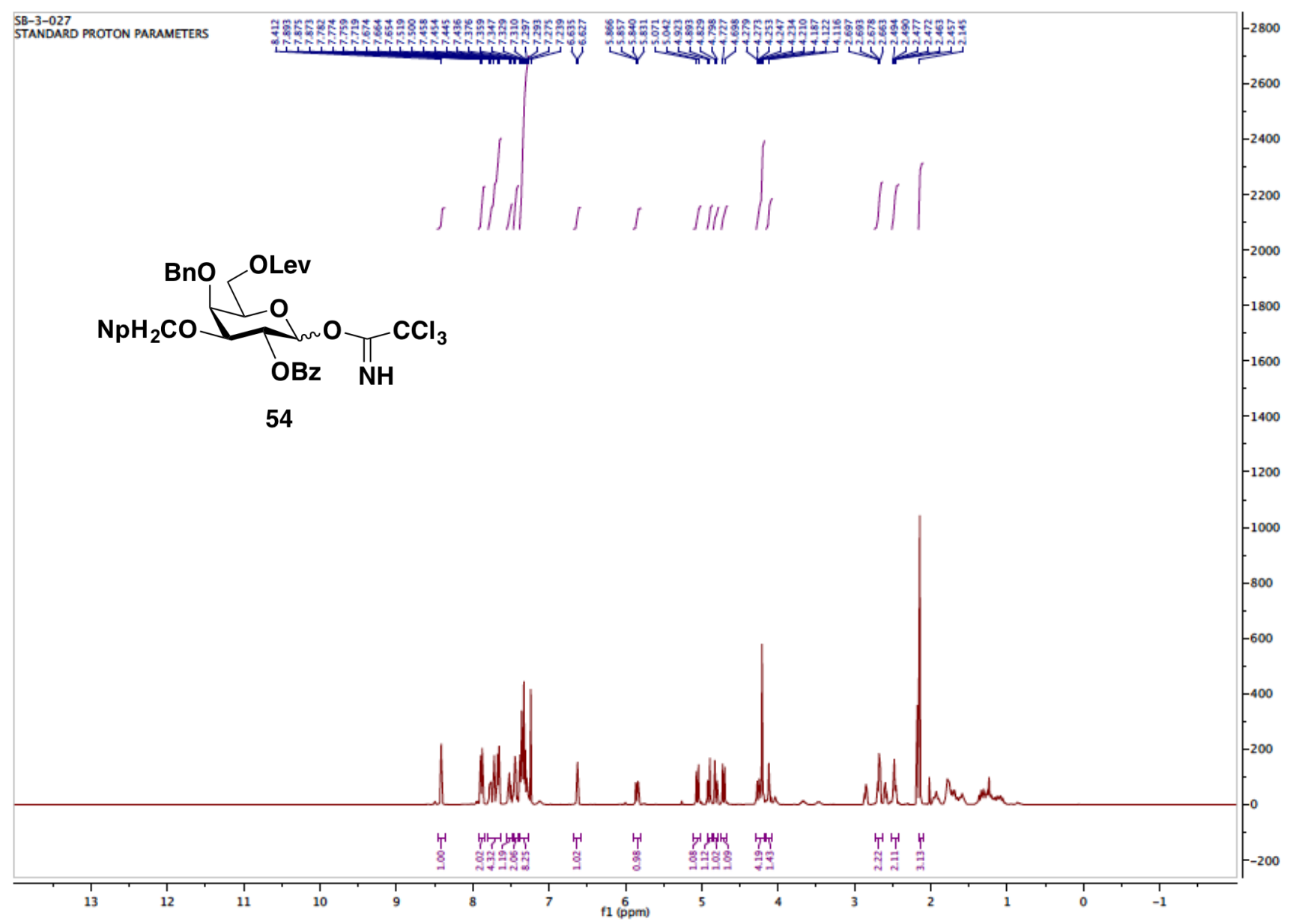

${ }^{\mathbf{1}} \mathbf{H}$ NMR (400 MHz) spectrum of compound 54 in $\mathrm{CDCl}_{3}$ 


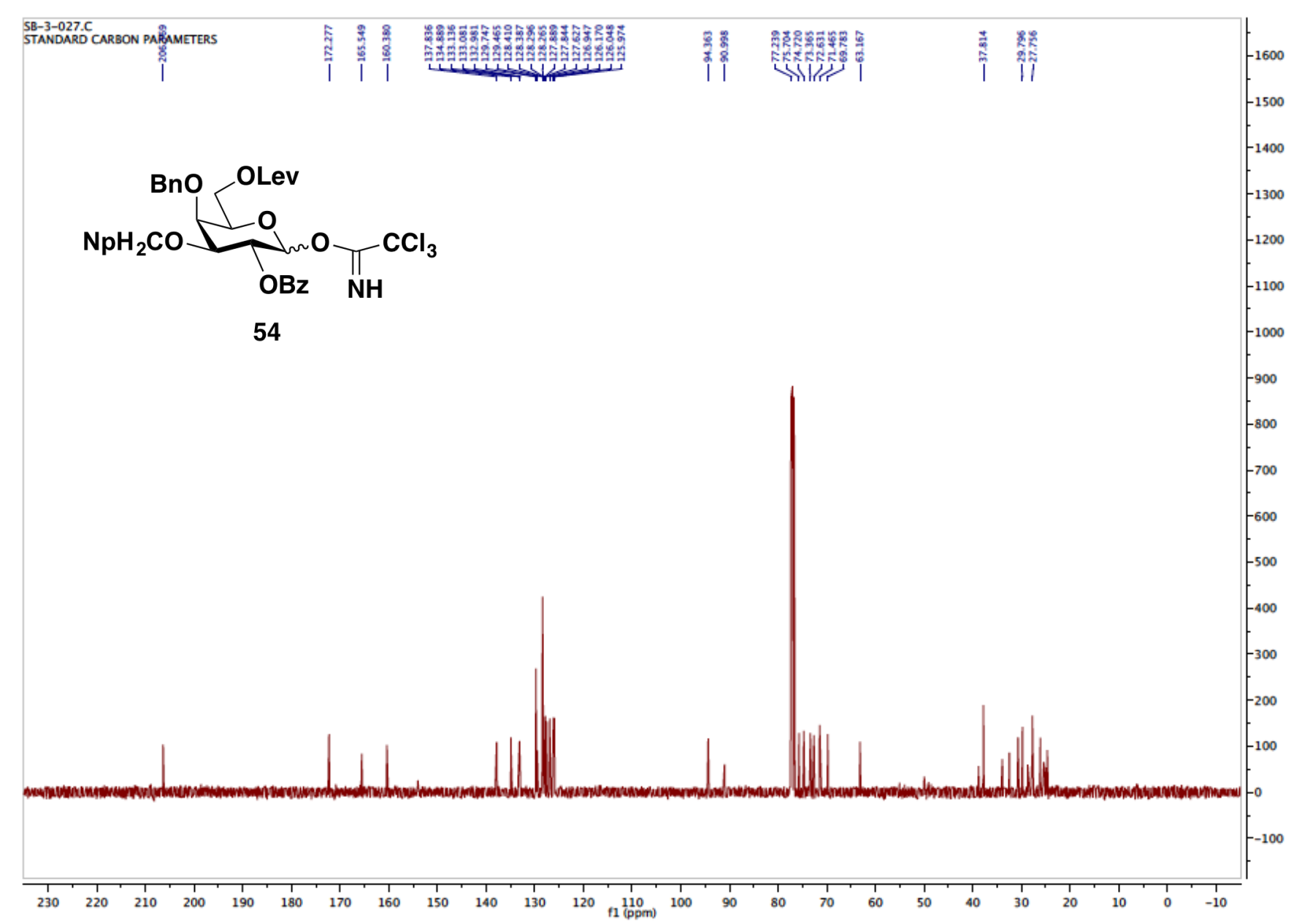

${ }^{13} \mathbf{C}$ NMR (101 MHz) spectrum of compound 54 in $\mathrm{CDCl}_{3}$ 


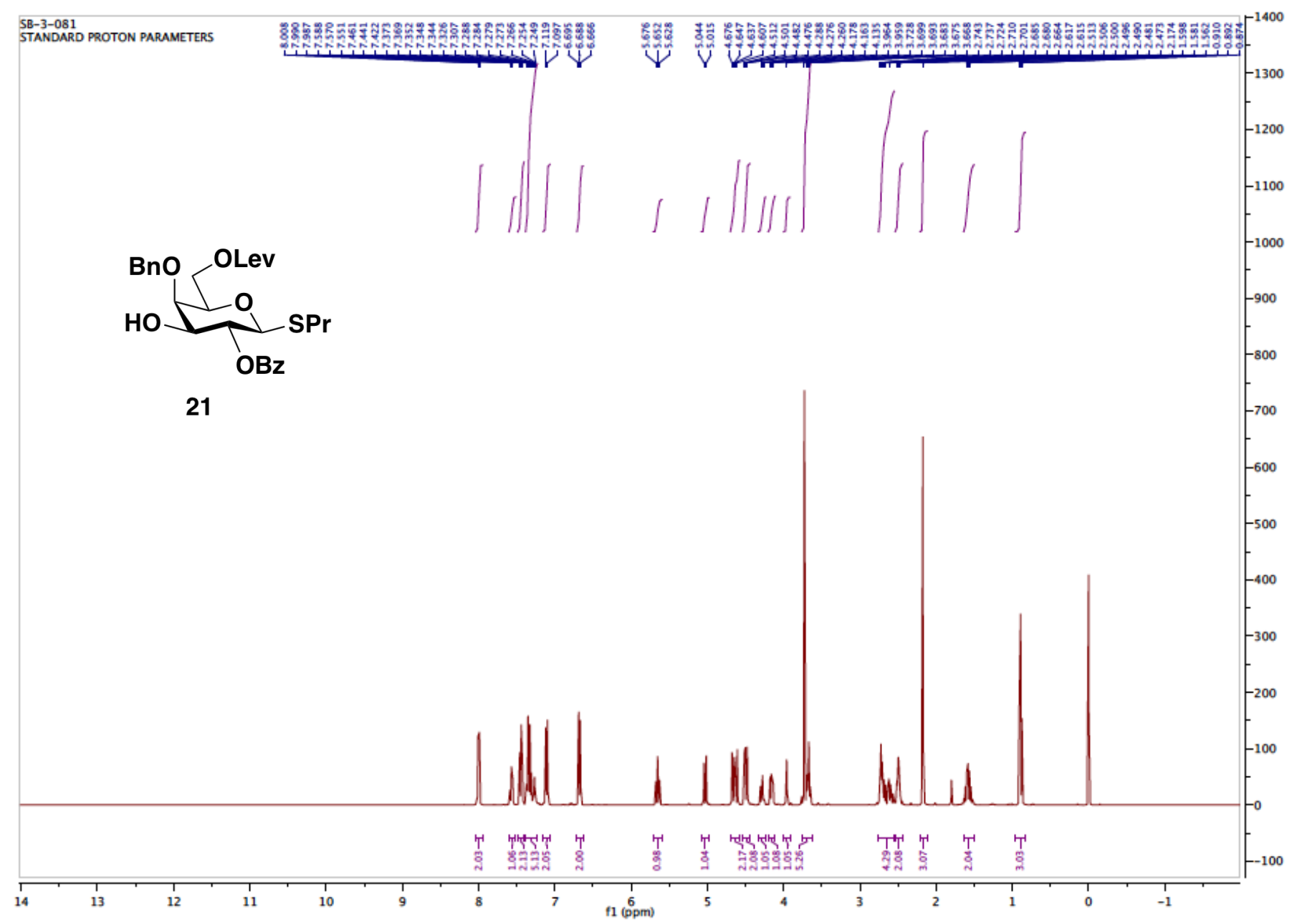

${ }^{1} \mathbf{H}$ NMR (400 MHz) spectrum of compound 21 in $\mathrm{CDCl}_{3}$ 


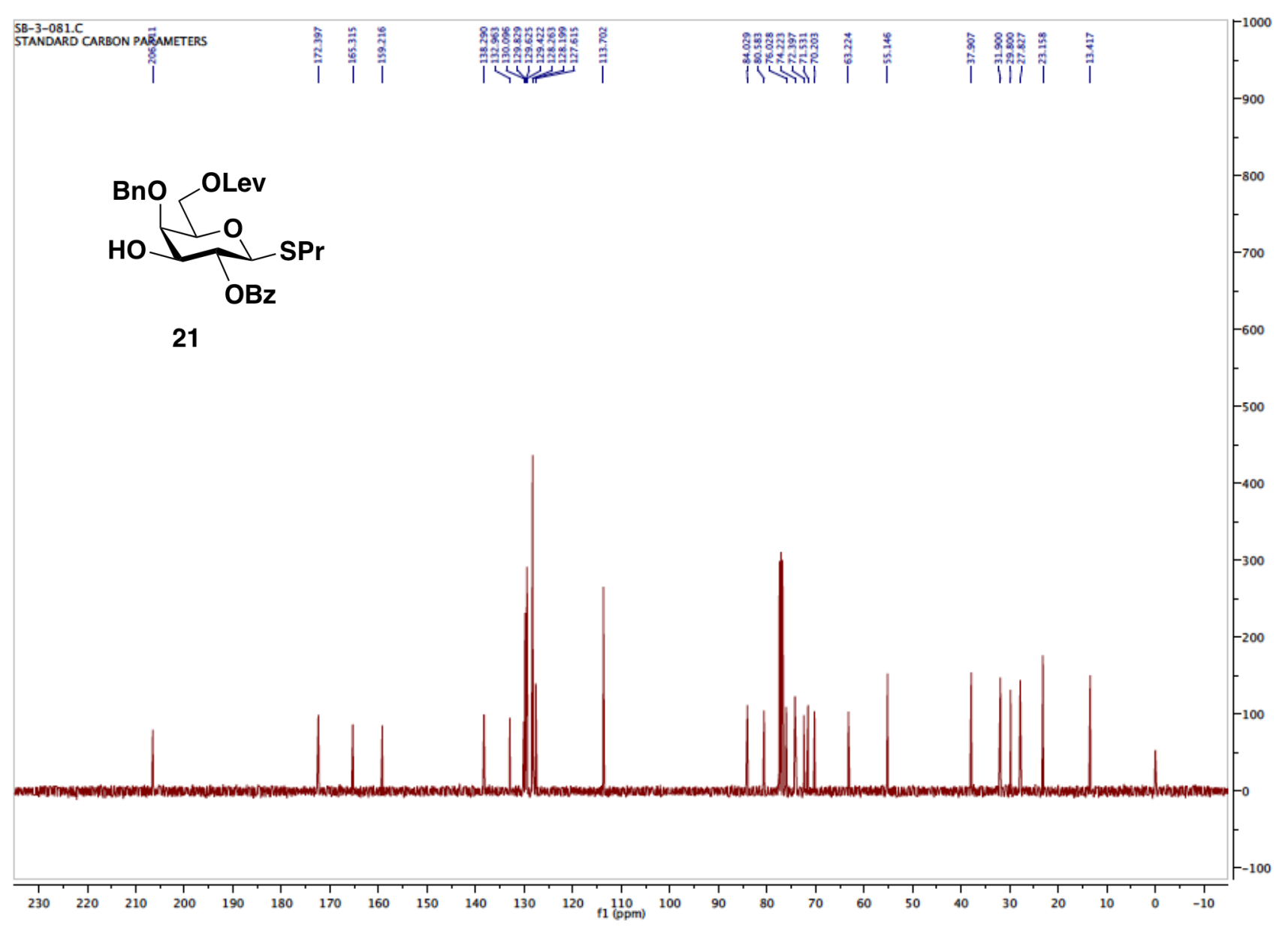

${ }^{13} \mathbf{C}$ NMR (101 MHz) spectrum of compound 21 in $\mathrm{CDCl}_{3}$ 


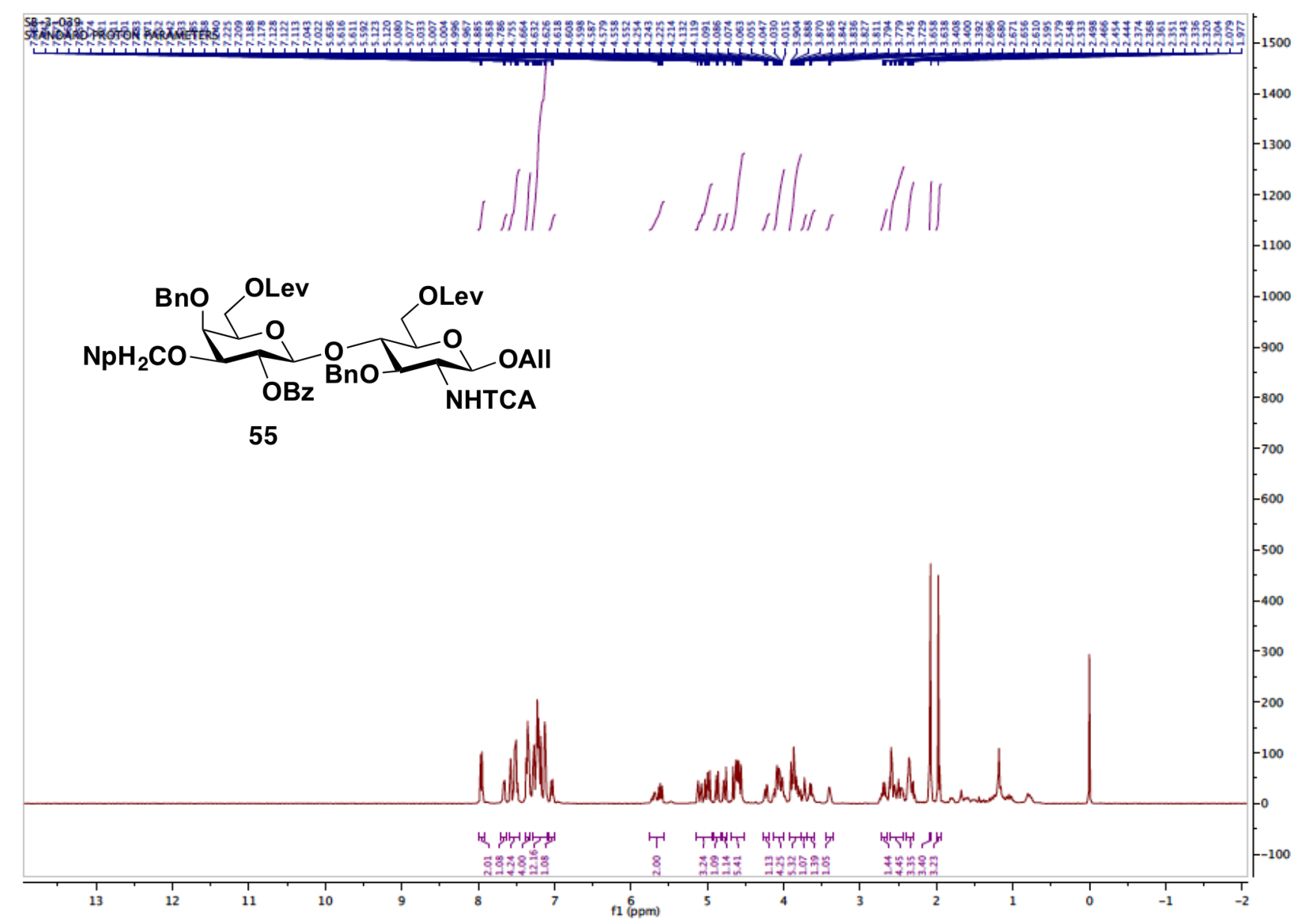

${ }^{1}$ H NMR (400 MHz) spectrum of compound 55 in $\mathrm{CDCl}_{3}$ 


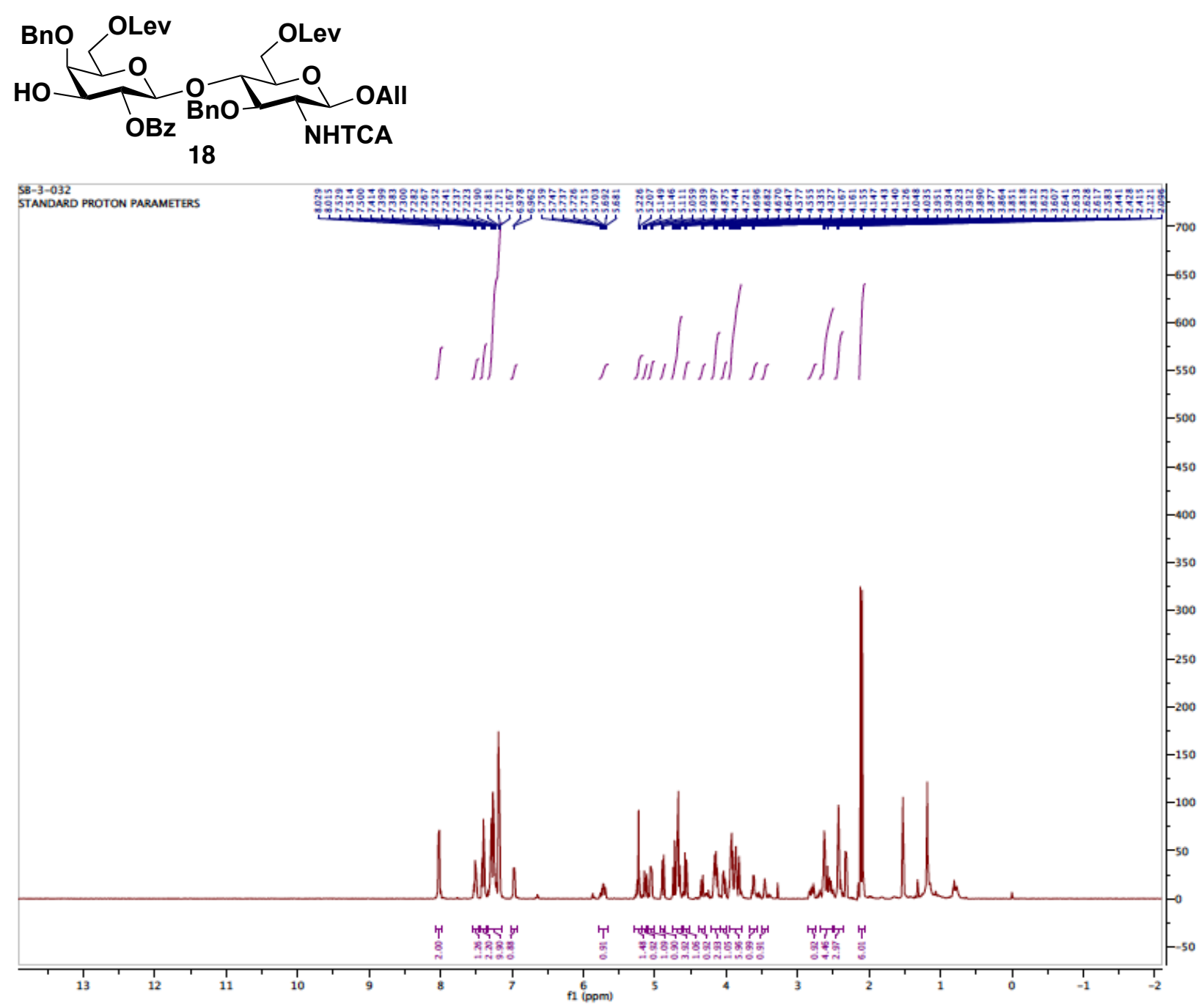

${ }^{1}$ H NMR (500 MHz) spectrum of compound 18 in $\mathrm{CDCl}_{3}$ 


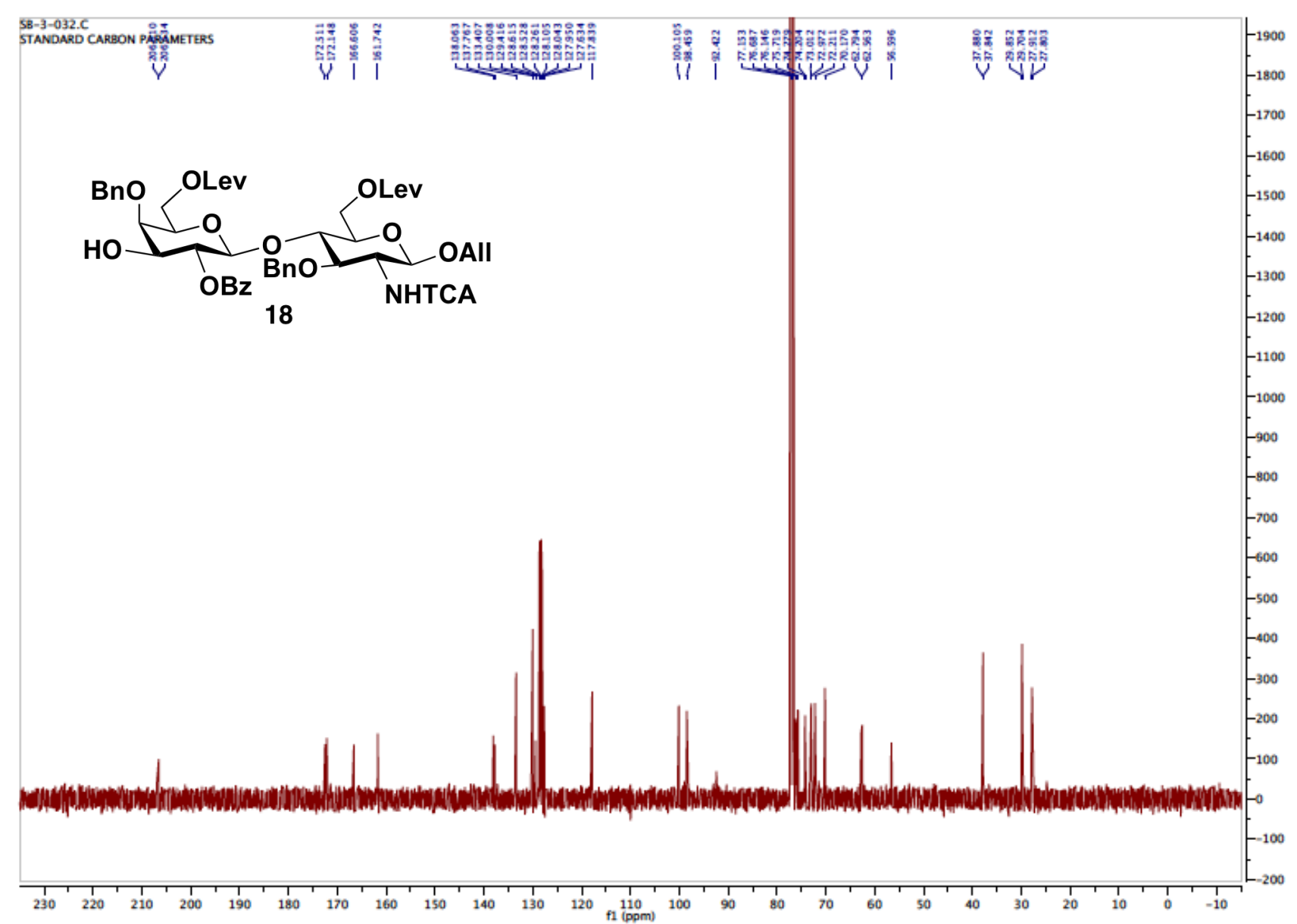

${ }^{13}$ C NMR (126 MHz) spectrum of compound 18 in $\mathrm{CDCl}_{3}$ 


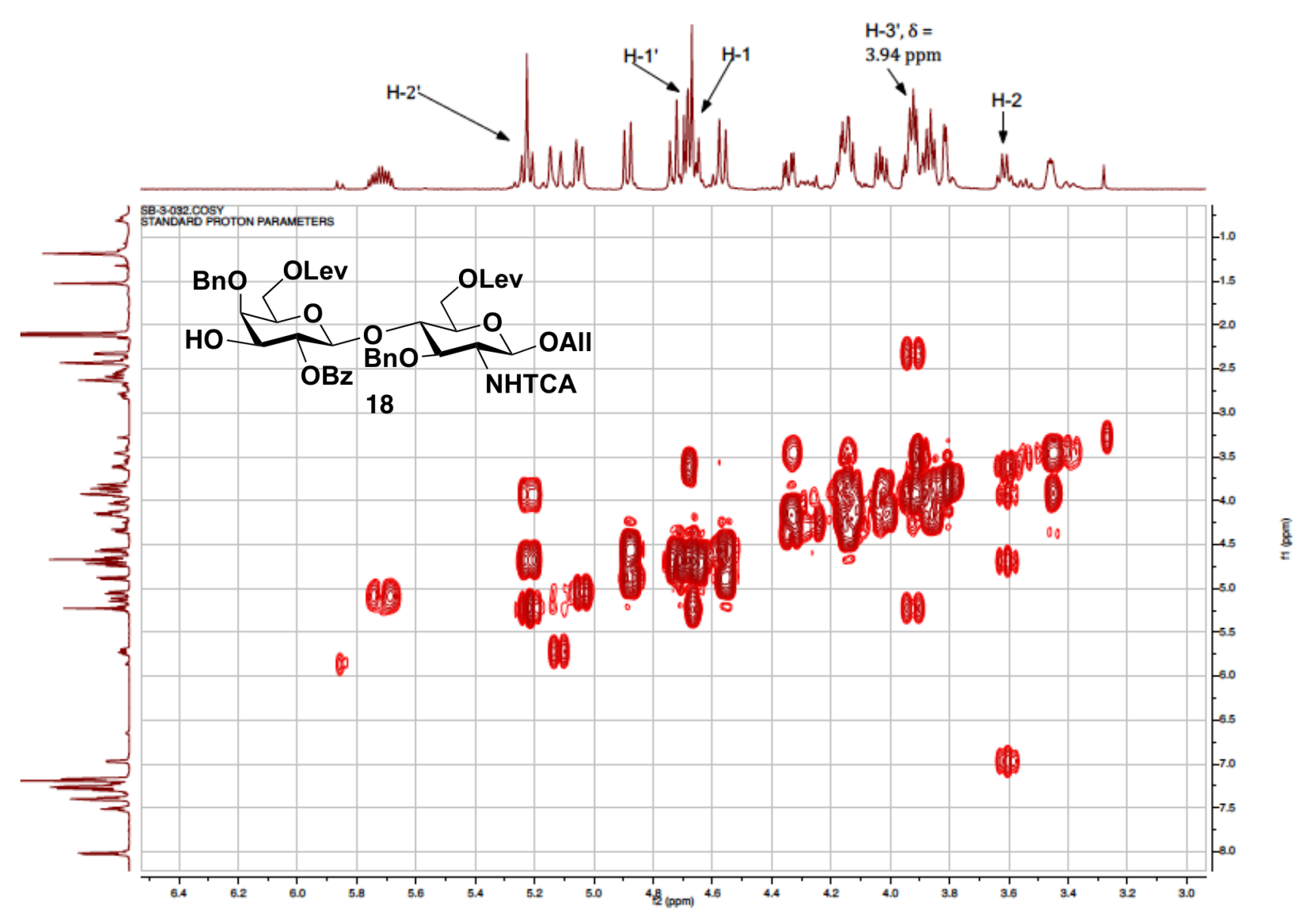

${ }^{1} \mathbf{H}-{ }^{1} \mathbf{H}$ COSY NMR (500 MHz) spectrum of compound 18 in $\mathrm{CDCl}_{3}$ 


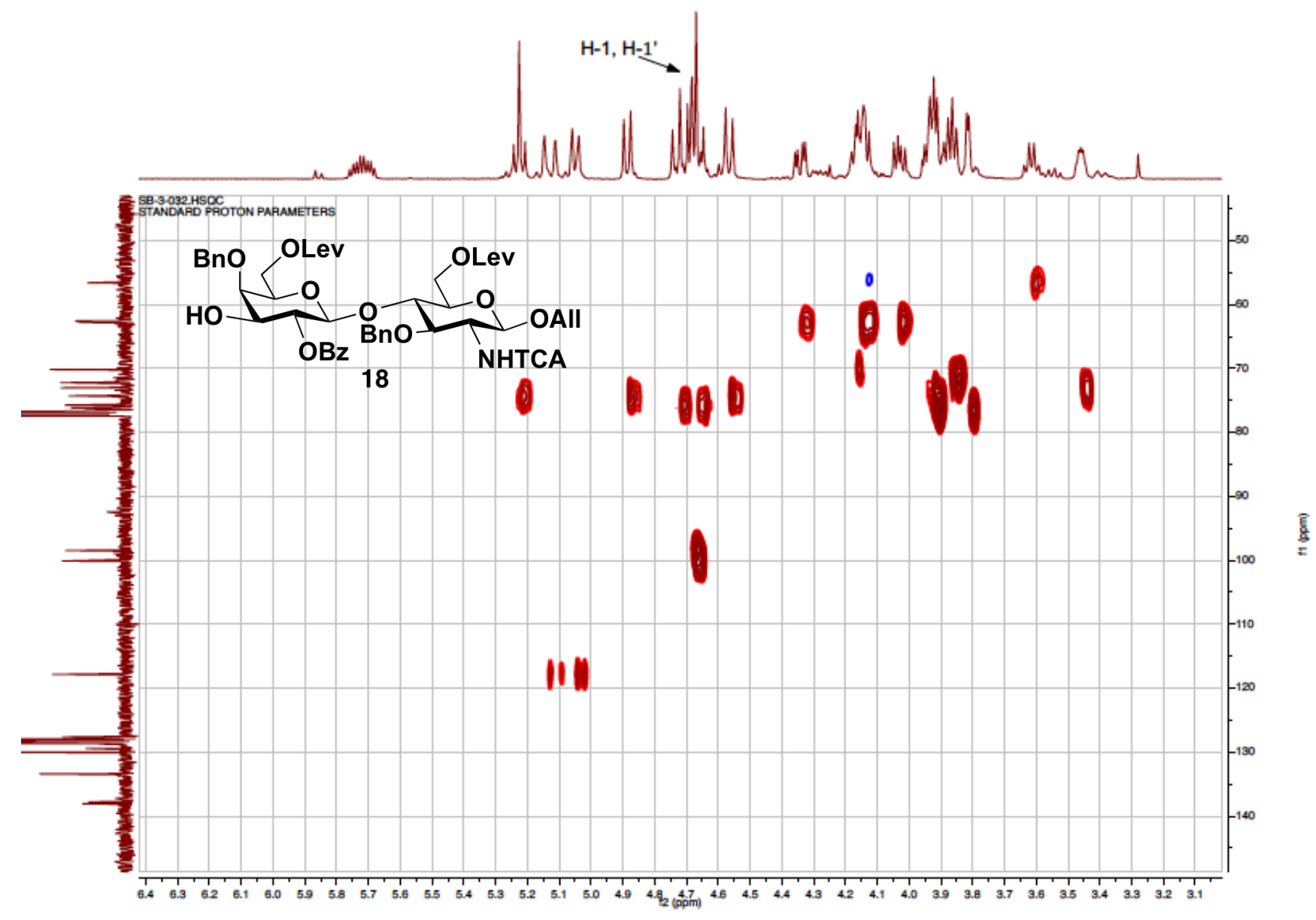

${ }^{1} \mathbf{H}-{ }^{13} \mathbf{C}$ HSQC NMR (500 MHz) spectrum of compound 18 in $\mathrm{CDCl}_{3}$ 


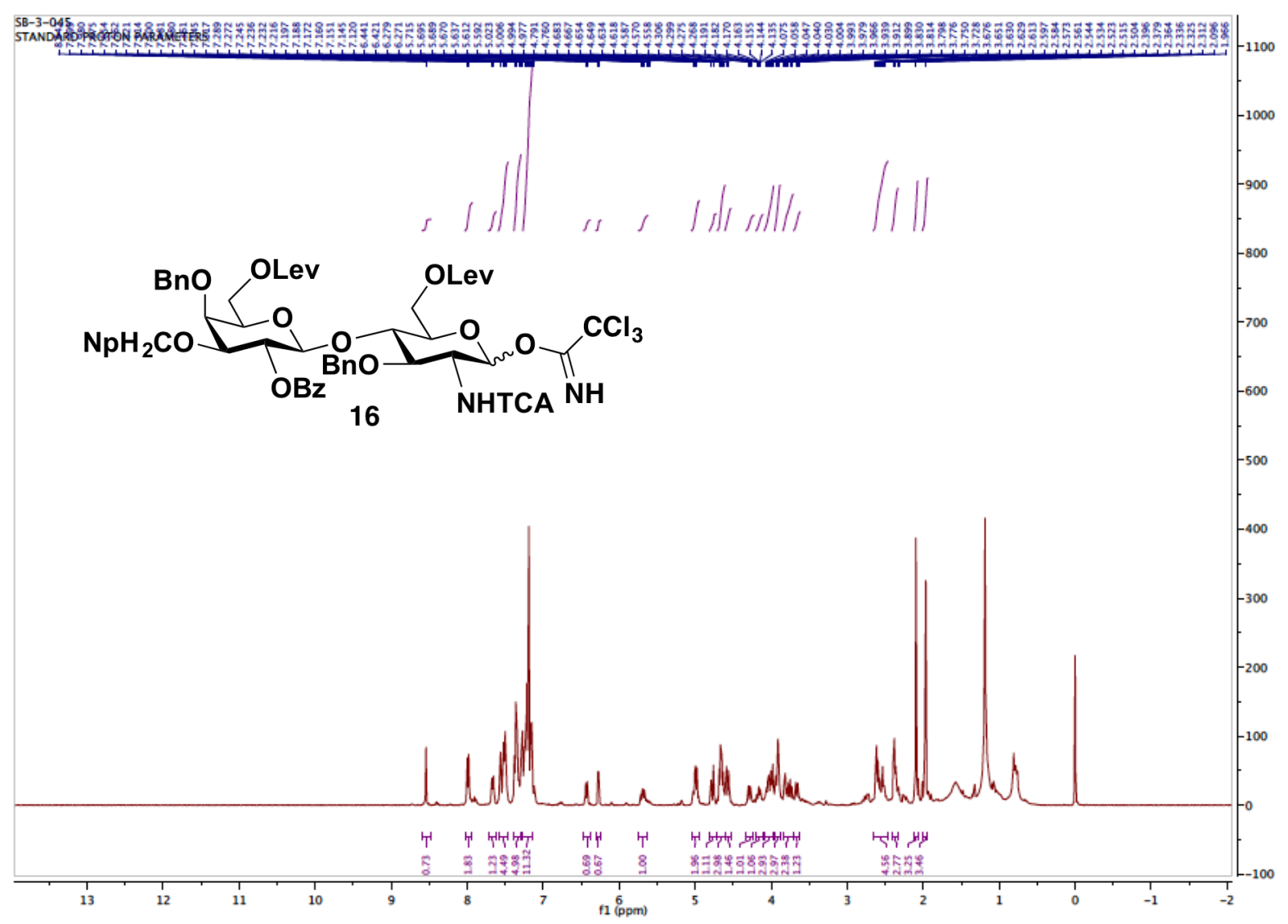

${ }^{1} \mathbf{H}$ NMR (400 MHz) spectrum of compound 16 in $\mathrm{CDCl}_{3}$ 


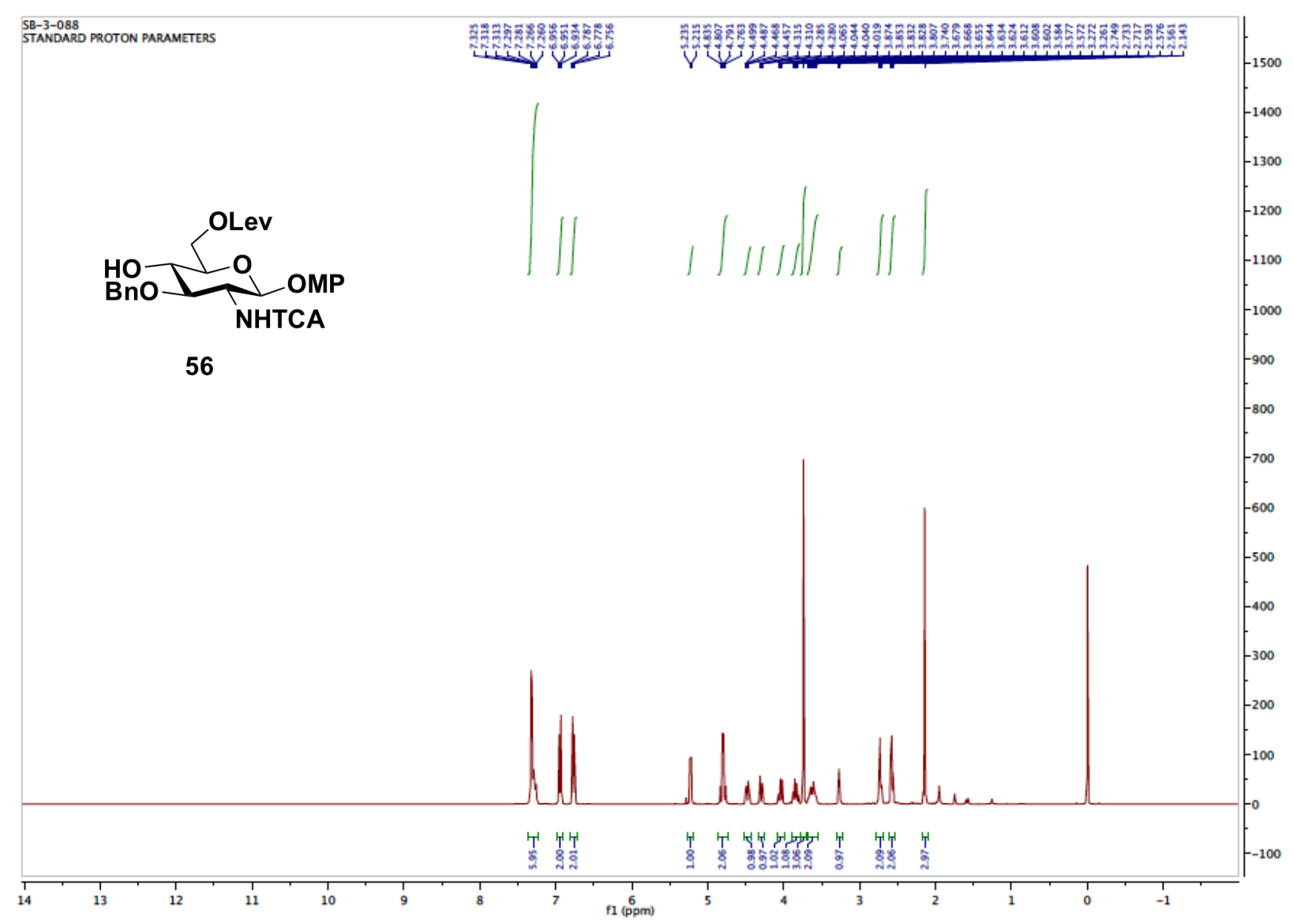

${ }^{1} \mathbf{H}$ NMR (400 MHz) spectrum of compound 56 in $\mathrm{CDCl}_{3}$ 


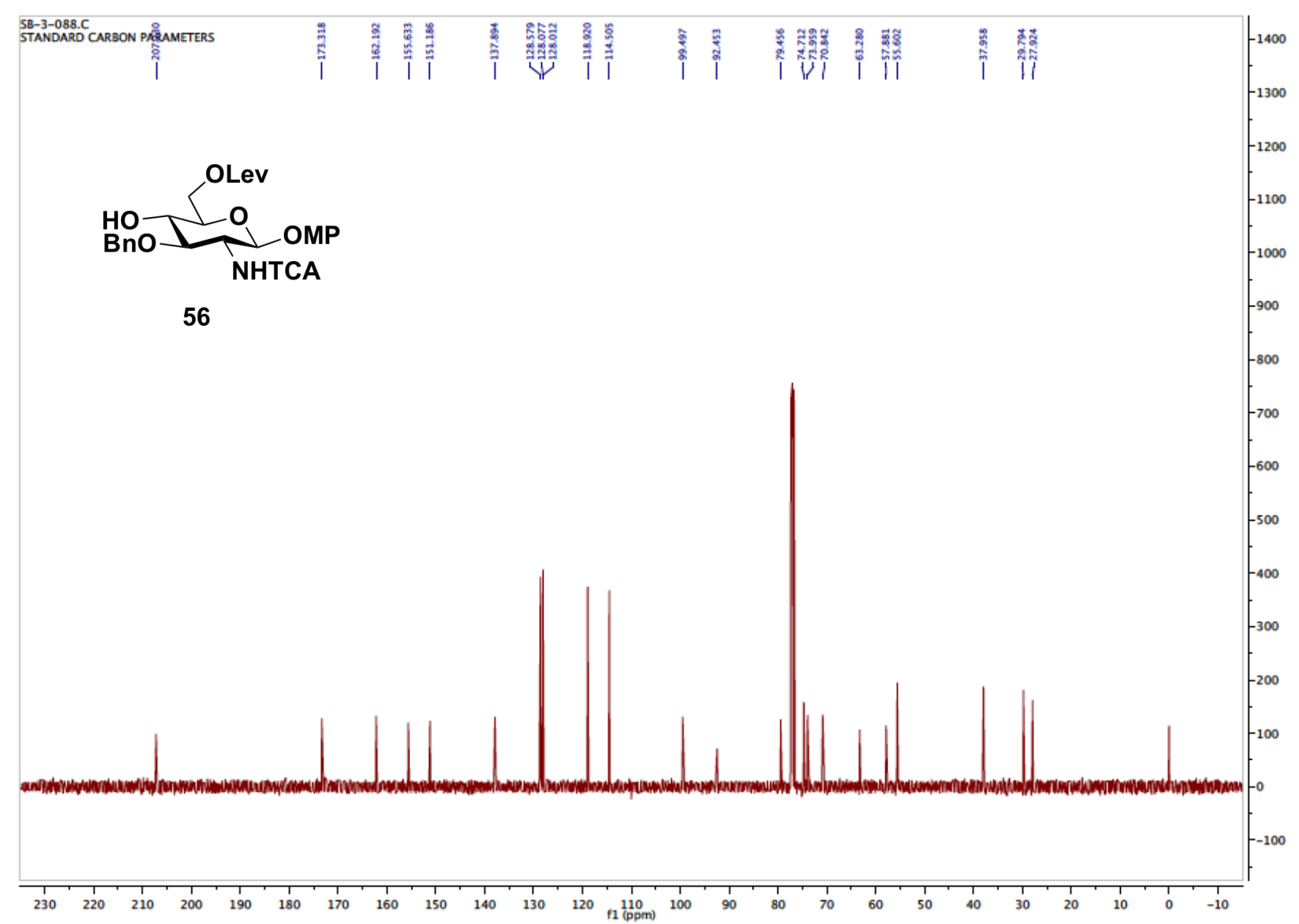

${ }^{13}$ C NMR (101 MHz) spectrum of compound 56 in $\mathrm{CDCl}_{3}$ 


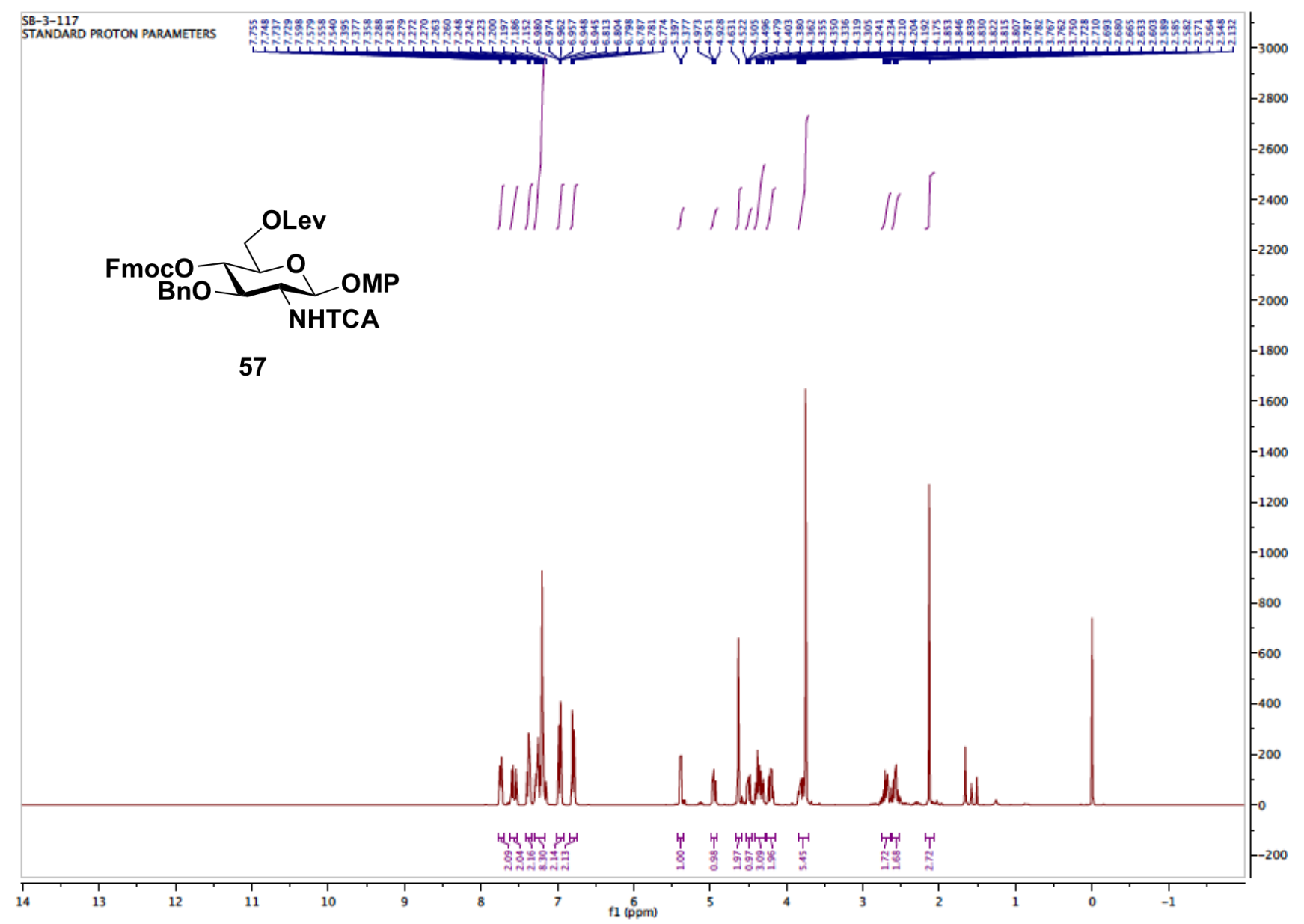

${ }^{1} \mathbf{H}$ NMR (400 MHz) spectrum of compound 57 in $\mathrm{CDCl}_{3}$ 


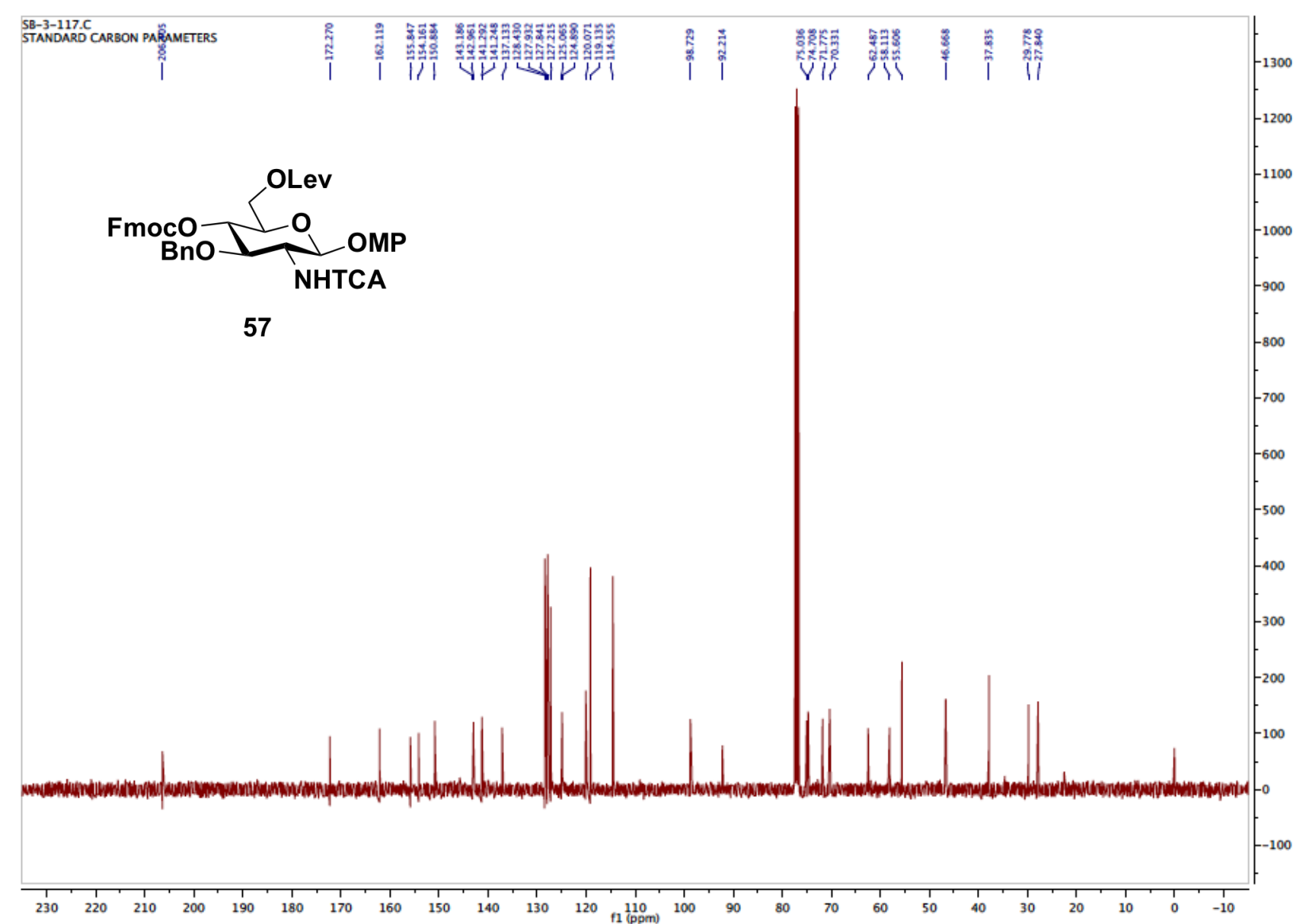

${ }^{13} \mathbf{C}$ NMR (101 MHz) spectrum of compound $\mathbf{5 7}$ in $\mathrm{CDCl}_{3}$ 


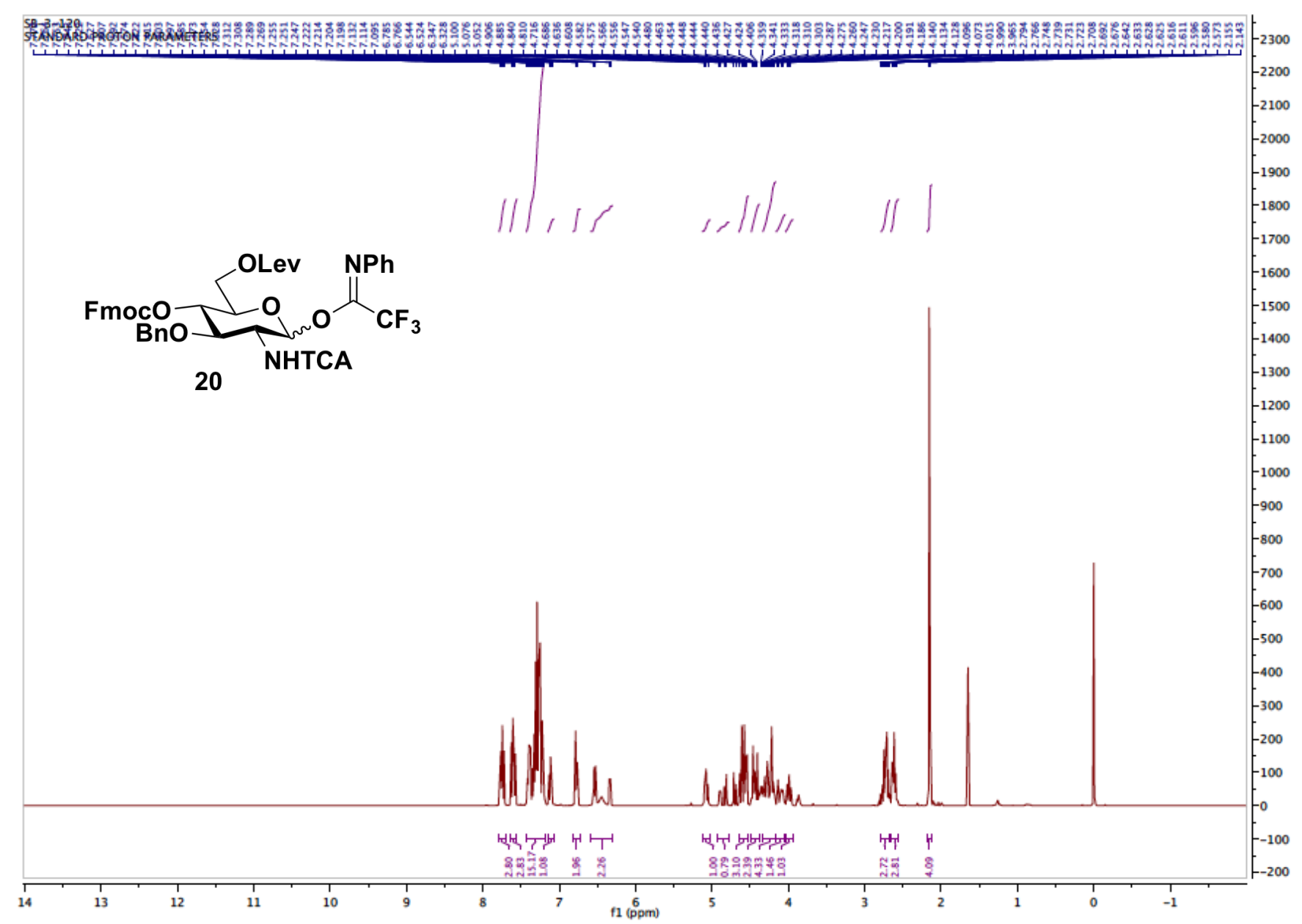

${ }^{1} \mathbf{H}$ NMR (400 MHz) spectrum of compound 20 in $\mathrm{CDCl}_{3}$ 


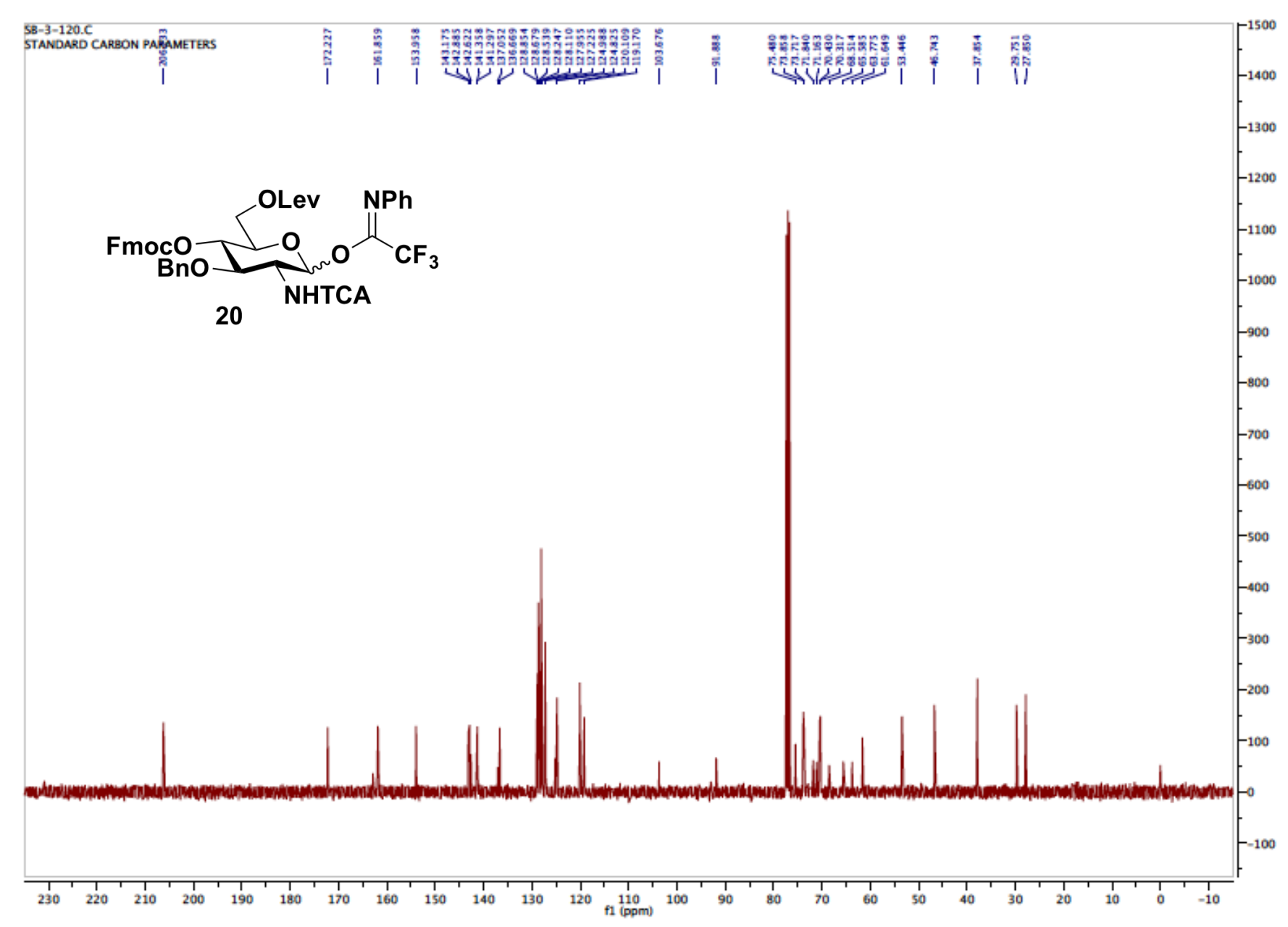

${ }^{13} \mathbf{C}$ NMR (101 MHz) spectrum of compound 20 in $\mathrm{CDCl}_{3}$ 


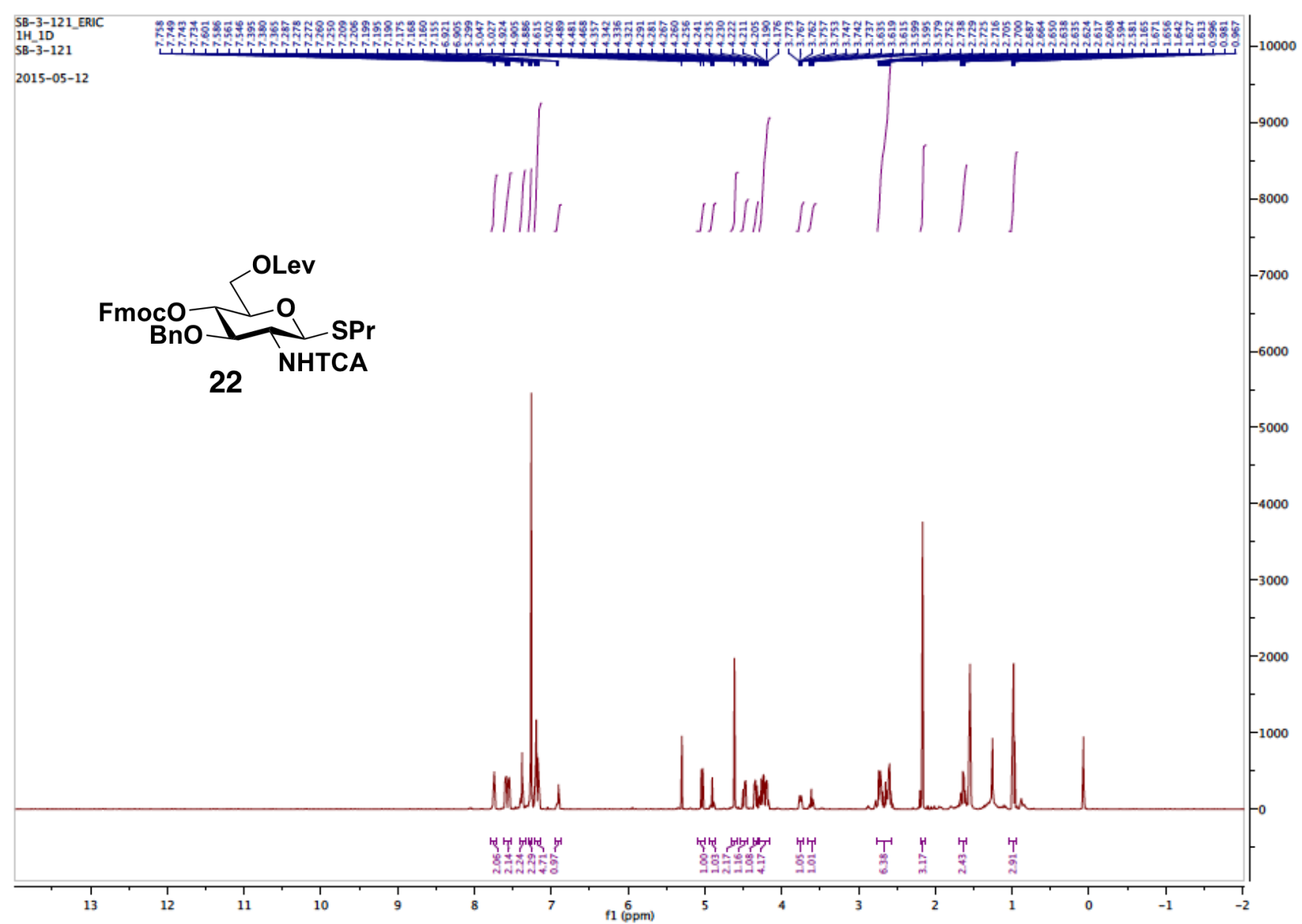

${ }^{1} \mathbf{H}$ NMR (500 MHz) spectrum of compound 22 in $\mathrm{CDCl}_{3}$ 


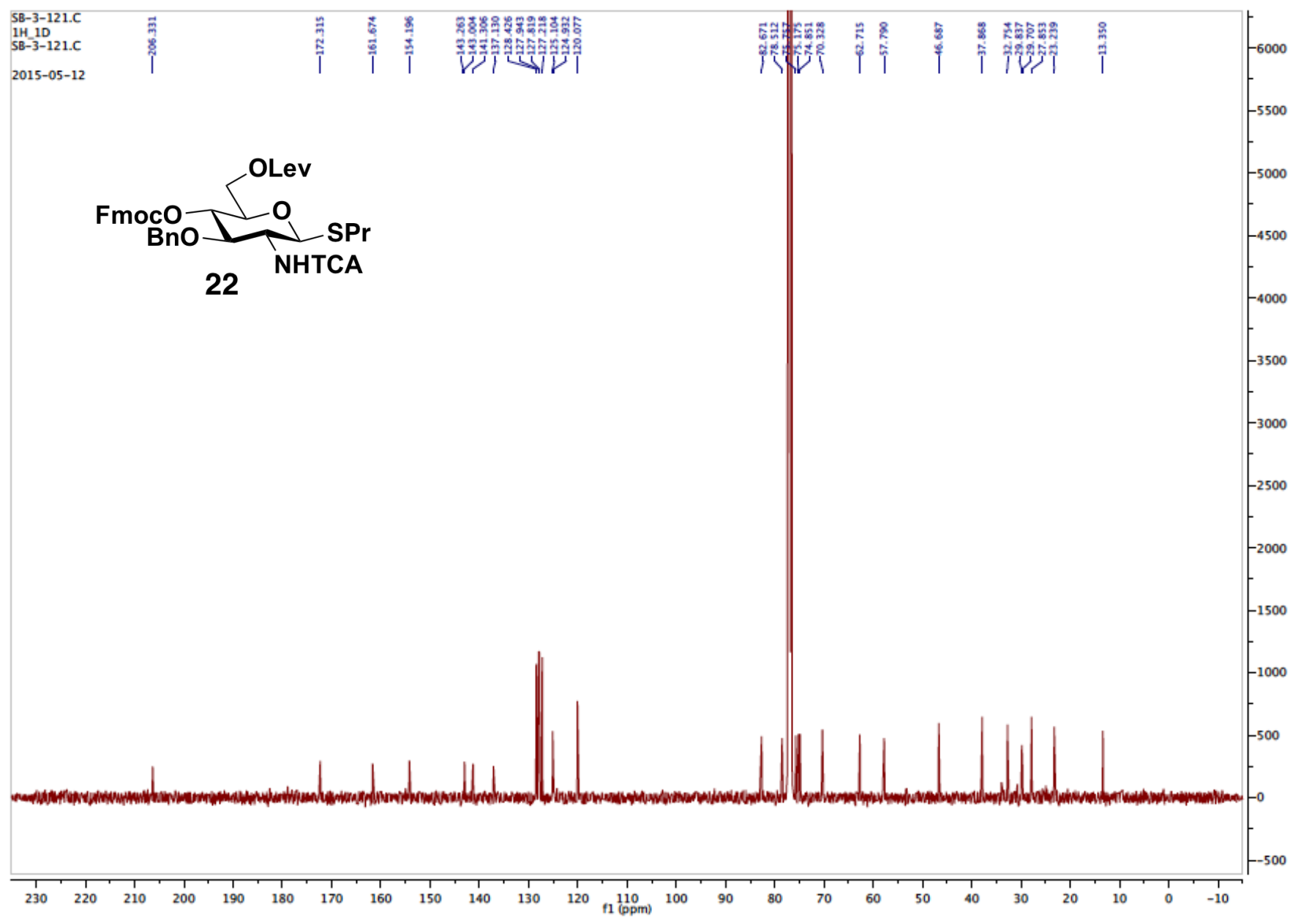

${ }^{13} \mathrm{C}$ NMR (126 MHz) spectrum of compound 22 in $\mathrm{CDCl}_{3}$ 


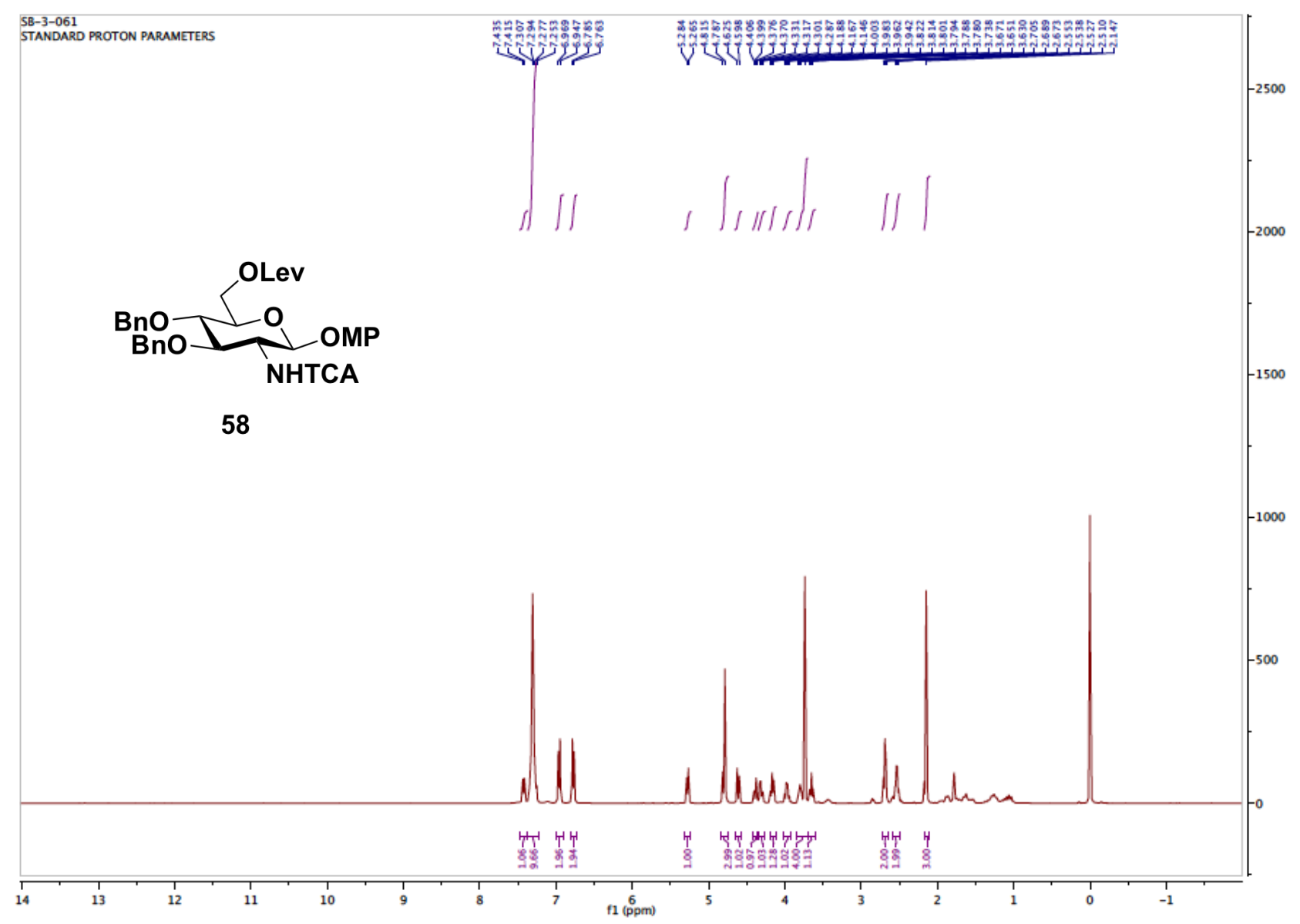

${ }^{1} \mathbf{H}$ NMR (400 MHz) spectrum of compound 58 in $\mathrm{CDCl}_{3}$ 


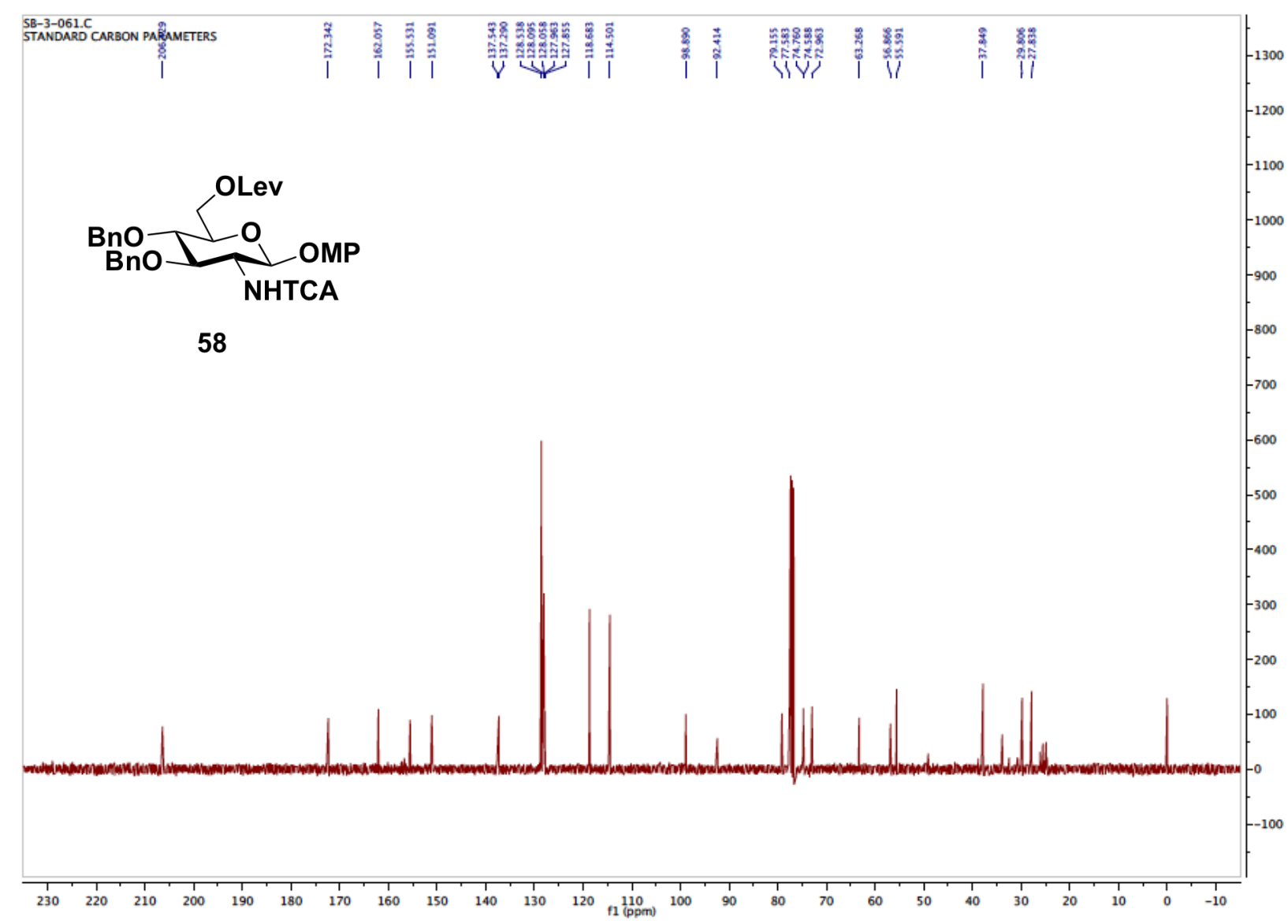

${ }^{13} \mathbf{C}$ NMR (101 MHz) spectrum of compound $\mathbf{5 8}$ in $\mathrm{CDCl}_{3}$ 


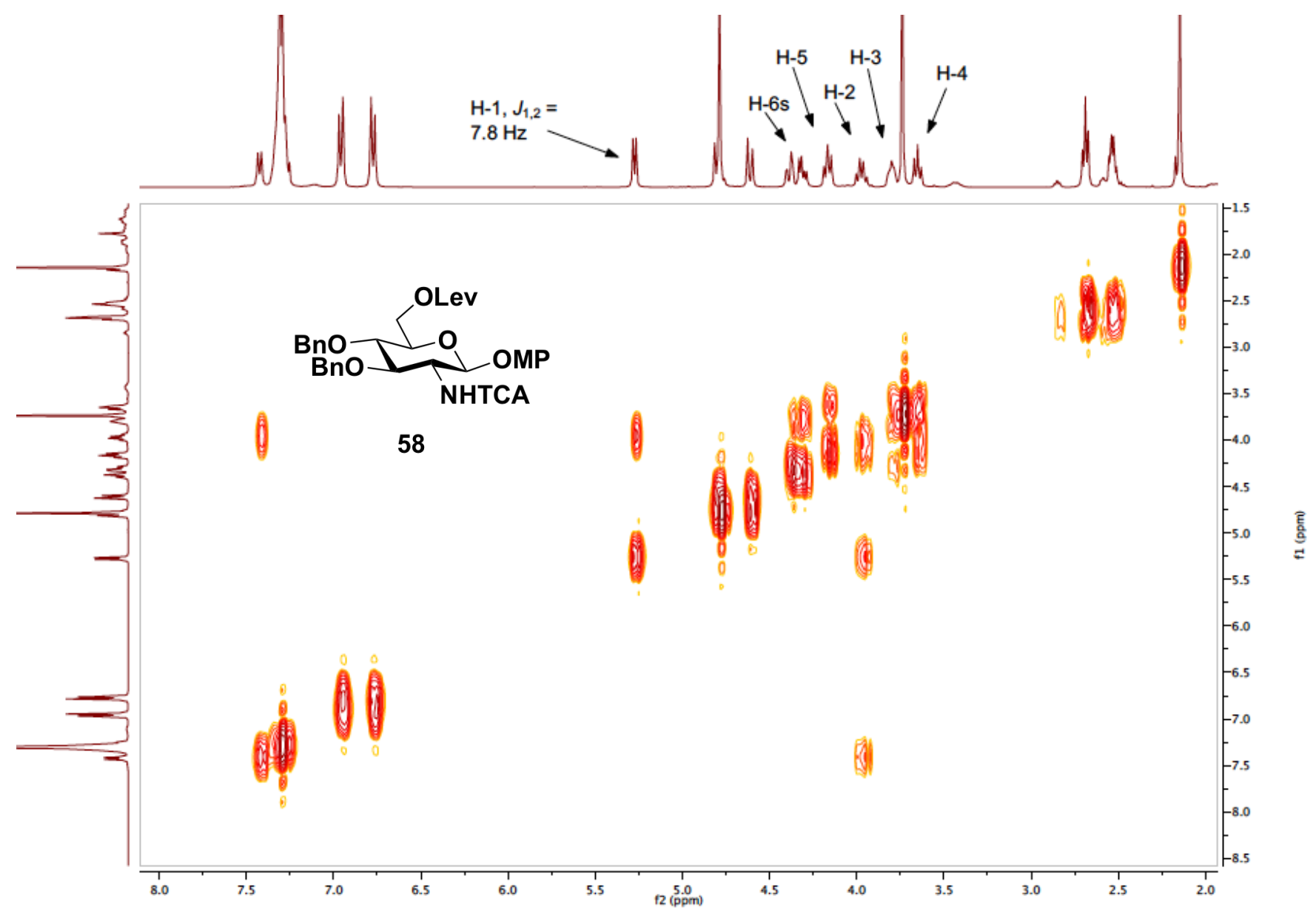

${ }^{1} \mathbf{H}$ - ${ }^{1} \mathbf{H}$ COSY NMR (400 MHz) spectrum of compound $\mathbf{5 8}$ in $\mathrm{CDCl}_{3}$ 


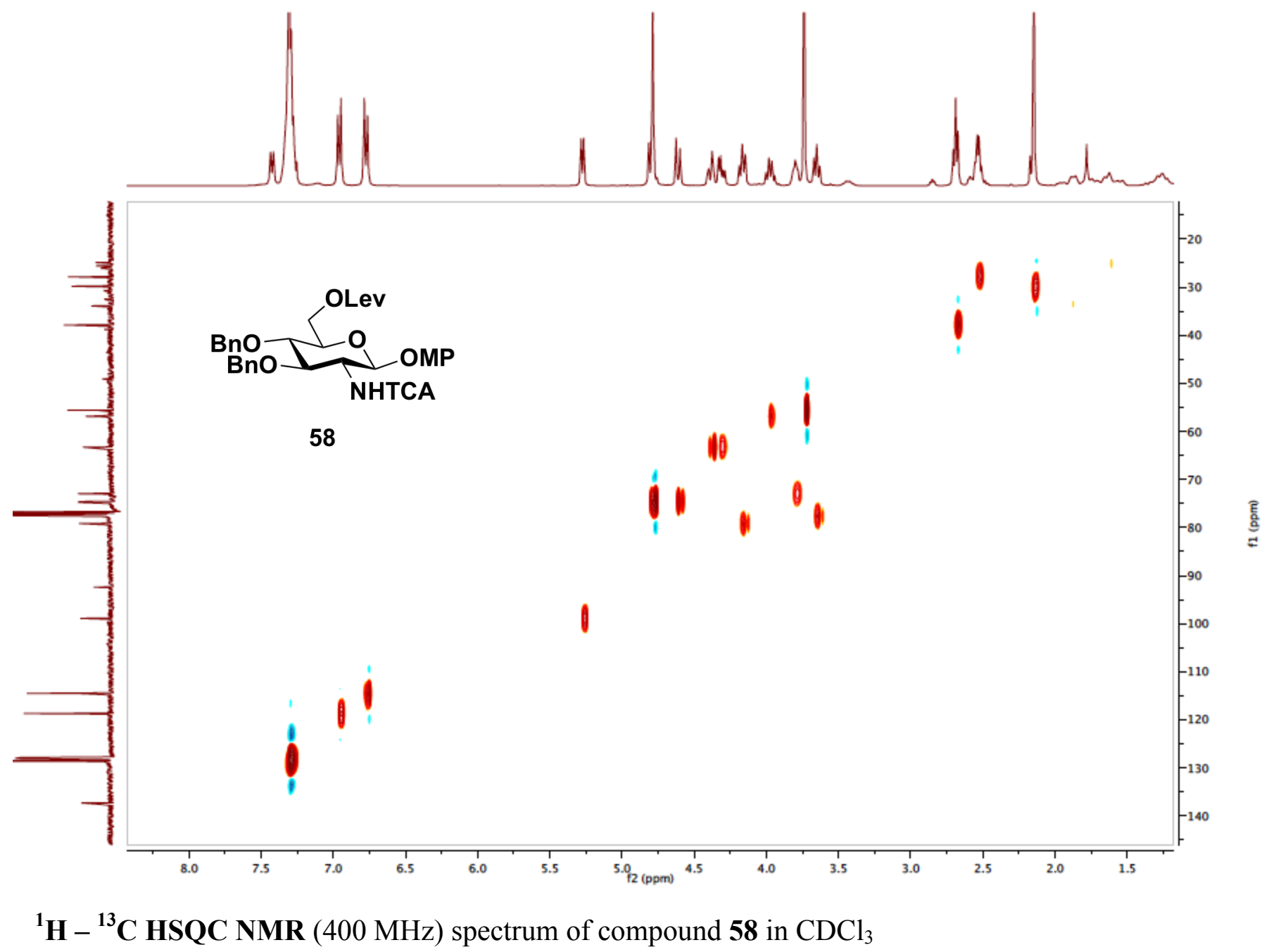

140 


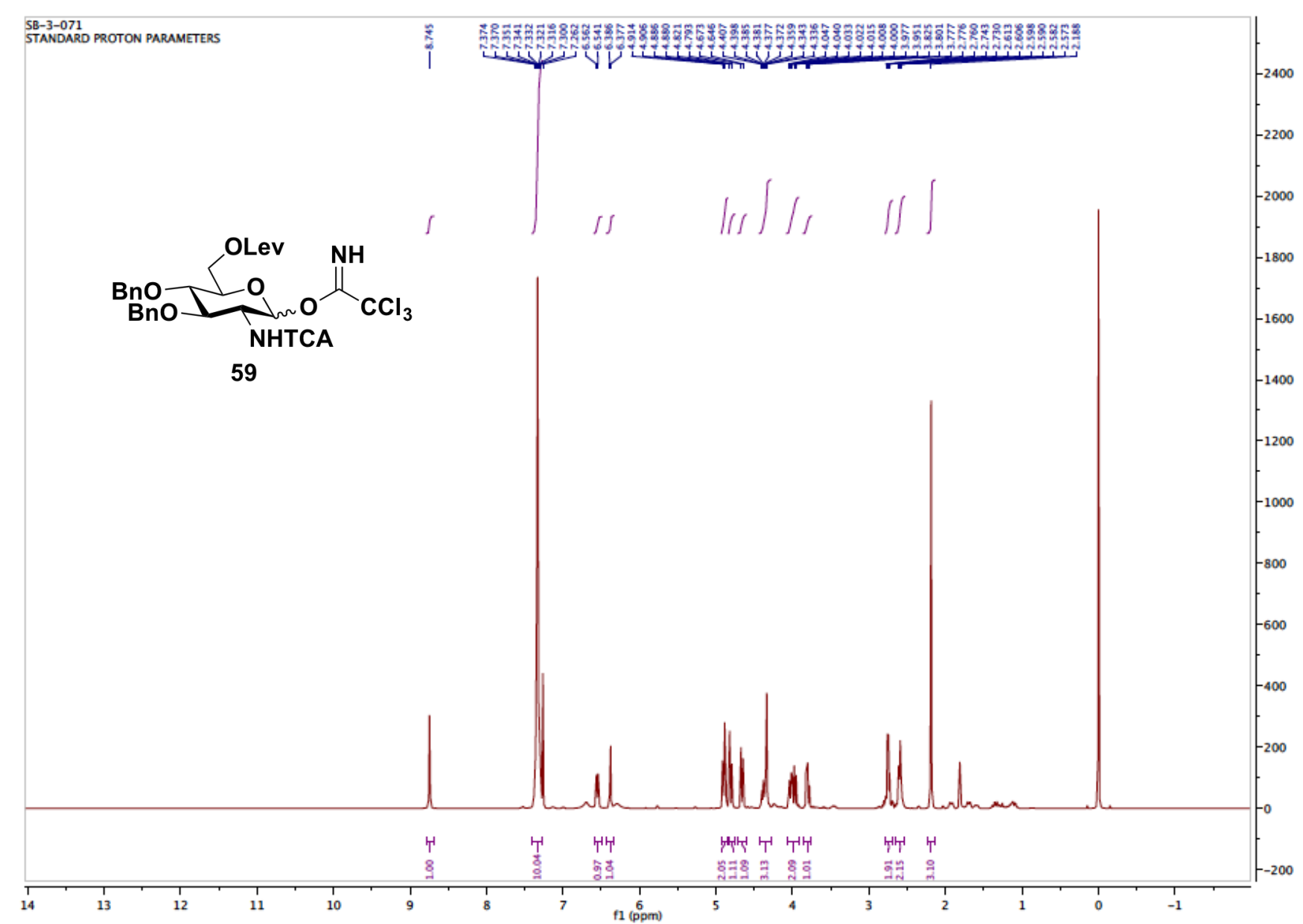

${ }^{1} \mathbf{H}$ NMR (400 MHz) spectrum of compound 59 in $\mathrm{CDCl}_{3}$ 


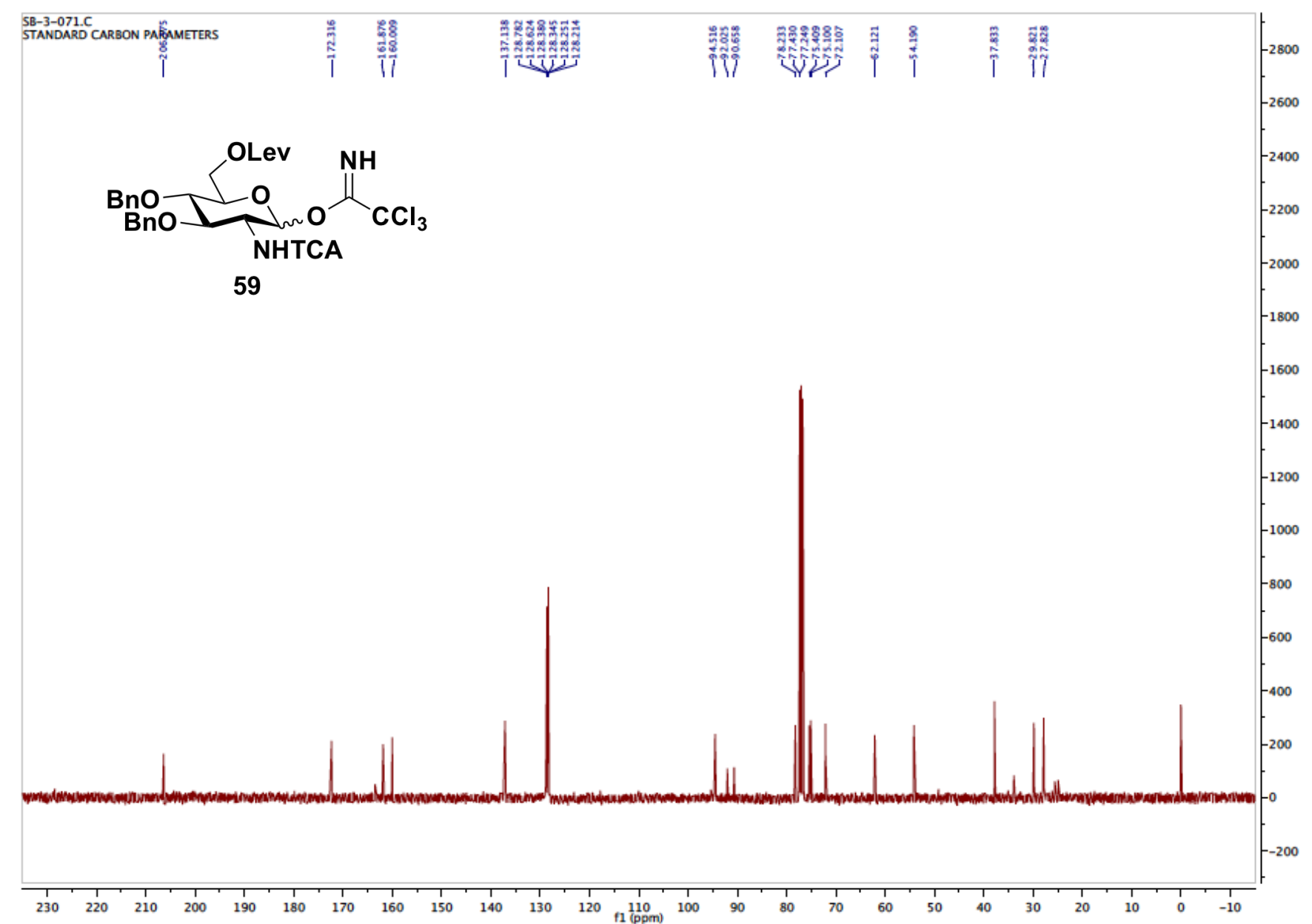

${ }^{13} \mathbf{C}$ NMR (101 MHz) spectrum of compound 59 in $\mathrm{CDCl}_{3}$ 


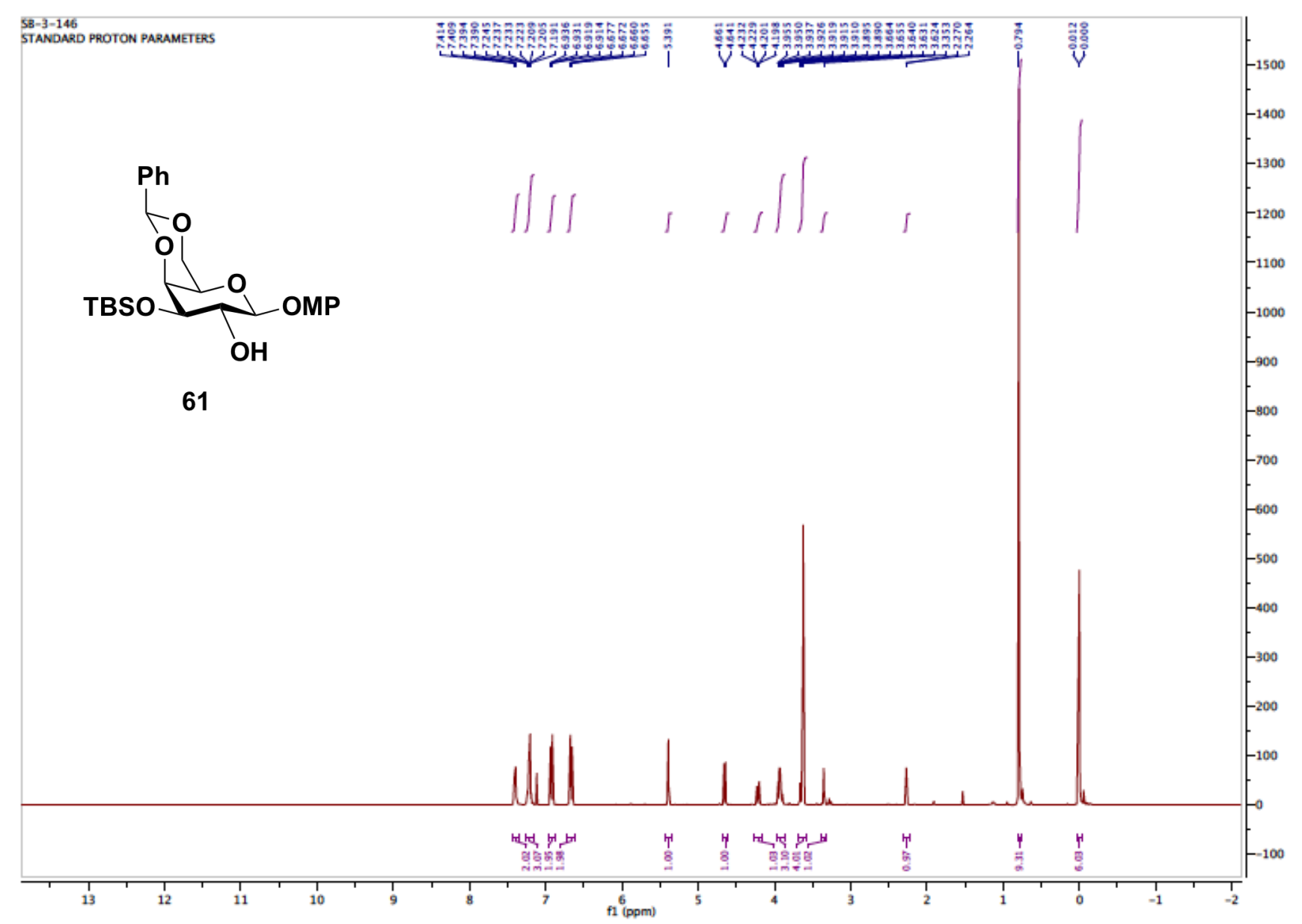

${ }^{1} \mathbf{H}$ NMR (400 MHz) spectrum of compound 61 in $\mathrm{CDCl}_{3}$ 


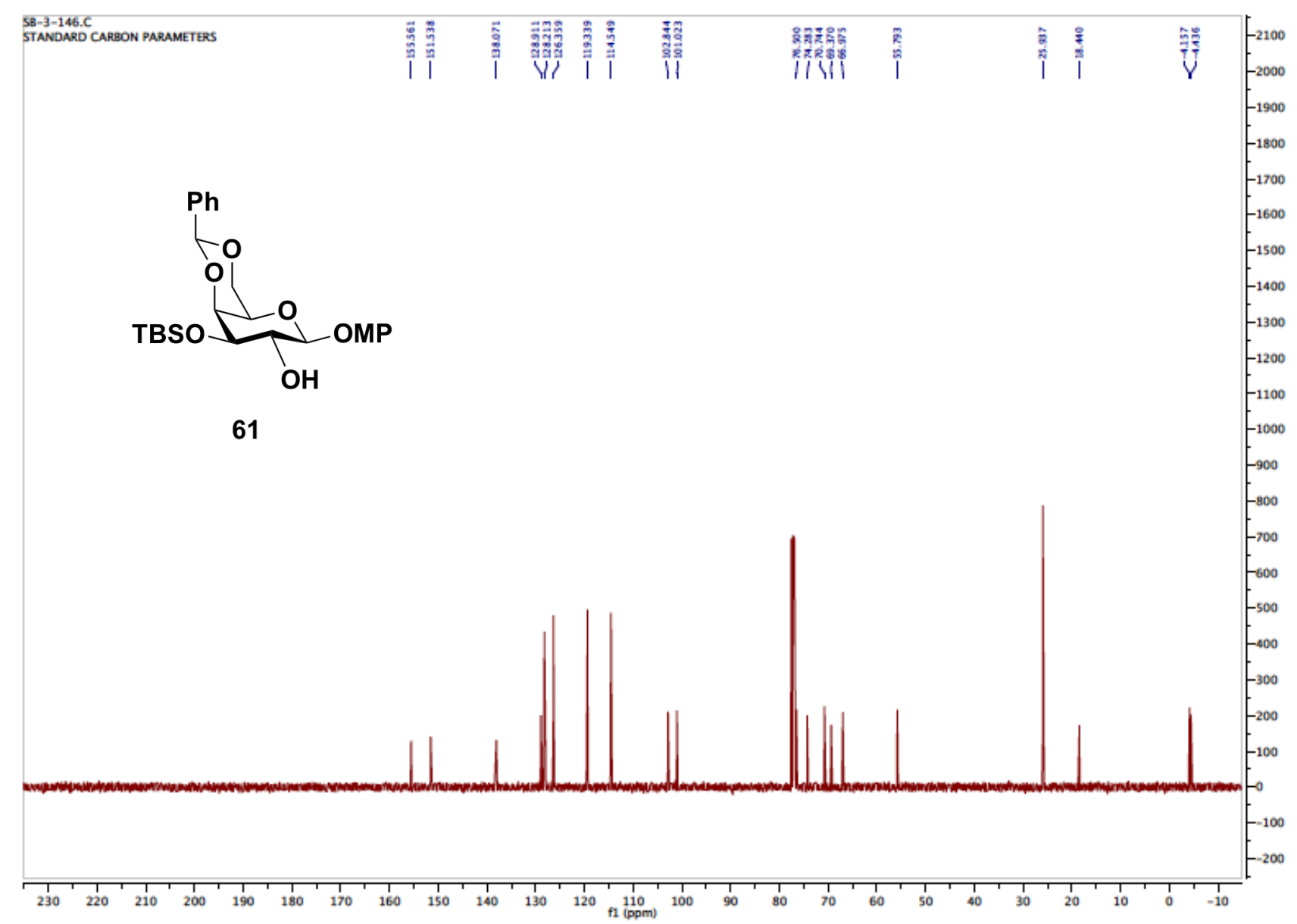

${ }^{13} \mathbf{C}$ NMR (101 MHz) spectrum of compound $\mathbf{6 1}$ in $\mathrm{CDCl}_{3}$ 


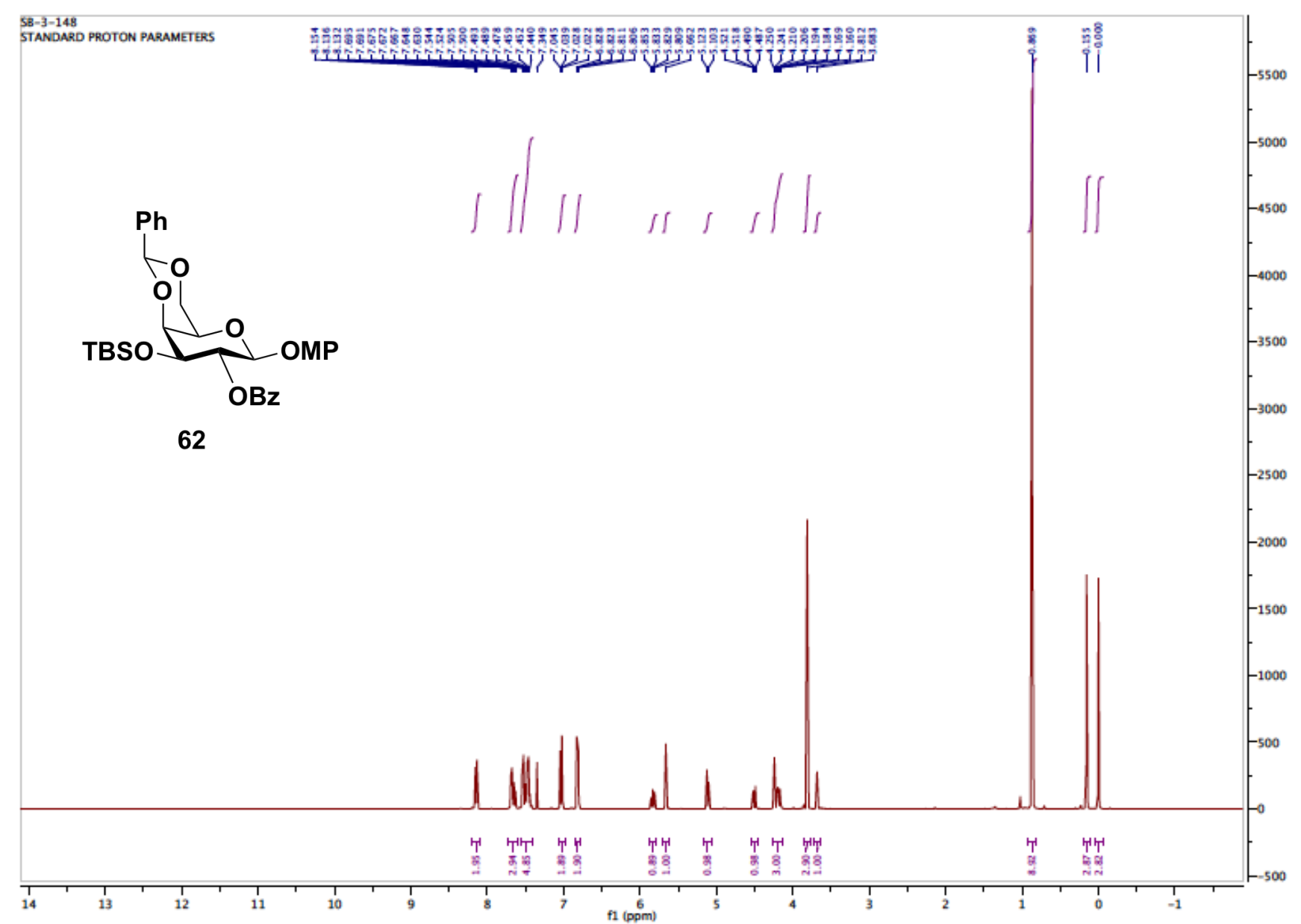

${ }^{1} \mathbf{H}$ NMR (400 MHz) spectrum of compound 62 in $\mathrm{CDCl}_{3}$ 


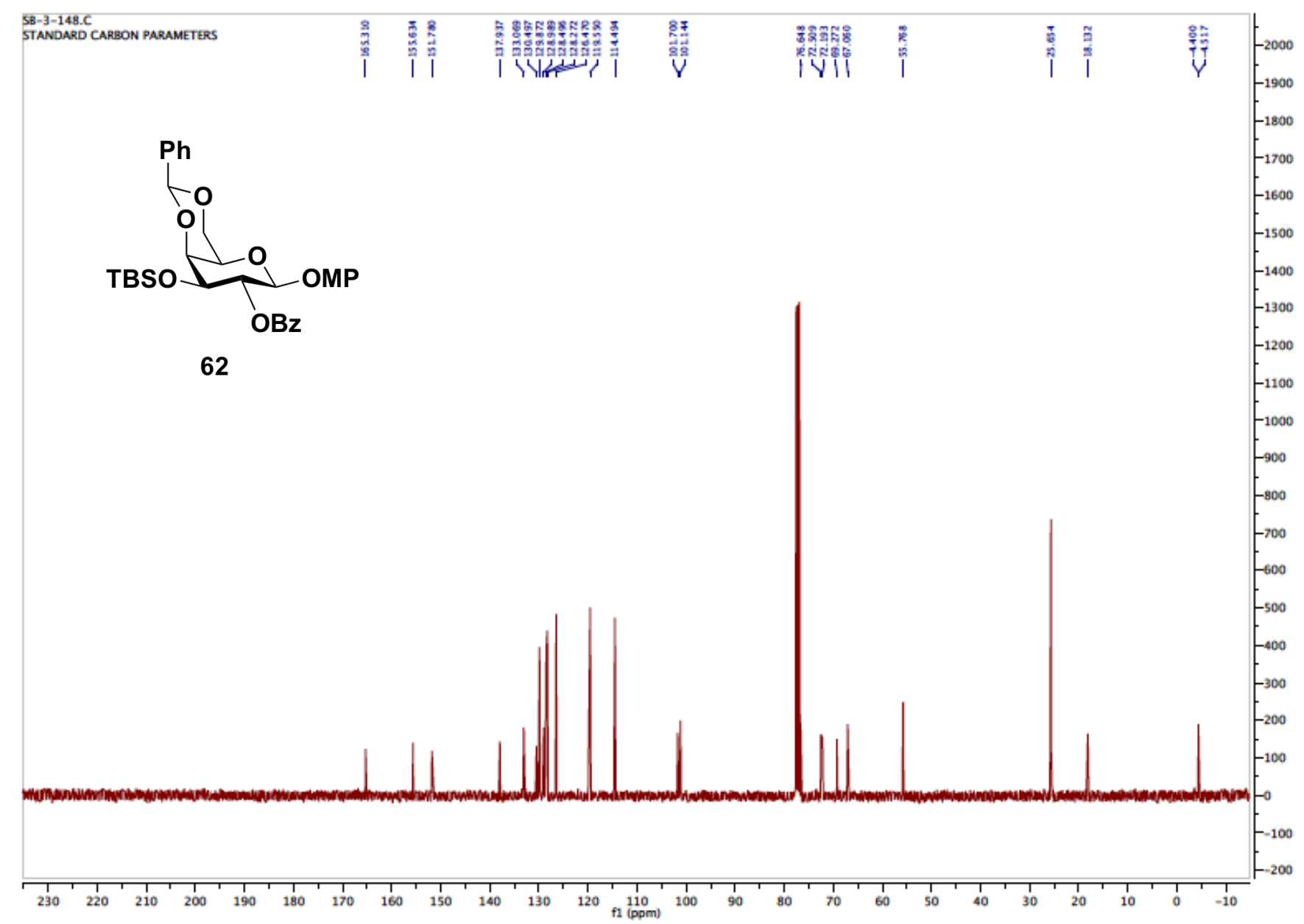

${ }^{13} \mathbf{C}$ NMR (101 MHz) spectrum of compound 62 in $\mathrm{CDCl}_{3}$ 


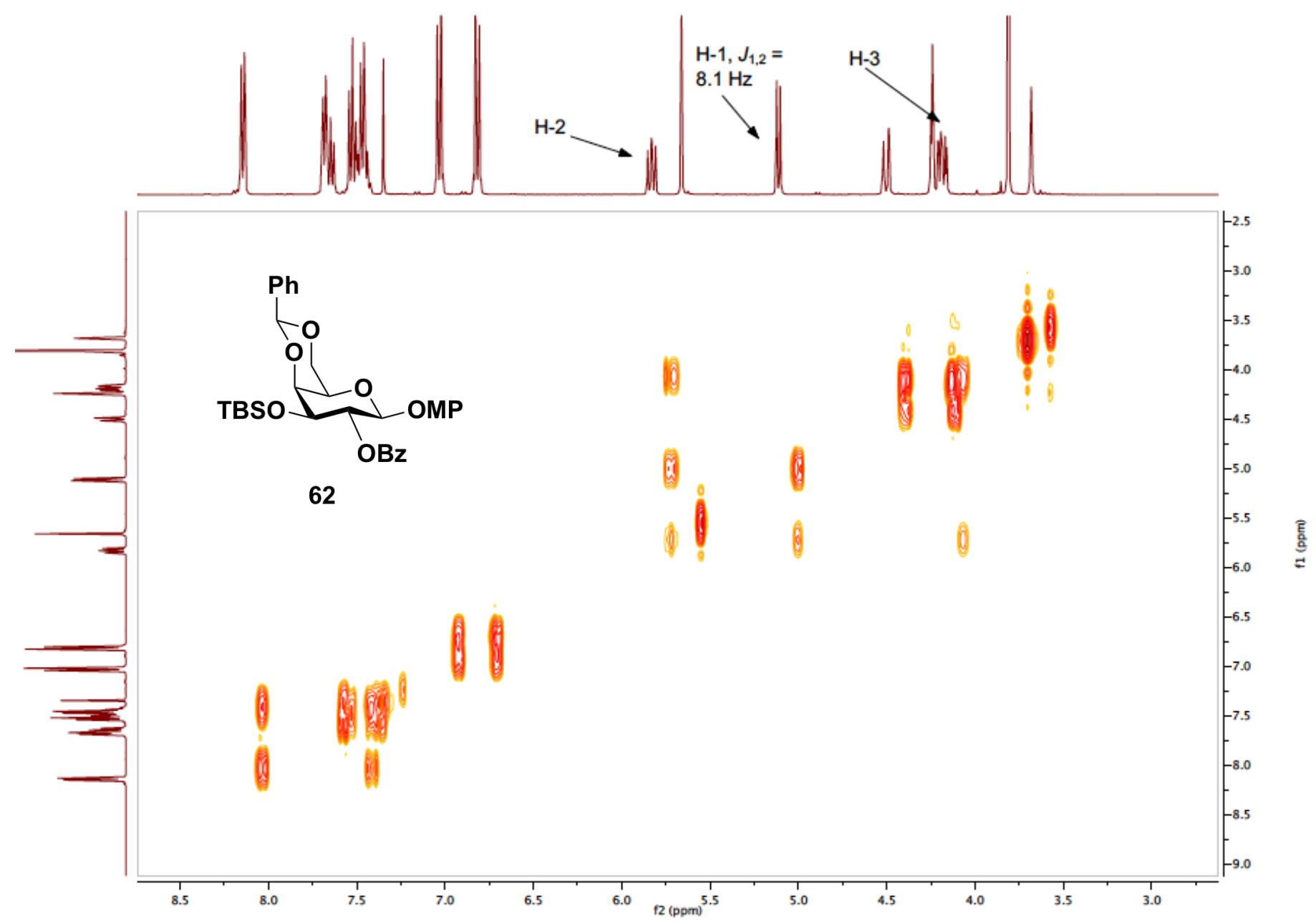

${ }^{1} \mathbf{H}$ - ${ }^{1} \mathbf{H}$ COSY NMR (400 MHz) spectrum of compound 62 in $\mathrm{CDCl}_{3}$ 


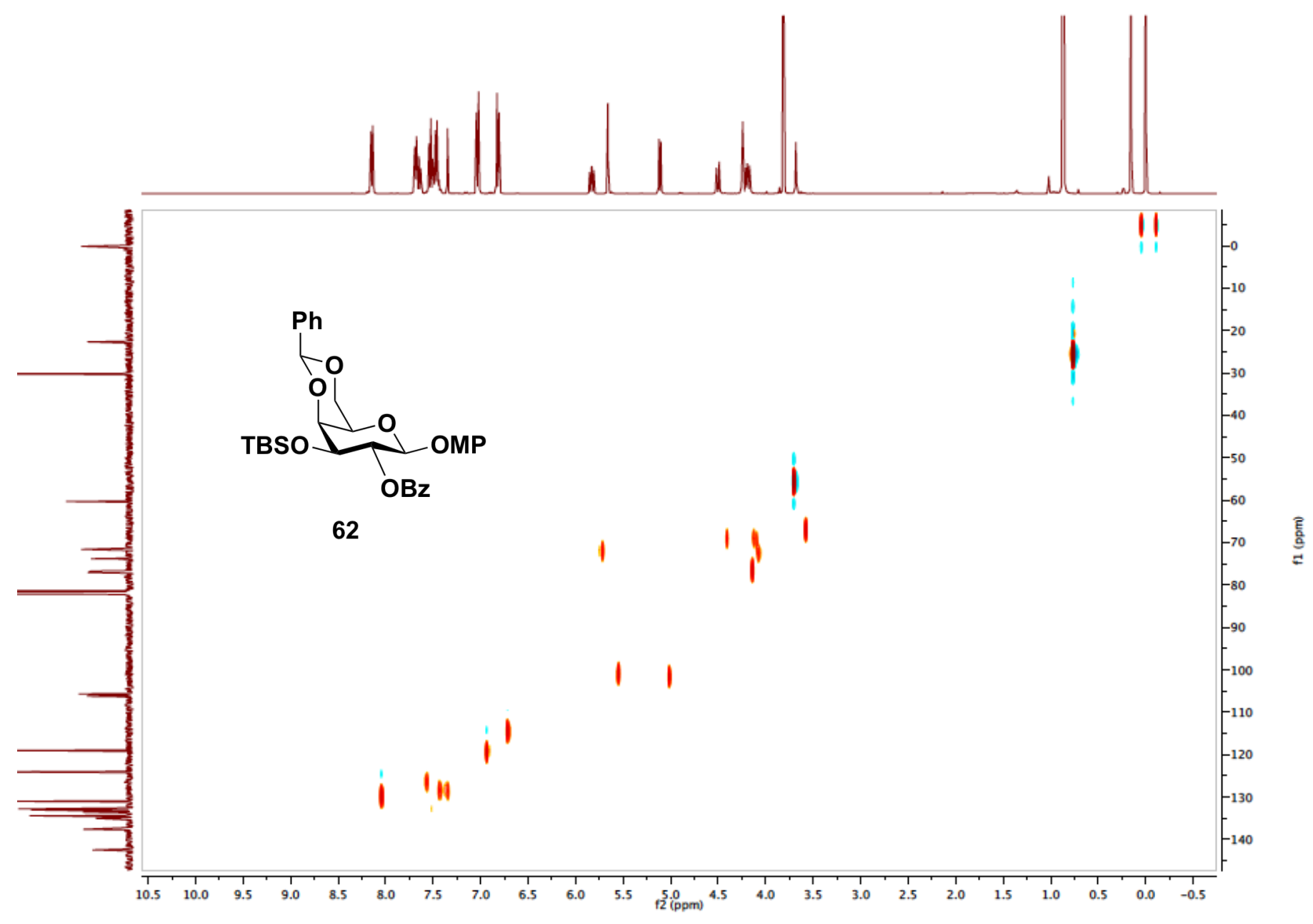

${ }^{1} \mathbf{H}-{ }^{13} \mathbf{C}$ HSQC NMR (400 MHz) spectrum of compound 62 in $\mathrm{CDCl}_{3}$ 


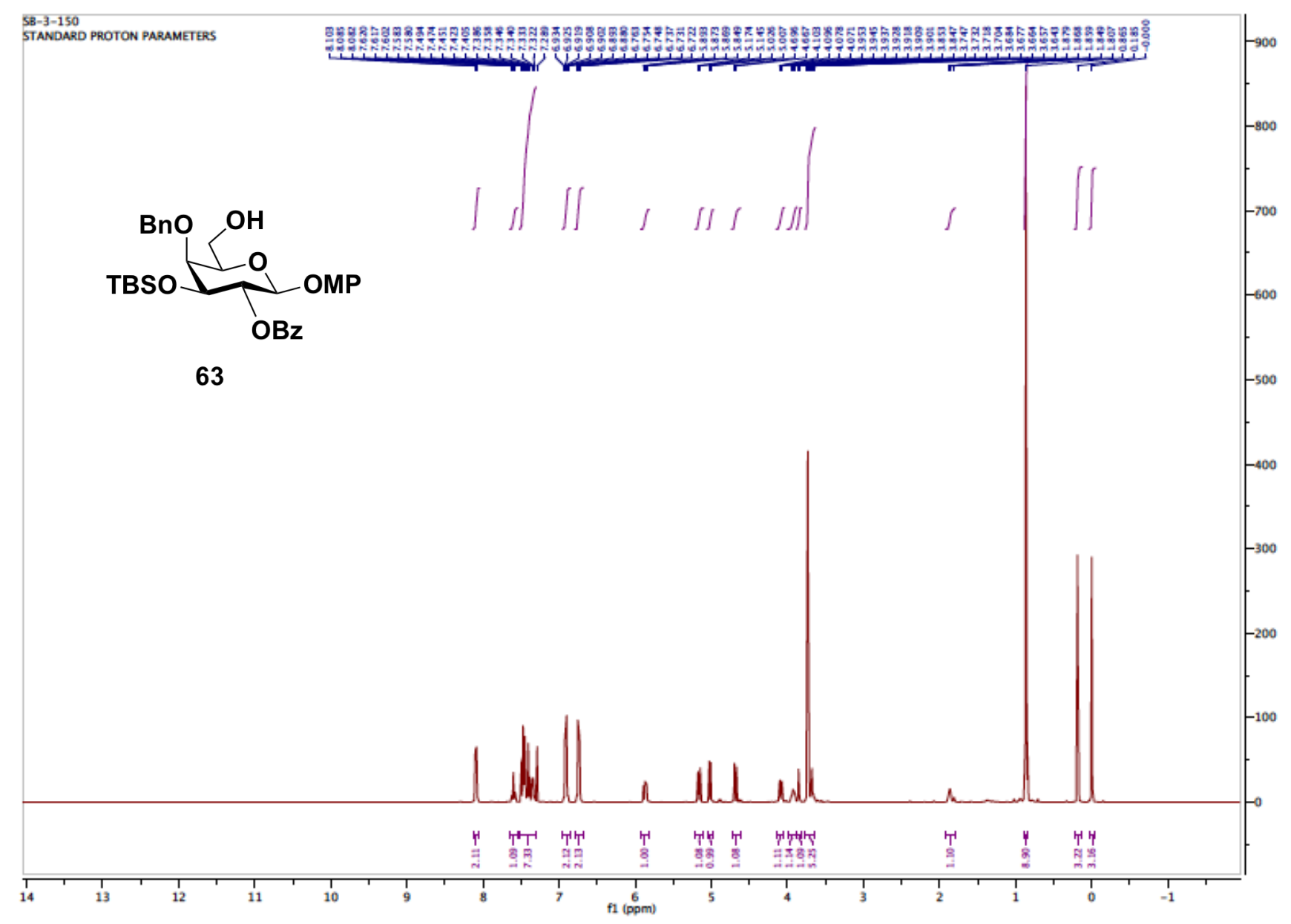

${ }^{1}$ H NMR (400 MHz) spectrum of compound 63 in $\mathrm{CDCl}_{3}$ 


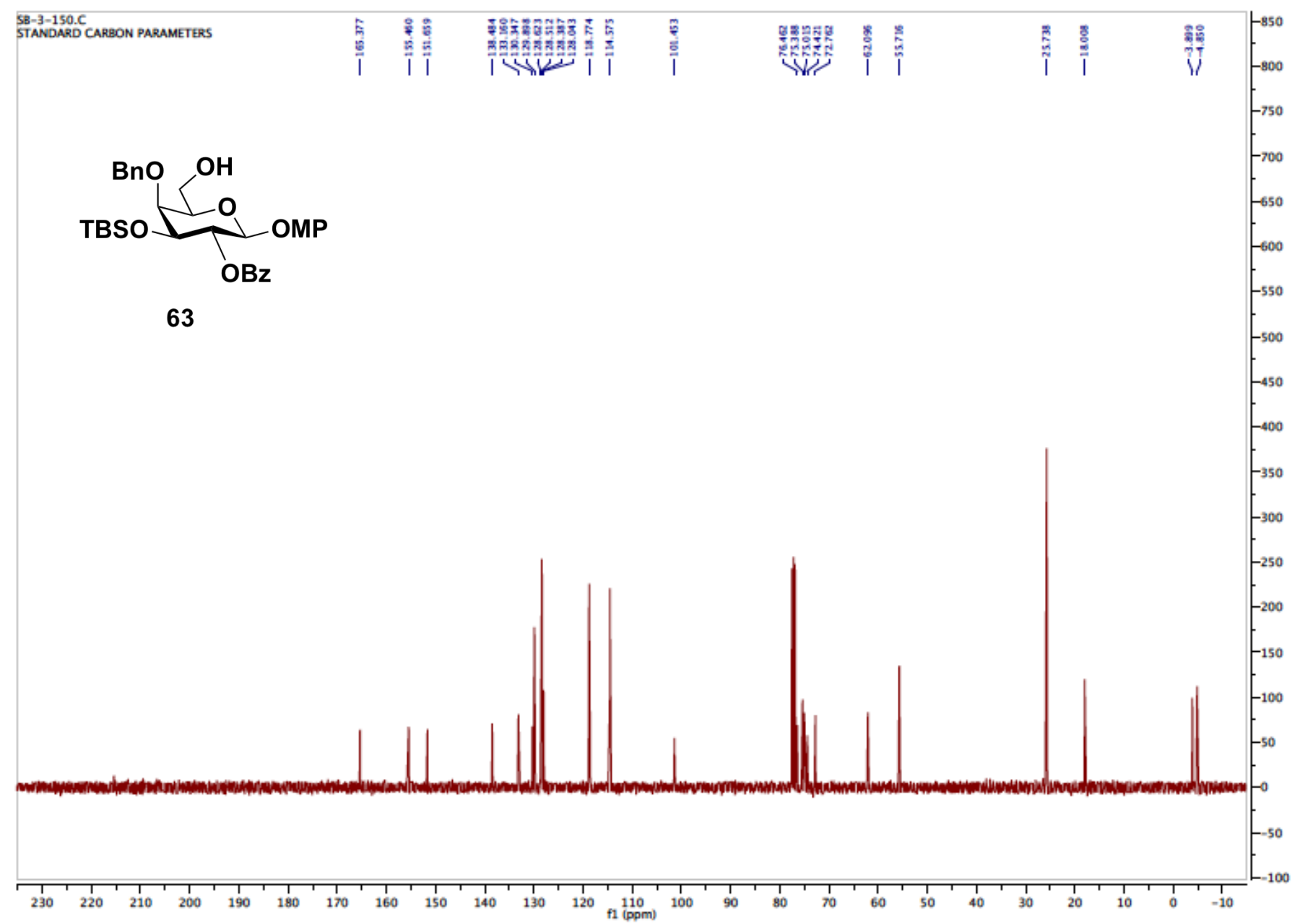

${ }^{13} \mathbf{C}$ NMR (101 MHz) spectrum of compound $\mathbf{6 3}$ in $\mathrm{CDCl}_{3}$ 


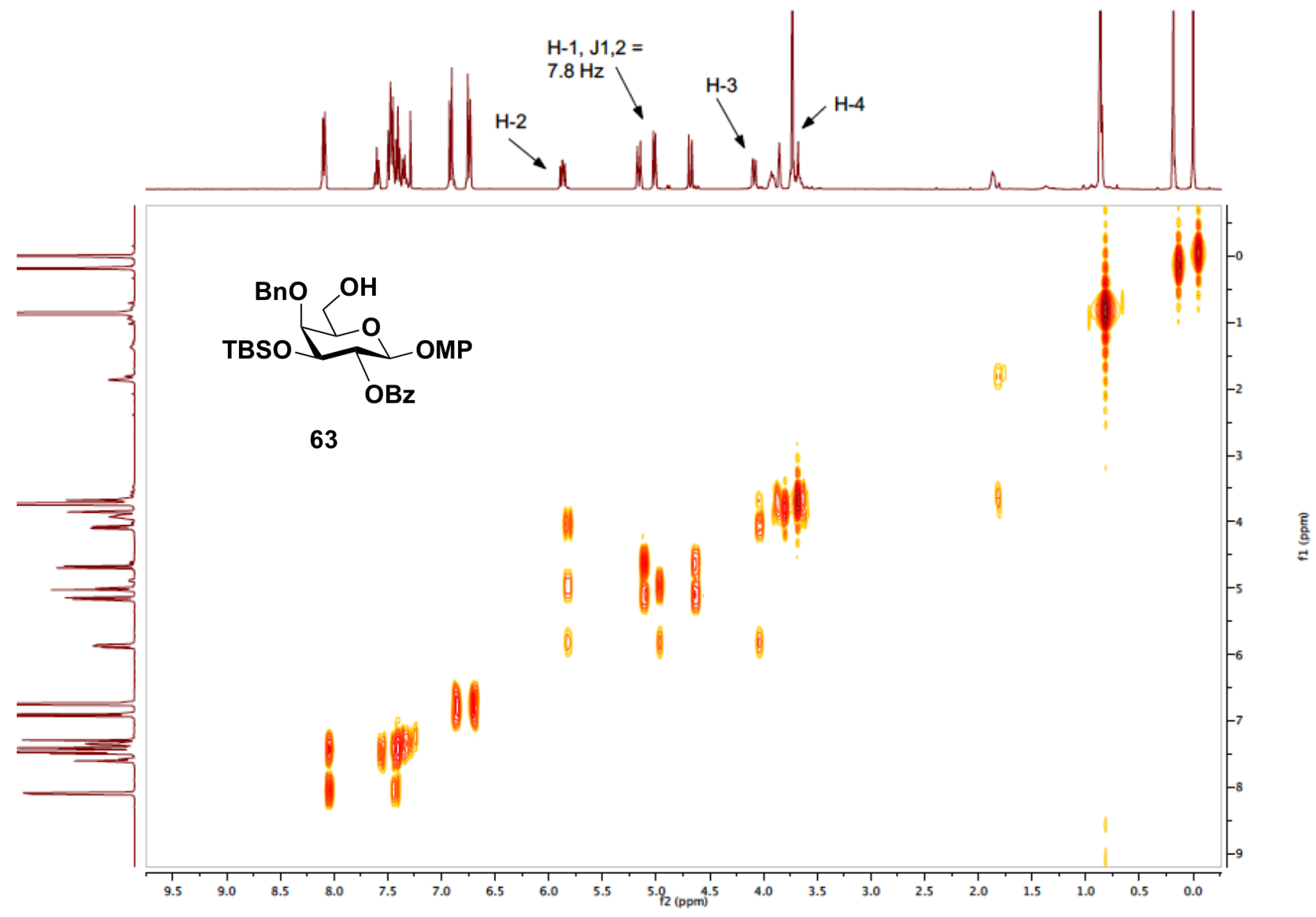

${ }^{1} \mathbf{H}$ - ${ }^{1} \mathbf{H}$ COSY NMR (400 MHz) spectrum of compound 63 in $\mathrm{CDCl}_{3}$ 


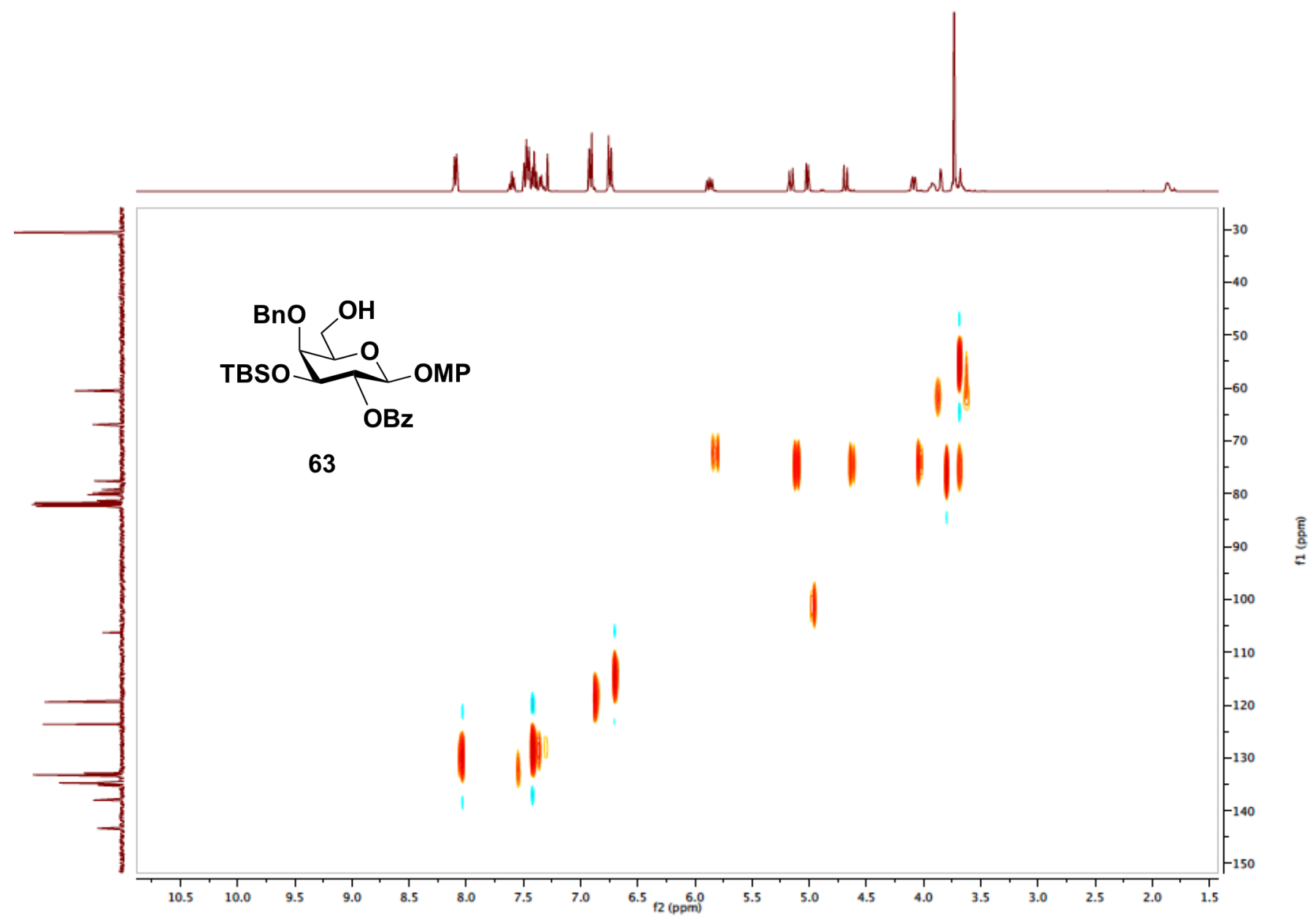

${ }^{1} \mathbf{H}-{ }^{13} \mathbf{C}$ HSQC NMR (400 MHz) spectrum of compound $\mathbf{6 3}$ in $\mathrm{CDCl}_{3}$ 


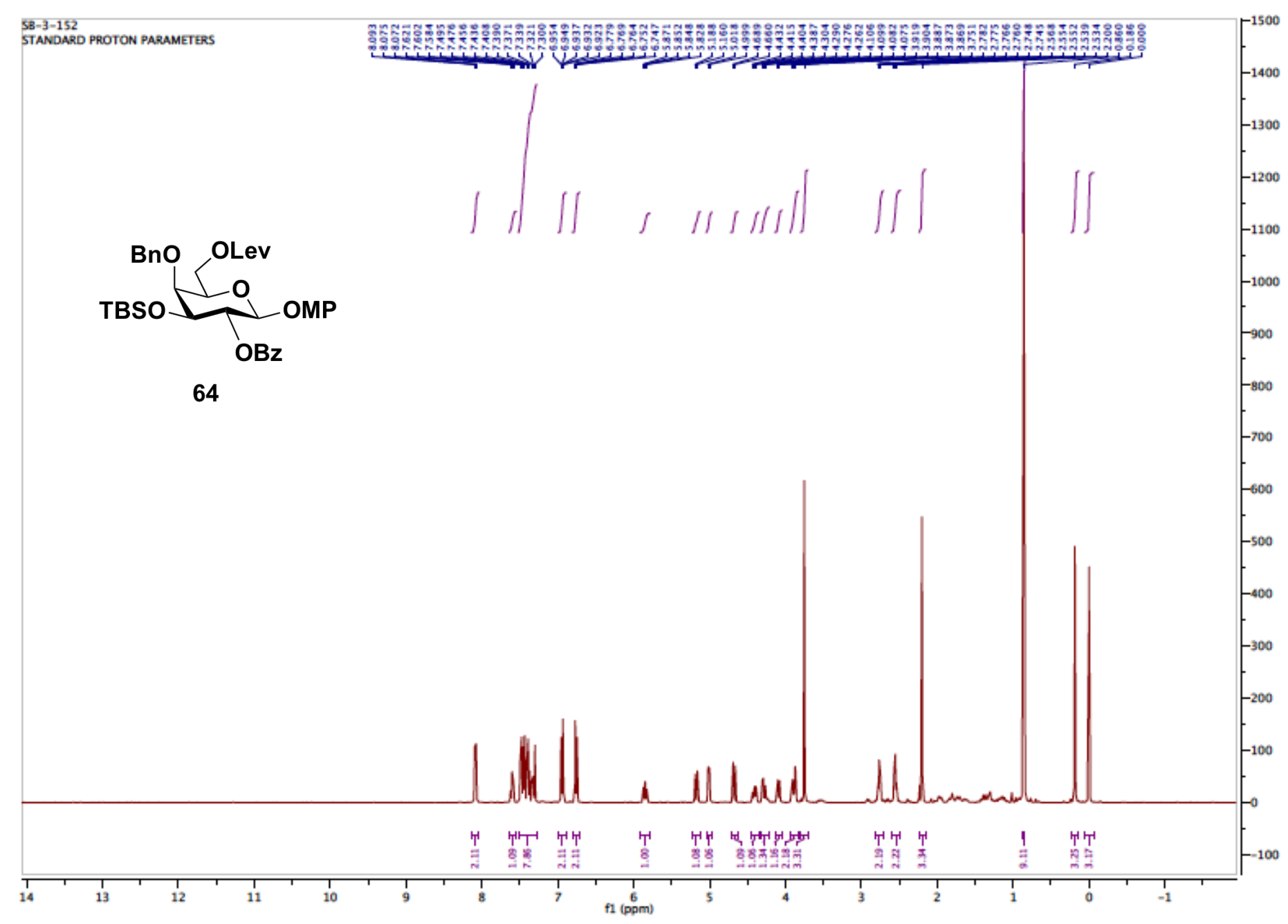

${ }^{1} \mathbf{H}$ NMR (400 MHz) spectrum of compound 64 in $\mathrm{CDCl}_{3}$ 


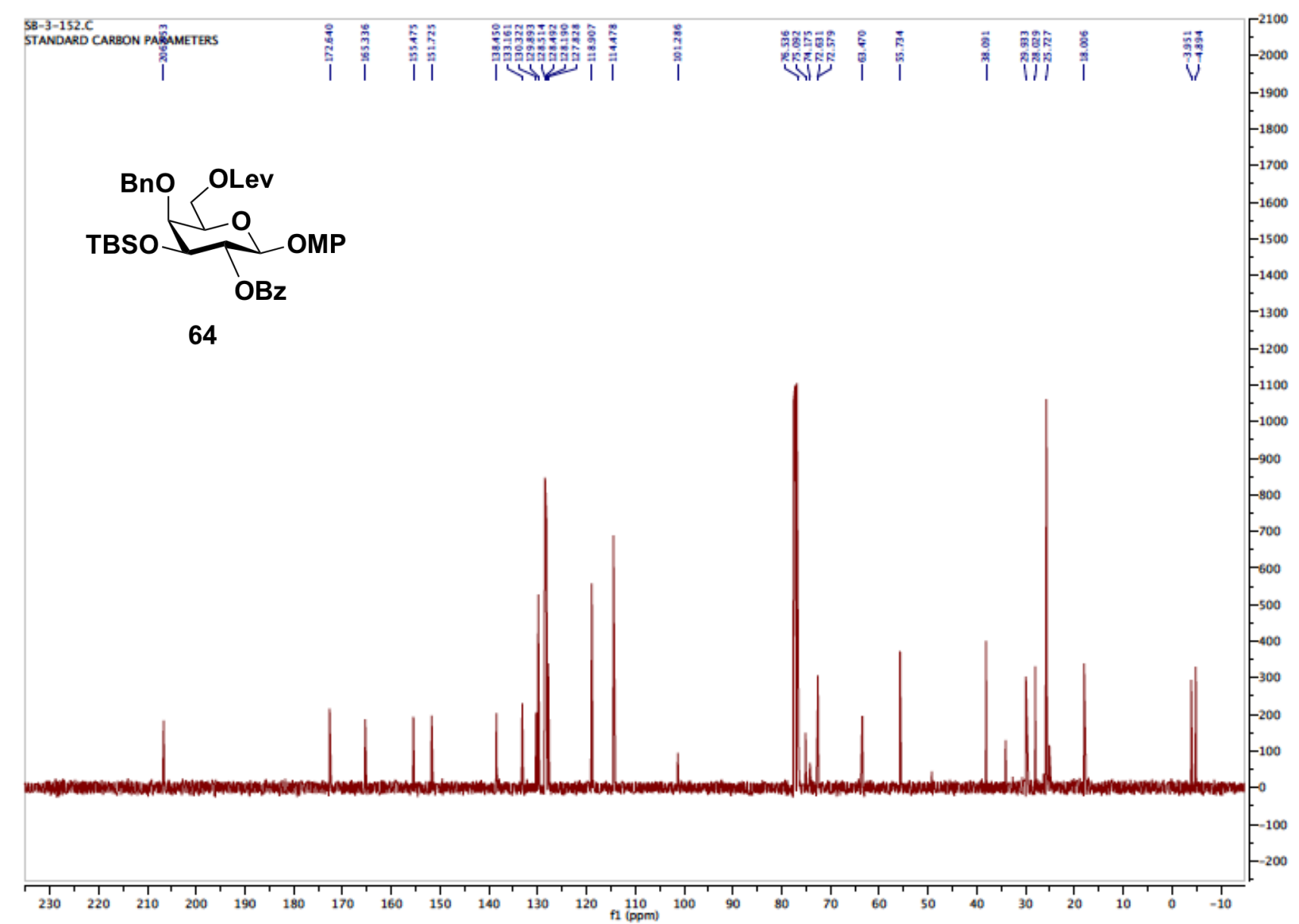

${ }^{13} \mathrm{C}$ NMR (101 MHz) spectrum of compound 64 in $\mathrm{CDCl}_{3}$ 


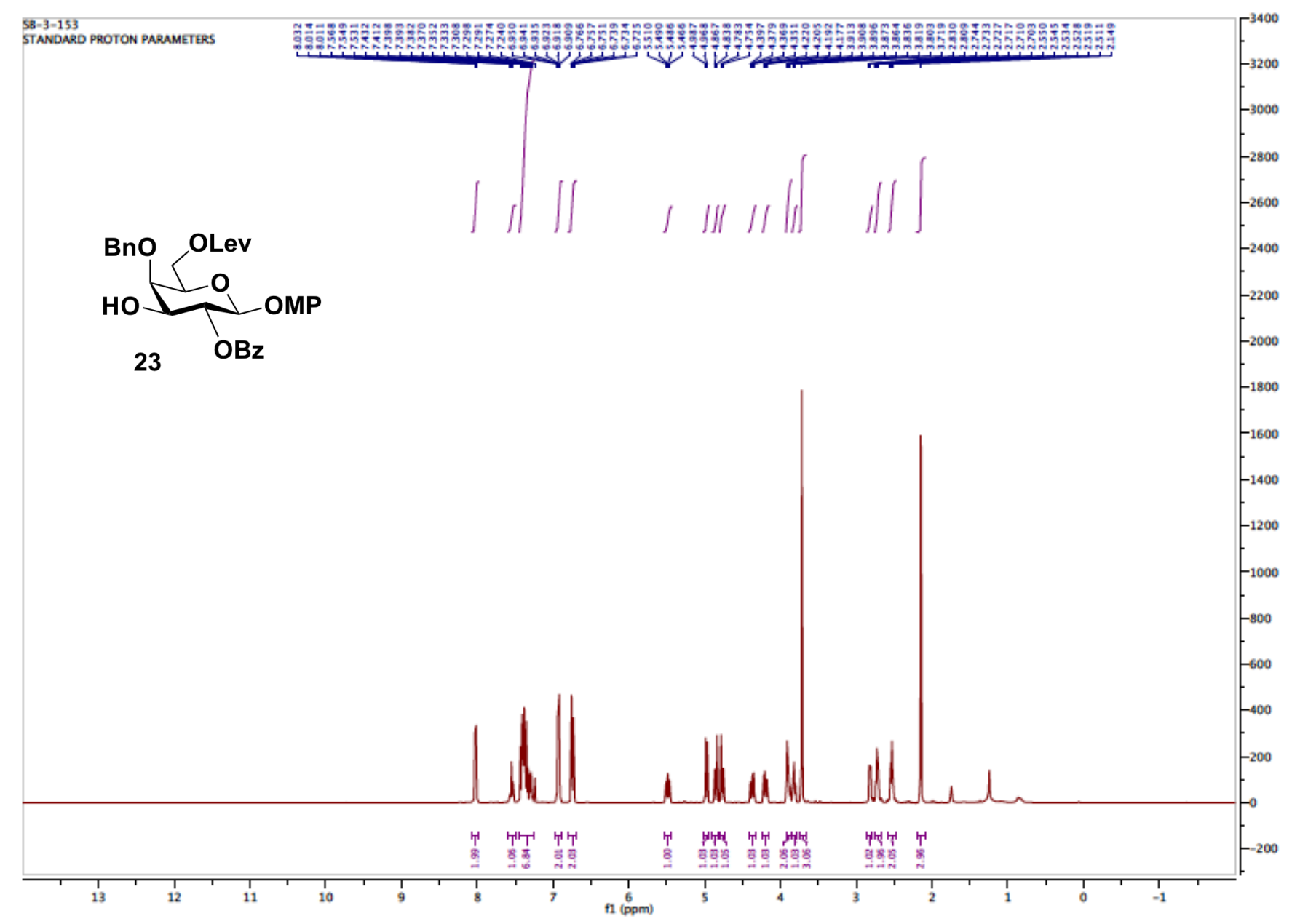

${ }^{1} \mathbf{H}$ NMR (400 MHz) spectrum of compound 23 in $\mathrm{CDCl}_{3}$ 


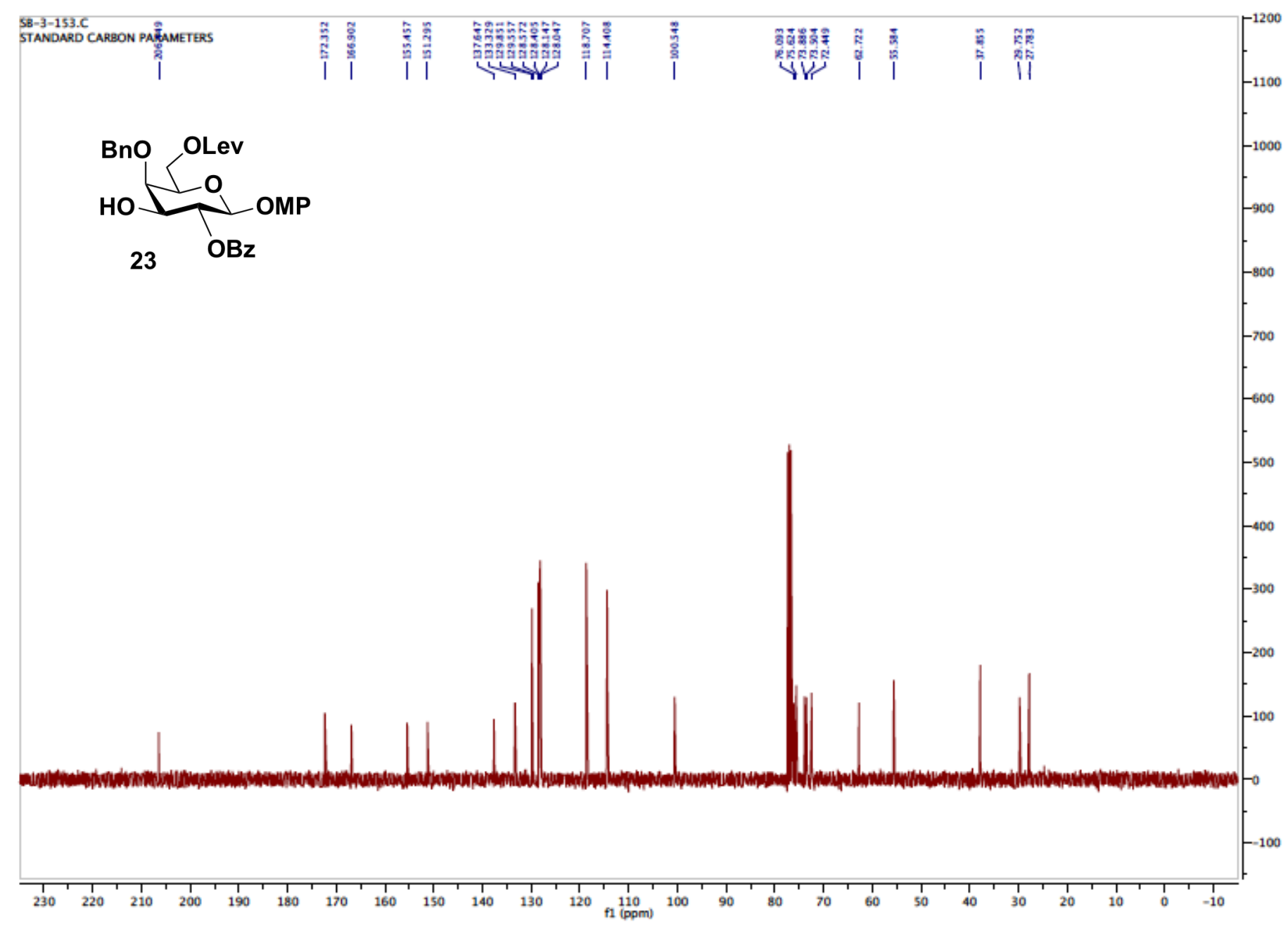

${ }^{13} \mathbf{C}$ NMR $\left(101 \mathrm{MHz}\right.$ ) spectrum of compound 23 in $\mathrm{CDCl}_{3}$ 


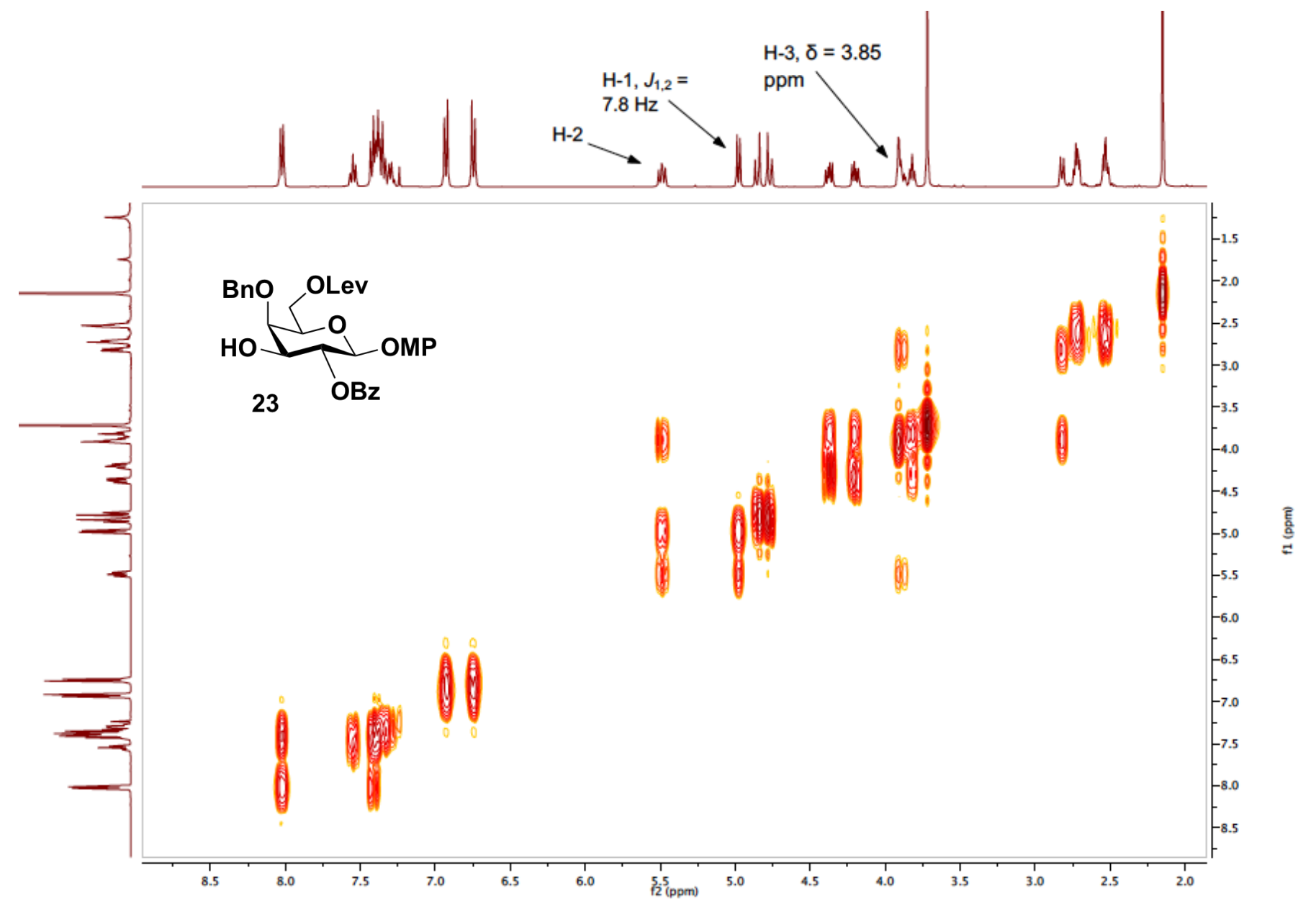

${ }^{1} \mathbf{H}-{ }^{1} \mathbf{H}$ COSY NMR (400 MHz) spectrum of compound 23 in $\mathrm{CDCl}_{3}$ 


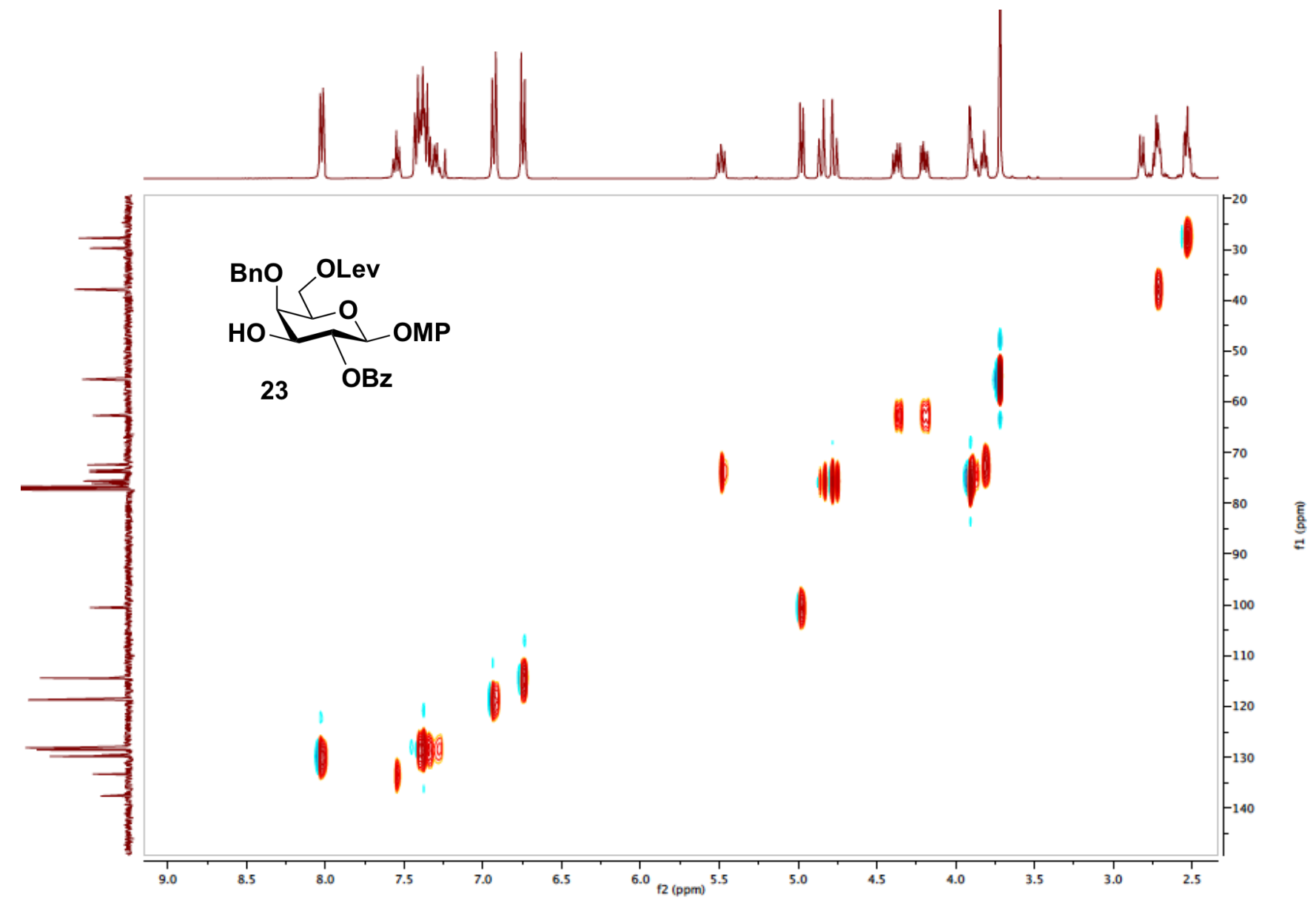

${ }^{1} \mathbf{H}-{ }^{13} \mathbf{C}$ HSQC NMR (400 MHz) spectrum of compound 23 in $\mathrm{CDCl}_{3}$ 


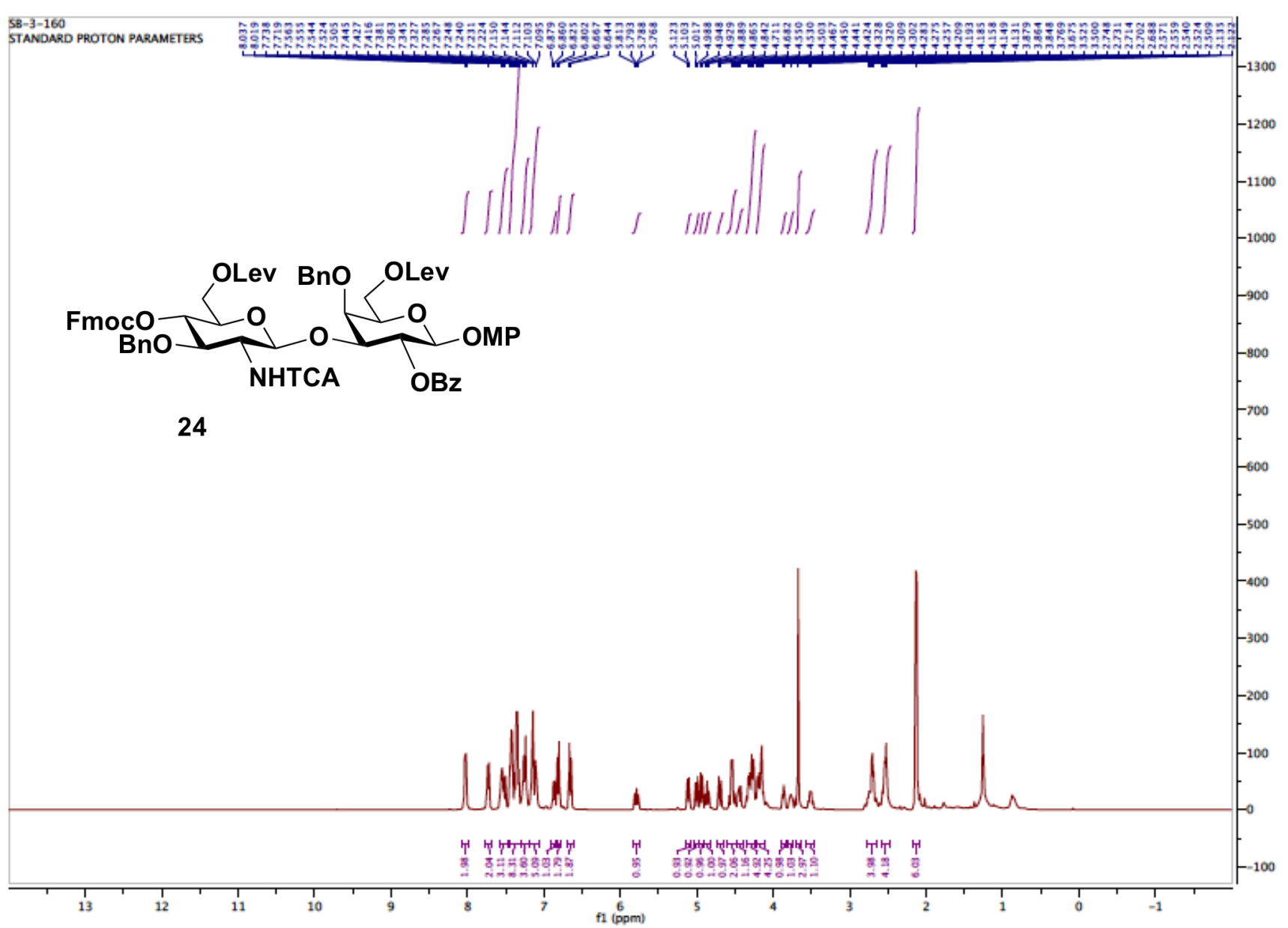

${ }^{1} \mathbf{H}$ NMR (400 MHz) spectrum of compound 24 in $\mathrm{CDCl}_{3}$ 


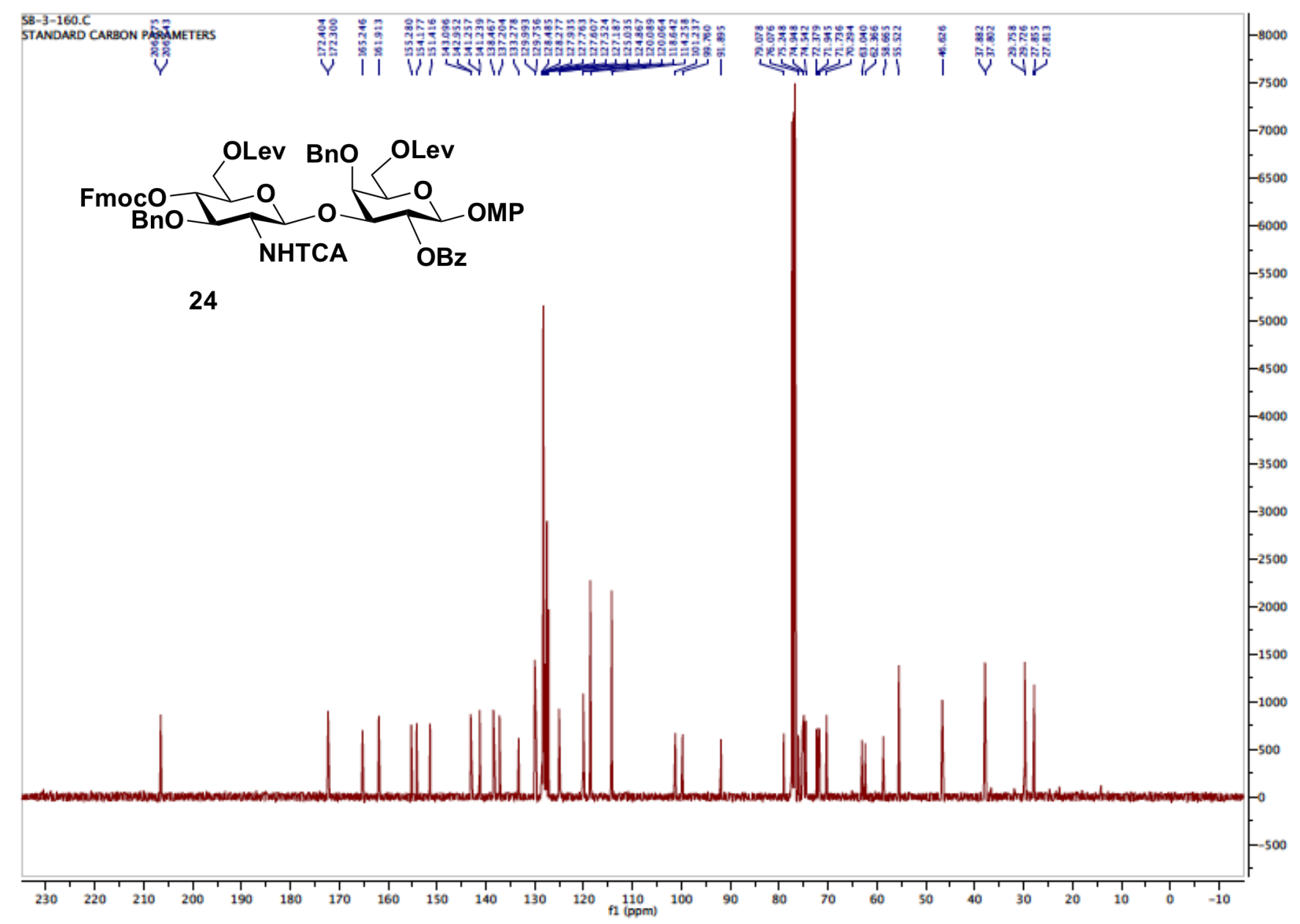

${ }^{13} \mathbf{C}$ NMR (101 MHz) spectrum of compound 24 in $\mathrm{CDCl}_{3}$ 


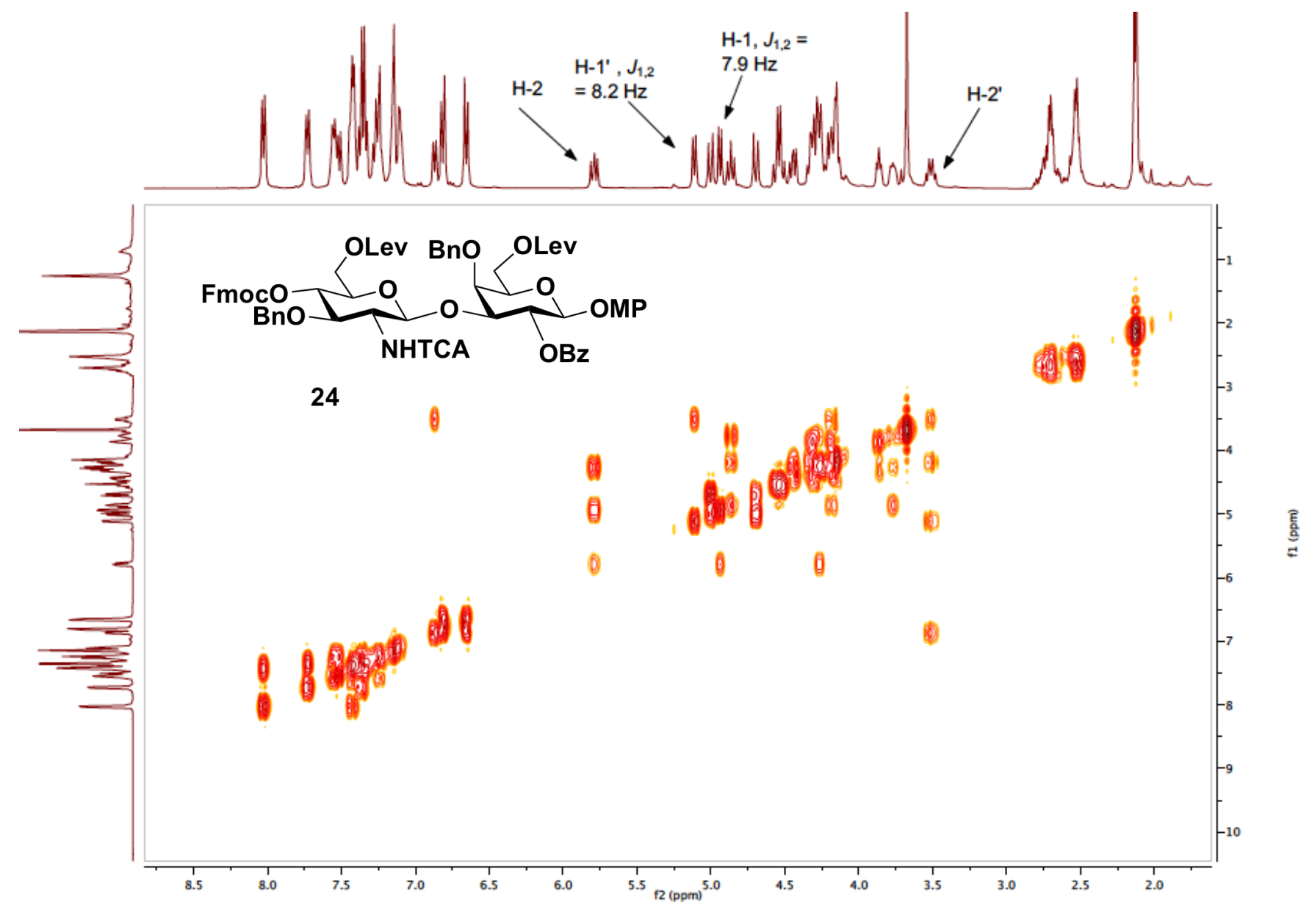

${ }^{1} \mathbf{H}-{ }^{1} \mathbf{H}$ COSY NMR (400 MHz) spectrum of compound 24 in $\mathrm{CDCl}_{3}$ 


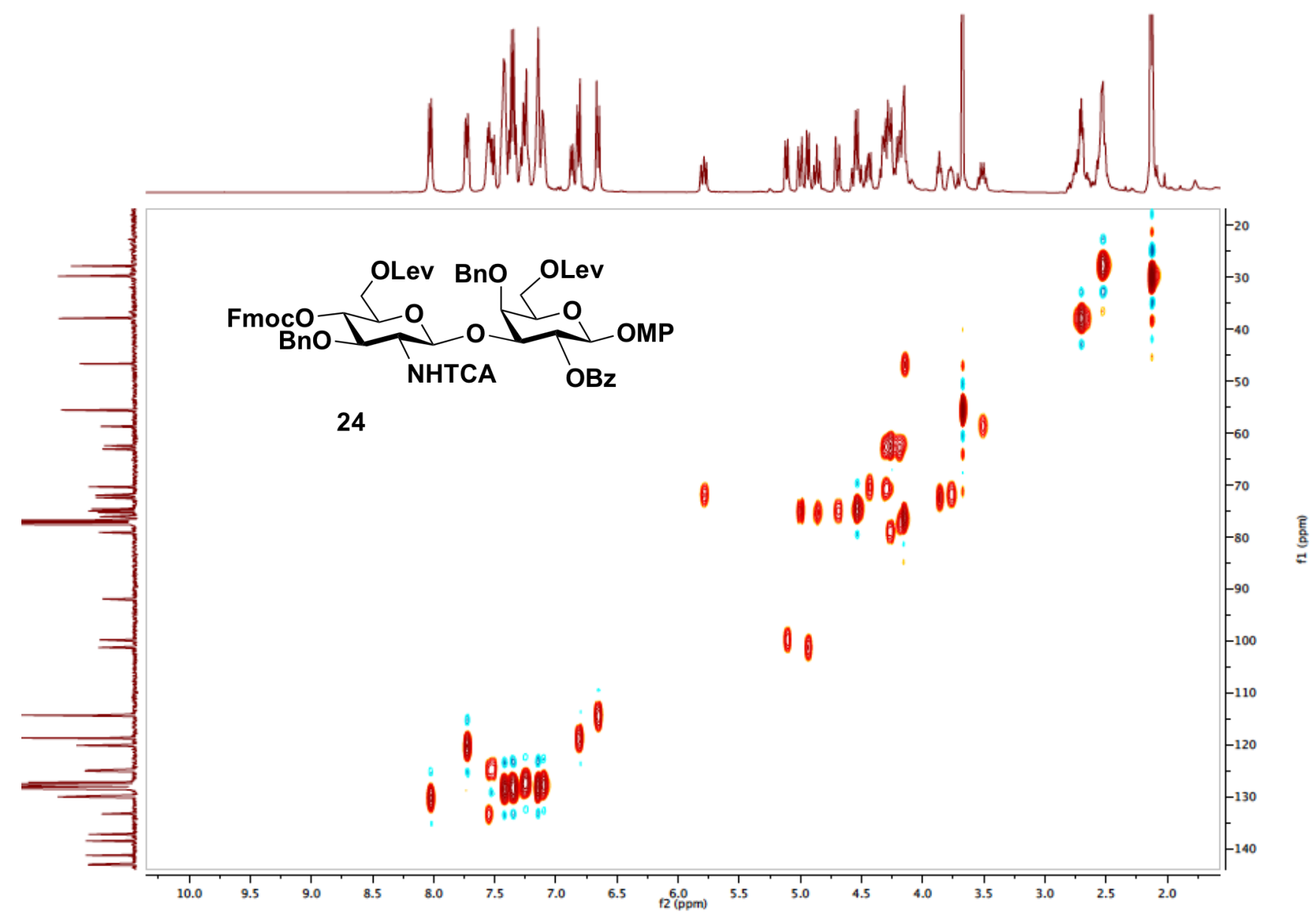

${ }^{1} \mathbf{H}-{ }^{13} \mathbf{C}$ HSQC NMR $(400 \mathrm{MHz})$ spectrum of compound 24 in $\mathrm{CDCl}_{3}$ 


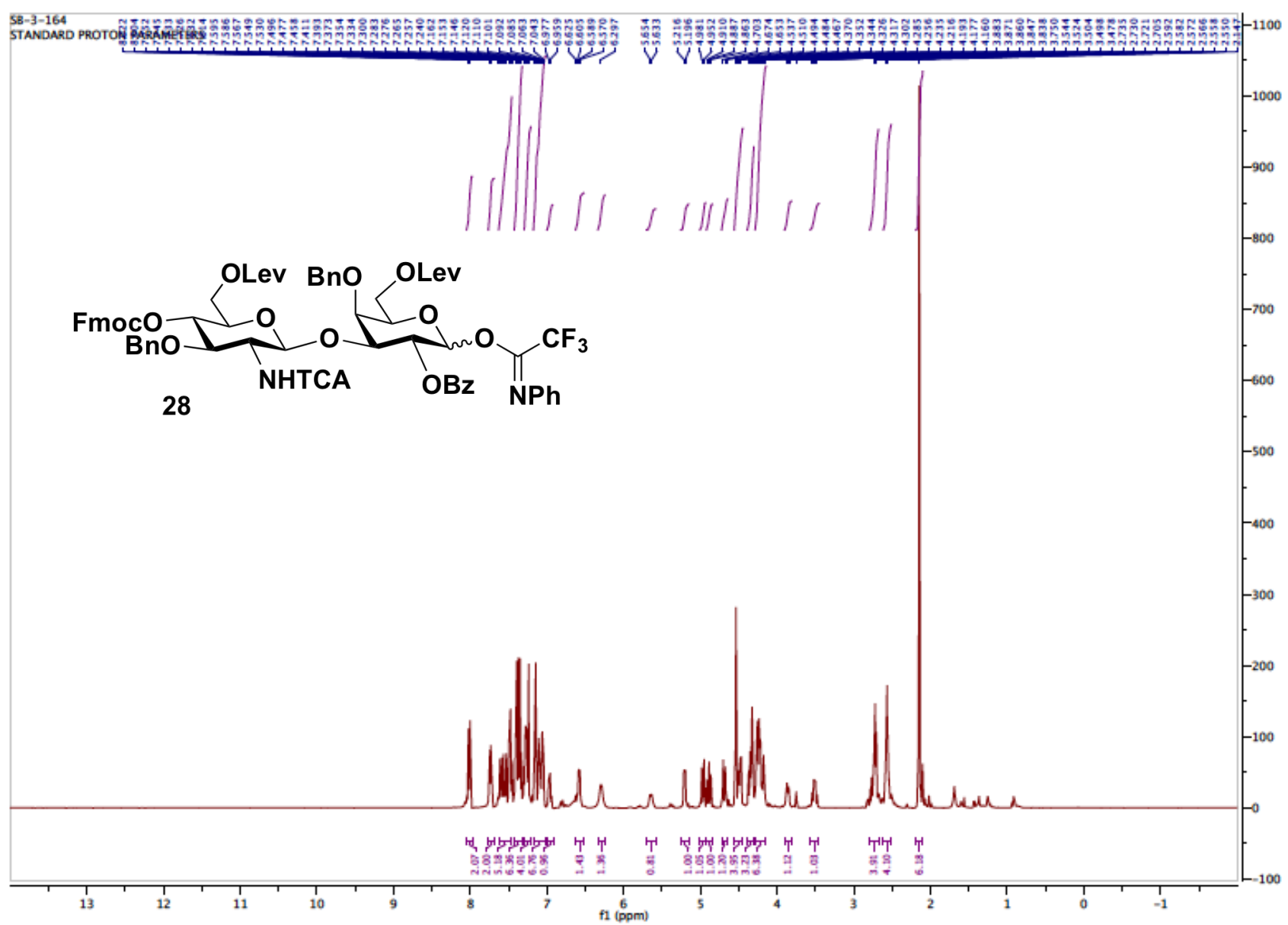

${ }^{1} \mathbf{H}$ NMR (400 MHz) spectrum of compound 28 in $\mathrm{CDCl}_{3}$ 


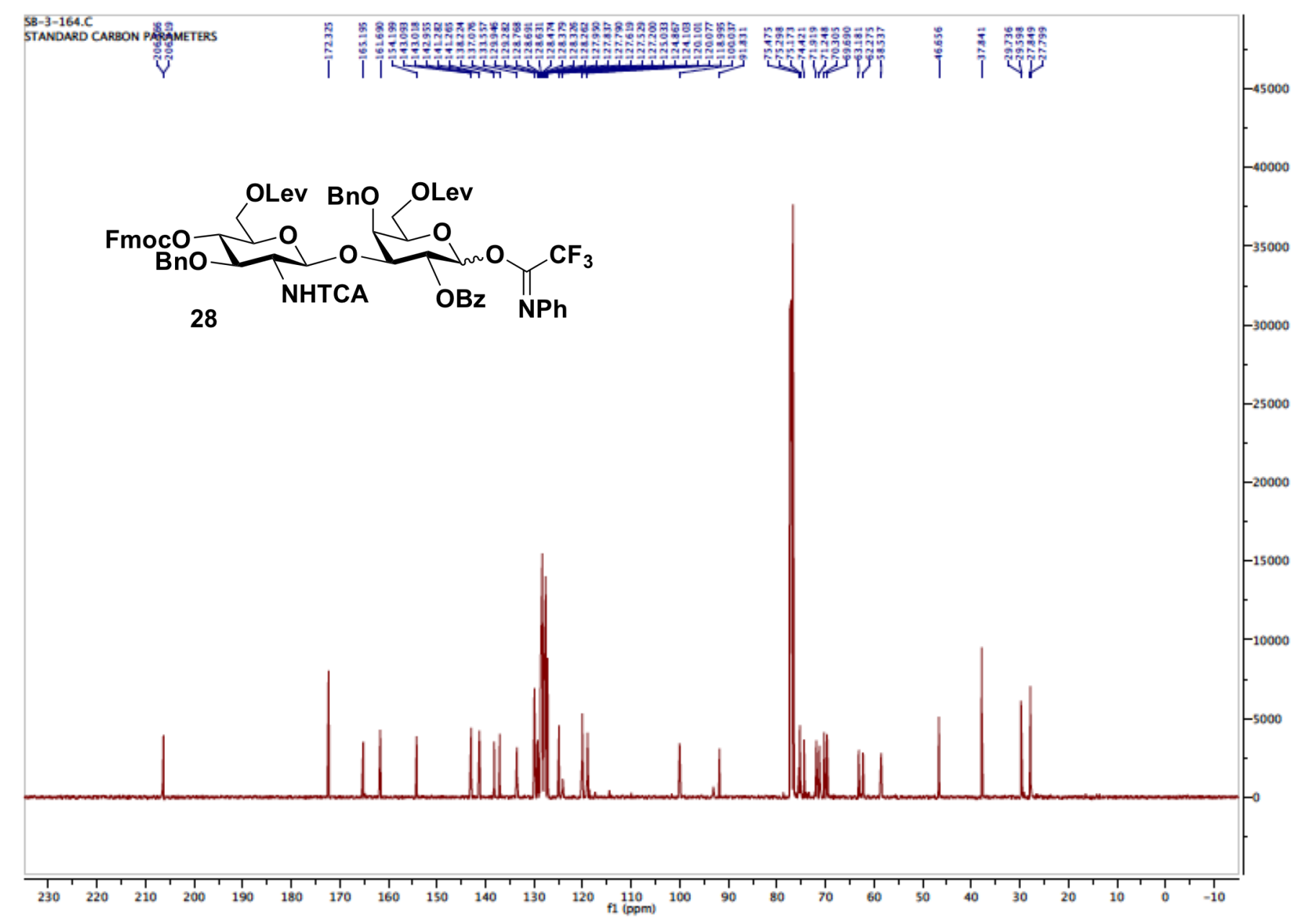

${ }^{13} \mathbf{C}$ NMR (101 MHz) spectrum of compound 28 in $\mathrm{CDCl}_{3}$ 


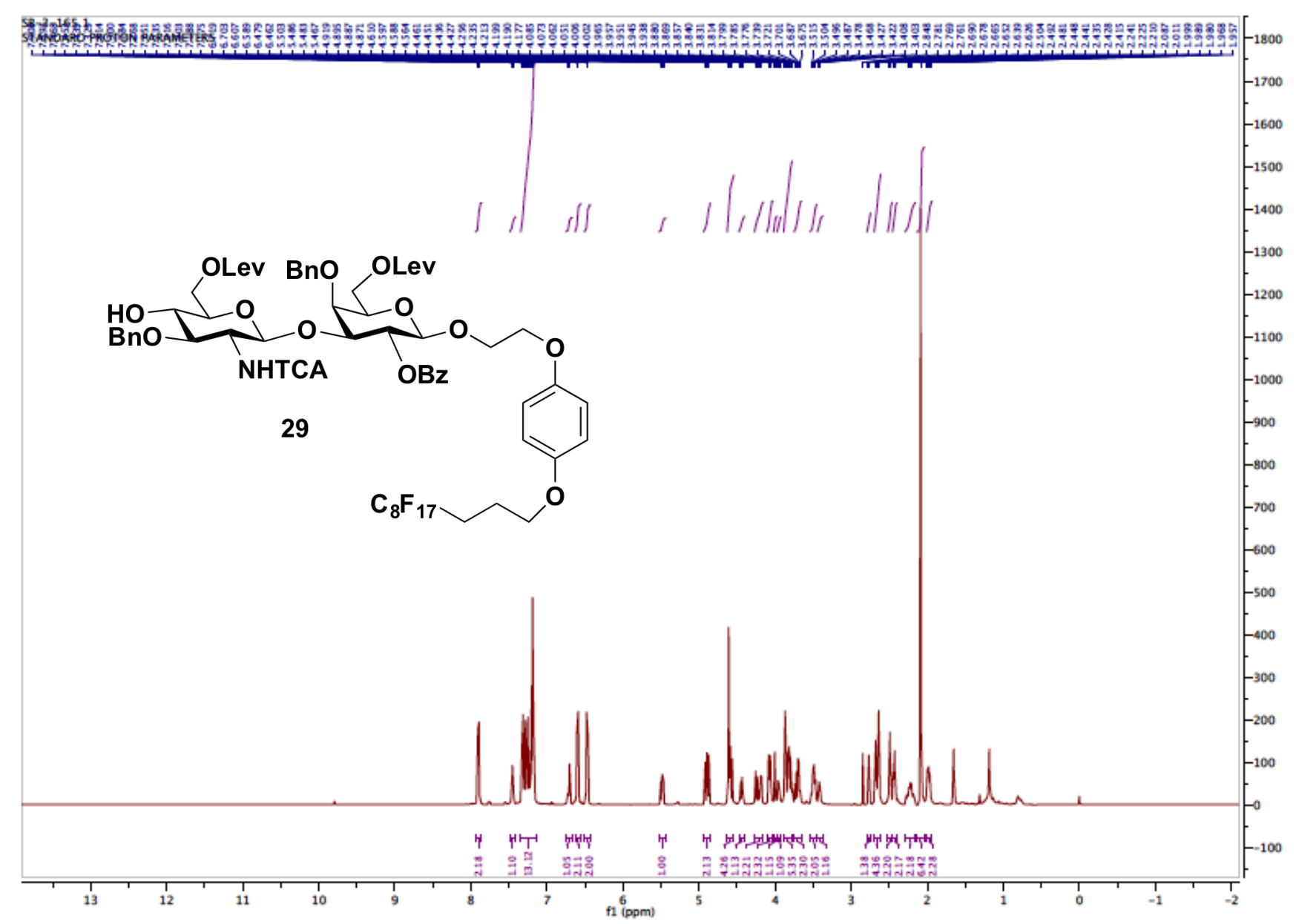

${ }^{1} \mathbf{H}$ NMR (500 MHz) spectrum of compound 29 in $\mathrm{CDCl}_{3}$ 


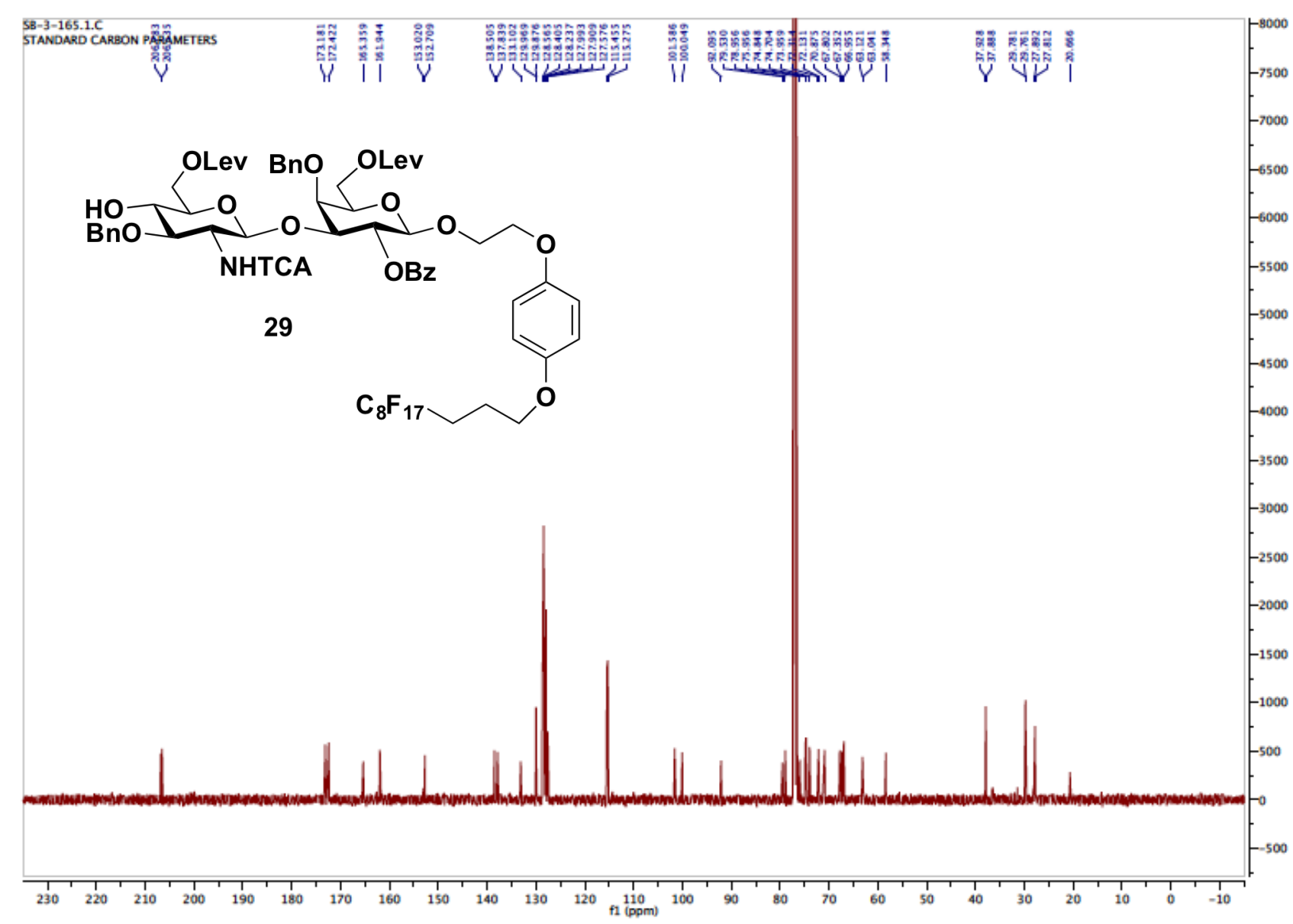

${ }^{13} \mathbf{C}$ NMR (126 MHz) spectrum of compound 29 in $\mathrm{CDCl}_{3}$ 


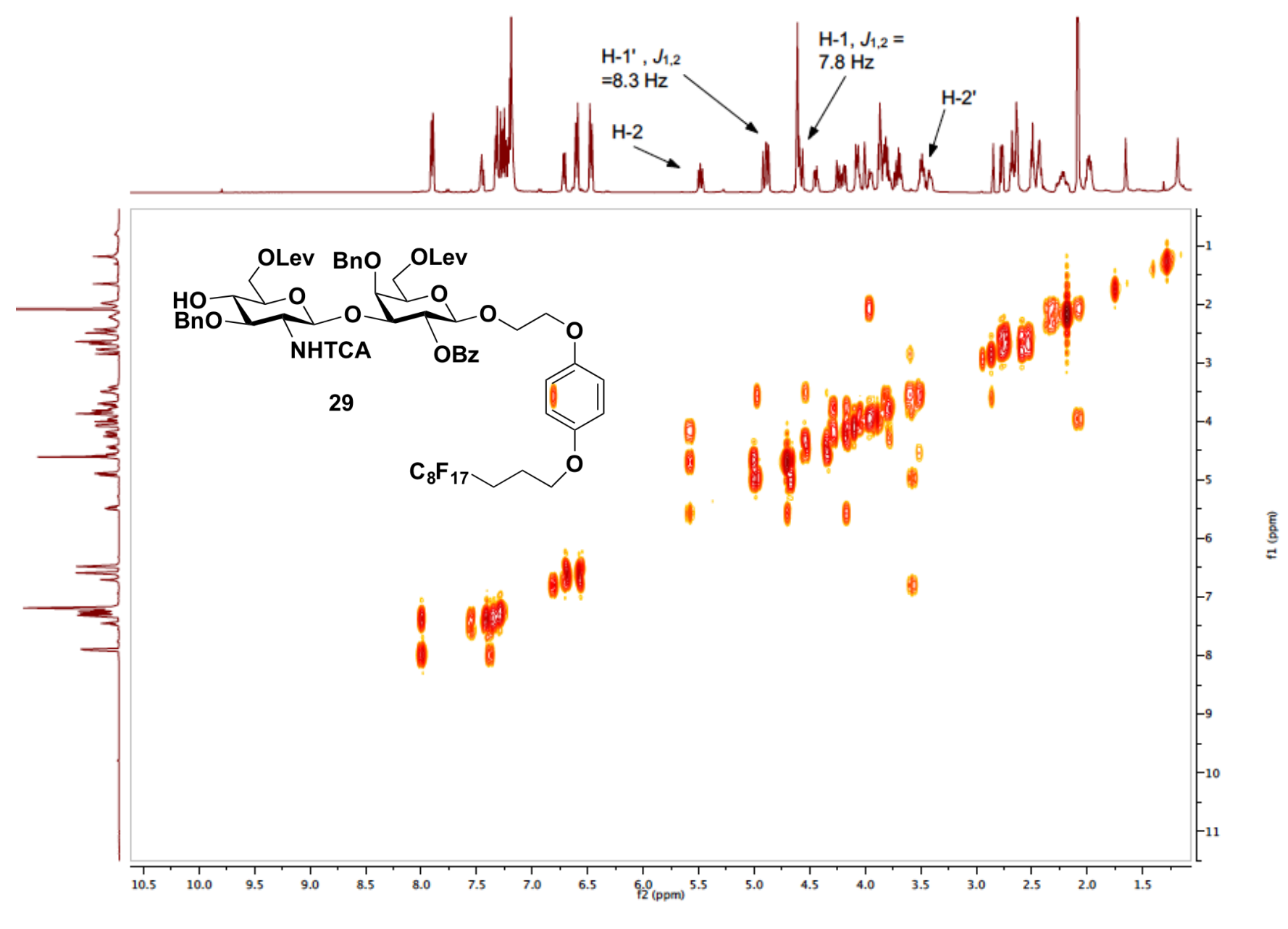

${ }^{1} \mathbf{H}-{ }^{1} \mathbf{H}$ COSY NMR (500 MHz) spectrum of compound 29 in $\mathrm{CDCl}_{3}$ 


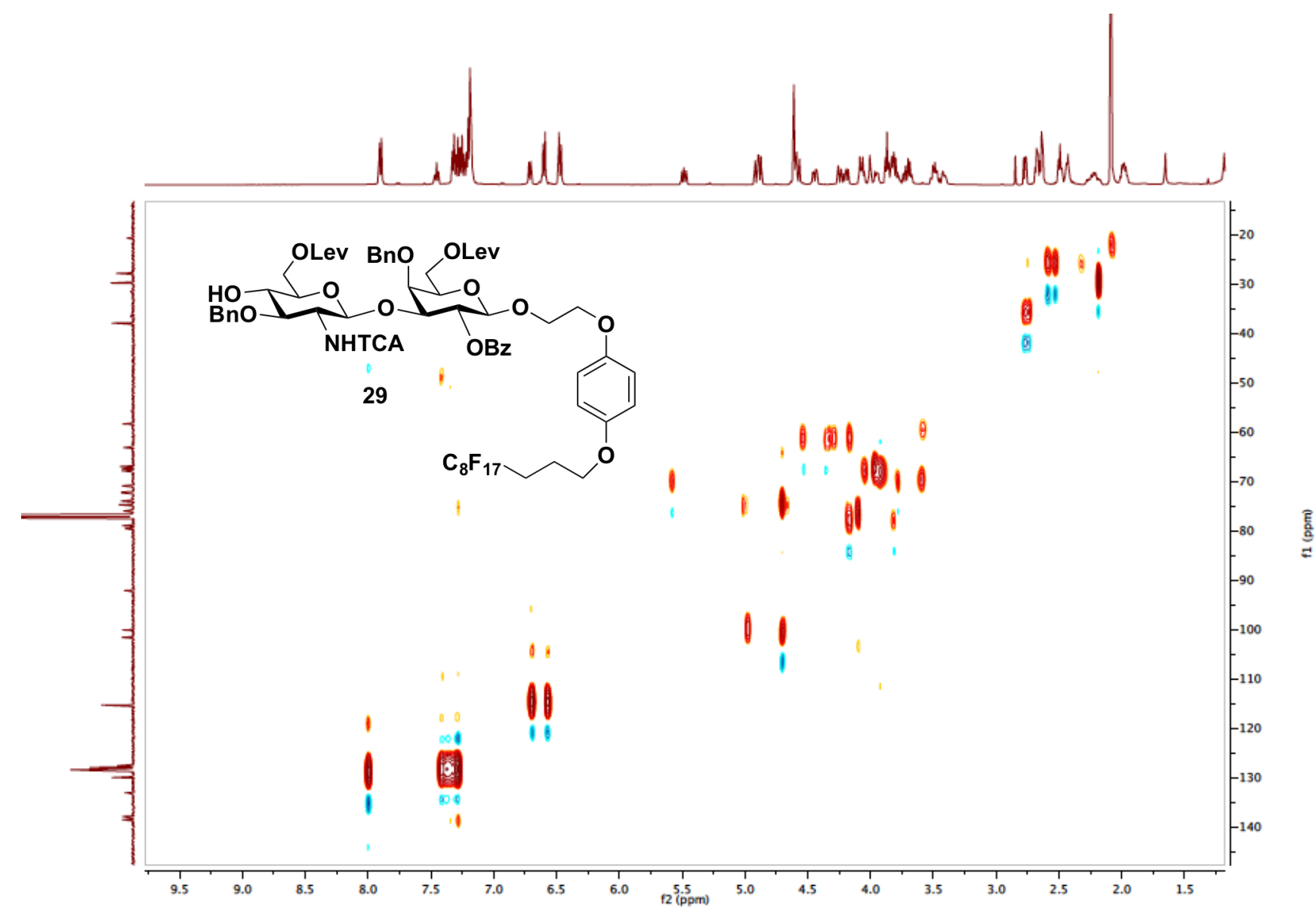

${ }^{1} \mathbf{H}-{ }^{13} \mathbf{C}$ HSQC NMR (500 MHz) spectrum of compound 29 in $\mathrm{CDCl}_{3}$ 


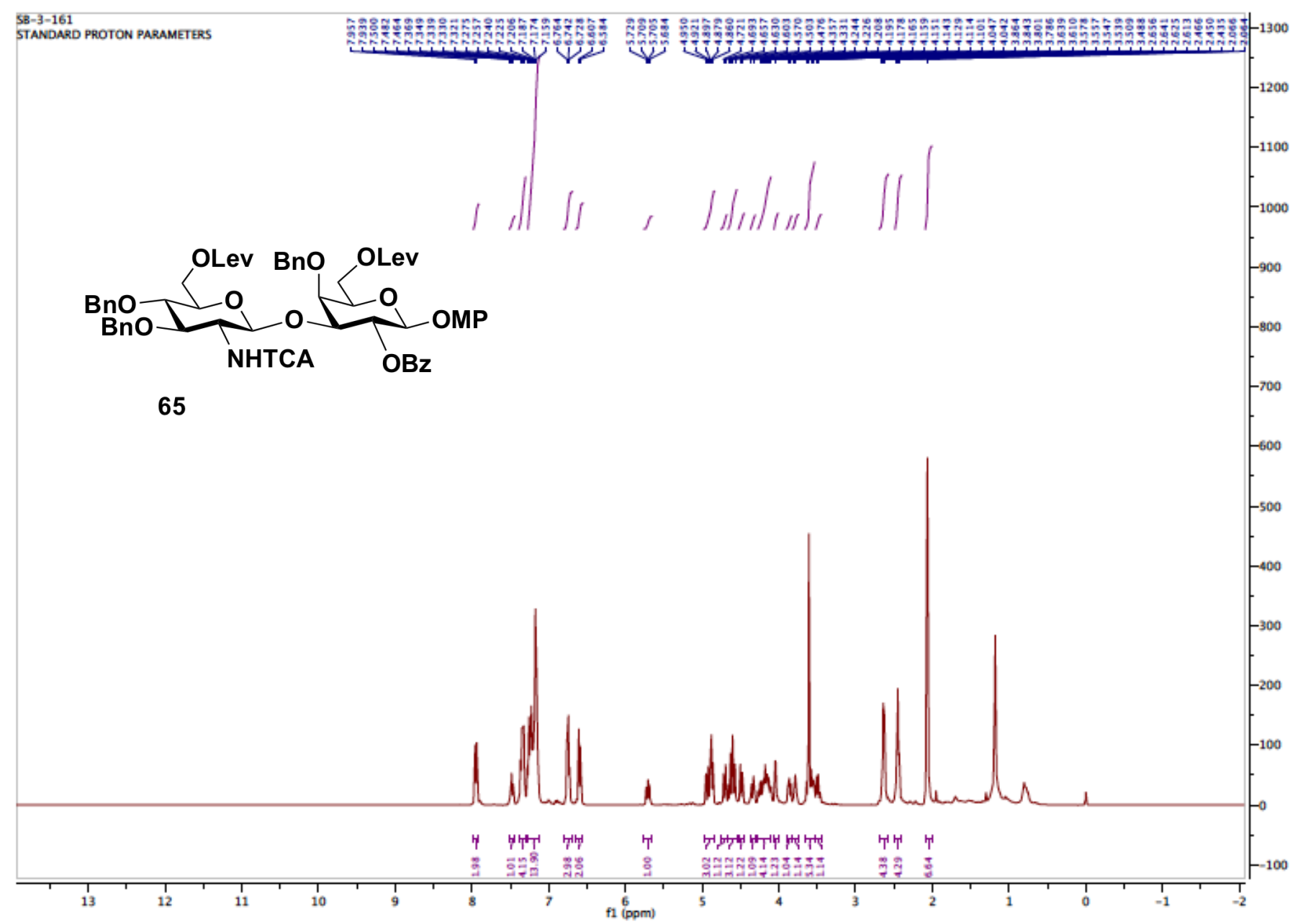

${ }^{1} \mathbf{H}$ NMR (400 MHz) spectrum of compound 65 in $\mathrm{CDCl}_{3}$ 


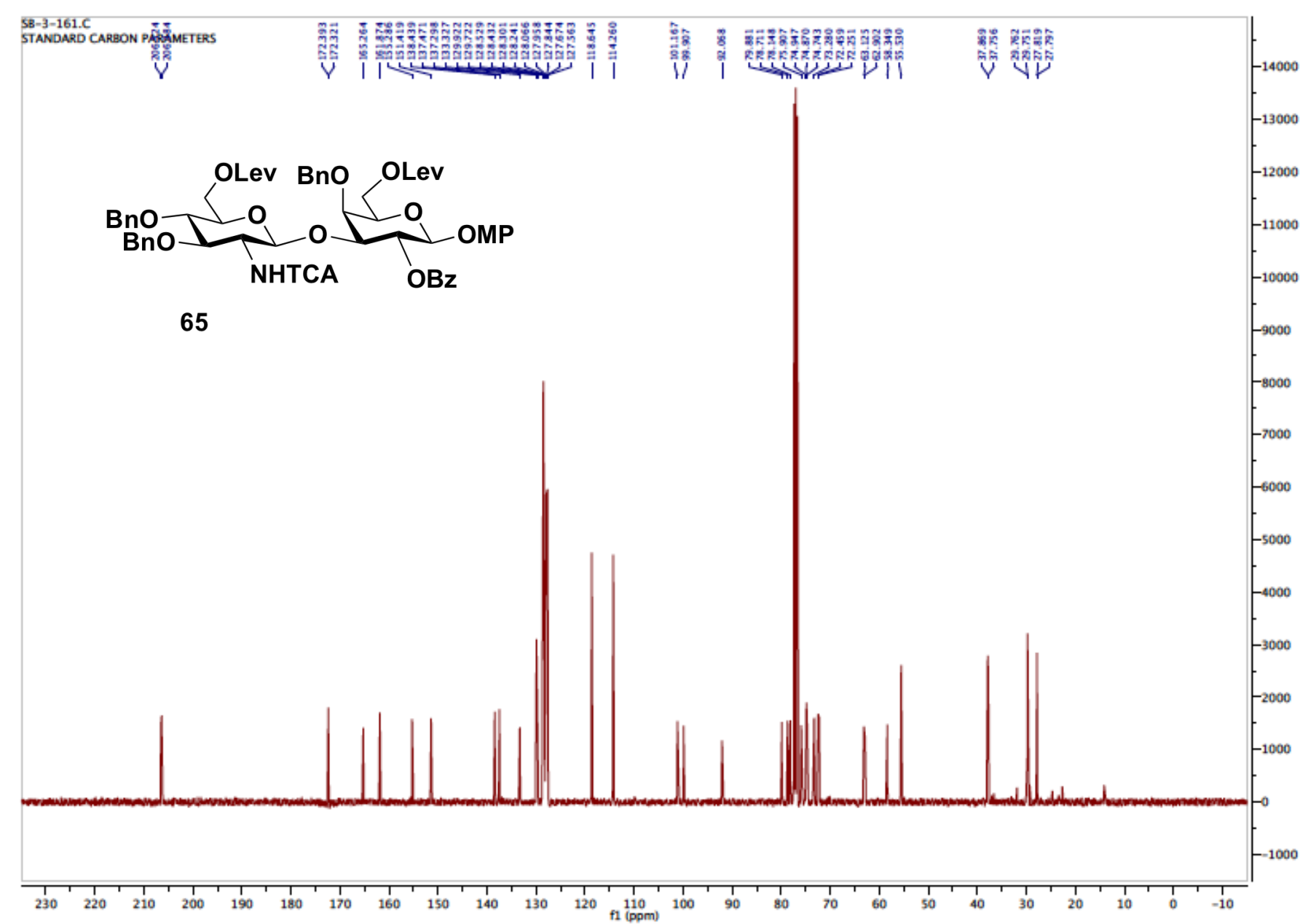

${ }^{13} \mathbf{C}$ NMR (101 MHz) spectrum of compound 65 in $\mathrm{CDCl}_{3}$ 


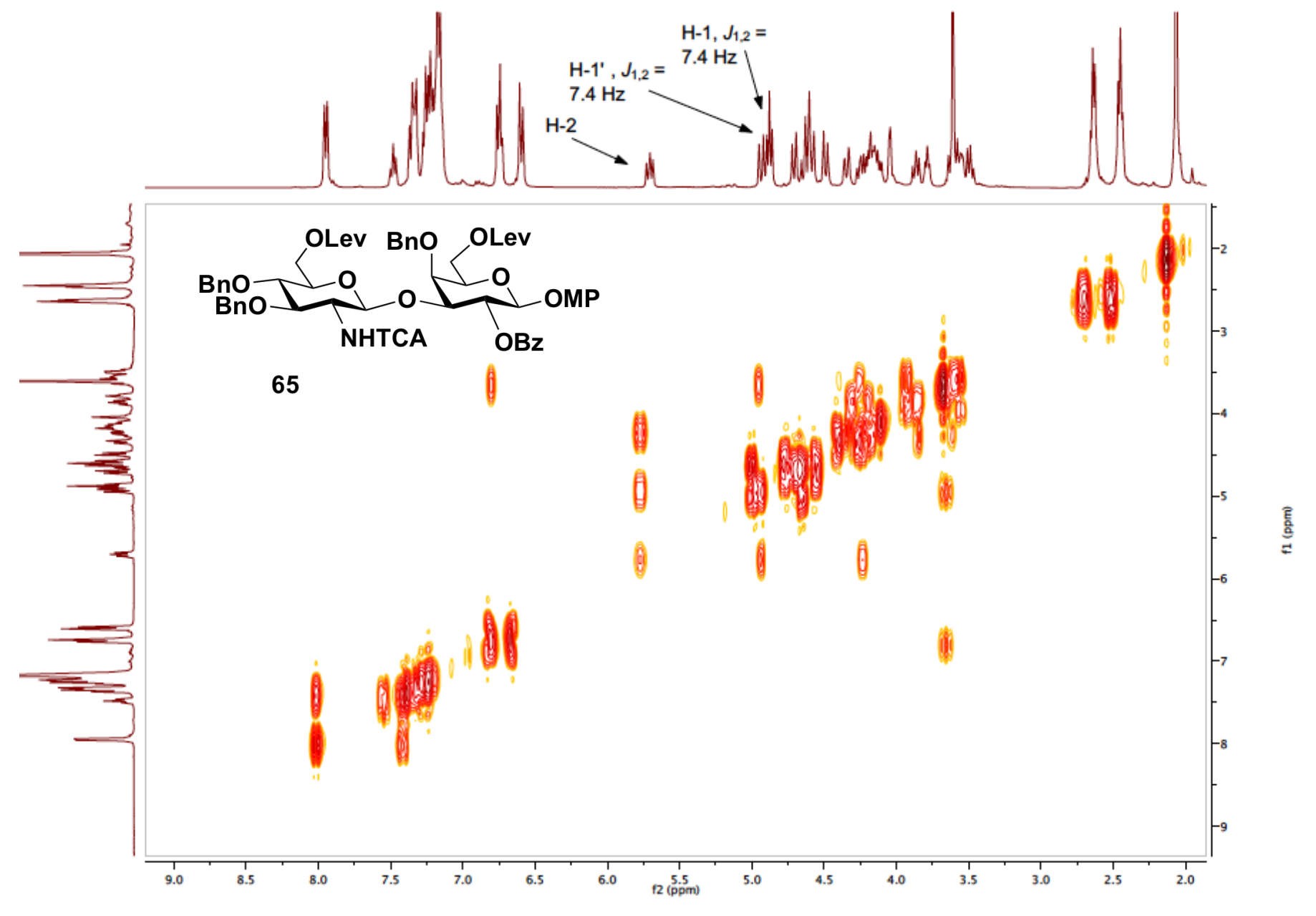

${ }^{1} \mathbf{H}$ - ${ }^{1} \mathbf{H}$ COSY NMR (400 MHz) spectrum of compound 65 in $\mathrm{CDCl}_{3}$ 


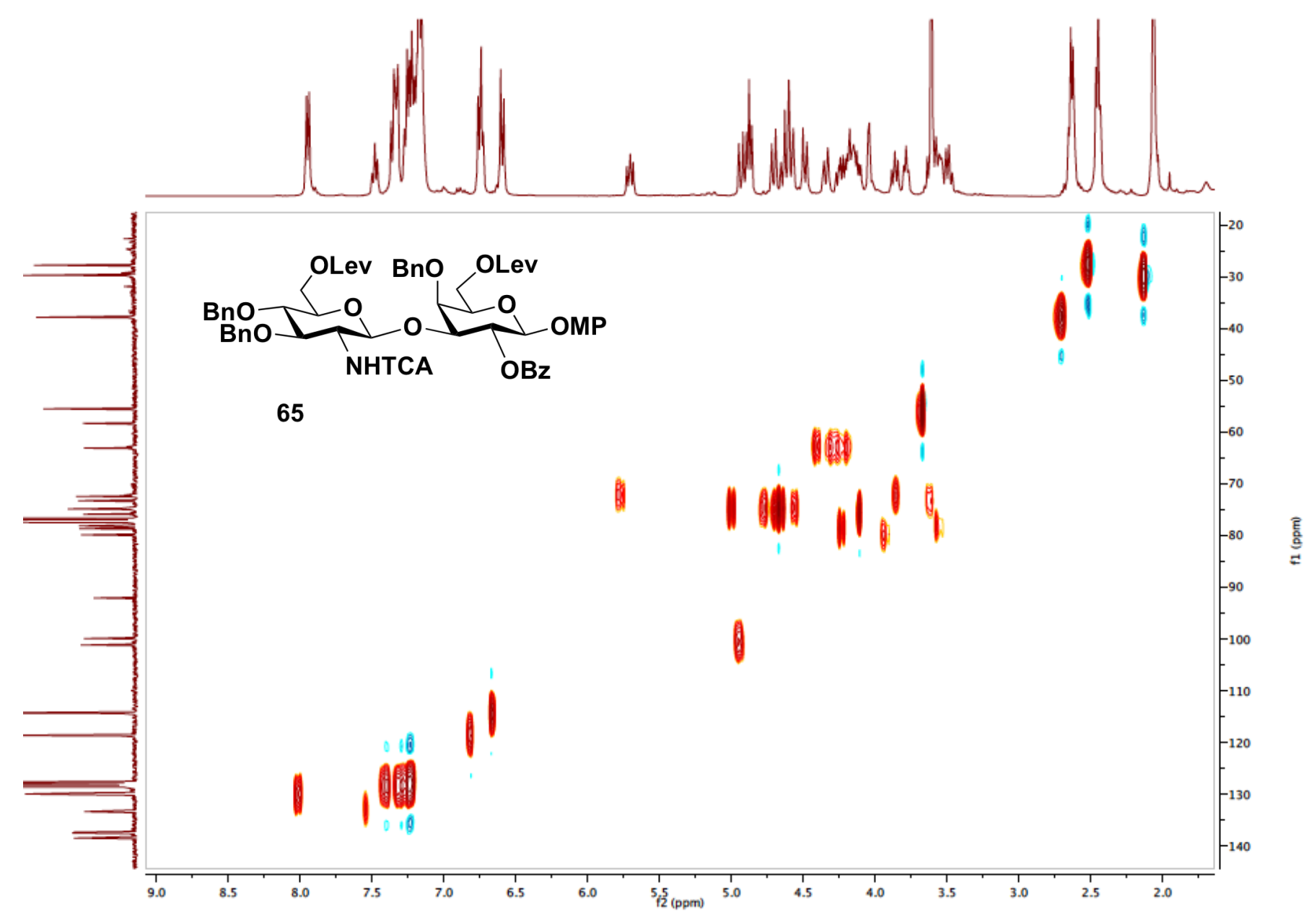

${ }^{1} \mathbf{H}-{ }^{13} \mathbf{C}$ HSQC NMR (400 MHz) spectrum (expanded) of compound 65 in $\mathrm{CDCl}_{3}$ 


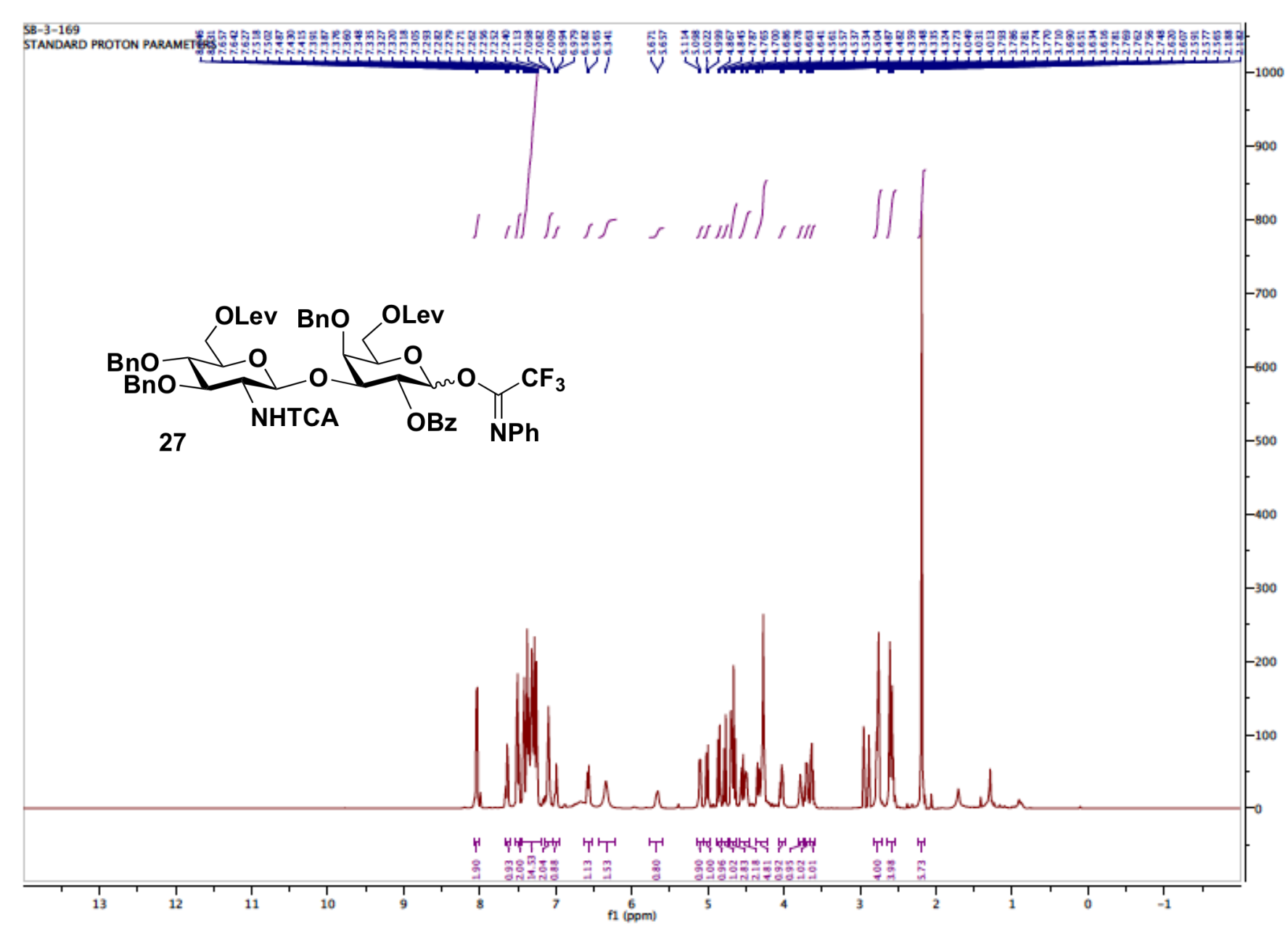

${ }^{1} \mathbf{H}$ NMR (400 MHz) spectrum of compound 27 in $\mathrm{CDCl}_{3}$ 


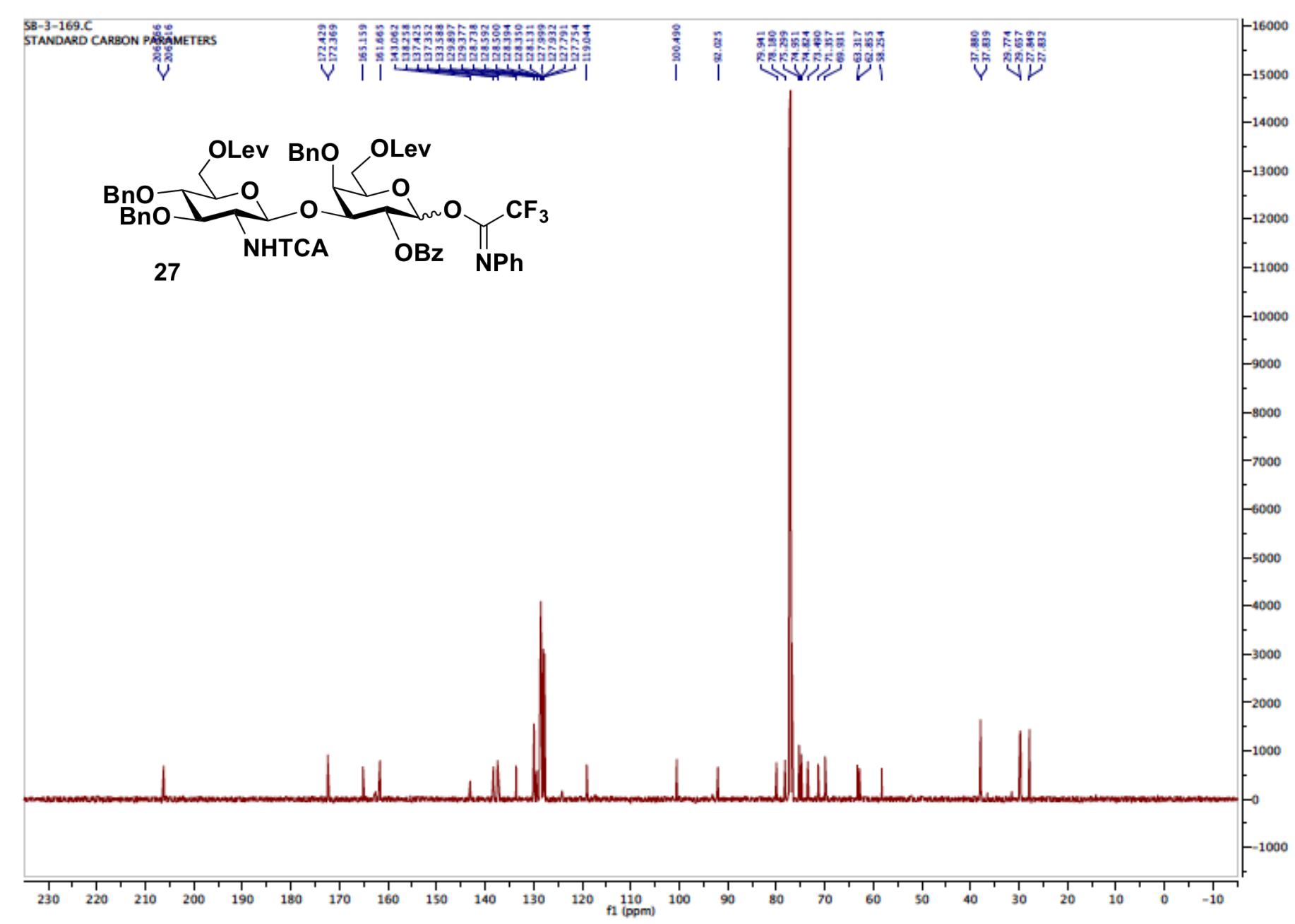

${ }^{13} \mathbf{C}$ NMR (101 MHz) spectrum of compound 27 in $\mathrm{CDCl}_{3}$ 


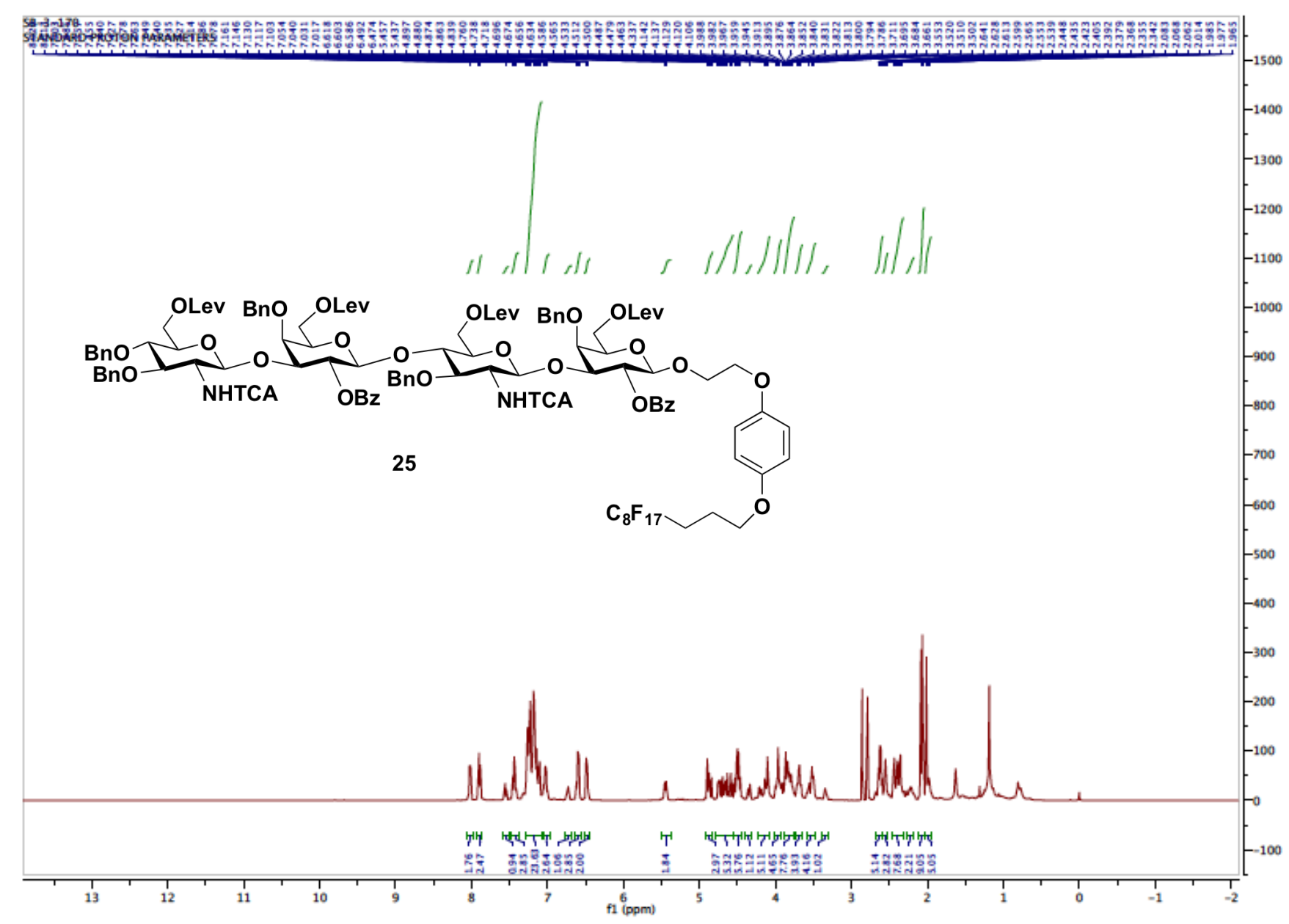

${ }^{1} \mathbf{H}$ NMR (500 MHz) spectrum of compound 25 in $\mathrm{CDCl}_{3}$ 


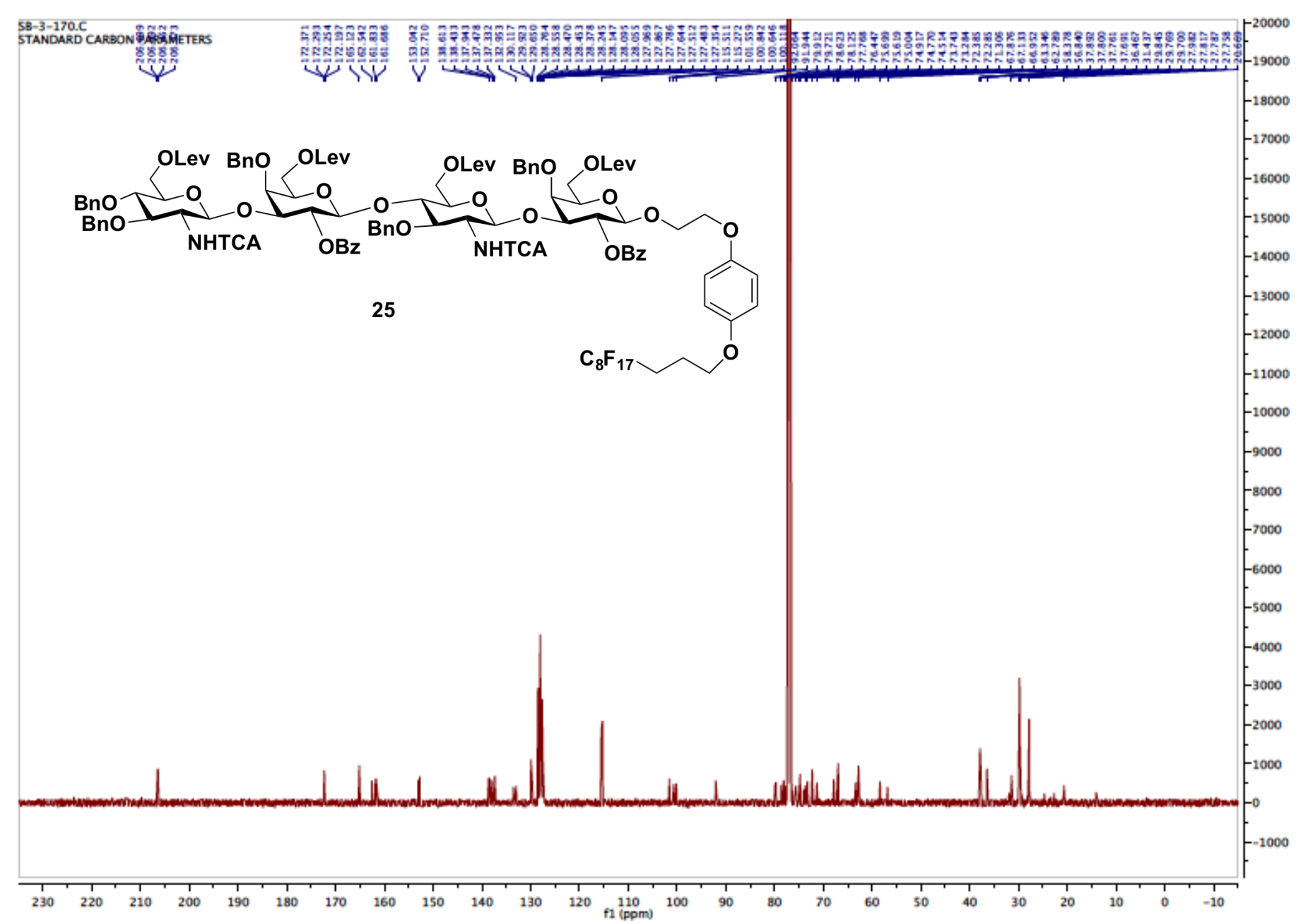

${ }^{13} \mathbf{C}$ NMR (126 MHz) spectrum of compound 25 in $\mathrm{CDCl}_{3}$ 


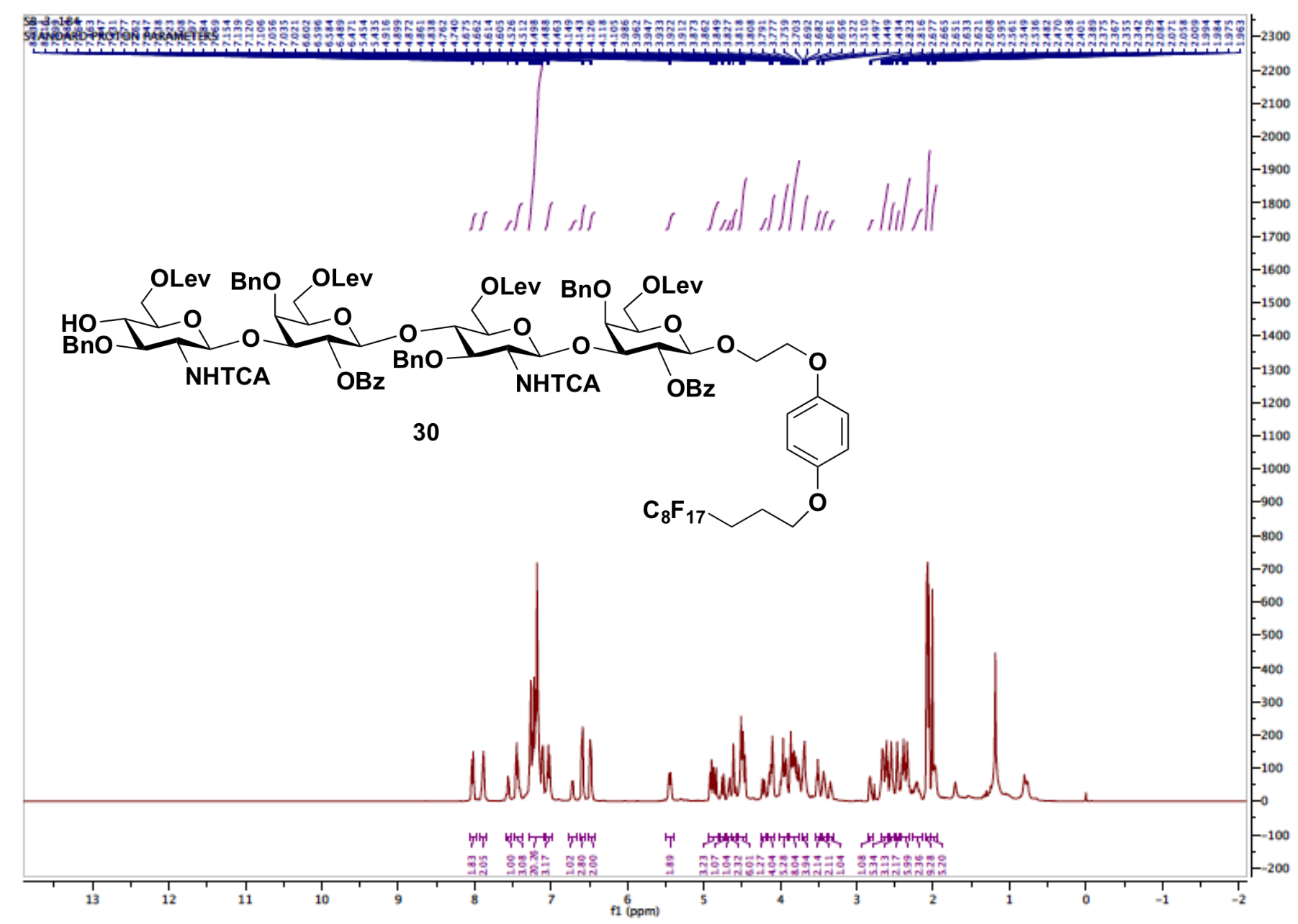

${ }^{1} \mathbf{H}$ NMR (500 MHz) spectrum of compound 30 in $\mathrm{CDCl}_{3}$ 


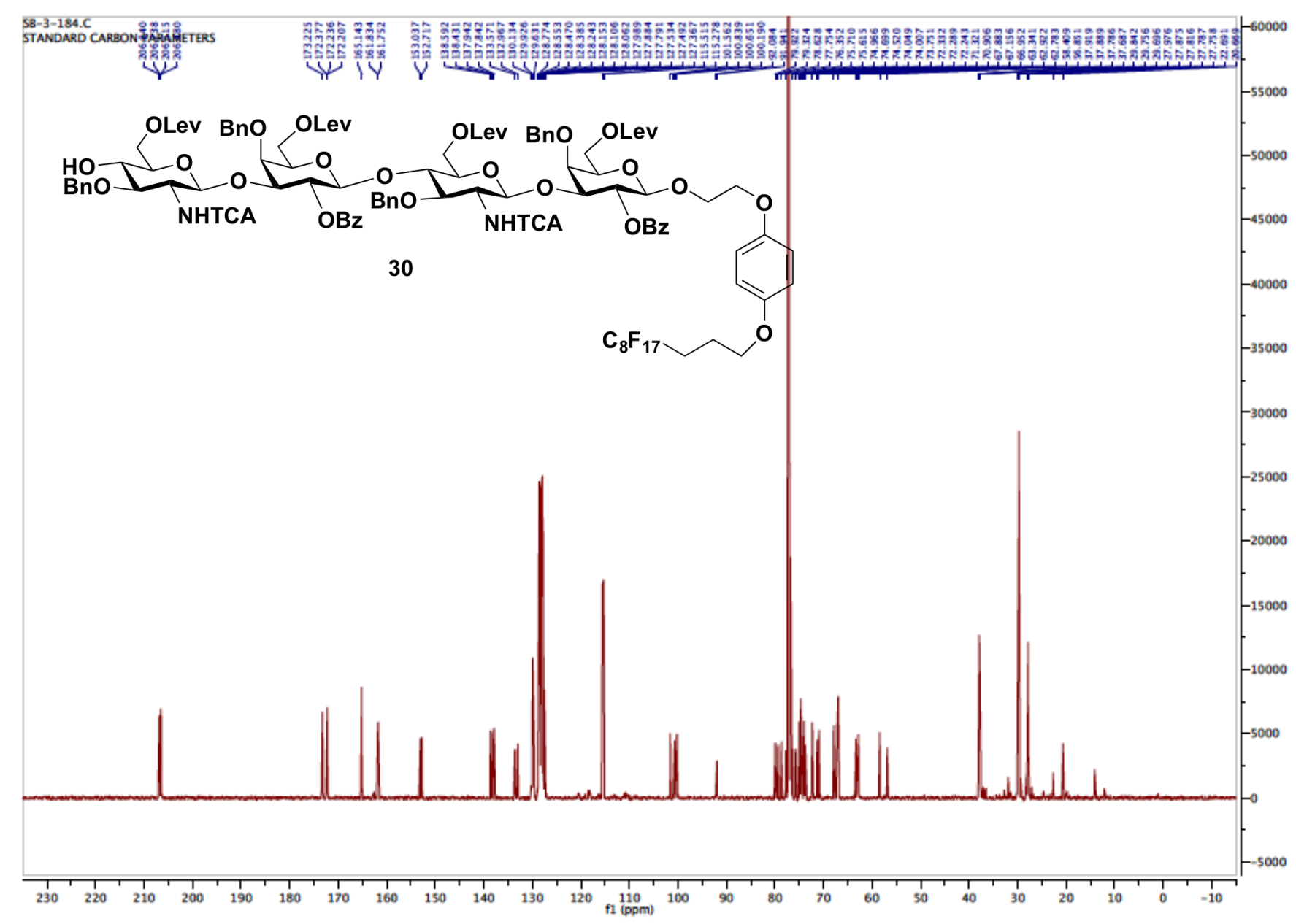

${ }^{13} \mathbf{C}$ NMR (126 MHz) spectrum of compound 30 in $\mathrm{CDCl}_{3}$ 


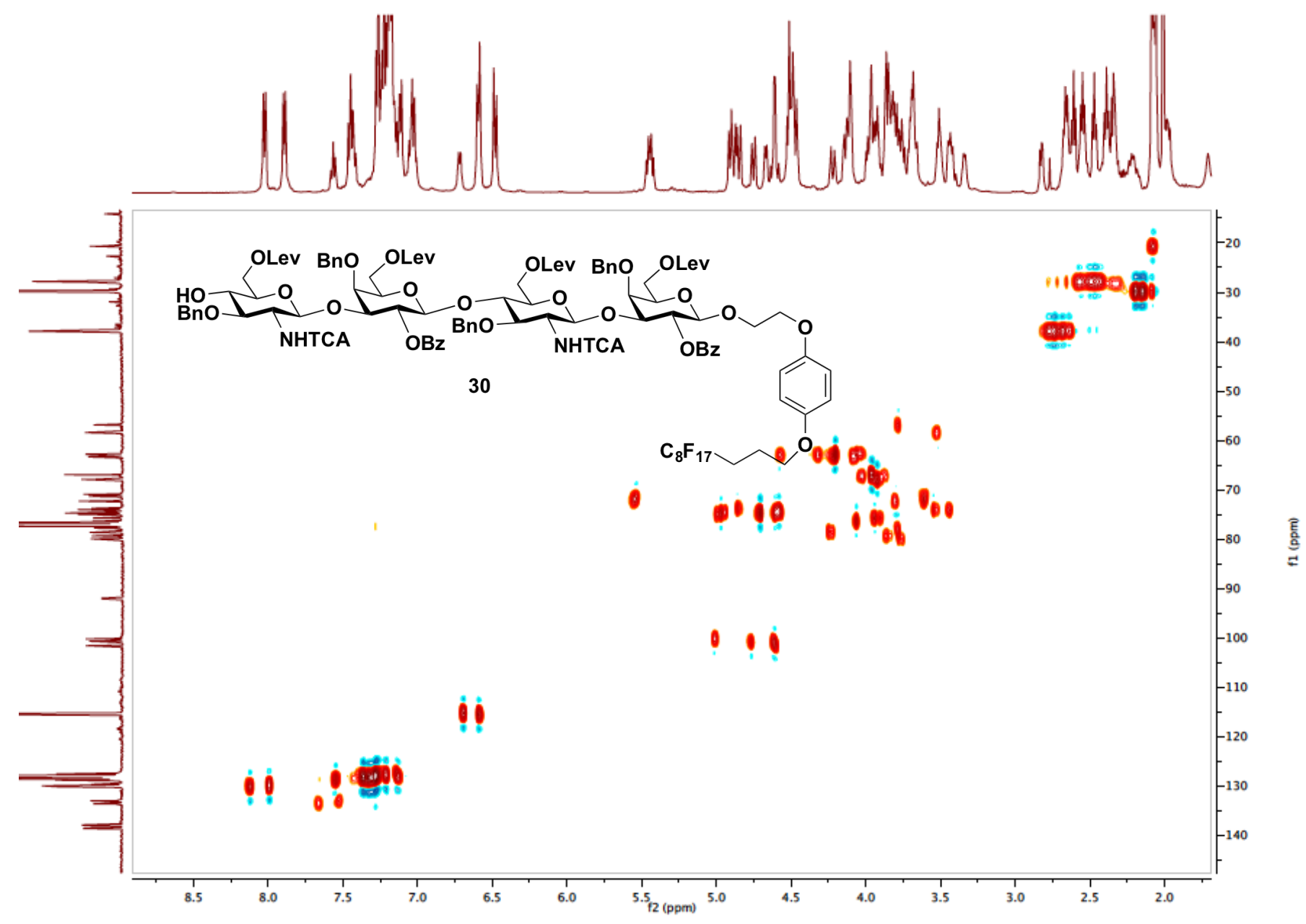

${ }^{1} \mathbf{H}-{ }^{13} \mathbf{C}$ HSQC NMR (500 MHz) spectrum of compound 30 in $\mathrm{CDCl}_{3}$ 


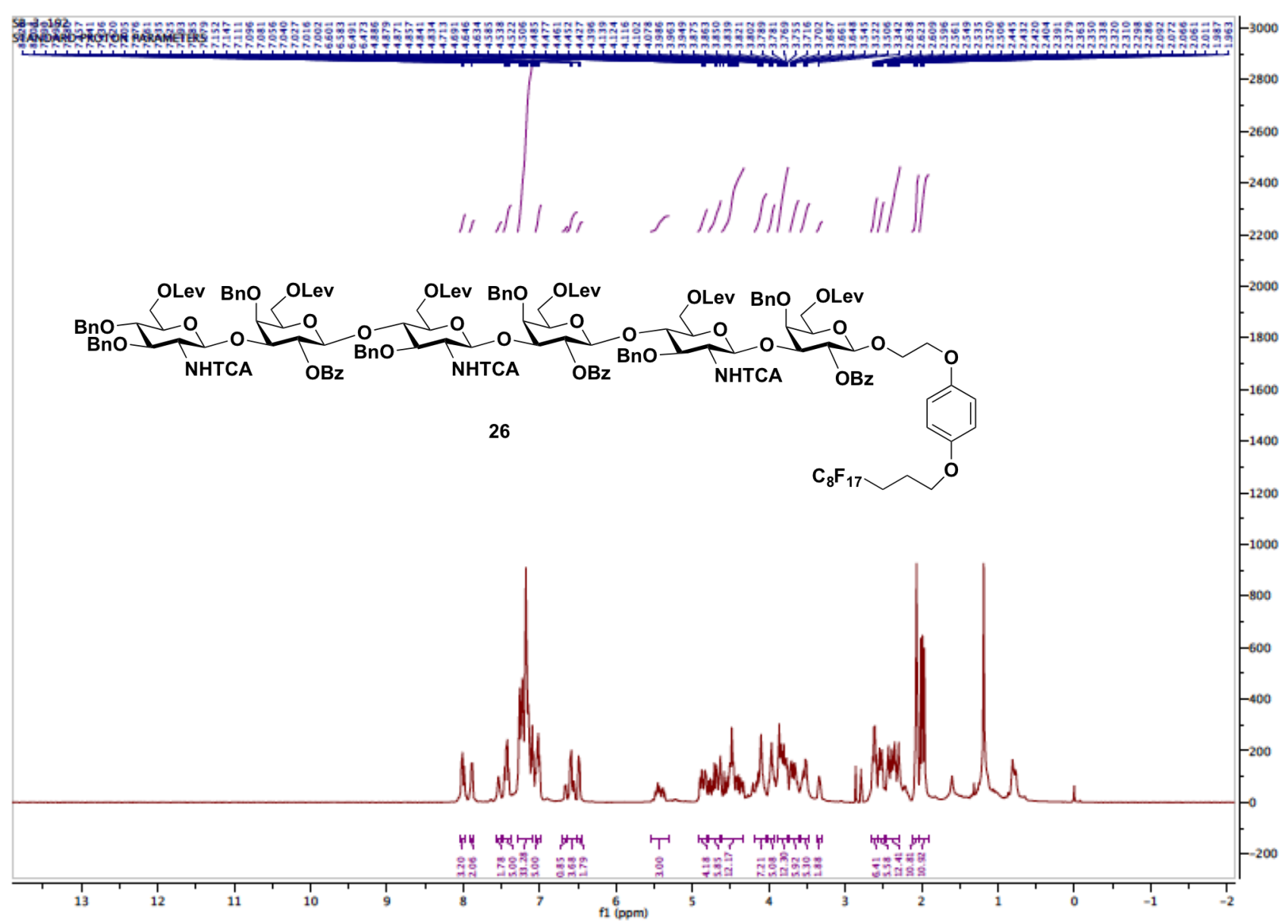

${ }^{1} \mathbf{H}$ NMR (500 MHz) spectrum of compound 26 in $\mathrm{CDCl}_{3}$ 


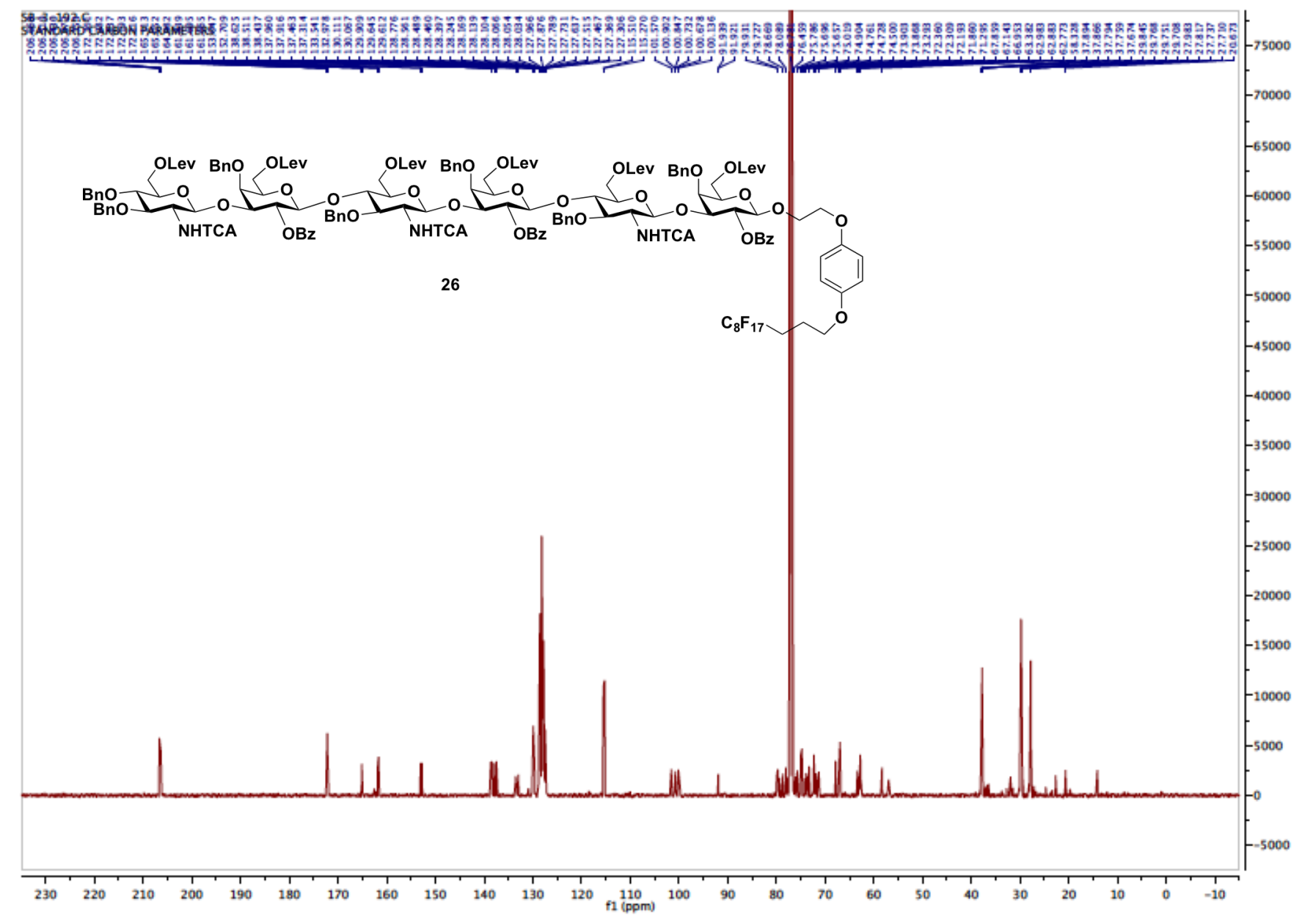

${ }^{13} \mathbf{C}$ NMR (126 MHz) spectrum of compound 26 in $\mathrm{CDCl}_{3}$ 


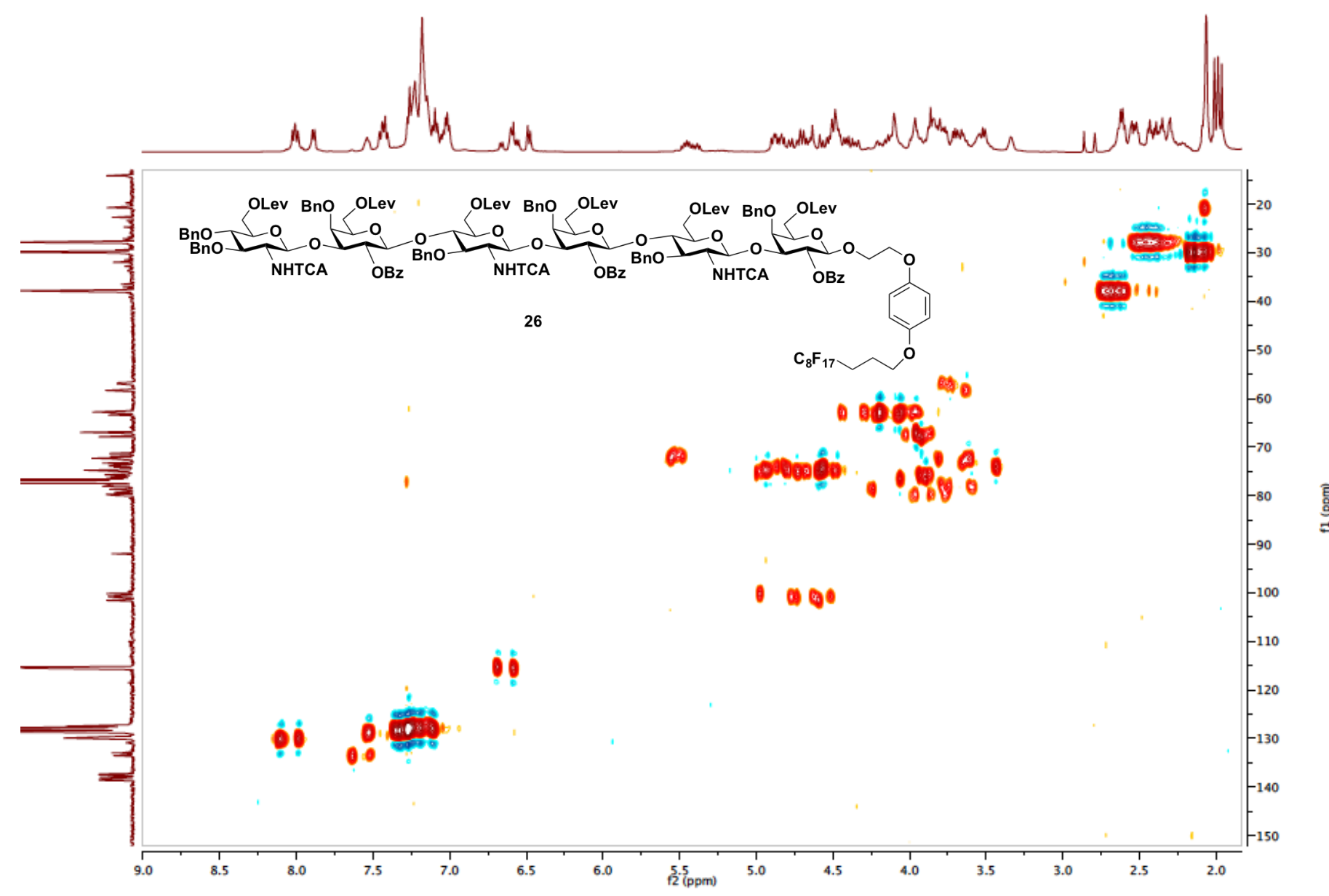

${ }^{1} \mathbf{H}-{ }^{13} \mathbf{C}$ HSQC NMR (500 MHz) spectrum of compound 26 in $\mathrm{CDCl}_{3}$ 


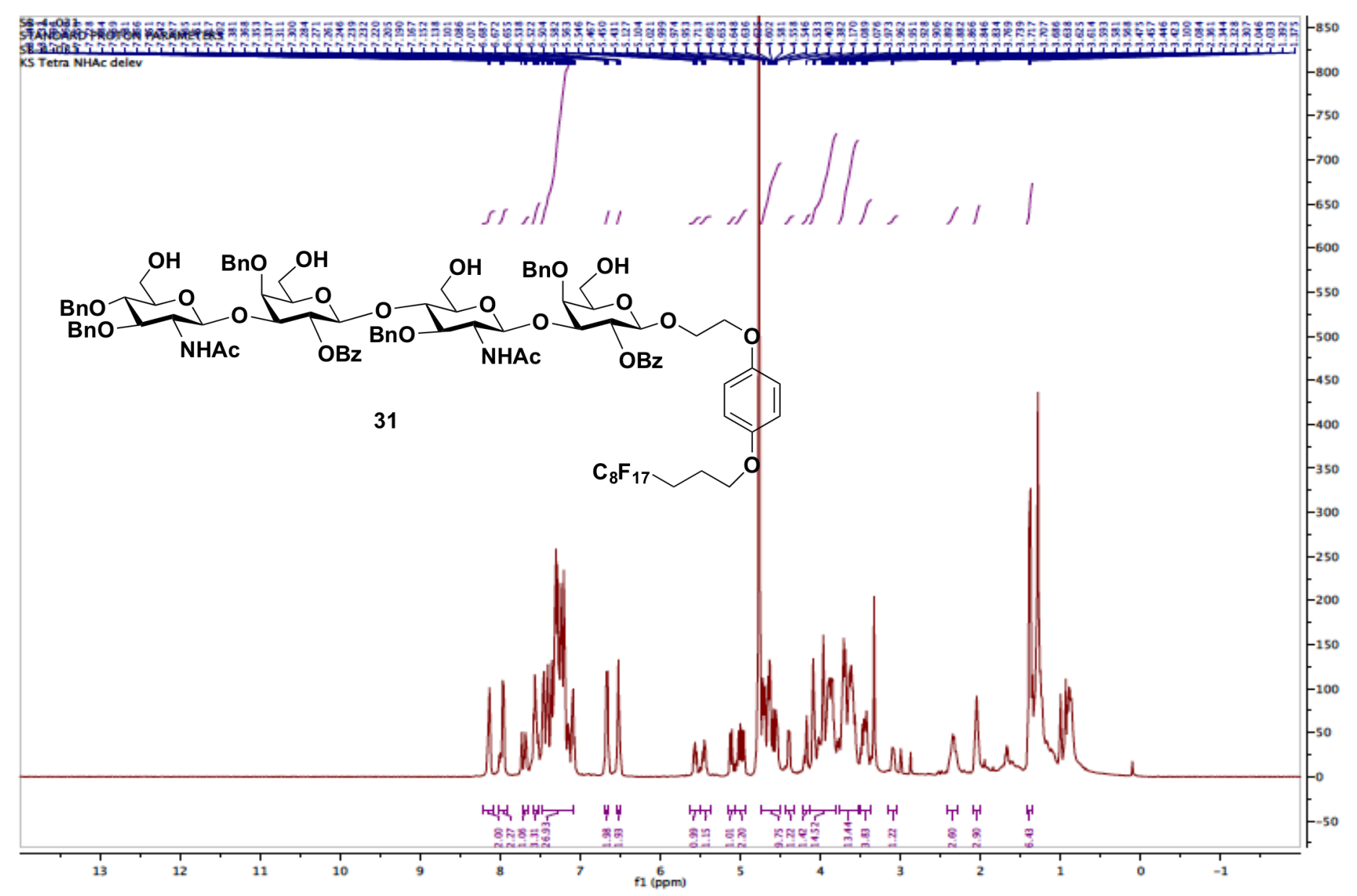

${ }^{1} \mathbf{H}$ NMR (500 MHz) spectrum of compound 31 in $\mathrm{CD}_{3} \mathrm{OD}$ 


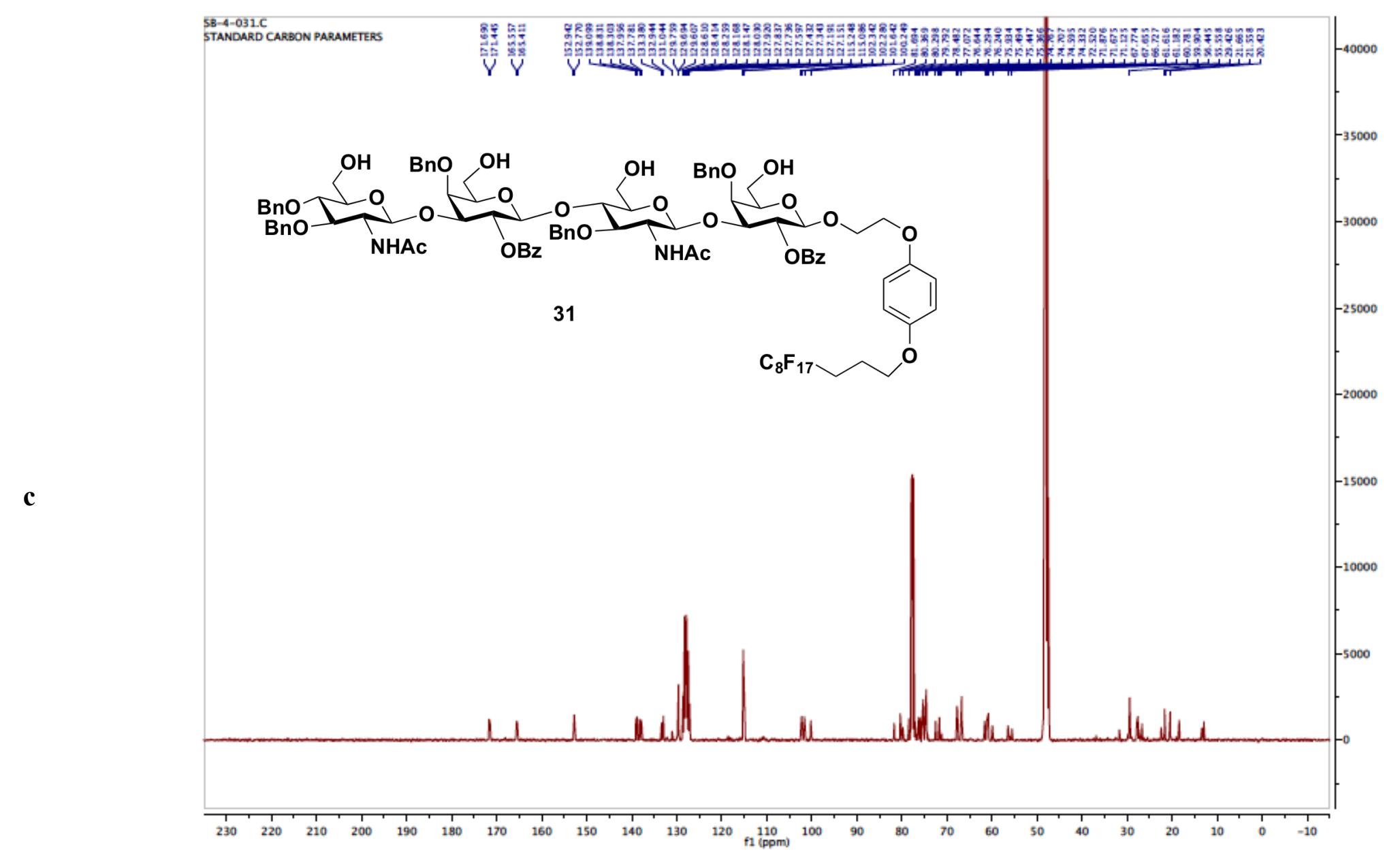

${ }^{13} \mathbf{C}$ NMR (126 MHz) spectrum of compound 31 in $\mathrm{CD}_{3} \mathrm{OD}$ 


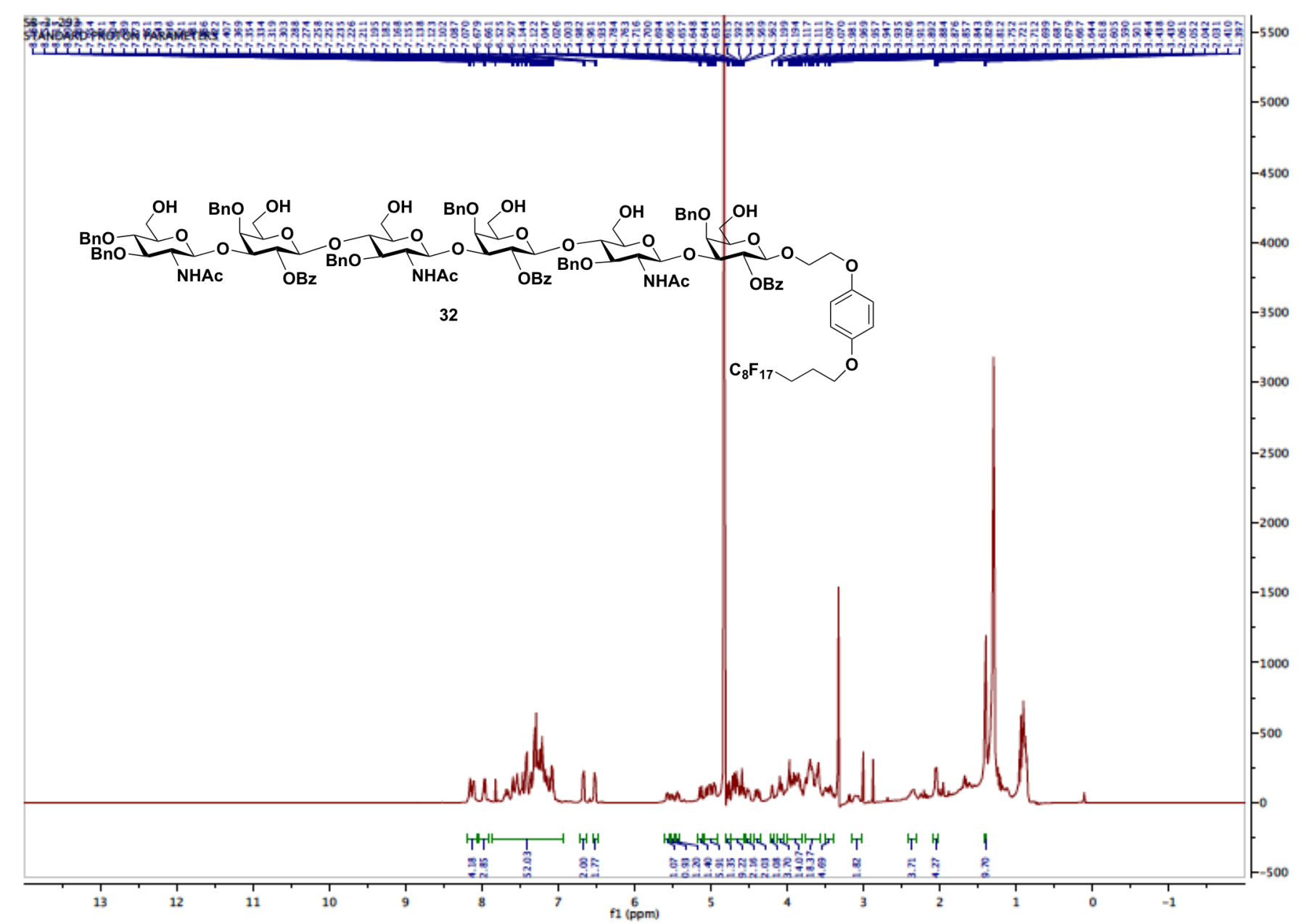

${ }^{1} \mathbf{H}$ NMR (500 MHz) spectrum of compound 32 in $\mathrm{CD}_{3} \mathrm{OD}$ 


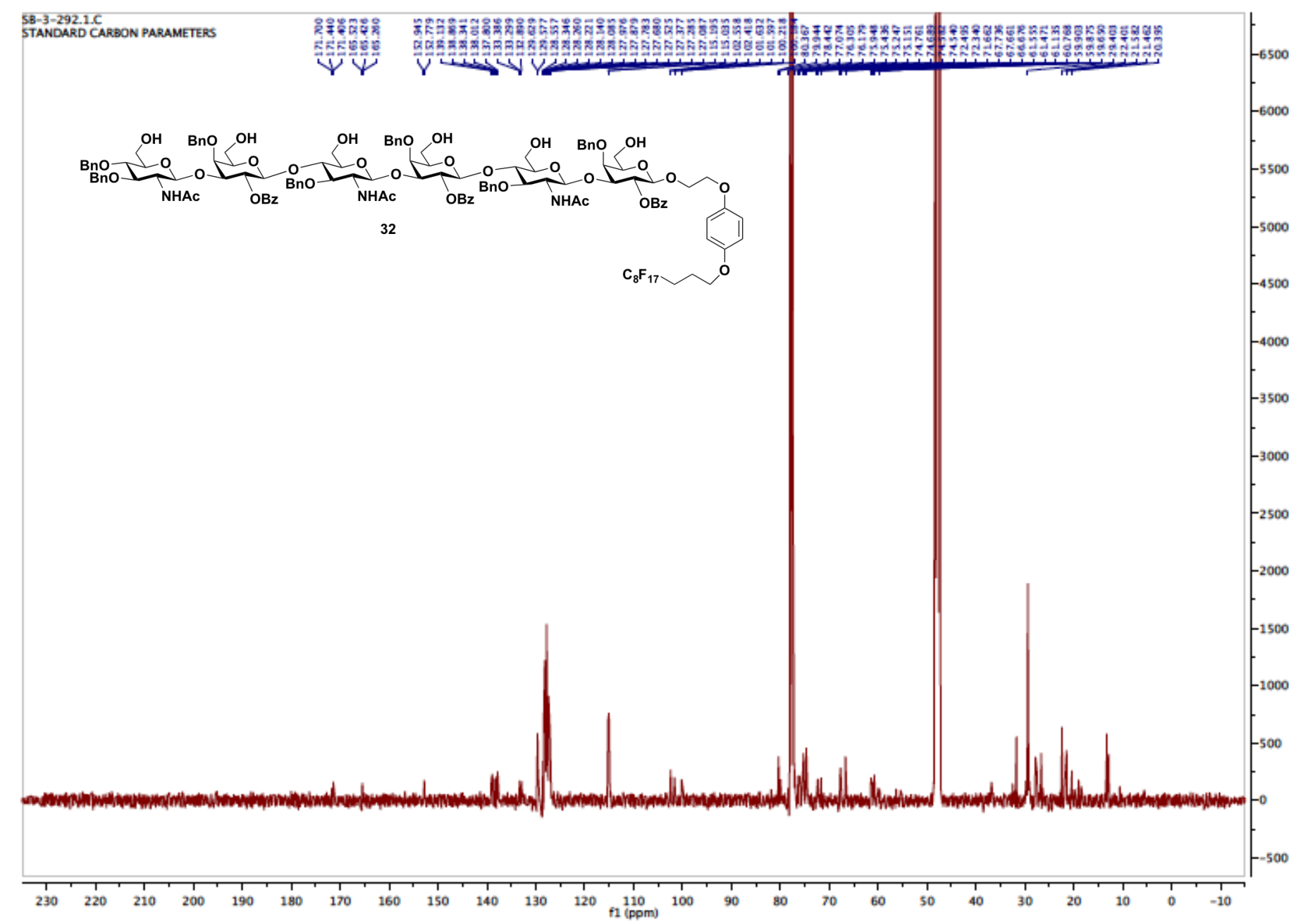

${ }^{13} \mathbf{C}$ NMR (126 MHz) spectrum of compound 32 in $\mathrm{CD}_{3} \mathrm{OD}$ 


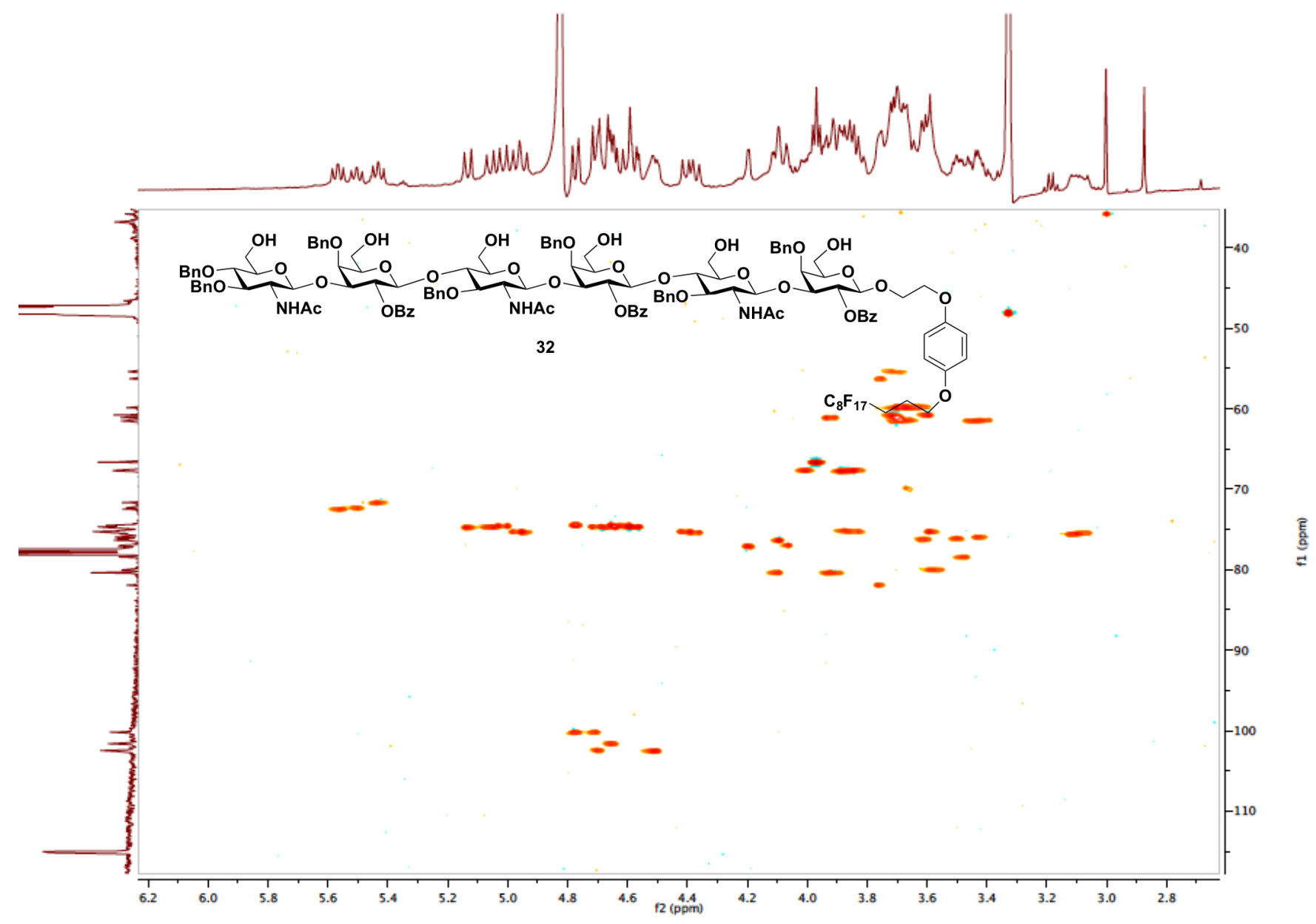

${ }^{1} \mathbf{H}-{ }^{13} \mathbf{C}$ HSQC NMR (500 MHz) spectrum of compound 32 in $\mathrm{CD}_{3} \mathrm{OD}$ 


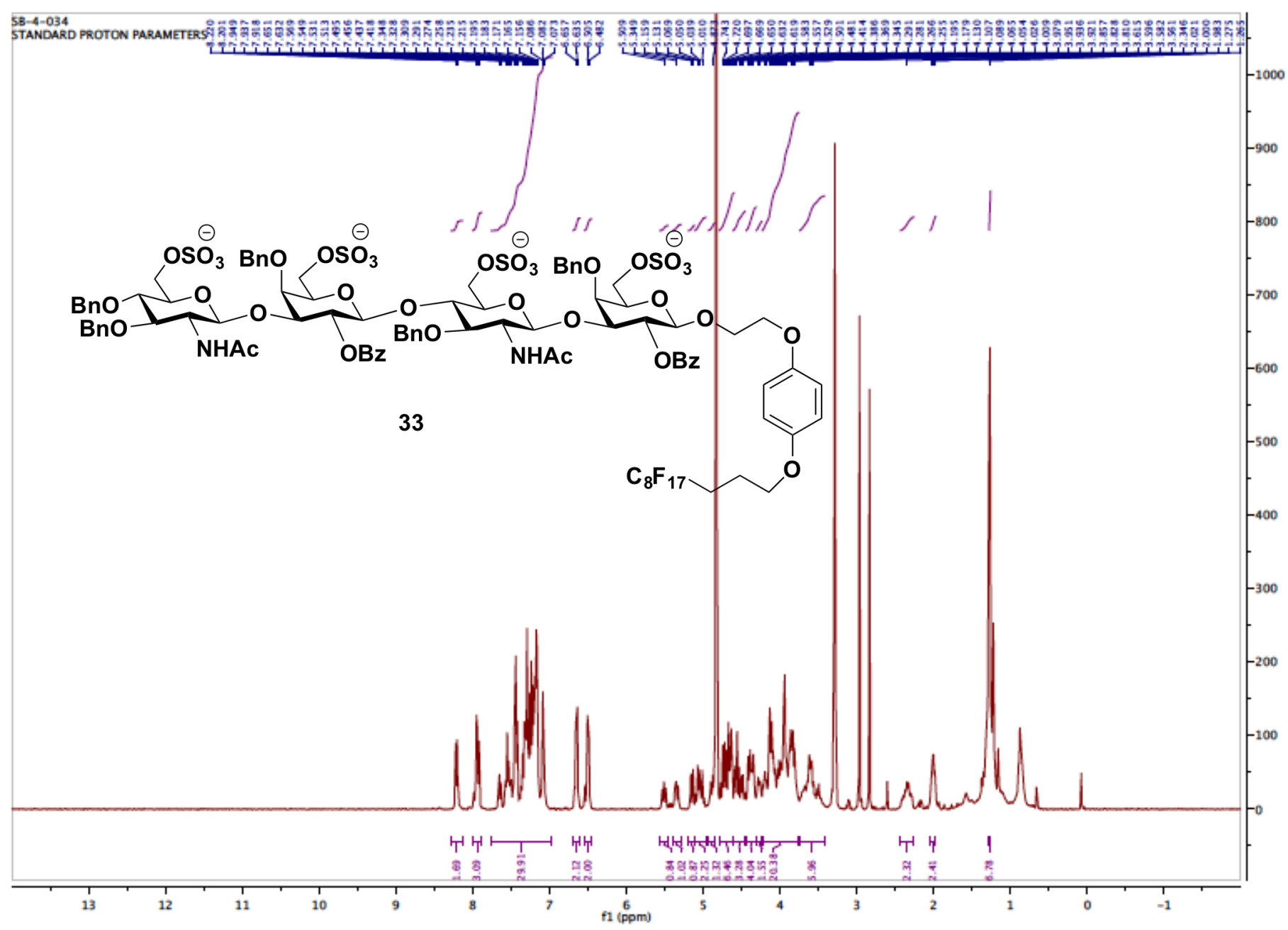

${ }^{1} \mathbf{H}$ NMR (500 MHz) spectrum of compound 33 in $\mathrm{CD}_{3} \mathrm{OD}$ 


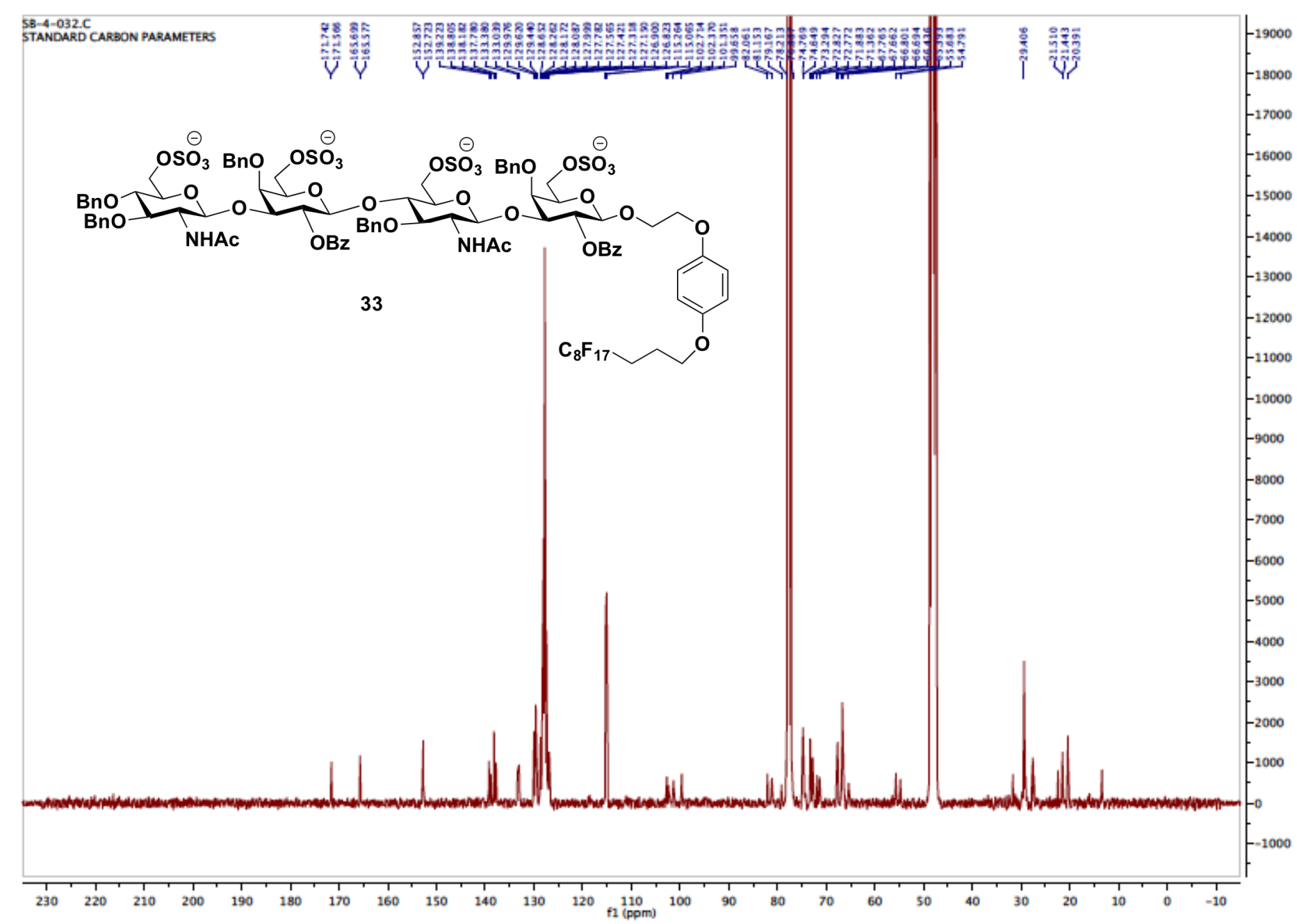

${ }^{13} \mathbf{C}$ NMR (126 MHz) spectrum of compound 33 in $\mathrm{CD}_{3} \mathrm{OD}$ 


\section{High-resolution Mass Spectrometry Data (HRMS)}

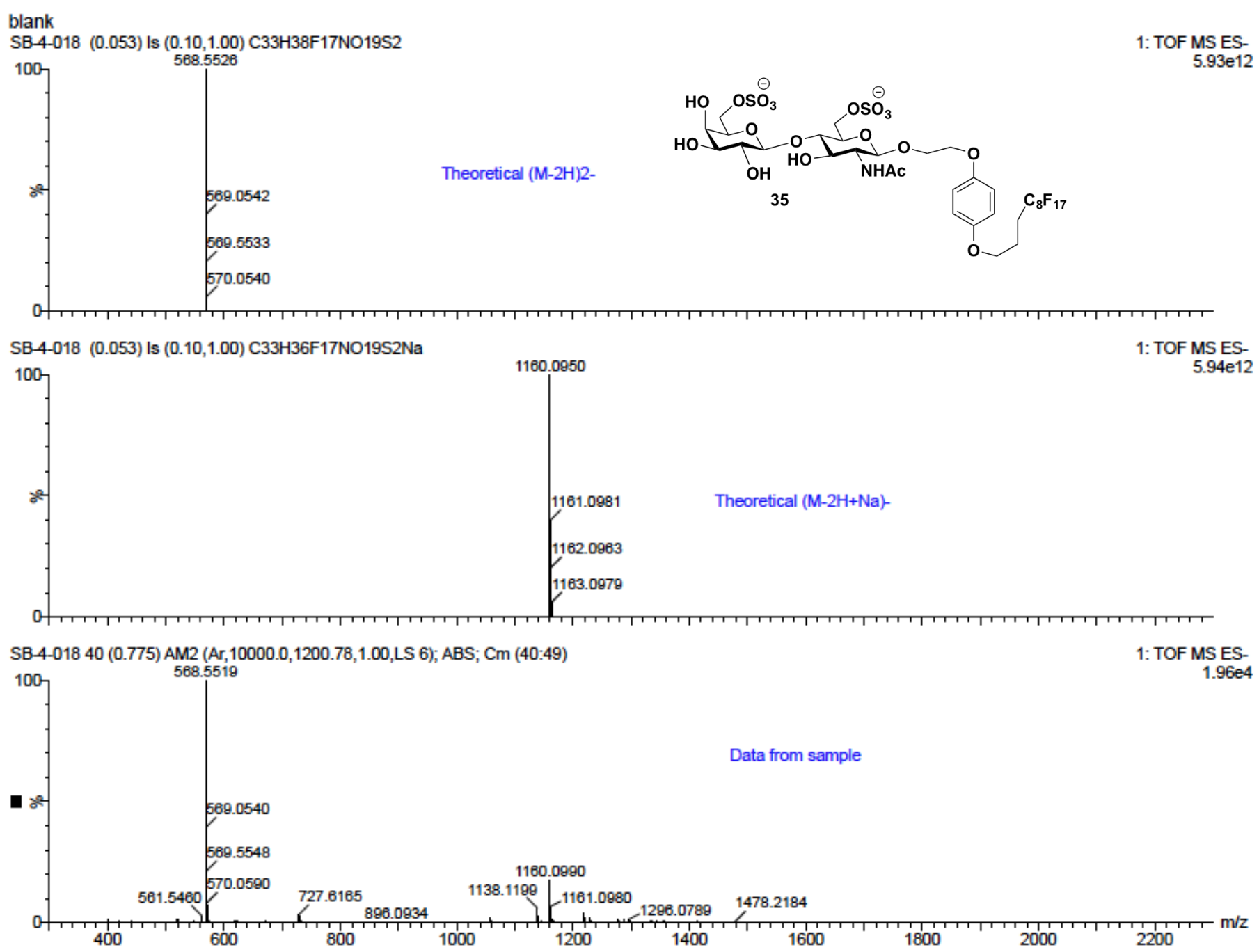

HRMS-ESI-TOF (m/z) spectrum of compound 35 


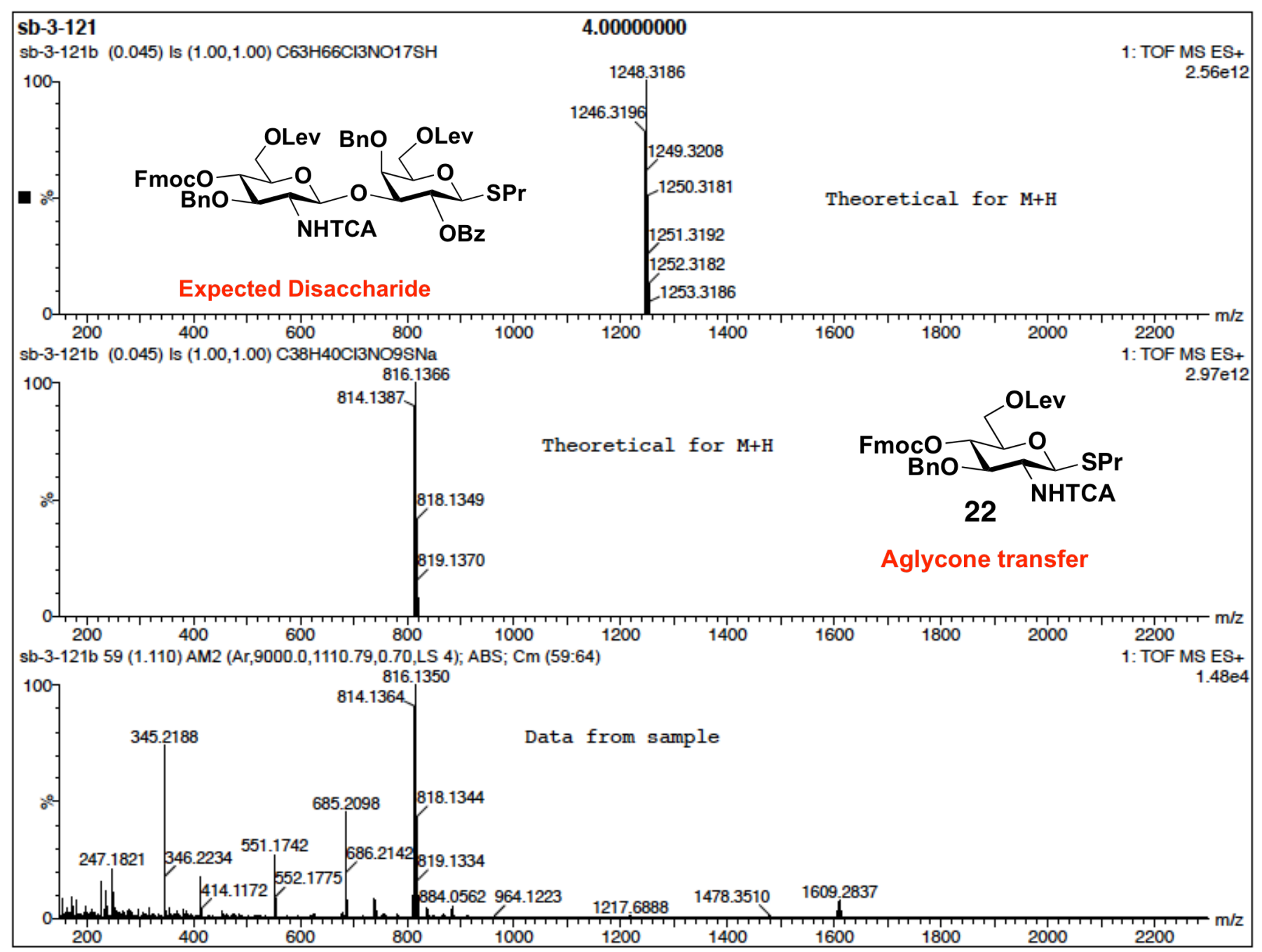

HRMS-ESI-TOF (m/z) spectrum of compound 22 
sb-3-196

jtr-vi-dimeothe $(0.036)$ is $(1.00,1.00) \mathrm{C} 161 \mathrm{H} 174 \mathrm{~F} 17 \mathrm{~N} 3 \mathrm{O} 48 \mathrm{Na}$

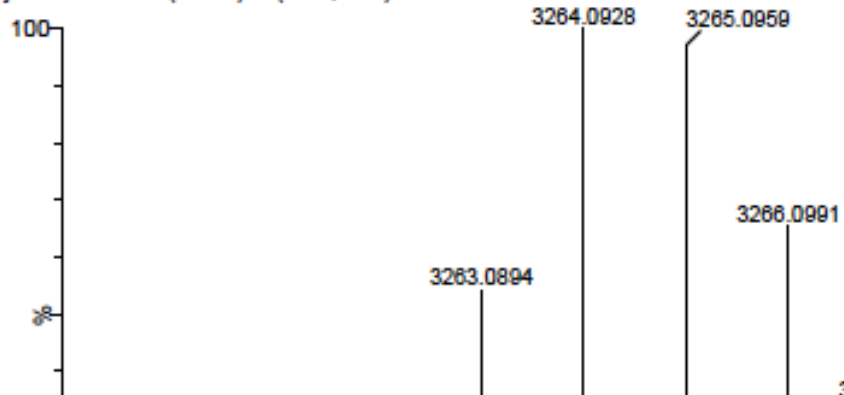

Theoretical for $\mathrm{M}+\mathrm{Na}$

1: TOF MS ES+

2.67e12

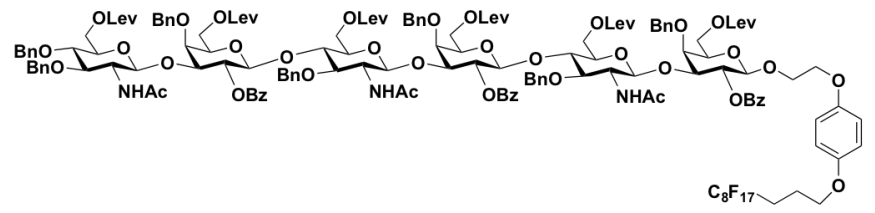

sb-3-196 16 (0.407) AM2 (Ar,9000.0,3286.39,0.70,LS 4); Cm (12:16)

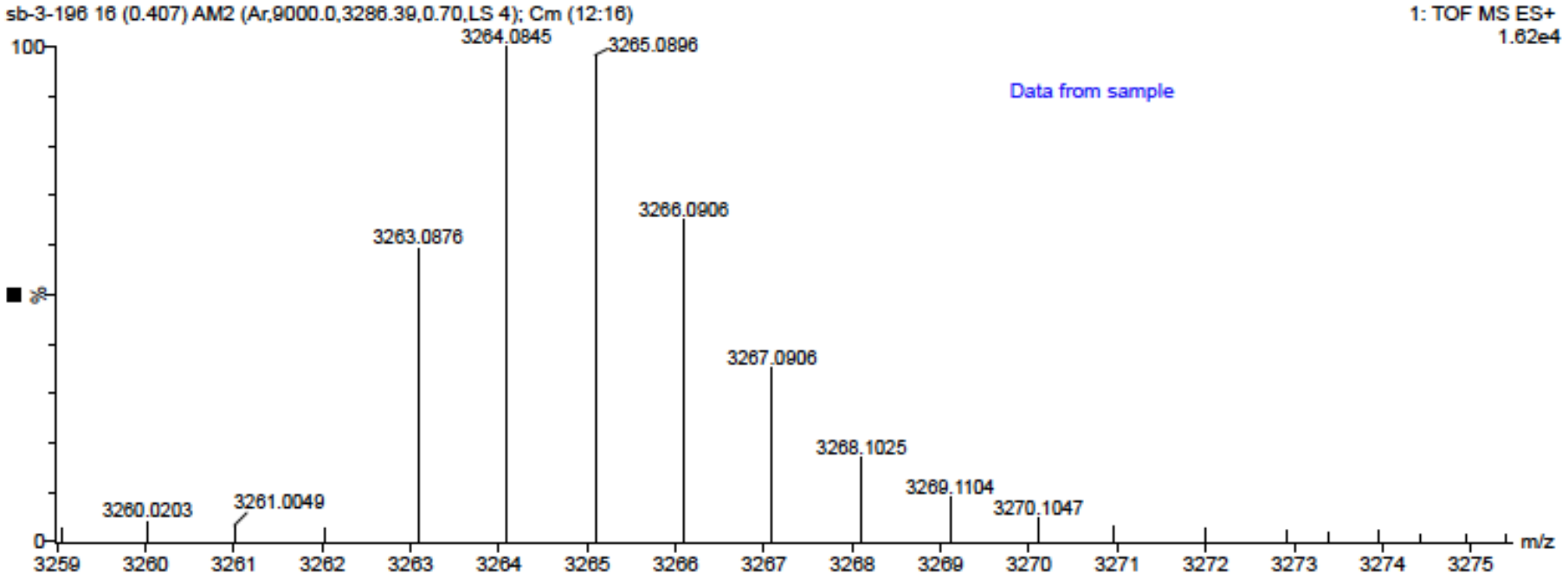

192 


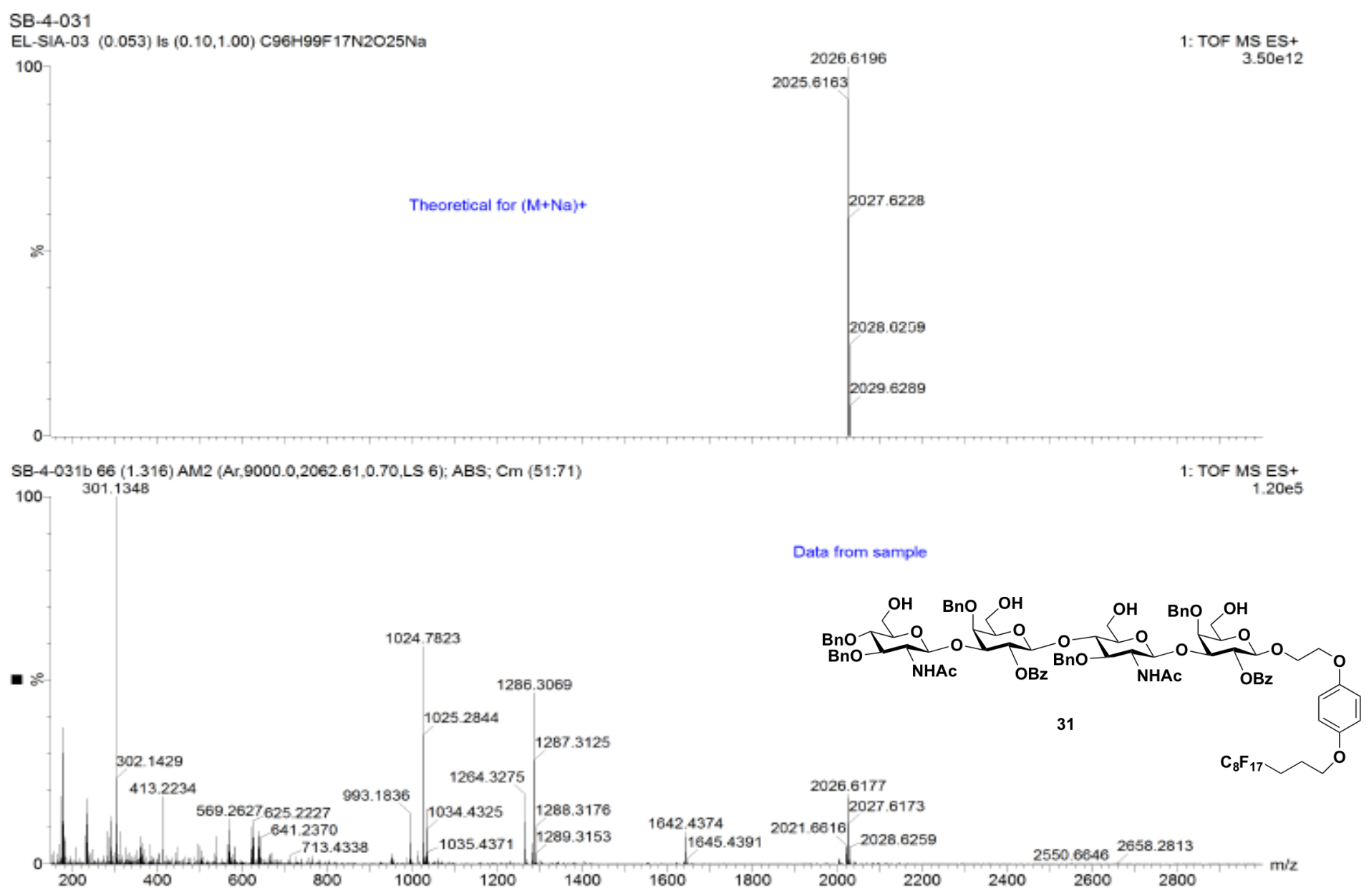

HRMS-ESI-TOF (m/z) spectrum of compound 31 


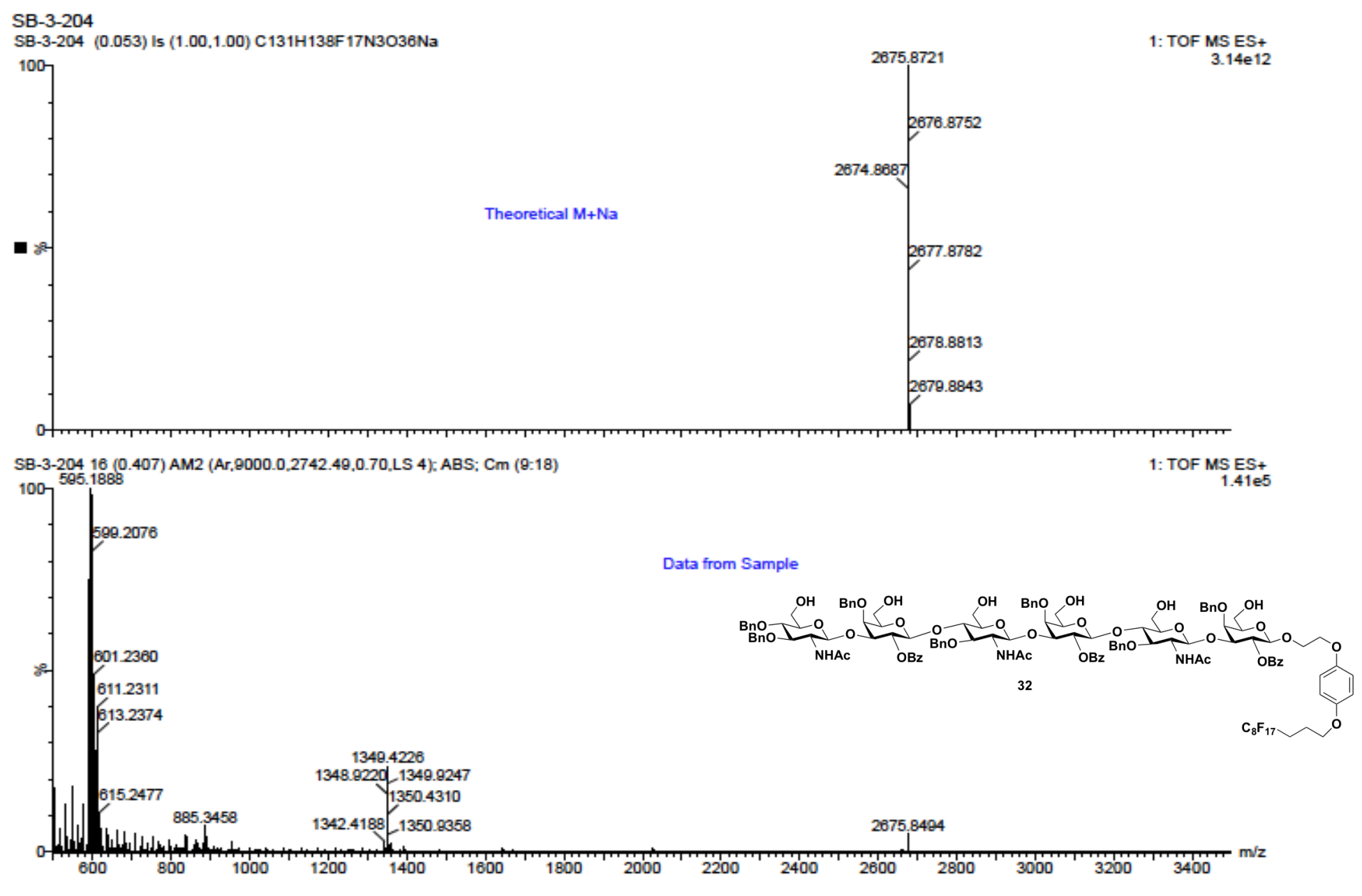

HRMS-ESI-TOF (m/z) spectrum of compound 32 


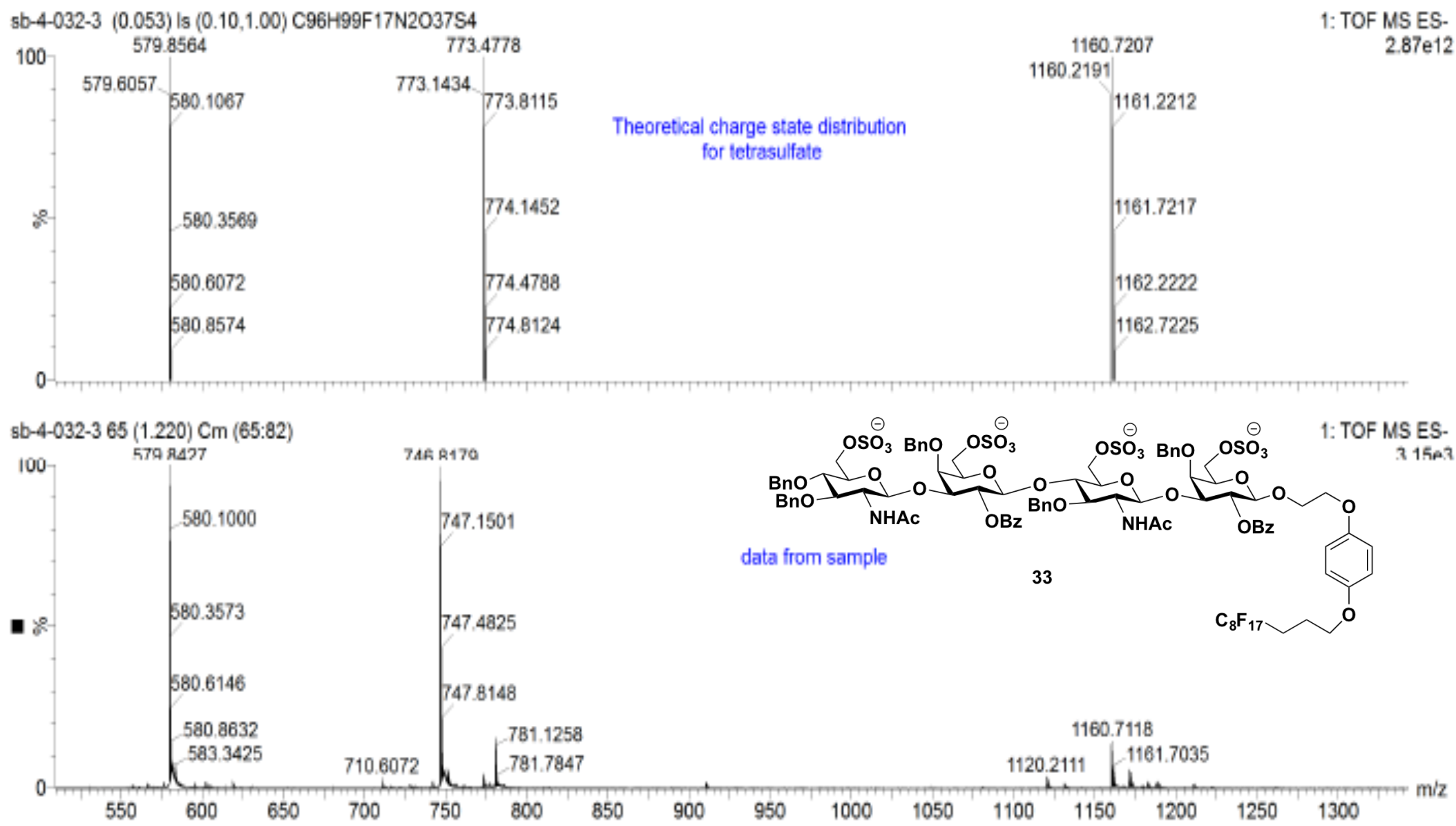

HRMS-ESI-TOF $(\mathrm{m} / \mathbf{z})$ spectrum of compound 33 


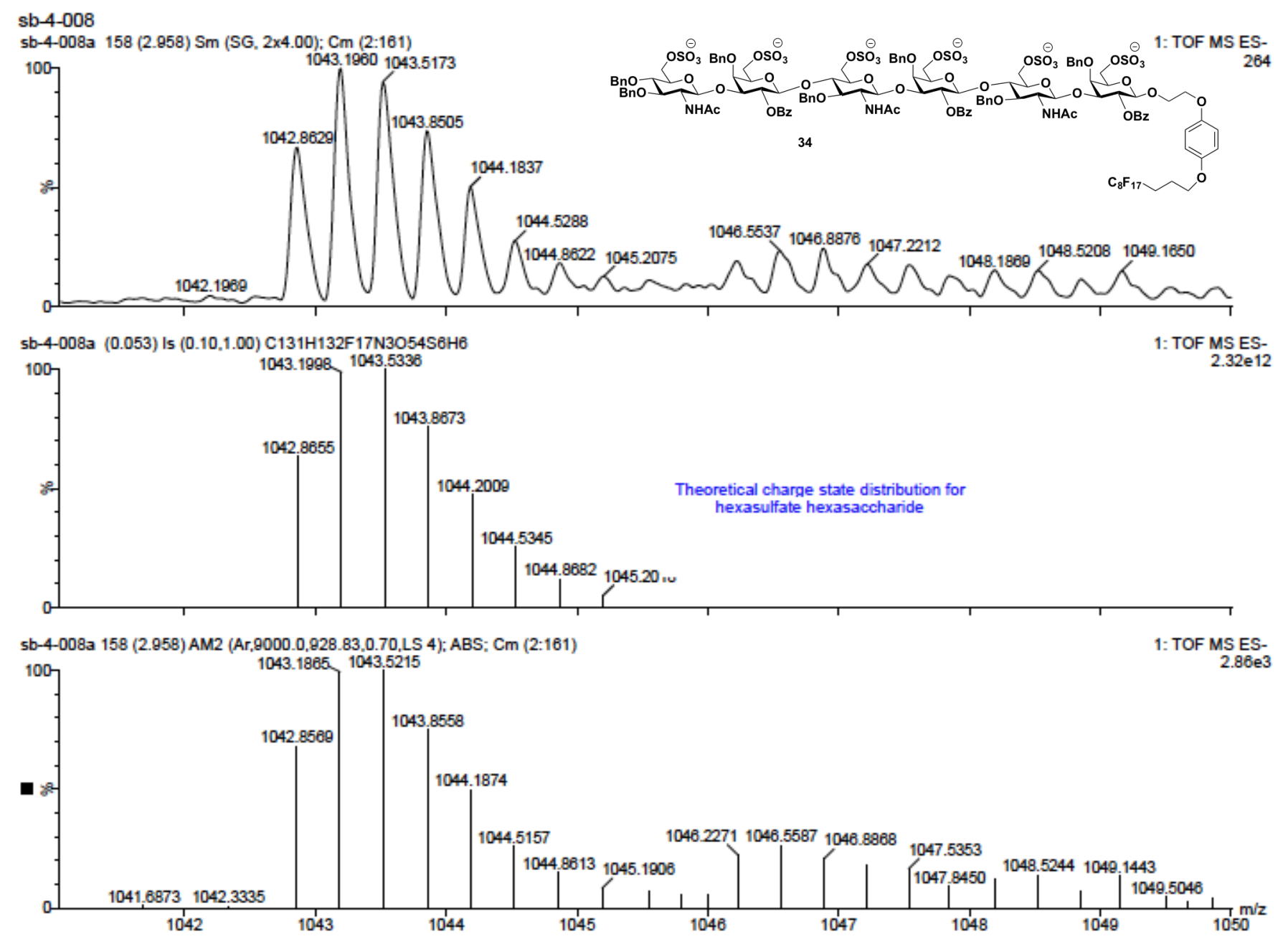

HRMS-ESI-TOF $(\mathrm{m} / \mathbf{z})$ spectrum of compound 34 